*ak FMIS View/Frint Document Cover Sheet tow

This document was retrieved from the Documentation and Records Management (DRM) ISEARCH System. It is intended for Information only and may not be the most recent or updated version. Contact a Document Service Center (see Hanford Info for locations) if you need additional retrieval information.

Accession \#: D195067913

Document \#: SD-WM-ER-521

Title/Desc:

STRUCTRUAL ANALYSIS DESIGN \& EVALUATION OF MOCKUP PLATFORM MONORAIL \& TANK PLATE CUT OUT [SEC 1 OF 3]

Pages: 102 
This document was too large to scan as a whole document, therefore it required breaking into smaller sections.

DOCUMENT NUMBER: $5 D-\omega m-E P-521$

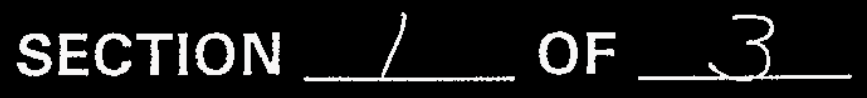

TITLE: STructural Analysis Desigh í ElaluaTion of mackup plat form monorail t Tant PLaTe CuT-04T

DATE: $12-19-95$

ORIGINATOR: I S. Hundal

CO:

RECIPIENT:

CO:

REFERENCES: 


\begin{tabular}{|l|l|}
\hline $\begin{array}{l}\text { 2. To: (Receiving Organization) } \\
\text { Integrity Assessment }\end{array}$ & $\begin{array}{l}\text { 3. From: coriginating organization) } \\
\text { Integrity Assessment }\end{array}$ \\
\hline 5. Proj./Prog./Dept./Div.: & 6. Cog. Engr.: \\
DST Robotic UT Inspection & T. S. Hundal (373-4438) \\
\hline
\end{tabular}

8. Originator Remarks:

For review and approva]

11. Receiver Renarks:
4. Related EDT No.:

N/A

7. Purchase Order No.:

N/A

9. Equip./Component No.:

$N / A$

10. System/Bldg./Facility: 200 Area Tank Farms

12. Hajor Assm. Dwg. Ho.:

H-3-306027

13. Permit/Permit Application No.: N/A

14. Required Response Date:

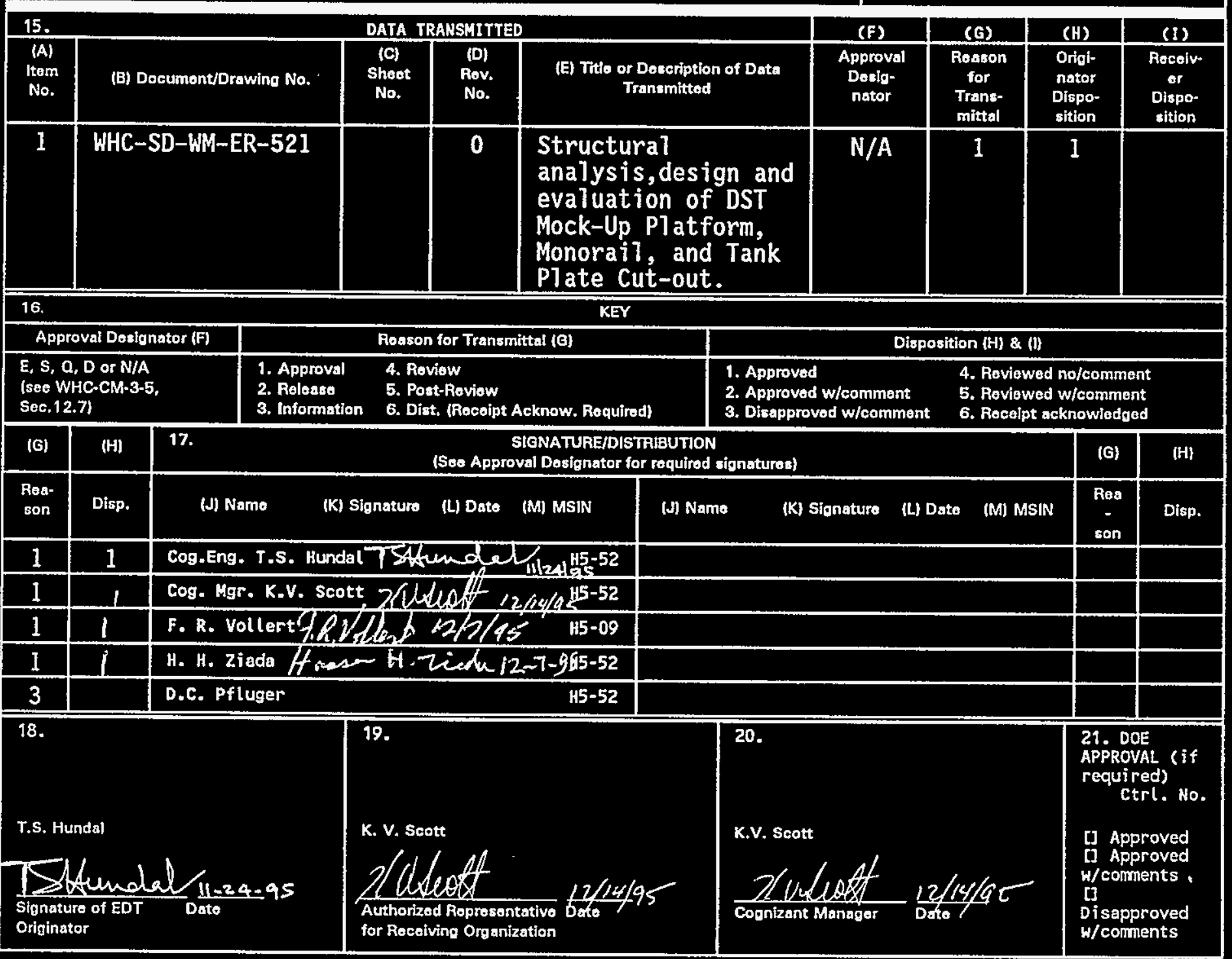




\title{
STRUCTURAL ANALYSIS, DESICN AND EVALUATION OF MOCK-UP PLATFORM, MONORAIL, AND TANK PLATE CUT-OUT
}

\author{
T. S. HUNDAL \\ WHC, Richland, WA 99352 \\ U.S. Department of Energy Contract DE-AC06-87RL10930 \\ EDT: 613853 \\ Org Code: 74750 \\ B\&R Code: 35EW31207 \\ UC: 721 \\ Charge Code: $N 1029$ \\ Total Pages: 288289
}

Key Words: Double She11 Tank, DST, Mock-up platform, structural seismic analysis, Robotic UT inspection, CMA, seismic, design, finite element analysis, monorail, hoist, trolley, plate cut-out.

Abstract: Platform - Structural analyses were performed for design seismic, live and dead load combinations for the freestanding platform over the partial DST mock-up section. The platform is to be used for Robotic ultrasonic inspection of the tank wall. It is a free standing structure anchored to floor slab with Hilti Kwik bolts.

Monorail - The Monorail, trolley and hoist arrangement was designed and provided for accidental Robot fall.

Plate cut-out - The primary tank plate cut-out section was designed to validate the Robot test results.

TRADEMARK DISCLAIMIER. Reference herein to any specific commercial product, process, or service by trade name, trademark, manufacturer, or otherwise, does not necessarily constitute or imply its endorsement, recommendation, or favoring by the United States Government or any agency thereof or its contractors or subcontractors.

Printed in the United States of Anerica. To obtain copies of this document, contact: WHC/BCS Document Control Services, P.0. Box 1970, Mailstop H6-08, Richland WA 99352, Phone (509) 372-2420; Fax (509) 376-4989.
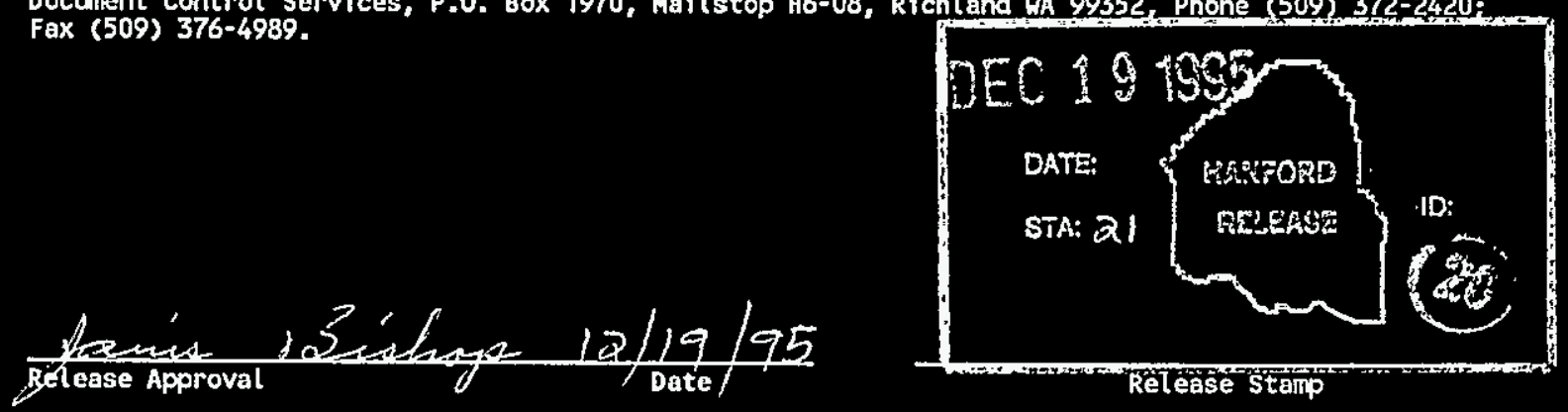

Approved for Public Release 
CONTENTS

Page

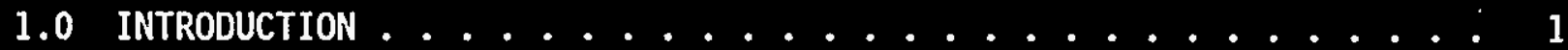

2.0 CONCLUSIONS AND RECOMMENDATIONS .............. 1

3.0 MATERIALS AND ALLOWABLE STRESSES ............. 2

3.1 STRUCTURAL STEEL STRENGTH AND ALLOWABLE STRESSES $\ldots \ldots$

3.2 WELD ALLOWABLE STRENGTH ................ 3

4.0 CONFIGURATION AND LOADS ...................... 3

5.0 STRUCTURAL ANALYSES AND RESULTS ............. 4

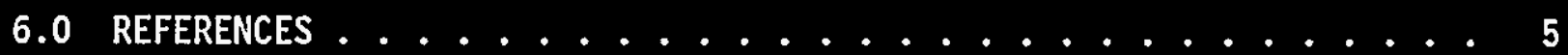

\section{FIGURES}

Figure 1 - DMP Frame Assembly ............... 6

Figure 2 - CMA Frame Assembly .................. 7

Figure 3 - DMP and CMA Frame Assembly $\ldots . . . . .28$

\section{APPENDICES}

APPENDIX A - FINITE ELEMENT ANALYSES. . . . . ....... A-1 APPENDIX B - DESIGN EVALUATION OF MEMBERS AND JOINTS. . ........ B-1 APPENDIX C - MONORAIL DESIGN AND DETAILS. ........... C-1 APPENDIX D - PRIMARY TANK PLATE CUT-OUT DESIGN AND DETAILS. $\because \cdots$ 
AUTHOR'S NOTE:

This report was initially prepared for the analysis of Mock-up P7atform only but later, two more analyses were performed and are included in Appendix $C$ and Appendix $D$ for Monorail Design and Details, and Primary Tank Plate Cut-0ut Design and Details, respectively.

So accordingly, it will be noticed that the main body of the report represents the platform analys is whereas the Appendices $C$ and $D$ describe analyses for Monorail and Primary Tank Plate Cut-out. This was done to avoid the cost of writing two SDS and two EDTs. 
WHC-SD-WM-ER-521

Rev. 0

\author{
STRUCTURAL ANALYSIS, DESIEN AND EVALUATION \\ OF \\ DST WOCK-UP PLATFORY
}

\title{
1.0 INTRODUCTION
}

Structural analyses have been performed for the Hanford Site Double-She11 Tank (DST) mock-up platform (DMP) mounted on the concrete floor slab of 300 Area, highbay building 337. This DMP accommodates the Cable Management Assembiy (CMA) frame unit with the hoisting cable reel. The CMA frame stands on four jacks for raising and lowering the unit to suit the various DST riser heights. It is recommended that these jacks be directly bolted to DMP beams. Six loading cases were analyzed including the normal operational vertical deadweight plus live loads, and the deadweight combined with seismic response loads.

The DMP and CMA frame will be used with the DST partial mock-up to test and qualify a robotic ultrasonic examination system. The DMP is a braced steel space frame structure with mixture of welded and bolted joints. It is fabricated from various size structural shapes. The six DMP columns are anchored to the concrete floor with Hilti Kwik bolts through base plates. The CMA unit is prefabricated from structural steel tubes with welded connections, and stands on the support jacks. The objectives of the structural analyses include determining and evaluating the stresses in the frame members and the joints subject to required design seismic, live loads, and dead load combinations. Three dimensional equivalent static finite element analyses of the DMP were performed (ANSYS 1990) to determine the stresses in the members and forces in the joints. The output results were printed for member stresses and joint forces. For these analyses, it was necessary to presume the CMA support racks are anchored (bolted) to the DMP beams. The joint forces were used to calculate the bolt and weld stresses for the joints with methods from AISC 1989 and Blodgett 1982. The member, joints and weld stresses were evaluated and determined to be in conformance with AISC 1989 and AWS 1992, respectively.

\subsection{CONCLUSIONS AND RECOHIENDATIONS}

The DMP structural analyses (ANSYS) results are the structural member stresses and joint forces. The joint forces were then used to calculate the bolted and welded joint stresses. These calculated members, bolts and welds stresses are all within the allowable values. Each CMA support jack should be anchored (bolted) to the DMP beams with two $\frac{1}{2} "$ diameter SAE Grade 8 bolts.

\footnotetext{
'Trade Name: Hilti, Incorporated, Tulsa, Oklahoma.
} 


\subsection{WATERIALS AND ALLOMABLE STRESSES}

The drawings for the DMP (WHC 1995a), specify ASTM A36 steel for al1 structural members. For CMA frame, the structural steel tubes are ASTM A1020, (WHC 1995b). The steel plates and other components used are ASTM A36. The weld metal used has a specified minimum ultimate strength of 70,000 psi

(E70XX Electrodes).

\subsection{STRUCTURAL STEEL STRENGTH AND ALLONABLE STRESSES}

The elastic modulus, strength and allowable* stresses used for all the structural steel members and components of the DMP and CMA frames are as follows:

Modulus of Elasticity, E

ASTM A36 Stee1 for DMP frame:

U1timate Tensile Strength, $F_{u}$

Yield Strength, $F_{y}$

Allowable Axial Stress, $F_{\mathfrak{t}}$

A1lowable Bending Stress, $F_{b}$

Allowable Shear Stress, $F_{v}$
$=29,000,000$ psi

$=58,000 \mathrm{psi}$

$=36,000$ psi

$=0.60 \times F_{y}=21,600 \mathrm{psi}$

$=0.66 \times F_{y}=23,760 \mathrm{psi}$

$=0.40 \times F_{y}=14,400 \mathrm{psi}$

ASTM A1020 Stee1 for CMA frame:

UTtimate Tensile Strength, $F_{u}$ $=52,000$ psi

Yield Strength, $F_{y}$

$=38,000 \mathrm{psi}$

A1 lowable Axial Stress, $F_{t}$

$=0.60 \times F_{y}=22,800 \mathrm{psi}$

A1 lowable Bending Stress, $F_{b}$

$=0.66 \times F_{y}=25,080 \mathrm{psi}$

Allowable Shear Stress, $F_{v}$

$=0.40 \times F_{y}=15,200$ psi

* Allowable stresses may be increased by $33 \%$ for seismic load combinations. 


\subsection{MELD ALLOMABLE STRENGTH}

The ultimate strength and allowable stress for the welds joining the DMP structural members were established in accordance with the Structural Welding Code (AWS 1992), and the Manual of Steel Construction (AISC 1989). These forces per unit length values are determined based on the minimum ult imate strength $\left(F_{u m m}\right)$ of the weld metal, and the thickness $\left(t_{k}\right)$ of the weld as follows:

$$
\begin{aligned}
& \text { Weld Metal U1timate Strength, } F_{u m}=70,000 \mathrm{psi} \\
& \text { Fillet Weld Ultimate Strength, } F_{u m}=0.707 \times t_{w} \times F_{u m m} 1 \mathrm{bs} / \text { in } \\
& \text { Fillet Weld Allowable* Stress, } F_{w}=0.30 \times F_{u w}=14,800 \times t_{w} \text { 1bs/in }
\end{aligned}
$$

* Allowable stress may be increased by $33 \%$ for seismic load combinations.

\subsection{CONFIEURATION AND LOADS}

The configuration of the DMP structure was obtained from the design drawing H-3-306027 Sh.1 thru $7^{\star}$ (WHC 1995a). It is a braced steel space frame structure with welded and bolted joints. It is fabricated from various structural steel (A36) shapes and plates. The CMA frame assembly is fabricated from A1020 steel tube members as shown on drawing 9213-CMA-ASSY-001 (WHC 1995b).

In 7 ieu of the design load requirements in WHC 1992, the platform structure was analyzed for more realistic actual loads as described below.

The complete DMP assembly was analyzed for dead load plus 75 psf live load on the platform. The CMA frame supports the cable reel from two beams at the top. The reel carries about $6000 \mathrm{lbs}$ total operating load, including the robotic system. The complete structure was further analyzed for: two orthogonal horizontal seismic design response forces of $0.20 \mathrm{~g}$ and $0.06 \mathrm{~g}$ combined with the dead load; and a $0.133 \mathrm{~g}$ vertical seismic design response force combined with the dead load. These seismic forces are appropriate for the safety class 3 (SC3) of the platform (WHC 1992). 


\subsection{STRUCTURAL ANALYSES AND RESULTS}

Three dimension finite element static analyses were conducted for the DMP structure (ANSYS 1990) for the normal deadweight and live loads, and for deadweight combined with the seismic design forces. The frame was modeled as a mixture of welded and bolted connections. It was presumed that the CMA support jacks are bolted to the DMP beams. The six columns bases are anchored to the floor slab with Hilti Kwik bolts and the top of the columns are connected to platform beams with bolts. These columns are idealized as pinned joints.

Six load cases were run. This was necessary to ensure that the analyses results for the DMP will capture the maximum stresses in the members and the maximum forces at the joints. The finite element models are described in detail, and all analyses results are provided in Appendix $A$.

The analyses results given in Appendix $A$, include the forces and moments in the members and joints, and the member stresses. The bending, axial, shear, and combined stresses are calculated for each beam element in the DMP model. The maximum bending, shear, and combined stress values in the DMP members and connection joints for various load cases are listed in tables on pages A-221 through A-226 of Appendix A. Tables on page A-220 1ist the enveloped forces and stress values for all load cases used for the Appendix B design evaluation of members and joints.

Appendix $B$ is the structural design evaluation for the DMP and CMA members and joints using the ANSYS analyses results. The structural member stresses were determined to be with in the allowable 1 imits. The stresses in the bolted and welded joints were calculated using methods from AISC 1989 and Blodgett 1982. The calculated stresses in the boits and welds are with in the al lowable limits. 


\subsection{REFERENCES}

AISC, 1989, Manual of Steel Construction, Allowable Stress Design, Ninth Edition, American Institute of Steel Construction, Chicago; I11inois.

ANSYS, 1990, ANSYS-Engineering Analysis System, Rev, 4.4, Swanson Analysis Systems, Inc. Houston, Pennsylvania.

ASTM, 1989, Annual Book of ASTM Standards, American Society for Testing and Materials, Philadelphia, Pennsylvania.

AWS, 1992, Structural Welding Code-Stee7, ANSI/AWS D1.1-92, American Welding Society, Miami, Florida

Blodgett, 0. H., 1982, Design of Welded structures, James F. Lincoln Arc Welding Foundation, Cleveland, Ohio

DOE, 1989, Standard Architectural-Civil Design Criteria, HPS-SDC-4.1, Rev.11. Richiand, Washington.

UBC, 1991, Uniform Building Code, International Conference of Building officials, Whittier, California.

HHC, 1992, Specification for Double She17 Tank Mock-up Test Facility, WHC-S-0152, Revision 0.

HHC, 1995a, Double Shel7 Tank Mock-up Stand Assemb7y, Drawing No. H-3-306027 Sht. 1 thru 7.

WHC, 1995b, Double She11 Tank Inspection Final System Design Specification, (WHC-4375-SP-3.0) Vo1. 2, Design Drawings, Cable Management Assembly, Raytheon Engineers \& Constructors, Inc. and RedZone Robotics, Inc. 
WHC-SD-WM-ER-521

Rev. 0

Figure 1. DMP Frame Assembly.

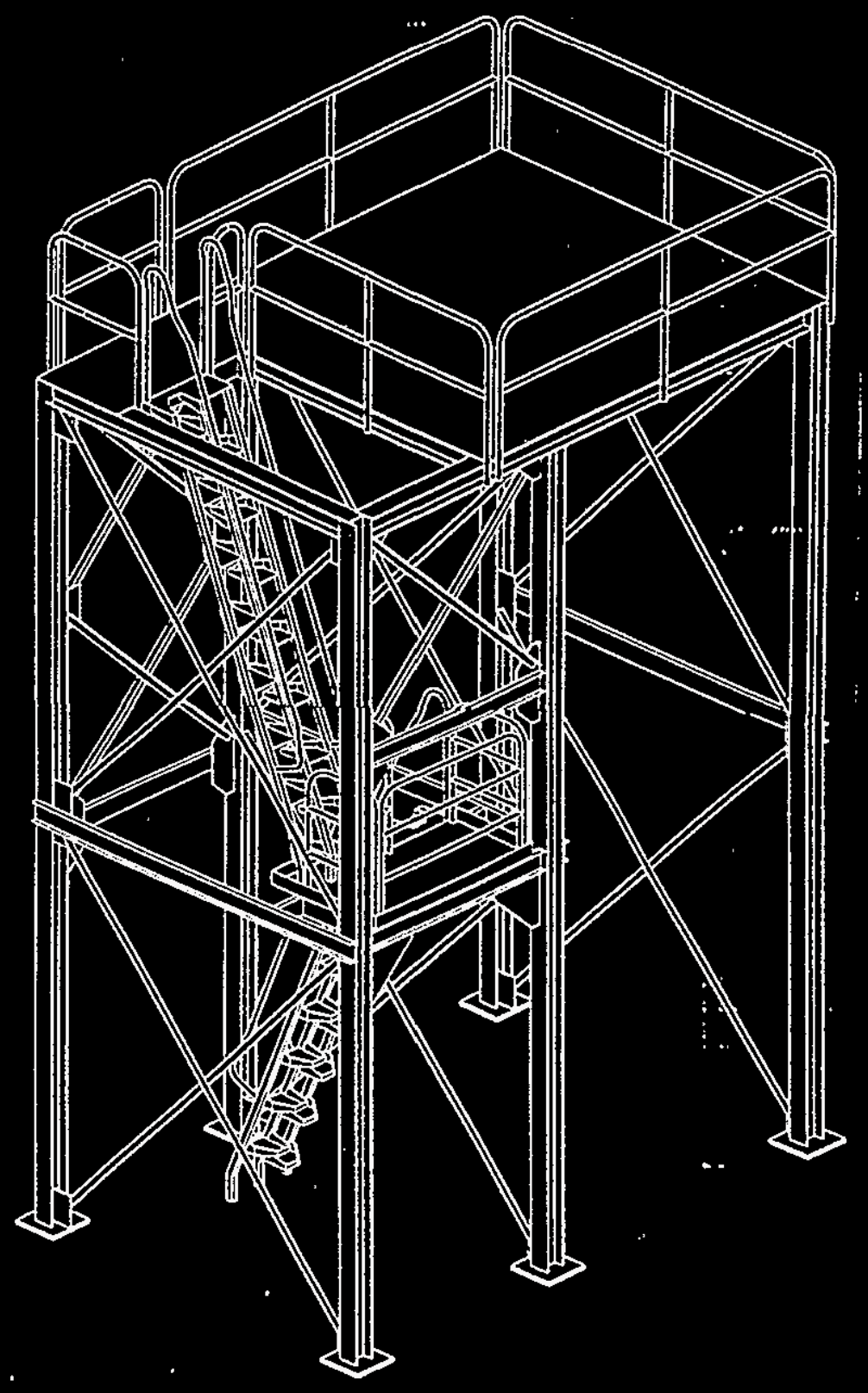


Figure 2. CMA Frame Assembly.

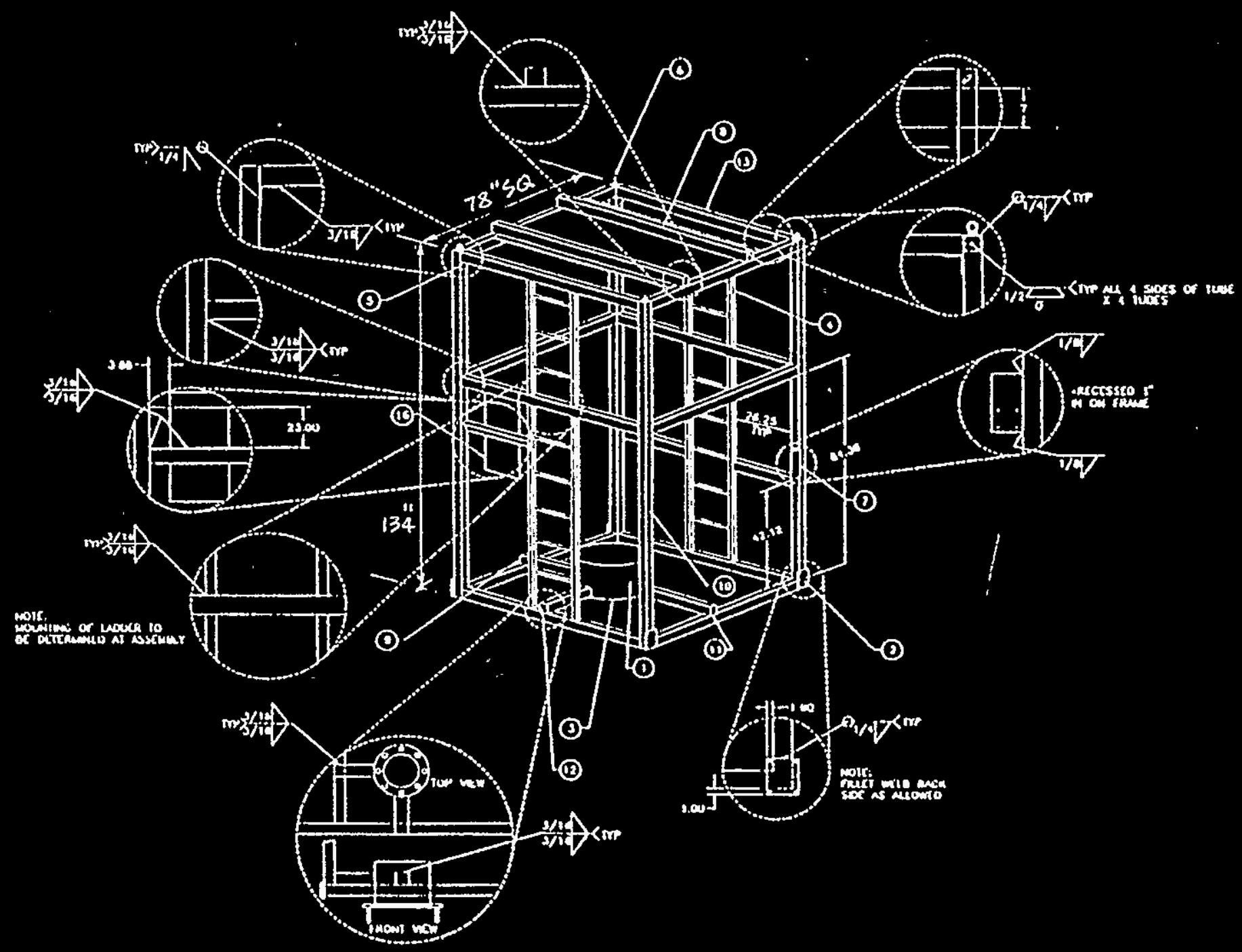


WHC-SD-WM-ER-521

Rev. 0

Figure 3. DMP and CMA Frame Assembly.

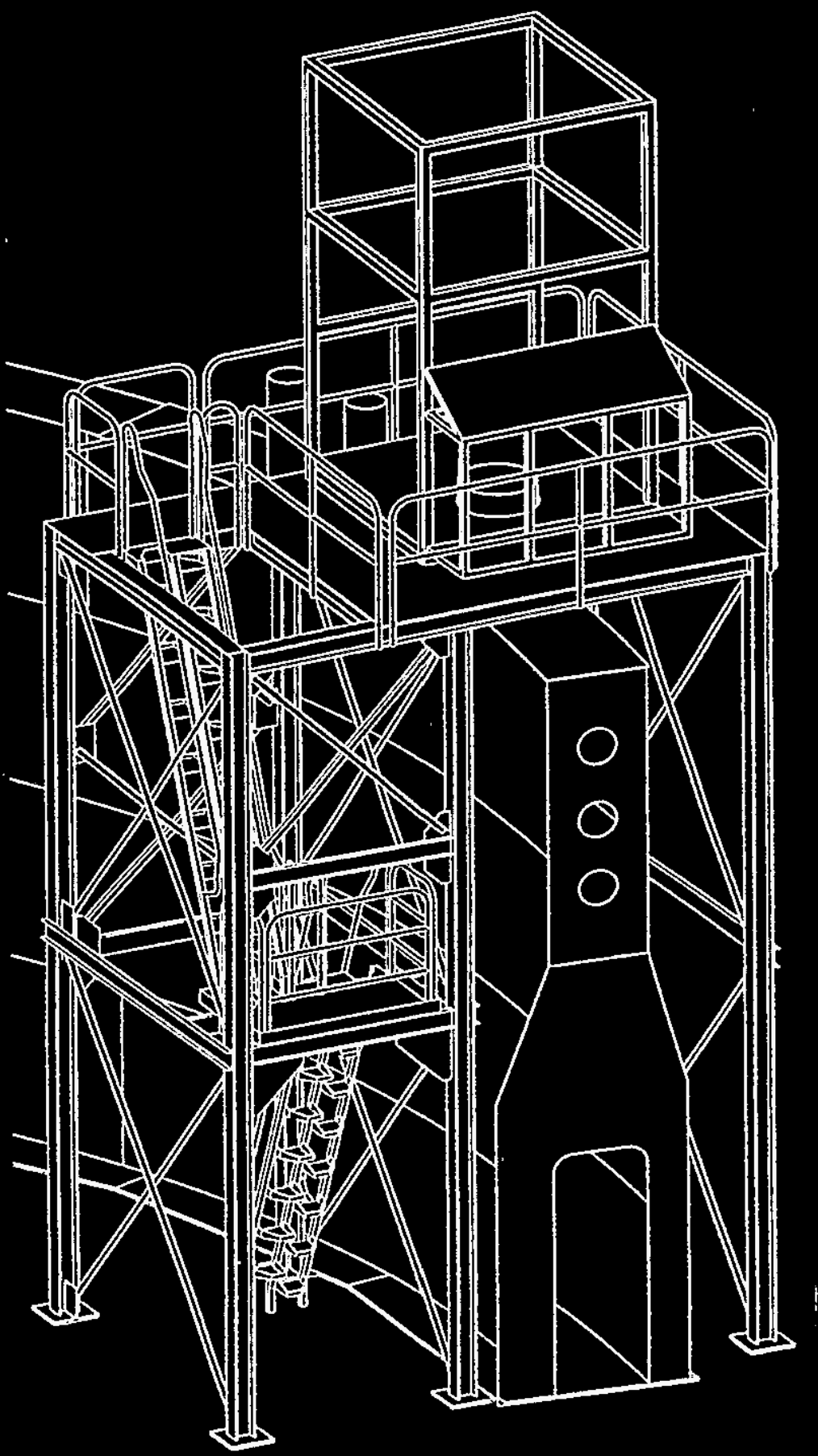


WHC-SD-WM-ER-521

Rev. 0

APPENDIX A

FINITE ELENENT ANALYSES 


\section{INDEPENDENT REVIEW CHIECKLIST}

Document Reviewed: Structural analysis, design and evaluation of DST Mock-up Platform

Author: T. S. Hundal

Document No. WHC-SD-WM-ER-521, R/0

Yes No N/A

[X] $[$ ] [ ] Problem completely defined.

[X ] [ ] [ ] Necessary assumptions explicitly stated and supported.

[X ] [ ] [ ] Computer codes and data files documented.

[Y ] [ ] [ ] Data used in calculations explicitly stated in document.

L ] [ ] [ ] Data checked for consistency with original source information as applicable.

[ ] [ ] [y] Mathematical derivations checked including dimensional consistency of results.

[X] $\quad$ [ ] [ ] Models appropriate and used within range of validity or use outside range of established validity justified.

[†] [ ] [ ] Hand calculations checked for errors.

[X] [ ] [ ] Code run streams correct and consistent with analysis documentation.

[X ] [ ] [ ] Code output consistent with input and with results reported in analysis documentation.

[Y $]$ [ ] $\quad[\quad]$ Acceptability limits on analytical results applicable and supported. Limits checked against sources.

[X] [ ] [ ] Safety margins consistent with good engineering practices.

[† ] [ ] [ ] Conclusions consistent with analytical results and applicable limits.

[ $f]$ [ $]$ [ ] Results and conclusions address all points required in the problem statement.

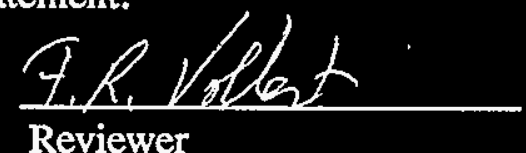

A-2

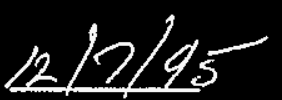

Date 
WHC-SD-WM-ER-521

Rev. 0

CONTENTS of APPENDIX - A

Section

A.1

A.2

A.3

A.4
Description

Finite Element Model

Applied Loads \& Material Properties

Finite Element Model ANSYS Inputs and Outputs

Summary of ANSYS output results
Page

A-4

A-5

A-13

A-220 


\section{A.1 FINITE ELEMENT MODEL}

The Double Shell Tank (DST) Mock-up platform (DMP) is mounted on the 300 Area Highbay building 337 floor. This platform is a braced space frame with a steel plate at its top serves as a working area for the Robot operation to be lowered into the DST annulus space and is anchored to the concrete floor with Hilti Kwik bolts. A Cable Management Assembley (CMA) prefabricated steel frame is mounted on the top of the DMP. The CMA frame is supported on four jacks to accomodate the varying heights of diffferent risers on top of the tanks. The DMP is made up of various A36 structural shapes and sizes. See Drawings H-3306027 Sh. 1 thru $7^{*}$ for details. The CMA is fabricated from A1020 tube steel. See Drawing 9213-CMA-ASSY-001 by RedZone Robotics, Inc., for details.

The purpose of the analysis is to determine the forces in the joints and stresses in the members mof the DMP and CMA. The analysis loads are: the deadweight od DMP and CMA frames and the hoisting reel weight (approx. $6000 \mathrm{lbs}$ ) including the weight of the Robot. To acccomplish this, three dimensional finite element model was generated for both frames using the ANSYS computer program. For seismic events, a $0.12 \mathrm{~g}$ free field horizontal accelration value was used as per SDC-4.1. Applied seismic loads were computed and applied as per UBC 1991 methods. The maximum computed horizontal accelration value was applied in one direction and a thirty percent of this computed value was applied in the other orthogonal horizontal dierection combined with the dead loads. Various boudary conditions were applied to reperesent the field coditions. See page A-14 for the model geometry and configuration. Six load cases were run to get the maximum reactions, forces and/or stresses in the joints and/or members. These load cases are as follows:

Load Case 1. DL + LL

Load Case 2. DL +0.2g (x) $+0.06 \mathrm{~g}(\mathrm{z})$

Load Case 3. DL $+0.2 \mathrm{~g}(-\mathrm{x})+0.06 \mathrm{~g}(\mathrm{z})$

Load Case 4. DL +0.06g (x) +0.2g (z)

Laod Case 5. DL +0.06g (x) +0.2g (-z)

Load Case 6. DL +0.133g (y)

(Note: $\mathrm{x}, \mathrm{y}, \mathrm{z}$ are the directions in which the seismic accelrations were applied to represent the seismic loads)

The output for maximum forces and/or stresses for joints and members were tabulated for each load case and the enveloped values were compiled in the final table. The final enveloped values were used to design and/or evaluate the joint and members. The design and evaluation of members and joints is given in Appendix B.

* To be issued 
WHC-SD-WM-ER-521

Rev. 0

A.2 APPLIED LOADS AND MATERIAL PROPERTIES 
(1) Drawing

(2) Doc. No.

(3) Page of

(4) Building

(5) Rev.

(6) Job No.

(7) Subject DST Mock-UP PLATroRen

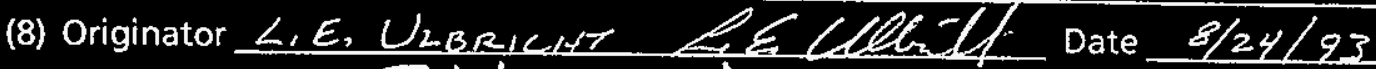

(9) Checker TSdenendal Date $7 / 10 / 95$

(10) LOAD CONEITIONS:

STATIC:

UNIFORM PLATFORM LOAD OF 75 HEF/Ft $=$ FOR LIGHT MANUFATIUEING Selsmic:

PLATFORM (UBC 1991, THBLE 23-A)

FOR SAFETY ClASS 3 COMPONENTS, SDC4.1 ( DOE I 989) SPECIFIES THE FOLLOWING SEISMIC DEEIGN LRTTERIA

$$
\begin{aligned}
& Z=0,1 Z_{g}=Z E R O P E R I O D \text { ACCCLERATION } \\
& I=1,25=\text { IMPORTANCE FACTCR }
\end{aligned}
$$

PER UCRL 1990, THE STATIL EQUIVALENT METHOD SPECIFIED BIY UBC 19\% IS APPROPEIATE FOR EQUIPMENT AND NON-SIRULTURAL ELenents (SC3\&SC4).

$$
\begin{aligned}
& F_{p}=z / C_{p} W_{p} \\
& \text { WHERE: } F_{D}=\text { THE EQUIVALENT STPTIC LDAD APPLIEO TO } \\
& \text { THE COMMONGNT TO REPAESAAM SEISMIC LOAUNG } \\
& C_{p}=2(.75)=1.5=\text { HORIFONTAL FORLEFACTOR PER } \\
& \text { TAELE Z3-P AND PARA. Z3SG(G) OF ULC 1991 } \\
& W_{P}=\text { SEISMIC DEAD LOAD: PER PARA, Z334A oT } \\
& \text { UBC 1991, THE } 75 \text { lo/fF" LIVE LOAD IS EXCLUDEW } \\
& F_{p}=0.12(1.25)(1.5) W_{p}=0.2 W_{P}
\end{aligned}
$$

THERETOEE, USE A HoRizONTHL ALCELERATrON of $a_{h}=0_{n} z_{g}$

THE VERTILAL ACLELERATIAN is $a_{V}=\frac{2}{3} a_{h}=\frac{3}{3}\left(0.2_{g}\right)=0.133 \mathrm{~g}$. (UCRL1990, SECT 4.4.1)

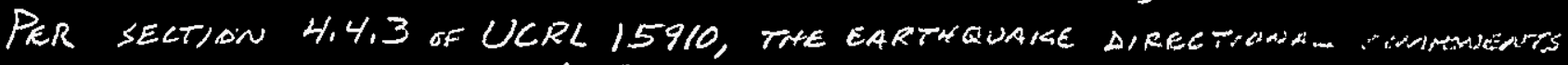
ARE COMBINEO BY APDING $100 \%$ of THE HORIFONTAL SEISMIC FCRCES IN ONE DIRELTION WITH 30\% OF THE HORICONTAL SEIMIC FORCE IN THE ORTTHOGONAL PIRECTION, ALSO, FOR SAFETY CLASS 3 COMPONENTS, THE VERTICAL SEISMIC ACCELERATION IS NOT COMBINES WITM THE HORIZONTAL ACCELERA TrOW.

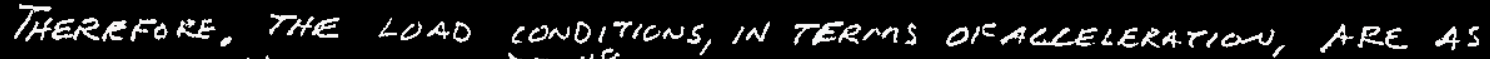
FOLLOWS. NOTE: THE $7516 f_{\text {Ft }}$ PLATFORMM LOACING DOES NET APPLY TO THE SEISMIC CONDitions.
r) $a_{x}=0.2 y$,
$a_{y}=\lg , a_{z}=0.069$
2) $a_{x}=0.069$,
$a_{y}=1$ g
$a_{z}=0.2 g$
NoTE:
3) $a_{x}=0$
$a_{y}=1.133 \mathrm{~g}, a_{z}=0$
$A-6$
Y IS VERTILAL 
Subject DST MOCK-UP PLATFORM

Originator 1 Statudal

Checker

Page 2 of

platform safety classification (SC) is 3

PLATFORM LOADING

$\frac{D L}{1 / 4} R=10.2 \mathrm{psf}$

$L L \quad=75.0$ pSf (RAf.UBC,91)

(1) $\left\{\begin{array}{l}D L+L L=85.2 p 5 f \quad \text { (STATIC) } \\ D L \text { of CMA }=6000 \mathrm{lbs}\end{array}\right.$

SEISMIC . (Ref. SDC-4.1)

$F_{p}=. z w_{p}$

$\therefore$ Three Seismic Loading cases

2) $a_{x}=2 g, a_{y}=\lg \quad a_{z}=.06 g$

3) $a_{x}=.06 g, a_{y}=\lg \quad a_{z}=.2 g$

4) $a_{x}=0 \quad a_{y}=1.133 \mathrm{~g} \quad a_{z}=0$

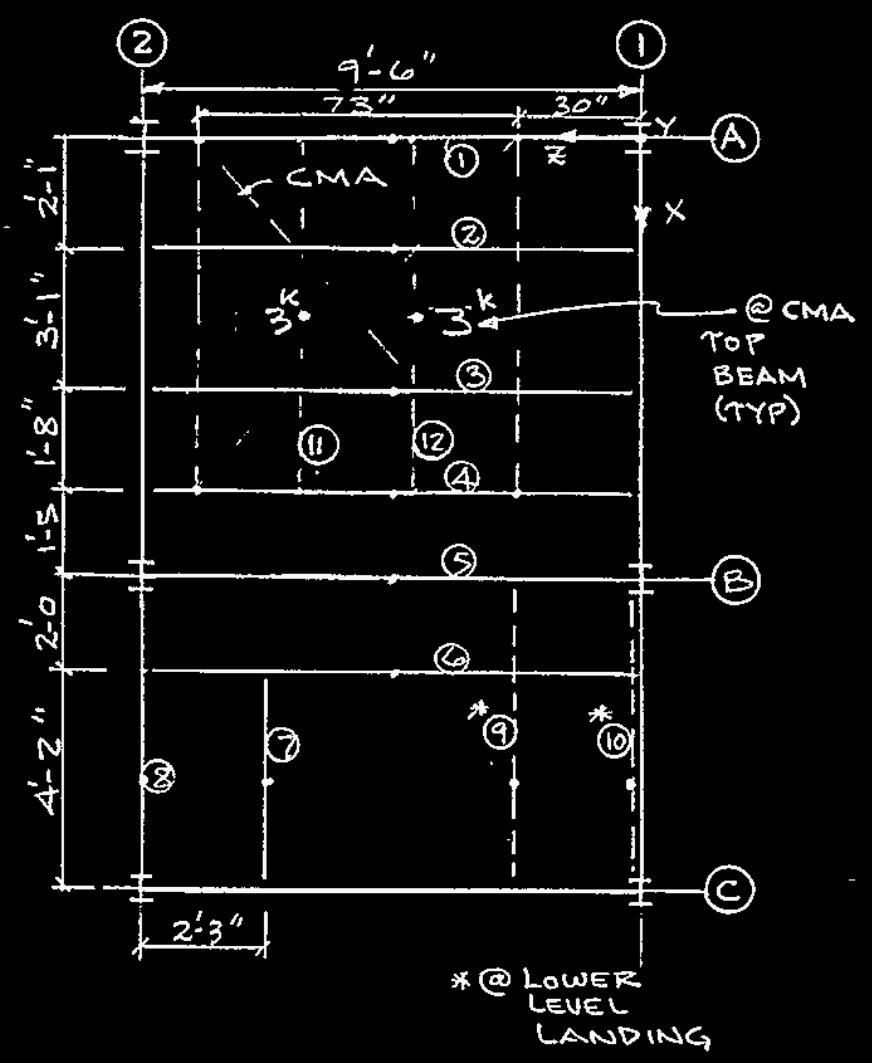

CALCULATE (DL+LL) for BEAMS C MID SPAN

$$
\begin{aligned}
& \text { BEAM } 1=85.2(9.5 \times 2.1) / 2=850^{\#} \\
& z=85.2(9.5 \times 5.2) / z=2104^{*} \\
& 3=85.2(9.5 \times 4.75) / 2=1922 \\
& 4=85.2(9.5 \times 3.1) / 2=1255 \\
& 5=85.2(9.5 \times 3.42) / 2=1384 \\
& 6=85.2(9.5 \times 2) / 2=809 \\
& 7 \$ 8=85.2(4.2 \times 2.25) / 2=403+150=553 \\
& 9 \neq 10=85.2(6.2 \times 2.25) / 2=594 t^{*} 300=894 \\
& \text { DL ONLY@10.2psf } \\
& 105 \neq \\
& 252 \\
& 230 \\
& 150 \\
& 166 \\
& 97 \\
& 48+* 150=198 \\
& 71+300=371 \\
& \text { * Dl of stairs (AAsSUmed) } \\
& 20^{4 / F_{+} \times 15^{\prime}}=300^{* 1} \\
& A-7 \\
& \text { BD-6400-060.1 (07/93) }
\end{aligned}
$$




\section{ANALYTICAL CALCULATIONS}

Rev. 0

Subject DST Mock-UP P.AFOEM

Originator

Checker

Page $Z$ of

Date $7-10-95$
Date

SEISMIC LOADS

$.29 \neq .06 g$ \& $133 \mathrm{~g}$

\begin{tabular}{|c|c|c|c|c|c|}
\hline BEAM & NODE & $F_{y}$ & $.2 F_{Y}$ & $.06 F_{Y}$ & $1.133 \mathrm{FY}_{Y}$ \\
\hline 1 & 20 & 105 & 21 & 6 & 119 \\
\hline$z$ & 23 & 252 & 50 & 15 & 286 \\
\hline 3 & 26 & 230 & 46 & 14 & 261 \\
\hline 4 & 29 & 150 & 30 & 9 & 170 \\
\hline 5 & 54 & 166 & 33 & 10 & 188 \\
\hline 6 & 55 & 97 & 19 & 6 & 110 \\
\hline 7 a 8 & 49,57 & 198 & 40 & 12 & 224 \\
\hline $9 \neq 10$ & 60,62 & 371 & 74 & 22 & 420 \\
\hline $11 \neq 12$ & 41,42 & 3000 & 600 & 180 & 3399 \\
\hline
\end{tabular}

$\mathbf{A}-\mathbf{B}$ 
(1) Drawing

(2) Doc. No.

(4) Building

(5) Rev.

(3) Page 4 \& of

(7) Subject DST Mock-UP PLATFORM

(6) Job No.

(8) Originator

(9) Checker

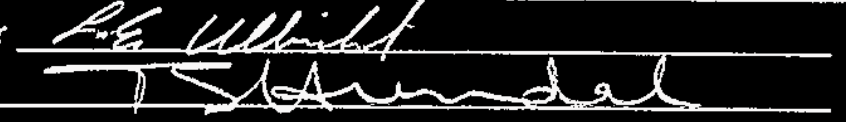

Date $\frac{8 / 24 / 93}{7 / 10 / 95}$

(10)

\section{Allowarle Stresses:}

Sratic:

STRUCTURAL MEMBERS: ASTM-A36 REF AISC 1989

TENSION: $F=0.6 F=0.6(36,000)=21,600 \mathrm{~kg} / \mathrm{m}=$

BENDING: $\bar{b}=0.6 F \mathrm{~F}=21,600 \mathrm{kf} / \mathrm{in} \mathrm{F}^{2}$ ar calculated case by case

SHEAR: $F_{V}=0,4 F_{Y}=0.4(36,000)=14,400 \mathrm{lbF} / \mathrm{In}^{2}$

COMPRESSION: $F \rightarrow$ BASED ON BUCKLING CRITERIA, EVKUATED CASE BY CASE

Plate: AstM -A36, REF A1SC 1989

Tension: $F_{T}=0.6 F_{y}=21,600 \mathrm{HF} / \mathrm{in}^{2}$

BENDING ABOUT MINOR AXIS: $F_{z}=0.75 F_{y}=0.75(36,000)=27,00016 \mathrm{f}_{\mathrm{g}} \mathrm{m}^{2}$

SITEAR: $\quad F_{V}=0.4 F_{y}=14,400 \mathrm{BbFlin}{ }^{2}$

WELOS; ETOXX ROO REFAHS 1989 , THBLE JZ.5

SHEAR: $F_{V}=0,3$ (NOM. TEMSILE STRENGTH DIE WELD METAL)

$$
F_{V}=0.3(70,000)=21,00016 \mathrm{f} / 1 \mathrm{n}^{2}
$$

BLLTS: REFAISC 1989 TABLE J3,2 U TABLE J3,7

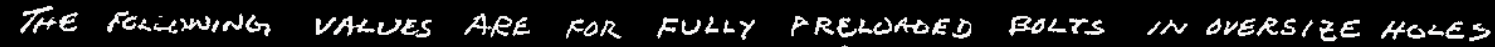
ASTM A3Z5 BOLTS $F_{T}=44,000 \mathrm{lbF} / \mathrm{mz}, F_{V}=15,00016 \mathrm{~F} / \mathrm{m}^{2}$

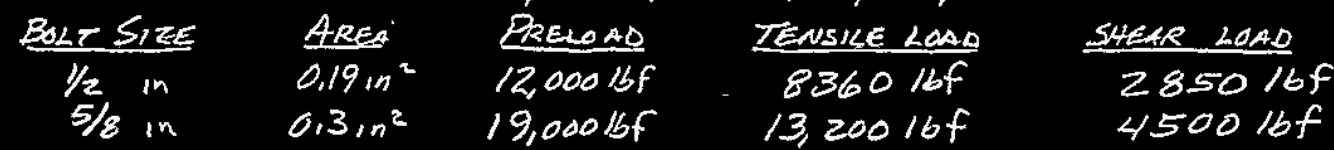

ANCHOR BOLTS: REF SDC $4 / 2$ (DOE 1992) TABLE/I

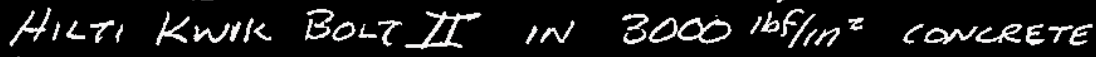

\begin{tabular}{|c|c|c|c|}
\hline BoLT SIZE & ENBEDMENT & TENGLE LOAO & Shear hoad \\
\hline $\begin{array}{l}5 / 8 \text { in } \\
5 / 8 \text { in }\end{array}$ & $\begin{array}{l}23 / 4 \mathrm{in} \\
4 \mathrm{in}\end{array}$ & $\begin{array}{l}1750.16 f \\
267016 f\end{array}$ & $\begin{array}{l}253016 f \\
275016 f\end{array}$ \\
\hline $3 / 4$ in & $31 / 4$ in & $217016 f$ & $341016 \mathrm{~F}$ \\
\hline $3 / 4$ in & $43 / 4$ in & $3870 \mathrm{lbf}$ & $371016 f$ \\
\hline
\end{tabular}

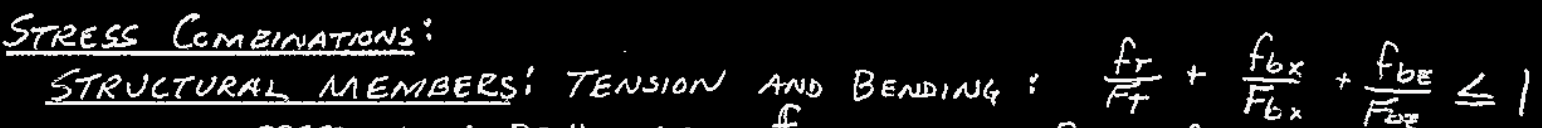
COMPRESSION AND BENLING: if $\frac{f_{c}}{F_{c}}<0.15, \frac{f_{c}}{F_{c}}+\frac{f_{d x}}{f_{b x}}+\frac{f_{b E}}{F_{b E}} \leq 1$

INTERACTION FoR BOLTS Q ANCHOR BOLTS:

$$
\frac{f_{\tau}}{F_{T}}+\frac{f_{V}}{F_{V}} \leq 1
$$

$$
A-9
$$


(1) Drawing

(4) Building

(2) Doc. No.

(3) Page 5 of

(7) Subject DST MOCK-UP PLATFORM

(5) Rev.

(6) Job No.

(8) Originator

(9) Checker

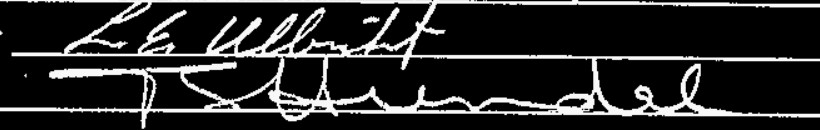

Date Date

$\frac{8 / 24 / 93}{7 / 10 / 95}$

(10)

\section{ALOWABLE STRESSES (CONT:):}

SESSMic:

PER AISC 1989, PARA A5,2 AND URC 1991, PARA, 2303(d), THE

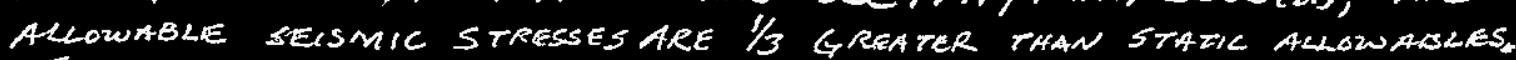
TAlS DOES wot APPLY TO ANCHOR BOLTS.

STRUCTURAL MEMBERS: ASTM-A36

TEnsion: $F_{T}=4 / \mathrm{s}(21,600)=28,80016 \mathrm{f} / \mathrm{ni}^{\mathrm{e}}$

Bending: $F_{6}=4 / 3(21,600)=28,800 \mathrm{kf} / \mathrm{m}^{2}$

SHEAR: $\quad F=4 / 3(14,400)=19,200 \mathrm{HF} / \mathrm{min}$

COMPRESSION: $E_{L}=4 / 3$ (FE FOR STATSC)

\section{Pate: Astm A36}

TENSION: $F_{T}=4 / 3(2,600)=28,80016 \mathrm{f} / \mathrm{in}^{2}$

BENDING (MANOR AXIS) : $F_{6}=4 / 3(27,000)=36,00016 \mathrm{f} / \mathrm{m} \mathrm{c}$

SHEAR: $F_{r}=4 / 3(14,400)=19,200 \mathrm{lbf} / \mathrm{in}^{2}$

WELDS: ETOXX RAD

SHEAR: $F_{V}=4 / 3(2,0 \infty)=28,000 \mathrm{HF} / \mathrm{rn}^{2}$

BoLTS:

TENSION : $F_{T}=\frac{4}{3}(4 y, \infty)=58,667 \mathrm{lbt} / \mathrm{m}^{2}$

SHEAR: $F_{V}=4 / 3(15,000)=20,000165 / \mathrm{ln}^{2}$

BOLTSIEE TEARUELOAD SHEAKLOAD

$1 / 2,{ }^{2} \quad 4 / 3(8360)=11,147 \mathrm{lbF} \quad 4 / 3(2850)=3800 \mathrm{lff}$

$5 / 8$ in $\quad 4 / 3(13,200)=17,600 / 6 \mathrm{f} \quad 4 / 3(4500)=6000 \mathrm{kff}$

ANCHOR BOLTS: HKB-IT IN 3000 ibflin=

No INCREASE FOR SEISMIC LOAD M SEE STATIC AWOWABLES

STRESS COMEINATIONS:

SAME AS FOR STATIC CONDitions 
(1) Drawing

(2) Doc. No.

(3) Page

Rev. 0

(4) Building

(5) Rev.

(6) Job No.

(7) Subject DST Macic-UP PLATroRM

(8) Originator

(9) Checker

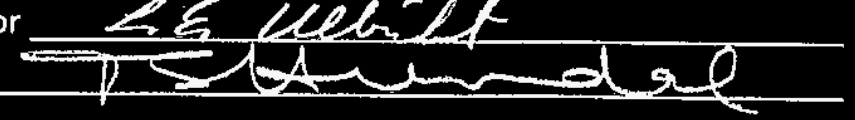

Date $\frac{8 / 24 / 83}{7 / 10 / 95}$

(10)

Sectional Propermes of StRUCTURAL MEMBER: REF AISC 1989

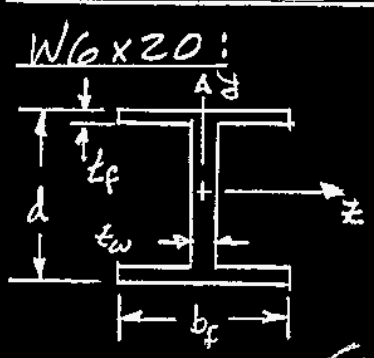

$$
\begin{aligned}
& A=5.87 \mathrm{~m}^{\mathrm{N}} \\
& d=6.2 \mathrm{~m} \\
& t_{w}=0.26 \mathrm{in} \\
& b_{f}=6.02 \mathrm{in} \\
& t_{f}=0.365 \mathrm{in}
\end{aligned}
$$

$I_{z}=41,4 \mathrm{in}^{4}$

$I_{y}=13.3 \mathrm{~cm}^{4}$

$r_{z}=2.66 \mathrm{in}$

$S_{z}=13.4 \mathrm{in}^{3}$

$s_{y}=4.41 \mathrm{in}^{3}$

SHEAR AREA $=A_{s}=d t_{\omega}=6.2(.26)=1.612 \mathrm{~m}^{2}$

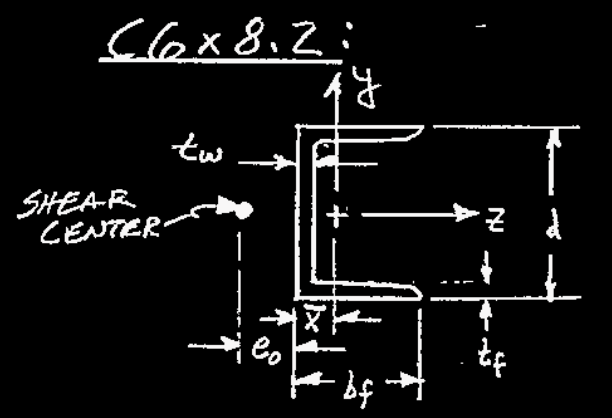

$$
\begin{aligned}
& A=2.4 \mathrm{~m}^{2} \\
& d=6 \mathrm{in} \\
& t_{w}=0.2 \mathrm{in} \\
& b_{f}=1.92 \mathrm{in} \\
& t_{f}=0.343 \mathrm{~m} . \\
& \bar{x}=0.511 \mathrm{in} \\
& e_{0}=0.599 \mathrm{in}
\end{aligned}
$$$$
I_{z}=13.1 \mathrm{in}^{4}
$$

$r_{y}=1.5 \mathrm{in}$

SHEAR AREA $=A_{5}=d t_{w}=6(.2)=1.2 \mathrm{in}^{2}$

\section{$\angle 2 \times 2 \times 1 / 4:$}

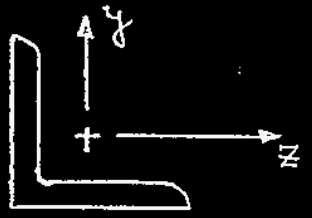

$$
\begin{aligned}
& A=0.938 \mathrm{in}^{2} \\
& r_{\min }=0.391 \mathrm{in} \\
& \omega t / f t=3.19 \mathrm{lbf} / \mathrm{ft}
\end{aligned}
$$

DECK PLATE:

$$
\text { 1/4" PLATE }
$$

\section{$T S 3 \times 3 \times 14(A-1020)$}

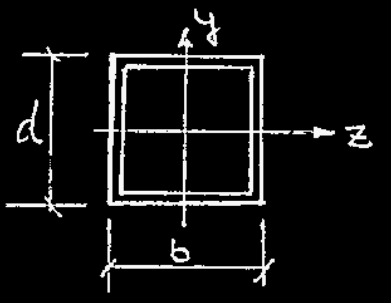

$$
\begin{array}{rlrl}
A=2.59 \mathrm{in}^{2}, & I_{y}=I_{z}=3.16 \mathrm{in}^{4} \\
d=b=3 " & J=5.35 \mathrm{in}^{4} \\
r & =1.10 "
\end{array}
$$

$S_{z}=4.38 \mathrm{in}^{3}$ $s_{y}=-492$ in $^{3}$ $r_{y}=0,537 \mathrm{in}$

$J=0.08 \mathrm{in}^{4}$ 
(1) Drawing

(2) Doc. No.

Rev. 0

(4) Building

(5) Rev.

(3) Page 7 of

(7) Subject DST Mack-UP RaTFORM

(8) Originator _.. $\$$ Date

(6) Job No.

(9) Checker

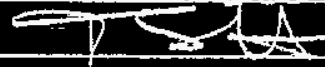
Date

$\frac{8 / 24 / 93}{7 / 10 / 95}$

(10)

\section{STATIC EVALUATION:}

The plateorm is analyzed using AWSys 4. La (ANSYS 1989). The INPUT LISTINGS AND RESLLTS ARE PESENTED IN THE ATRACHMONTS A SUMmary of tHE RESOLTIMUG MAXIMUM VALUES for streESES AND FORCES ARE TABULATEO ON PAGEA-ZZO. THE NOTATION IS AS FOLLOWL.

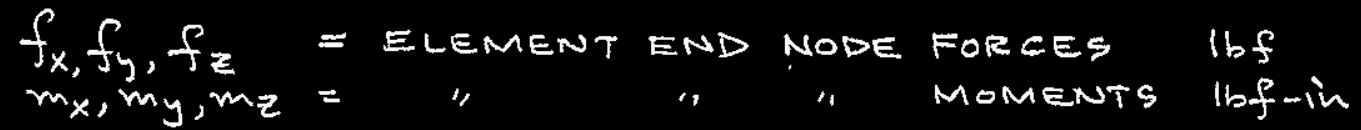

SAX = AXIAL STRESS IN STRULTORAL MEMEERS, Ibflinz

$S B Y=\frac{M_{Y}}{S Y}=$ BENDING STRESS INBEAMS AND COLUMNS, $\mid 6 F / / \mathrm{h}^{2}$

$S B Z=\frac{M Z}{S E}=$ BENDING STRESS in BEAMS AND LOLUMNS, / GF/lin'

$S G|=S A X+| S B Y|+| S B z|| b, F / \ln 2$

$S G B=S A X-|S B Y|-|S B Z|, \quad 16 F / i n^{2}$

PREFIXES I AND J, DENOTE START AND ENL NODE OF AN ELEMENT

TAE MAXIMUM STRESSES FOR BEAMS ANO COLUMNS ARE BASED ON THE MAXIMUNI OT SGI AND SG3 VALLES, AND AS SUCH THE REPORTED STRESSES OCCUR AT A SINGLE NODE. TAE INAXIMUM SHEAR FORLES REPORTED ARE TAE MAXIMUM VALUE FOR ALL ELEMENTS OF THAT TYPE, AND AS SWCH, THESE MAXIMUMS MAY NOT OCCUR AT THE SAME NODE. THEREFORE, BEAM cONNECrIONS DESIGNED FOR THE REPORTIED MAXMUM SHEAR FORCES WILL EE CONSERUATIVE.

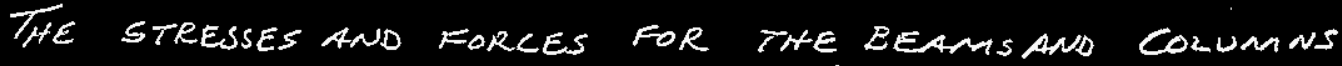
ARE REPORTED IN THE ELEMENT'S COORUINATE SYSTENAS SHOWN BELOW.
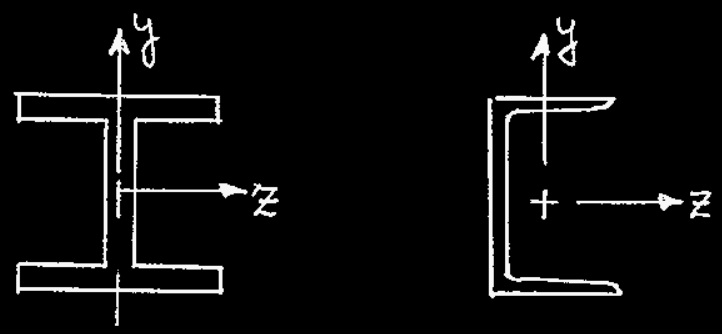

THe Baseflate reaction forces are reported in global LOORDINATES (Y-VERTICAL). THE NIAXININSS REPORTED IN THE FOLLOWING TABLE MAY NOT OCCUR AT THE SAMENOUE

$$
A-12
$$


HHC-SD-WH-ER-521

Rev. 0

A.3 FINTTE ELEMENT MODEL, ANSYS INPUTS AND OUTPUTS

A-13 

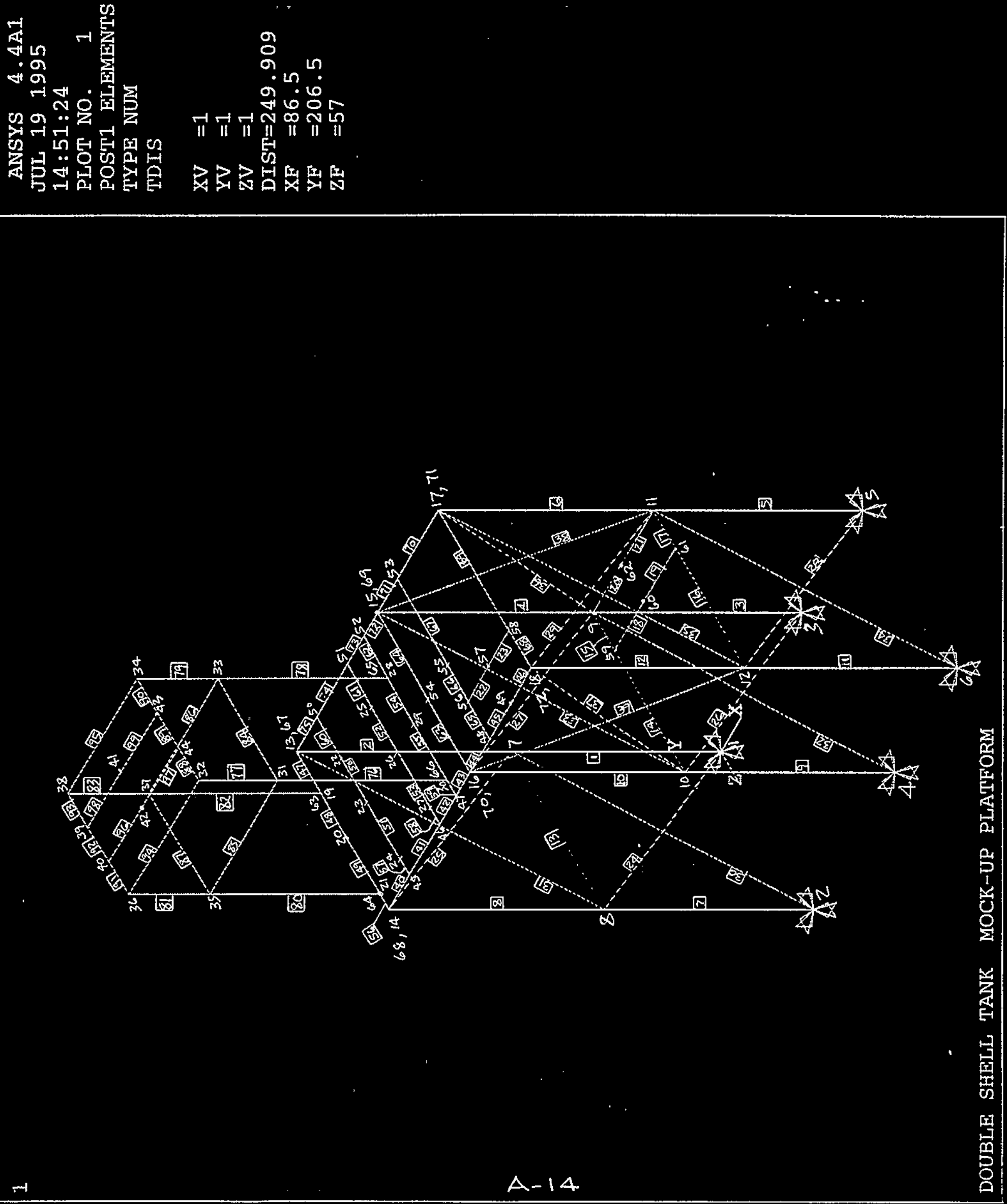


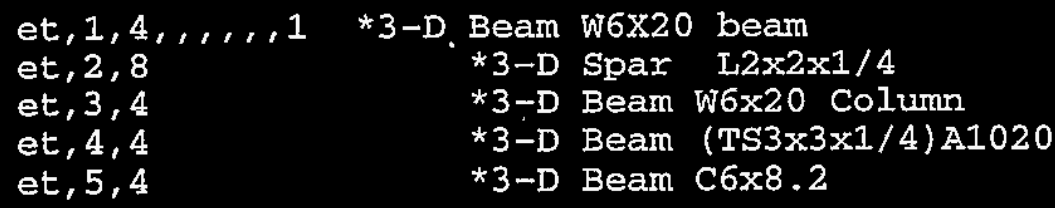

* Steel

*Mass density of steel 
$\mathrm{n}, 66,82,261,103$

$n, 67,261$

$n, 68,261,114$

$\mathrm{n}, 69,99,261$

Rev. Io

$\mathrm{n}, 70,99,261,114$

$n, 71,173,261$

$\mathrm{n}, 72,173,261,114$

Mat, 1

Type, 3

rea1, 3

*W6x20 columns

e, 1,7

e, 7,13

$e, 3,9$

$e, 9,15$

$e, 5,11$

$e, 11,17$

egen, $2,1,1,6,1$

Type, 5

real, 5

${ }^{*} \mathrm{C} 6 \times 8.2$

e, 7,8

e, 10,59

e, 59,9

e, 12,61

$e, 61,11$

$e, 59,60$

e, 60,61

e, 9,62

e, 62,11

$e, 56,57$

e, 57, 58

Type, 2

real, 2

$e, 1,8$

e, 7,14

egen, $3,2,24,25,1$

$e, 2,7$

e, 8,13

egen, $3,2,30,31,1$

$e, 9,17$

$e, 10,18$

$e, 11,15$

$e, 12,16$

Type, 1

real, 1

$e, 68,45$

e, 45,46

e, 46,47

$e, 47,70$

e, 70,48

e, 48,49

e, 49,72

e, 67,19

e, 19,20

$e, 20,21$

egen, $4,3,48,49,1$

e, 21,68

$e, 24,45$

$e, 27,46$

$e, 30,47$

$e, 22,50$

$e, 25,51$

$e, 28,52$

$e, 70,54$

*W6x20 Beams 
e, 54,69

e, 48,56

e, 56,55

WHC-SD-WM-ER-521

e, 55,53

Rev. 0

e, 72,58

$e, 58,71$

$e, 53,71$

$e, 53,69$

$e, 69,52$

$e, 52,51$

$e, 51,50$

$e, 50,67$

Type, 4

real, 4

$e, 66,31$

$e, 31,32$

$e, 65,33$

$e, 33,34$

e, 64,35

e, 35,36

e, 63,37

e, 37,38

$e, 31,33$

e, 31,35

e, 37,33

e, 37,35

$e, 32,44$

$e, 44,43$

$e, 43,34$

$e, 36,40$

e, 40,39

$e, 39,38$

e, 32,36

e, 38,34

e, 40,42

e, 42,44

$e, 39,41$

$e, 41,43$

$\mathrm{Cp}, 1, \mathrm{ux}, 19,63$ $C P, 2, u y, 19,63$

cp ; $3, \mathrm{uz}, 19,63$

$\mathrm{cp}, 4, \mathrm{ux}, 21,64$

$\mathrm{Cp}, 5, \mathrm{uy}, 21,64$

$\mathrm{cp}, 6, \mathrm{uz}, 21,64$

$\mathrm{cp}, 7, \mathrm{ux}, 28,65$

cp, 8, uy $, 28,65$

$\mathrm{Cp}, 9, \mathrm{uz}, 28,65$

$\mathrm{Cp}, 10, \mathrm{ux}, 30,66$

$c p, 11, u y, 30,66$

$\mathrm{cp}, 12, \mathrm{uz}, 30,66$

$\mathrm{cp}, 13, \mathrm{ux}, 13,67$

cp , 14, uy, 13, 67

$\mathrm{cp}, 15, \mathrm{uz}, 13,67$

$\mathrm{cp}, 16, \mathrm{ux}, 14,68$

$\mathrm{cp}, 17, \mathrm{uy}, 14,68$

$\mathrm{cp}, 18, \mathrm{uz}, 14,68$ $c p, 19, u x, 15,69$ cp, 20, uy $, 15,69$ Cp $, 21, \mathrm{uz}, 15,69$ $\mathrm{cp}, 22, \mathrm{ux}, 16,70$ $\mathrm{cp}, 23, \mathrm{uy}, 16,70$ $C P, 24, u z, 16,70$ Cp, 25, ux, 17, 71 cp, 26, uy, 17, 71 
itex, 1, 1, 1

$f, 20, f_{Y},-850$

$f, 23, f y,-2104$

$f, 26, f y,-1922$

$f, 29, f y,-1255$

$f, 54, f y,-1384$

$f, 55, f y,-809$

$f, 49, f y,-553$

$f, 57, \pm y,-553$

$\mathrm{f}, 60, \mathrm{fy},-894$

$f, 62, f y,-894$

$f, 41, f y,-3000$

$f, 42, \pm y,-3000$

acel,, 386.4

lwrite

$\mathrm{f}, 20, \mathrm{fy},-105$

$f, 23, f y,-252$

$f, 26, f y,-230$

$f, 29, f y,-150$

$\mathrm{f}, 54, \mathrm{fy},-166$

$\mathrm{f}, 55, \pm \mathrm{y},-97$

$f, 49, f_{Y},-198$

$\mathrm{f}, 57, \mathrm{fy}_{\mathrm{y}},-198$

$f, 60, f y,-371$

$f, 62, f y,-371$

$\pm, 41, f y,-3000$

$f, 42, f y,-3000$

$\$ \pm, 42, \mathrm{fx}, 600$

ace $1,-77.28,386.4,-23.18$

lwrite

$\mathrm{f}, 20, \mathrm{fy}_{\mathrm{y}},-105$

$f, 23, f y,-252$

$f, 26, f y,-230$

$\mathrm{f}, 29, \mathrm{fy},-150$

$f, 54, f_{Y},-166$

$f, 55, f y,-97$

$f, 49, f_{y},-198$

$f, 57, f_{Y},-198$

$f, 60, f y,-371$

$f, 62, f y,-371$

$f, 41, f y,-3000$

$f, 42, f y,-3000$

$\$ f, 20, E x,-21$

$\$ f, 23, \mathrm{fx},-50$

$\$ f, 26, \mathrm{fx},-46$

$\$ f, 29, f x_{x},-30$

$\$ \mathrm{f}, 54, \mathrm{Ex},-33$

$\$ \mp, 55, \mathrm{fx},-19$

$\$ f, 49$, fx, -40

$\$ \mp, 57, f x,-40$

$\$ f, 60, \mathrm{fx},-74$

$\$ f, 62, \pm x,-74$

$\$ f, 41, \mathrm{fx},-600$

$\$ f, 42, \mathrm{fx},-600$

acel, $77.28,386.4,-23.18$

Iwrite

$\mathrm{f}, 20, \mathrm{fy}_{\mathrm{y}},-105$

$\pm, 23, \pm y,-252$

$f, 26, f_{Y},-230$

$f, 29, f y,-150$

$f, 54, \mathrm{fy},-166$

$f, 55, f y,-97$

$f, 49, f_{y},-198$

$f, 57, f y,-198$

$f, 60, f y,-371$

$f, 62, f y,-371$

$\$ f, 20, f x, 6$

$\$ f, 23, \mathrm{fx}, 15$

$\$ f, 26, \mathrm{fx}, 14$

$\$ f, 29, \mathrm{fx}, 9$

$\$ \mathrm{f}, 54, \mathrm{Ex}, 10$

$\$ f, 55, \pm x, 6$

$\$ f, 49, f x, 12$

$\$ f, 57, \mathrm{fx}, 12$

$\$ \mathrm{f}, 60, \mathrm{Ex}, 22$

$\$ \mathrm{f}, 62, \mathrm{fx}, 22$

$f, 41, \pm y,-3000$

$\mathrm{f}, 42, \mathrm{fy},-3000$

$\$ \mathrm{f}, 41, \mathrm{fx}, 180$

$\$ \pm, 42, \pm x, 180$

ace $1,-23.18,386.4,-77.28$

Iwrite

$\mathrm{f}, 20, \mathrm{fy},-105$

$f, 23, f y,-252$

$\$ f, 20, \mathrm{fx}, 6$

$\$ f, 23, \mathrm{fx}, 15$
* (DL + LL) Static Analysis

$\$ f, 20, f z, 6$

$\$ f, 23, f z, 15$

$\$ f, 26, f z, 14$

$\$ f, 29, \mathbf{f z}, 9$

$\$ f, 54, \mathrm{Ez}, 10$

$\$ f, 55, \mathrm{fz}, 6$

$\$ f, 49, \mathrm{fz}, 12$

$\$ f, 57, \pm z, 12$

$\$ f, 60, \pm z, 22$

$\$ f, 62, f z, 22$

$\$ f, 41, f z, 180$

$\$ f, 42, f z, 180$

${ }^{*} \mathrm{DL}+\operatorname{Seismic}[.2 \mathrm{~g}(\mathrm{x}), \mathrm{lg}(\mathrm{y}), .06 \mathrm{~g}(\mathrm{z})]$

$\$ \mathrm{f}, 20, \mathrm{fz}, 6$

$\$ f, 23, f z, 15$

$\$ \mp, 26, f z, 14$

$\$ f, 29, \pm z, 9$

$\$ f, 54, f z, 10$

$\$ \mp, 55, \pm z, 6$

$\$ f, 49, f z, 12$

$\$ f, 57, \pm z, 12$

$\$ \pm, 60, \pm z, 22$

$\$ f, 62, f z, 22$

$\$ f, 41, f z, 180$

$\$ f, 42, \pm z, 180$

$* \mathrm{DL}+\operatorname{Seismic}[-.2 \mathrm{~g}(\mathrm{x}), 1 \mathrm{~g}(\mathrm{y}), .06 \mathrm{~g}(\mathrm{z})]$

$\$ f, 20, f z, 21$

$\$ f, 23, f z, 50$

$\$ \notin, 26, \pm z, 46$

$\$ f, 29, f z, 30$

$\$ f, 54, \pm z, 33$

$\$ f, 55, \pm z, 19$

$\$ \mp, 49, \pm z, 40$

$\$ f, 57, \mathrm{fz}, 40$

$\$ f, 60, f z, 74$

$\$ \pm, 62, f z, 74$

$\$ \mp, 41, \pm z, 600$

$\$ \mp, 42, \pm z, 600$

$*_{D L}+$ Seismic $[.06 g(x), \operatorname{Ig}(y), .2 g(z)]$

$\$ f, 20, f z,-21$

$\$ f, 23, f z,-50$ 
$\pm, 26, \mathrm{fy},-230$ $f, 29, f y,-150$ $f, 54, f y,-166$ $f, 55, \pm y,-97$ $f, 49, f y,-198$ $f, 57, f y,-198$ $f, 60, f y,-371$ $\pm, 62, f y,-371$ $f, 41, f y,-3000$ SE, $41, \pm x, 180$ $f, 42, f y,-3000$ \$f, $42, \mathrm{fx}, 180$

ace $1,-23.18,386.4,77.28$ lwrite fdele, a.l

$f, 20, f y,-119$ $\mathrm{f}, 23, \mathrm{fY}_{\mathrm{Y}},-286$ $f, 26, f y,-261$ $£, 29, f_{Y},-170$ $f, 54, f y,-188$ $f, 55, f y,-110$ $\mathrm{f}, 49, \mathrm{fy},-224$ $f, 57, f y,-224$ $f, 60, f y,-420$ $f, 62, f y,-420$ $\mathrm{f}, 41, \mathrm{fy},-3399$ $f, 42, f_{Y},-3399$ acel, 437.79 lwrite afwrite finish /input, 27 finish
$\$ \mathrm{f}, 26, \mathrm{fx}, 14$

$\$ f, 29, f x, 9$

$\$ \mathrm{f}, 54, \mathrm{fx}, 10$ $\$ \mathrm{f}, 55, \mathrm{fx}, 6$

$\$ \mathrm{f}, 49, \mathrm{fx}, 12$ $\$ \mathrm{f}, 57, \mathrm{Ex}, 12$

$\$ f, 60, \mathrm{fx}, 22$

$\$ f, 62, f x, 22$

$\$ \mathrm{f}, 41, \mathrm{fx}, 180$

$\$ f, 26, f z,-46$

$\$ f, 29, f z,-30$

$\$ f, 54, f z,-33$

\$f, $55, \mathrm{fz},-19$

$\$ E, 49, f z,-40$

$\$ \mp, 57, \pm z,-40$

$\$ f, 60, f z,-74$

$\$ \mathrm{f}, 62, \mathrm{fz},-74$

$\$ f, 41, \pm z,-600$

$\$ f, 42, f z,-600$

$\star \mathrm{DL}+\operatorname{Seismic}[.06 \mathrm{~g}(\mathrm{x}), 1 \mathrm{~g}(\mathrm{y}),-.2 \mathrm{~g}(\mathrm{z})]$
WHC-SD-WM-ER-521

Rev. 0 
/title, DOUBLE SHELI TANK MOCK-UP PLATFORM ANALYSIS

stress, ifx, 4, I \$stress, ify, 4, 2

stress, imx, 4, 4 \$stress, imy, 4, 5

stress, $\mathrm{jfx}, 4,7$ \$stress, $j \mathrm{fy}, 4,8$

stress, jmx, 4, 10\$stress, jmy, 4, 11

stress, isax, 4,13 \$stress, isby, 4,14

stress, jsax, $4,16 \$$ stress, $\mathrm{j}$ sby $, 4,17$

$$
\text { \$stress, ifz }, 4,3
$$

\$stress, imz, 4,6

\$stress, jfz, 4, 9

\$stress, $j \mathrm{mz}, 4,12$

\$stress, isbz, 4,15

stress, isg1, 4, 19 \$stress, isg3, 4, 20

\$stress, jsbz, 4, 18

stress, jsg1, 4, 21 \$stress, jsg3, 4, 22

stress, $\operatorname{sax}, 8,3$

store

set, 1

* create, mres

esel, type, 1

*w6x20 Beam

esort, isg1, , 1, 3

prstrs, isg1, ifx, ify, ifz, imx, imy, imz

esort, isg $3,1,3$

prstrs, isg3, ifx, ify, ifz, imx, imy, imz

esort, $j \operatorname{sg} 1,1,1,3$

prstrs, jsg1, jfx, jfy, jfz, jmx, jmy, jmz esort, jsg3, 1,3

prstrs, jsg3, j fx, jfy, jfz, jmx, jmy, jmz

esel, type, 2

$* \mathrm{~L} 2 \times 2 \times 1 / 4$

esort, $\operatorname{sax}, 1,3$

prstrs, sax

esel, type, 3

prrfor

esort, isg1, 1, 3

prstrs, isg1, ifx, ify, ifz, imx, imy, imz esort, isg3, 1,3

prstrs, isg3, ifx, ify, ifz, imx, imy, imz esort, jsg1, 1,3

prstrs, jsg1, jfx, jfy, jfz, jmx, jmy , jmz esort, $\mathrm{jsg} 3,, 1,3$

pxstrs, jsg3, j fx, jfy, jfz, jnx, jmy, jmz

esel, elem, $2,12,2$

prstrs, jfx, jfy, jfz, jmx, jmy, jmz

esel, type, 4

*w6x20 Column

* Reactions at column bases

esort, isg1, 1,3

*TS3 $3 \times 3 \times 1 / 4$ CMA Frame

prstrs, isg1, ifx, ify, ifz, imx, imy, imz esort, isg $3,1,3$

prstrs, isg 3 , ifx, ify, ifz, imx, imy, imz esort, $j$ sg $1,1,1,3$

prstxs, jsg1, jfx, jfy, jfz, jmx, jmy, jmz esort, $j \mathrm{sg} 3,1,3$

prstrs, jsg3, jfx, jfy, jfz, jmx, jmy, jmz esel, elem, 88,99

prstrs, ifx, ify, ifz, imx, imy, imz

prstrs, $j f x, j f y, j f z, j m x, j m y, j m z$

ese1, elem, $76,82,2$

*Forces at the 4-CMA jack bases

prstrs, ifx, ify, ifz, imx, imy, imz

esel, type, 5

* 6 6 8.2

esort, isg1, 1,3

prstrs, isg1, ifx, ify, ifz, imx, imy, imz

esort, isg $3,1,3$

prstrs, isg 3 , ifx, ify, ifz, imx, imy, imz esort, $j$ sg1, 1,3

prstrs, jsg1, jfx, jfy, jfz, jmx, jmy, jmz esort, jsg3, 1,3

prstrs, jsg3, jfx, jfy, jfz, jmx, jmy, jmz

* end

loutp, static

/title,DST MOCK-UP PLATFORM STATIC ANAIYSIS

*use, mres

/outp, seismx

$A-20$

the top of columns

\author{
Cur trame
}


set, 2

/title,DST MOCK-UP PLATFORM SEISMIC ANALYSIS [DL + $0.2 \mathrm{~g}: \mathrm{x}+.06 \mathrm{~g}: \mathrm{z}] \quad$ WHC-SO-HM-ER-521
*use, mres

loutp, seism-x

set, 3

/title,DST MOCK-UP PLATFORM SEISMIC ANALYSIS [DL $+0.2 \mathrm{~g}:-\mathrm{x}+.06 \mathrm{~g}: \mathrm{z}$ ]

*use, mres

loutp, seismz

set, 4

/title,DST MOCK-UP PLATFORM SEISMIC ANALYSIS. [DL +.06g: $\mathrm{x}+.2 \mathrm{~g}: \mathrm{z}]$

*use, mxes

loutp, seism-z

set, 5

titile, DST MOCK-UP PLATFORM SEISMIC ANALYSIS [DL $+.06 \mathrm{~g}: \mathrm{x}+.2 \mathrm{~g}:-z]$

*use, mres

loutp, seismy

set, 6

/title,DST MOCK-UP PLATFORM SEISMIC ANALYSIS [DL +.133g:y]

*use, mres

loutp, model

/title, DST MOCK-UP PLATFORM FRAME MODEL

set, 1

nali

eal1

nlist

elist

finish 


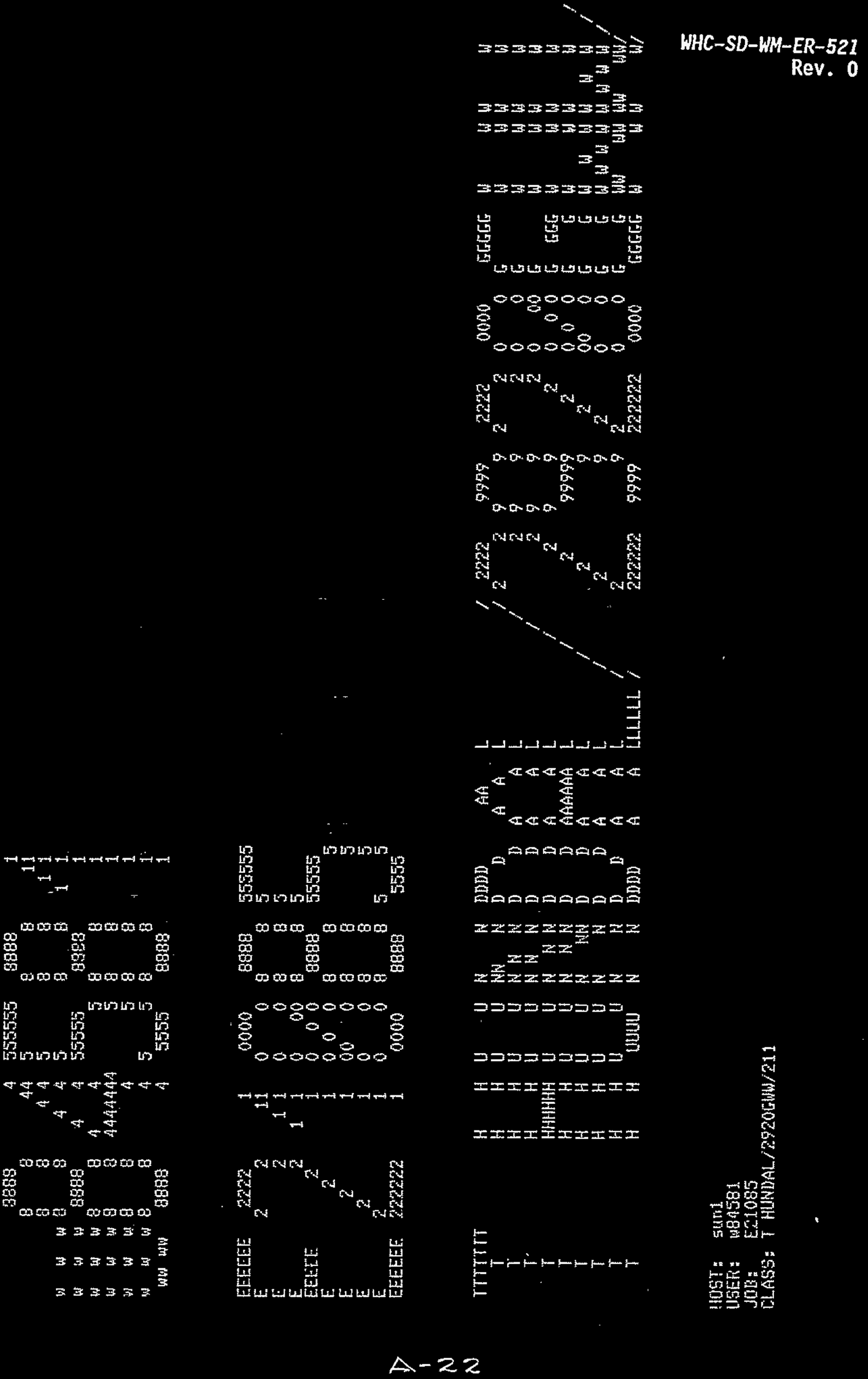


AHSYS - EHGIHEEFEHE AHALYSIS, GYSIEM FEUISIOH 4.4

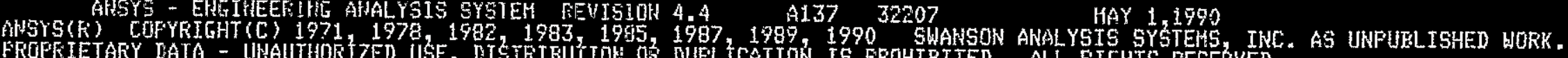

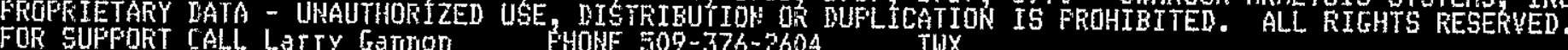

15. 1.264 AUG $1,1995 \mathrm{CF}=$

1.600
WOHE $X$

$.00000000 \mathrm{E}+00$ $.00000000 \mathrm{E}+00$ 79.000000 99.000000 173.00000 173.00000 $.00000000 E+00$ $.00000000 \mathrm{E}+00$ 97.000000 99.000000 173.00000 173.00000 $.00000000 E+00$ $.00000000 E+00$ 97.000000 99.000000 $77 \times 000000$
173.00000 173.00000 $.00000000 E+00$ $.00000000 E+00$ $.00000000 E+00$ 25.000000 25.900000 25,000000 62.000000 62.000000 62.000000 62.000000 82.000000 82.000001 82.000000 2.000000 82.000000 82.000000 82.000000 .06000000E +00 $.00000000 E .00$ $.00000000 E+00$ .00000000E +00 $.00000000 E+00$ $.00000000 \mathrm{E}+00$ 41.000000 41.000000 82.000000 82.000000 25.000090
$.00000000 E+00$ $00000000 \mathrm{E}+00$ $.000007000+00$ $.00000000 E+00$ $.00000000 \mathrm{E}+00$ $.00000000 E+00$ 129.00000 $12 \% .00000$ $12 ? .00000$ 129.00000 127.00000 129.00000 261.00000 261.00000 281.00000 281.00000
261.00000 261.00000 261.00000 261.00000 261.00000 261.00000 261.00000 ist.00000 261.00000 261.00000 261.00000 261.00000 261.00000 261.00000 261.00000 365.00000 413.00000 365.00000 413.00000 365.00000 0000 413.00000 413.00000 413.00000 413.00000 413.00000 417.00000 251.00000
$.00000000 E+00$ 114.00000 $.00000000 \mathrm{E}+00$ 114.00000

$.00000000 E+00$ 114.00000

$.60000000 \mathrm{E}+00$

114.00000

$00000000 \mathrm{E}+00$ 114.00000

.00000000 E 100

$1 \pm 4.00000$

$.00000000 \mathrm{E}+00$

114.00000

$.00000000 \mathrm{E}+00$

1.14 .00000

$.00000000 \mathrm{E}+00$

114.00000

30.000000

57.000000

103.00000

30.000000

57.000000

103.00000

30,000000

57000000

103.00000

30.000000

57.000000

$103 n 00000$

1.03 .00000

103.00000

30.000600

30.000000

103.00000

103.00000

30.000000

30.000000

54.000000

$7 \% .000000$

54.000000

79.000000

54.000000

79.000000

114.00000
THXY

THYZ

$00000000 E+00$ $.00000000 \mathrm{E}+00$ $.00000000 E+00$ $.00000000 E+00$ $00000000 E+00$ $00000000 E+00$ $00000000 \mathrm{E}+00$ $00000000 E+00$ $.00000000 \mathrm{E}+00$ $.00000000 E+00$ $.00000000 E+00$ $.00000000 E+00$ $00000000 \mathrm{E}+\mathrm{TO}$ $.00000000 \mathrm{E}+00$ $.00000000 \mathrm{E}+00$ $00000000 E+00$ $.00000000 E+00$ $.00000000 E+00$ $.00000000 \mathrm{E}+00$ $.00000000 \mathrm{E}+00$ $00000000 \mathrm{E}+00$ $00000000 \mathrm{E}+00$ $00000000 E+00$ $.00000000 \mathrm{E}+00$ $.00000000 E+00$ $.00000000 \mathrm{E}+00$ $.00000000 E+00$ $.00000000 \mathrm{E}+00$ $00000000 E+00$ $.00000000 E+00$ $.00000000 \mathrm{E}+00$ $.00000000 E+00$ $.00000000 E+00$ $.00000000 \mathrm{E}+00$ $.10000000 \mathrm{E}+00$ $00000000 \mathrm{E}+00$ $00000000 E+00$ $.00000000 E+00$ $.00000000 E+00$ $-00000000 \mathrm{E}+00$ $.00000000 E+00$ $.00000000 \mathrm{E}+00$ $00000000 \mathrm{E}+00$ $00000000 \mathrm{E} \cdot 00$ $.00000000 \mathrm{E}+00$
$.00000000 E+00$ $.00000000 E+00$ $.00000000 \mathrm{E}+00$ $.00000000 E+00$ 00000000 E +00 $.00000000 E+00$ $.00000000 \mathrm{E} \cdot 00$ - $00000000 \mathrm{E}+00$ $-00000000 E+00$ - $00000000 \mathrm{E}+00$ $.00000000 \mathrm{E}+00$ $.00000000 \mathrm{E}+00$ $.00000000 \mathrm{0}+00$ $.00000000 E+00$ $.00000000 E+00$ $.00000000 E+00$ $.00000000 E+00$ $.00000000 \mathrm{E}+00$ $00000000 \mathrm{E}+00$ $.00000000 \mathrm{E}+00$ .00000060 E t 00 - DOCDOC + 00 $.00000000 \mathrm{E}+00$ $.00000000 \mathrm{E}+00$ $.00000000 \mathrm{E}+00$ $.00000000 \mathrm{E}+00$ $.00000000 \mathrm{E}+00$ $.00000000 \mathrm{E}+00$ .00000000 E 300 $.00000000 E+00$ $.00000000 E+00$ $.00000000 E+00$ .00000000E+00 $.00000000 \mathrm{E}+00$ $.00000000 \mathrm{E}+00$ $.00000000 \mathrm{E}+00$ $.00000000 \mathrm{E}+00$ .000000000 E 00 $.00000000 E+00$ $.00000000 \mathrm{E}+00$ $00000000 \mathrm{E}+00$ $.00000000 E+00$ $.00000000 \mathrm{E}+00$ , $00000000 \mathrm{E}+00$

\section{THXZ}

$.000000000 \mathrm{E}+00$ $.00000000 \mathrm{E}+00$ $.00000000 E+00$ $.00000000 E+00$ .00000000 E +00 $.00000000 \mathrm{E}+00$ $.00000000 E+00$ $.00000000 E \div 00$ $.00000000 E+00$ $.00000000 E+00$ $.00000000 E+00$ 00000000 E +00 $00000000 \mathrm{E}+00$ $00000000 \mathrm{E}+00$ $.00000000 E+00$ .00000000 E +00 $.00000000 \mathrm{E}+00$ $.00000000 \mathrm{E}+00$ $.00000000 \mathrm{E}+00$ 00000000 E +00 00000000 E +00 .00000000 E +00 $.00000000 E+00$ $.00000000 E+00$ $.00000000 \mathrm{E}+00$ .00000000 E +00 00000000 E+00 .00000000 E +00 .00000000 E +00 - 00000000E:00 $.00000000 \mathrm{E}+00$ .00000000 E +00 -ODOO0000E +00 .00000000 E +00 .00000000E +00 $.000000000+00$ $.00000000 E+00$ $.00000000 E+00$ - 00000000 E +00 .00000000 E + 00 .00000000 E +00 00000000 E + 00 $00000000 E+00$ .00000000E t00 
AHETS - EHGIHEEFIKG A

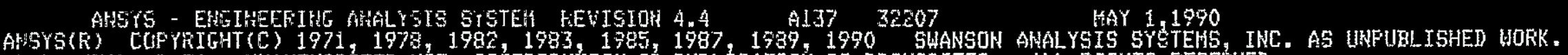

HAY 1,1990

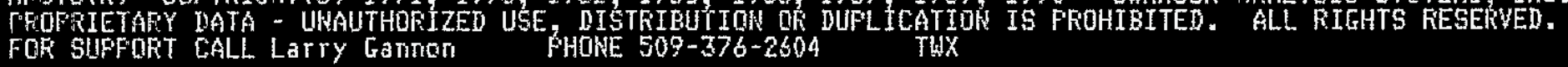

DGUUELE BHELL TAHK HOCK-UJP FLATFORH

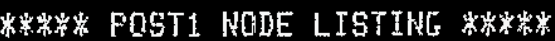

NODE X

Y

7

$\begin{array}{ll}46 & 62.000000 \\ 47 & 82.000000 \\ 48 & 1.2 .00000 \\ 49 & 148.00000 \\ 50 & 23.000000 \\ 51 & 62.000000 \\ 52 & 92.000000 \\ 53 & 123.00000 \\ 54 & 99.000000 \\ 55 & 123.00000 \\ 56 & 123.00000 \\ 57 & 148.00000 \\ 58 & 173.00000 \\ 59 & 99.000000 \\ 60 & 136.00000 \\ 61 & 173.00000 \\ 67 & 136.00000 \\ 63 & .00000000 E+00 \\ 64 & 00000000 E+00 \\ 65 & 82.000000 \\ 66 & 82.000000 \\ 67 & .00000000 E+00 \\ 68 & 00000000 \mathrm{E}+00 \\ 69 & 99.000000 \\ 70 & 97.000000 \\ 71 & 173.00000 \\ 72 & 17.00000 \\ 6 & \end{array}$

PRIHT REHEFIT LISTING

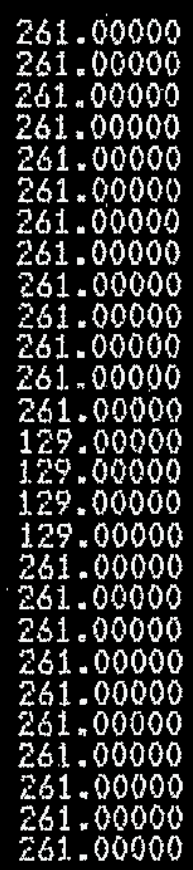

261.00000
261.00000
261.00000
261.00000
261.00000
261.00000
261.00000
261.00000
261.00000
261.00000
261.00000
261.00000
129.00000
1.29 .00000
127.00000
129.00000
261.00000
261.00000
261.00000
261.00000
261.00000
261.00000
261.00000
261.00000
261.00000
261.00000

THXY

THYZ

$00000000 E+00$ $.00000000 E+00$ $00000000 \mathrm{E}+00$ $.00000000 \mathrm{E}+00$ $.00000000 \mathrm{E}+00$ $.00000000 \mathrm{E}+00$ $.00000000 \mathrm{E}+00$ $.00000000 \mathrm{E}+00$ $.00000000 E+00$ $.00000000 \mathrm{E}+00$ $.00000000 \mathrm{E}+00$ $.00000000 \mathrm{E}+00$ $.000000000 \mathrm{E}+00$ $.00000000 \mathrm{E}+00$ $.00000000 E+00$ $.00000000 E+00$ $.00000000 E+00$ 0000000 E - 0000000 e $.00000000 E+00$ $.00000000 E+00$ $.00000000 \mathrm{E}+00$ $.00000000 E+00$ $.00000000 \mathrm{E}+00$ 0000000 E +00 . $0000000 \mathrm{E}+0$ .0000000 E +00 $.00000000 \mathrm{E}+00$
$.00000000 \mathrm{E}+00$ $00000000 E+00$ $.00000000 \mathrm{E}+00$ $.00000000 \mathrm{E}+00$ $.00000000 E+00$ $.00000000 E+00$ $.00000000 E+00$ $.00000000 E+00$ $.00000000 E+00$ $.00000000 E+00$ $.00000000 \mathrm{E}+00$ $.00000000 E+00$ $.00000000 E+00$ $.00000000 E+00$ $.00000000 E+00$ $.00000000 E+00$ $.00000000 E+00$ $.00000000 \mathrm{E}+00$ * $.00000000 \mathrm{E}+00$ $.00000000 \mathrm{E}+00$ $.00000000 E+00$ $.00000000 E+00$ $.00000000 \mathrm{E}+00$ $.00000000 \mathrm{E}+00$ $.00000000 E+00$ $.00000000 \mathrm{E}+00$
THXZ

$.00000000 E+00$ $.00000000 E+00$ $.00000000 E+00$ $.00000000 \mathrm{E}+00$ $00000000 E+00$ $.00000000 E+00$ $.00000000 E+00$ $.00000000 E+60$ $.00000000 E+00$ $.00000000 E+00$ $00000000 \mathrm{E}+00$ - 00000 E $.00000000 E+00$ $-00000000 E+00$ $.00000000 E+00$ .00000000 E +00 $.00000000 \mathrm{E}+00$ $00000000 \mathrm{E}+00$ .00000000 E +00 .00000000 E +00 $.00000000 E+00$ $.00000000 E+00$ $\because 00000000$ E 400 .00000000 E +00 $.00000000 \mathrm{E}+00$ $.00000000 \mathrm{E}+00$ $.00000000 \mathrm{E}+00$ 
Rev. 50

8

害虽

Put

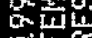

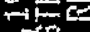

-

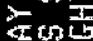

50

전

zo

분

4

5

要

9

产

M

Bsis

w

M

$\mathrm{P}^{2}$

战我

in

4to $\operatorname{los}_{0 \rightarrow 1}$

$\mathrm{st}_{-1}=\frac{1}{2}$

1

$\cos =$

षita

类管

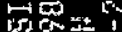

$\Leftrightarrow \rightarrow$ 声

$x_{i}=m$

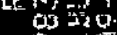

$x \rightarrow=$

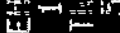

i.r.

in

is ${ }^{m}$

$\rightarrow \infty=$

30

I 34

称

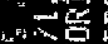

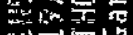

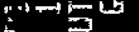

i.

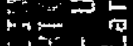

$=1$

- $\because$ 匠声

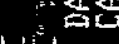

$\therefore:$

$\therefore$ 踏

$=i$

$\because \frac{w}{4}$

$\because$ 政

“点

00000000000000000000000

$\sin 000$

雚

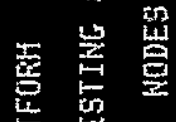

西

는

? 空 案

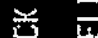

空

$\times \quad$ 约

$\Rightarrow \quad \underline{I}$

$\stackrel{2}{\prime}$

* 5

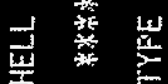

出 夏

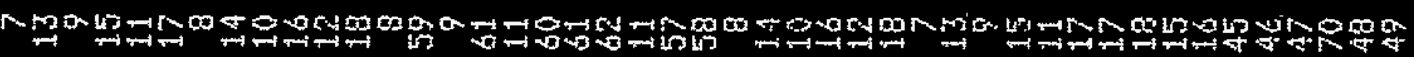

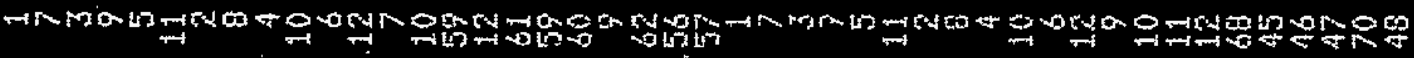

000000000000000000000000000000000000000000000

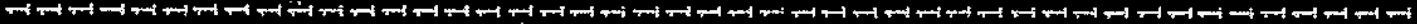

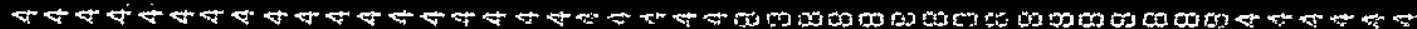

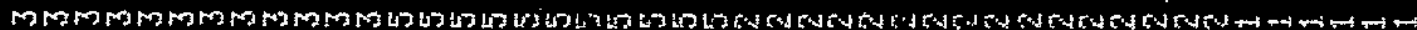
s.

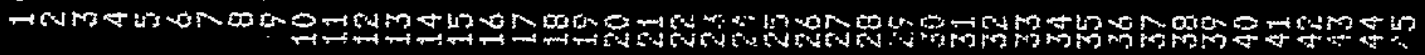

$$
A-25
$$


000000000000000000000000001000000000000000000

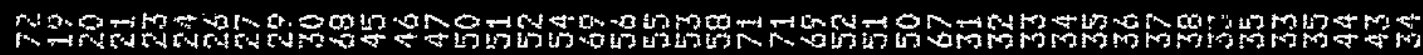

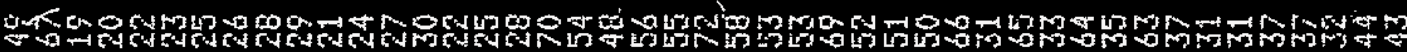

000000000000000000000000000000000000000000000

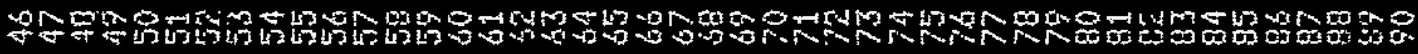


8

co

通

본

인

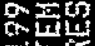

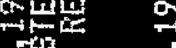

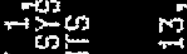

똔도

罗

,

定标

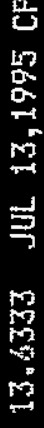

总会 学

s

格

oi

Din

wis

鯧象

可的事

$x$

:

$\sin 8$

75

我俄

of

궁요

的的

cose

w

$\mathrm{cos}^{2-4}$

nos

$\rightarrow \lim _{\substack{0 \\ 0}}$

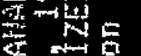

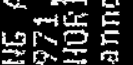

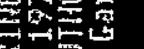

可

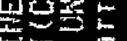

둔:

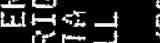

1

45

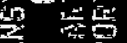

政安

tore on

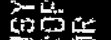

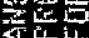

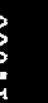

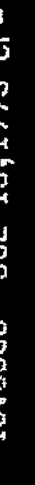

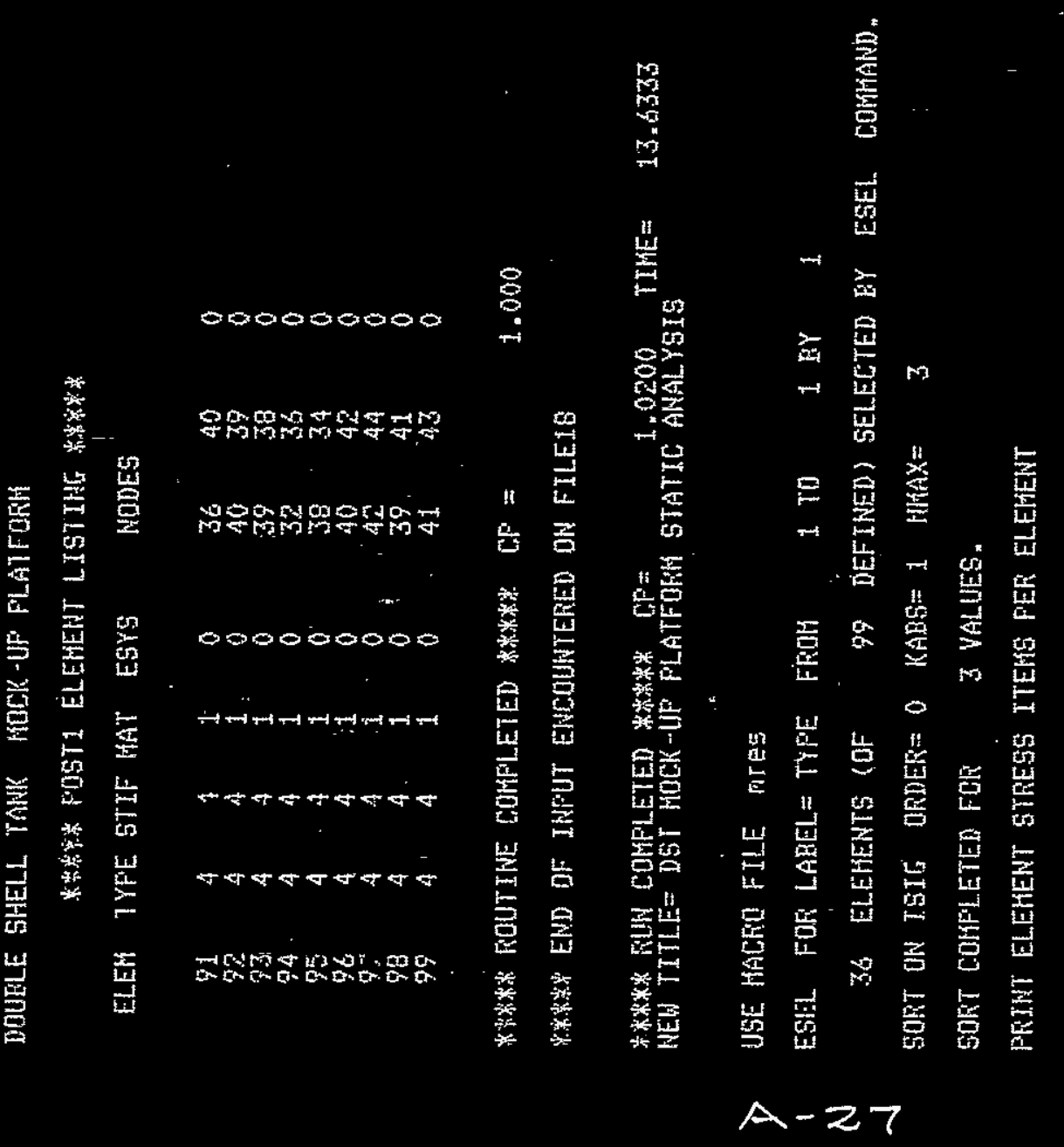



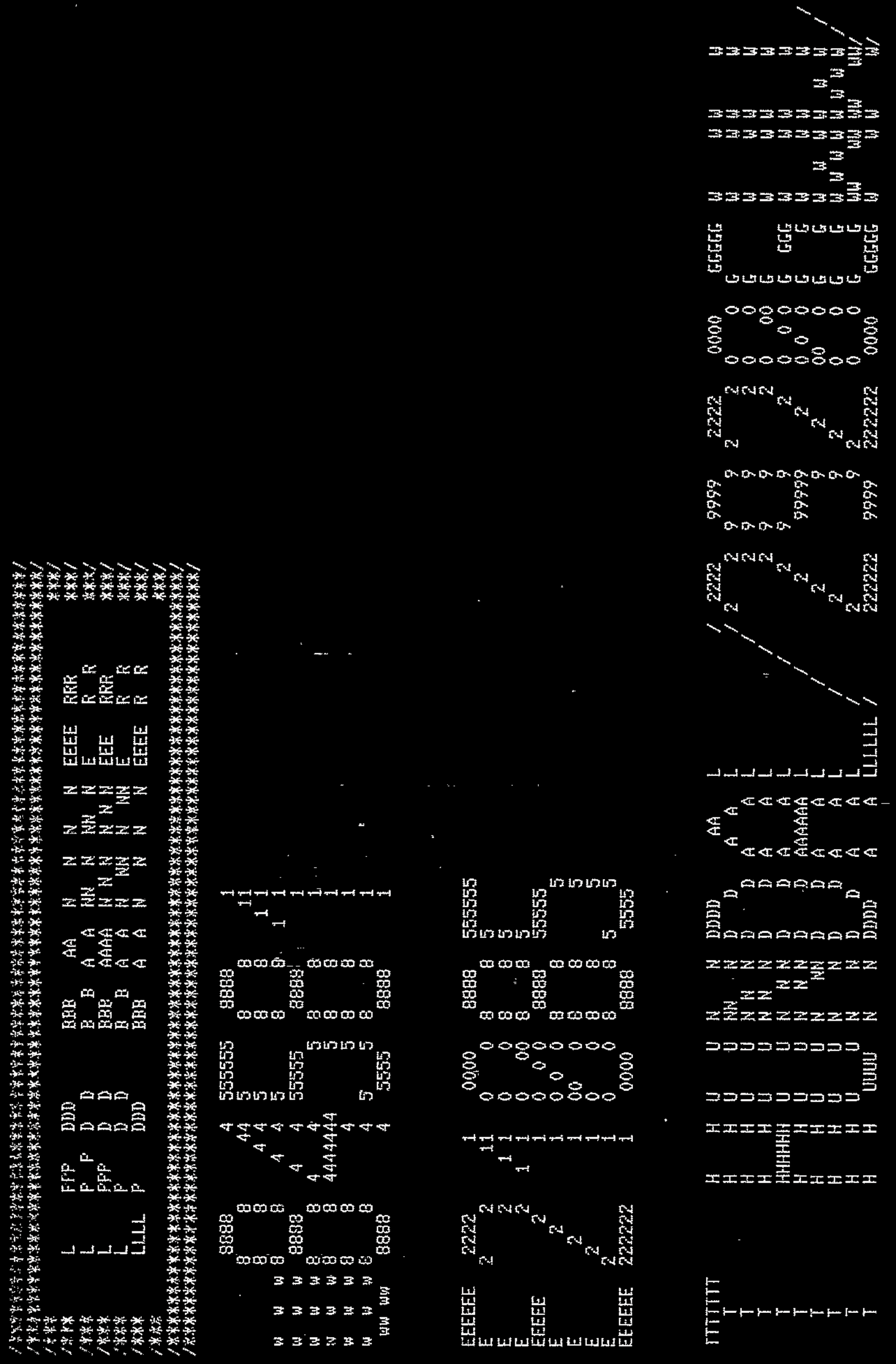
WHC-SD-WM-ER-521

Rev. 0

$\frac{2}{2}$

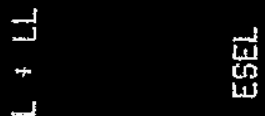

南 $\quad \rightarrow$ 学

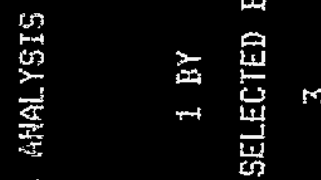

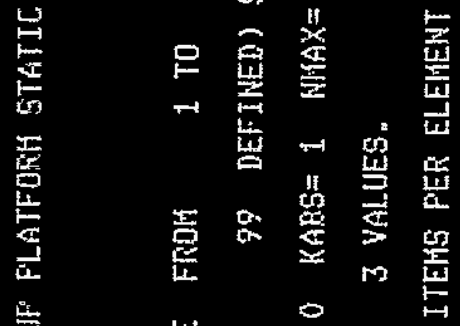

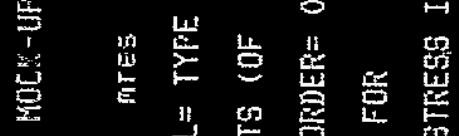

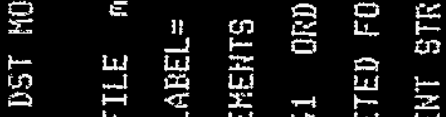

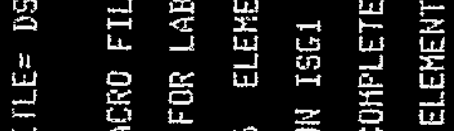

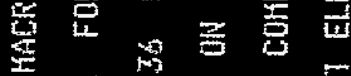

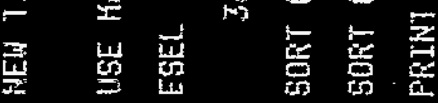

$A-29$ 
总,

g

焉桌

원

맨단

${ }_{-10}^{-12}$

-

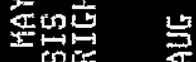

竞素

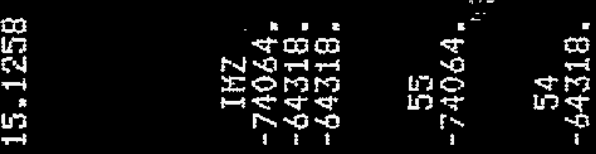

话垈

今

空

证

옵

4

峞

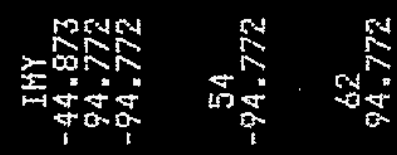

arts

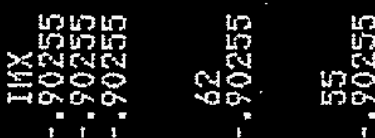

굴둥웡

본준

ind

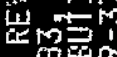

象幽品

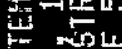

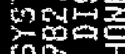

$a^{24}$

出

vos

匍

禹要

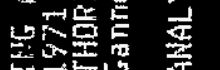

$\rightarrow \rightarrow 5$

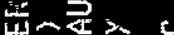

불돈

본도에

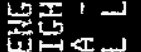

, 잔다

音的

는

织

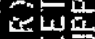

6ै

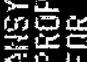

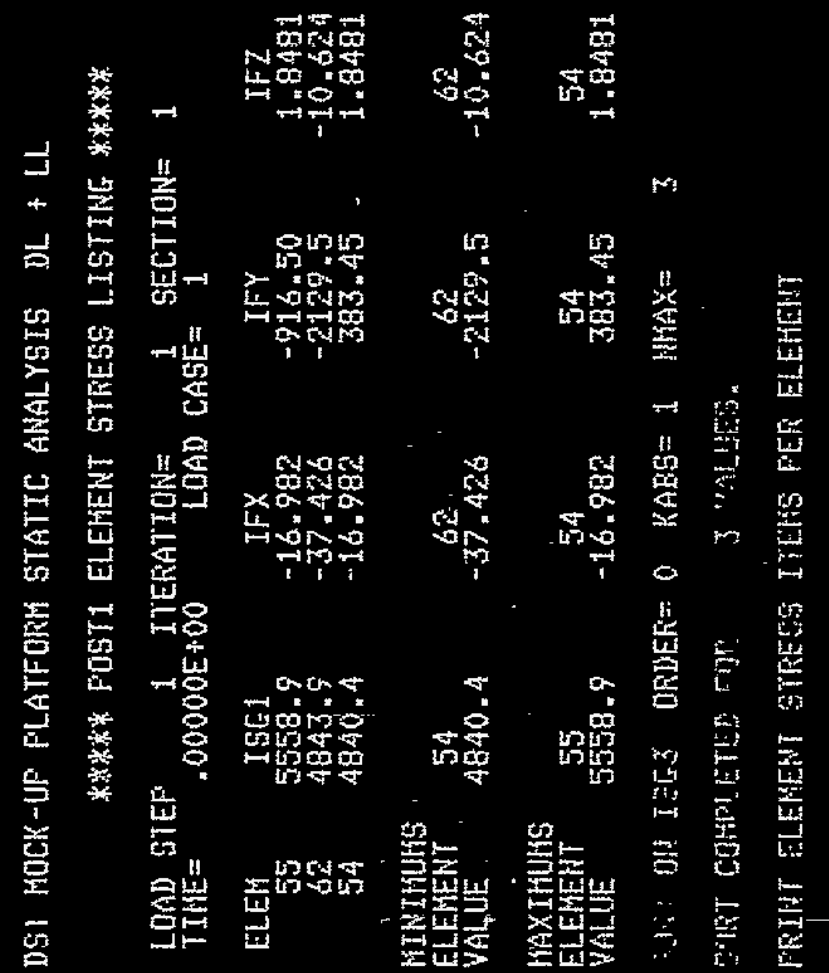




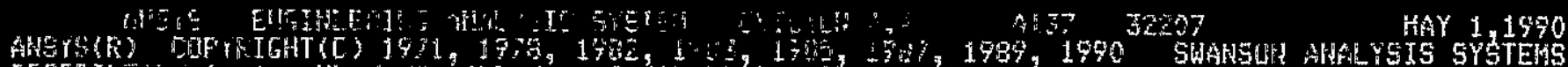

TFOFEIET

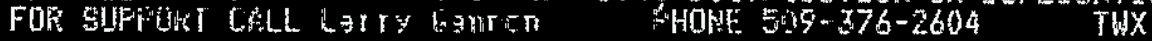

DST MOCK -UF PLATFORH STATIC, AHALYSIS DL + LL

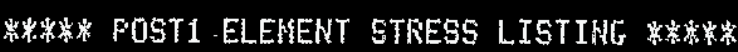

LOAD STEP $\quad 1$ ITERATION= $\frac{1}{1}$ SECIIOH $=1$

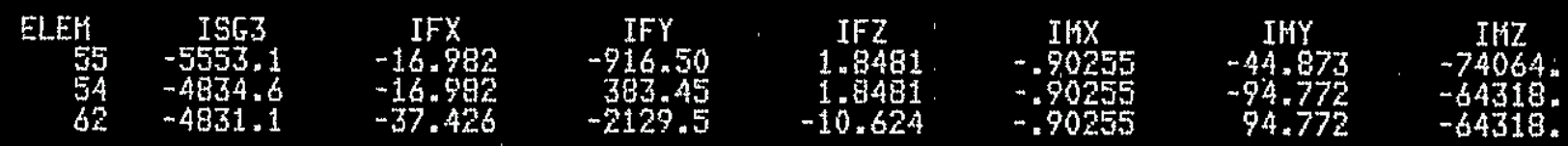

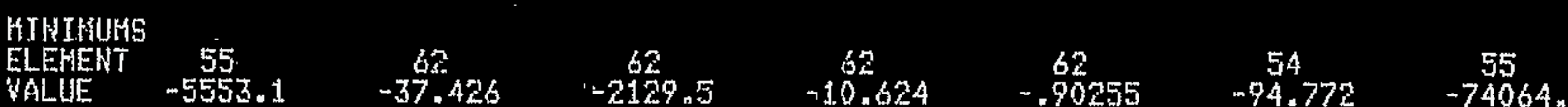

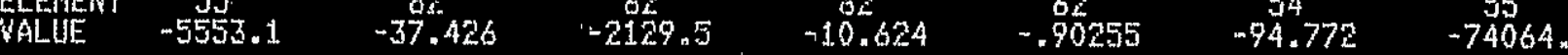

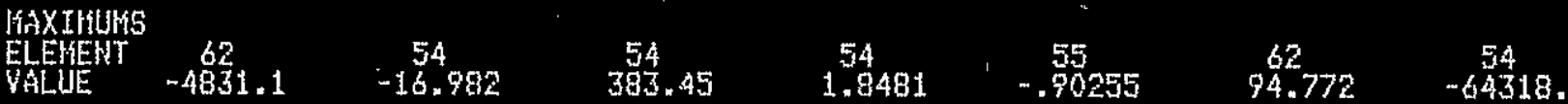

SORT ON JGG 1 QRDER $=0 \quad$ KABS $=1$ HHAX $=3$

SORT COHPLETED FOR 3 VALUES.

FRTHT ELEHEHT STFESS ITEHS FER ELEHENT 
8

贸

昰望

문

Pon

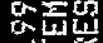

$\rightarrow-1$

का का का

实证

븐도

焉

공효

骂

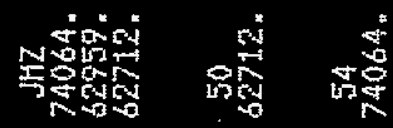

$1 \frac{5}{3}$

的空

लि

初

음

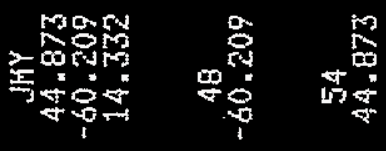

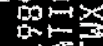

${ }^{\infty}$

때뭉

농용

$\frac{d}{c}$

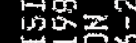

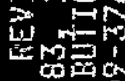

때요

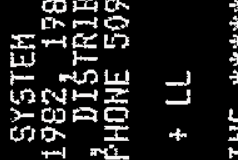

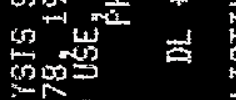

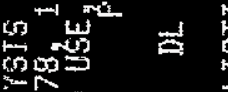

的焉

象茄

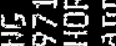

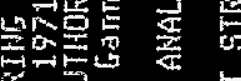

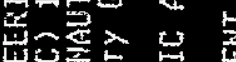

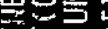

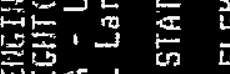

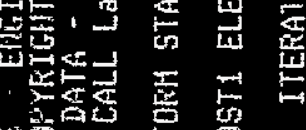

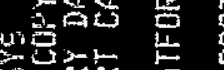

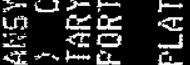

네요

bx

可密

$\frac{5}{3}$

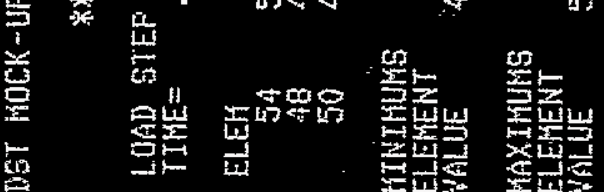

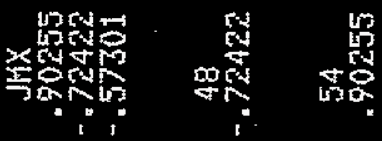

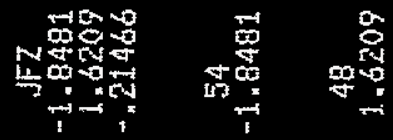

눌

F

topan

Mint

용

$m$

范

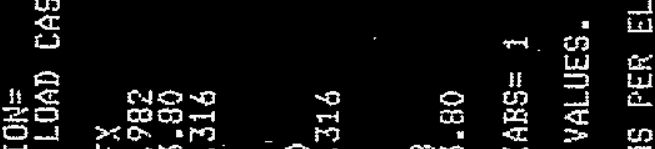

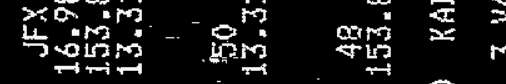

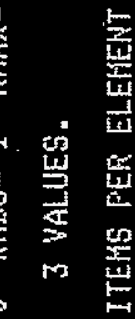

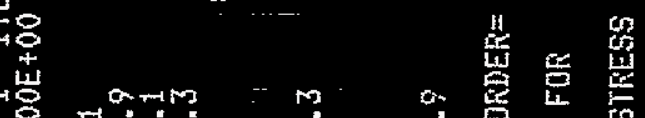

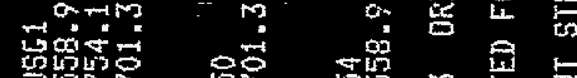

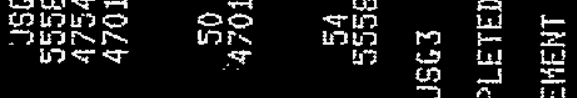

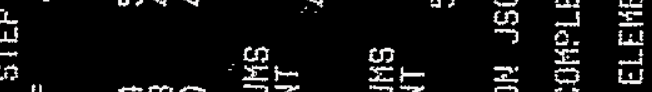

点 点 
th

$\sum_{i=1}$

$\rightarrow$

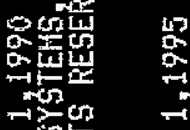

$\rightarrow$ cor

玨时旨

整严

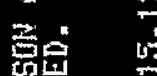

苟

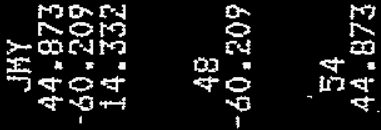

$\rightarrow a$

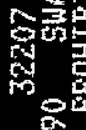

$\alpha_{0 \rightarrow n}^{2}$

음응

tis

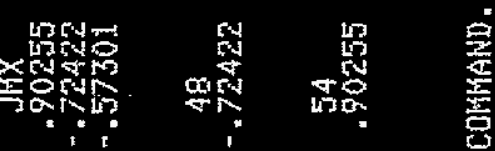

\&田

돈

this

3 r

14

$\cos _{0}$

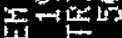

town

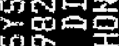

63 ,

is w

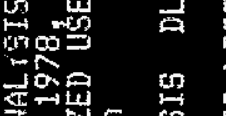

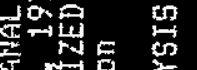

包贶富

武些要

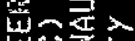

넌든돈든

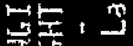

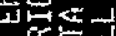

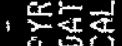

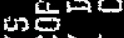

4 동

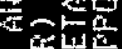

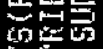

驾总

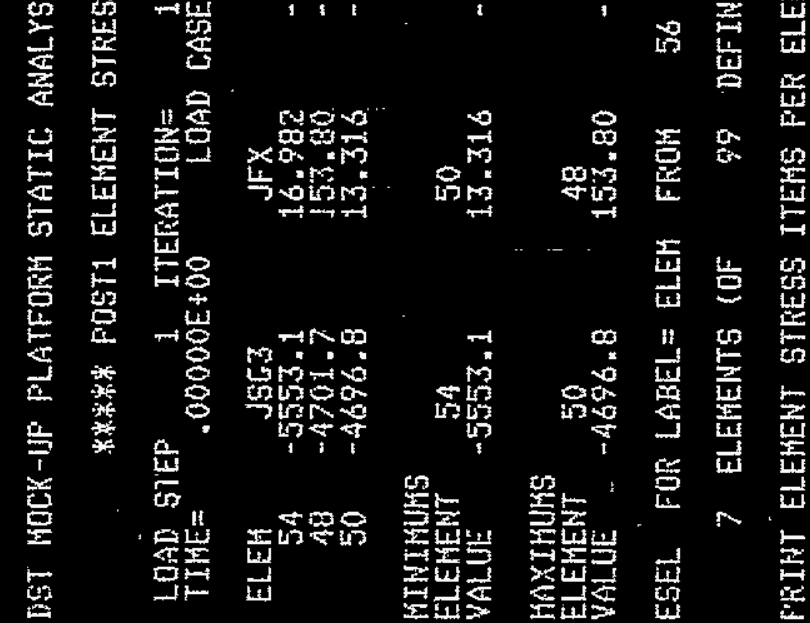


$\underset{7}{7}$

哭

密要

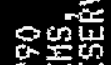

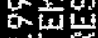

$\rightarrow \rightarrow=$

$\rightarrow i_{\mathrm{tin}}$

可的密

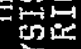

$\frac{5}{4}$

78070

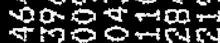
N⿴囗十丁: 500 mois a da

की

$\frac{1}{2}$

嵒

곰ㅂㅂㅂ

4apsiga

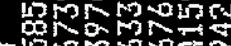

soming

intw

要

욤

要出

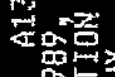

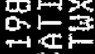

amorame

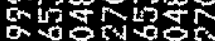

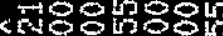

s.mon

Noros

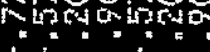

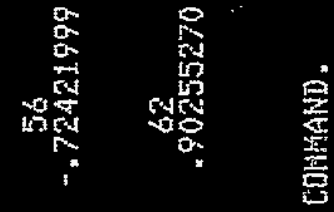

紐制总

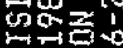

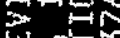

뭉ํำ

월

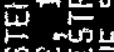

tox

wis

崖然

崖然

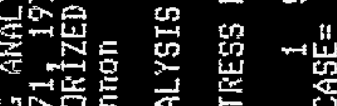

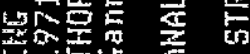

$\rightarrow \rightarrow 5$

出的

空

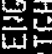

단단

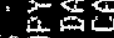
is

然

c5ta

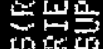

岛能

政

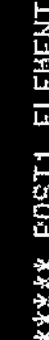

索

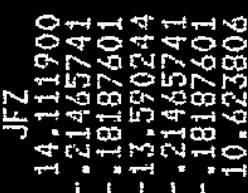

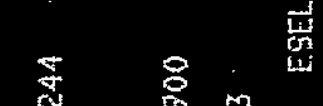

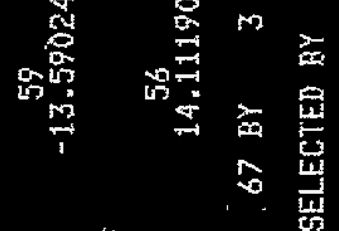

t8 -140

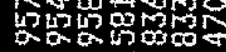

L

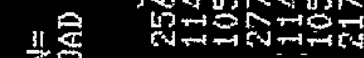

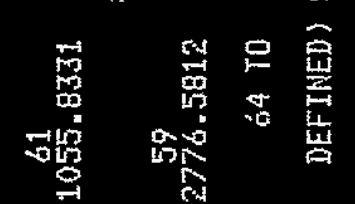

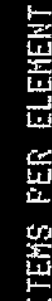

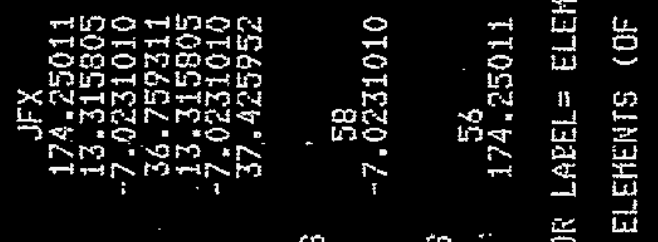

点

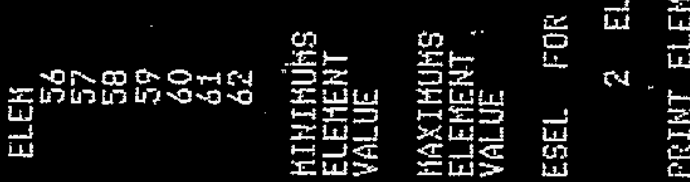

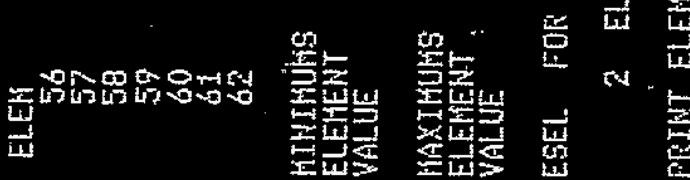


密高 竞

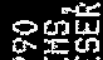

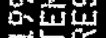

it

에

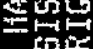

岸

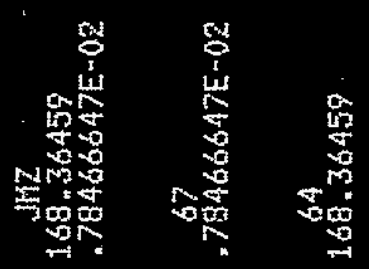

变步

哭

喜空

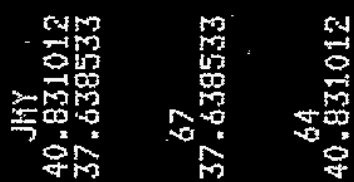

象

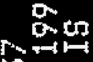

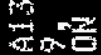

여을

$\rightarrow$ 송

N-1

象铻

+7 品

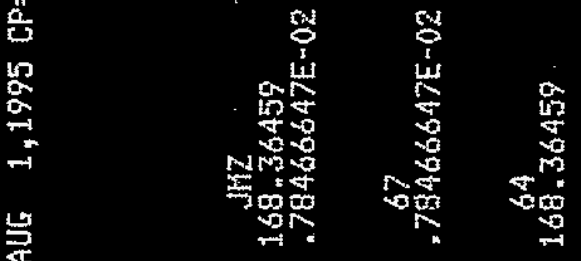

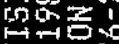

$=15$

ma

두요요

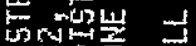

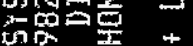

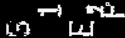

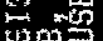

$\rightarrow$ in

到悬

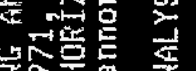

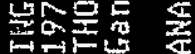

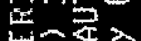

분돈는

年, 出

Int

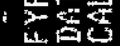

in

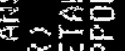

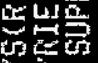

知总皆

点

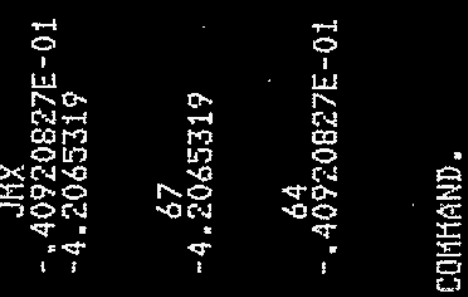

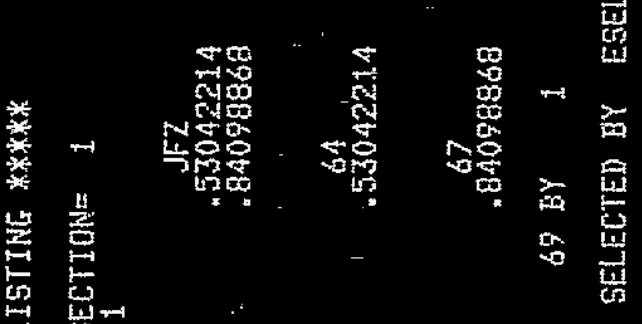

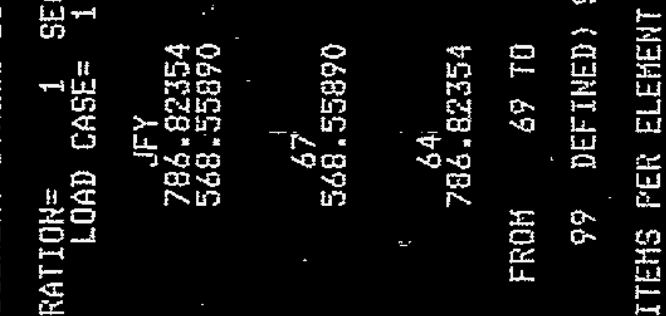

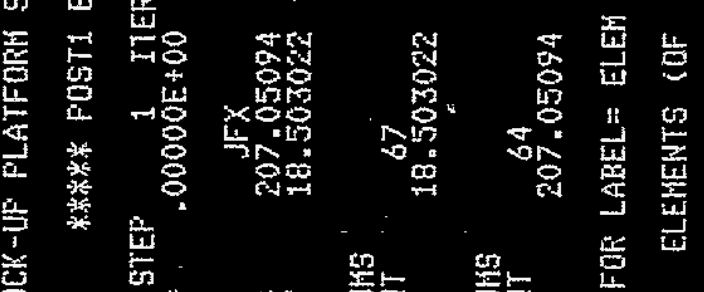

高

翣

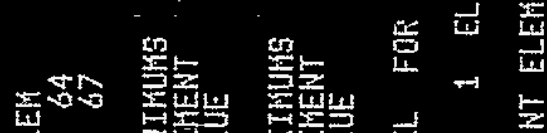

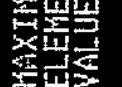

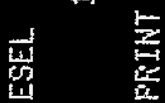


FHEY - EIGIHEERIHG AUALYSTS GYGTEM FEVIGIDH 4.3

A137 32207

MAY 1,1990

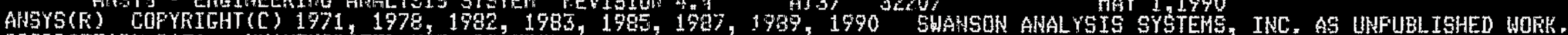
FROFIETARY DATA - UWAUTHOKIZED USE, DISTRIEUTION ON DUFLLCATIDK IS PRDHIBITEN, ALL RIGHTS RESERVED.

FOK SUFPORT CALL LaTrY Gannor FHONE 509-376-2604 TUX

DST HOCK-UF FLATFORH STATIC AHALYSIS DL + LL

15.1258 AUt $1,1995 \mathrm{CF}=$

.430

****** POST1 ELEHENT STRESS LISTINL $* * * * * *$

LOAD STEF 1 ITERATION= $\frac{1}{\text { TIME }}=$ SECTION= 1

$\begin{array}{ccccccc}\text { ELEH } & \text { JFX } & \text { JFY } & \text { JFZ } & \text { JWK } & \text { JHY } & \text { JHZ } \\ 89 & -10.764487 & 164.73693 & 2.1267978 & 19.390014 & 106.52648 & 30.013878\end{array}$

HINIMULIS

$\begin{array}{lccc}\text { ELEMENT } & 69 & 69 & 69 \\ \text { VALUE } & -10.764487 & 164.73693 & 2.1267\end{array}$

(1)

HAXIMUHS

ELELUER

$-10.764487$

164.73693

2.1267978

$19^{69} .390014$

106.52648

69

ESEL FOR LABEL = ELEN FROM

47 To

$47 \mathrm{BY} 1$

19.390014

106.52648

1. ELEHENTS (OF 99 DEFINED) SELECTED BY ESEL COHMAHD.

PRIM1 ELEEENT STRESS IIEHS PEF ELEHENT 
是

th

$\sum_{i=1}^{n}$

站

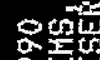

aw

$\rightarrow$

的㱐

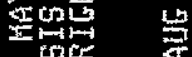

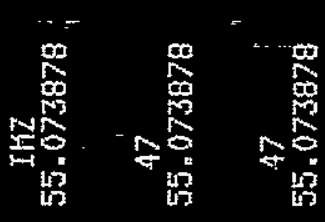

$\frac{1}{\frac{1}{x}}$

品

营局骂

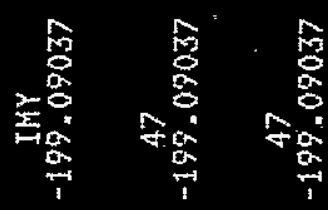

$\mathrm{sin}^{-1}$

为

总地

40

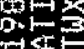

$\rightarrow$ 造

की

证

공

tis

$\lim _{\rightarrow \rightarrow+\infty}$

if

무용

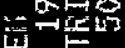

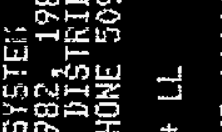

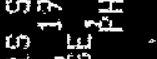

的的点

50

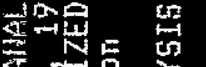

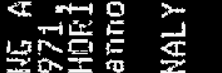

$\rightarrow-5$

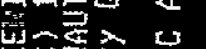

뻔온

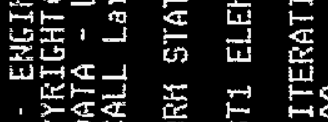

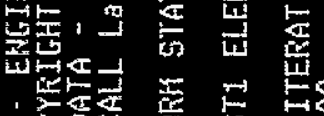

总

in

tion

5

엔대

togen

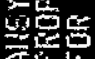

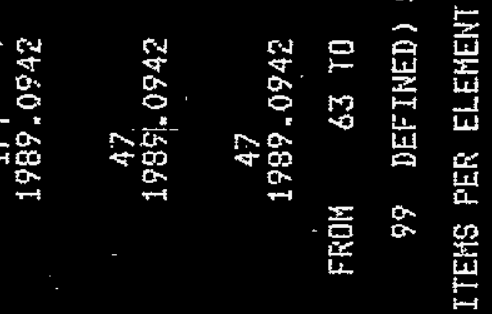

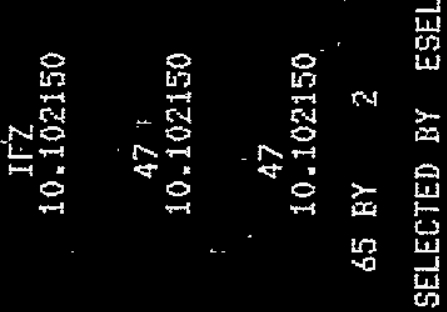

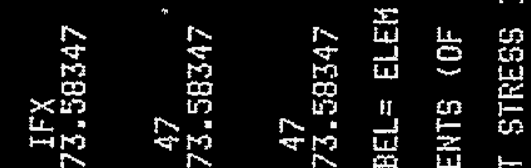

生菜 兵

$\frac{1}{3} * \frac{11}{13}$

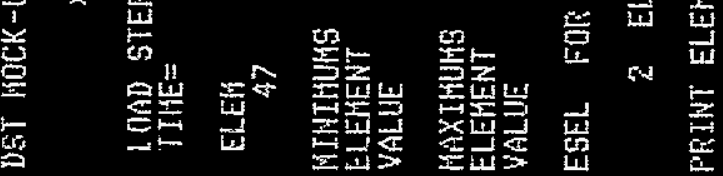


AHIGYS - ENG IHEERTHE AHALYSIS SYSTEH REVISTOH 4.4

A137 32207

HAY 1,1990

AHSYS(F) COFYKIGHT(C) $1971,1978,1982,1983,1985,1987,1989,1990$ SWANSON AHALYSIS SYSTEMS, INC. AS UNNPUELISHED WDRH, FFOFFIETARY DATA - UAAUTHORTZED USE, ISISTKIEUTION OR DUFLICATION IS FROHIBITED. ALL RIGHTS RESERVED.

FOR SUFFIJRT CALL LarTy Gaman FHOHE 509-376-2604 THX

DST HOCK-UF FLATFOFH STATIC ANALYSIS DL + LL

15.1258 AUG $1,1995 \mathrm{CP}=$

.430

****** FOST1 ELEKENT STRESS LISTING * * ****

LOAD STEF
TIME $=$
.00000 ITERATION=

ELEH IFX IFY IFZ

$63-207.05094 \quad 786.96769 \quad-.5304221$

$65 \quad-18.970191 \quad 722.58625 \quad-4.2780505$

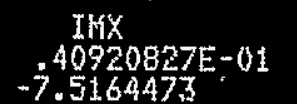

IHY 19,67111

$\operatorname{IMZ}$

$\begin{array}{rr}139.22089 & -160.14811 \\ 1.6715960\end{array}$

HINIKUMS

ELEMEHT

$-207.05094, \quad 722.58625$

$-4.2780505$

$-7.55164473$

19.637111

63

HAXIMUHS

ELEME

65
-19.970191

786.96767

$-635$

4092

65

ESEL FOR LABEL= ELEH FKOH $69 \mathrm{TO}$

68 EY 1

1 ELEHENTS (DF 99 DEFINED) SELECTED BY ESEL COHAND.

FRINT ELEHEHT STRESS ITEHS FER ELEHENT 


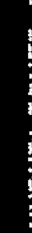

焉

윤

:

总早

ong

는

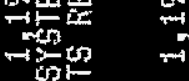

$>\cos$

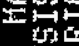

营

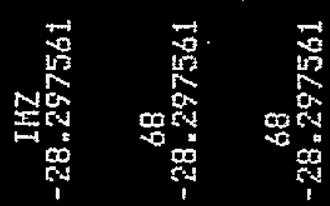

줄단

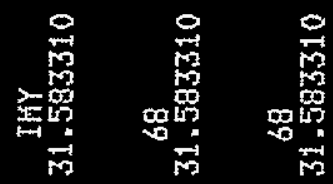

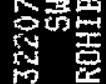

$\mathbb{8}^{-4}$

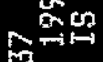

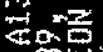

舆倩

$\rightarrow-\frac{\pi}{5}$

$+\infty$

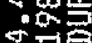

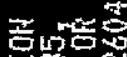

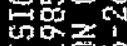

$\sum_{i=0}$

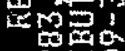

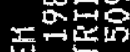

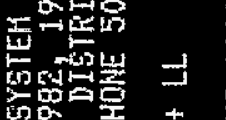

$\lim _{\rightarrow 1}$

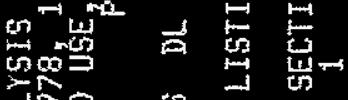

的的

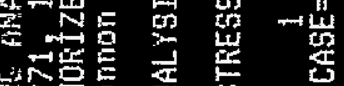

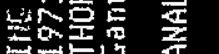

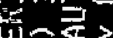

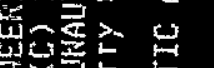

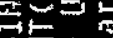

존군:-

, tests

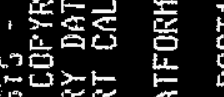

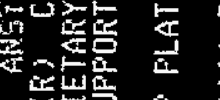

ํํ욤

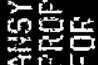

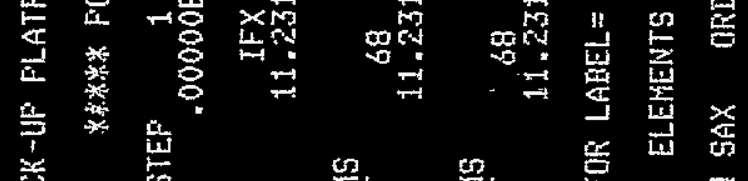

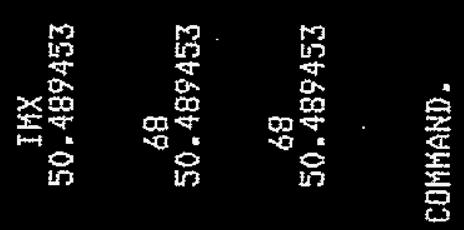

논

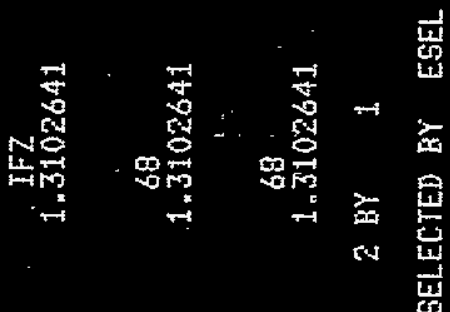

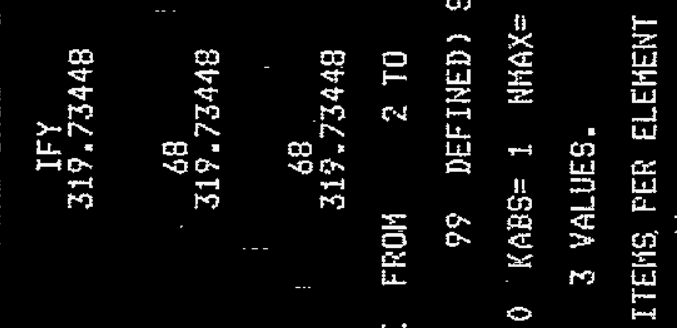

w

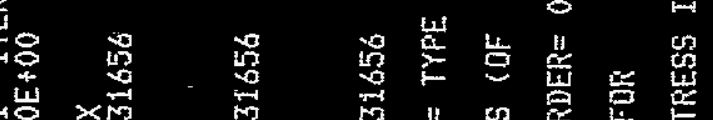

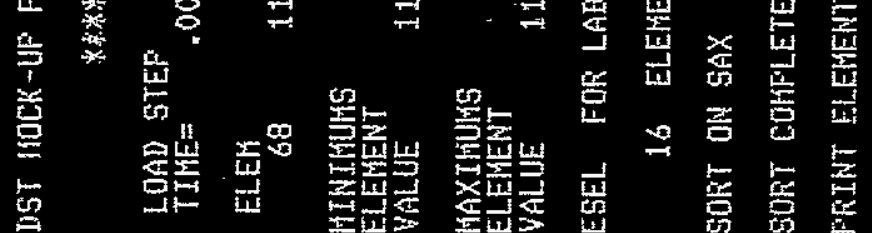

$A-39$ 
㕸

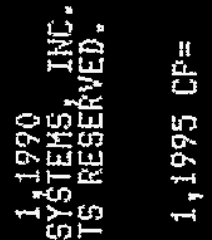

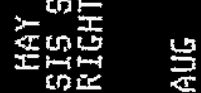

2

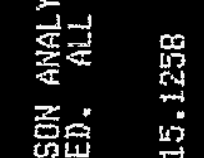

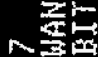

为

象

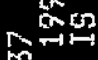

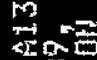

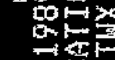

象

t器高

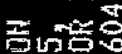

t要

$\rightarrow \rightarrow 0$

悉此?

axis

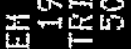

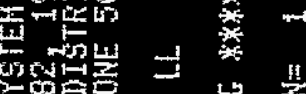

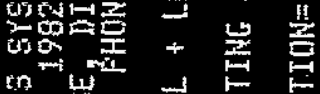

ติำ

尚

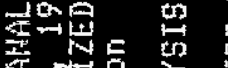

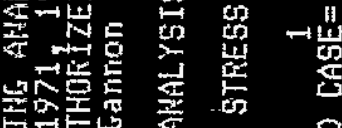

焉

形舟

4 웅

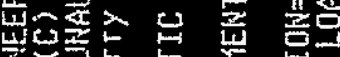

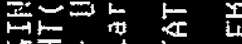

鼓, 可出

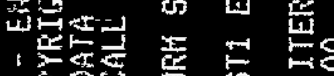
o

西距焉要

点兵

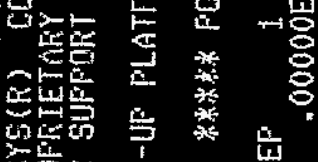

㕝

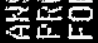

늘

登

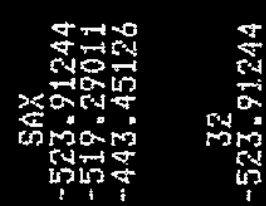

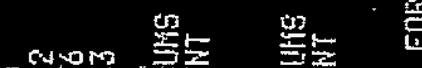

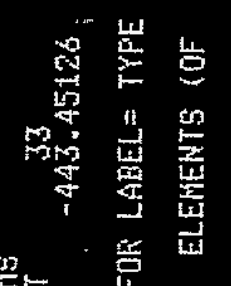

茄

$-1$

on

$m$.

믄

m

琶窟

surems

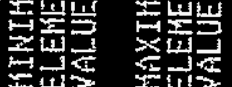

s

s

蒠 
遥量

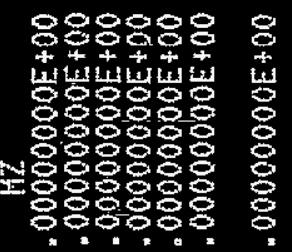

至二

좀요

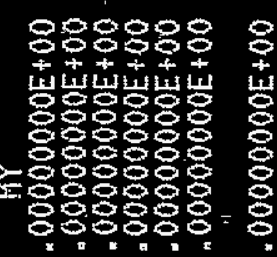

N

anen

min

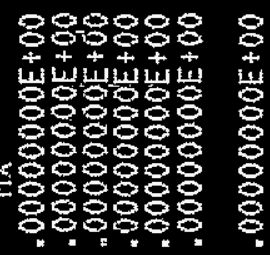

$+\infty$

둔용

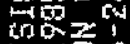

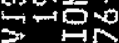
$3=\mathbb{m}$

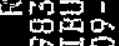

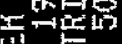
cosu

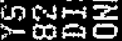
in $4^{4}$ 500

음

$$
\text { 焉 }
$$

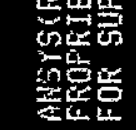$$
\text { 望 }
$$

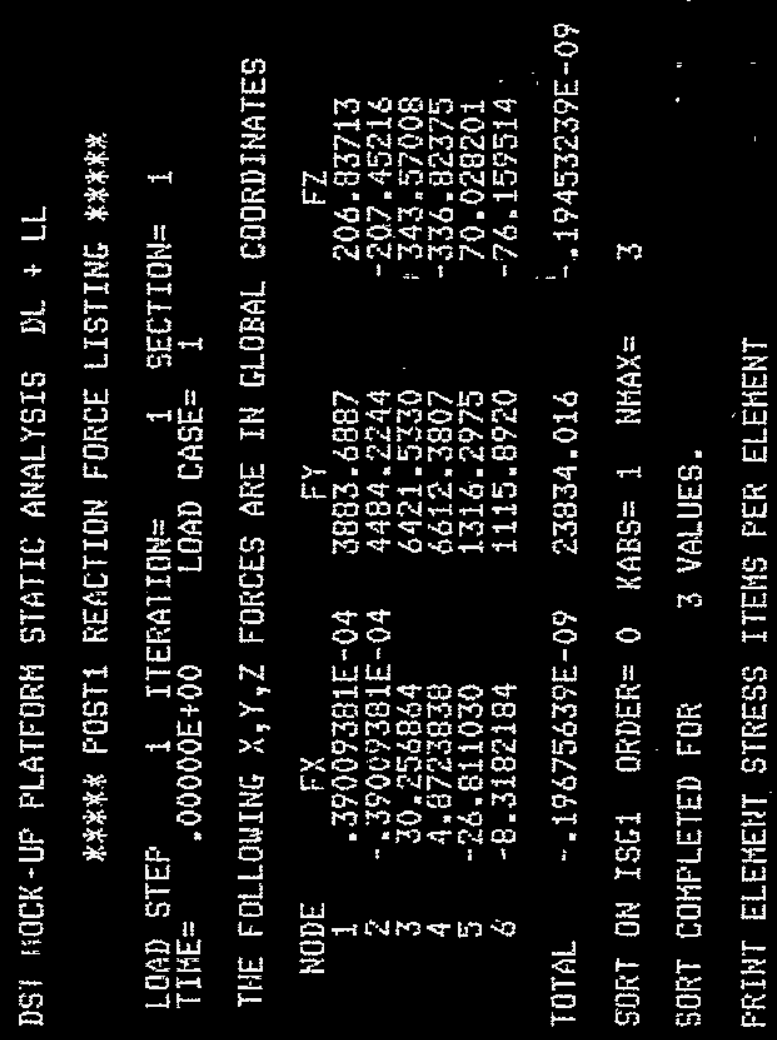


营

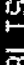

爱

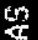

这

\&us

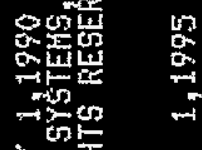

$\therefore$ 든

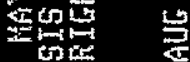

整震

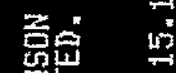

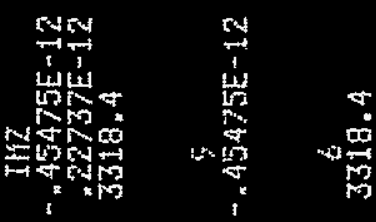

我

묠

9880

章离

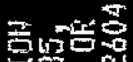

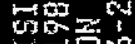

$\leftrightarrow \rightarrow$

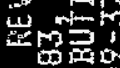

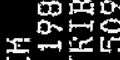

wentus =

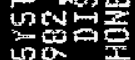

(1) ${ }^{x \rightarrow 1}$

$\cos =$

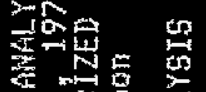

幽㑒焉专

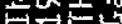

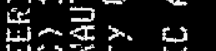

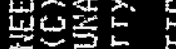

눈, 1

证保

1 不震

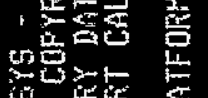

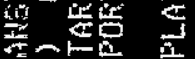

乎暗

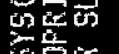

害祒

䆑

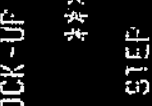

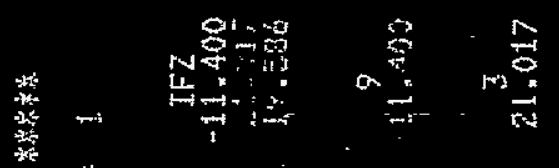

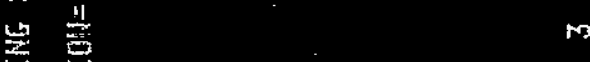

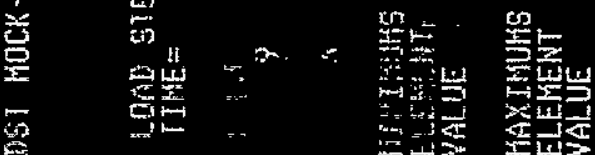

$\sum_{m \rightarrow \infty}^{\infty}$

$\underset{1}{x}$

and

$5 \pm$

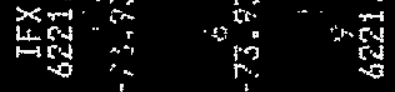

点

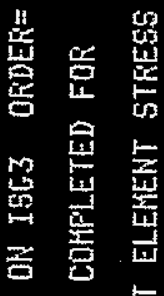

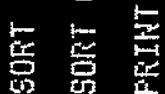


AHSYS - EHGIHEEEING AHALYSTS SVSTEH REVISIOH 4.4

A137 32207 HAY 1,1990

ANSYS(F) COFYFIGHT(C) $1971,1978,1992,1983,1985,1967,1999,1990$ 5WANSOK ANALYSIS SYSTEMS, IKL. AS UNFUFLISHED WOFK.

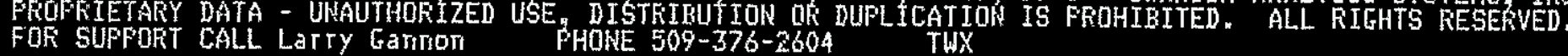

DST HOCK - UF FLATFOFH STATIC ANALYSIS DL + LL

****** FOST1 ELEHENT STRESS LISTING ******

LOAD STEF $\stackrel{1}{1}$ TTERATIEN- $\stackrel{1}{1}$ SECTIOK $=1$

$\begin{array}{rrrrr}\text { ELEH } & \text { ISG3 } & \text { IFX } & \text { IFY } & \text { IFZ } \\ 4 & -1660.5 & 4677.3 & -29.013 & 19.313 \\ 10 & -1362.3 & 5843.2 & 4.6952 & -10.728 \\ 9 & -1059.8 & 621.2 & -4.8724 & -11.400\end{array}$

$\begin{array}{lll}\text { IHX } & \text { IHY } & \text { IKZ } \\ .41633 E-16 & -2549.3 & -3829.8 \\ .71094 E-14 & 1416.0 & 819.77 \\ .00000 E+00 & .11369 \mathrm{E}-12 & -.45475 E-12\end{array}$

HithIHUHS

ELEFERIT

$-1660.5$

4677.3

$-27.013$

$-11.400$

$.000005+00-2549.3$

$-3829.8$

HAXIMUHS

$\begin{array}{llcc}\text { ELEHEWT } & 9 & 9 & 9 \\ \text { VALUE } & -1059.8 & 921.2 & 4.6952\end{array}$

$19^{4} .313$

$.2009 \mathrm{E}-14$ 10 1416.0

619.77

SORT ON JSE 1 ORDEF $=0$ RABS $=1$ NHAX $=3$

SORT COHFLETED FOR

3 VALUES.

FFIMT ELEHENT STRESS ITEHS PER ELEMENT 
8

哭

i

告

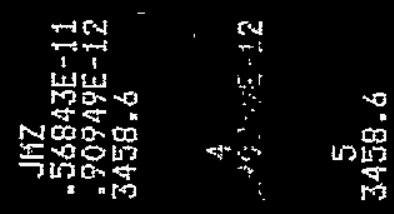

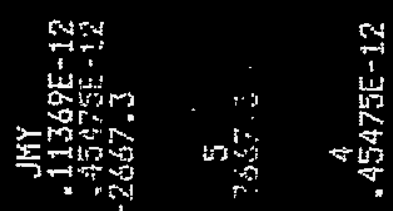

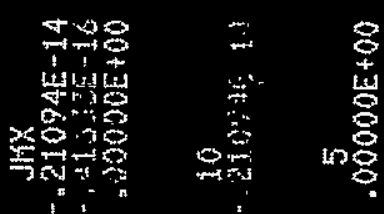

몽뭉용

is

$\lim _{0 \rightarrow \infty}$

405

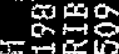

要州的

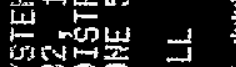

6⿻一𠃋十

$w^{-1} w^{-m}$

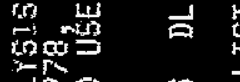

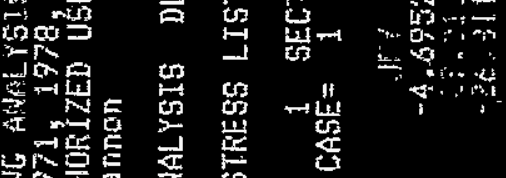

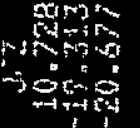

$\underbrace{\infty}_{0}$

造密

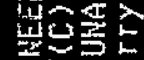

넨도에

, 空政

, 空近它

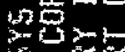

题突

내볼

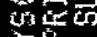

整统

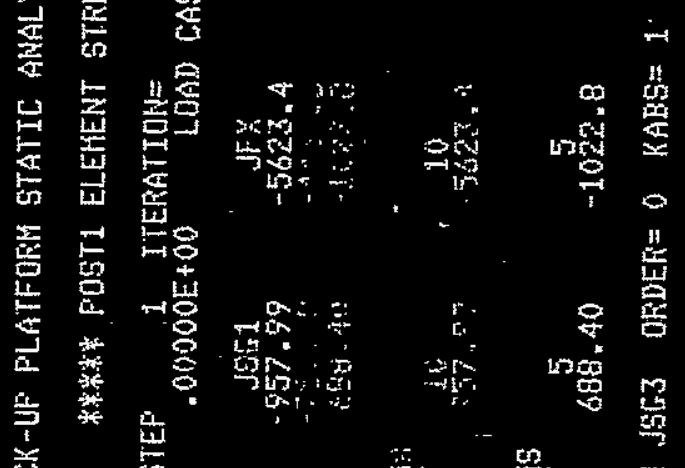

ming

集点

i

兽

袋

일 
$\frac{8}{8}$

罡

幽

क्षिए

車战它

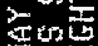

the

$\frac{\frac{1}{x}}{\frac{1}{4}}$

些。

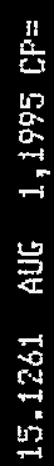

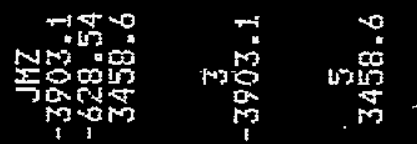

s.

数

$\Omega^{\frac{0}{4}}$

Sos

뭉

D車玨

$\rightarrow$ 乐

$x^{n-1}$

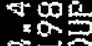

푹 전동

$0_{0}=0$

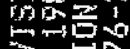

tin

mas

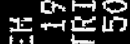

선

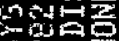

$\lim _{\rightarrow \rightarrow 1}$

in

$\lim _{0}$ of

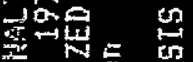

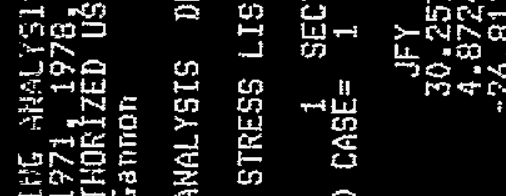

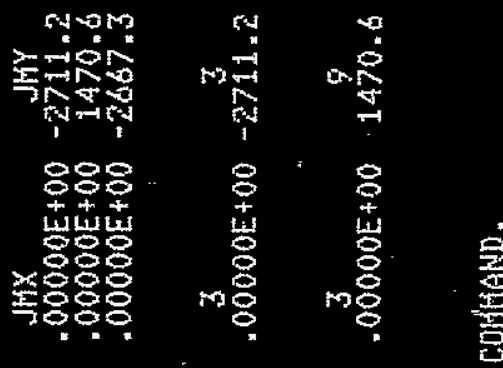

焉东

원돈

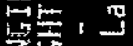

立心

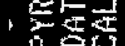

$x_{i=15}$

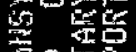

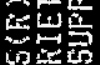

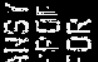

: od 嵌

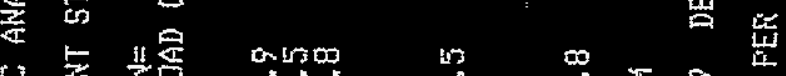

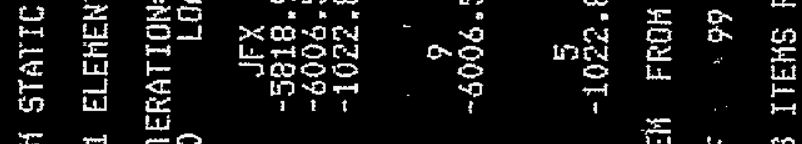

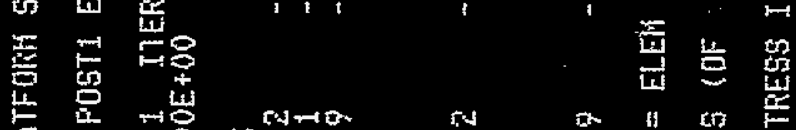

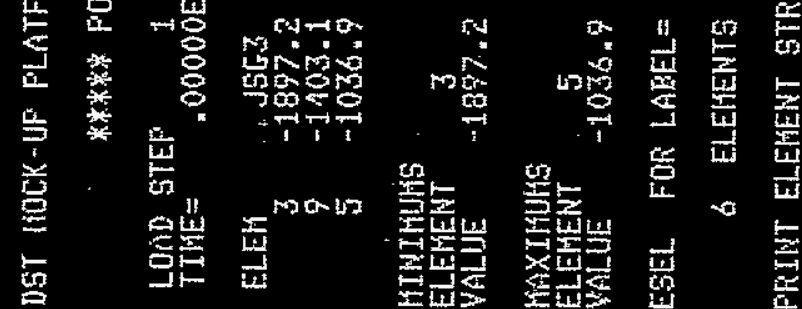


\&

装

놈욤

ovin

내는

a

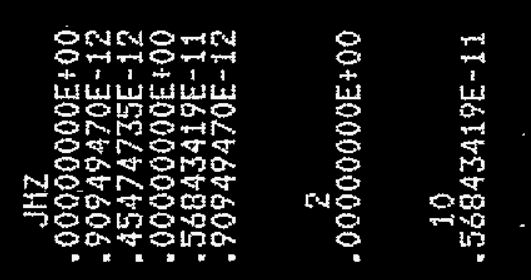

2 in

똔든

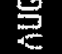

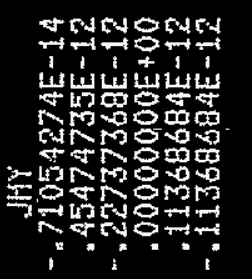

का

온

总的

证些

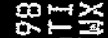

8용ㅇㅇㅇㅛ

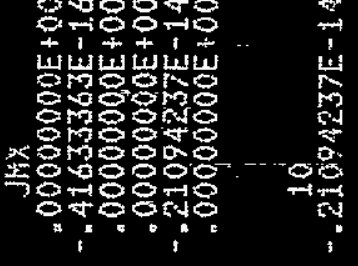

$\frac{8}{8}$

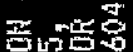

the

$\rightarrow \infty$

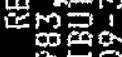

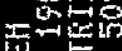

ton 2.

in

thos

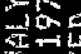

整栗要

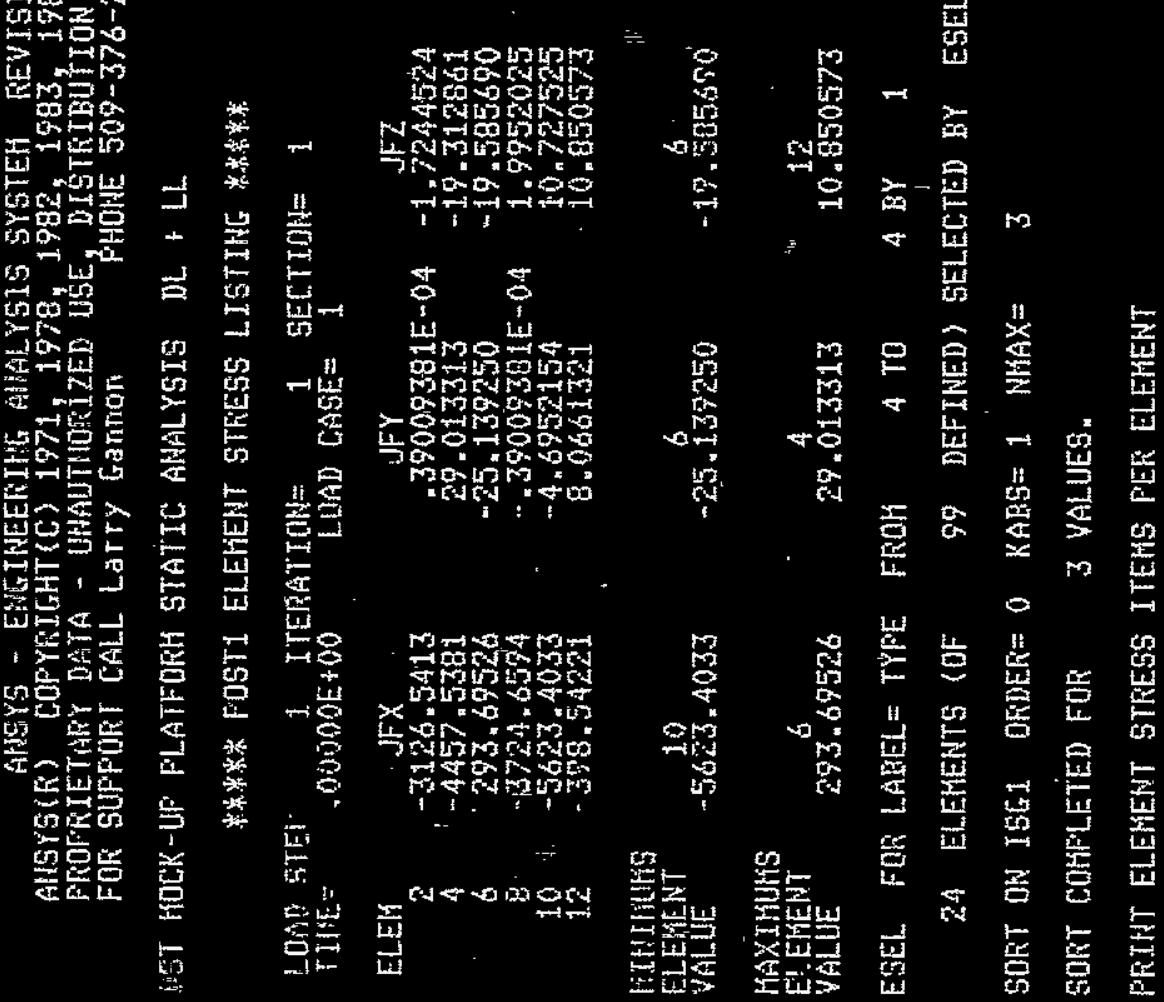

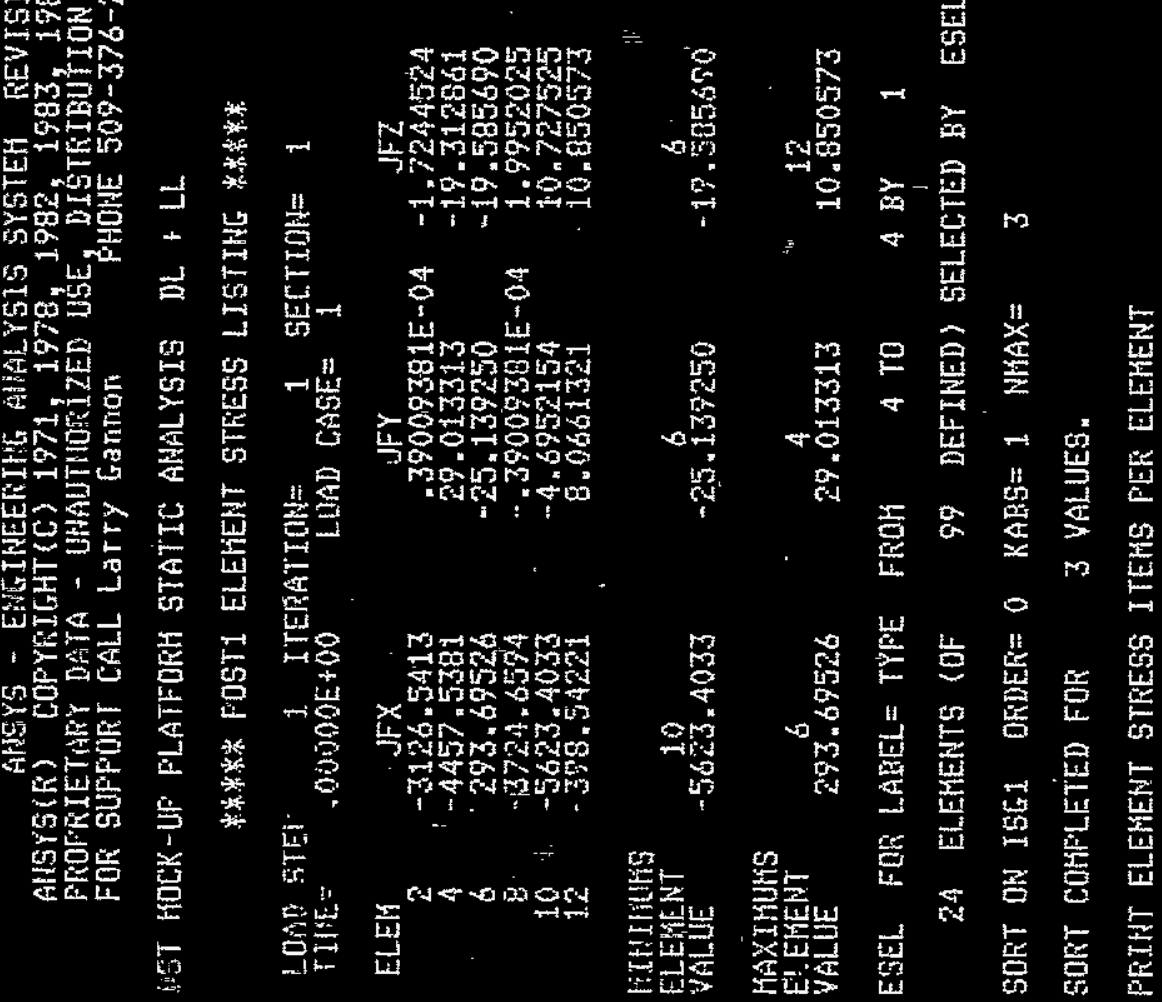

些'

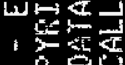

n옹

5

近此要

的国它

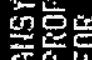




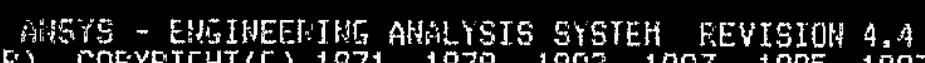

A137 32207

HAY 1,1990

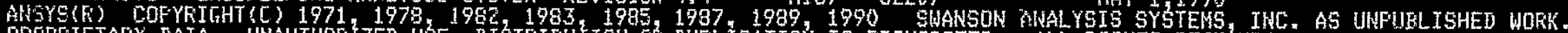
PRUPRIETARY DATA - URAUTHORIZED USE, DISTRIBUIION OR DUFLICATIOR IS FFOHIBITED. ALL RIGHTS REGERVED.

FOK SUPPOFT CÁLL Larty Gamion PHONE 509-376-2604 THX

IST HOCK-UF PLATFOEH STATIC AHALYSIS DL + LL

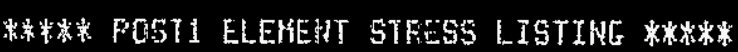

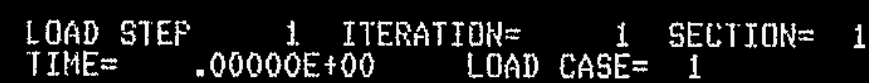

\begin{tabular}{|c|c|c|c|c|c|c|c|}
\hline $\begin{array}{c}\text { ELEFi } \\
99 \\
79 \\
92\end{array}$ & $\begin{array}{l}1361 \\
23647 . \\
20407 \\
2945.0\end{array}$ & $\begin{array}{c}\text { IFx } \\
2.4326 \\
2.4334 \\
467.28\end{array}$ & $\begin{array}{c}\text { IFY. } \\
-1499.6 \\
-1500.4 \\
6.3346\end{array}$ & $\begin{array}{c}\text { IFZ } \\
59401 \\
.99401 \\
-1.9121\end{array}$ & $\begin{array}{c}I H X \\
38.810 \\
38.818 \\
-30.343\end{array}$ & $\begin{array}{c}\text { IHY } \\
-8.8971 \\
9.8977 \\
53.085\end{array}$ & $\begin{array}{c}\text { If12 } \\
-47702 . \\
-47702 . \\
-19171 .\end{array}$ \\
\hline
\end{tabular}

\begin{tabular}{|c|c|c|c|c|c|c|c|}
\hline ELEUE & $\begin{array}{l}92 \\
8945.0\end{array}$ & $\begin{array}{c}79 \\
2.4326\end{array}$ & $\begin{array}{c}97 \\
-1500.4\end{array}$ & $\begin{array}{c}92 \\
-1.9121\end{array}$ & $\begin{array}{r}92 \\
-30.343\end{array}$ & $\begin{array}{c}95 \\
-8.8971\end{array}$ & $\begin{array}{r}99 \\
-4770\end{array}$ \\
\hline
\end{tabular}

MAXIHUHS

ELEMEHT

$\begin{array}{cc}99 \\ 22647 . & 967.28\end{array}$

$\frac{92}{2} 3346$

.99701

97
38.81 .8

$\frac{72}{53.085}$

92
-191.71

SONT the IGS : OROEK $=0$ KABS $=1$ NHAX $=3$

SORT COHFLETED FUA 3 VALUES,

D. THEHEUT STRESS ITEHS FER ELEHENT 
$\stackrel{8}{8}$

$\frac{15}{6}$

空

ํ.

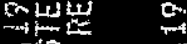

$\rightarrow$

㱐

논당

楚

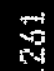

종분

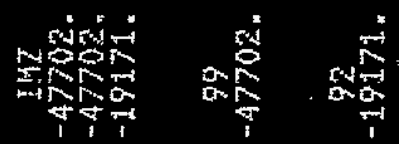

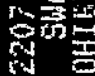

mo

要

.

a

类

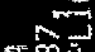

땅

돈동

in

$\rightarrow \rightarrow+5$

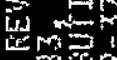

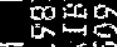

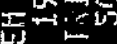

6ne

$\because+n$

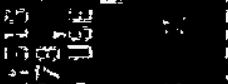

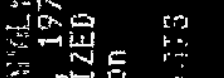

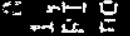

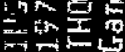

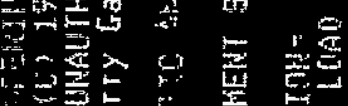

$\rightarrow=3$

政

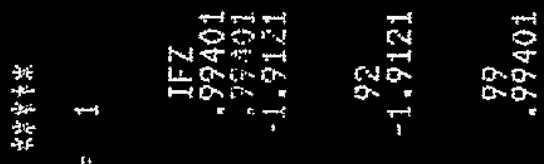

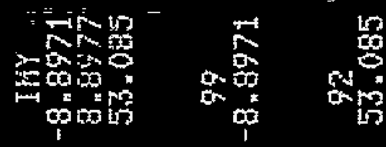

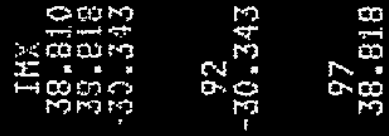

=

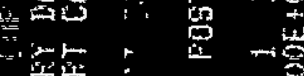

政要

a

籍

Lis

$\frac{5}{513}$

$\infty \pi$

ras

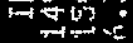

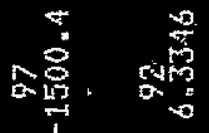

紊

$-1 \quad \overrightarrow{\mathrm{w} i}$

要

$\sin$

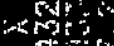

जारी

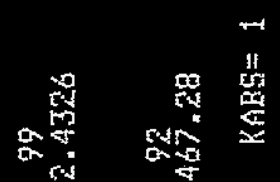

薄

$\rightarrow \frac{8}{5}$

营 点

$\operatorname{man} 0^{\infty}$

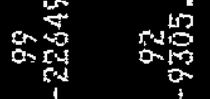

悹

望

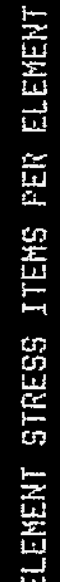

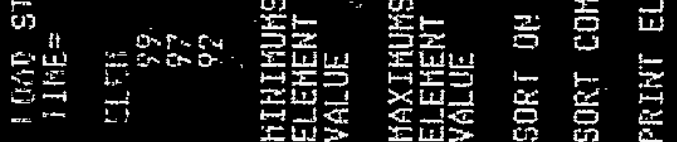


AH5IS - EHEIHEERI AHALYSTS BYSTEH REVISIOH 4.4

A137 32207

HAY 1,1990

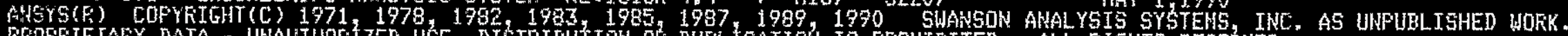

PTOFFIEIARY DATA - UNAUTHORIZED USE, DISTRIEUTIOH DI: DUFL ICATION IS FFOHIBITED. ALL. RIGHTS RESERVED.

BST HOCK-IP PLATFORH STATIC AHALYSIS DL + HL

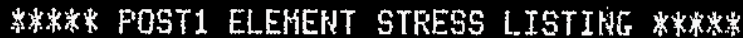

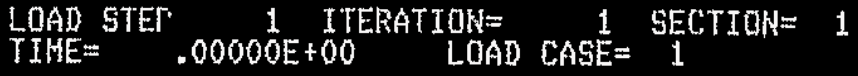

ELEN
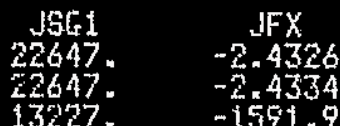

JFY

$-1.500 .4$

$\begin{array}{rrr}-2 & 4334 \\ -1591.9 & -1489.96 & -799401 \\ 4 & -869.00\end{array}$

JWX

$-38.818$

$-5.1264$

$.34 Y$
8.8971
-8.8977
18068

J7M7

47702 :

96
-38.818

96
-8.8977

177092

ELEMENT

13727

77
$-1591=9$

98
-1500.4

98
-.99401

77

ELEHENT

98
22647

98
-2.4328

77
-288.99

489.00

77
-5.1264

18068 .

98

SORT ON JAG3 ORDEF $=0$ KAES $=1$ WHAX $=3$

SORT COHPLETEB) FOR 3 VALLUES.

PFINT ELEHEHT STFESS ITEHS FER ELEHEHT 
AHSYS - EHGIHEEFIHI AHALYSIS SYSTEH FEYISIOH 4.4

A 137323207

KAY $1,1,990$

AUT $1,1978,1982,1983,1985,1987,1989,1970$ SWANSOH ANALYSIS SYSTEHS, ING. AE UNFUELTSHED WOKK.

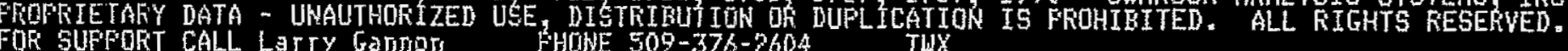

DST HOCK-UF FLATFOKH STATIC ANALYSIS DL + LL

15.1261

ALIG $1,1995 \mathrm{CF}=$

.500

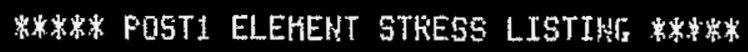

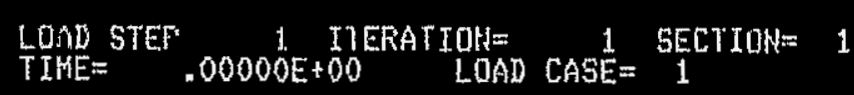

\begin{tabular}{|c|c|c|c|c|c|c|c|}
\hline $\begin{array}{r}\text { ELEH } \\
98 \\
78 \\
77\end{array}$ & $\begin{array}{l}5553 \\
-22649 \\
-22649 \\
-14456\end{array}$ & $\begin{array}{c}w F X \\
-2.4326 \\
-2.4334 \\
-1.591 .9\end{array}$ & $\begin{array}{c}\text { JFY } \\
-1500.4 \\
-1497.6 \\
-288.99\end{array}$ & $\begin{array}{c}\sqrt{1 F} \\
-.97401 \\
-.99401 \\
469.00\end{array}$ & $\begin{array}{r}51 X \\
-38.810 \\
-30.818 \\
-5.1264\end{array}$ & $\begin{array}{r}\text { WY } \\
8.8971 \\
-8.8977 \\
18068 .\end{array}$ & $\begin{array}{c}142 \\
47702 \\
47702 . \\
11092 .\end{array}$ \\
\hline
\end{tabular}

KINIHLHSTS

ETEHEH

$\begin{array}{ccccc}98 & 77 & & 98 & 96 \\ -22645 & -1591.9 & -1500.4 & -.97401 & -38.818\end{array}$

$\begin{array}{cc}76 & 77 \\ -8.3977 & 11092\end{array}$

MAXINUWHS

ELEHENT

77

$00^{\circ}$

(1)

$-2.4326$

77
-288.99

$469.00 \quad-57.1264$

18068

47702.

ESEL FOR LABEL = ELEH FroH 88 TO 99 BY 1
12 ELEHENTS LOF
99 DEF INED) SELECTED BY
EGEL COMHAND.

PRINT ELEHENT STRESS ITEHS PER ELEHENT 
옹

\&

产焉

站

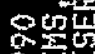

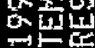

$\rightarrow=0$

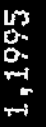

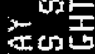

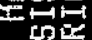

놀

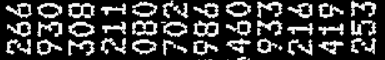

Nrin

Nam smogar

oratammate

난

等

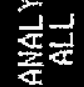

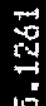

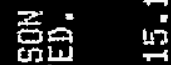

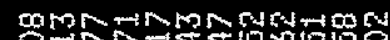

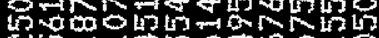

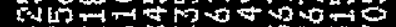

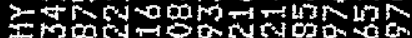

mond

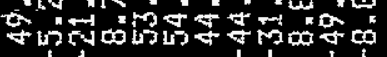

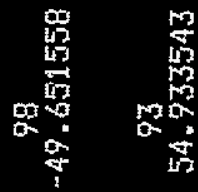

舵

密的

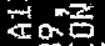

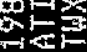

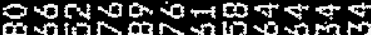

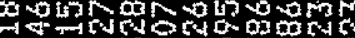

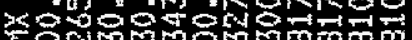

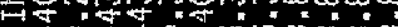

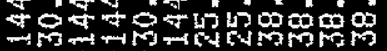

然

标要

些跑要

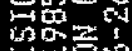

$\Rightarrow \rightarrow$

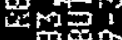

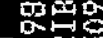

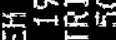

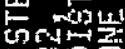

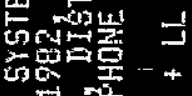

$0^{-1} \ln ^{2}$

nog

줄

№

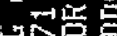

곤달

(4)

붕중

踏工

넌도

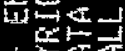

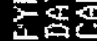

15:

然

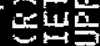

पुलि

非

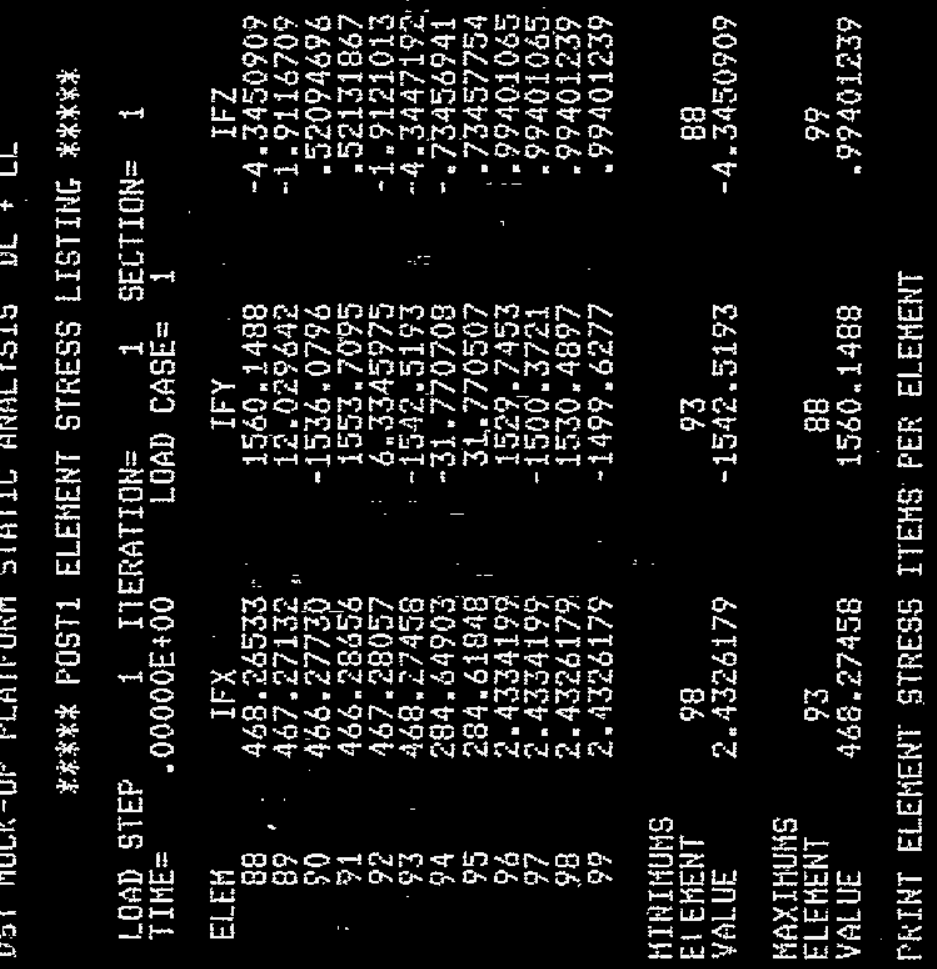


$\stackrel{9}{4 ! 3}$

몯

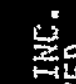

竞

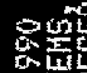

antw

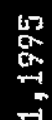

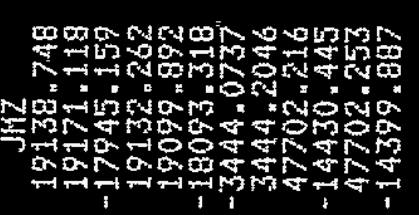

$\frac{\infty}{3}$

$\frac{1}{d}$

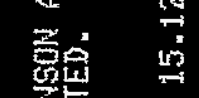

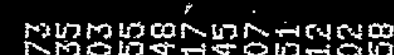
DMOR

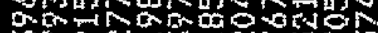

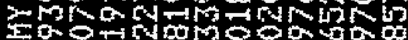

$\therefore$ 的

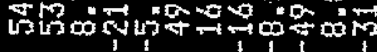

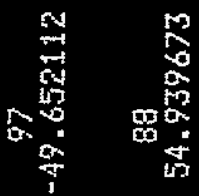

웅

$\lim _{\substack{\infty \\ m \rightarrow \infty}}$

如象

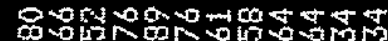

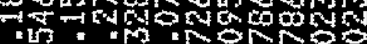
х8

今이의

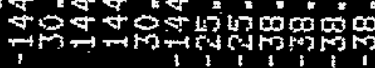

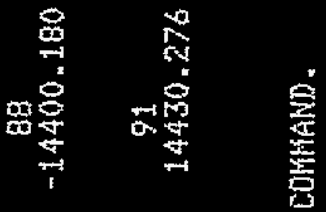

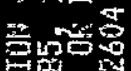
要要

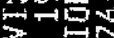

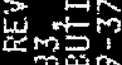

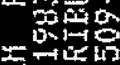

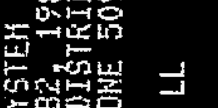

6 6

$\omega^{\rightarrow-1}$

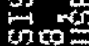

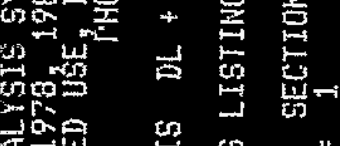

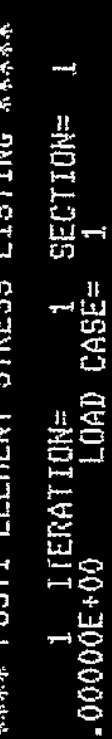

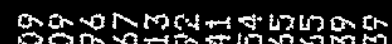

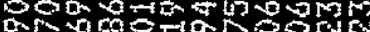

W

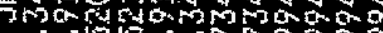

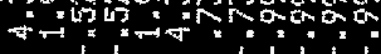

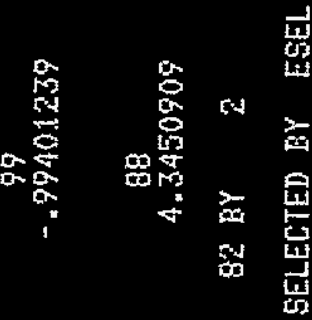

Non

त5

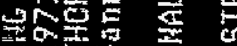

mite

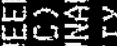

int

通要

, sis

的岂舟

药

政

的崖

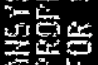

荘

-

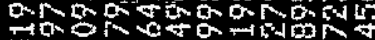

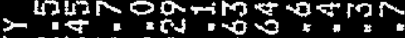

H

$\rightarrow$ thon

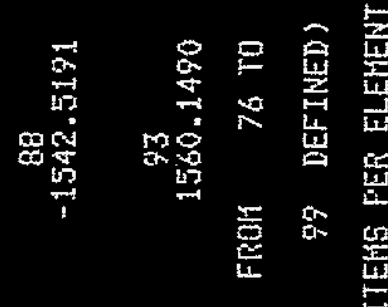

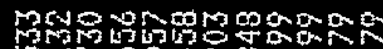

An

xentidy

4 .

कर

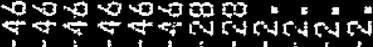

要

告

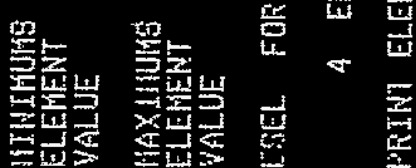

$$
A-52
$$


옥

桇

卧息

닌

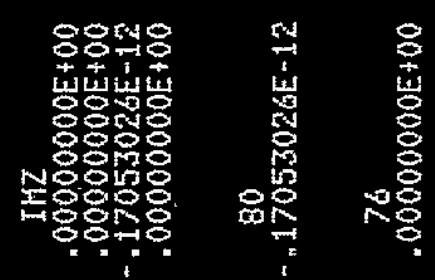

栾需

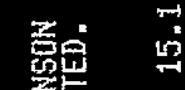

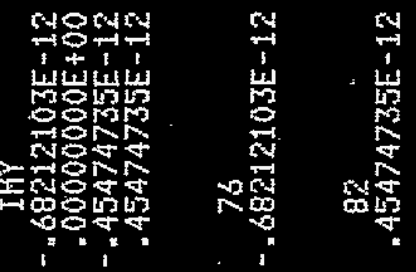

量

复

政的

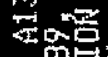

总证肴

는

4

하늘

器

낼

(2)

ㄴ.

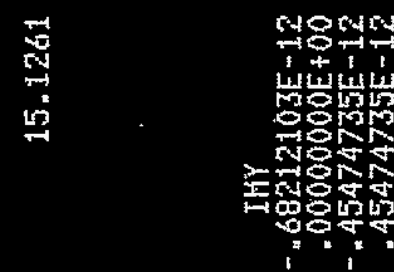

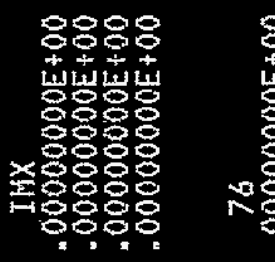

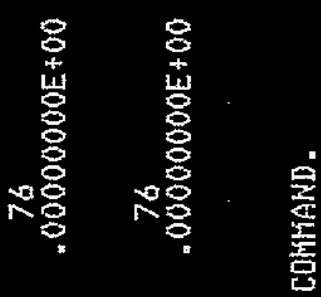

$\lim _{\rightarrow \rightarrow \infty}$

$x_{i=\infty}$

这的?

$x_{0 \rightarrow 3}$

wro

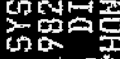

$\mathrm{Bn}^{n+1}$

$5 \operatorname{sen}$

远面

$x$

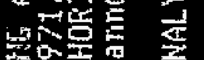

$\Rightarrow$ 때현

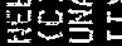

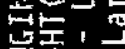

焉

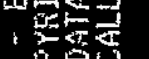

is

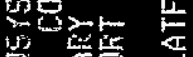

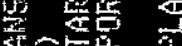

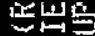

的兽家

운룬

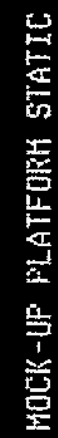

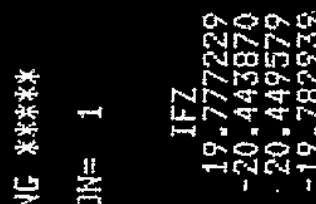

$p \quad 2 \quad \frac{\vec{w}}{w}$

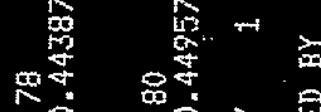

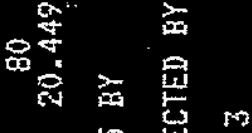

監点

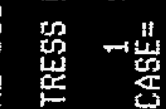

上㝵

题题

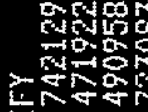

-irici

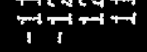

ris

的䓌

.

击

$F \underset{i=1}{\stackrel{m}{5}}$

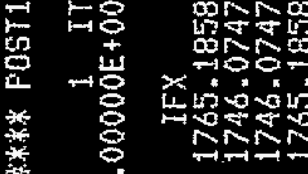

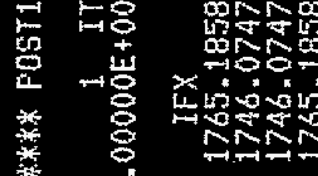

赢

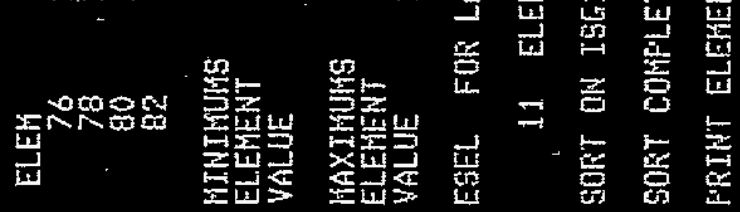

$A-53$ 


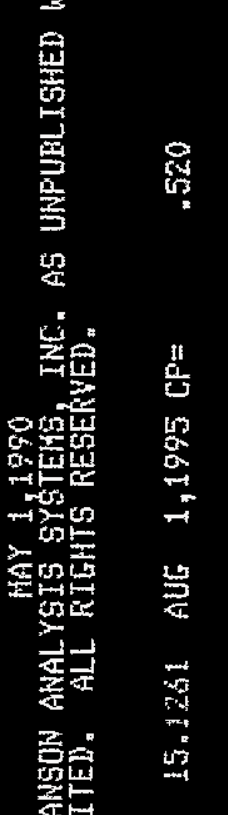
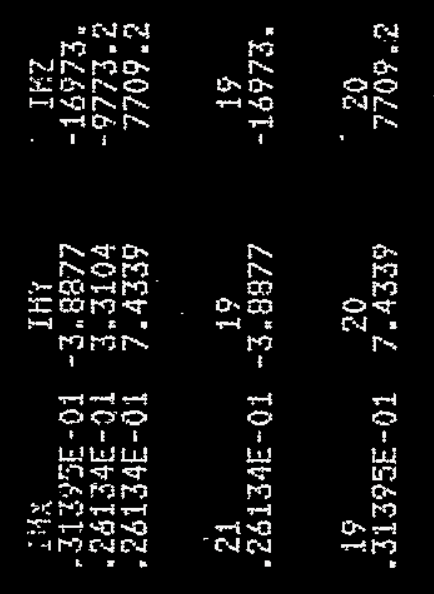

管为要

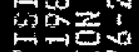

证为?

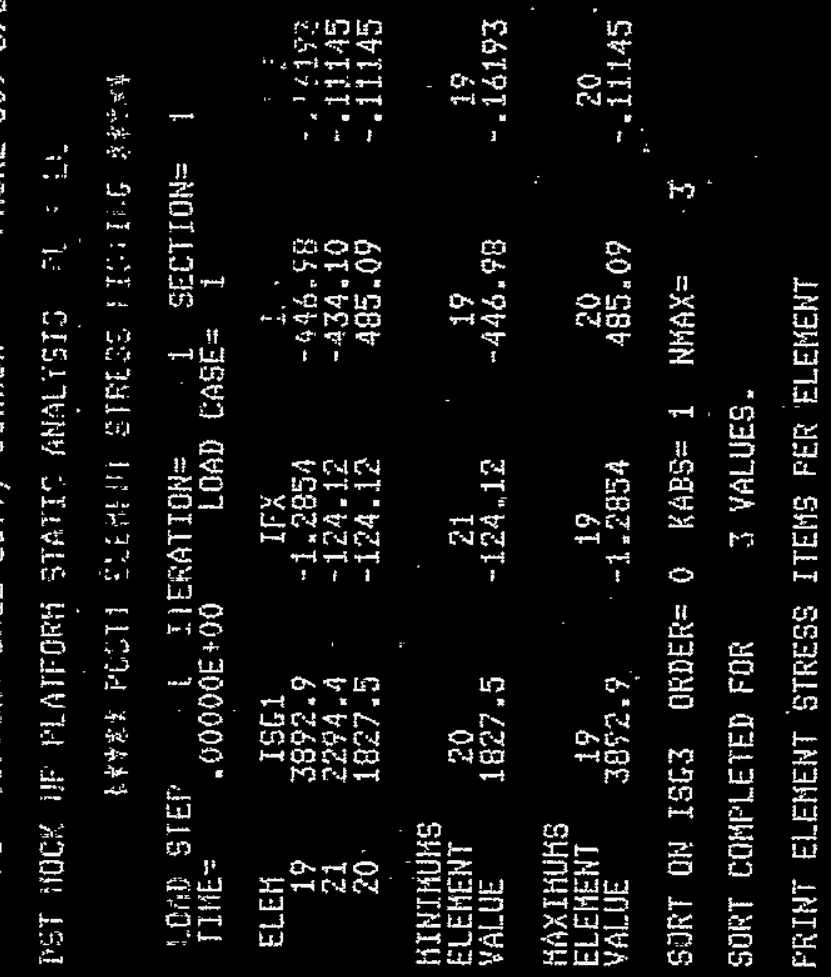

$$
\text { A- } 54
$$


-

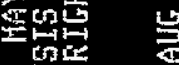

迹势

觜出

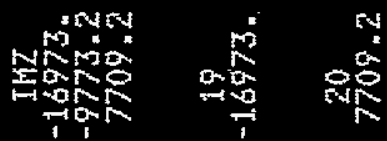

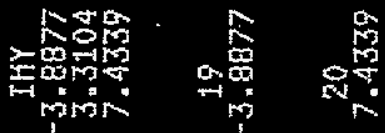

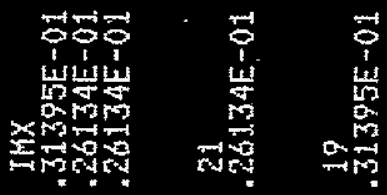

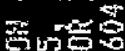

thic

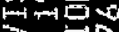

$=m$

cas

논

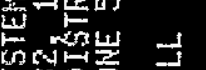

2000

$\rightarrow$

15 th

ting

슨 क

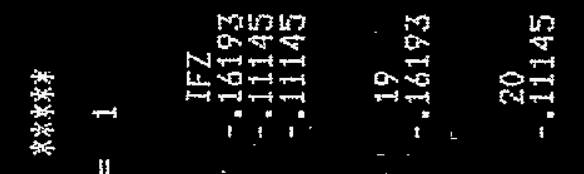

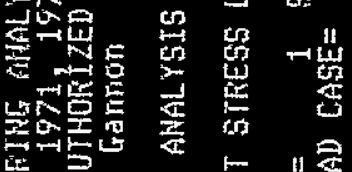

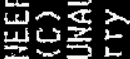

논,

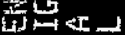

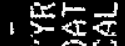

부의.

的若

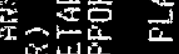

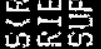

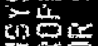

눈

点

空

H $\quad$ god

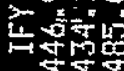

$\underset{4}{\infty}$

$m$

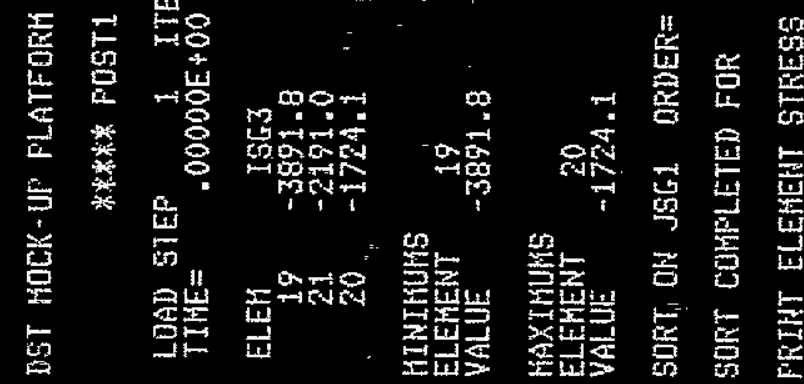


:

哭

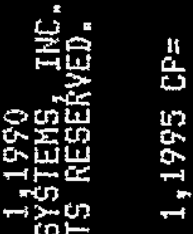

空密

$\infty$

这要

喜完

要

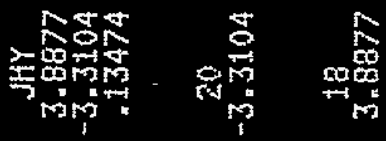

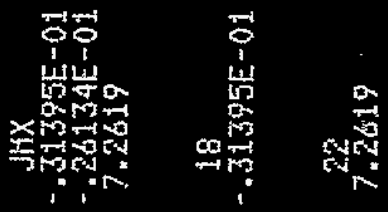

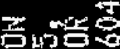

as

$\sin ^{-i n}$

win

o

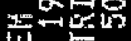

$5 \cos u$ -

${ }_{1 \rightarrow 1}$

tim

$\lim _{x \rightarrow \infty}$

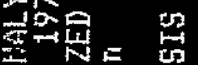

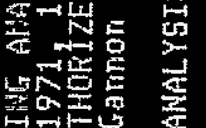

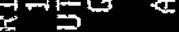

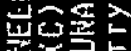

政, i

近地-

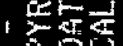

$\cos _{1 \rightarrow 0}$

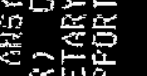

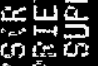

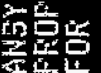

* $\rightarrow$ क्ष

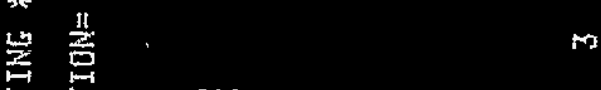

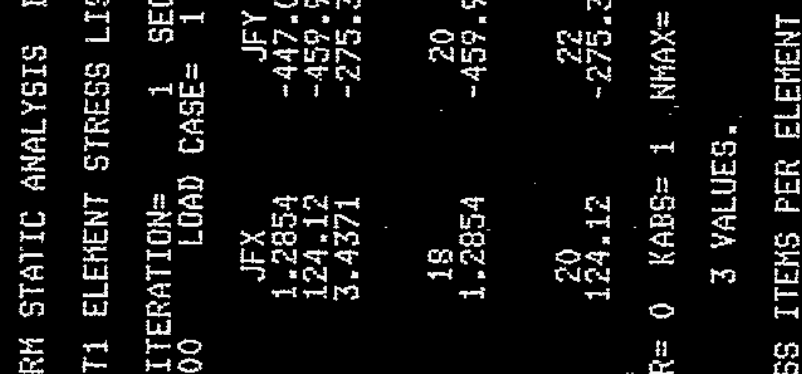

S욤요

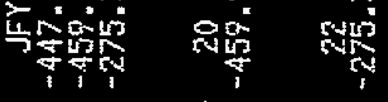

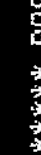

点

音

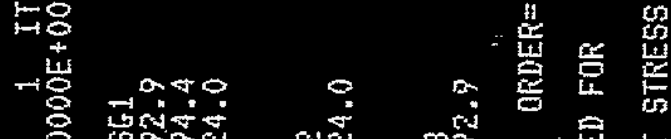

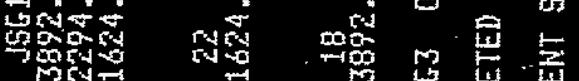

a m

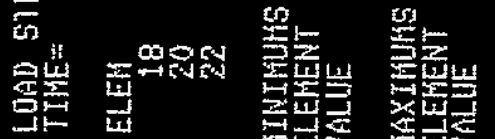

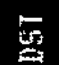

tows 
AHSYS - EILIHEEFIHI ANALYSTE SYSTEH FEVISTOH A A

A137 32:207 HAY 1.1990

AHSYS(R) COFYFIGHT(C) $1971,1978,1982,1983,1985,1997,1989,1990$ SWANSON ANALYSIS SYSTEMS, INL. AS UNFULLISHED UDRK.

FROFFIETARY DATA - UNAUTHORIZZED USE DISTRIBUTIOK OR DUPLICATIOK IS FROHIBITED. ALL RIIGHTS RESEKVED.

DST MOCK-UF FLATFOFH STATIC ANALYSIS DL + LL

15.1261 AUG $1,1995 \mathrm{CF}=$

.540

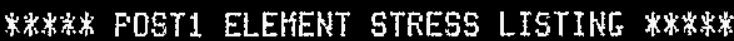

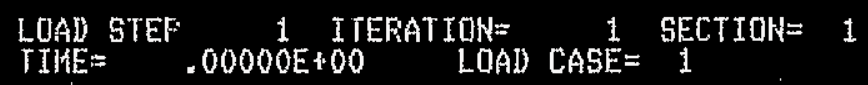

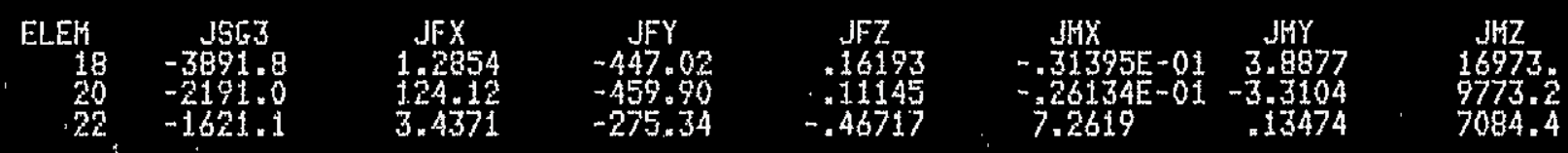

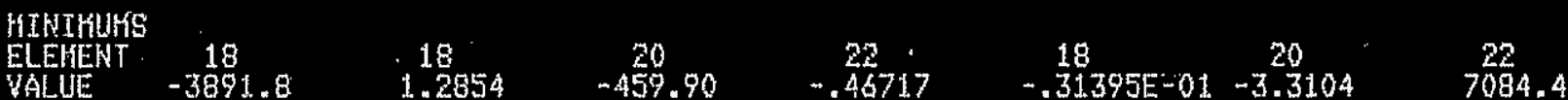

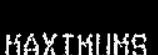

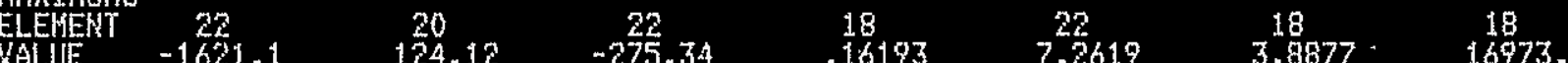

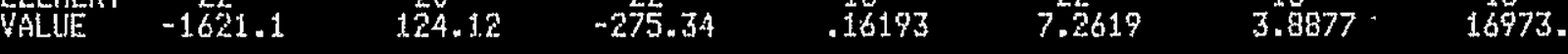

ESEL FOR LAEEL = ELEH FFOH $18 \mathrm{TO} \quad 22 \mathrm{BY} \quad 4$

2 ELEHENTS (OF 99 DEFINED) SELECTED BY, ECEL COHHAND.

PRINT ELEHENT STRESS ITEMS FER ELEHENT 


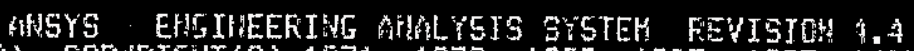

A137 32207

AHSYS(F) COFYFIGHT(C) $1971,1078,1982,1793,1985,1987,1989,1990$ SWANSOH ANALYSIS SYSTEMS, INC. AS UKFUBLISHED WORK.

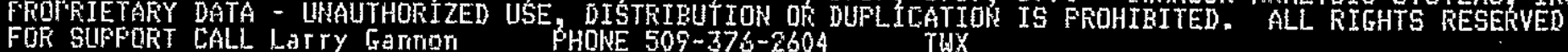

DST HOCK-UF FLATFOHH STATIC AKALYSIS DL + LL

15.1261 AlIG $1,1995 \mathrm{CF}=$

$\$ 540$,

***** FOSTI ELEHENT STFESS LISTING $*$ ***:*

TOMD STEF, 1 TTERATION= $\frac{1}{1}$ SECTIOK $=1$

$\begin{array}{cccc}\text { ELEH } & \text { IFX } & \text { IFY } & \text { IFZ } \\ 18 & -1.2853841 & 472.20434 & -16193440 \\ 22 & -3.4370618 & 282.35393 & 46716892\end{array}$

22

$3.437061 \%$

272.35393

.46716892

$\operatorname{IHX}$
$31394996-01$
.7018727

IMY

2. 1038737

11.813959

Inz

MINIHULIS

ELEMENT

$-3.4370617$

202.35393

., 18193440

$-7.2618729$

$-11.2213959$

11.725979

MAXIKUHS

ELEYEN

$-1.2853841$

472.20434

.46716892

19
$-31394996 E-01$

18
2.1038737

11.722979

ESEL FOF LABEL= ELEH FFOH $19 \mathrm{TO} \quad 23 \mathrm{BY} \quad 4$

2 EIEKENTS (OF 99 DEFINED) SELECTED BY ESEL COMHAND.

PRINT ELEKENT STRESS ITEMS PER ELEMEHT 
THSY3 - EMGIHEEFTH AMULYSTS SYSTEH FEVISIOH 4.4

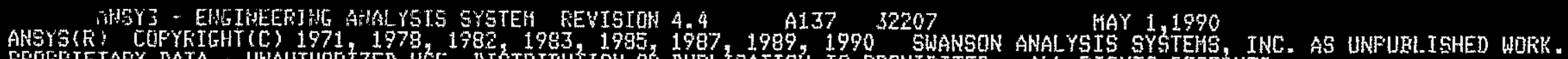

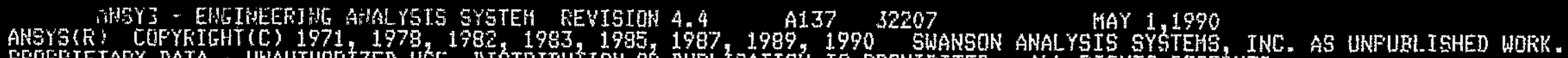

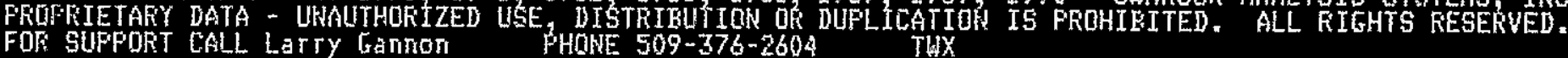

15.1261 AUG $1,1995 \mathrm{CF}=$

DST HOCK-UF FLATFORH STATIC ANALYSIS DL + LL

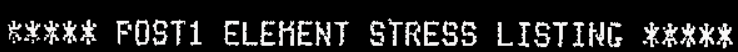

LOAD STEF' $\frac{1}{1}$ ITEFATIOK= $\frac{1}{1}$ SECTION= 1

$\begin{array}{ccccccc}\text { ELEW } & \text { JFX } & \text { JFY } & \text { JFZ } & \text { JHX } & \text { JHY } & \text { JHZ } \\ 19 & \frac{1}{3} .2853841 & : 472.16614 & .16193440 & -31394996-01 & 9.9792720 & -31.013205 \\ -23 & 3.4370619 & , 294.69019 & .46716892 & 7.2618729 & -11.544488 & -69.879467\end{array}$

HINTHUHS

ELEMENT

$1.2853841 \quad 294.6$

294.68019

1

ELEKENT

VALIIE

UISE LODA STEF

3.23750619

$472 \cdot 19614$

$-23$

$-.31394996 E-01$

23

$-11.544488$

23

DISPLACEHENT. STOKEO FOR 72 NODES

***WARIITE * ***

DATA FOR STRESS LABEL IFX HAS NOT BEEN STORED FOR ALLO ELEHENTS

23019

9.19792720

$-31.013205$

IFX DATA FOR TYFE 2. STIF 8. ELEMENTS UILL BE SET TO ZERO

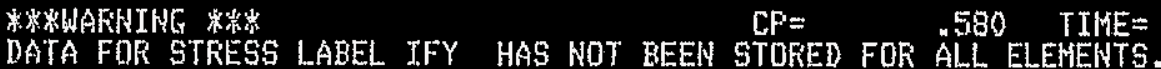

15.12611

DATA FOR STRESS LABEL IFY HAS NOT BEEN STOFED FOR ALL ELEHENTS.
IFY DATA FOR TYFE

in WATA FUR STRESS LABEL IFZ HAS NOT BEEN STORED FOK ALL ELEHEHTS.

(1) IFZ DATA FOR TYFE 2 STIF 8 . ELEHENTS WILL BE SET TO ZERO

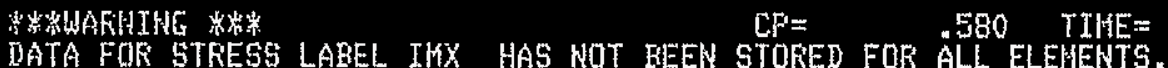

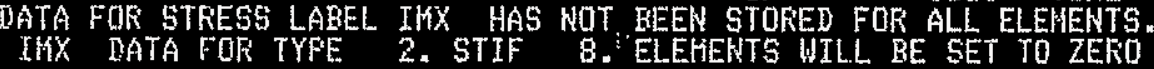

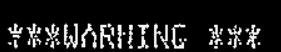

DATA FOF SYHESS LABEL IHY HAS WOT FEEN STORED FOE ALLO ELEHENTS.

15.1261 .1

IMY IBATA FOR TYPE 2 . STIF 8 . ELEHENTS UILL HE SET TO ZENO

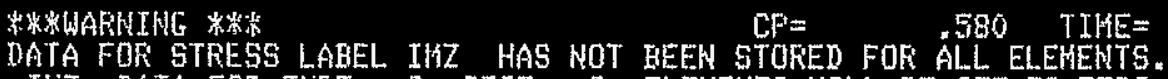

DATA FOK STRESS LAAEL IHZ HAS NOT BEEN STOFED FOF ALL ELEHENTS.
ITZ. DATA FOR TYFE

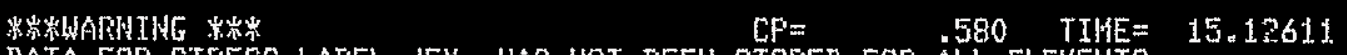

DATA FOR STKESS LAEEL JFX HAS NQT BEEN STORED FOR ALL ELEMENTS

15.12611

1.5.12611

15.12611

JFX DATA FOR TYFE 2. STIF B. ELEHENTS UILL BE SET TO ZERO

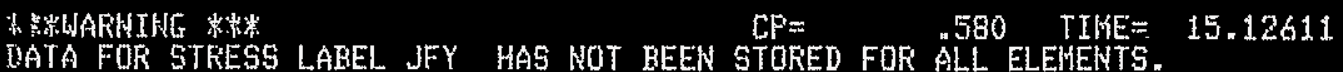

DATA FOR STRESS LAREL JFY HAS NOT BEEN STORED FOR ALL ELEHENTS.
JFY DATA FOR TYPE

BHWAFHIHE

ONTA FOK STKESS LABEL JFZ HAS NOT GEEN STORED FOR ALL ELEMENTS.

JFZ DATA FOR TYFE 2. STIF B. ELEHEHTS WILL BE SET TO ZERO

W WHANIIIS *

cpis:

.580 TIHE:

15.12611 
WHC-SD-WM-ER-521

Rev."O

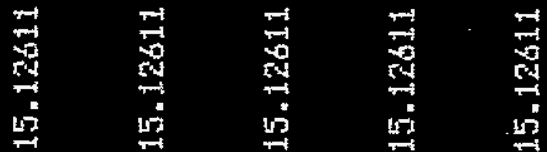

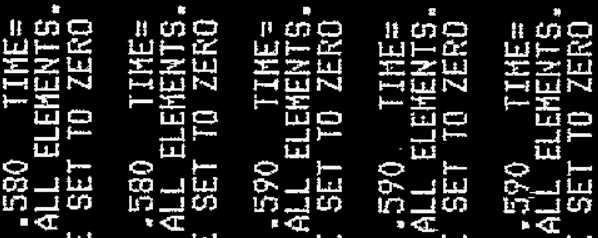

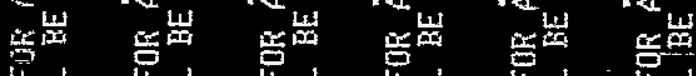

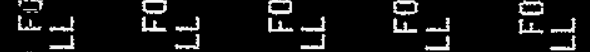

量

星

是是

昰象

11 苚

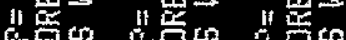

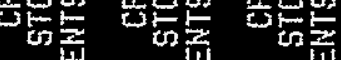

经的

눈?

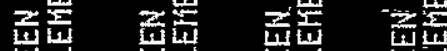

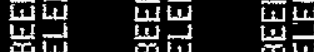

질

빕

密:

需出

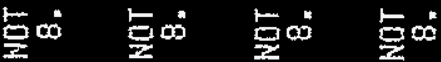

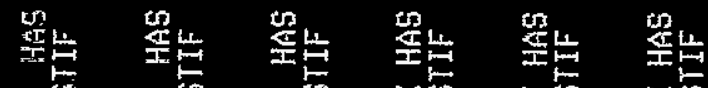

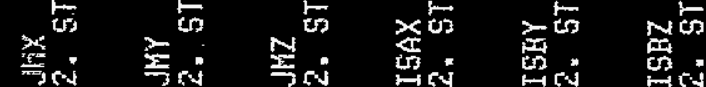

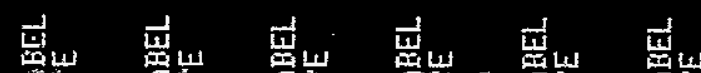

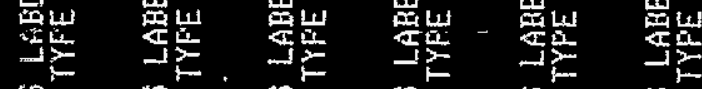

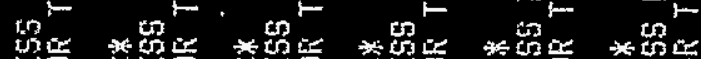

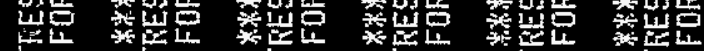

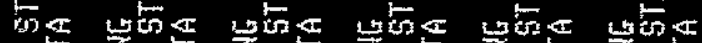

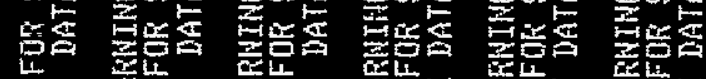

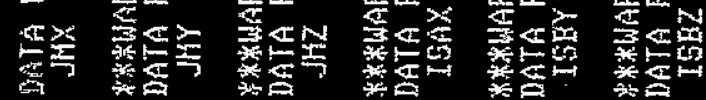


Rev. 0

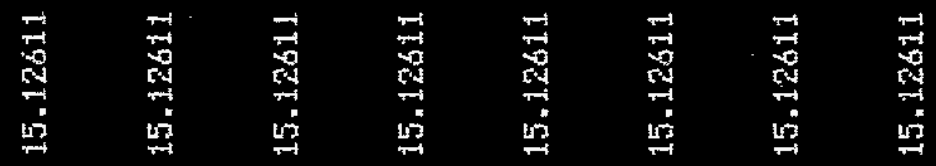

불

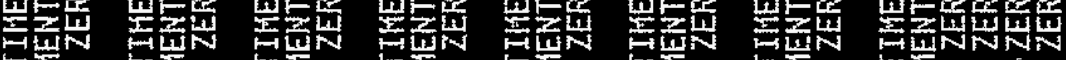

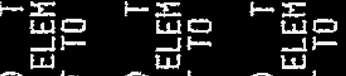

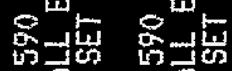

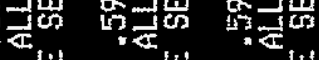

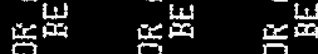

II

恶

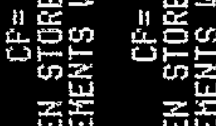

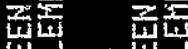

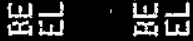

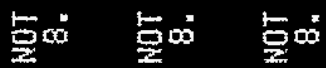

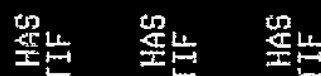

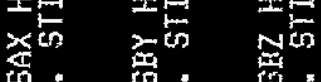

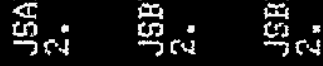

窝

단

*是它

,

我密

$5^{2}$

我姑

te
画山

in

墭要

*

$\sin 6$

焉点乐

它西

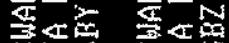

我影

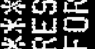

its

호응동

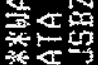

내뭉

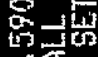

눈

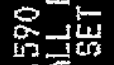

$\frac{1.10}{w .3}$

$0^{\circ-1}$

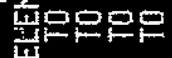

Q_tutetutus

出 崖

뜰

点而

它恶

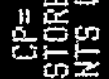

恶

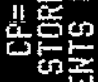

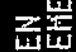

㟧击

$5 \infty^{\circ}$

50 50 50

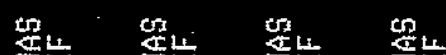

itiv

近

恶迹

us

*

Lects

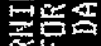

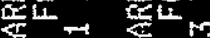

要边

倧吉

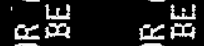

응

学雪

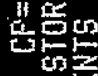

曹

존닌

崮㟧

C.

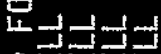

il

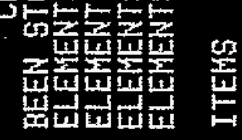

五む்ன்ன்

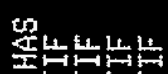

conten

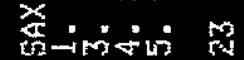

ज्ञ

焉出 璐

in

速幽要

एध一

㱐䍃

남

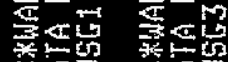

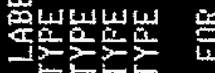

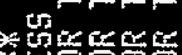

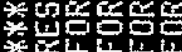

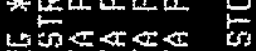

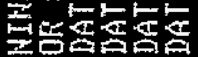

类

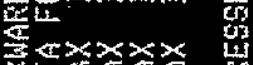

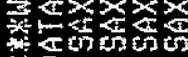

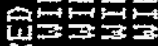

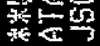

照区

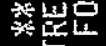

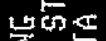

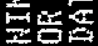

j4⿻

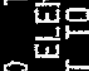

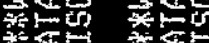

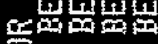

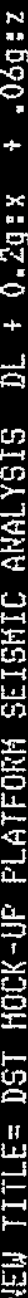

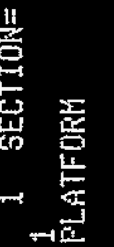

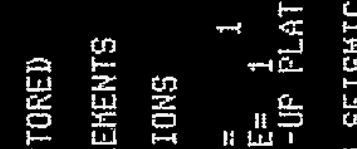

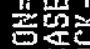

넌동

Te

$\stackrel{\infty}{\rightarrow}$

兵

$\varliminf_{i=1}$

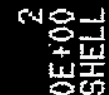

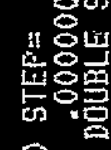

菭․ㅐ

常

频

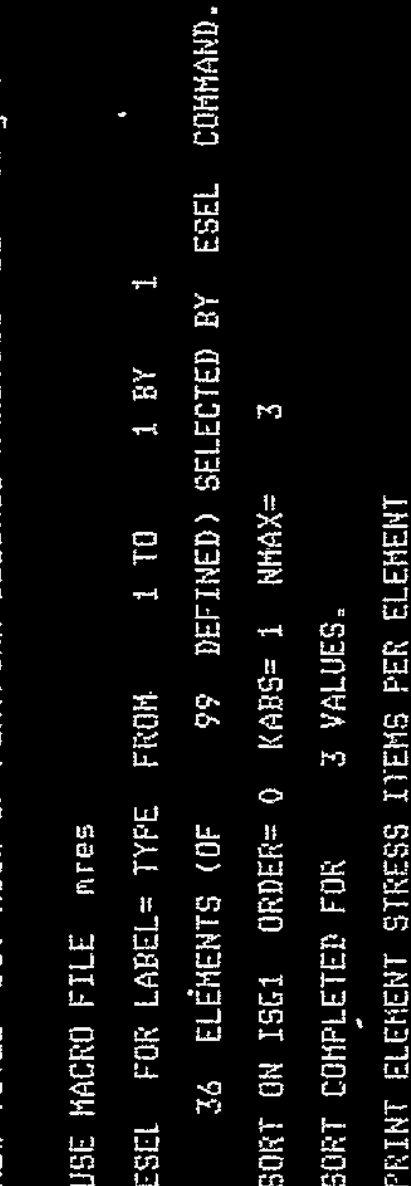

$$
A-61
$$


哭

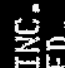

党

$\stackrel{20}{2}$

证然

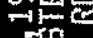

Thts

更的要

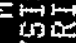

$\frac{1}{2}$

总室 些

总

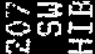

는

s.

ot

总的

B?

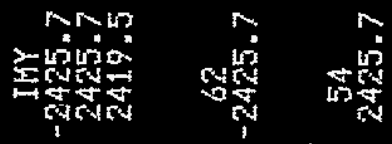

$\cos _{x \rightarrow 1} x$

난

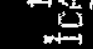

T象

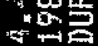

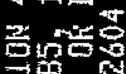

농

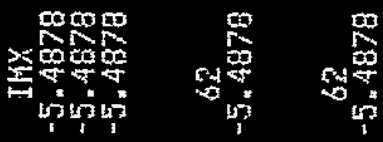

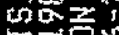

:

岩的?

然果

का

잘

* men

新

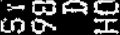

$\left.i^{-1}\right)^{n-1}$

Tion

$+$

늘

ton

兽

焉- $>$

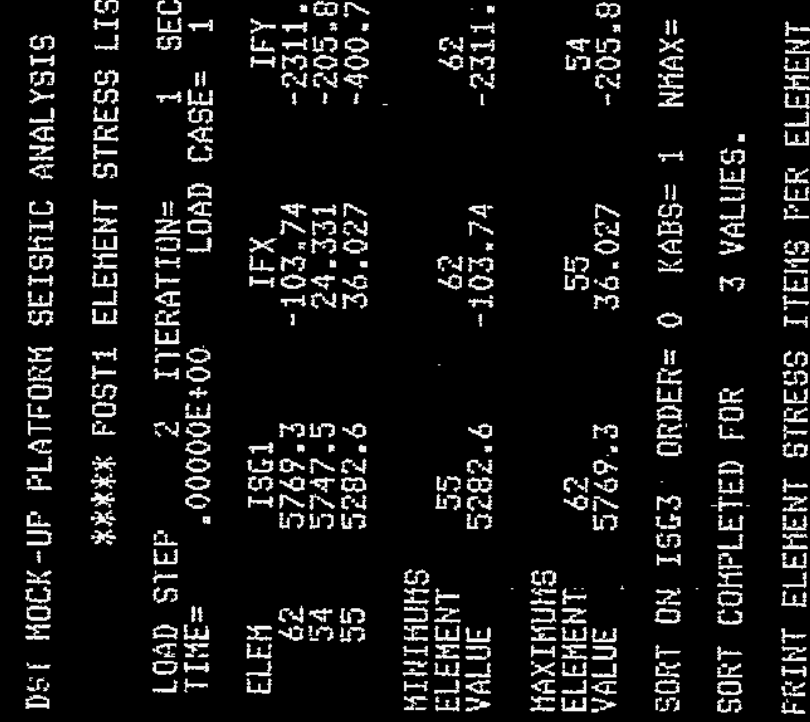

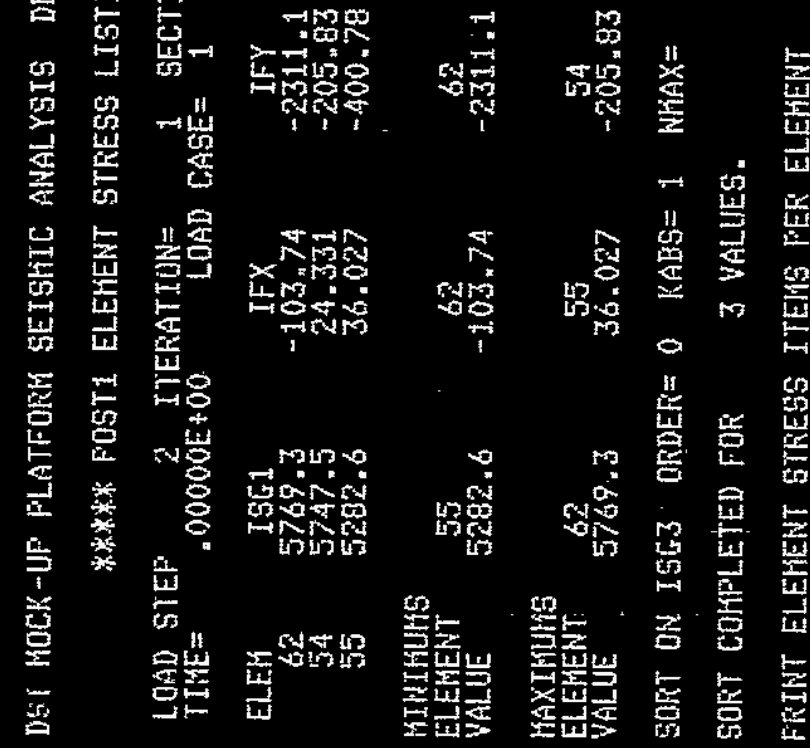

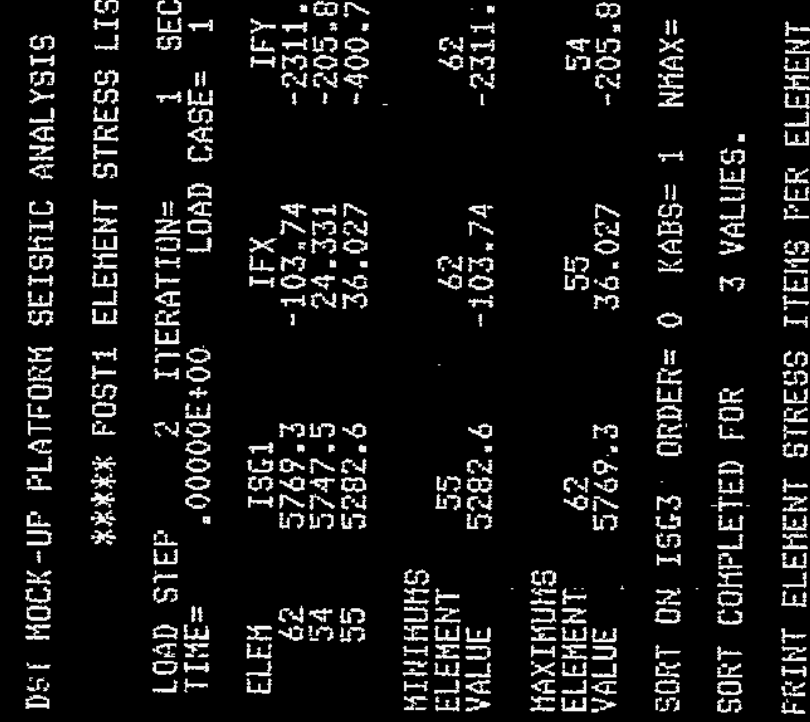

象昌

욜

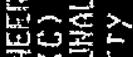

$\rightarrow$

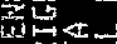

i 단단

$\operatorname{nis} 0$

整铝 毛步

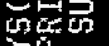

路品 
0

g

害㤩

ochin

ton

$\rightarrow$ tin

$\rightarrow$ in

and

oto

运

余迹

좀웅

$\underset{40}{40}$

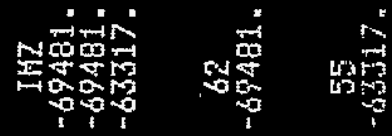

0 的的

为

묭

and

m

ogt

开

50

\&

登明兵昰

象

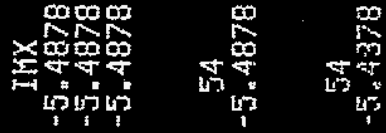

$\cot _{x \rightarrow 1}$

w

的焉要

州來的

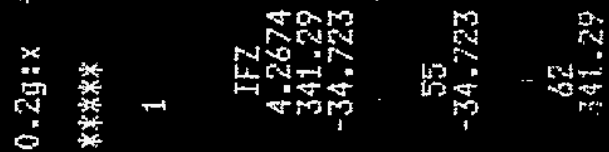

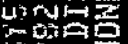

of ${ }^{7-1}{ }^{n}$

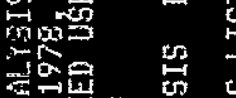

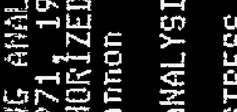

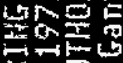

可而山

至致要

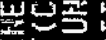

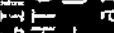

馬1

, 些些姑

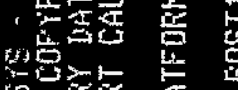

包要

和山娄

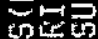

实点

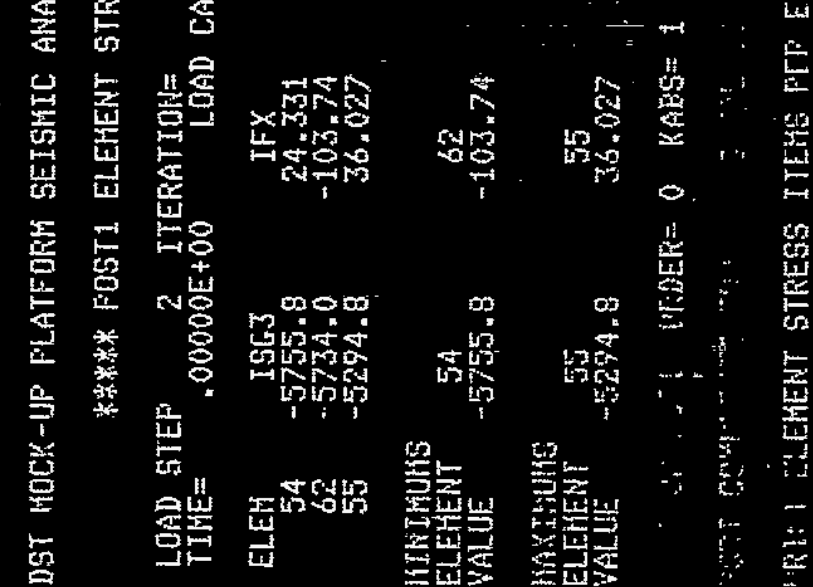


HOF

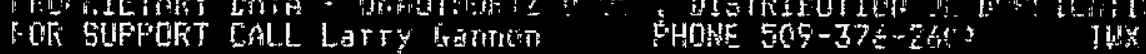

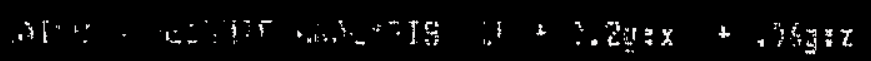

****** FUST1 ELEHENT STRESS LISTING *****

LUAD STEP 2000 ITEFATION= $\frac{2}{1}$ SECTTON=

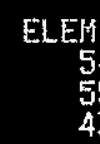

$$
\begin{gathered}
5951 \\
5284.1 \\
3378
\end{gathered}
$$$$
\begin{gathered}
\text { JFX } \\
-27.027
\end{gathered}
$$$$
-27.027
$$$$
-863.85
$$

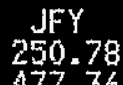

477.36

39.45 .8

JFZ 50.037
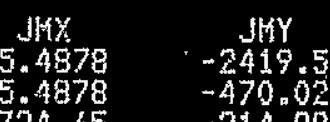

4.112

324.65

$-470.02$

43120.

MTNILUME

ELEMEK

43
2692.1

$-863.85$

254.78

$-211.29$

5.4878

54
-2419.5

$-37268$

MAXIKUHS

VLEYEN

5254 . $\quad-54.027$

$\begin{array}{cc}43 & 55 \\ 3945.8 & 50.039\end{array}$

324.65

43
-214

SOKT ON JSG3 ORUER $=0$ KAES $=1$ NHAX $=3$

SORT COKPLETED FOF

3 VALUES.

FRIHT ELEMEHT STRESS ITEHS PER ELEMENT 


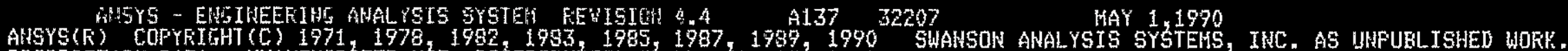

A137 32207

MAY 1,1970 FROFFIETAFY DATA - UNALTHOKIZED USE, DISTRIBUTIOH OK DUFLICATIOR IS FROHIBITED, ALL RIGHTS RESERVED.

FOR SUPPORT CALL LaTTY GamTON PHOHE 509-376-2604 THX

DST HOCK-IJP FLATFQRH SEISHIC AHALYSIS DL + 0.2g: $+.06 \mathrm{~g}: \mathrm{z}$

15.1261 AUG $1,1995 \mathrm{CF}=$

.620

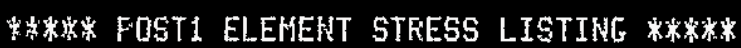

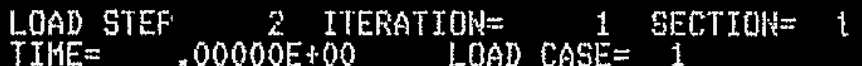

\begin{tabular}{|c|c|c|c|c|c|c|c|}
\hline $\begin{array}{c}\text { ELEEf } \\
54 \\
55 \\
43 \\
43\end{array}$ & $\begin{array}{r}5963 \\
-5293.3 \\
-3342.1 \\
-2996.4\end{array}$ & $\begin{array}{c}\text { JFX } \\
-27.027 \\
-40.621 \\
-863.85\end{array}$ & $\begin{array}{l}\text { JFY } \\
250.78 \\
477.38 \\
3945.8\end{array}$ & $\begin{array}{c}\text { JFZ } \\
4.7227 \\
50.039 \\
-211.29\end{array}$ & $\begin{array}{c}J 1 Y X \\
5.4878 \\
5.4878 \\
324=65\end{array}$ & $\begin{array}{c}J 14 Y \\
-2419.5 \\
-470.02 \\
-214.99\end{array}$ & $\begin{array}{c}J H Z \\
63317 \\
43120 . \\
-37269 .\end{array}$ \\
\hline
\end{tabular}

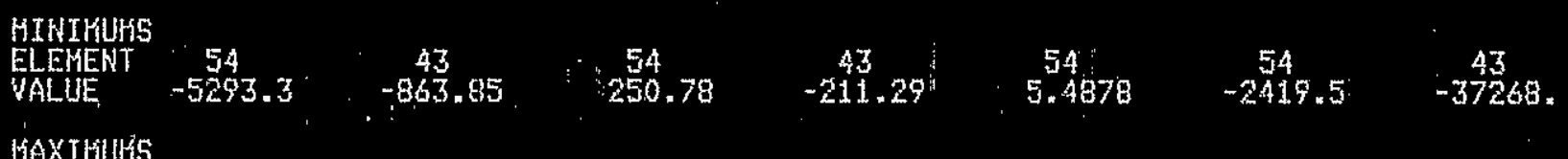

\begin{tabular}{|c|c|c|c|c|c|c|c|}
\hline ELEMENT & 43 & 27.027 & $\frac{43}{3945.8}$ & 50.550 & $\begin{array}{c}43 \\
324.65\end{array}$ & $\begin{array}{c}43 \\
-214.99\end{array}$ & $\frac{54}{63317}$ \\
\hline
\end{tabular}

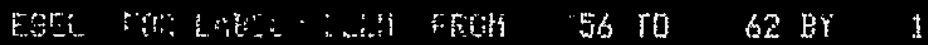

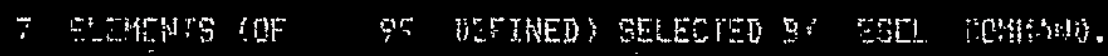

".." ‥ 
暂

哭

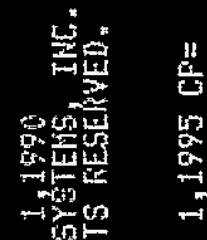

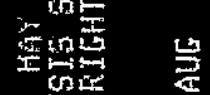

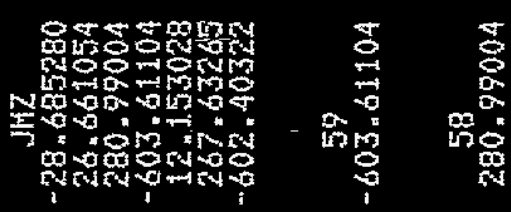

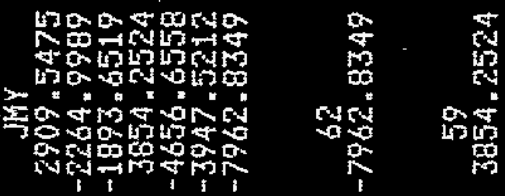

rit

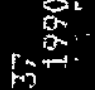

tin.

踏

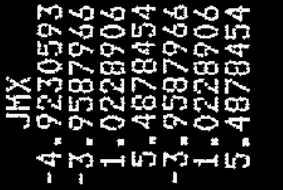

要

잔,

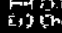

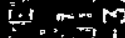

sing

, 然

‥

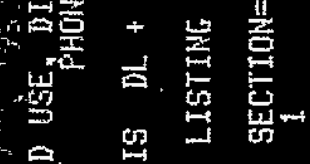

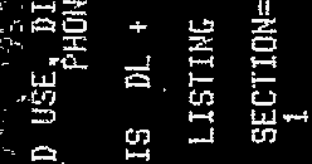

$x \quad$ Fangatas Br.

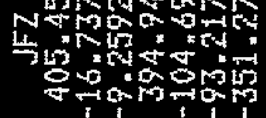

요

焉 要

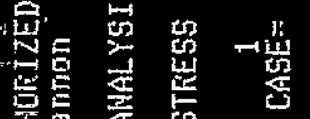

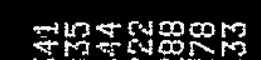

mopotido

3000000

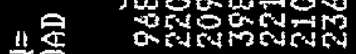

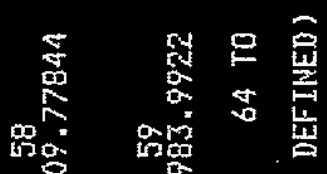

品营 点

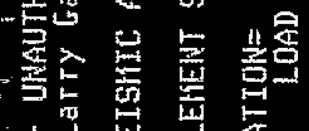

1 i

돈단

量

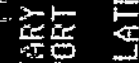

出空

政

跐

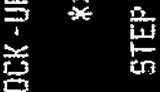

욜

䓢

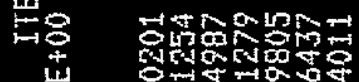

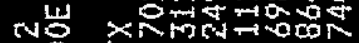

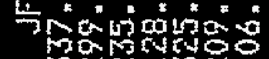

总 哭

堅

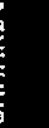

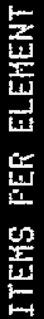

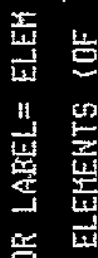

总

$\frac{1}{42}$

A -66 


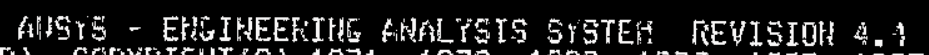

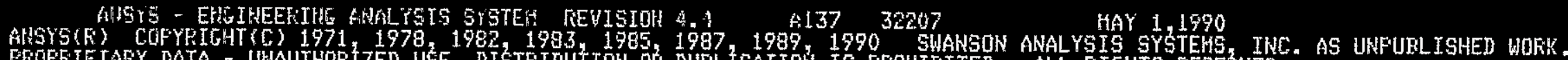

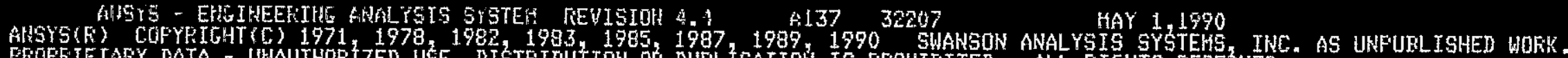

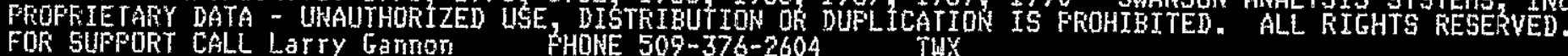

DST KOCK-UF PLATFORH SEISMIC ANALYSIS DL +0.2g: $+.06 \mathrm{~g} z$ ***** FOST1 ELEHENT STRESS LISTING ******

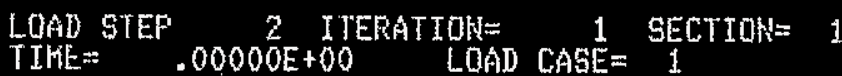

\begin{tabular}{|c|c|c|c|}
\hline 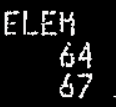 & $\begin{array}{c}J F \times \\
305.84344 \\
23.765065\end{array}$ & $\begin{array}{c}\text { JFY } \\
177.90003 \\
170.83057\end{array}$ & $\begin{array}{c}\text { JFZ } \\
-57.586415 \\
-56.29050\end{array}$ \\
\hline
\end{tabular}

MITIHUWS

ELEHENT

$$
\begin{array}{ll}
67 & 67 \\
23.765865 & 170.83057
\end{array}
$$

$-57.586415 \quad-6.7413946$

$-21.64 .1349$

1. 1487131

HAXT任UHS

ELEHENT

64

309.8 .4344

177.90003

$-56.279058$

$=040783521$

$-2107.4312$

64

[ESFL FOR LABEL= ELEH FROH 69 TO 69 BY 1

1 ELEHENTS (OF 99 IHEFIHED) SELLCTED BY ESEL COHWAND.

PFINT ELEHEHT STFESS ITEITS FER ELEHENT 
8

\&

$\sum_{i=1}^{n}$

Sin

En. Wu

-

的电

论

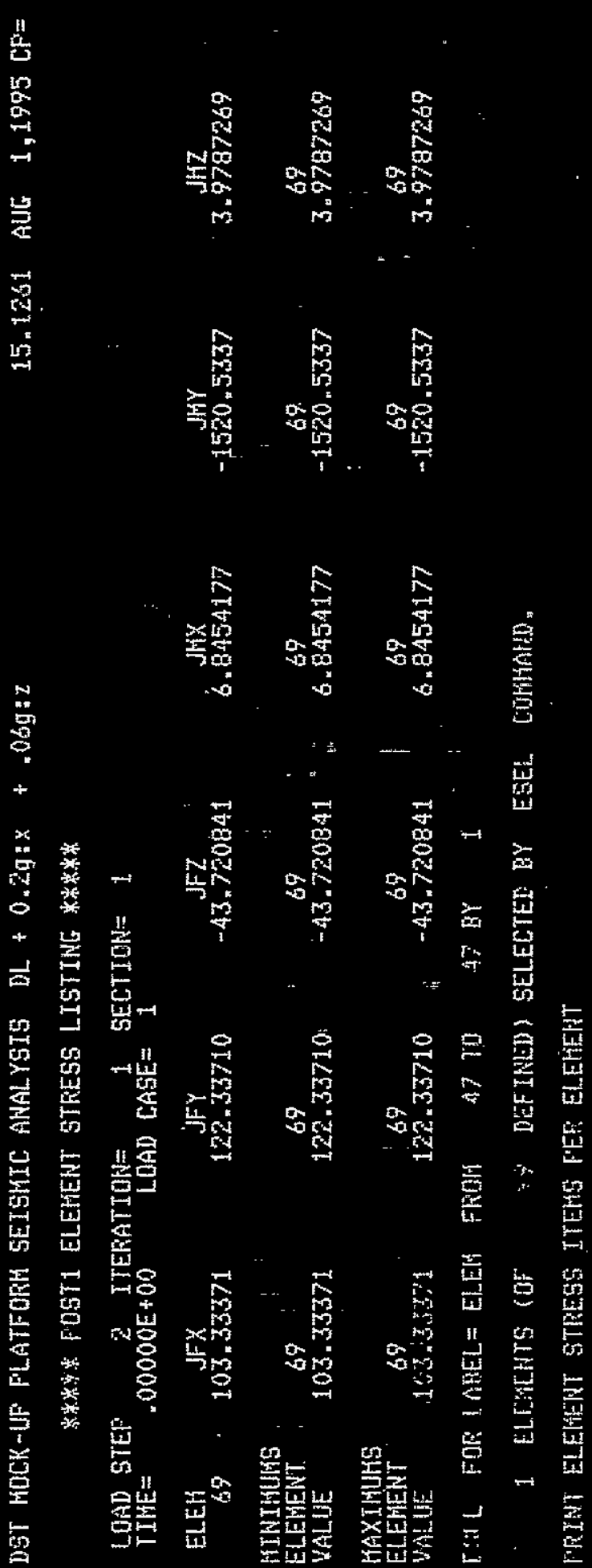

整自

密点

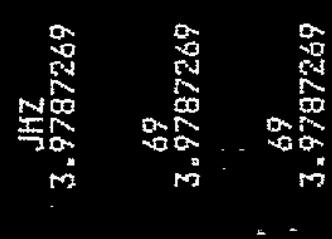

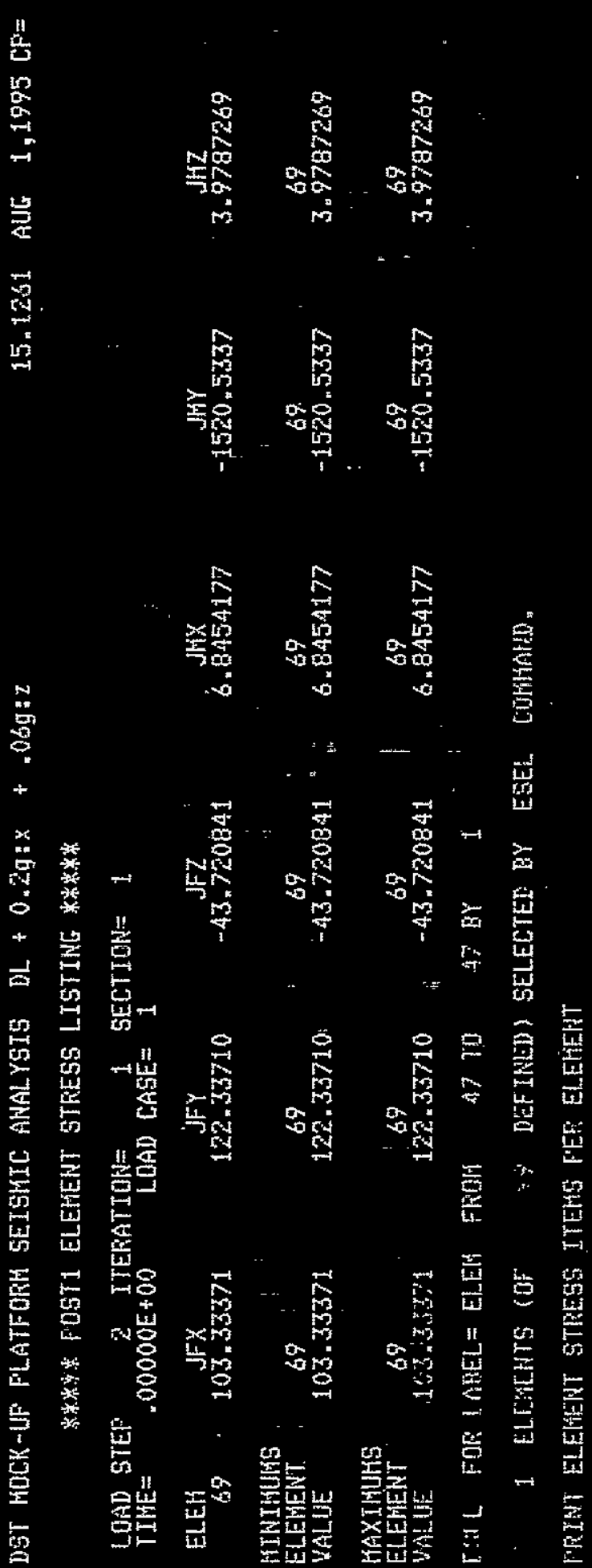

i)

I

sin

in

orre

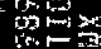

- in

$x=$

的要

을

in 13

$\Rightarrow$

as

$x_{x \rightarrow 0}=0$

minu

的是

hit

(1)

$\rightarrow$

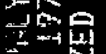

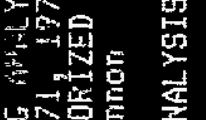

的晴要

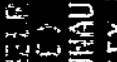

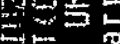

焉, 可

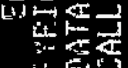

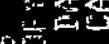

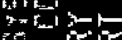

$=\ldots$

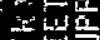

的些宛

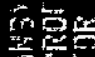


8

th

密

논

$\underset{4}{2}$

농

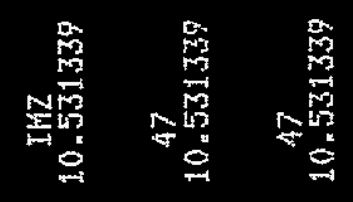

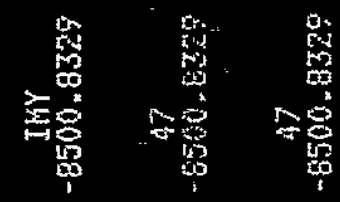

$a^{\frac{x}{2}}$

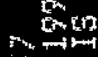

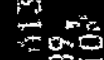

照的象

$\rightarrow<$

$+8$

$\rightarrow \rightarrow$

瑟证语

드은

$\rightarrow=0$

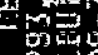

$=2-7$

$5 x x^{2}$

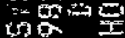

or 10

aros

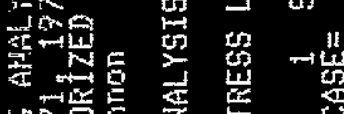

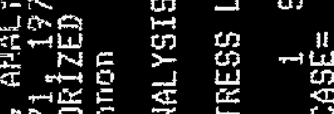

的是要

행존전

政

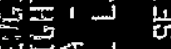

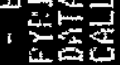

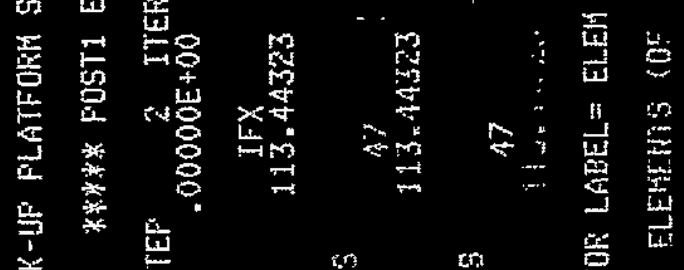

$\rightarrow$ wis

两

분쪽

60

的要点

뭉

急营

草

क

㟧-

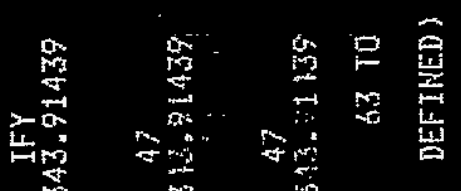

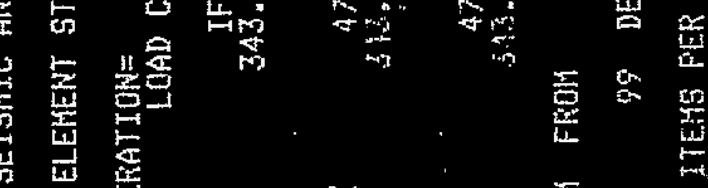

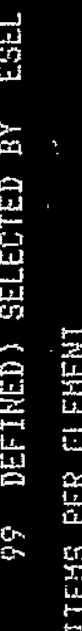

告 岕

总 焉 
옹

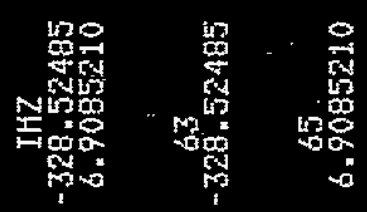

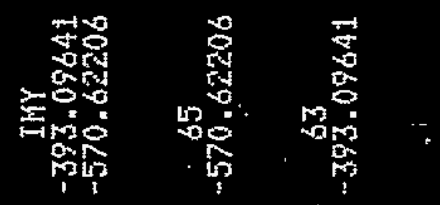

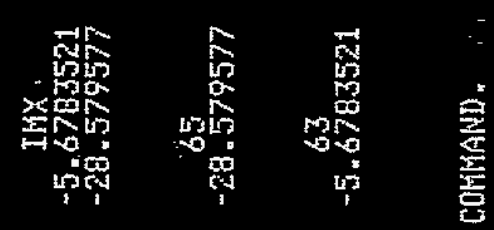

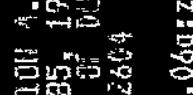

$\begin{array}{r}N \\ 5 \\ \hline\end{array}$

$5^{-1}$

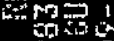

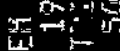

matis o

$-2$

$=-m^{2}$

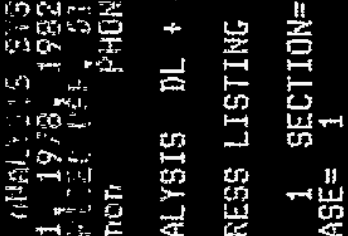

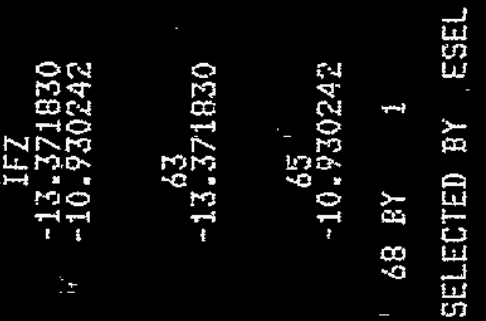

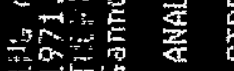

7 .

的经起

.

.

4

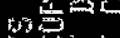

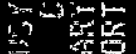

$=5, \frac{1}{6}$

놉용

ne

竤

密

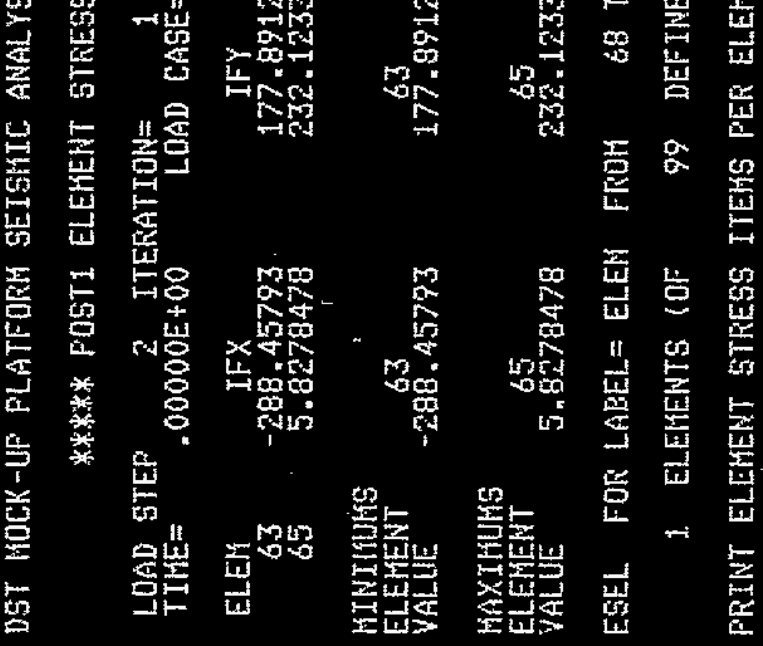


웅

to

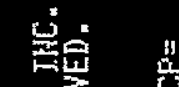

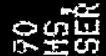

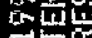

$\rightarrow-5$

당

的

xtrit

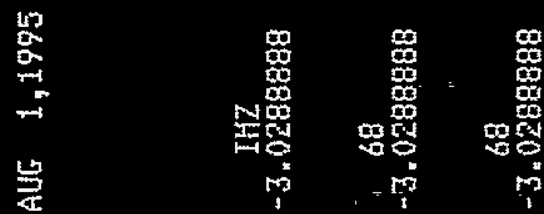

竞费

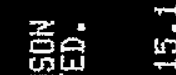

穿

S言

酷

of

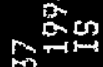

줄

맘늘

단다

ब大ed

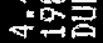

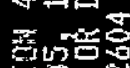

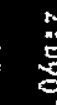

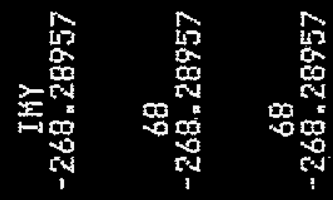

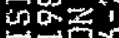

내요

的承

The

witu

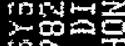

os

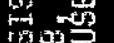

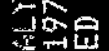

No

事

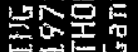

딴단

봃을

단,

페

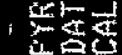

ge.

穿

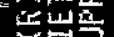

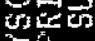

数新

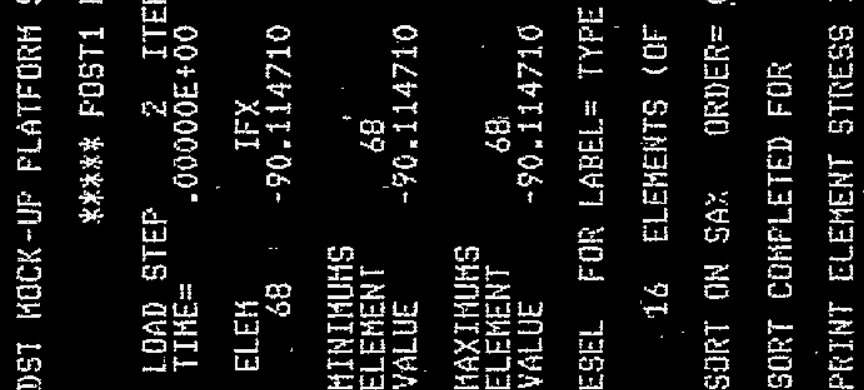

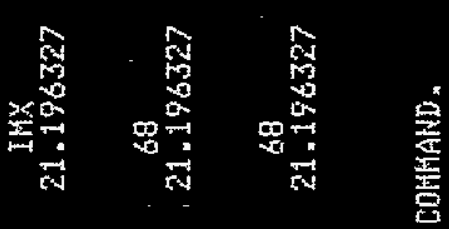

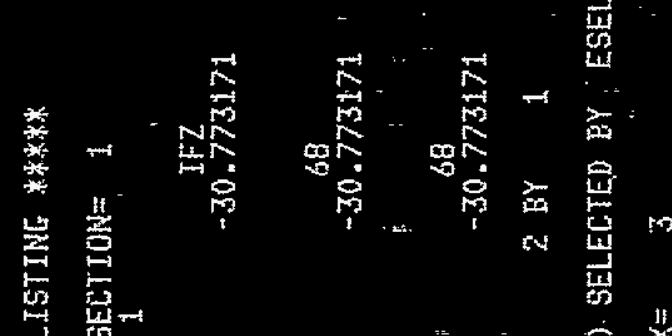

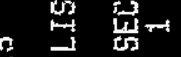

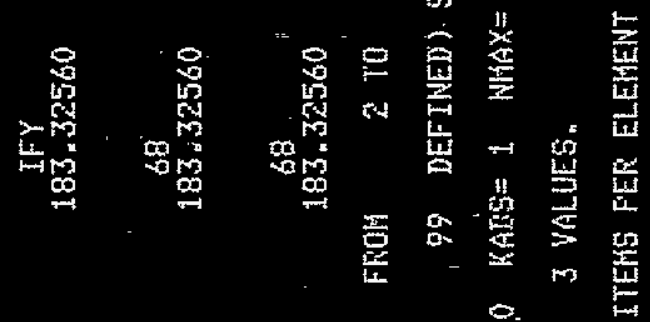

분 


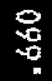

a

这望

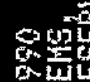

$\rightarrow$

$\rightarrow$

20

程的

0당

迹娄

곯

r.te

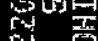

我

intin

$\tan$

aㅏ을

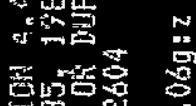

뭉

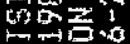

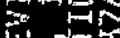

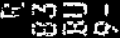

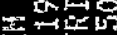

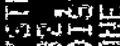

내봉

6)

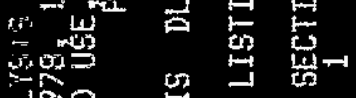

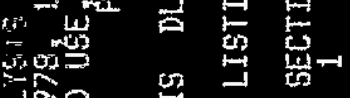

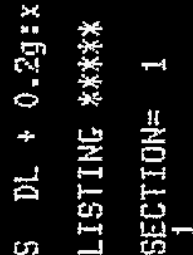

资墨

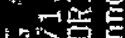

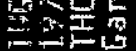

然

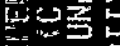

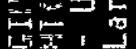

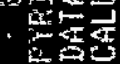

군

政

isis

.

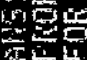

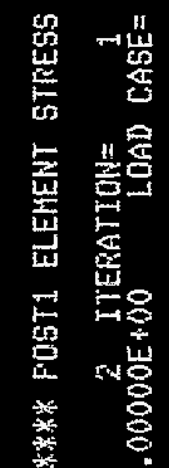

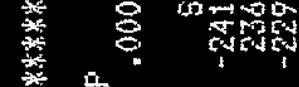

善

尊皆

면

$\therefore$ 整 些

点

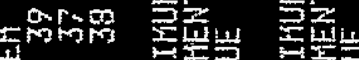

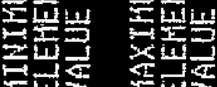

崺 $^{-\frac{5}{4}}$

峘

퐁

茹

車

봃

을

旨

獣

ordo

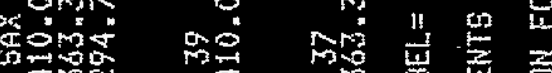

峷

严? 


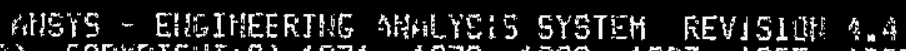
A 137320 MAY 1,1970

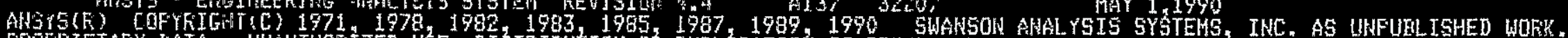

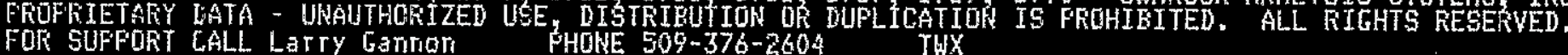

IYST HOCK-UP PLATFOFH SEISHIE AHALYSIS DL + $0.2 \mathrm{~g} 7 \mathrm{x}+.06 \mathrm{~g} Z$

***** FOST1 REACTION FORCE LIGTING *****

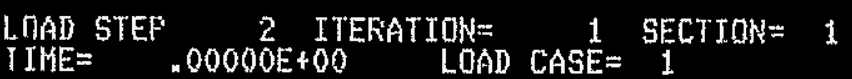

THE FOLLOWING $X_{Y} Y, Z$ FORCES ARE IN GLOBAL COORDTNATES

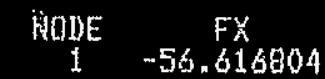

$$
\begin{aligned}
& \text { + } 2 \quad-56.616004 \\
& 3 \quad-794.03812 \\
& 4 \quad-606.45787 \\
& 6 \quad-617.36442
\end{aligned}
$$

TOTAL.

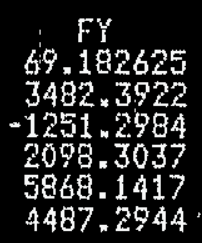

14754.016

$$
\begin{gathered}
F 2 \\
-219.66510 \\
-369.76837 \\
-108.69349 \\
-1.46 .90850 \\
260.53036 \\
-281.48738 \\
-884.89248
\end{gathered}
$$

GORT OH ISGI OKDEF $=0$ KABS $=1$ NKAX $=$

SORT LOHPLETED FOR

3 VALUES.

$$
\text { VY. }
$$

$.00000000 E+100$ $.00000000 E+00$ $00000000 \mathrm{E}+00$ $.00000000 \mathrm{E}+00$ $.00000000 E+00$ $.00000000 E+00$

$.00000000 \mathrm{E}+00$
WY $.00000000 E+00$ $.00000000 \mathrm{E}+00$ $.00000000 \mathrm{E}+00$ $.00000000 \mathrm{E}+00$ $.00000000 E+00$ $.00000000 E+00$

$.00000000 \mathrm{E}+00$
112

$00000000 \mathrm{E}+00$ $.00000000 E+00$ $.00000000 \mathrm{E}+00$ $.00000000 \mathrm{E}+00$ $.00000000 E+00$ $.000000005+00$

$.00000000 \mathrm{E}+00$

PRINT ELEFENT STRESS ITEHS PER ELEHEKT 
8

r

趈

2몸

문분

$\rightarrow$

20

ent

宸

空空

좋웅

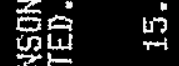

点

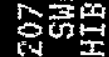

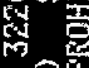

落地

$\mathrm{M}$

xac

마말

is

- 50

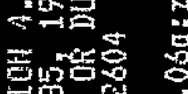

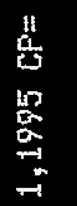

峞

क思

$\rightarrow-0$

㟧舟

器要要

品

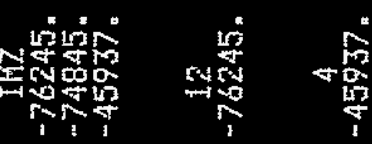

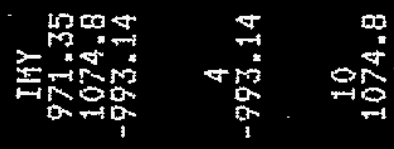

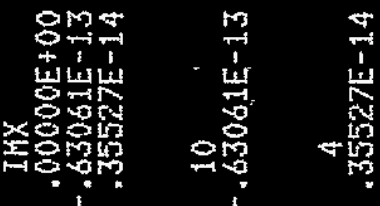

- 때

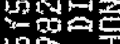

$\operatorname{lin}^{2}$

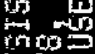

-

空可点

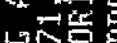

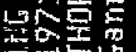

군굴

변온

분도

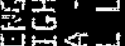

1 둔

을

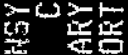

原

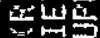

0

嚁

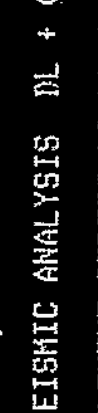

气

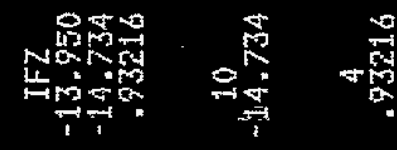

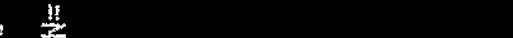

58

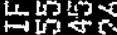

世的

$\infty$
4
4

$\infty$

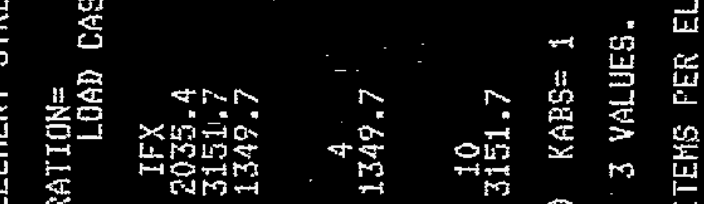

$\leftarrow$ 点

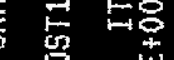

的 的

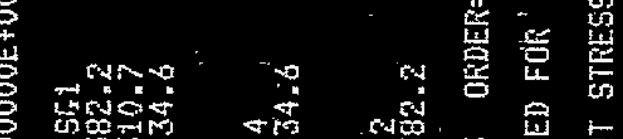

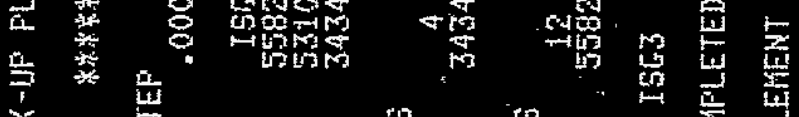

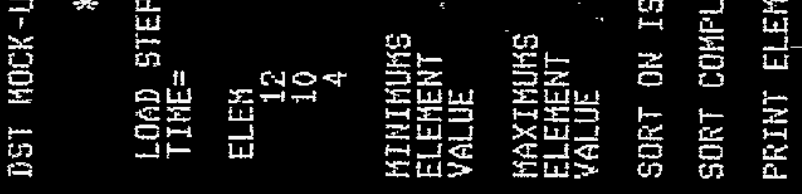




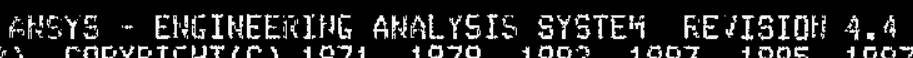

A.37 32207

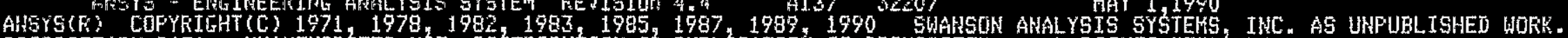
FEOFFIETARY DATA - UNAUTHORIZED USE, DISTRIEUTION OR DUFLICATION IS FROHIBITEI. ALL RIGHTS RESERVED.

FOF SUFFORT CALL LaTrY ramion PHOAE 509-376-2604 TWX

DST HOCK-UP PLATFOFH SEISHIC AHALYSIS DL + $0.2 \mathrm{~g}: \mathrm{x}+.06 \mathrm{~g}: \mathrm{z}$

**w* FOST1 ELERENT STFESS LISTING ******

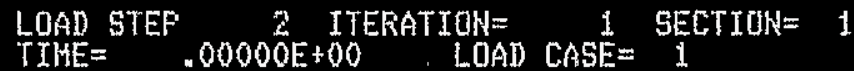

\begin{tabular}{|c|c|c|c|c|c|c|c|}
\hline $\begin{array}{l}\text { ELEH } \\
10 \\
12 \\
4\end{array}$ & $\begin{array}{r}1953 \\
-6384.5 \\
-6275.7 \\
-3894.4\end{array}$ & $\begin{array}{c}\text { IFX } \\
3151.7 \\
2035.4 \\
1349.7\end{array}$ & $\begin{array}{c}\text { IFY } \\
-545.03 \\
-555.64 \\
-326.04\end{array}$ & $\begin{array}{c}\text { IFZ } \\
-14.734 \\
-1.3 .750 \\
.93216\end{array}$ & $\begin{aligned} & \text { IHX } \\
&-.63061 E-13 \\
& . 00000 E+00 \\
&- 35527 E-14\end{aligned}$ & $\begin{array}{c}14 Y \\
1074.8 \\
971.35 \\
-993.14\end{array}$ & $\begin{array}{c}I H Z \\
-74845 \\
-76245 \\
-45037\end{array}$ \\
\hline
\end{tabular}

HIHTHUHS

ELEMENT

10
-6384

$\begin{array}{cc}4 \\ 1349.7 & -555.64\end{array}$

10

$.35527 E-14-993.14$

-45637 .

KAXIYHUHS

ELERENT

$-3894$

$\begin{array}{lr}10 & 4 \\ 3151.7 & -326.04\end{array}$

.9321 .6

$-.63031 E-13-993.14$

$-7645$.

SORT ON JSG1 ORDEF $=0$ KAES $=1$ WHAX $=$

SORT COMFLETED FOR 3 VALUES.

PFIHT ELEKEEHT STRESS ITEMS PER ELEKEENT 
8

哭

望息 芒

요에

知畐

$\prod_{i \rightarrow \infty}$

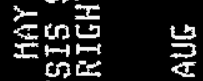

尊盒嵒

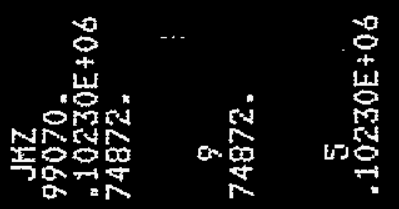

S敋

N

oin

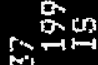

证空

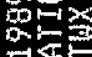

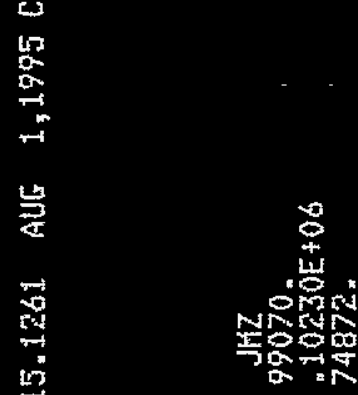

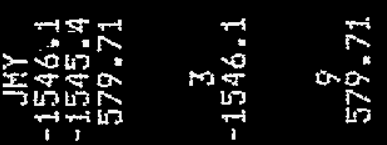

齿

-

뭉

$n$
0
0
0

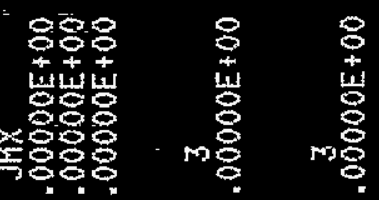

虽品

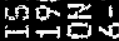

要

嘼象象

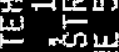

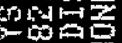

का

$\cos ^{n-1}$

in

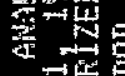

송

돈

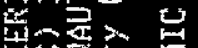

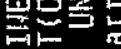

군

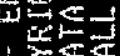

, >

문은

湈

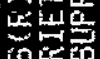

䱡

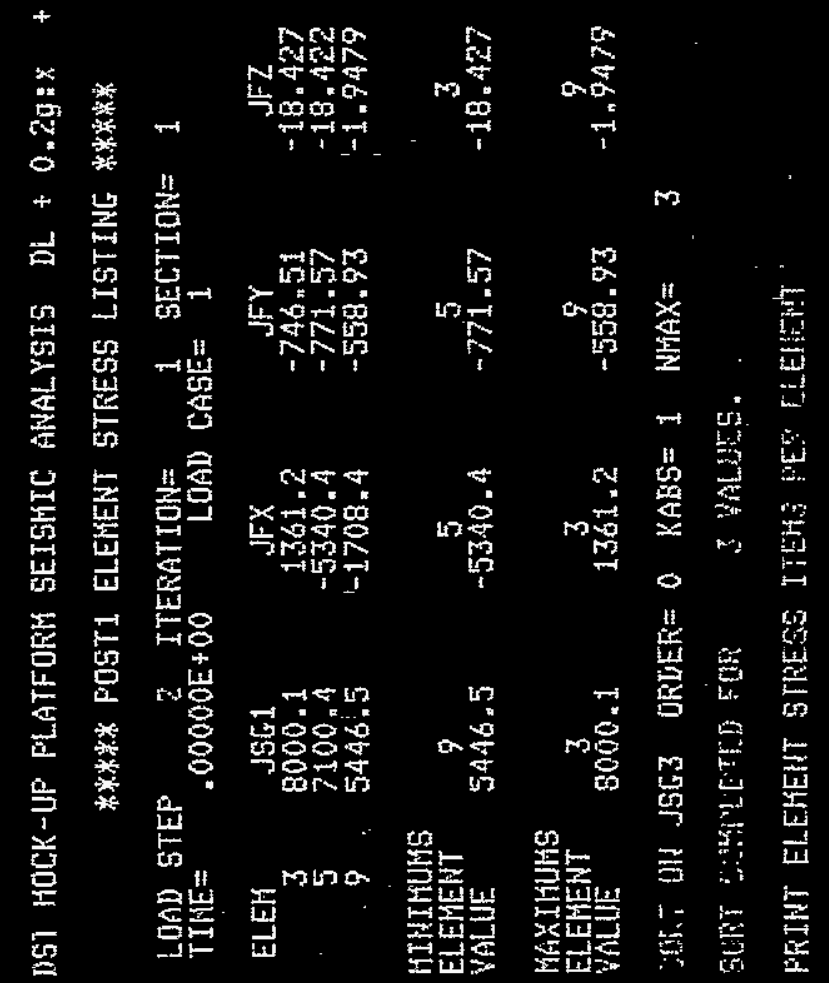


$\stackrel{9}{0}$

果

产

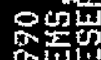

$\prod_{\rightarrow \rightarrow-1}^{\infty}$

的空

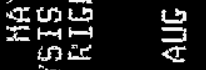

焉

舜嵒

$\stackrel{1:}{2}$

$\underset{21}{20}$

$\stackrel{4}{\stackrel{4}{4}}$

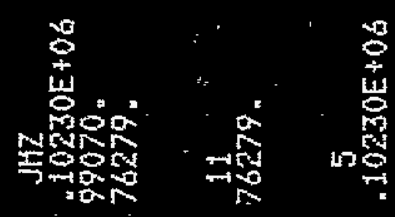

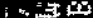

3

i.

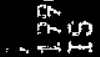

$\approx \pi$

㤎证爸

잔

단

$\therefore$

$=$ का

5

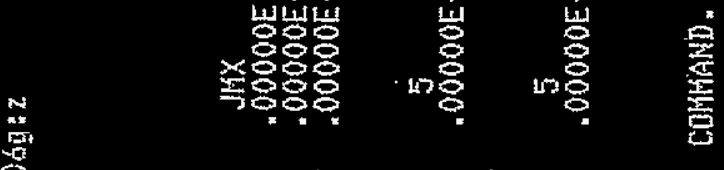

is

$4 x^{2}$

留新

in

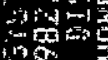

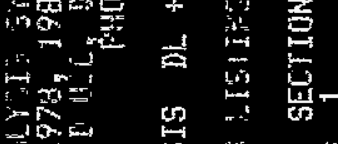

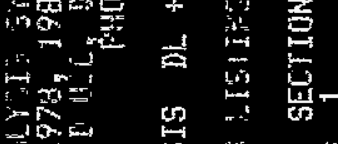

tis.

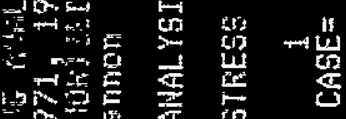

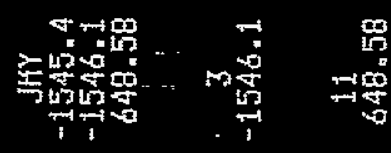

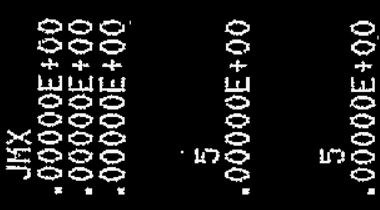

$=-\rightarrow$

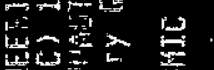

난.

整焉

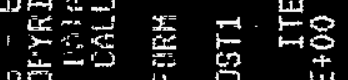

点包点

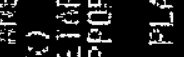

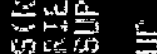

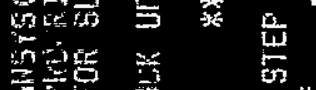

No

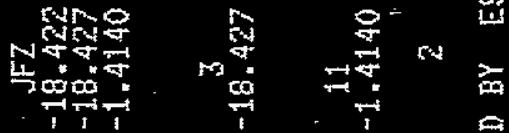

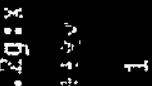

:

㟧

istum

交

$4 \operatorname{lig}^{2}$

17.

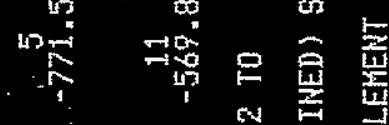

ㄴ.

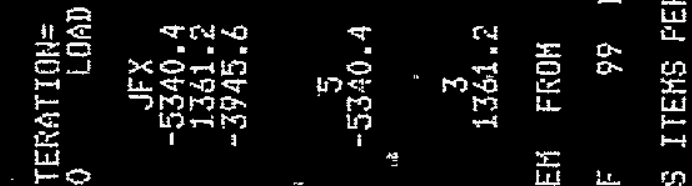

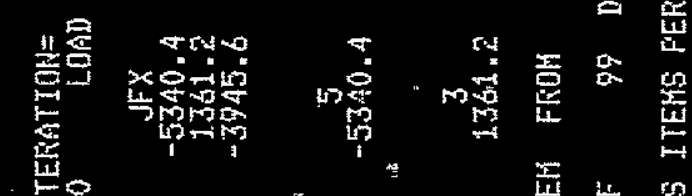

崖

8 a.

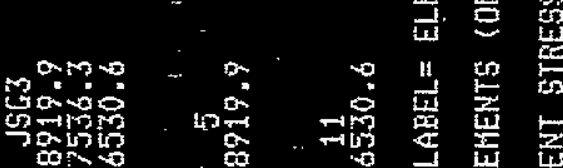

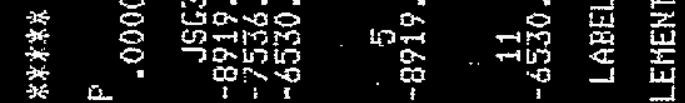

点 的昜

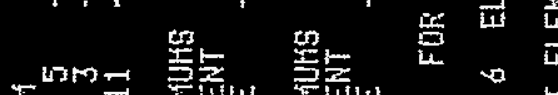

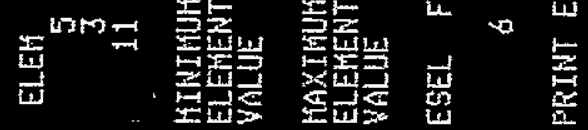


용

$x$

$\stackrel{5}{*}$

11

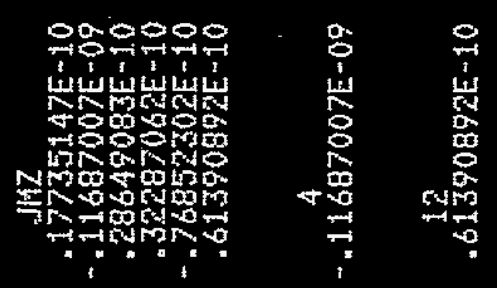

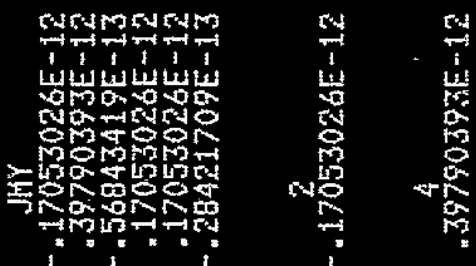

$m$ 蛋

and

zan

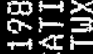

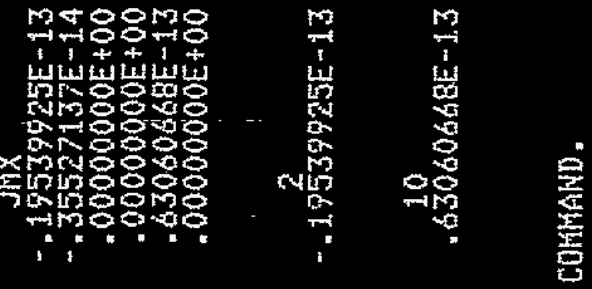

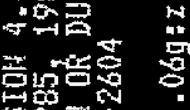

wo

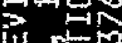

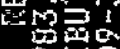

$x \rightarrow$

$5, n_{0}$

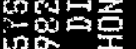

in

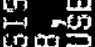

品稳

象豆焉

논올

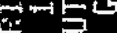

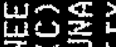

눈도의

些

; 紫然 nots 被骂

然

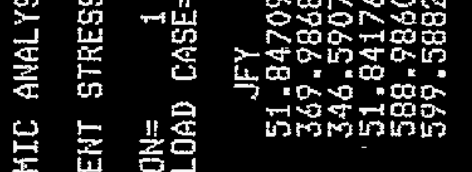

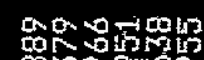

comago.

Nowm

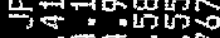

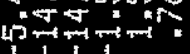

는

+ 닐

실

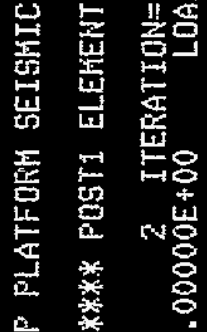

돈는

vosucos

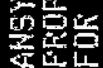

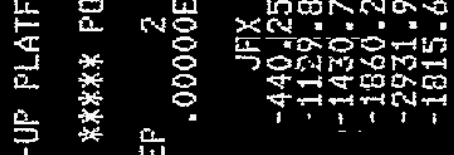

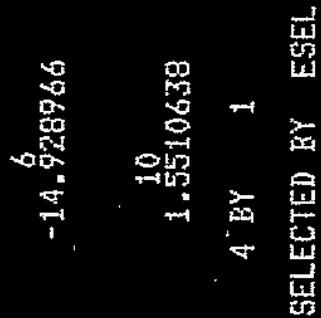

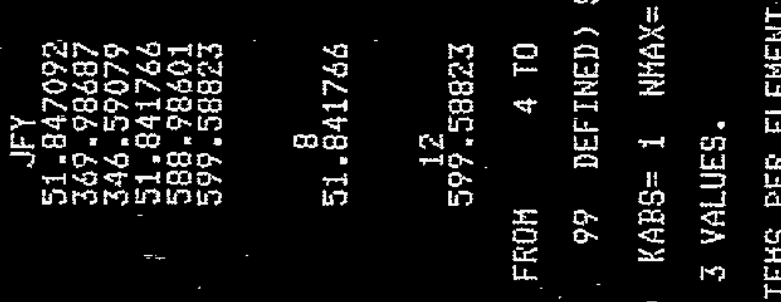

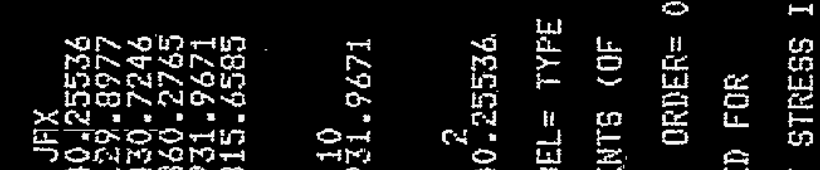

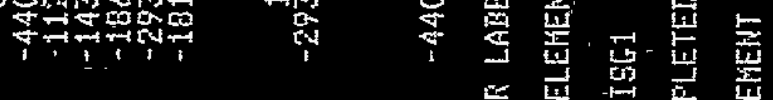

莺

$5 \quad$ St:

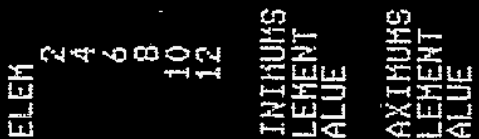

운

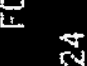

首

공 웅

$-5$

涌高

虽 
용

en

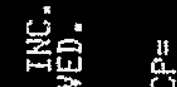

$00 \frac{14}{10}$

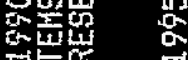

$\rightarrow>0$

Cot

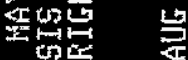

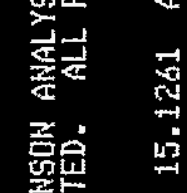

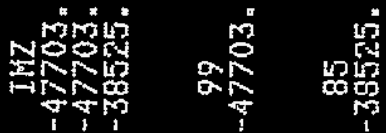

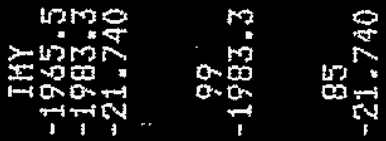

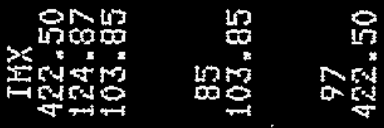

풀용

How

$\rightarrow \rightarrow \infty$

w $m$

क뭉

点

* +

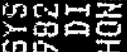

+ ب 韭

$x^{n+1} \min ^{n}$

$\cos 20$

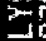

$\operatorname{lom}_{n}$

点m

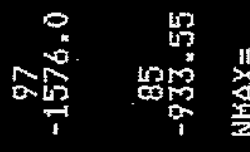

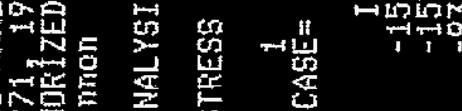

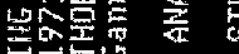

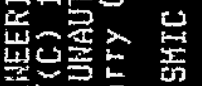

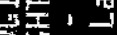

㟶

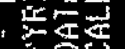

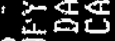

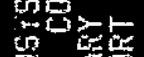

正政

돈설

tsing

紫兽

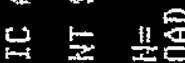

至是

- $\rightarrow$ को

品歪

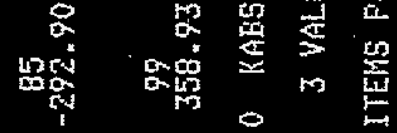

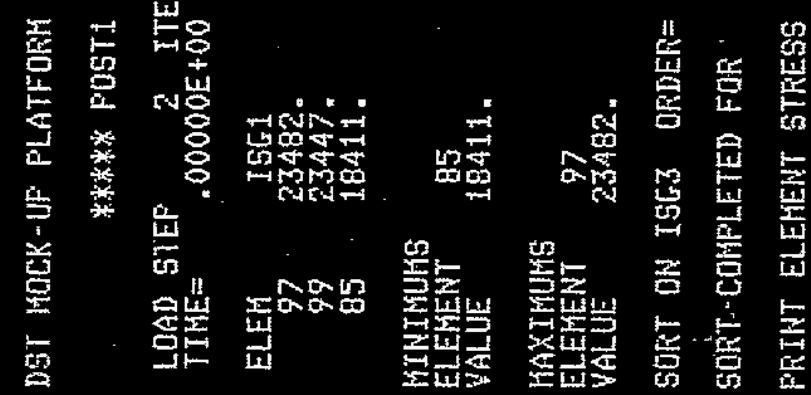




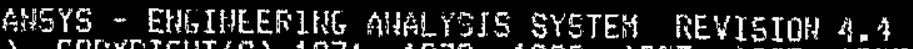

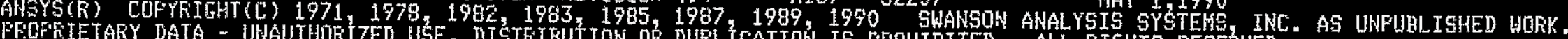
FEOFFIETARY DATA - UEAUTHORIZED USE DISTFIBUTION OR DUFLICATIOH IS FROHIBITED. ALL RIGHTS RESERVED.
FOR SUFPORT CALL La TY GaTmOT

DST HOEK-UF PLATFORH SEISMIC ANALYSIS DL + 0.2g: $\mathrm{x}+.069: 2$

*w*** FUST1 ELEHENT STRESS LISTING ******

LDAD STEP $=2$ ITERATIOK: 2000 LAD CASE $=\frac{1}{1}$ SECTION= 1

\begin{tabular}{|c|c|c|c|c|c|c|c|}
\hline $\begin{array}{r}\text { ELEH } \\
99 \\
97 \\
85\end{array}$ & $\begin{array}{r}1563 \\
-23724 . \\
-23672 . \\
-18184 .\end{array}$ & $\begin{array}{c}\text { IFX } \\
358.93 \\
245.93 \\
-292.90\end{array}$ & $\begin{array}{c}\text { IFY } \\
-1570.8 \\
-1576.0 \\
-433.55\end{array}$ & $\begin{array}{c}1 F Z \\
48.396 \\
1.45 .72 \\
-.59766\end{array}$ & $\begin{array}{c}\text { IHX } \\
124.87 \\
422.50 \\
103.85\end{array}$ & $\begin{array}{c}\text { IHY } \\
-1983.3 \\
-1965.5 \\
-21.740\end{array}$ & $\begin{array}{c}162 \\
-47703 \\
-47703 . \\
-39525\end{array}$ \\
\hline
\end{tabular}

HINIHUKS

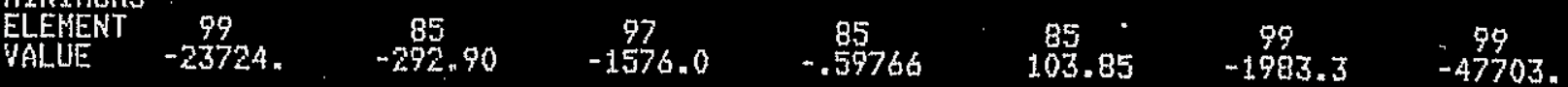

KAXTHUHB

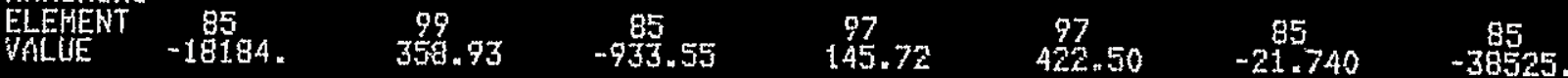

SORT DN JSG1 GRDER $=0$ KABS $=1$ NHAX $=3$

SORT COHFLETED FOK 3 VALUES:

FFIHT ELEHENT STRESS ITEHS PEF ELEMEHT 
$\stackrel{8}{?}$

喿

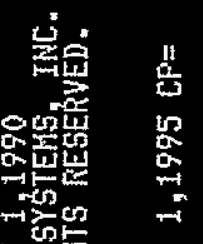

का

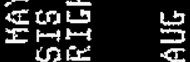

变古

磞离

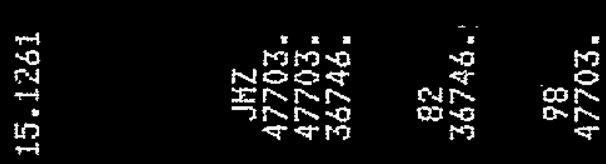

옥

窝

용

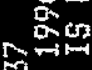

따을

였-1졸

똔

No

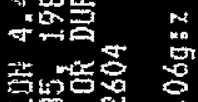

$\operatorname{tin}_{x \rightarrow 1} x^{2}$

is

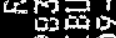

폰

in.

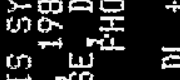

wing

瑟点

날

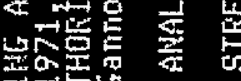

त्त.

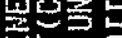

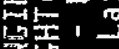

武空

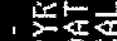

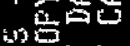

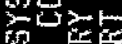

等窟

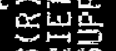

çis

㩆空

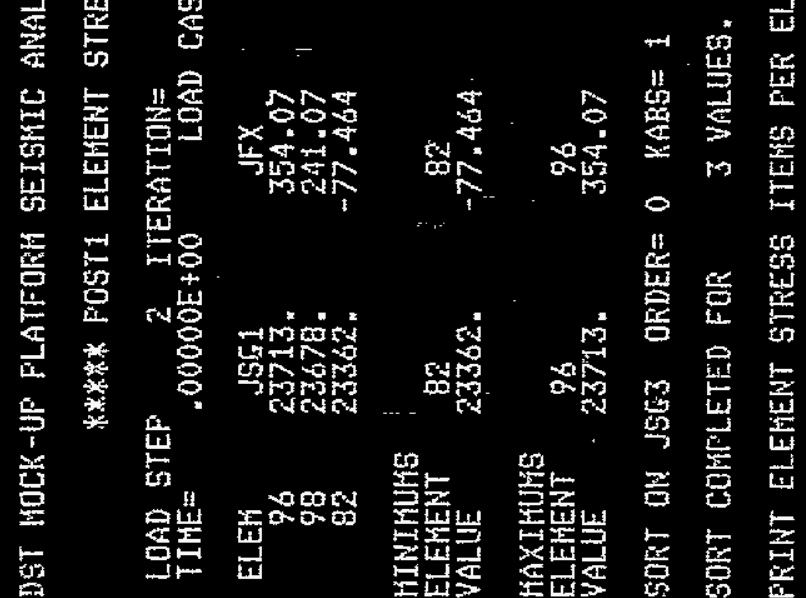




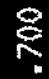

哭

$\sum_{\substack{i=1 \\ 1}}^{5}$

竞

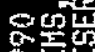

네는

$x_{i=1}$

$>$

요난

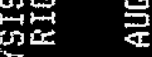

空表

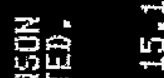

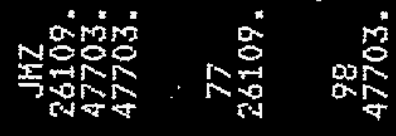

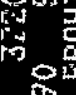

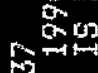

正

\%을

gis

-4

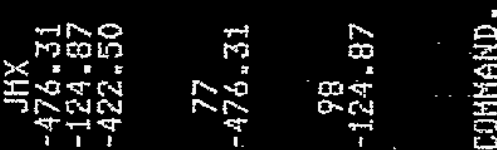

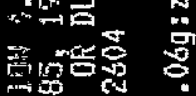

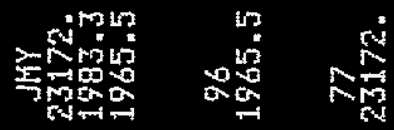

जis?

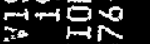

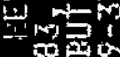

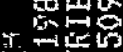

iti 5

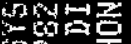

$\lim _{\substack{x \rightarrow \\ x}}$

$\operatorname{lig}_{i=1}^{n-4}$

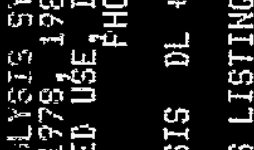

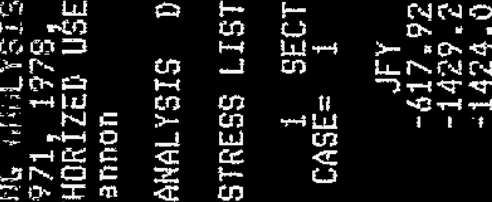

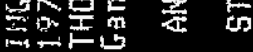

政

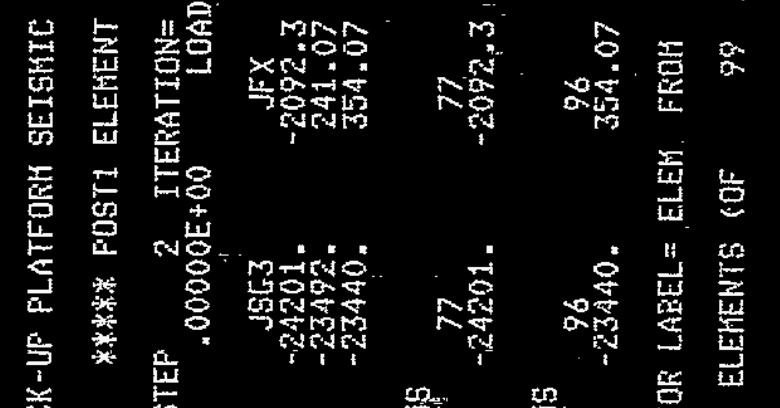

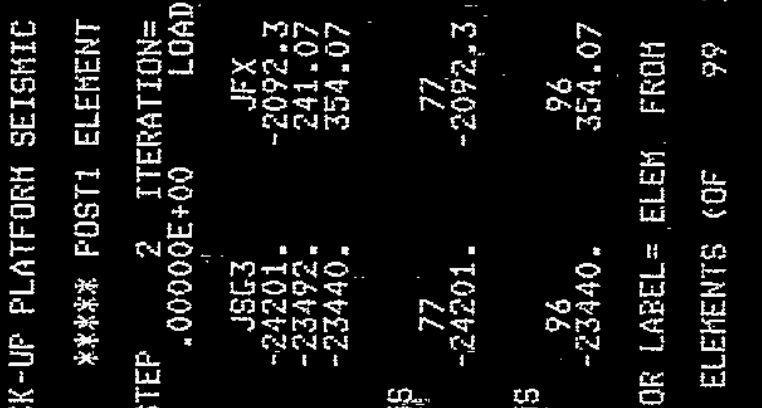

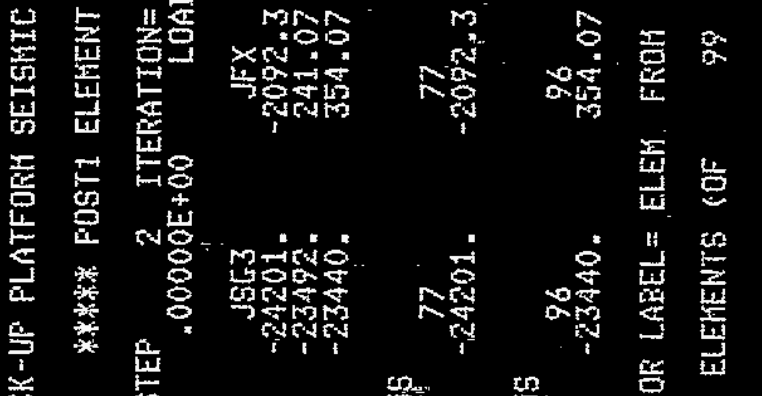

语:

运过

in후의

造政

c

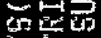

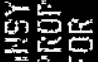

站皆

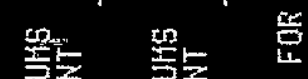

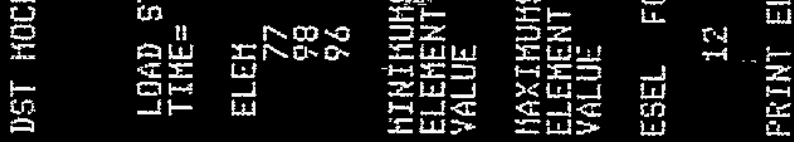


$\stackrel{0}{1}$

哭

焉事

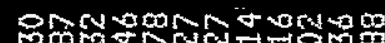

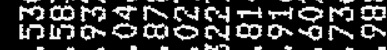

Then

cos

岁

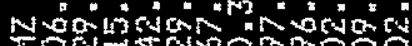

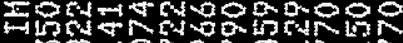

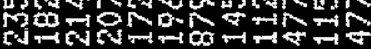

器

势考

穿

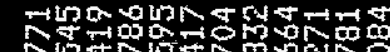

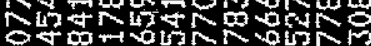

In

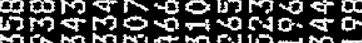

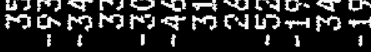

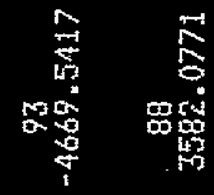

mit

(1)

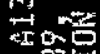

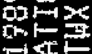

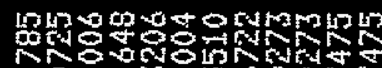
TSO Xom $=2$ ind

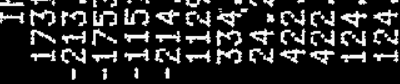

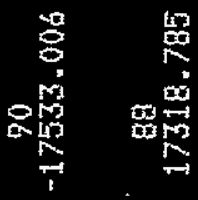

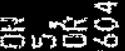
wo in

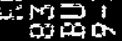

드료

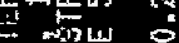

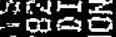

की

की

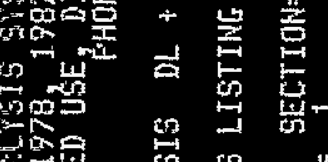

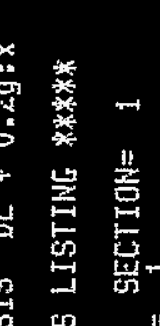

紫

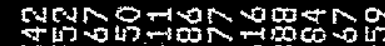

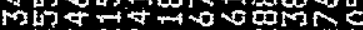

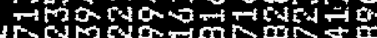

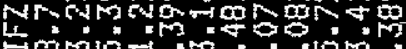

gingan

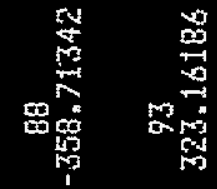

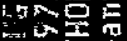

列

可震

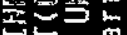

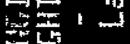

进结卉

象舟

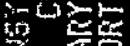

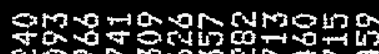
SOSNONWEN 1. L

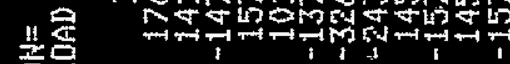

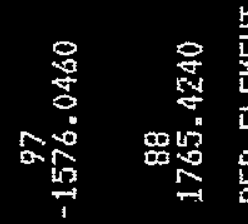

章

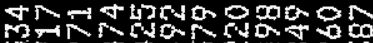

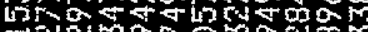
$\times 04=0700000$

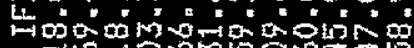

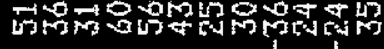

点

旨

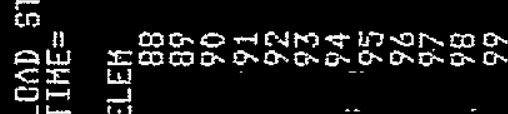

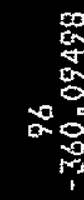

骂

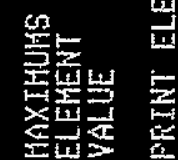

$A-83$ 


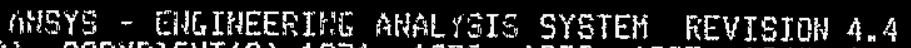

AHSYS(E) COFYFIGHT(C) $1971,1978,1982,1983,1985,1987,19891900$ SWANSON ANALYSIS SYSTEHS, IHC. AS UNPUBLISHED WOFK.

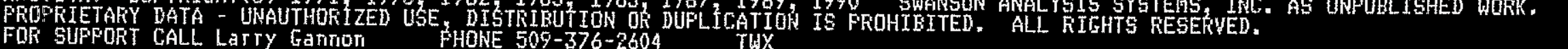

DST MOCK-UP FLATFORH SEISHIC ANALYSIS DL + 0.2g:X + .06g:

******* FOST1 ELEMEHT STRESS LISTING *****

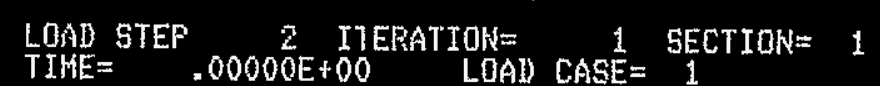

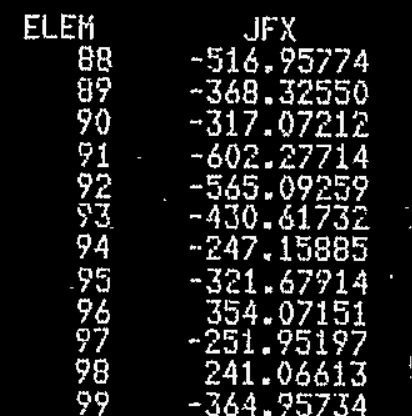

ifIHIHUNS

ELEHENT

VALISE:

$$
-602.27714
$$

$$
\begin{array}{r}
J F Y \\
-1747.7943 \\
-123.26669 \\
1495.3262 \\
-1555.7444 \\
-83.308846 \\
1393.5923 \\
266.63987 \\
303.76733 \\
-1423.9540 \\
1.606 .1634 \\
-1429.1541 \\
1600.9632
\end{array}
$$
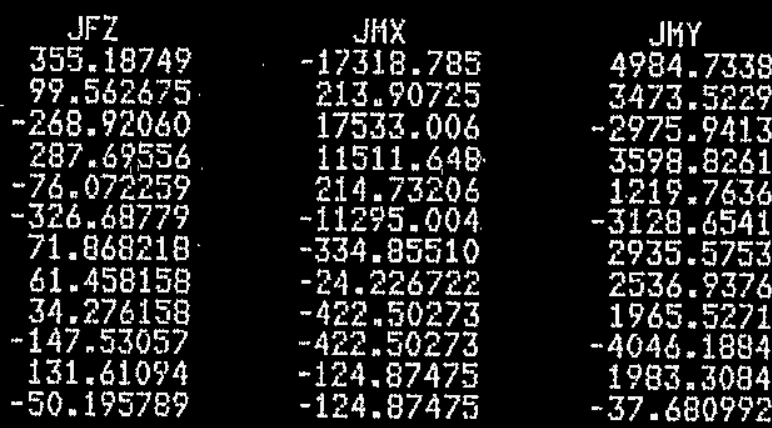

93
-326.68779

$-17318.785$

HAXIHUHS

ELEHENT
YALUE

\section{6}

354.07151

88
-1747.7943

97

1606.1634

\begin{abstract}
$\frac{88}{355.18749}$
\end{abstract}
90

17533.006

g2 BY 2

4 ELEHENTS (OF 99 DEFIHED) SELECTED BY ESEL LOHHAND.

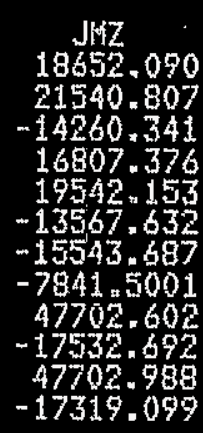

97

$-17532.692$

89

4984.7338 


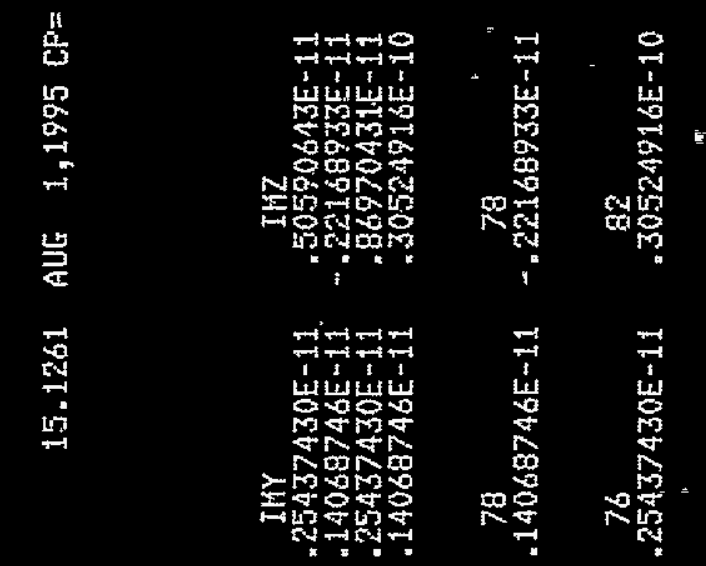

空

要

F

눈

$\sum_{i=1}^{3}$

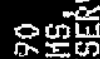

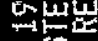

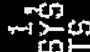

$\cos$

$\leq$

$\frac{1}{4}$

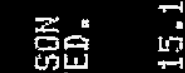

$-$

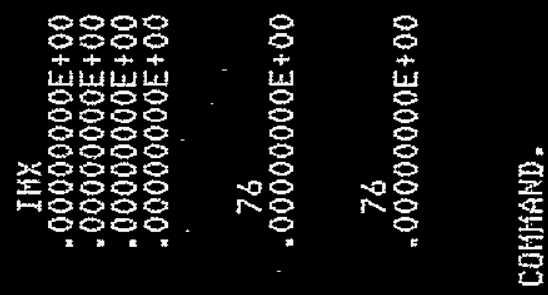

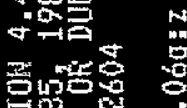

Ho

㟧的为

cowa

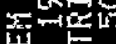

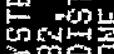

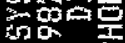

$\cos ^{-12}$

品的 क

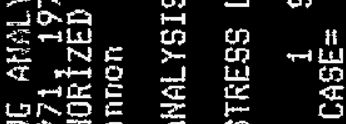

논온

焉承

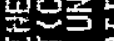

넌,

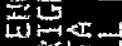

⿶⿶凵

政

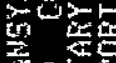

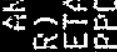

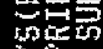

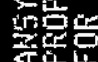

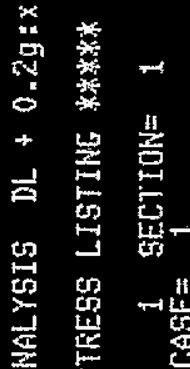

Nogin

लिक्ष

NaOo

$\ln _{10}^{2}=$

$\operatorname{low}_{i \rightarrow 1}$

焉

$\frac{\substack{9 \\ \frac{8}{5}}}{3}$

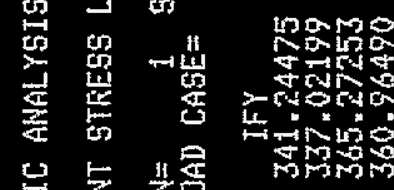

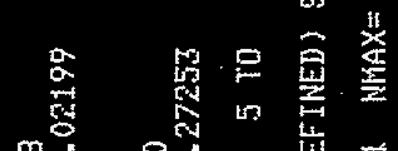

只 管

ctis

焉

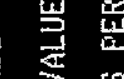

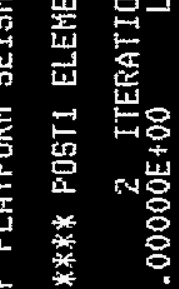

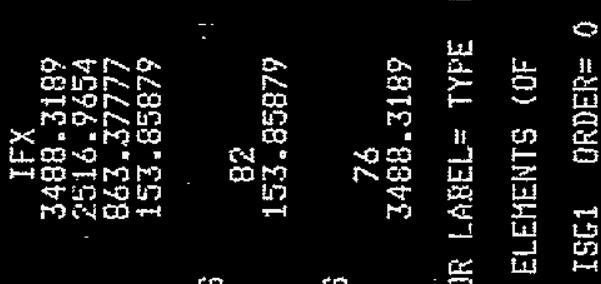

$m$

起

站

acions.

趾 紫

2

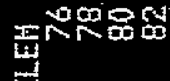

$\dddot{7}$

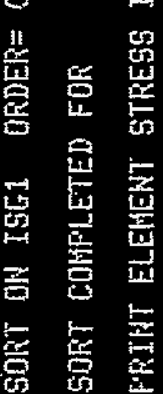


AHSYS - EHEINEERING AHALYSIS SYSTEH FEVISIOH 4.4 13732207 HAY 1,1990

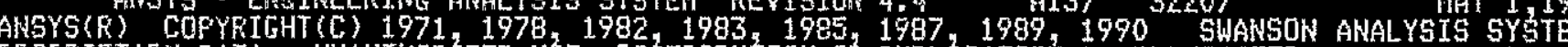

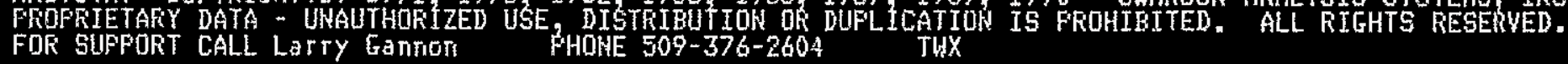
TWX

DST HOCK-UF PLATFORH SEISHIC AHALYSIS DL $+0.2 \mathrm{~g}: \mathrm{x}+.06 \mathrm{~g}: \mathrm{z}$

15.1261 AUG $1,1995 \mathrm{CP}=$

, 730

****** FOST1 ELEHENT STRESS LISTING ******

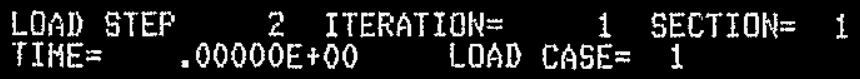

ELEH

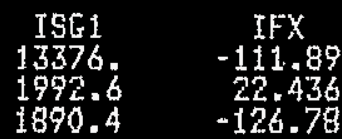

IFY
-1309.1
-187

$\begin{array}{cc}\text { IFZ } & \text { IHX } \\ -30.032 & .35708 \\ -5.3124 & .32374 \\ 61.747 & -50.460\end{array}$

IHY

846.59

-235.76
-870.22

IWZ

$-197.10 \quad 61.747$

$-7315.6$

HIIUIHUKS

ELEHENT

189

$-126.78$

20
-1309.1

$-30.03$

15
-50.469

15
-870.22

$-53082$

HAXIHUHS

ELEKENT

1.20 $33 \% \quad \frac{19}{22.436}$

15
-187.10

61.747

.30708

846.59

$-2780.3$

SONT DU IGG3 ONDEF $=0$ KABS $=1$ NHAX $=3$

SORT COHPLETED FOF 3 VALUES:

FRINT ELEHENT STRESS ITEHS PEF ELEHEHT 
崡

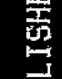

票

P

$x$

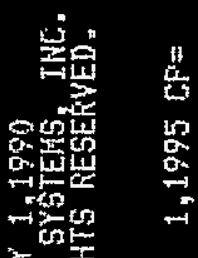

2

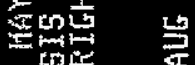

这梳

空些

$\underset{n}{m}$

象焉

$\lim _{i \rightarrow 1}$

의

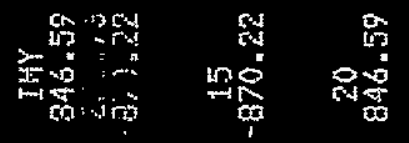

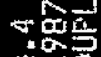

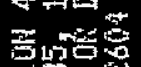

510

3 ,

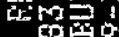

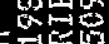

蒠

toves

mo웅

$t_{i}^{\rightarrow 1}$

H人

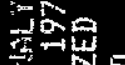

$\operatorname{lit}_{i=0}$

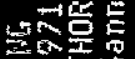

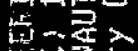

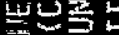

$4=19$

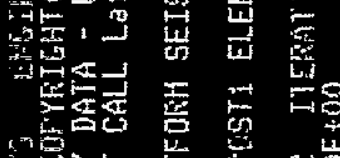

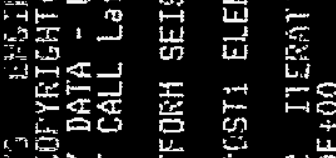

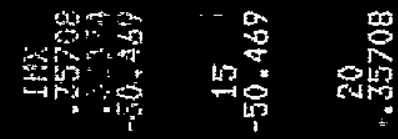

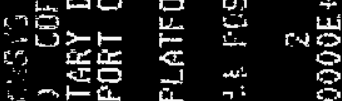

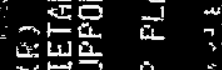

b它品

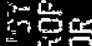

站

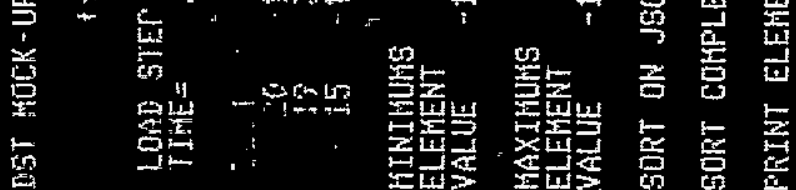

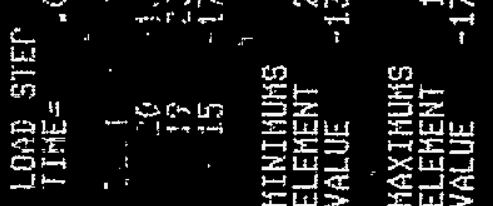

mon

+ 委 - - m

웅

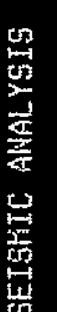

要

is

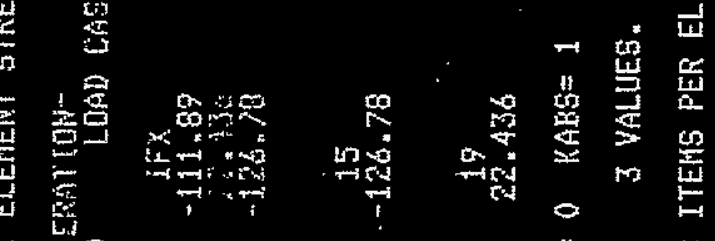


AHSYS - EHGIHEEFIHG ANALYSIS SYSTEH EEVISIOH 4.4

13732207

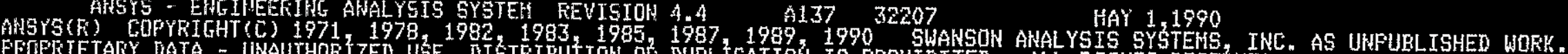
FUR SUFFORT CALL L UNAUTHORIZED USE, DISTRIGUTION OF DUFLICATIOA IS PROHIBITED. ALL KIGHTS RESERVED.

DST HOCK-LFF PLATFORH SEIGHIC ANALYSIS DL +0.2g:x +.06g: *****: FOST1 ELEMENT STRESS LISTING *

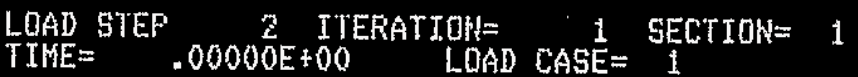

\begin{tabular}{|c|c|c|c|c|c|c|c|}
\hline $\begin{array}{r}\text { ELEH } \\
21 \\
1.9 \\
15\end{array}$ & $\begin{array}{c}3561 \\
14234 \\
2023.4 \\
1807.6\end{array}$ & $\begin{array}{c}\text { JFX } \\
27.812 \\
51.564 \\
127.88\end{array}$ & $\begin{array}{c}J F Y \\
1730.5 \\
-183.05 \\
205.48\end{array}$ & $\begin{array}{c}J F Z \\
5.0100 \\
27.312 \\
-65.423\end{array}$ & $\begin{array}{rl} & J H X \\
-.35708 & =32374 \\
-.32374 & 50.469\end{array}$ & $\begin{array}{c}\text { JKY } \\
449.96 \\
235.76 \\
-846.59\end{array}$ & $\begin{array}{c}542 \\
-59383 \\
7315.6 \\
-2539.6\end{array}$ \\
\hline
\end{tabular}

HTHIMUHS

\section{HAXIHUHAS}

$1807.6 \cdot 27.812-183.05$

$-65.42$

$-.35708$

15

HAXIHUTAS

$\frac{21}{4234} \quad 1 \frac{15}{27} .8$

${ }_{12}^{2} \frac{20.5}{230.5}$

27.312

50.469

$-846.59$

$-59383$

SORT OH JSG3 ORDER= O KABS= 1 NHAX =

SORT COHFLETED FOK 3 VALUES.

FRIKT ELEKENT STRESS ITEHS FER ELEHENT 


\section{*alls View/Frint Document Cover Sheet tow}

This document was retrieved from the Documentation and Records Manaqement (DRM) ISEARCH System. It is intended for Information only and may not be the most recent or updated version. Contact a Document Service Center (see Hanford Info for locations) if you need additional retrieval information.

Accession \#: D195067914

Document \#: SD-WM-ER-521

Title/Desc:

STRUCTRUAL ANALYSIS DESIGN \& EVALUATION OF MOCKUP PLATFORM MONORAIL \& TANK PLATE CUT OUT [SEC 2 OF 3]

Pages: 102 
This document was too large to scan as a whole document, therefore it required breaking into smaller sections.

DOCUMENT NUMBER: $5 D-\omega m-E P-521$

\section{SECTION 2 OF 3}

TITLE: STrucTural Analysis Desigh E EValuation of mock-up platform monorail I Tank PLaTe CuT-OUT

DATE: $12-19-95$

ORIGINATOR: I S. Hundal

CO:

RECIPIENT:

CO:

REFERENCES: 
$\stackrel{8}{\frac{1}{2}}$

架

$\dot{t i}$

幽焉 类

앙뭄

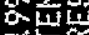

$\rightarrow-4$

$\rightarrow$ con $\rightarrow$

致密

$x$

$\stackrel{9}{3}$

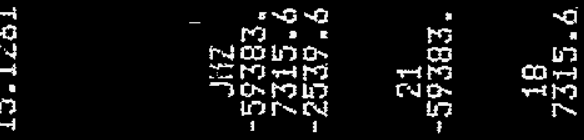

क

赶乎

고ㅇㅛㅛ

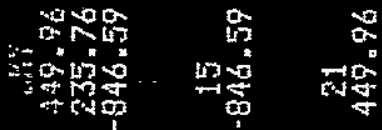

照

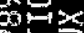

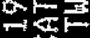

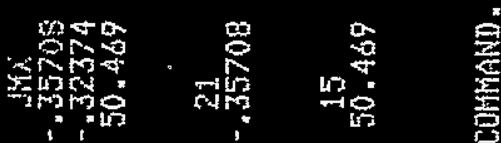

s하는

为

总。

急

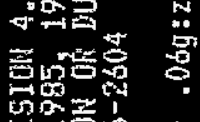

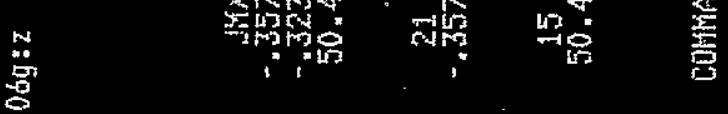

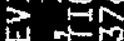

政象

도요

$+$

네는

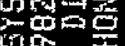

${ }_{i n}^{-1}$,

ming

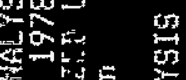

$n^{-1}-5$

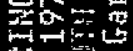

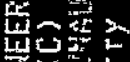

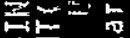

곤

, 这运

的部要

3

政

돈네

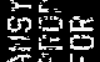

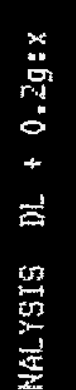

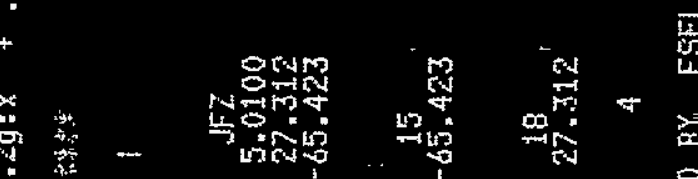

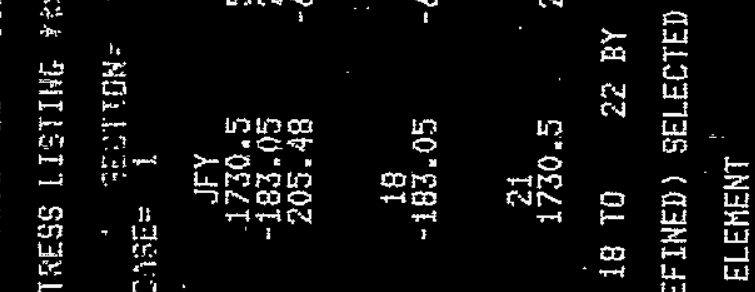

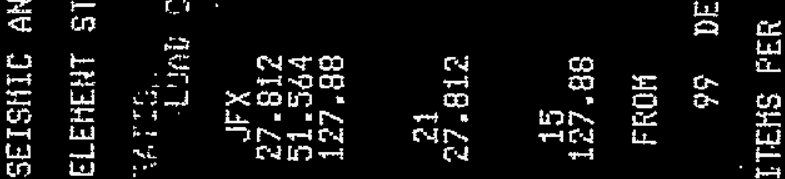

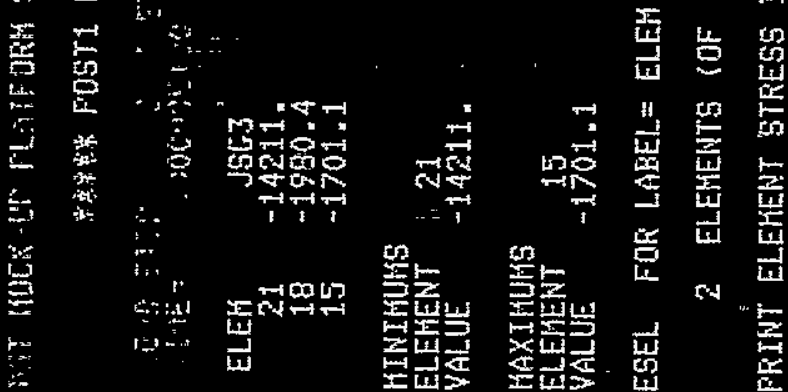


AHSYS - EHGIMEEEIHE AHALYSIS SYSTEH REVISIOH 4.4

AHSYS(F) COFYFIGHT(L) $1971,1978,1992,1783,1985,1987,1989,1990$ SWANSON ANALYSIS SYSTEHS, INC. AS UNFFELISHED WOEK. FROPKIETARY DATA - UHAUTHOFIZED USE, DISTRIEUTION OR DUFLICATIOK IS FROHIBITED. ALL RIGHTS RESEEVED.

FUK SUFFERT CALL Larry Gannon PHONE 509-376-2604 TUX

DST HOCK - UF FLATFORH SEISMIC AHALYSIS DL $+0.2 \mathrm{~g}: \mathrm{x}+.06 \mathrm{~g}: \mathrm{z}$

****** FOST1 ELEHENT STRESS LISTING $* * * * * *$

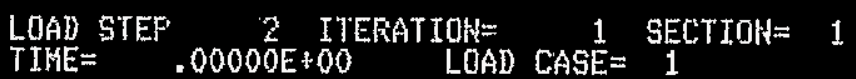

$\begin{array}{ccc}\text { ELEF } & \text { IFX } & \text { IFY } \\ 18 & -56.601013 & 208.23236 \\ 22 & -10.271055 & 116.16264\end{array}$

HINIKUHSS

ELEHEHT

VALLIE

$-56.601013$

$11 \frac{22}{6} \cdot 16264$

IFZ
-28.82328
-12.200204

ThX
.35373893
-.74922899

IMY
802.74501
214.10089

147
-78.933895

HAxisuth

ELEMEN

$-10.271055$

208.23236
$-28.98232$
$-.74722899$

22

14.10089

$\frac{18}{32373893}$

802.74501

23 BY 4

2 ELEIENTS SOF 99 DEFINED) SELECTED BY ESEL COHHAND.

PRTHT EIFHEHT STRESS ITEHS PEF ELEHENT 
品

秃

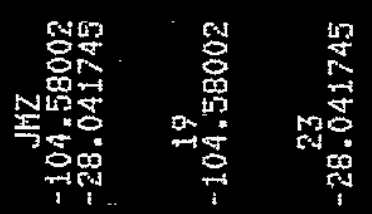

案孝

竞然

领

움

$\lim _{\rightarrow \rightarrow \infty}$

mas

唯标皮

$+5$

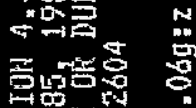

$\stackrel{N}{0}$

$=-\infty$

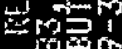

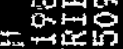

4 con

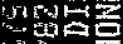

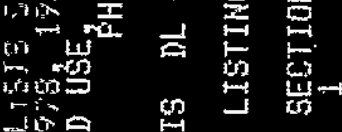

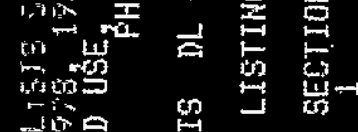

$\frac{\pi}{3}$

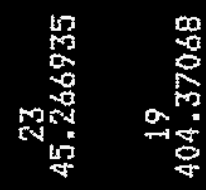

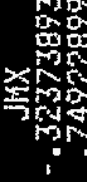

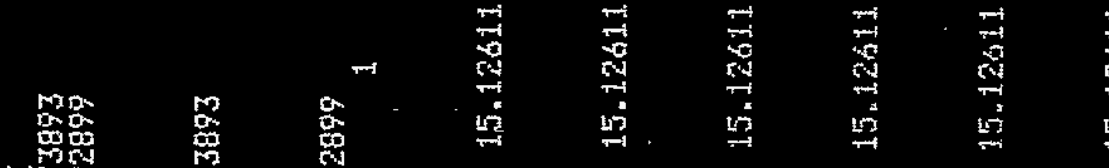

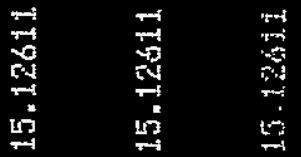

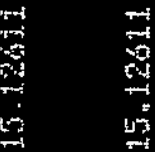

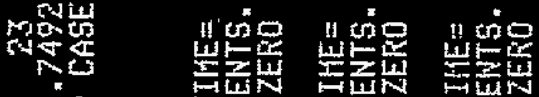

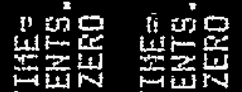

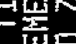

官

密e 喓

谞品

품ำ

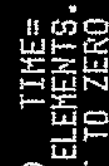

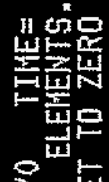

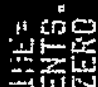

is

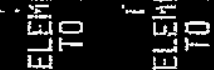

$\rho_{-1}^{5} \rho_{-1}^{w}$

P.

x

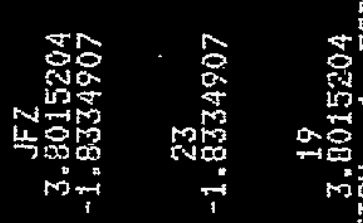

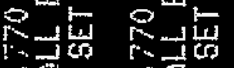

군

竞点

8

$8-1$

$\because$

列

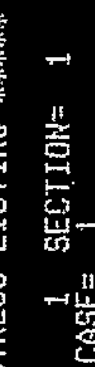

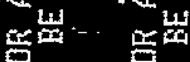

些出

동

동

동복

록

봉

올

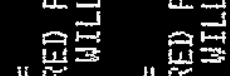

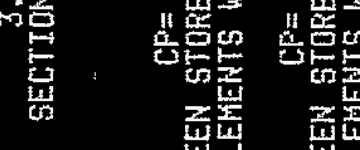

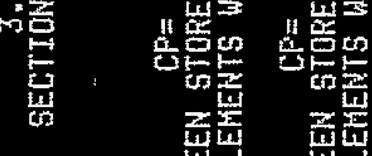

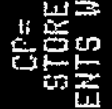

복

w云

案岗

댄문

눌

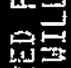

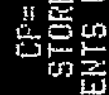

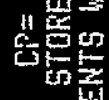

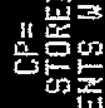

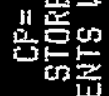

要空

蒠密

吾悹

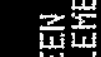

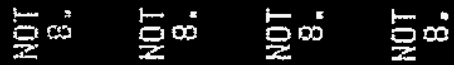

$\sum_{\operatorname{mon}}$

$\frac{1}{3}$

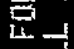

릴

6

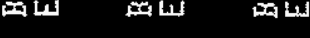

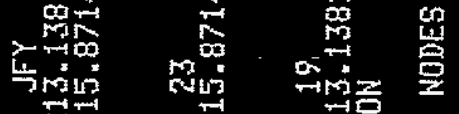

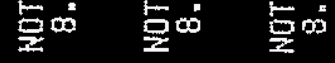

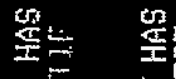

突

농

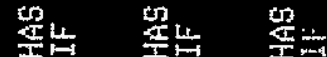

蛋点

乫庶

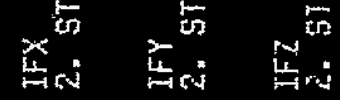

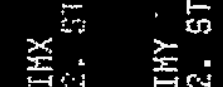

논

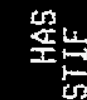

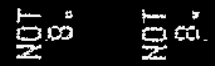

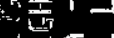

한렁

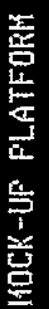

留

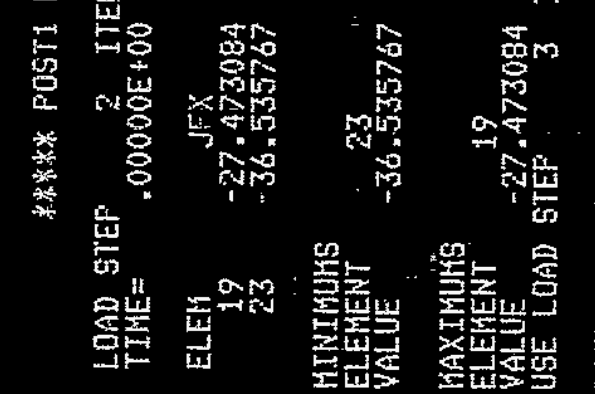

要

营

$\frac{d}{11}$

要憵 焉点 要

*

* 这

点的

* 난

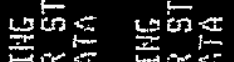

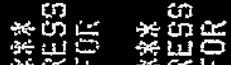

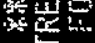

象:

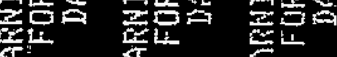

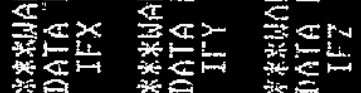

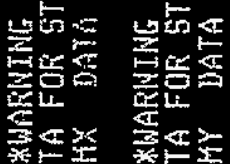

迹杜

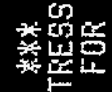

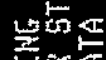

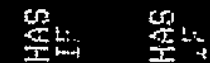

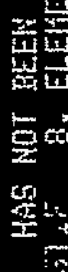

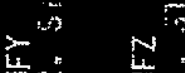

0

19

$$
A-91
$$




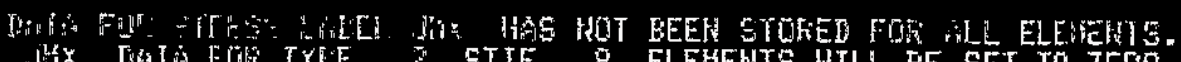

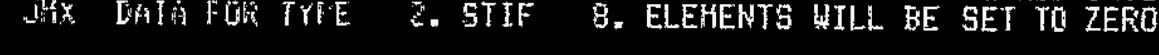

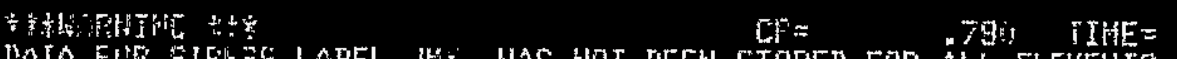

DAIA FUF SIFLSE LABEL JH HAS WOT BEEN GTORED FOR ALL ELEHENTS.

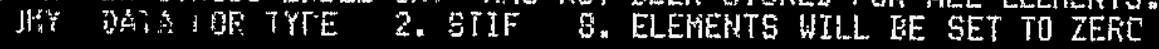

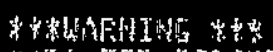

$\mathrm{Pr}=$

780 TIHE=

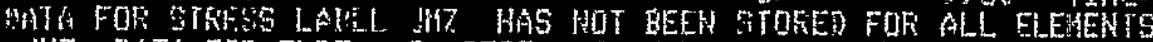

JHZ DATA FOK TYFE 2. STIF 8. ELEHEHTS WILL DE SET TO ZETO

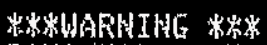

$C F=$

780 TTHE=

$19+241$

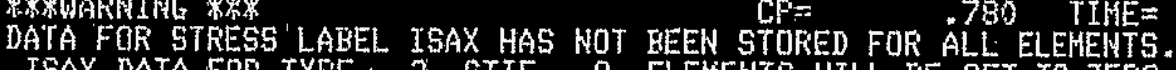

ISAX DATA FOR TYFE: 2. STIF 8. ELEMENTS UILL BE SET TO ZEFÓ

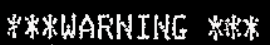

CF:

.780 TIHE $=$

DATA FOR STRESS LAEEL ISBY HAS NOT BEEH STORED FOR ALL ELEHENTS.

ISEY DATA FOF TYPE " 2. STIF 8. ELEHENTS WILL BE SET TO ZERO

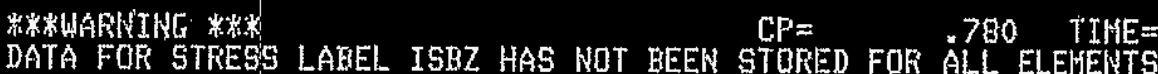

ISBZ DATA. FON TYFE
2. STIF 
$\prod_{i=1}^{+\infty}$

in

$\underset{7}{-1}$

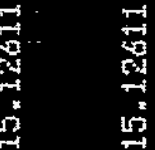

要

焂点

害

$\infty_{1+1}^{4}$

잠 0

觜峛

西至

1) 空

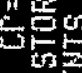

in

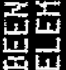

눙ㅇ

tos

要

觜

㟧以

的

*触

int

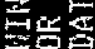

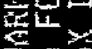

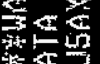

究

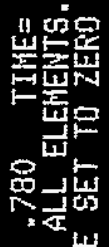

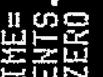

- 崖0

8 击占

积

近

봉

造战

눈ㅎㄴ

原車

思望要

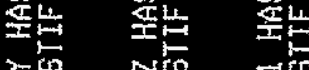

要

些㟧

몬교

I1 丠

造焉的

素窟

옹

1

嶎幽

变

*结皆

눌

牙要要

단

浣

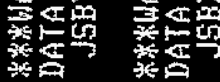

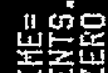

㟧的

直

骂出

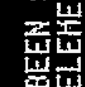

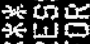

딴니

c4is

的空

*

就的

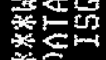

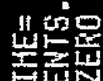

逗0

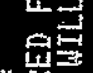

11 焉电

造管的

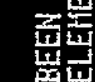

空实是

卙。

욤내

正㟧

象览

造崖

兽些

恶

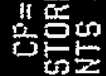

"

눈

我齿

然象

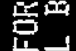

뵤로

11 总的

他点

需盇

崖嵌

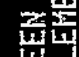

恶嘀

䒠文

50

논

占点

这地

농

눈

受岕

品

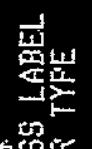

㟧缌

这览
爱热
善

$*$

非嵒它

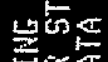

봉돈

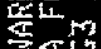

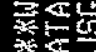

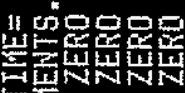

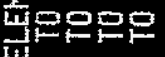

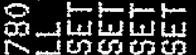

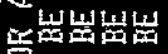

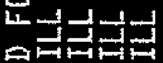

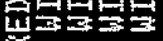

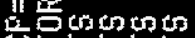

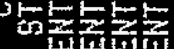

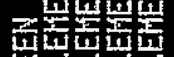

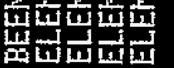

异む゙ஷ゙ஷ゙せ

五比造

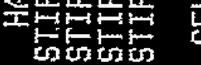

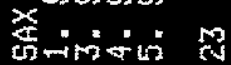

南

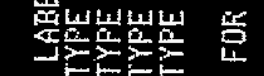

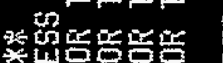

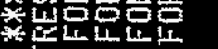

$10<4<6$

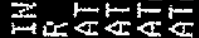

난

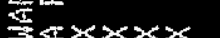

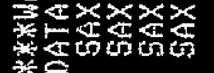

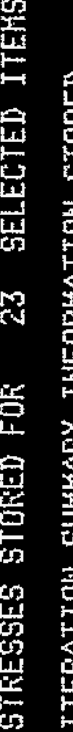

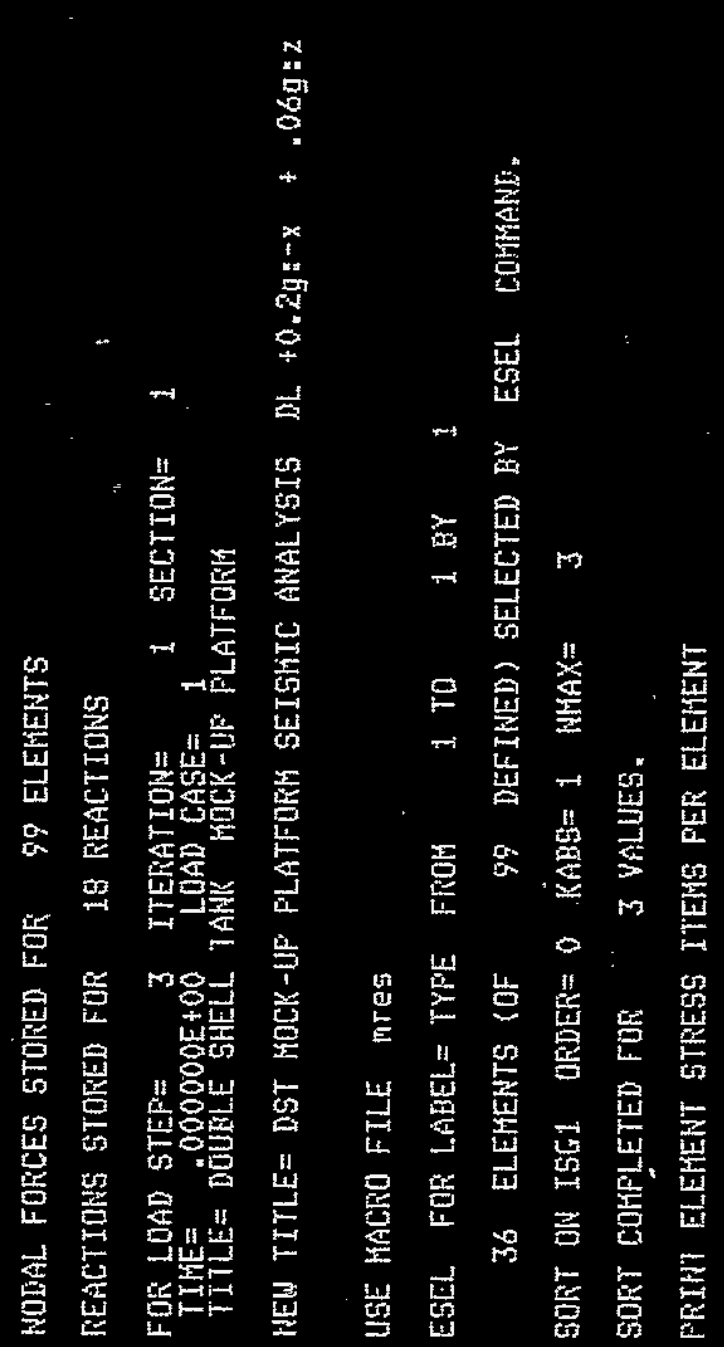

$$
A-q 3
$$




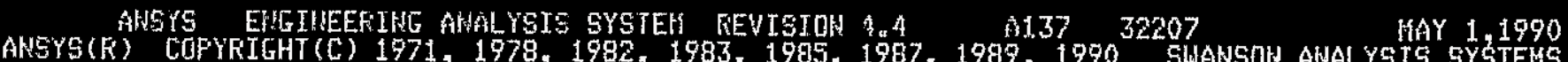

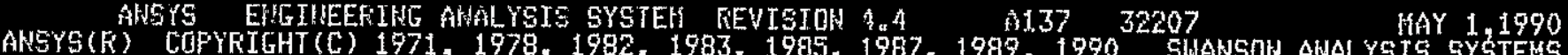

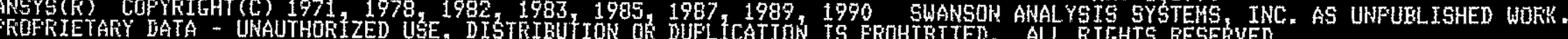
FOR SUFPORT CALL LaTTY GaTIOT PHONE $509-376-2604$ THX

DST HOCK-UP FLATFERH SEISHIC ANALYSIS DL +0.2g:-x + .06g:

15.1261 ALG $1,1995 \mathrm{CP}=\quad .790$

****** POST 1 ELEKENT STRESS LISTIHG * $^{*} * * *$

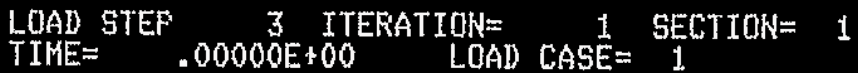

\begin{tabular}{|c|c|c|c|c|c|c|c|}
\hline $\begin{array}{r}\text { ELEK } \\
48 \\
49 \\
56\end{array}$ & $\begin{array}{c}\text { ISt1 } \\
5944.6 \\
5357.8 \\
3429.0\end{array}$ & $\begin{array}{c}\text { IFX. } \\
-433.17 \\
-424.48 \\
-333.12\end{array}$ & $\begin{array}{c}\text { IFY } \\
-225.94 \\
-375.89 \\
-3947.5\end{array}$ & $\begin{array}{c}\text { IFZ } \\
9,1017 \\
39.092 \\
395.95\end{array}$ & $\begin{array}{l}\text { IHX } \\
.91852 \\
.91852 \\
.91852\end{array}$ & $\begin{array}{c}\text { IKY } \\
-3090.2 \\
-2723.1 \\
-372.63\end{array}$ & $\begin{array}{c}\text { INZ } \\
-69064 . \\
-62357 . \\
-43305\end{array}$ \\
\hline NIHUMS & & & & & & & \\
\hline $\begin{array}{l}\text { EHENT } \\
\text { LUE }\end{array}$ & $\begin{array}{c}56 \\
3429.0\end{array}$ & $\begin{array}{r}48 \\
-433.17\end{array}$ & $\begin{array}{c}56 \\
-3947.5\end{array}$ & $\begin{array}{c}48 \\
9.1017\end{array}$ & $\begin{array}{c}56 \\
.91852\end{array}$ & $\begin{array}{c}48 \\
-3050.2\end{array}$ & $\begin{array}{c}48 \\
-69064 .\end{array}$ \\
\hline XIHU⿴囗十⺝ & & & & & & & \\
\hline EMENT & $\begin{array}{c}48 \\
5944.6\end{array}$ & $-3 \frac{56}{33} \times 12$ & $\begin{array}{r}48 \\
-225.94\end{array}$ & $\frac{56}{395} \times 95$ & $\begin{array}{c}48 \\
.91852\end{array}$ & -572.63 & $-4 \frac{56}{305}=$ \\
\hline
\end{tabular}

SOFT ON ISG3 ORDER $=0$ KAES $=1$ WHAX $=3$

SORT COMPLETED FOR 3 YALUES.

FRINT ELEHENT STKESS ITEHS PER ELEHENT 


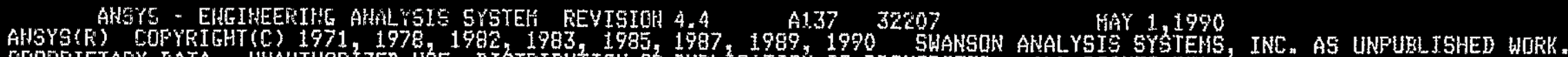

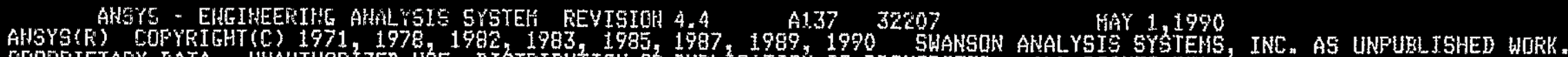

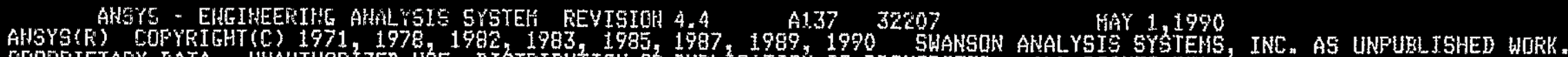

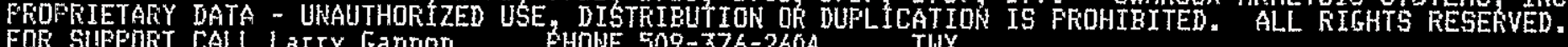

DST HACK-UF PLATFORH SEISHIC AHALYSIS DL $+0.2 g:-x+.06 g: z$

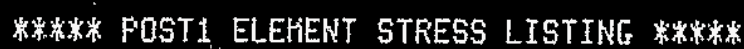

LOAD STEF
TIHE $=0$ ITERATION=
LOAD CASE $=$
1

\begin{tabular}{|c|c|c|c|c|c|c|c|}
\hline $\begin{array}{r}\text { ELEEH } \\
48 \\
49 \\
56\end{array}$ & $\begin{array}{r}1963 \\
-5797 \cdot 1 \\
-5213 \cdot 2 \\
-3315.5\end{array}$ & $\begin{array}{c}I F X \\
-433.17 \\
-424.48 \\
-333.12\end{array}$ & $\begin{array}{c}\text { IFY } \\
-225.94 \\
-375.89 \\
-3947.5\end{array}$ & $\begin{array}{c}\text { IFZ } \\
9.1017 \\
39.092 \\
395.95\end{array}$ & $\begin{array}{l}\text { THX } \\
.91852 \\
.91852 \\
.91852\end{array}$ & $\begin{array}{l}\text { IHY } \\
-3090.2 \\
-2723.1 \\
-572.63\end{array}$ & $\begin{array}{c}\text { IHZ } \\
-60084 \\
-68357 \\
-43305\end{array}$ \\
\hline
\end{tabular}

MINIMUKS

ELEHENT

$48 \quad 48 \quad 58$

VALLIE :

48
-5797.

$-43.17$

$-3947.5$

9.1017

56

HAXIHUH'

EALEENT -36

$-336.12 \quad-225,94$

355.95

48

SOFT ON JSG1 ORDER $=0$

KABS $=1$ NHAX $=$

SORT COMPLETED FOK

3 VALLUES:

PFINT ELEHENT STFESS ITEMS PEF ELEHENT 3 


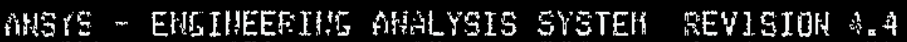

$4137 \quad 32207$

MAY 1,1990

AHSYS $(F)$ COFYRIGHT(C) $1911978,198 \%, 1993,1995,1997,1989,1990$ SWAASON ANALYSTS SYSTEMS, INC. AS UNFUELISHED WOFK.

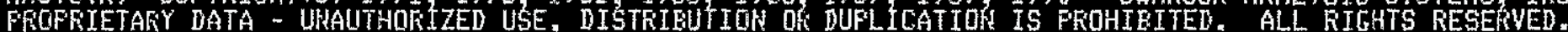

FOF SUFPOFT CALL LaTTY GanTOR FHONE 509-376-2604 THX

DST MOCK-UF PLATFORH SEISHIC ANALYSIS DL +0.2g:-x $+.06 \mathrm{g:z}$

15.1261 AUIS $1,1995 \mathrm{CF}=$

.810

*

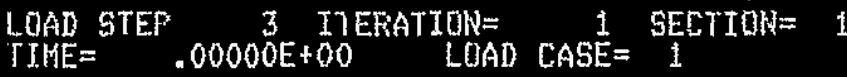

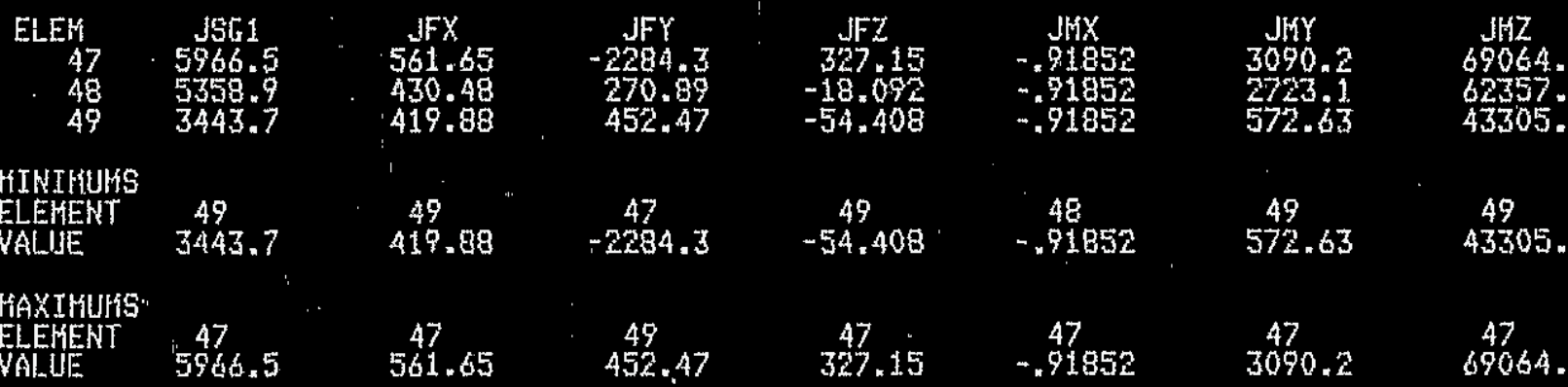

SORT ON JSG3 ORDER $=0$ KABS $=1 \quad$ NHAX $=3$

SORT COHFLETED FOR 3 VALUES.

FRINT ELEHENT STRESS ITELS PER ELEHENT

3


$\underset{\infty}{\infty}$

tos

$\sum^{2}$

ont

메는

mis

$\rightarrow$ in

눈

这卉

总电

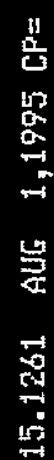

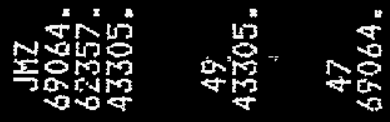

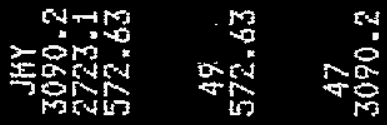

总品

喜舟

5

I

$\cos x$

$\rightarrow \sin ^{2}$

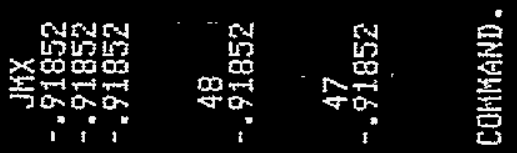

사

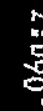

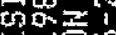

내

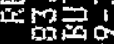

다눙

w

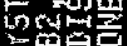

tom

(6) 出

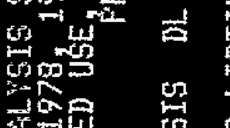

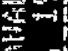

따은

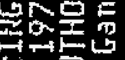

FT:

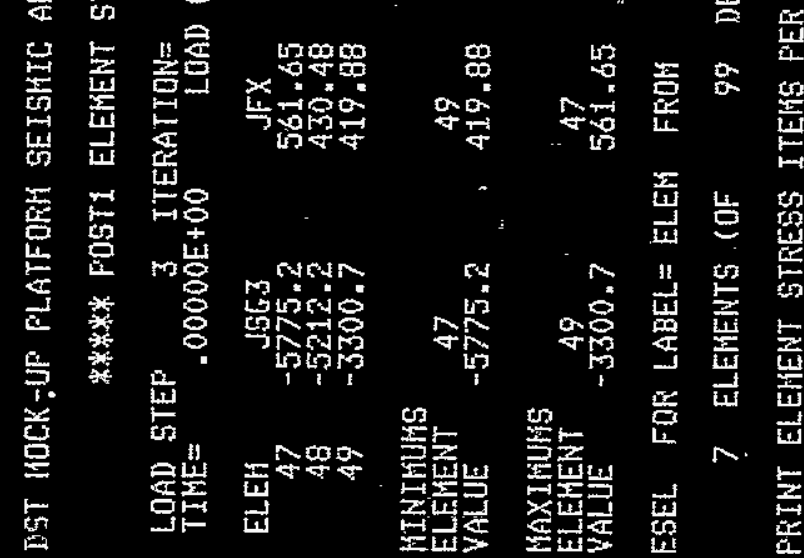


AHEIS. EHGIILEEFIHE AIAL YSIS GYETEK RCUISION 4.4

AHSYS(R) COFYKIGHT(C) $1971,1979,1982,1983,1985,1987,1989,1990$ SWANSON ANALYSIS SYSTEYS, INC. AS UHPUELISHED HDFK. FROFRIETARY DATA - UNAUTHORIZED USE, DISTRIBUTION OR DUFLICATIOK IS FROHIBITED, ALL FIRHTS RESERVED.

FOR SUFPORT CALL LaTTY Gannon PHONE 509-376-2604 THX

DST HOCK-UP FLATFOFH SEISHIC AHALYSIS DL +0.2g:-x $+.06 \mathrm{gzz}$

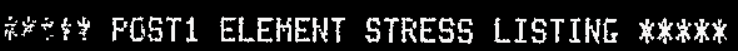

LOAD STEF
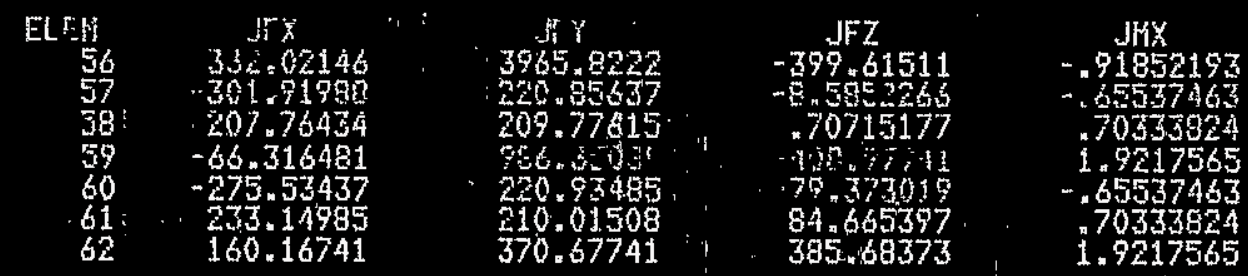

顸Y

$-3902 \times 5731$

$1398,193 \mathrm{~A}$

$-3416.6046$

$3+100 \times 1502$

108.1502

9174.2945

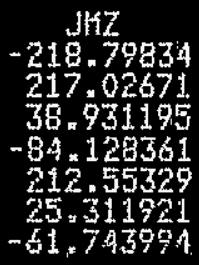

HINIMUNG

ELEMENT

$$
-301.91988
$$

209.77615

$-408$

$-408.97941$

56

HAXIHUHS

ELEHENT

332.02146

56

385.68373

1.9217565

$-3802.9931$

56

$-218.79834$

ESEL FOR LABEL = ELEH FFOH

6470

$67 \mathrm{BY} \quad 3$

$91.72 .2945 \quad 217.02671$

2 ELEIENTS (OF 97 DEFIHED) SELECTED BY ESEL COHHAND.

PRINT ELEKENT STRESS ITEHS PER ELEHENT 
ํㅗㅇ

喿

象望

oxin

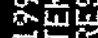

Then

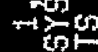

act

理昆比

点

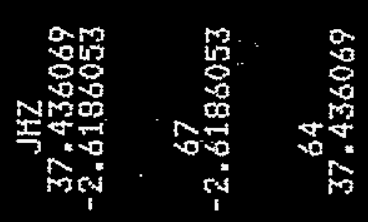

哉

突它

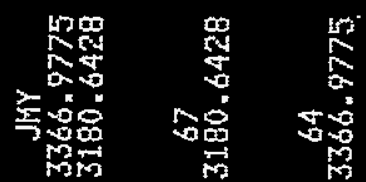

8

and

남

器

$\rightarrow$ 近

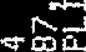

$x \rightarrow 0$

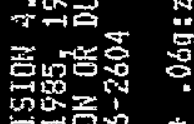

$3 \rightarrow$

络m象

gas?

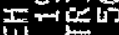

ตำ놈

成定

$x_{i=1}^{n}$

至

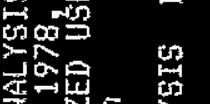

$\frac{\pi}{\tau}$

구늘

놇어ㅁㅗㅗㄴ

단

⿶ㅓㅇ존

证, 品

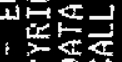

w害田

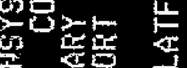

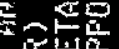

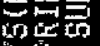

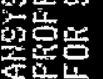

焉

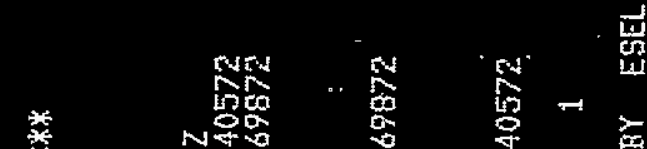

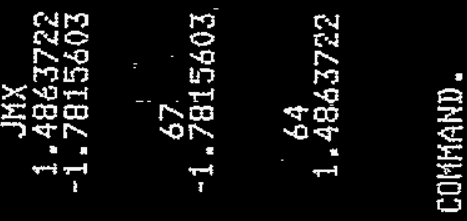

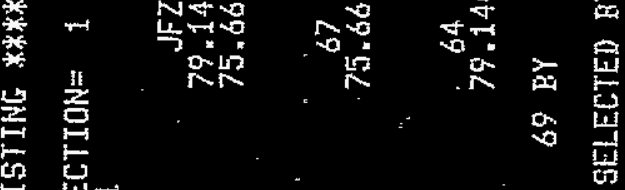

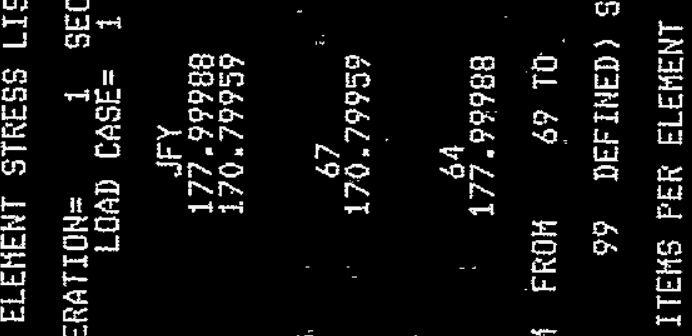

琴

章

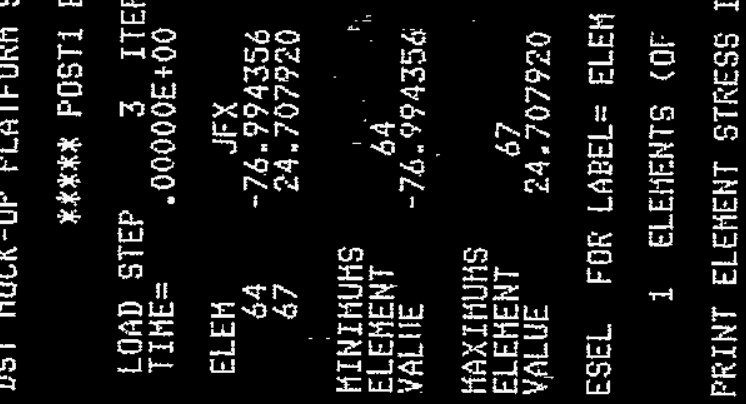

$\stackrel{5}{9}$ 
옳

몬

包密

$\frac{n}{\omega}$

osit

مu

a

돈곤

inc 肎

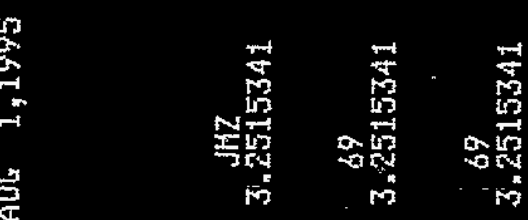

焉装

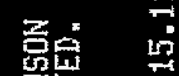

要

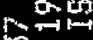

人和

좀

mo

我

赑>

맘살

nt-

뭉

츨

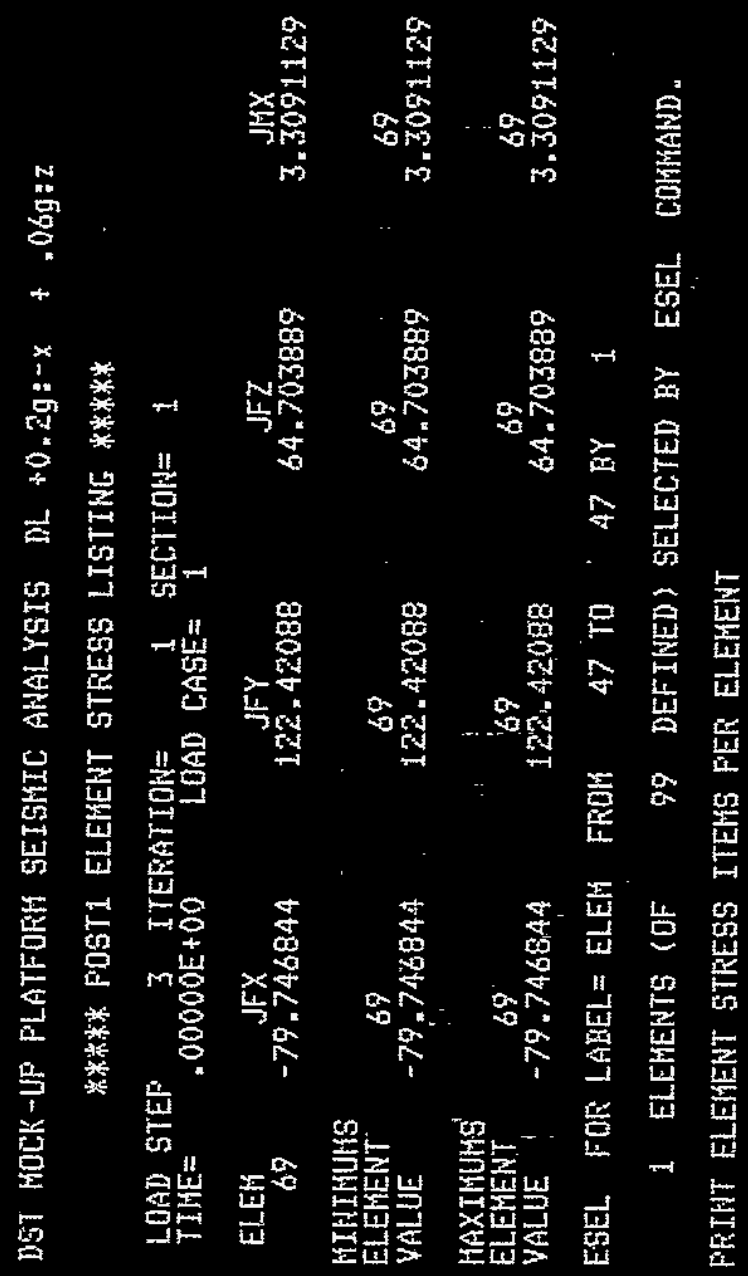

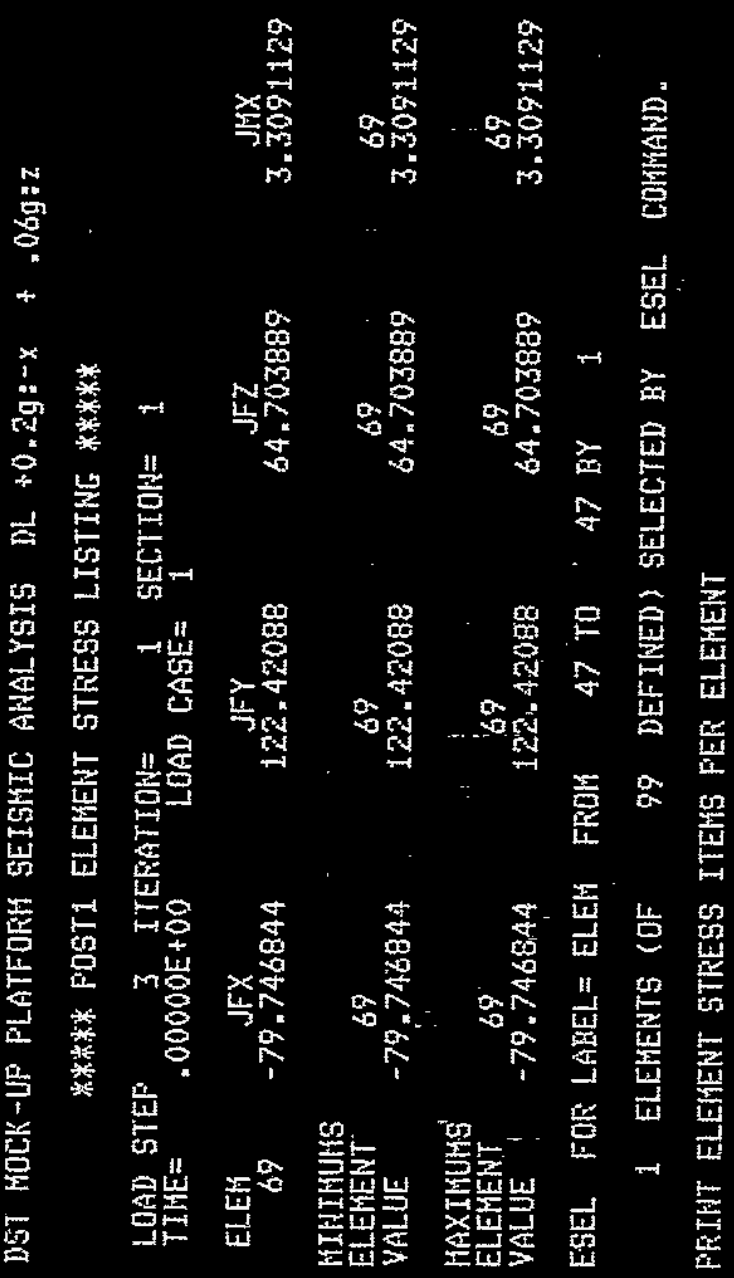

$x_{x \rightarrow 1}$

ist

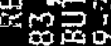

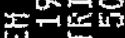

5 m5

의울

${ }_{10}+\frac{7}{2}$

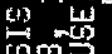

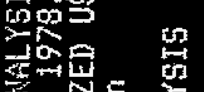

선돌

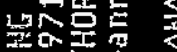

들

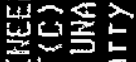

단, 四

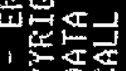

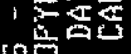

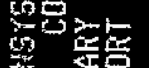

紫

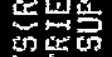

놀

$A-100$ 
AHSYG - EHETHEEKIWG ANALYSIS SYSTEH KEVISIOH 4,4

AWSYS(R) COFYKIGHT(C) $1971,1978,1982,1983,1985,1987,1989,1990$ SWANSOH ANALYSIF SYSTEHS, IHC. AS UNPUBLISHED UOFK.

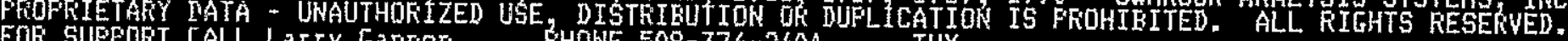

DS1 IIUCK-UP FLATFOEKH SEISMTC ANALYSIS DL $+0.2 \mathrm{~g}:-\mathrm{x}+.06 \mathrm{~g}: 2$ TWX

***** FOSTI ELEHENT STRESS LISTING *****

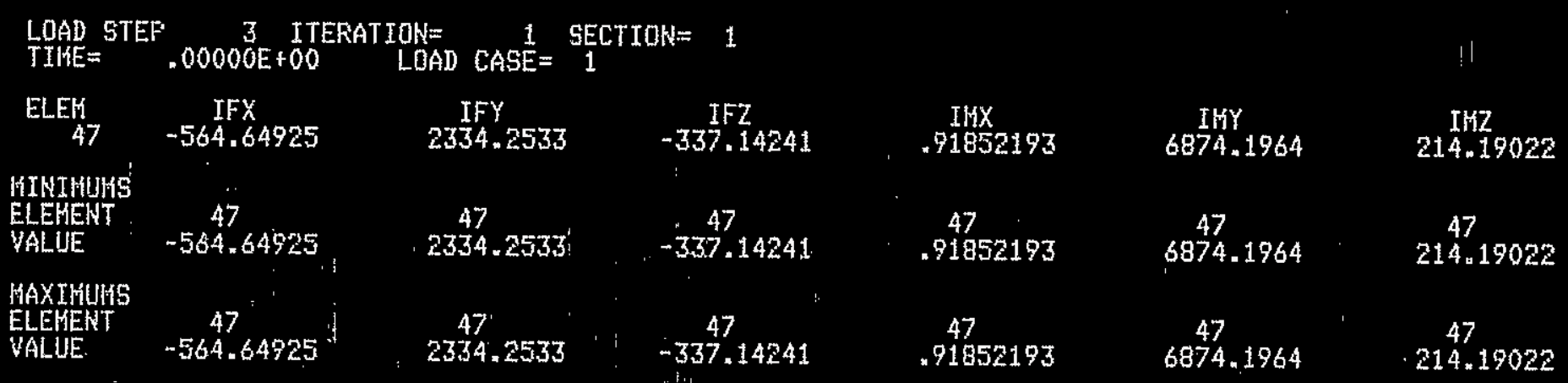

ESEL FOR LABEL = ELEH FROH 63 TO 65 BY 2

2 ELEHENTS (OF 99 DEFINED) SELECTED BY ESEL COHHAND.

PRINT ELEHENT STRESS ITEHS FER ELEHENT 


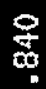

er

完.

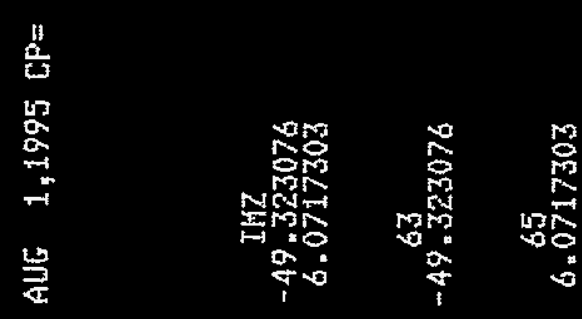

牙

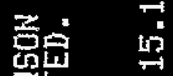

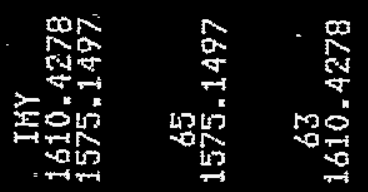

为

空国

要

욤

Ding

< 일

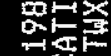

倠

\&国

$\therefore$

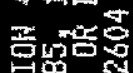

둥

문옹

in

$\operatorname{man}_{0 \rightarrow 0}$

든

tow ow

워웅

${ }_{40}^{1+1}$

14

욘

空 $=$

두을

业些

눈

볼논

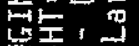

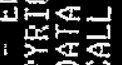

i热军

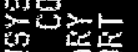

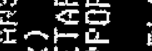

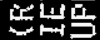

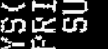

记占学

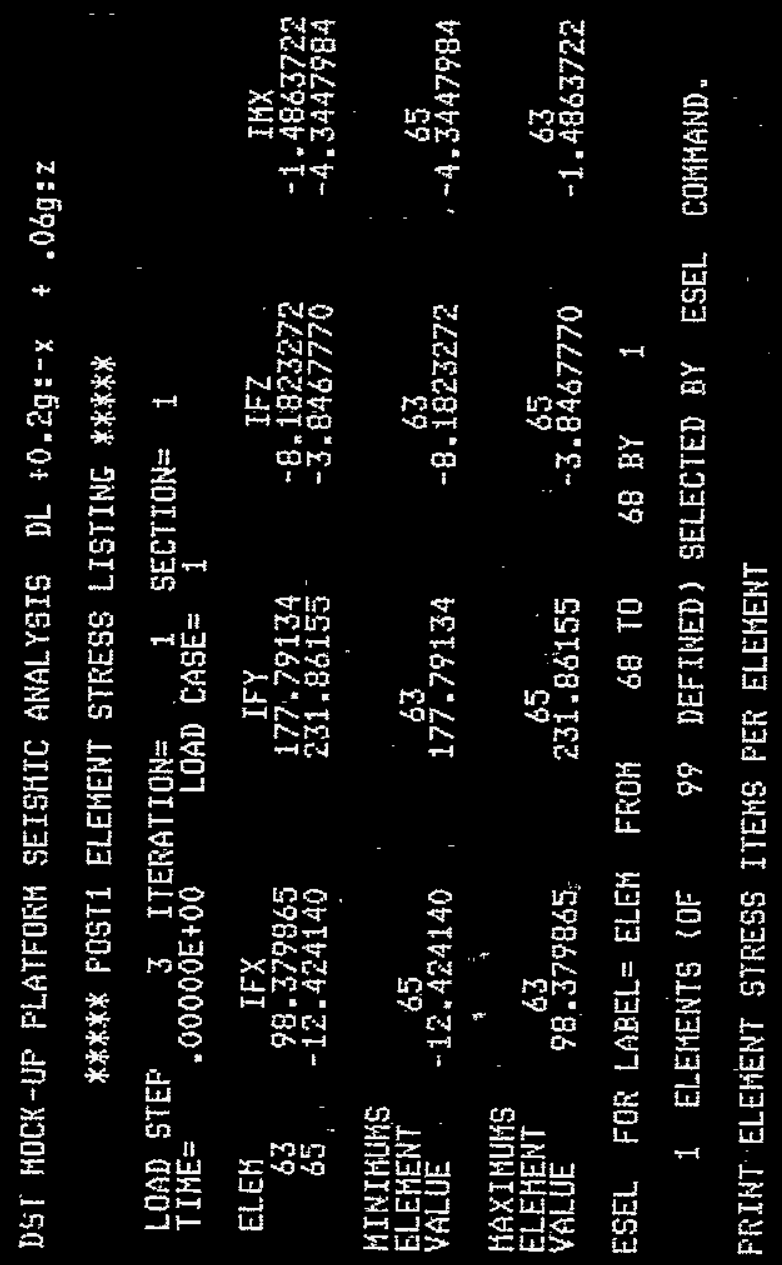

$A-102$ 
ANSYS - EXGIHEEFIHG ANALYSIS SYGTEH EEVISIOH 4.4

$A 13732207$

HAY 1,1990

ANSYS(K) COFYFIGHT(C) $1971,1978,1702,1983,1985,1987,1999,1990$ SWANSUN ANALYSIS SYSTEMS, IRL. AS UNFUBLISHED WURK.

FROFKIETARY DATA - UNAUTHORTZED USE, DISTRIEUTION OR DUFLICATION IS FROHIBITED. ALL RIGHTS RESERVED.

DST HOCK-UF FLATFORK SEISHIC AHALYSIS DL +0.2gn-x + .06g:z

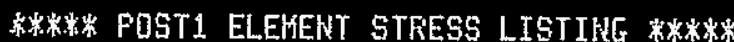

LOAD STEF 20000 ITERATION= 1 SECTIOH= 1

$\begin{array}{ccccccc}\text { ELEH } & \text { IFX } & \text { IFY } & \text { IFZ } & \text { IHX } & \text { IHY } & \text { IHIZ } \\ 68 & 110.27578 & 183.53454 & 5.1963282 & 10.174337 & 1331.0923 & -3.7174474\end{array}$

HTWTHUS

ELEHERT

68

VALIUE:

110.27578

68

183.53454

5.1963282

68

MAXIHUMS

ELEEENT

VALUE:

$110.27578 \quad 183.53454$

1331.0923

$-3.7174474$

ESEL FOR LABEL_= TYPE FFOH

2 To

68
5.1963282

68

10.174337

$\frac{68}{1331.0923}$

$-3.7174474$

I6 ELEHENTS (OF 99 DEFINED) SELECTED BY ESEL COHHAND.

SORT ON SAX ORDER $=0$ KABS $=1$. NHAX $=3$

SURT COMPLETED FOK

3 VALUES.

PRIUT ELEMENT STRESS ITEMS FER ELEHENT 


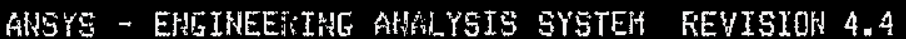

A137 32207 HAY 1,1970

WSYS(K) COFYGIGHT(C) $1971,1978,1987,1983,1985,1987,1989,1990$ SHANSON ANALYSIS SYSTEHS, INC. AS UNFUFLISHED WORK.

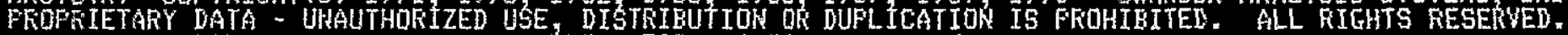

FOF SUFFORT CALL Larty Ganmon PHOKE 509-376-2604 TWX

DST HOCK-UF FLATFORH SEISHIC AHALYSIS DL +0.2g:-x $+.06 g: z$

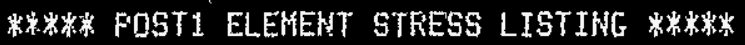

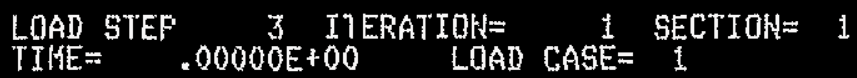

$\begin{array}{cc}\text { ELEH } & 54 X \\ 37 & -2355.5475 \\ 39 & 2334.3229 \\ 30 & -2189.6574\end{array}$

HTWIMUHS

ELEHEKT

VALUE

$$
-2355.5475
$$

MAXIHUHSO

ELEREVIT

$$
2334
$$

3.3422

ESEL. FOK LABEL= TYPE FRUH

3 TO $3 \mathrm{BY} 1$

12 ELEHENTS (DF 99 DEFIMED) SELECTED BY EGEL COMMAND.

FRINT REACTION FORCES FER HODE 
8

星

望要

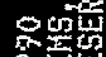

에난

-

질

我的

늘

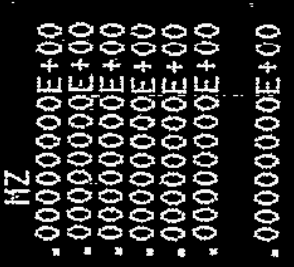

安数

突到

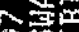

ont

空

ot

密的

본

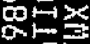

$\underset{1<}{2}$

888888
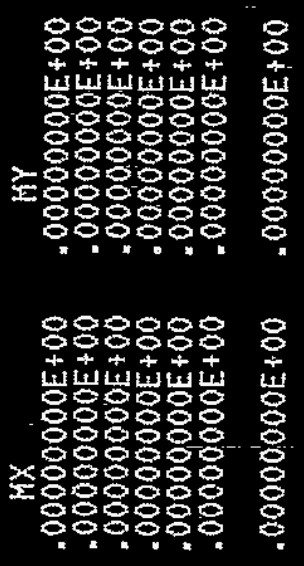

글

品我象

$\Leftrightarrow$

is

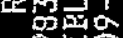

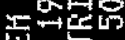

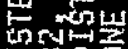
$x \mathrm{x}=0$ 的害

내에

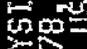

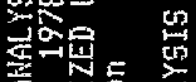

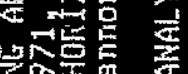

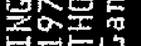

든

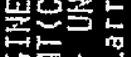

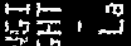

, $7 \leq \frac{1}{3}$

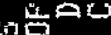

w证

똥

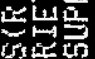

案案

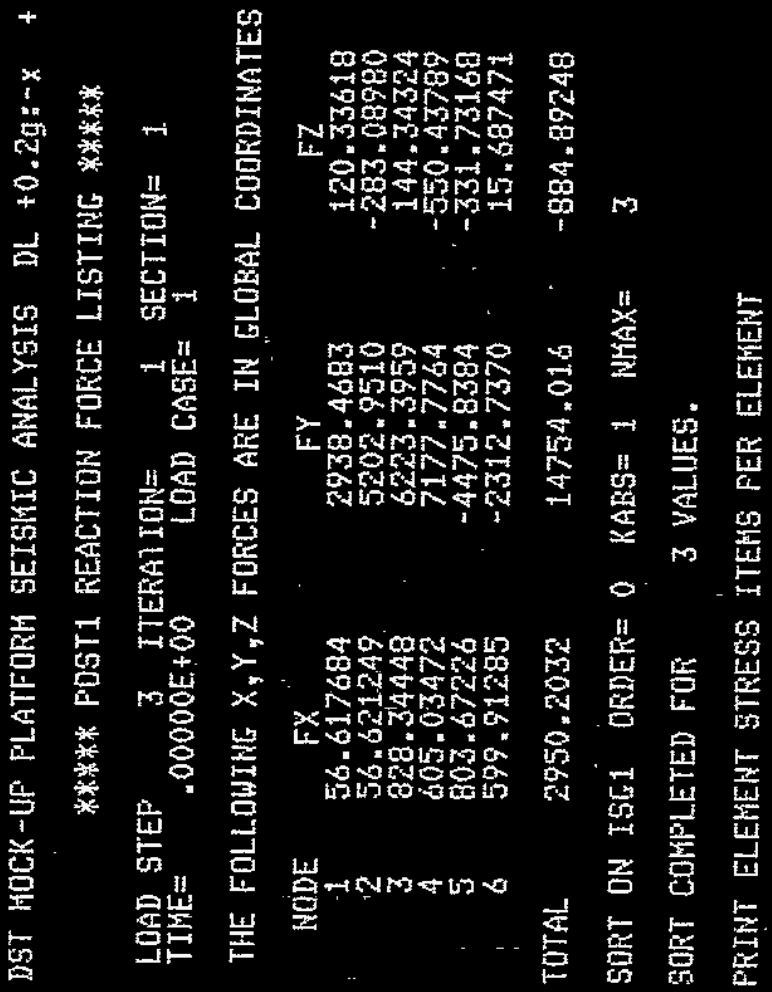


염

恕

蕰

Costin

혼넨

$\lim _{i=0}^{n-1}$

$x$

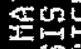

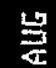

$\frac{1}{\frac{1}{4}}$

染

in

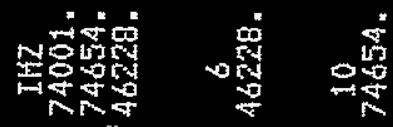

min

in

要

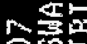

존

웅

然en

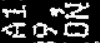

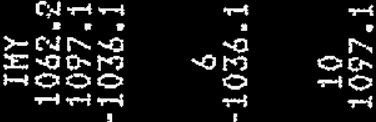

些象

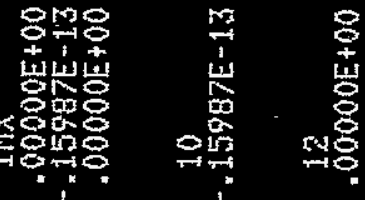

登此要

네에

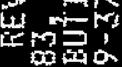

in

is

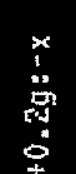

昰的䍃

Gid

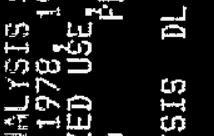

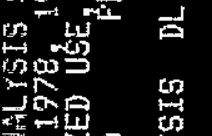

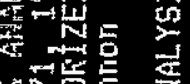

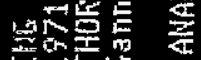

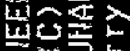

둔

네넌

1 些要

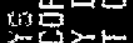

造焉

$\sum_{0=0}^{2}$

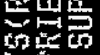

贯密要

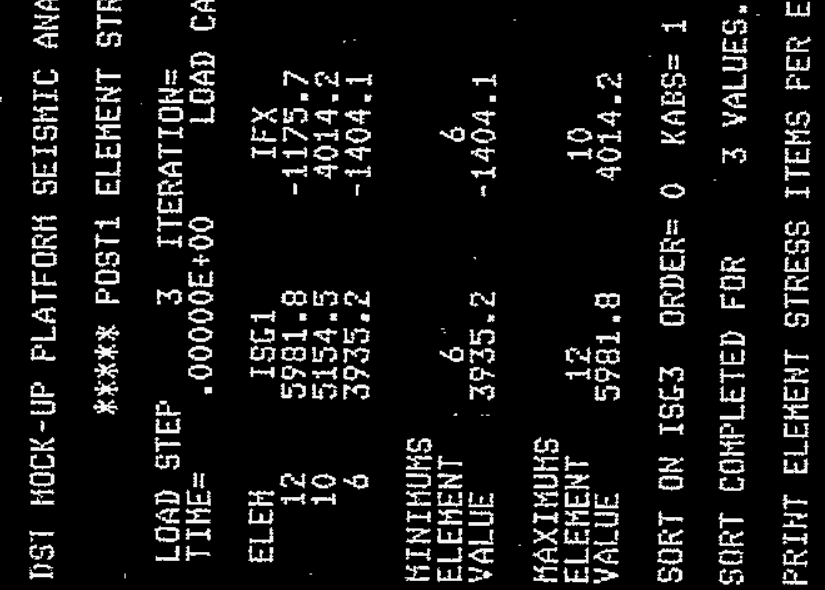


ALSY - EHTIHEERIHG AHAL YSTS SYSTEH REVISIOH 4.4

A137 32207

HAY 1,1990

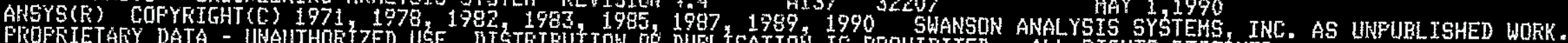
FOR SUPPORT CALL LarTy GamON

DET HOCK-UP FLATFORH SEISHTC AHALYSIS DL $+0.2 \mathrm{~g}:-x+.06 \mathrm{~g}: \mathrm{z}$

15.1261 AUG $1,1995 \mathrm{CP}=$

.860 ****** FOST1 ELEHENT STRESS LISTING $*$ ******

LOAD STEF $\quad 3$ ITERATIOHE $={ }_{\text {LOAD }}^{3}$ CASE $=\stackrel{\text { SECTION= }}{1} 1$

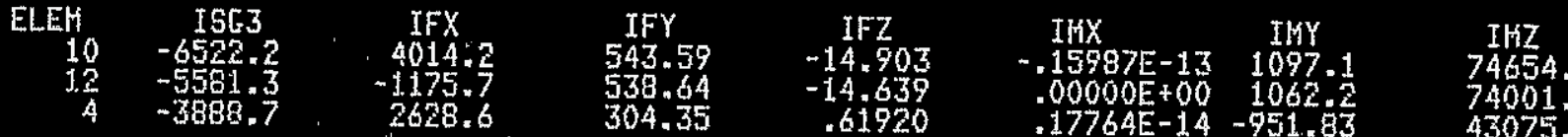

$.17764 E-14-951.83 \quad 43075$

$\begin{array}{lcccccc}\text { ELEHENT } & 10 & 12 & 12 & 10 & 10 & 4 \\ \text { VALUE } & -6522.2 & -1175.7 & 304.35 & -14.903 & -.15957 E-13-951.83 & 43075 .\end{array}$

KAXIKÍlKS

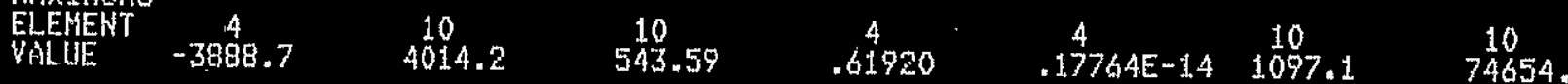

SORT ON JSG1 DKDEFE $=0$ KAES $=1$ NHAX $=3$

SORT COMFLETED FOR 3 VALUES.

FRIUT ELEHENT STRESS ITEHS PER ELEHENT 
AHGYS - EHGIHEERIAG AHAL TSIE SYSTEH REV1SIOH 4.4

A137 32207 N W HAY 1,1990

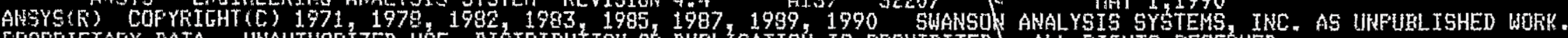

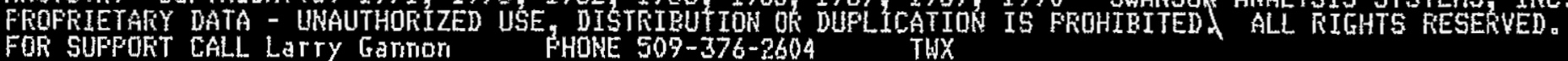

DST HOCK-UF FLATFORH SEISHIC ANALYSIS DL +0.2g:-x +.06g:z

15.1261 AUG $1,1995 \mathrm{cP}=$

.870

****** FOSTI ELEHENT STRESS LISTING $* * * * *$

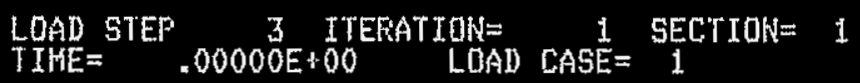

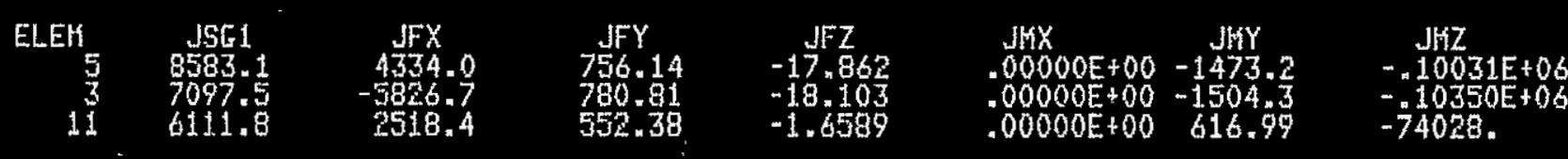

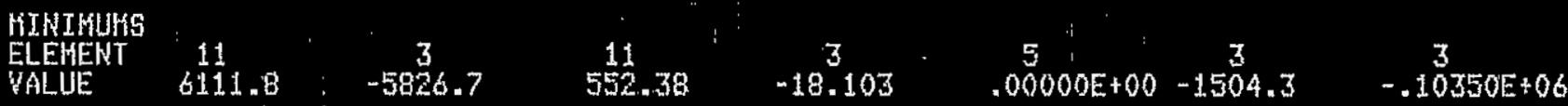

HAXIHUKS

ELEYENT

$8583.1 \quad 4334.0 \quad 780.81$

$-1 \frac{11}{6599}$

$.00000 E+00 \quad 6116.99$

$-74029$

SORT OH JSG3 ORDEFE-O KABS $=1$ NWAX $=3$

SORT COHPLETED FOR 3 Y YALUES.

PFITST ELEHENT STRESS ITEHS PER ELEHENT 
量

怘

哭

焉焉

䆛

政

的里

물

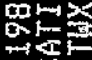

난

总窟

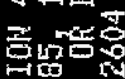

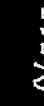

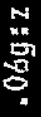

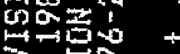

i⿱

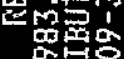

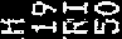

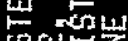

oscints

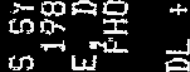

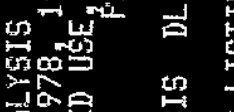

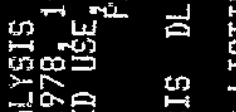

量

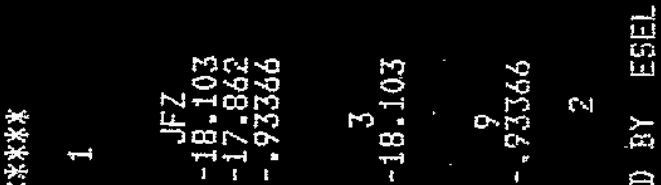

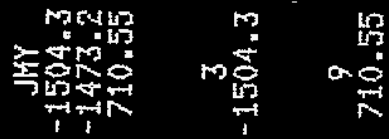

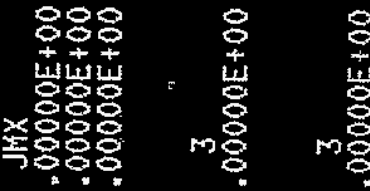

曾

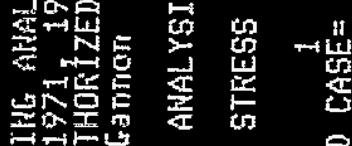

要入象

논

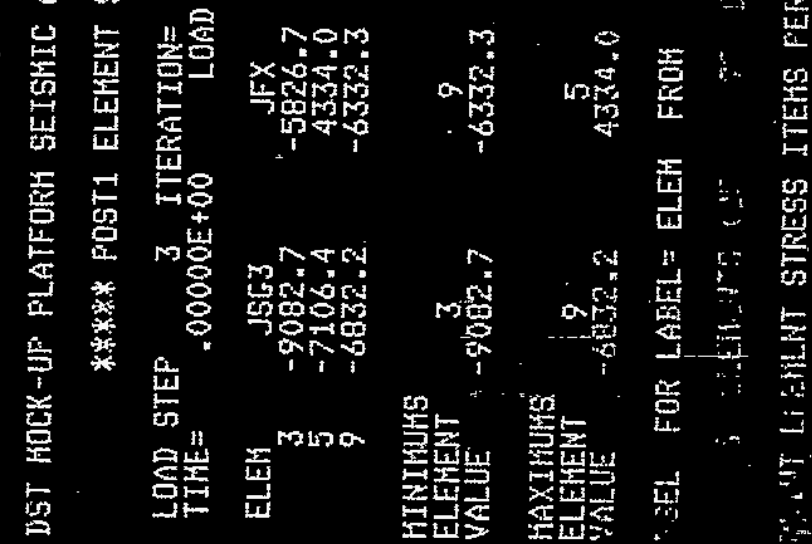

m $8+$ 出

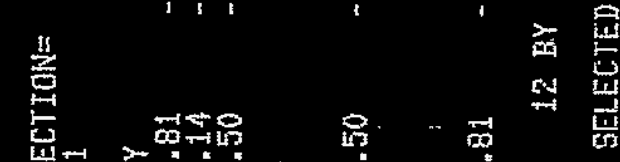

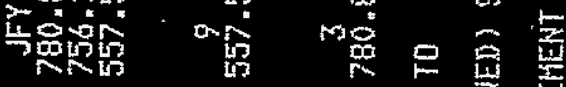

政

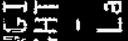

तो

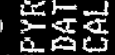

$38>1$

然

든한

知昆宛

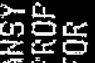


8

पृ

点是

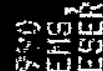

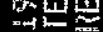

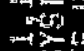

$-5$

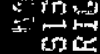

놀

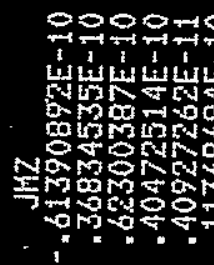

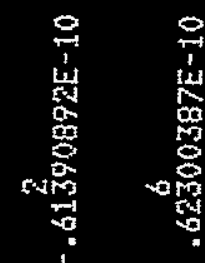

要

$\overrightarrow{5}$

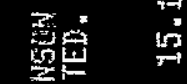

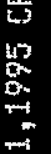

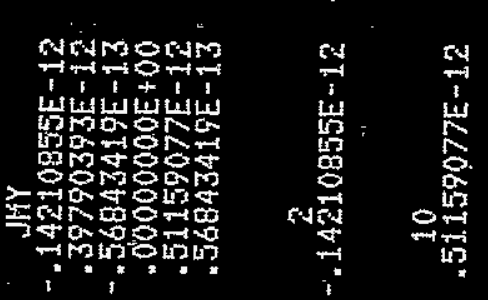

폴

ets

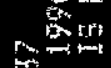

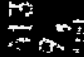

$x_{x \rightarrow+\infty}^{\infty}$

구웅

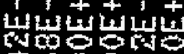

tring

幽的8

品N

$\infty_{x \rightarrow \infty}$

givis

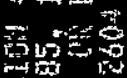

害

0

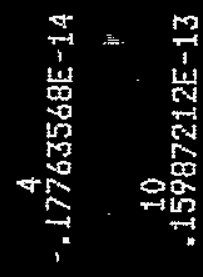

蜜

$\lim _{\rightarrow \rightarrow 1}$

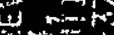

忽业是

ii

(3)

60

$x^{n-1}$ en

then

急为

要,

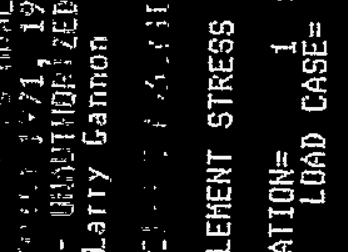

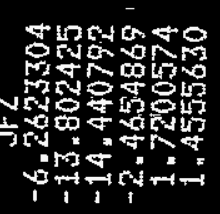

突

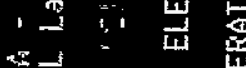

程起

酷

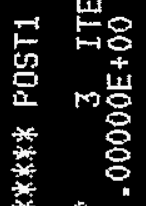

站

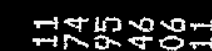

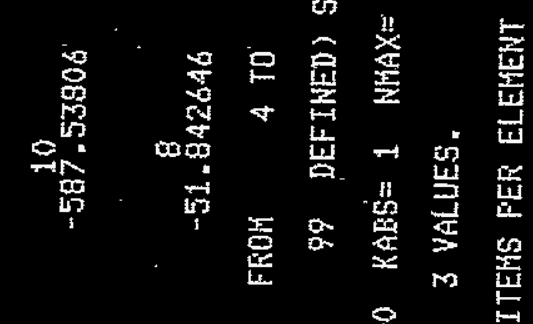

obman

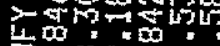

जिए人日

点

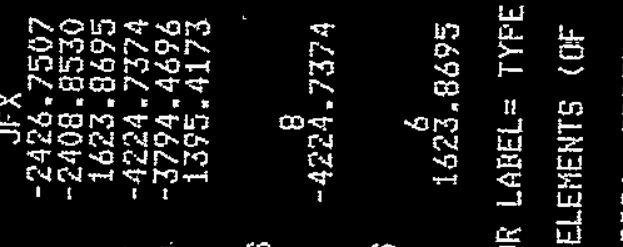

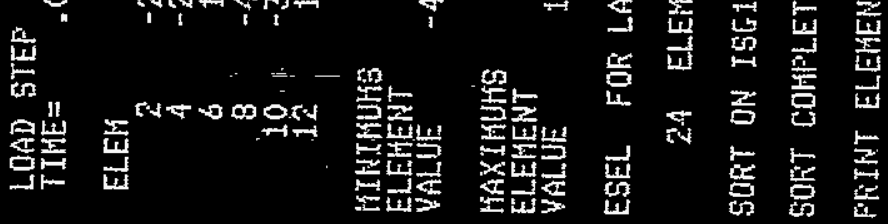


욣

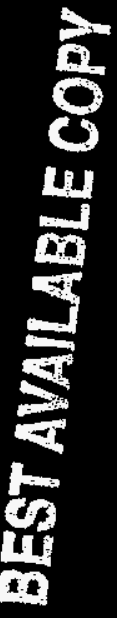

옹몸

政

焉to

$\operatorname{lin}^{2 x}$

Ia

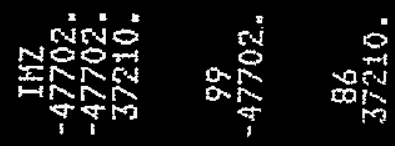

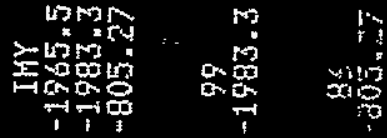

몽잘

따달

$\nabla \mathrm{D}^{2}$

ㅁำ

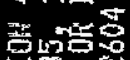

is

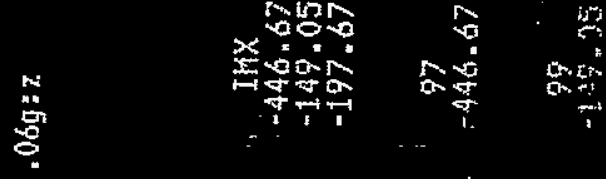

개을

is

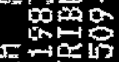

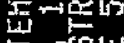

$+$

索

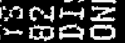

is

${ }_{0}^{T-4 w^{m}}$

崖

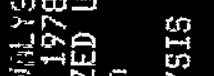

舟昆

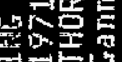

㐘入

跑第会

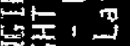

w的山

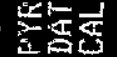

u告的西

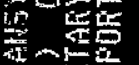

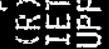

gen

㩆唯

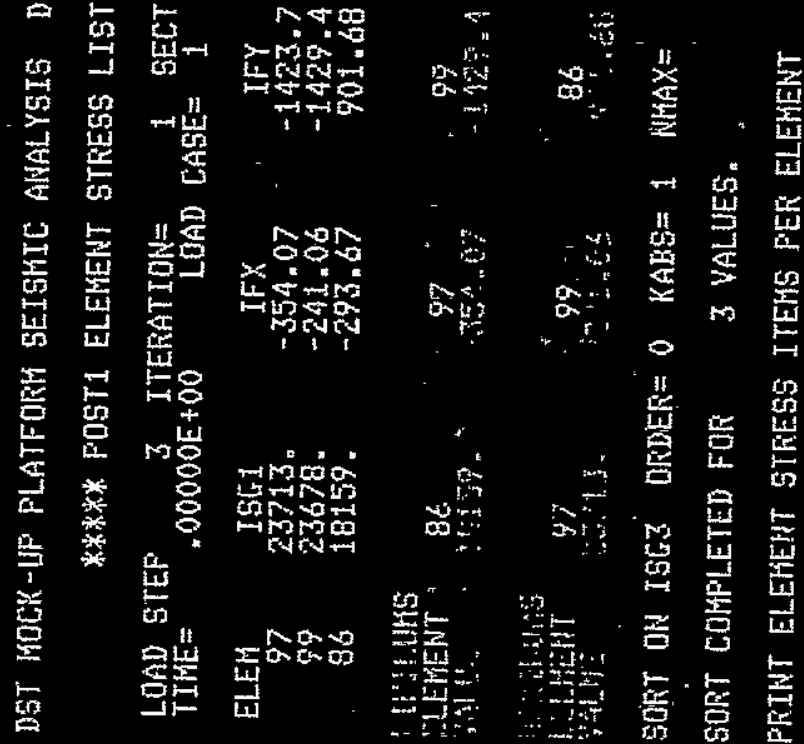




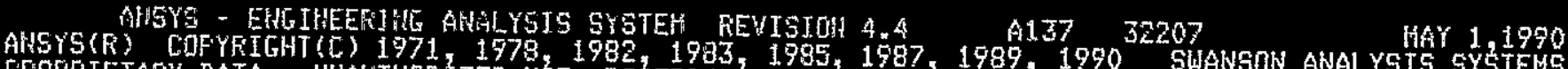

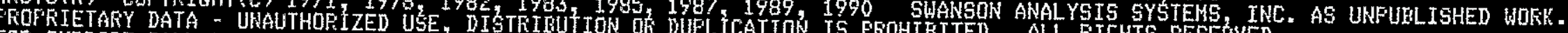
FOR SUFFORT CALL LarTy GaIrTOT PHOHE $509-376-2604$ TWX

DST MOCK-UP PLATFORH SEIGHIC AWALYSIS DL $+0.2 \mathrm{gg}-\mathrm{x}+.06 \mathrm{~g}: \mathrm{z}$

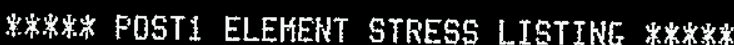

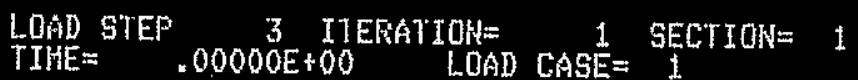

$\begin{array}{cr}\text { ELEF } & \text { ISG3 } \\ 99 & -23492 . \\ 97 & -23439 \\ 06 & -17932 .\end{array}$

MINIFULS

ELEFENT

VALUE:

99
2342

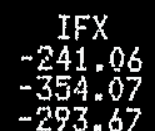

IFY
-1429.4
-1423.7

901.68

99

$-354.07$

$-1429.4$

MAXIHUHS

ELEKENT $\quad 86$

79
-241.06

$\frac{86}{901.68}$

SORT DK JSG1 ORDER $=0$ KABS $=1$ NHAX

3

SORT COHPLETED FOF

3 VALUUES.

FFINT ELEMENT STRESS ITEMS PER ELEHENT
$\frac{\operatorname{IFZ}}{131.0}$

33.676

13.370

86

8.370

139.01

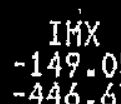

$-446.67$

$-197.67$

97

$-446.67$

$-149.05$

$-905.27$

IHY
-1983.3
-1965.5

$-1965.27$

90

99
-1993.3
$-47702$

$-47702$

37210 .

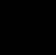


8

in

焉焉

窝

$\lim _{\substack{a \\ \operatorname{mon}}}$

的点

아물

$\rightarrow$

a宜

40.

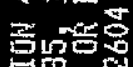

产

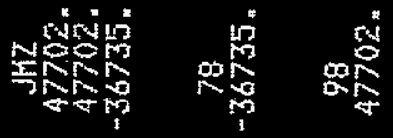

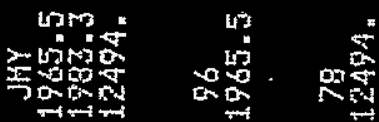

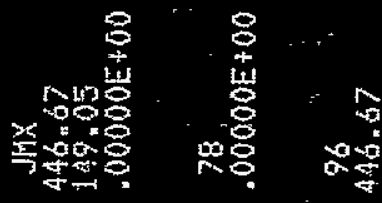

법조의

in

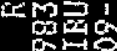

는

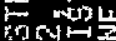

360

$\rightarrow$ 논

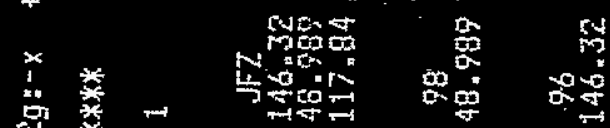

$\cos x$

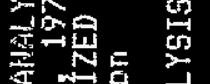

此要

国宽

空

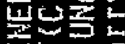

본면

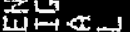

$1 \frac{1}{5}$

$\operatorname{ton} 5$

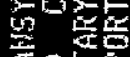

4

崖

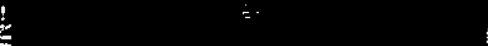

空

势

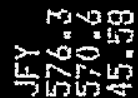

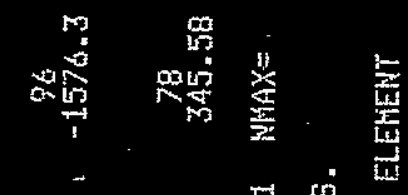

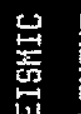

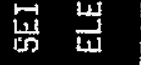

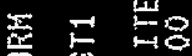

夏总

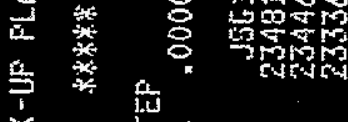

皆的

总

空至

놈

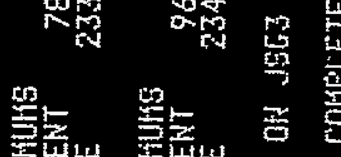

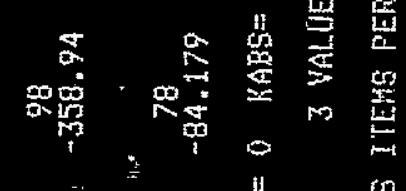

4tyos

$\underline{3}$

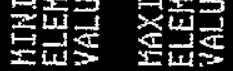

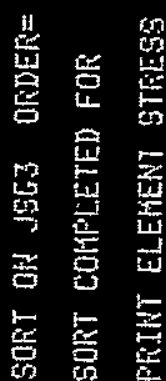


占昱

에에

$\rightarrow=$

$\stackrel{3}{2}$

的空

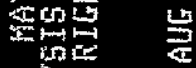

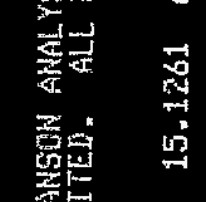

票

s

案率

昰

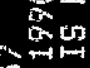

다용

맘줄

ज小

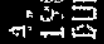

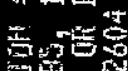

兑

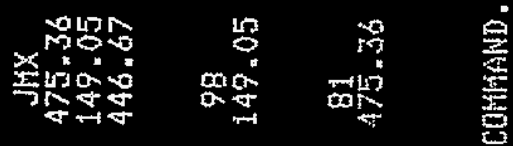

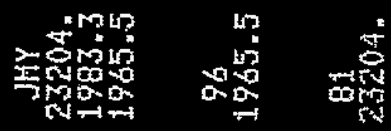

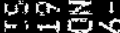

i. $\rightarrow$

'

$\therefore$ 的昆

it

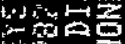

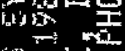

ot

givo

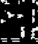

触点

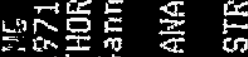

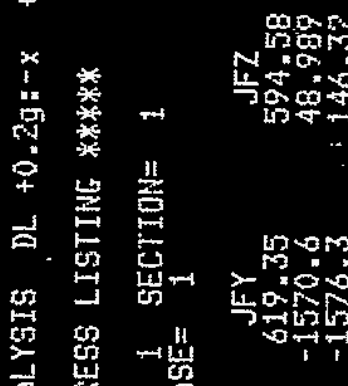

$3+5$

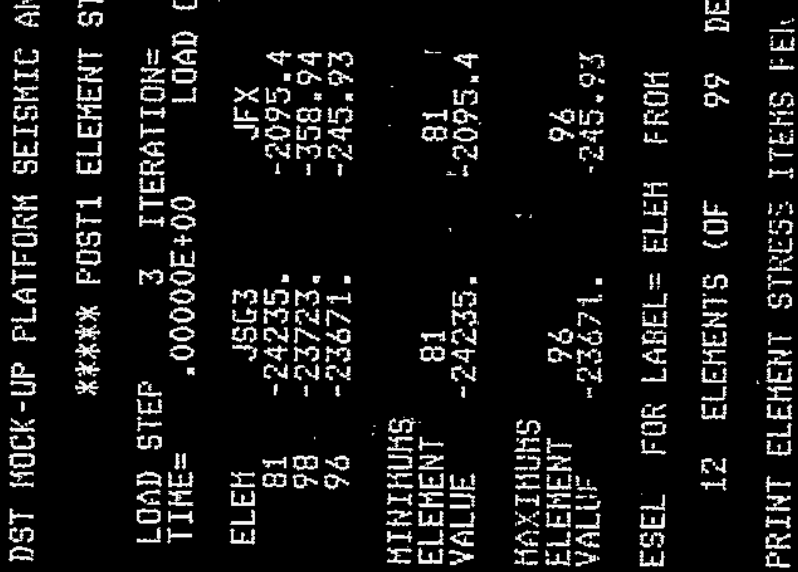

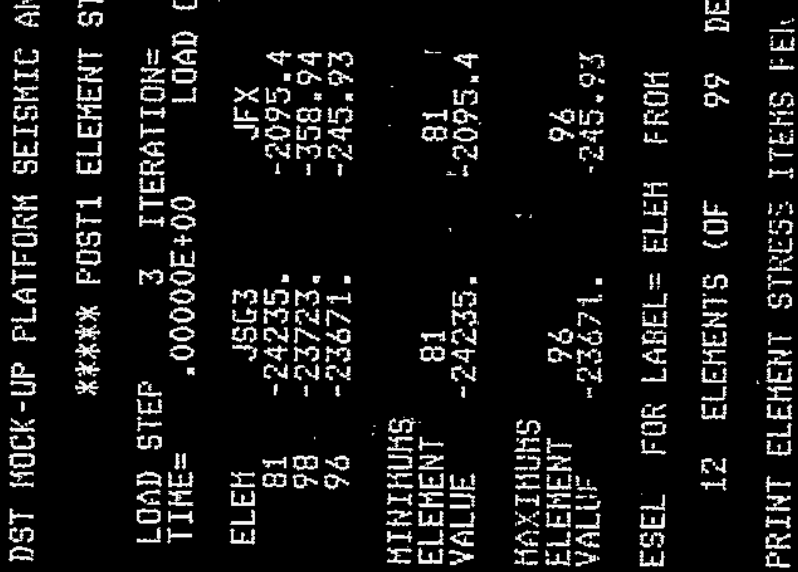

- c

wis

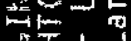

踏

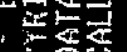

19

四点

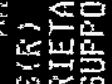

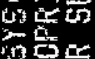

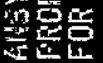


8

毠

运量 品

o

券需

ritect

$\rightarrow \rightarrow \infty$

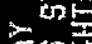

近保

品

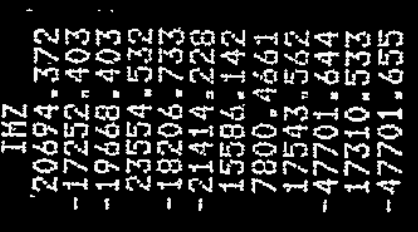

骂

2

武

㩆

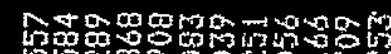

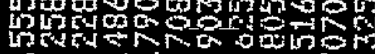
>ñan

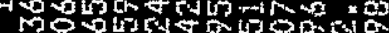
Bromangenta

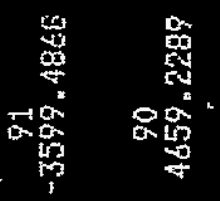
要 $\lim _{i \rightarrow n}$

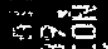

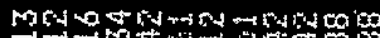

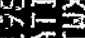

inter a가에

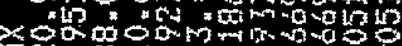
s.t.

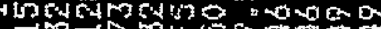
जी

否 要

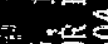

몽

-is?

in

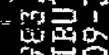

(n)

term

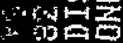

$x_{-i}$

(1) w

$x_{3}$

㱐

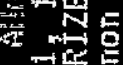

구울

要要

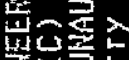

$x_{1 \rightarrow 2}$

3,9

, 象兴过

pis

tis

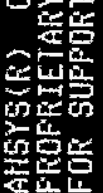

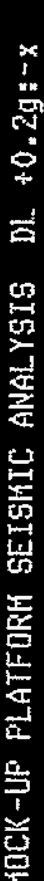

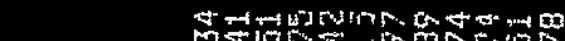

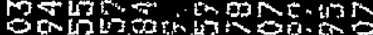

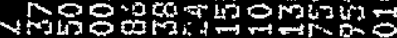

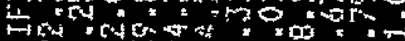

wrop

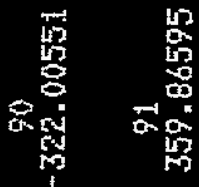

鯤

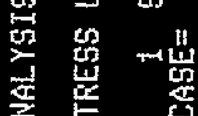

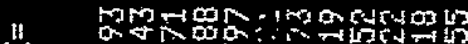

artoris

m80 7

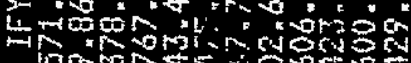

空完

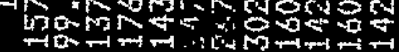

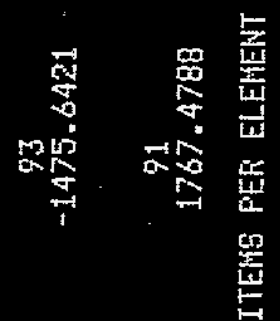

돈

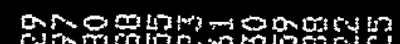

Sing

1070

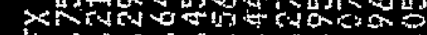

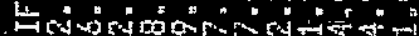

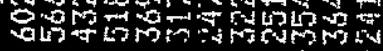

稟

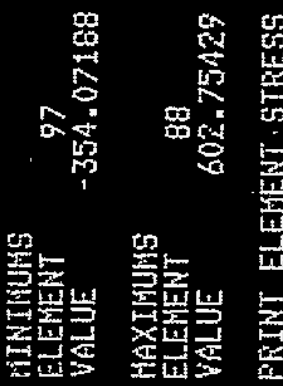




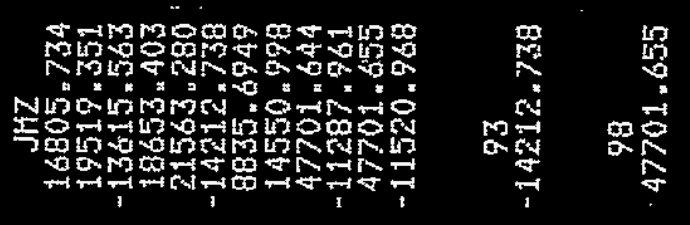

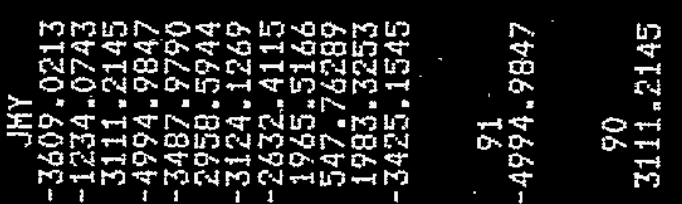

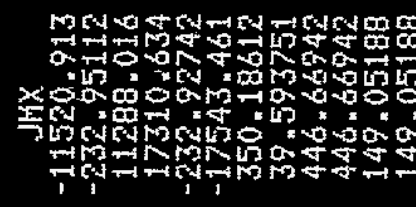

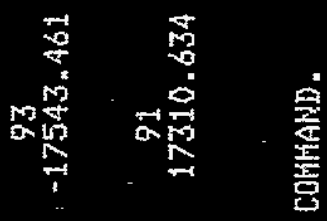

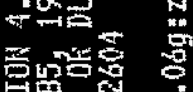

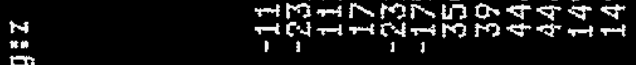

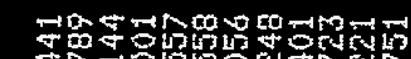

TMन

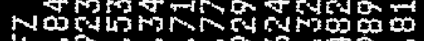

70

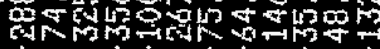

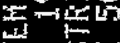

n⿴囗十心

ing

$\omega^{-1}$

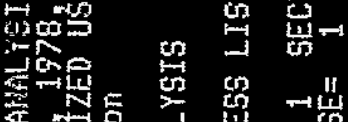

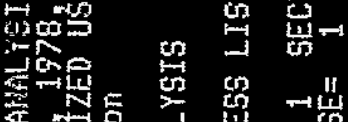

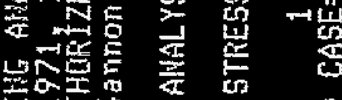

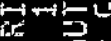

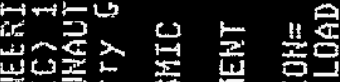

난

它年,

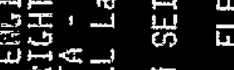

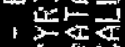
능현

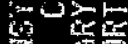

200909

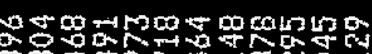

movar

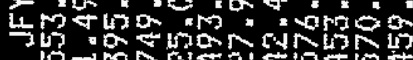

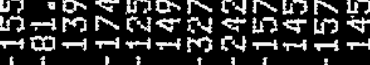

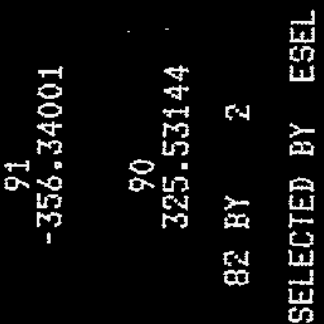

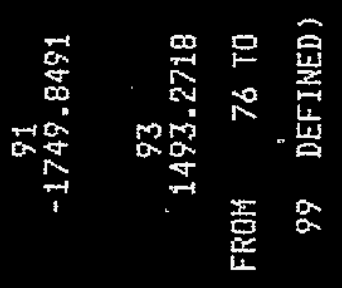

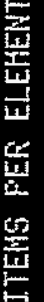

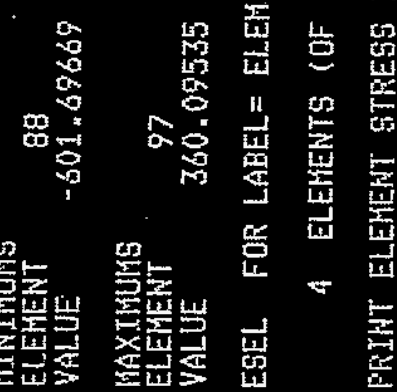


웅

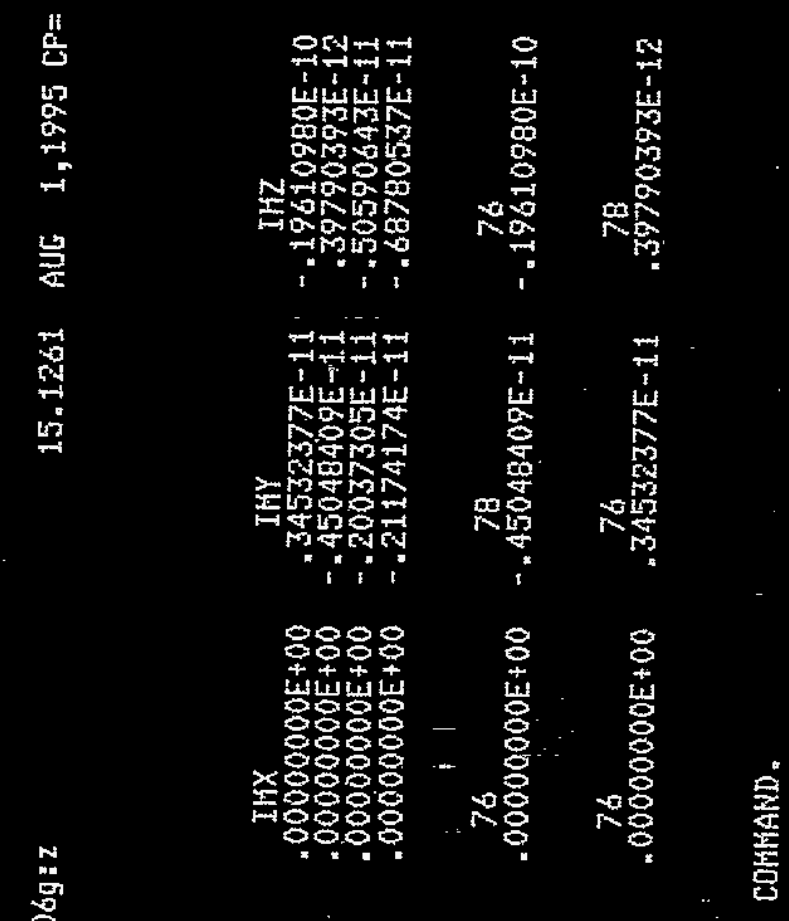

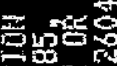

tsis

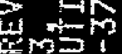

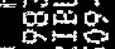

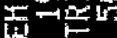

$\tan 4$

- man

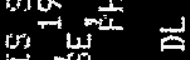

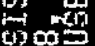

悀。

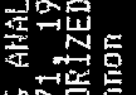

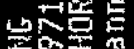

$\rightarrow \rightarrow E$

出可?

본, 西

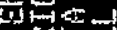

, 进

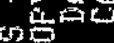

索然

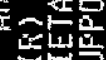

무음

혼돈

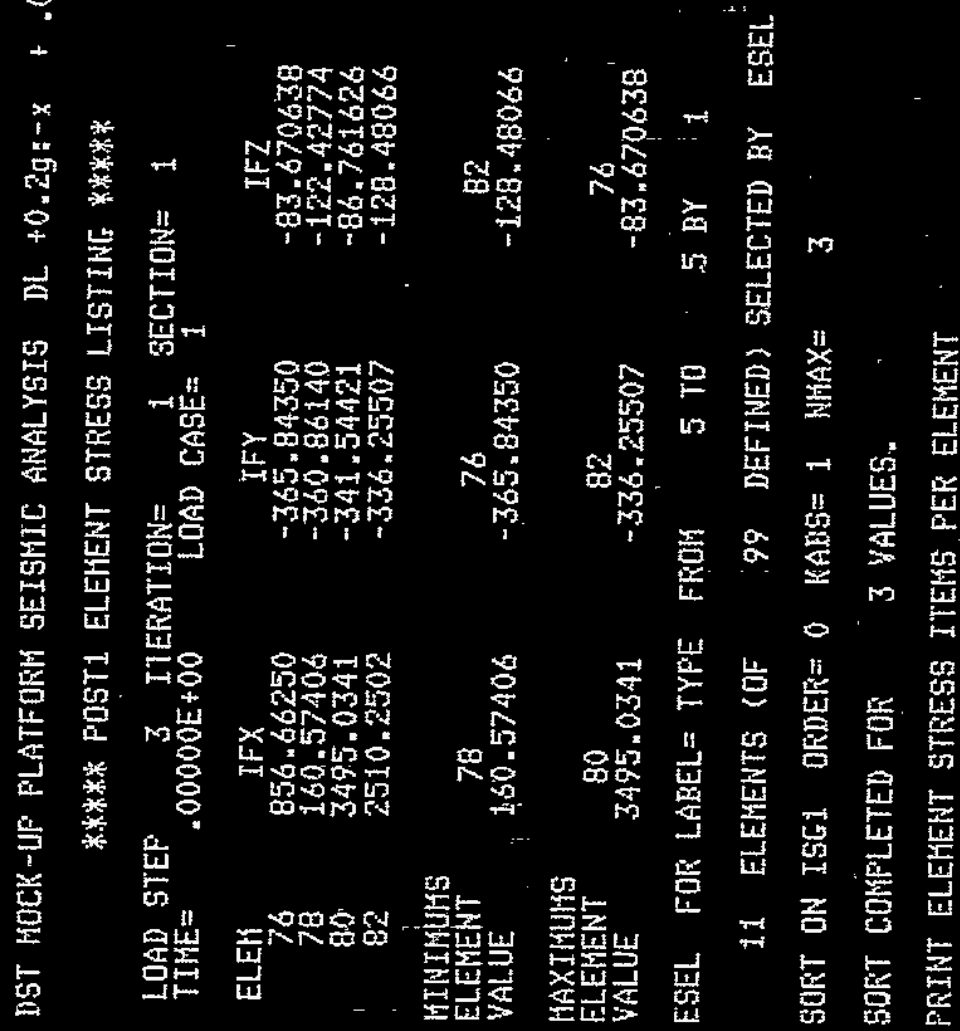

$A-117$ 
怘

운

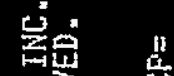

on

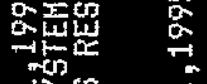

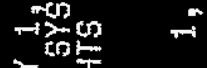

的运岁

童热

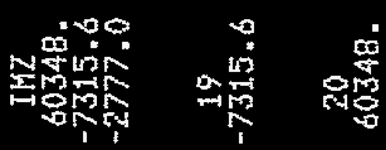

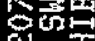

究

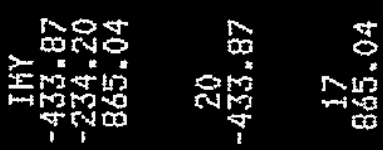

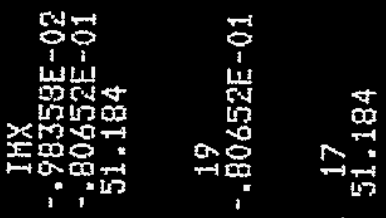

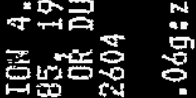

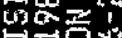

는

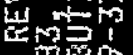

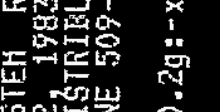

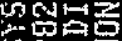

un

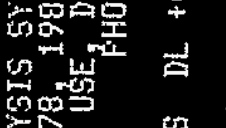

$\infty_{-1}$

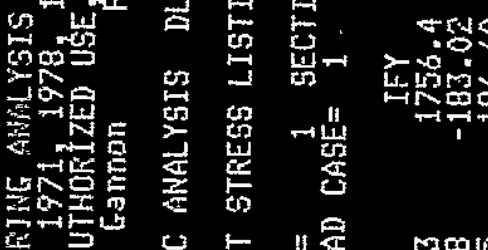

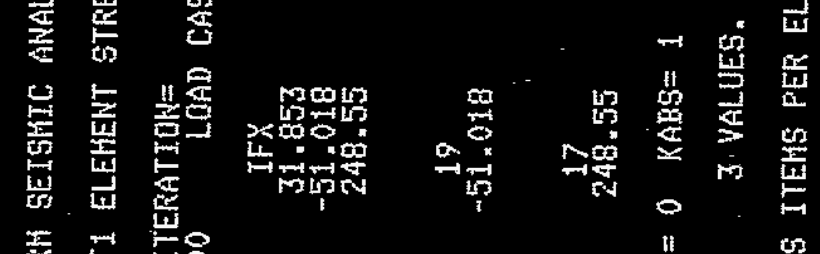

논,

要

, 近娄

g

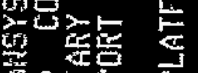

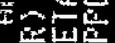

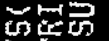

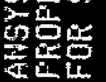

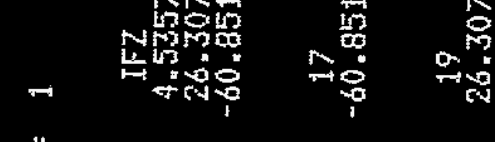

m岁

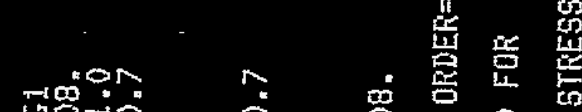

م然

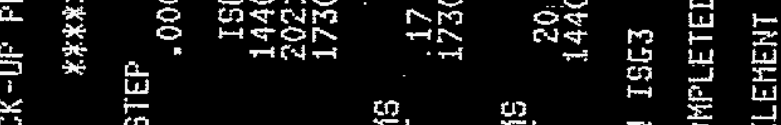

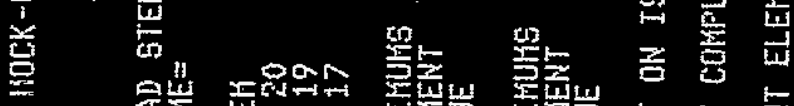

$\stackrel{\mathrm{Eg}}{\mathrm{E}}$

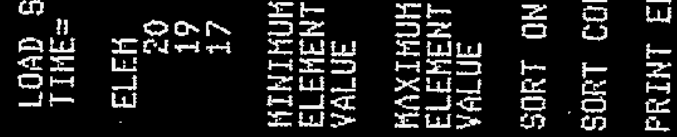


AHSYS - EHGIHEEFTHG ALALYSIO GYSTEH REVISIOH 4.4

A 13732307

HAY 1,1990

AHSYS(R) COFYRIGHTLC) $1971,1978,1982,1983,1985,1787,1799,1990$ SHANSON ANALYSIS SYSTEMS, INC. AS UNFUBLISHED HOFK.

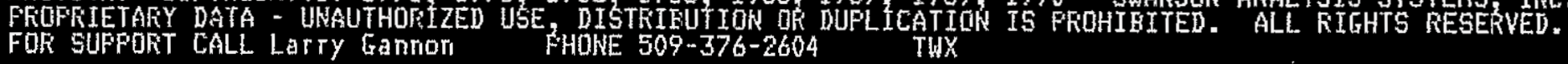
THX

DST HOCK-UF FLATFOFA SEISHIC AHALYSIS DL +0.2g:- $\mathrm{g}+.06 \mathrm{g:z}$

15.1261 AUG $1,1995 \mathrm{CP}=$

.920

*

LOAD STEP 0003 ITERATION= $\stackrel{1}{1}$ SECTION= $=1$

ELE:

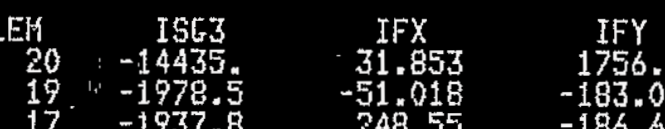

83.0

IFZ

4.5357
26.307

IHXX

$-.98358 E-02-433.87$

$-80652 \mathrm{E}-01-3 \mathrm{Cu}^{-20}$

$51.184 \quad 865.04$

InZ

$-7315.6$

MIWIHUHS

ELEMENT

20
-1443

$\begin{array}{cc}19,018 & -17 \\ -51.018 & -186.60\end{array}$

$-60.951$

$-2777.0$

HAXIHLHSS

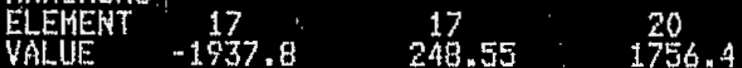

$\begin{array}{ccc}17 & 19 & 20 \\ -60.851 & -.80652 E-01 & -433.87\end{array}$

$-7315.6$

26.307

$59+178$

865.04

20348

SOKT OKS JSGI ORDER $=0$ KABS $=1$ WHAX $=$

3

SORT COMPLETED FOF

3 VALLUES.

PRINT ELERENT STFESS ITEHS PEF ELEHENT 
要要

点电

考泼

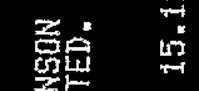

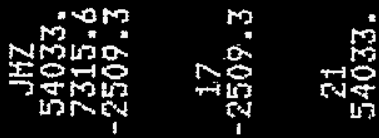

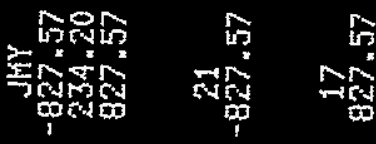

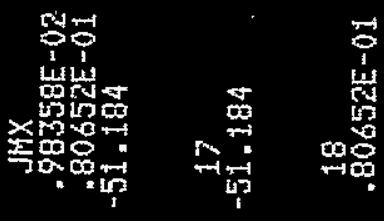

害的乘宫

4
뭉
+

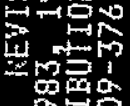

에의

要

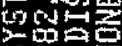

$\rightarrow$ 至

$\lim _{\infty}^{\infty}$

man in

정ㅎㅇ

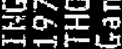

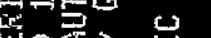

宸包蛋的

勿, 可

击心我

1 这安

$\log _{3}$

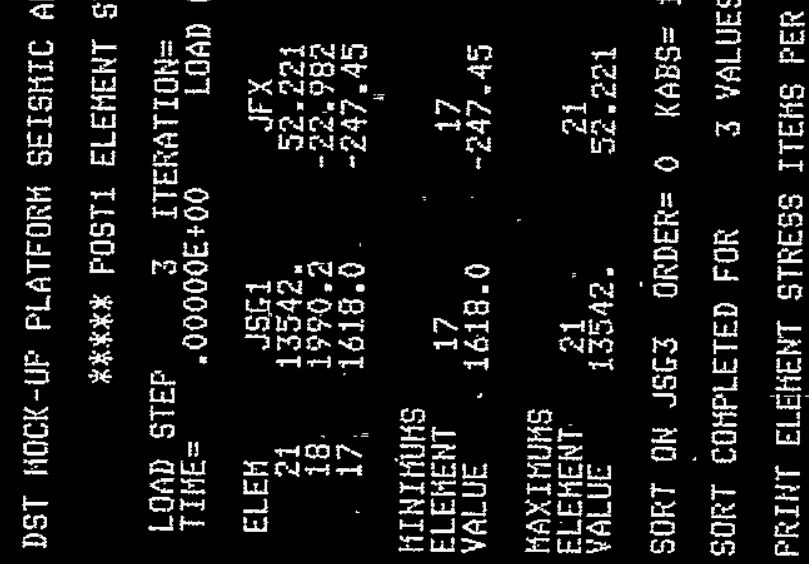


焉

案

要商

空

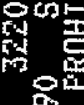

要昆

的话

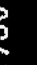

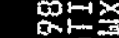

$\rightarrow<F$

$x$

멸

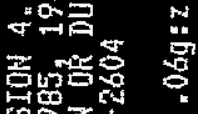

Nकूa

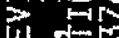

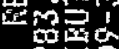

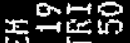

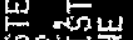

땜ำ

th

$\operatorname{lom}_{0}^{\infty}$ का

运是

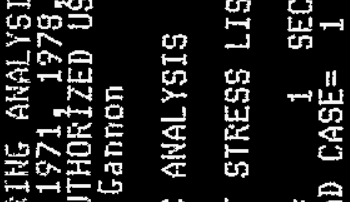

जô

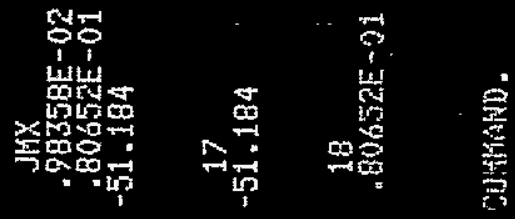

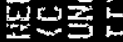

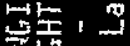

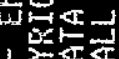

焉过

붕 $>$

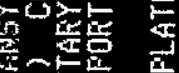

는

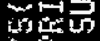

整统

䆑吉

당

농옹

Tsis

MEN - A 8

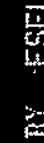

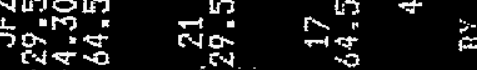

空点

$\underset{c i n}{200}$

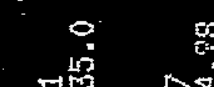

豆畺

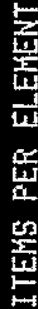

बis

a.

音

mascos

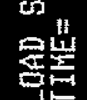

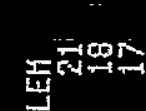

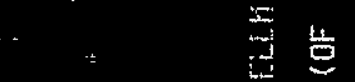

然

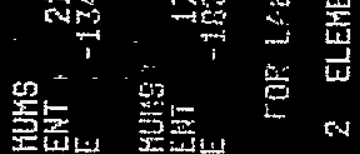

旨

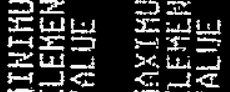

in

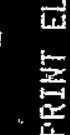

$$
A-121
$$




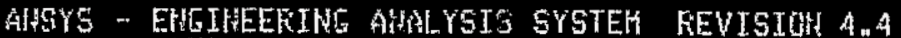

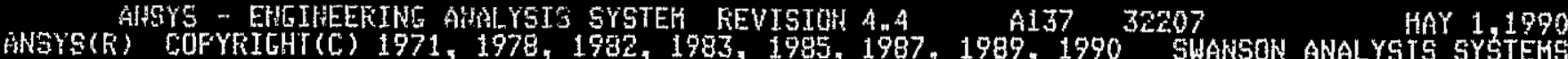

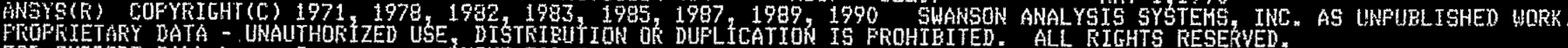
FDF SUFPORT CALL LaTrY Gammon FHONE 509-376-2604 $\mathrm{TWX}$

DST HUCK-UP FLATFORH SEISHIE ANALYSIS DL +0.2g:-x + .06g:

15.1261 AUG $1,1995 \mathrm{CF}=$

.940

****** FOST1 ELEHENT STRESS LISTINE *****

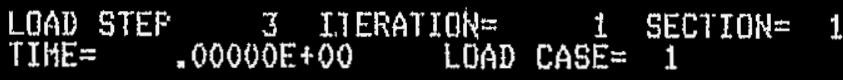

$\begin{array}{cccc}\text { ELEH } & \text { IFX } & \text { IFY } & \text { IF } \\ 18 & 28.019022 & 213.16043 & 2.79 \\ 22 & 14.864850 & 115.8691 . & 5.10\end{array}$
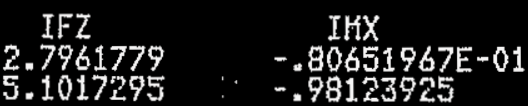

IHY

$.9061967 E-01-365.60875$

IHZ

10543119

HIIIFIUHS

ELEHENT

$14 \times 64850 \quad 11586991$

$2.7861779 \quad-.981 .23925 \quad-365.60875$

6.2263587

HAXIHUAS

ELEHENT

18

$21 \frac{18}{3} .16043$

5.2217295

$-.80651967 E-01-222.60203$

105.43119

ESEL. FOK LABEL= ELEH FROM 19 TO 23 BY 4

2 ELEHENTS (OF 99 DEFINED) SELECTED GY ESEL COHHAND.

PRINT ELERENT gTRESS ITEHS FER ELEHENT 
AHSYS - EHGIHEEFTHE AHAL YSIS SYSTEH REVISIOH 4.4

A 13732207

HAY 1,1990

ANSYS(K) CQFYFIGHT(C) $1971,1978,1982,1783,1985,1987,1999,1990$ SWANGON ANALYSIS SYSTEHS, INC. AS UNFUHL ISHED WORK.

FFUFFIETARY DATA - UHAUTHORIZED USE, DISTRIRUT ION OK DUFLICATION IS FROHIBITED. ALL RIGHTS RESENVD.

DST HOCK-LPP FLATFORH SETSHIC ANALYSIS DL +0.2g:-x + .06g:z

15.1261 AUG $1,1995 \mathrm{CP}=$

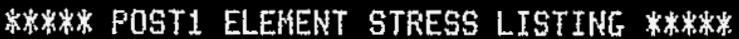

LOAD STEF 0003 TTERATIOKL $=\frac{1}{2}$ SECTION $=1$

\begin{tabular}{|c|c|c|c|c|c|c|}
\hline $\begin{array}{l}\text { ELEH } \\
19 \\
23\end{array}$ & $\begin{array}{c}J F X \\
56.055076 \\
31.941972\end{array}$ & $\begin{array}{l}\text { JFY } \\
208.21006 \\
116.16420\end{array}$ & $\begin{array}{c}J F Z \\
-27.817986 \\
-19.143424\end{array}$ & $\begin{array}{l}\text { JHX } \\
.80651967 \mathrm{E}-01 \\
.98123925\end{array}$ & $\begin{array}{c}\mathrm{JMY} \\
-767.11160 \\
-393.52680\end{array}$ & $\begin{array}{c}\frac{14 Z}{71.732706} \\
-13.483450\end{array}$ \\
\hline $\begin{array}{l}\text { HTHIMUNG } \\
\text { ELEKEHT } \\
\text { VALEE }\end{array}$ & 31.2341972 & $11 \frac{23}{6} \cdot 16420$ & $\begin{array}{c}19 \\
-27.817886\end{array}$ & $.80651967 E-01$ & $\begin{array}{l}19 \\
-787.11160\end{array}$ & $\begin{array}{l}23 \\
-13.483450\end{array}$ \\
\hline $\begin{array}{l}\text { WAXIKLMS } \\
\text { ELEHENT } \\
\text { VALLEE } \\
\text { USE LOAD }\end{array}$ & ${ }^{50} \cdot 19$ & $\frac{19}{208.21006}$ & 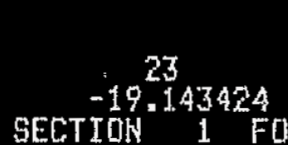 & LOAD CASE & -383.52680 & 77.732706 \\
\hline
\end{tabular}

DISFLACEMENT STORED FOR 72 NDDES

* *

DATA FOR STRESS LABEL IFX HAS NOT BEEN STOKED FOR ALL ELEMENTS.

IFX DATA FOR TYFE 2. STIF 8. ELEKENTS WILL BE SET TO ZEKO

* * * *WARNTHE $*$ *

7 DATA FOR STRESS LAEEL IFY HAS NOT BEEN STOFED FUR ALL ELEMENTS.
IFY DATA FOR TYFE 2. STIF 8 . ELEHEHTS WTLL BE SET TO ZERO

N WATAARHIHG w

W IFZ DATA FOK TYPE 2. STIF 8. ELEHENTS HILL BE SET TO ZEFO

* * WAFEIING *

DATA FOR STHESS LAEEL IMX HAS NOT BEEN STOFED FOE ALL ELEMENTS.

IHX DATA FOF TYFE 2. STIF 9 . ELEHENTS WILL, BE SET TO ZEFO

W*WHARNING *

DATA FOR STFESS LAEEL IHY HAS NOT BEEN STORED FOR ALL ELEMEHTS.

IHY DATA FOR TYFE 2, GTIF 8. ELEHENTS WILL BE SET. TO ZEKO

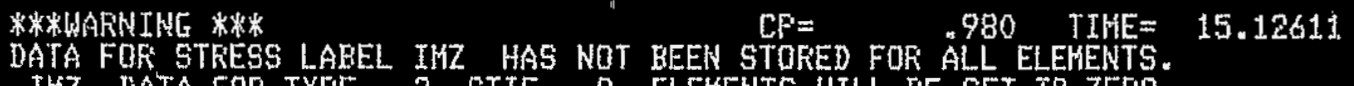

IHZ. DATA FUR TYFE 2 , STIF 8. ELEHENTS WILL BE SET TI ZERO

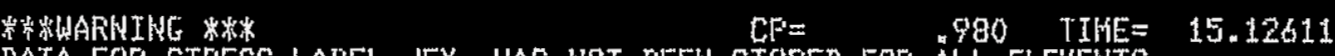

DATA FOR STRESS LABEL JFX HAS NQT REEN STOFED FOR ALL ELEHENTS.

JFX DATA FUK TYFE 2. STIF 8. ELEHEVTS WILL BE SET TO ZERO"

* *WWANUING * * W DATA FOR STFESG LABEL JFY HAS NOT GEEN STOFED FOR ALL ELEHENTS.
JFY DATA FOR TYFE

W* DATA FOR STRESS LABEL JFZ HAS NUT BEEN STORED FOR ALL ELEMENTS.

IF $Z$ DATA FOR TYFE 2. STIF 8 . ELEHEHTS WILL. BE SET TO ZEFO 


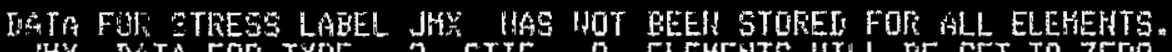

WHX DHTA FOF TYPE 2 "STIF 8. ELEHENTS WIUL EE SET TO ZERO"

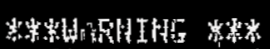

$\mathrm{CP}=$

980 TIHE= 13.12611

DATA FUK STRESS LABEL JMY HAS NOT BEEN STOKED FOK ALL ELEHENTS

JHY DATA FOR TYFE 2. STIF 8, ELEHEHTS HILL BE SET TO ZERO

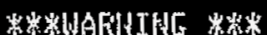

$C P=\quad .980$ TIHE $=15.12611$

DATA FOR STFESS LABEL JMZ HAS KOT EEEN STOEED FOR ALL ELEMEWTS.

JHZ DATA FOE TYPE 2 . STIF B. ELEHENTS HILL BE SET TO ZERO

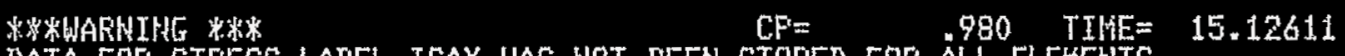

DATA FOK STFESG LABEL. ISAX HAS NOT BEEN STORED FQR ALL ELEIENTS.

ISAX DATA FOR TYPE 2 . STIF 8. ELEHENTS WILL HE SET TO ZERO

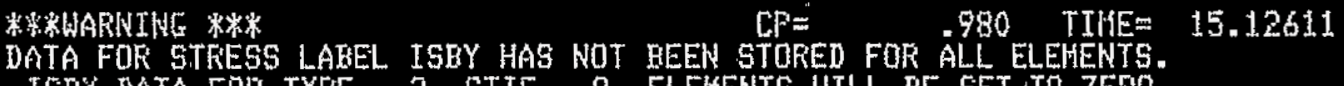

ISRY DATA FOK TYFE 2. STIF 8. ELEMENTS UILL BE SET TO ZERO

S*OTHARHTNG $*$ W

CF: $=$

980 TIHE $=15.12611$

DATA FOR STRESS LABEL ISBZ HAS NOT BEEN STOKED FOR ALL ELEMENTS

ISEZ DATA FOR TYFE 2. STIF 8. ELEHENTS WILL BE SET TO ZERO 


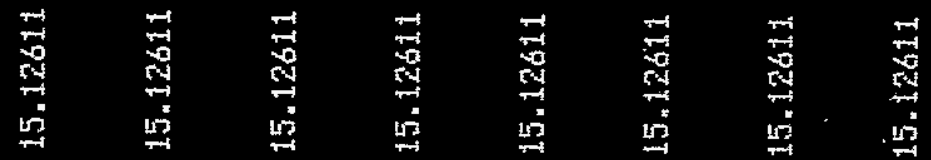

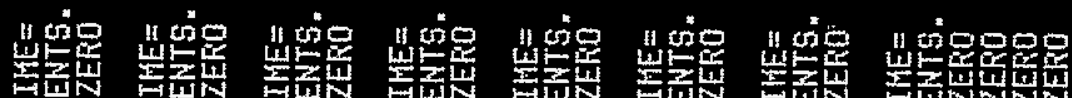

覃

焉的

要品

분은

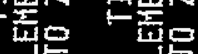

w罂吅

낸단

Fir

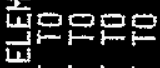

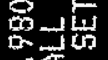

鱼势

类哲

$\circ$

$0^{i+5}$

해물

붐ํㄹ

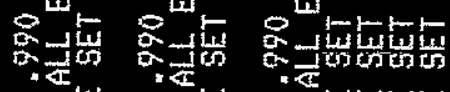

岩

落卖高

广र

<L

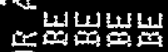

突这

突岗

案西

愿离

동

동몀

器出

E.te

量密

불

用

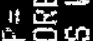

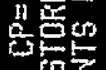

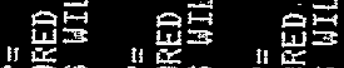

望夏

空焉

11

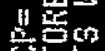

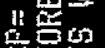

11 若

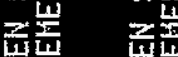

负

另䖯

0

运跑

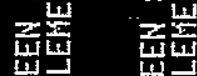

6

its

th 55

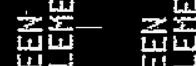

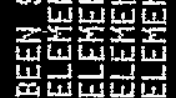

要证

要业

票㟧

垔式

5)

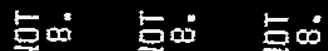

t⿹

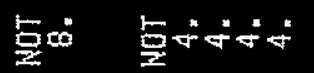

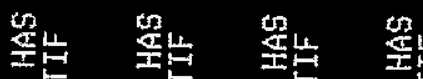

$\pm 0$

$>-6$

玨贴

空.

密

舟要

路L

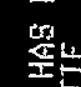

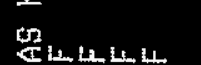

탕

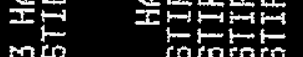

觔

荡

高

丽 $\frac{1}{1}$

해

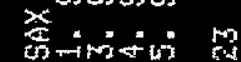

要空

*留留

望望

*彩竞

究究

穿止

*起证

崔点

*它证

는 4

${ }^{2}$

봉옹

安要

这转

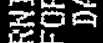

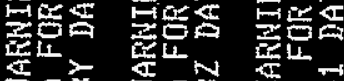

证窇

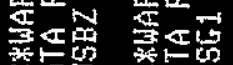

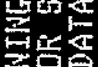

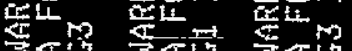

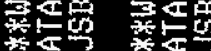

造悹

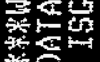

if

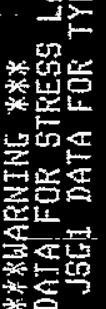

焉电

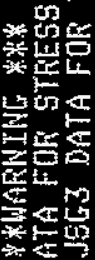

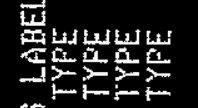

为留

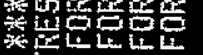

$\omega \mathrm{cr} \ll \ll \ll$

의

西然

(1)

*

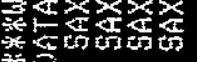

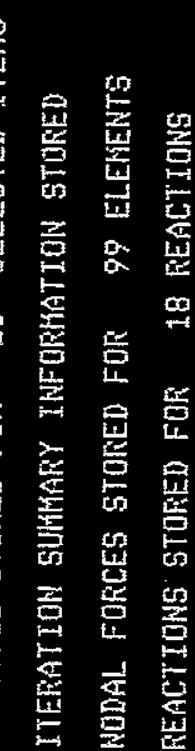

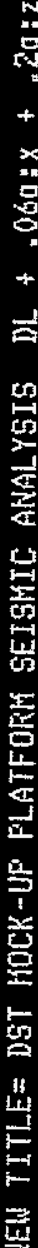

窟

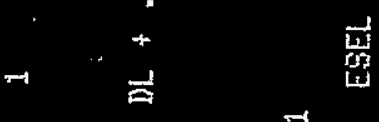

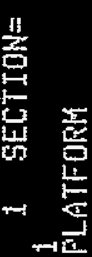

$+3$

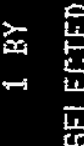

$\rightarrow \underset{0}{0}$

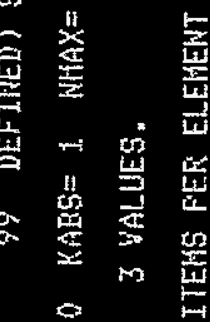

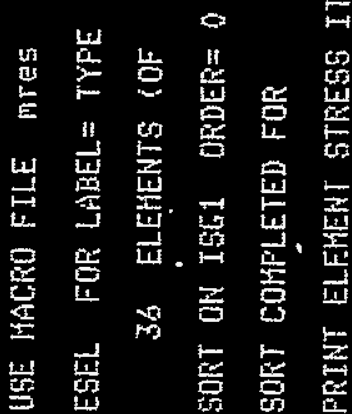


8

g

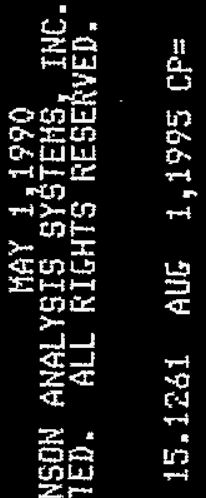

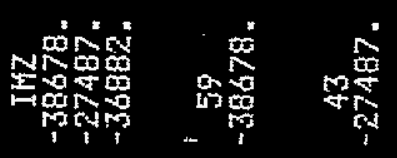

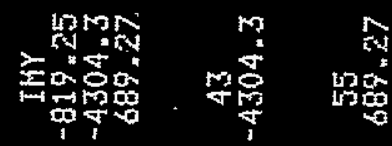

ram

aㅏㄴ졸

$\operatorname{lic}_{n \rightarrow 1}$

वक्षि

말

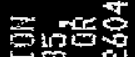

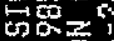

$\rightarrow \rightarrow \infty$

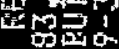

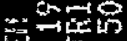

win w

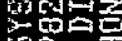

wa

要出

this

돈보

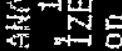

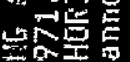

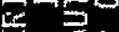

崖色这各

昰, 骂

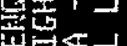

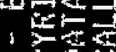

岁向心

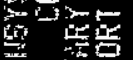

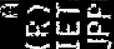

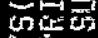

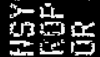

苞

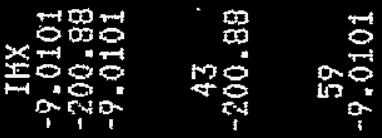

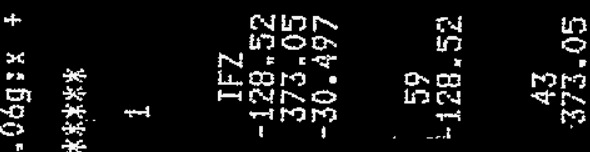

+ 皆

$\Rightarrow$

要

画-

>

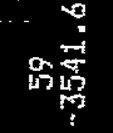

in

落望

무

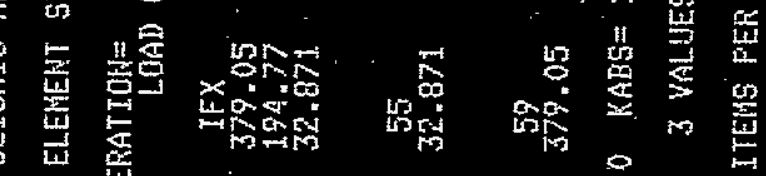

탕

点点恶

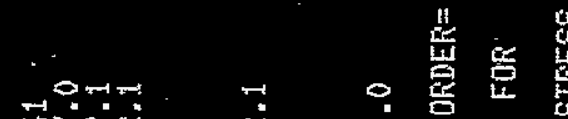

至

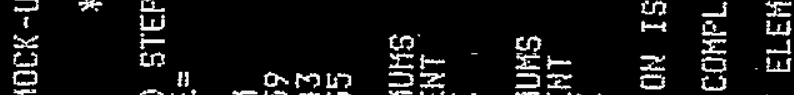

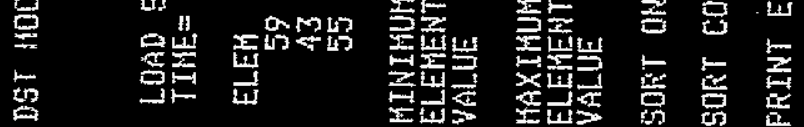


8

\&

穿密

䠛 贺

슨

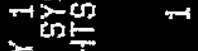

崖

焉击

좀

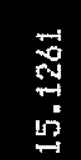

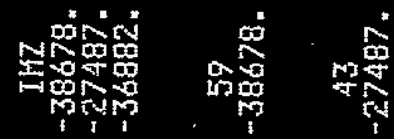

s

橆

욤

要留

물

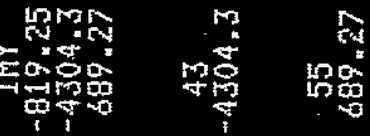

맘늘

$\rightarrow$ 近

$-6$

$x_{x \rightarrow 0}$

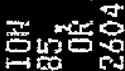

芴

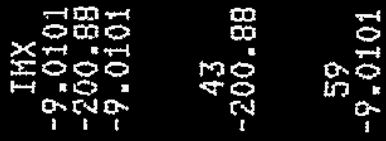

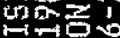

i

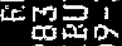

Ento

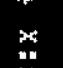

范

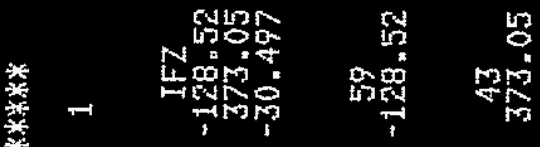

tond

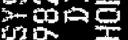

$\sin ^{-1} \mathrm{H}^{-\frac{1}{2}}$

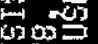

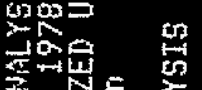

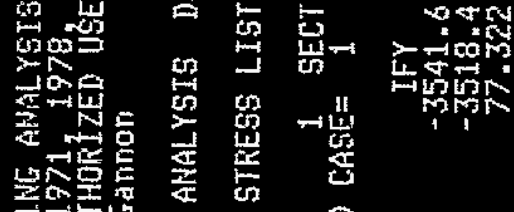

章

$\cdot r$

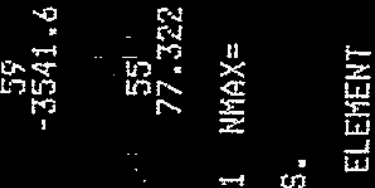

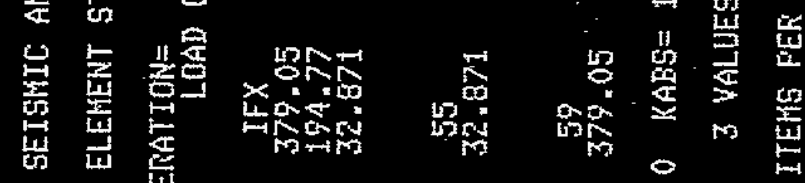

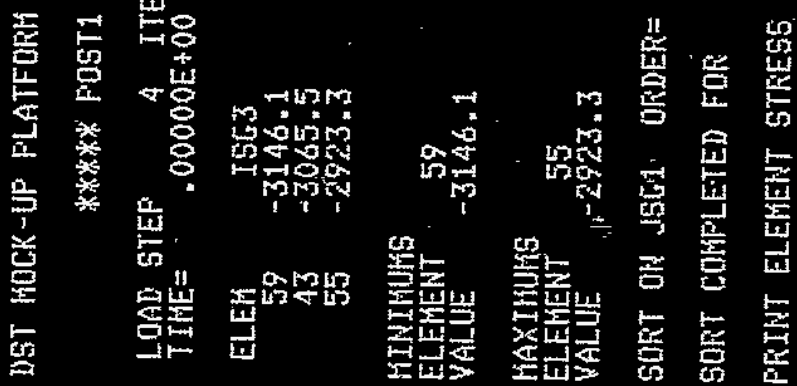


魚

架

通

oxing

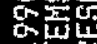

$\rightarrow-x_{0}$

正的

的究

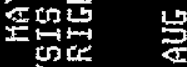

喜势

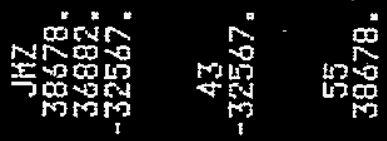

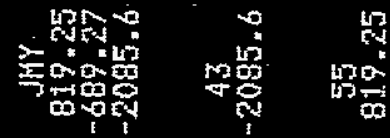

$m_{\rightarrow \rightarrow \infty}$

I무용

$\frac{3}{5}$

最

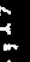

融

묘

$\operatorname{an}_{n \rightarrow 1}$

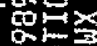

$\rightarrow$

नी金

물

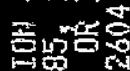

占心

Hiv

og

$x=-15$

昰

grvte

由人

的

$x=$

整苗

昰

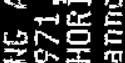

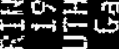

嵌金不

些步

분동

实止步

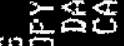

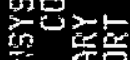

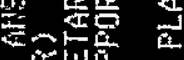

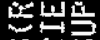

किष्ठ

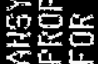

N Join wa

+
$x$
0
0
0

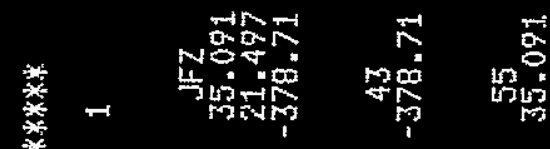

+ 紧览

这

窇

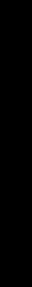

留

蜲

5

党

势

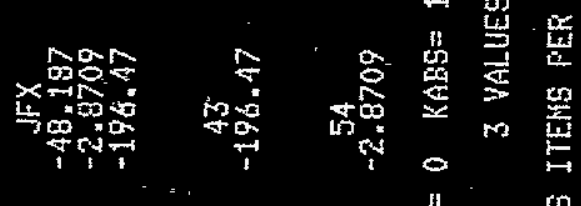

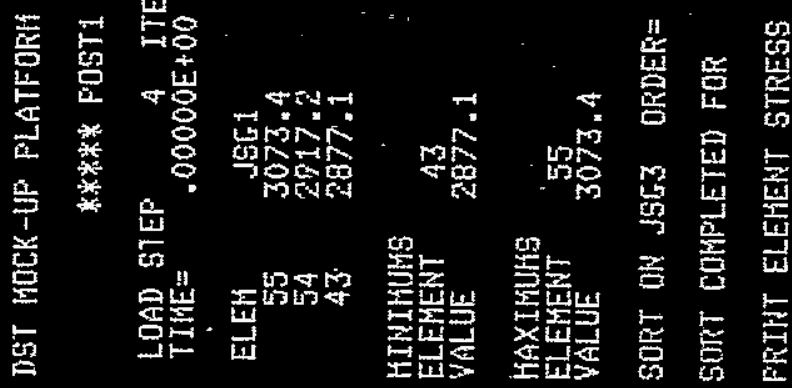


AHSYS - ENGIHEEFIHE AHALYSIS SYSTEH FEVISIUH 4.4

A137 32207 MAY 1,1990

ANSYS(R) COFYRIGHTCC) $1971,1978,1992,1983,1985,1987,1989,1990$ SWANSON ANALYSIS SYSTEMS. INC. AS UNFUELISHED UOKK. FOR SUFFORT CALL LarTy GañON PSHONE $509-376-2604$

DST HOCK -UF PLATFORH SEISHIC ANALYSIS DL $+.06 \mathrm{~g}: \mathrm{x}+.2 \mathrm{~g}: \mathrm{z}$ THX

***** FUST1 ELEHENT STRESS LISTING ******

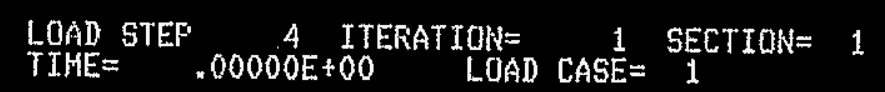

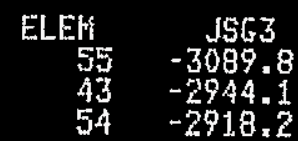

$$
\begin{gathered}
J F X \\
-48.187 \\
-19.4 .47 \\
-2
\end{gathered}
$$
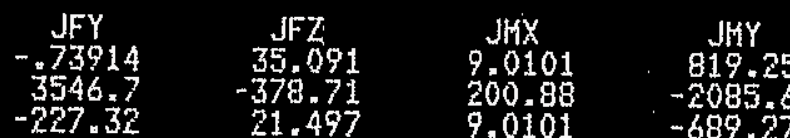

$-2085$.

$-689.27$

$316 Z$
38678

HINIHUKS

ELEKENT $\quad 55$

43

ALLE

$-196.47$

$-227.32$

$-378.71$

9.0101

43

$-2085.6$

$-32567$

MAXIMUKS

ELEHEN

$-294.2-2.8409$

$3536.7 \quad 355.091$

200.8

ESEL FOR LABEL= ELEH FFOH

56 To

$62 \mathrm{BY}$

819.25

55

7 ELEMENTS (OF " 99 DEFINED) SELECTED BY ESEL COMHAND.

FRINT ELEHEHT STRESS ITEMS PER ELEMENT 
要

tra

穿富

$\frac{n}{5}$

요욤

aitit

$\rightarrow \operatorname{con}$

똔돈

留

눌

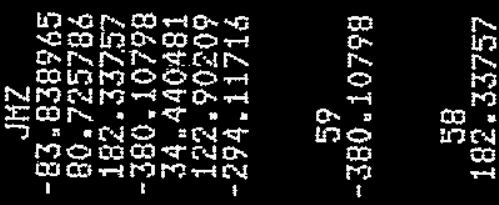

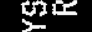

먼

贾至

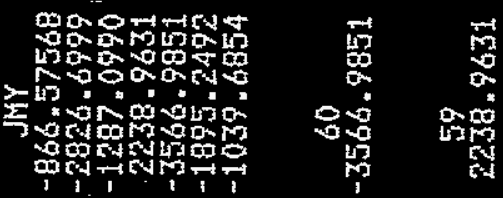

$m_{n \rightarrow \infty}^{\infty}$

植

$\cos _{\rightarrow \infty}$

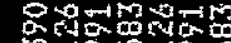

Mracotar

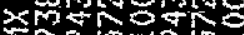

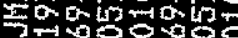

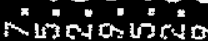

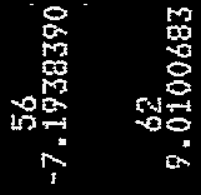

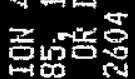

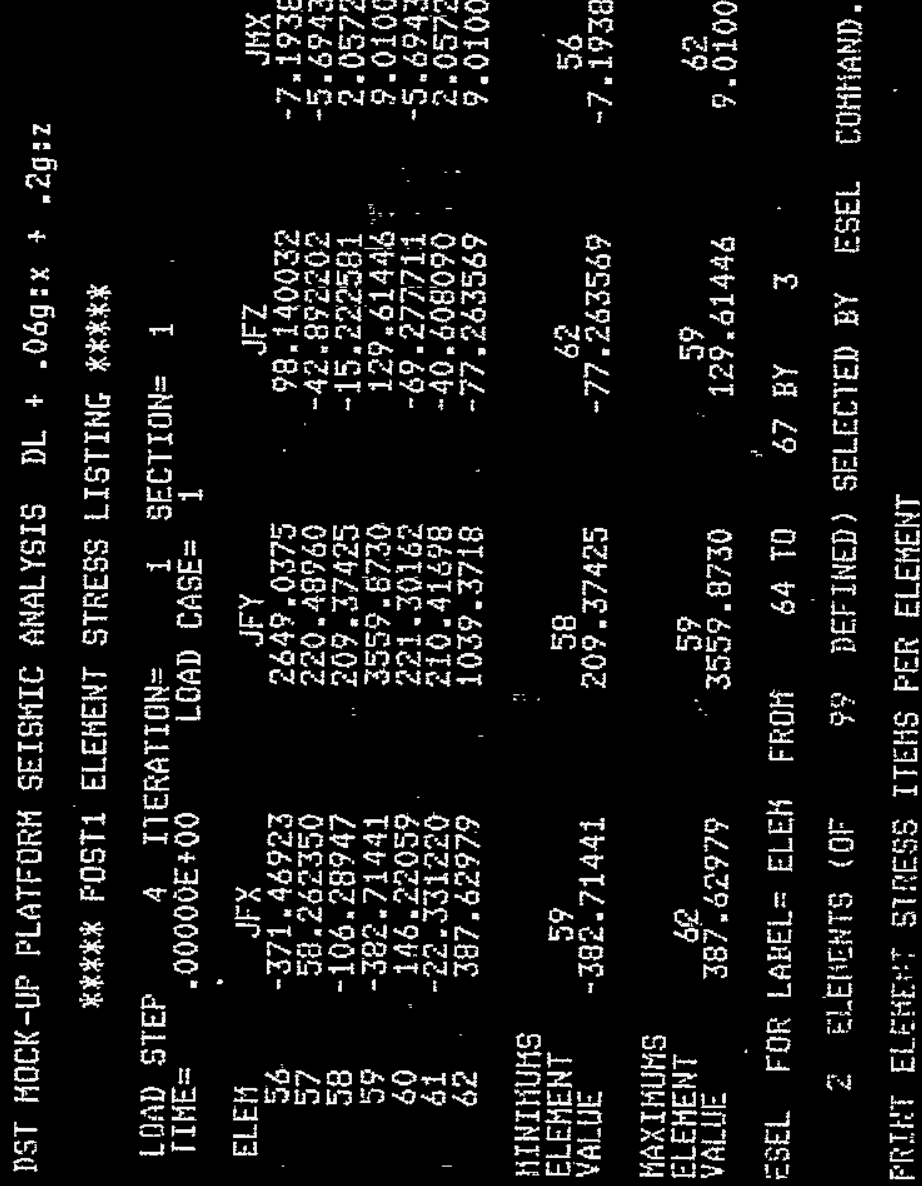

$\rightarrow$ m

का

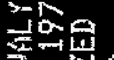

事

동동

的正

和

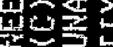

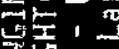

进必正

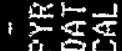

以늠

co

政点

约的

驼是 


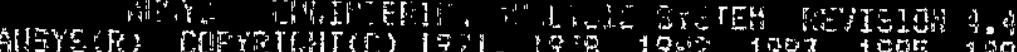

A137 32207

HAY 1,1990

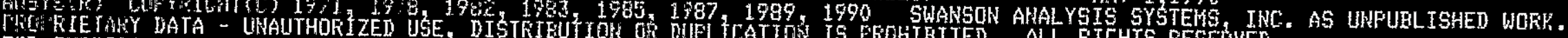
FOF SUPFOR CALL Larry Gamman FHOHE 509-376-2604 DUFLATL

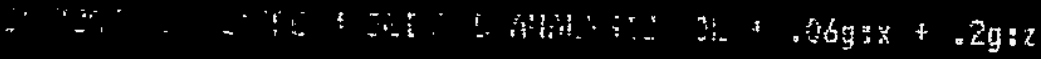

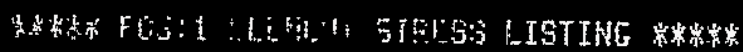

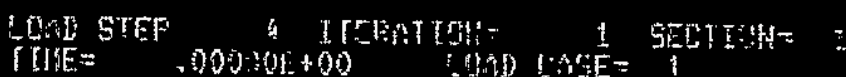

\begin{tabular}{|c|c|c|c|c|c|c|}
\hline $\begin{array}{l}\text { ELEH } \\
64 \\
67\end{array}$ & $\begin{array}{c}34 \\
57.149910\end{array}$ & 170.76671 & $\begin{array}{c}.5 \mathrm{FZ} \\
14.009931 \\
9.4420188\end{array}$ & 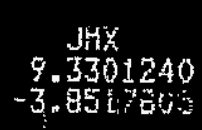 & $\begin{array}{l}114 \gamma \\
1147.0750 \\
839.758\end{array}$ & $\begin{array}{c}\text { Jhiz } \\
162.97132 \\
2.9751492\end{array}$ \\
\hline IIHULAS & & & & & & \\
\hline EENT & 57.149910 & 170.76671 & $\begin{array}{c}67 \\
7.4428188\end{array}$ & -3.8517805 & 838.76652 & 2.6751492 \\
\hline EMEN & & & & & & \\
\hline & 205.47665 & 178.28853 & 14.009831. & 9.04301 .240 & 1.1 .47 .0750 & " 162.97132 \\
\hline
\end{tabular}

ESEL FOR LABEL = ELEH FROM 69 TO $69 \mathrm{BY} 1$

1. ELEHENTS (DF 99 DEFINED \%. SELECTED BY ESEL COHHAND.

PRTHT ELEHENT STREG: ITEMS FER ELEHENT 
密

$\frac{11}{4}$

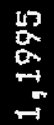

때는

$\rightarrow$

的密崖

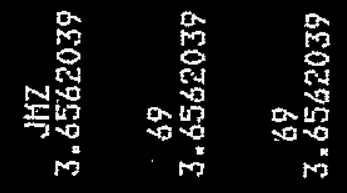

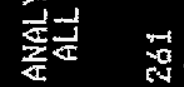

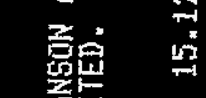

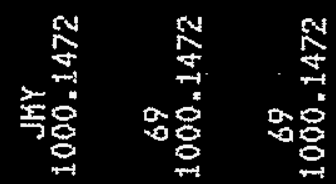

管

임

总的

Ig 글

드논

넌

解

플

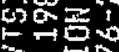

$\sum_{\rightarrow=0}$.

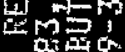

문웅

는

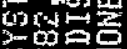

in⿴囗十

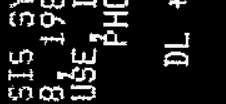

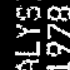

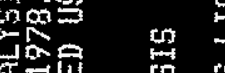

m po p

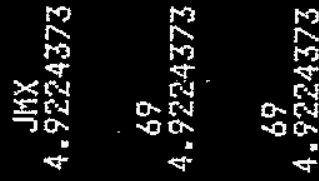<smiles>[B][B]</smiles>

$\lim _{\rightarrow \rightarrow 0}$

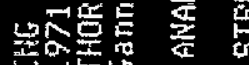

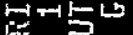

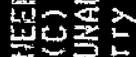

본동

政过

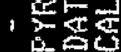

25

造语

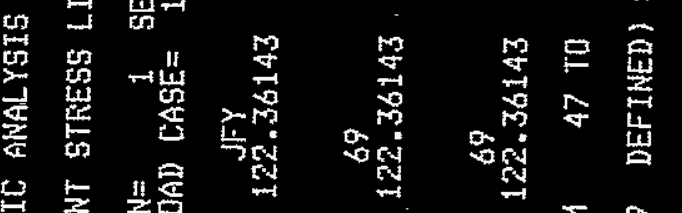

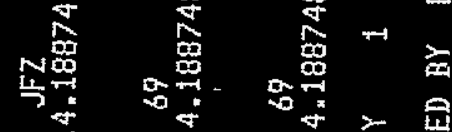

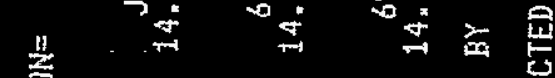

悹

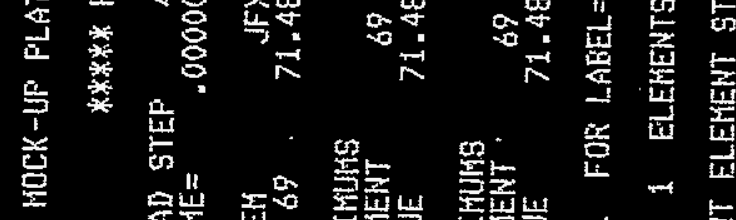

语 
AWSY - EHGINEEFTH AHALYSIS SYSTEH REVISIDH 4

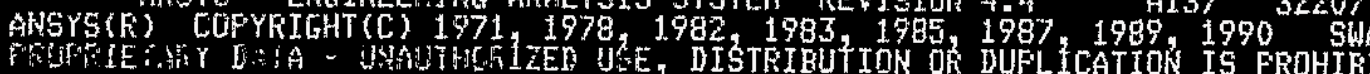

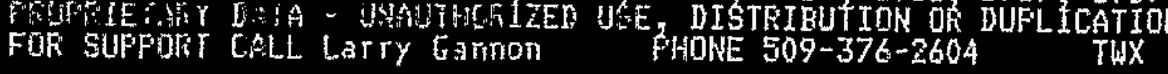

DST HOCK-UF FLATFORH SETSHIC ANALYSIS DL + .06g: $x+$,2g: $z$

HAY 1,1990

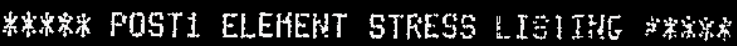

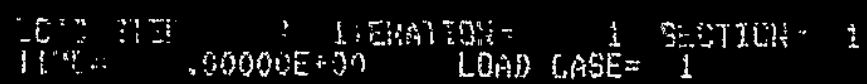

$\begin{array}{rcccccc}\text { ELEH } & \text { IFX } & \text { IFY } & \text { IF } & \text { IFY } & \text { IHY } & \text { IKR } \\ 47 & -389.60724 & 408.82099 & 154.09362 & 7.1933390 & -4551.7695 & 32.828089\end{array}$

MIHIHUIS

ELEKGENT

47
-389.

47.60724

47

HAXIMUITS

ELEMENT

VALUE

47
-389.60724

408.82099

154.09362

7.1938390

47
-4551.7695

32.828089

ESEL FOR LABEL = ELEM FROH,

$408.82099 \quad 154.09362$

7.1938390

$-477.7695$

47

32.829089

2 ELEHENTS (OF 99 DEFIHED) SELECTED BY ESEL COHHAND.

PRINT ELEHENT STRESS ITEHS PER ELEHENT 
AHEYS - EHGINEERING ANALYSIS SYSTEH FEVISTOH 4.4

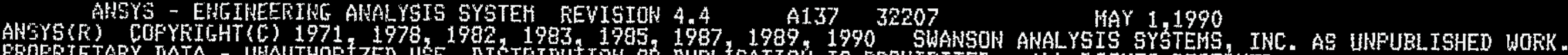

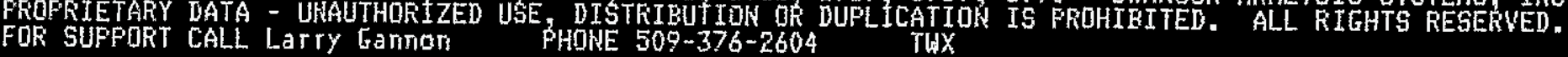

DST HOCK-UF FLATFOFH SEISHIC ANALYSIS DL +.06g: $+.2 g: z$

15.1261 AUE $1,1995 \mathrm{CP}=\quad 1.040$

****** POST1 ELEHENT STRESS LISTING *****

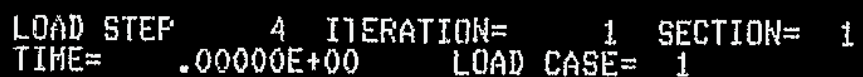

\begin{tabular}{|c|c|c|c|c|c|c|}
\hline $\begin{array}{l}\text { LEH } \\
63 \\
65\end{array}$ & $\begin{array}{c}\text { IFX } \\
-134.51840 \\
15.486442\end{array}$ & $\begin{array}{c}\text { IFY } \\
177.50269 \\
232.11174\end{array}$ & $\begin{array}{c}\text { IFZ } \\
-35.395340 \\
-19.618559\end{array}$ & $\begin{array}{c}\text { IfX } \\
-7.3301240 \\
-23.939212\end{array}$ & $\begin{array}{c}\text { IHY } \\
1669.0197 \\
1089.4235\end{array}$ & $\begin{array}{c}\text { IHZ } \\
-207.76424 \\
10.220135\end{array}$ \\
\hline
\end{tabular}

HIKIHLISS

ELEHENT

$-134.51840$

177.50269

$-35.395340$

$-23.939212$

1089.4235

$-207.76424$

HAXTHIHS

ELEHEHT

15.486442

232.11174

$-18.618559$

$-9.63301240$

1669.0197

65
10.220135

EOEL FOR LAFEL= ELEM FROM 68 TO $68 \mathrm{BY}$

1. ELEHENTS (OF 99 DEFTHED) GELFCTED BY ESEL COHHAHD,

FRIHT ELEHENT STEESS ITEHS PER ELEHENT 
논

8

政

密

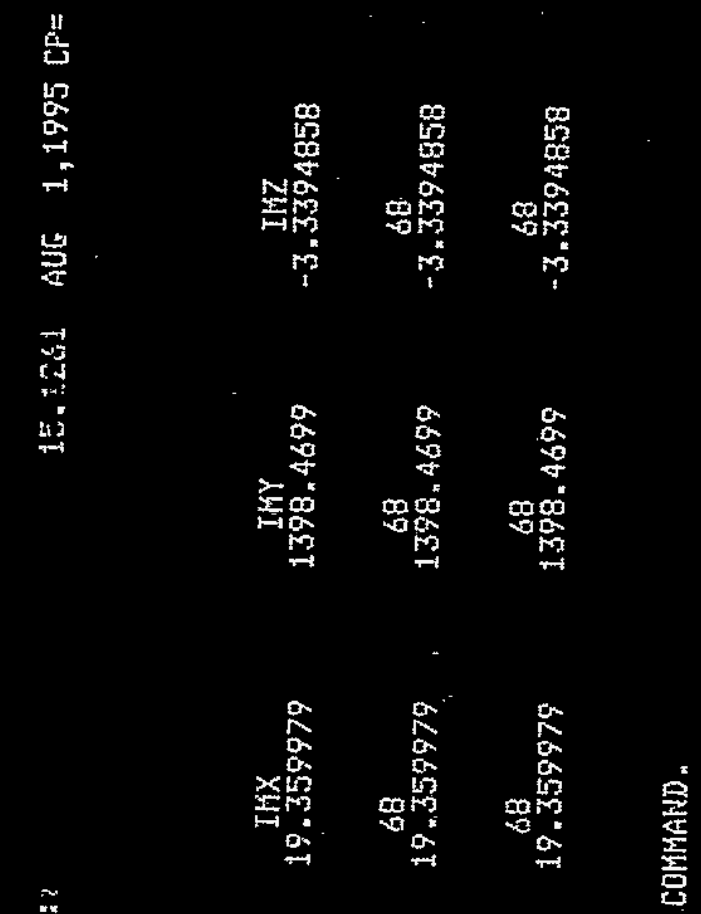

Cosis

포난

$\frac{\frac{1}{d}}{\frac{1}{4}}$

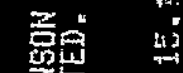

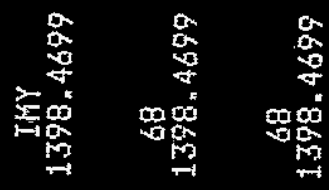

골

min

我

叫要焉

넌

Nit

$\rightarrow x^{x}$

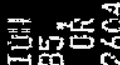

tas

然

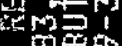

$\cos _{n \rightarrow 4}$

int 5

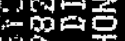

$\operatorname{lot}_{x \rightarrow 1}$

$30 \frac{11]}{3013}$

in

象点

$\Rightarrow$ 管

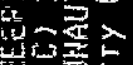

式年

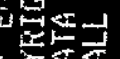

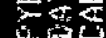

$\ln _{0 \rightarrow 1}$

4 积

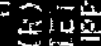

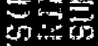

业的

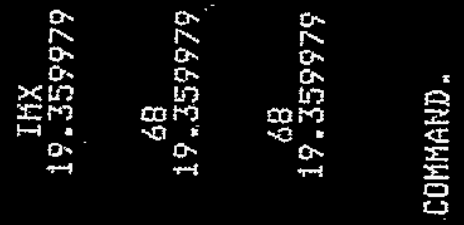

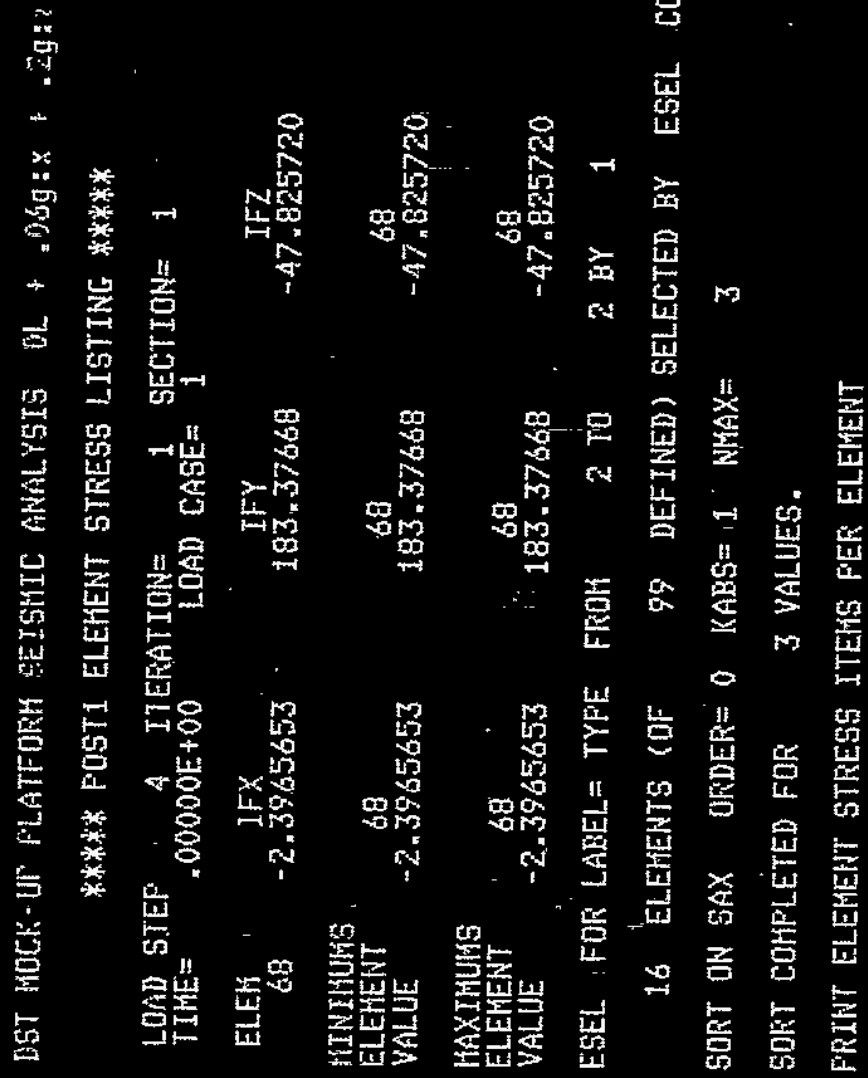

$$
+
$$


AHSTS - EHCINEERING AHAL STS SISTEH REVISIOH 4.4

A137 32207 HAY 1,1990

ANSYS(F) COFYKIGHT(E) $1971,1978,1982,1983,1985,1987,1989,1990$ SWANSON ANALYSIS SYSTEHS, INE. AS UNFUELISHED WDFK.

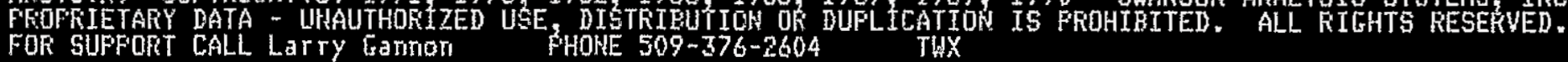

DST HOCK-UP FLATFORM SEISHIC AHALYSIS DL $+.06 \mathrm{~g}: \mathrm{x}+.2 \mathrm{~g}: \mathrm{z}$

15.1261 AUG $1,1995 \mathrm{CF}=$

1.050

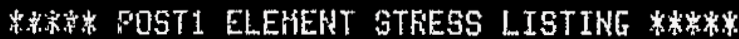

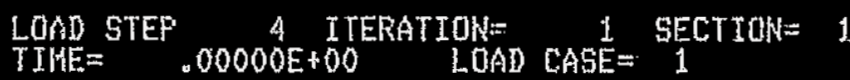

ELEHY $\quad$ SAX

$30 \quad-1250.6438$

$32-1056 \cdot 1,338$

HINIHUHS

ELEHENT

VALUE

30

$-1250.6430$

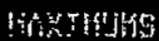

ELEIET

$-105 \frac{32}{54} \cdot 1238$

ESEL FOR LADEL $=$ TYFE FROH $: 3$ TO 3 BY 1

12 ELEHENTS (OF 99 DEFINED) SELECTED BY ESEL COHKAND.

PRINT REACTION FORCES PER WODE 
AHSYS - EHGINEERTHE WHAL YSIS SPSTEH REVISTDI 4.4

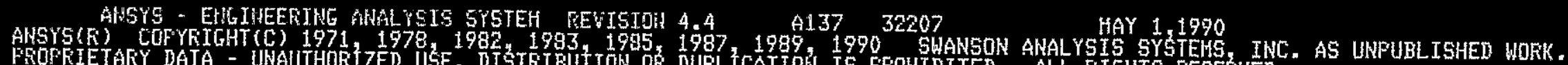

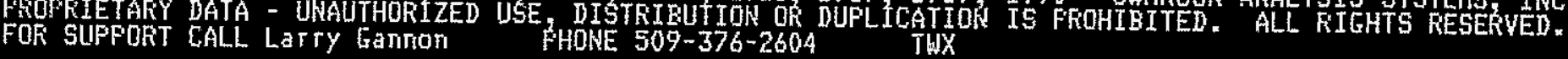

DST HOCK-UF' FLATFORH SEISHIC ANALYSIS DL +.06g: $\mathrm{x}+.2 \mathrm{~g}: \mathrm{z}$

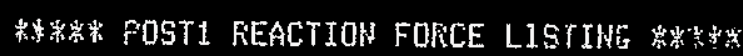

$$
15.1261 \text { ALG } 1,1995 \mathrm{CP}=\quad 1.050
$$

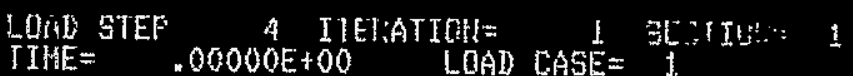

THE FOLLOWING $X, Y, Z$ FORCES ARE IH GLOAAL COOREINATES

$$
\begin{array}{cc}
\text { HODE } & F \times \\
1 & -16.980901 \\
2 & -16.984913 \\
3 & -218.68692 \\
4 & -189.992 .27 \\
5 & -243552601 \\
6 & -179.66146
\end{array}
$$

TITAL -584.89248

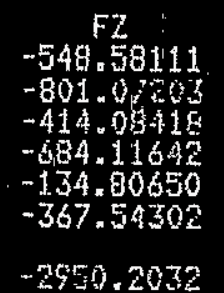

3

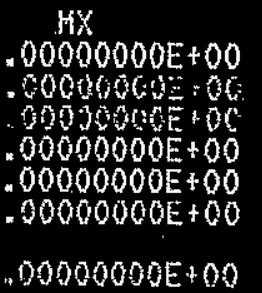

14754.015
BY

$.00000000 E+00$ 00000000E +00 00000000 +0 Do000000 +00 (00000000 + OO -0000000 to $.00000000 E+00$

$.00000000 \mathrm{E}+00$
$M Z$

$.00000000 E+00$ $.000000005+00$ $-00000000 \mathrm{E}+00$ . 0000000 $\because 0000000$ : 100 $.00000000 \mathrm{E}+00$ $.00000000 E+00$

$.00000000 E+00$

SORT COHPLETED FOR 3 VALUES.

FRINT ELEHEHT STRESS ITEHS PER ELEHENT 


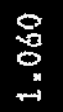

装

管

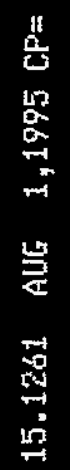

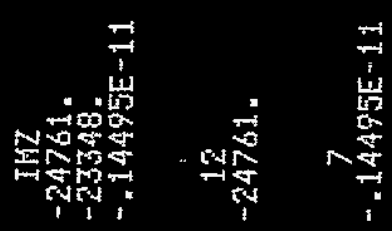

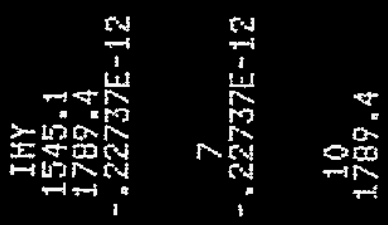

$\rightarrow$

물

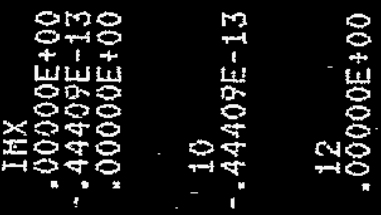

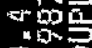

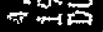

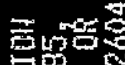

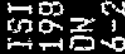

Un

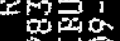

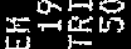

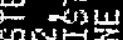

tots

$\cos ^{2} \rightarrow \sin ^{2}$

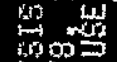

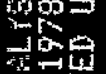

站证

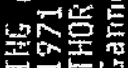

然

뻥존

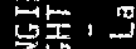

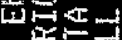

, 딴달

ons

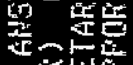

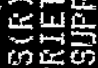

울울

8

+
$\times$
0
0

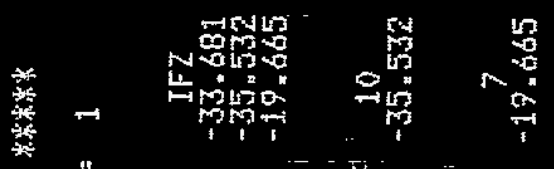

+ 恶焉 m

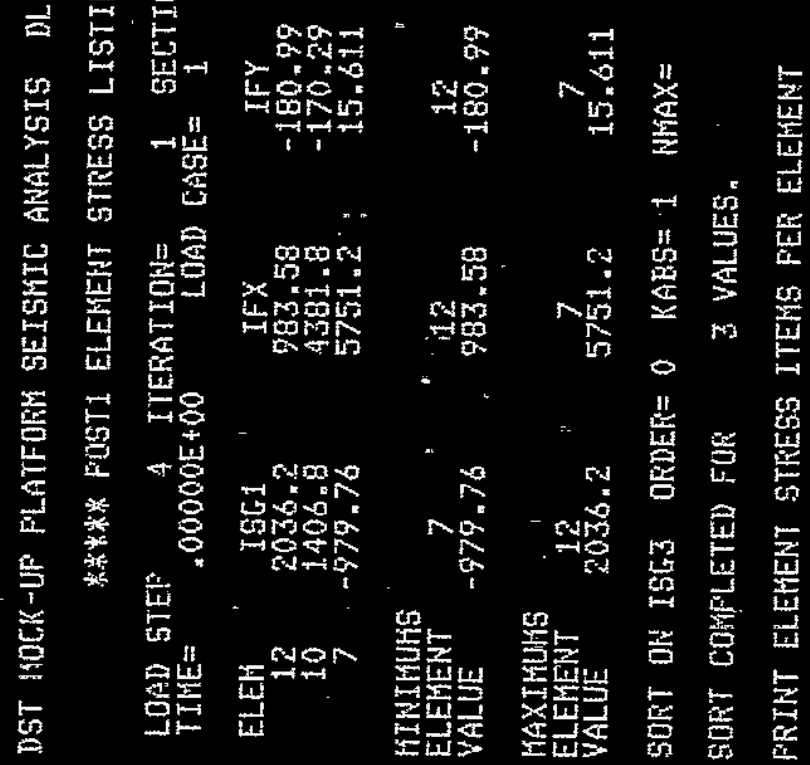




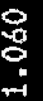

留

密雷

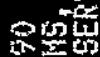

on

who

ine

这路

竞要

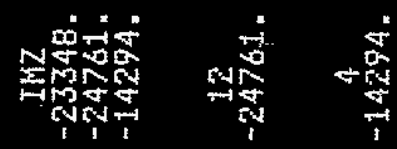

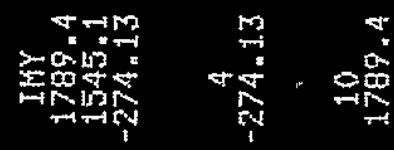

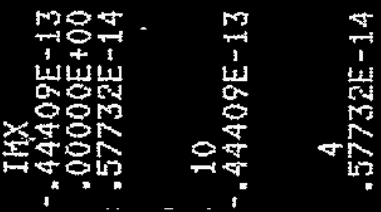

此

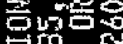

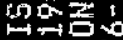

要,

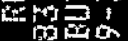

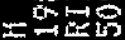

minu

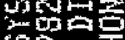

on ${ }_{4 \pi}$

思的

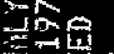

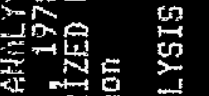

폴동

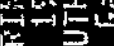

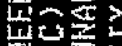

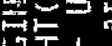

罡'

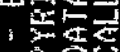

竞要

is

은

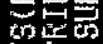

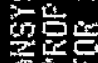

范

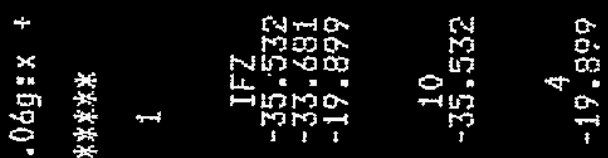

$+$

농

点

70

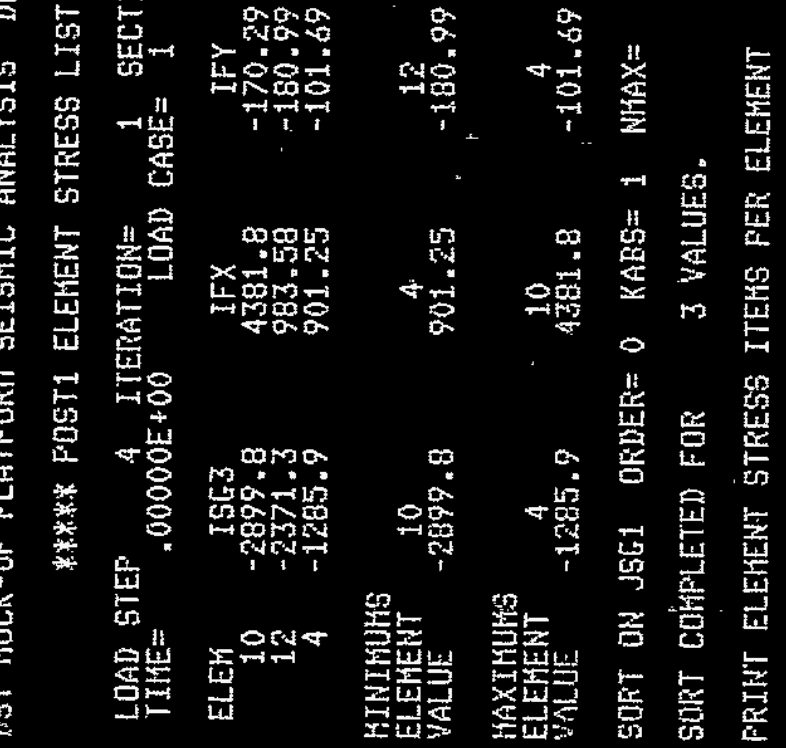

$m$ 
窝点

近志

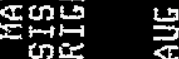

素直

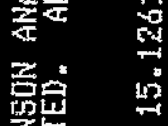

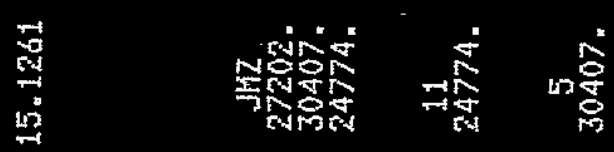

s.m

공

o

gon

$r_{i=1}$

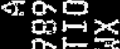

武些

st

$7 \infty$

폽

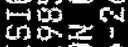

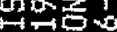

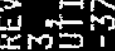

哭雅

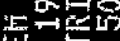

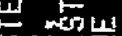

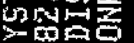

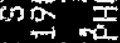

th

$\operatorname{trn} x=$

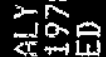

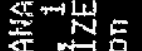

$x \rightarrow \frac{x}{6}$

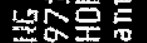

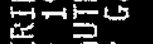

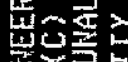

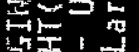

눈돈,

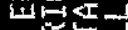

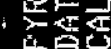

is

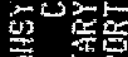

标要

敞是昆

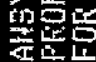

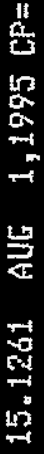

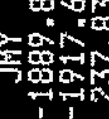

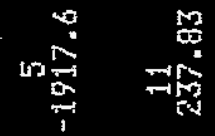

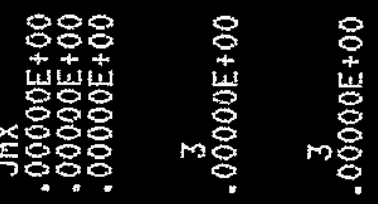

品

要

$\frac{1}{3}$

$\frac{m}{4}$

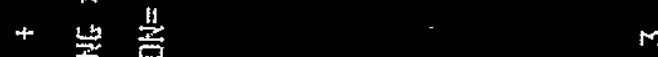

b

焉

崖皆

量

5

总还

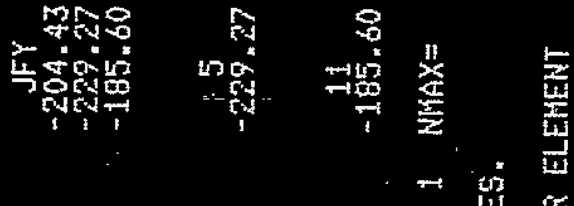

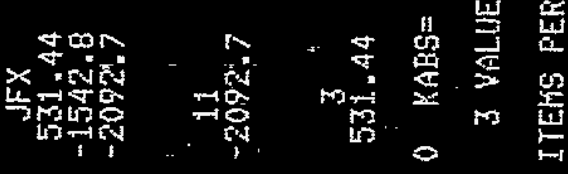

$=\stackrel{5}{ \pm}$

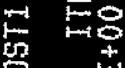

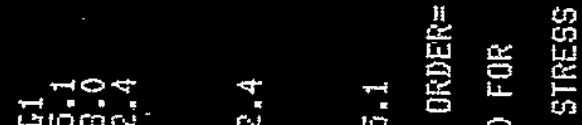

it

䟓

兽

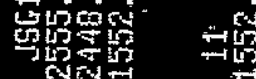

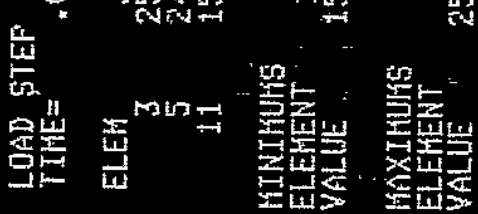

盁

学 竞

点 
8

望

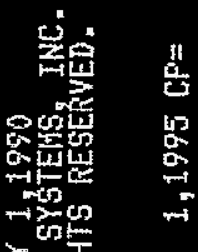

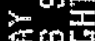

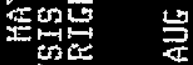

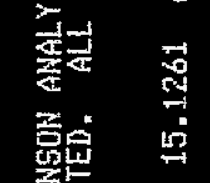

Nong a

sis

令 草

욤

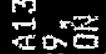

鄂稀

요

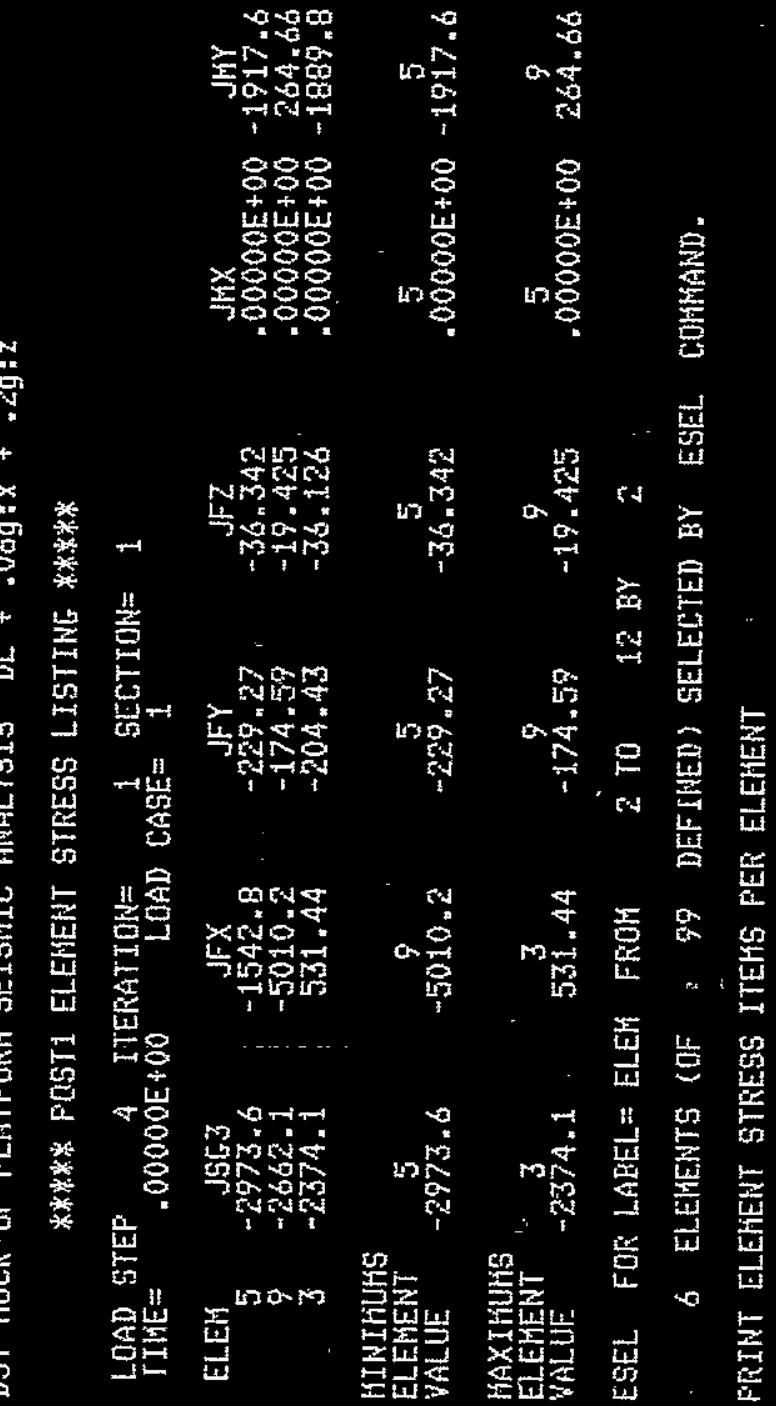

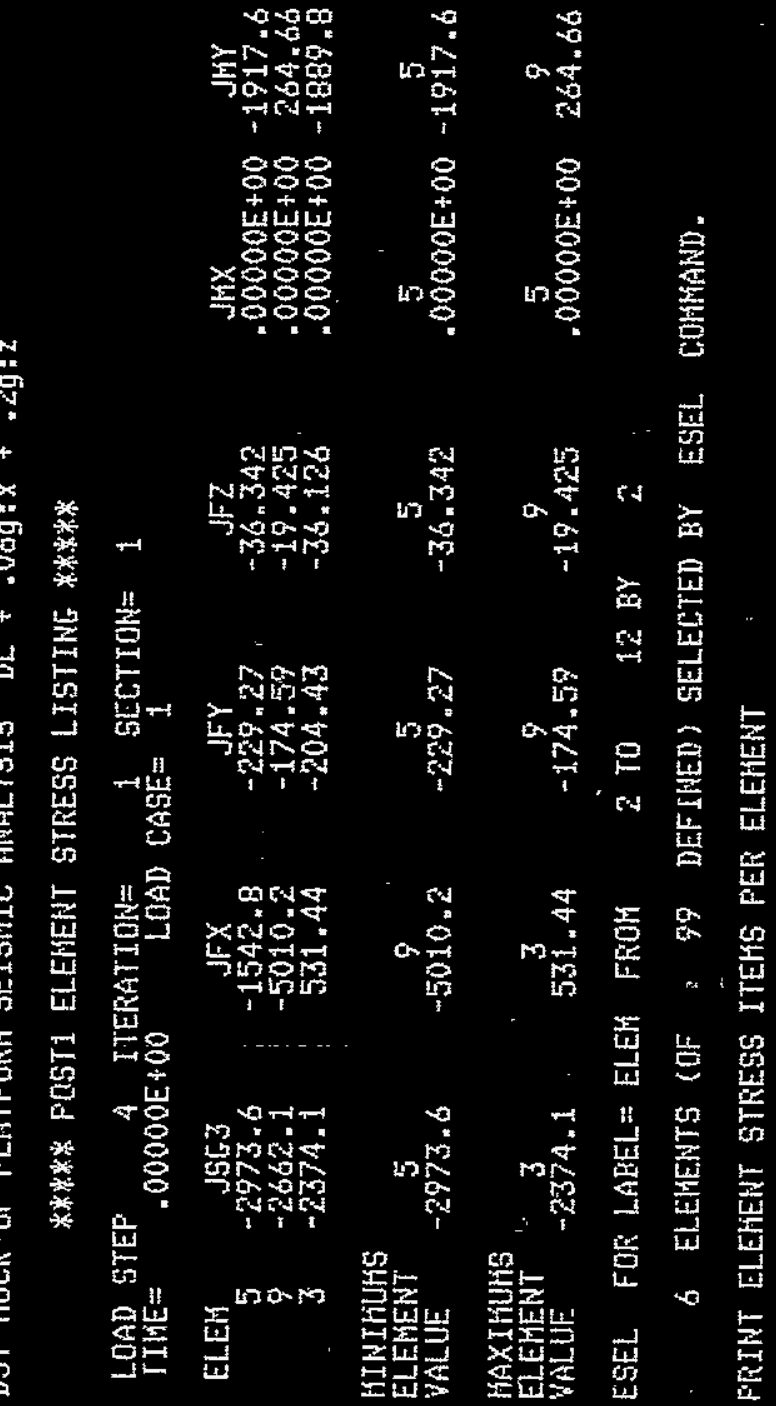

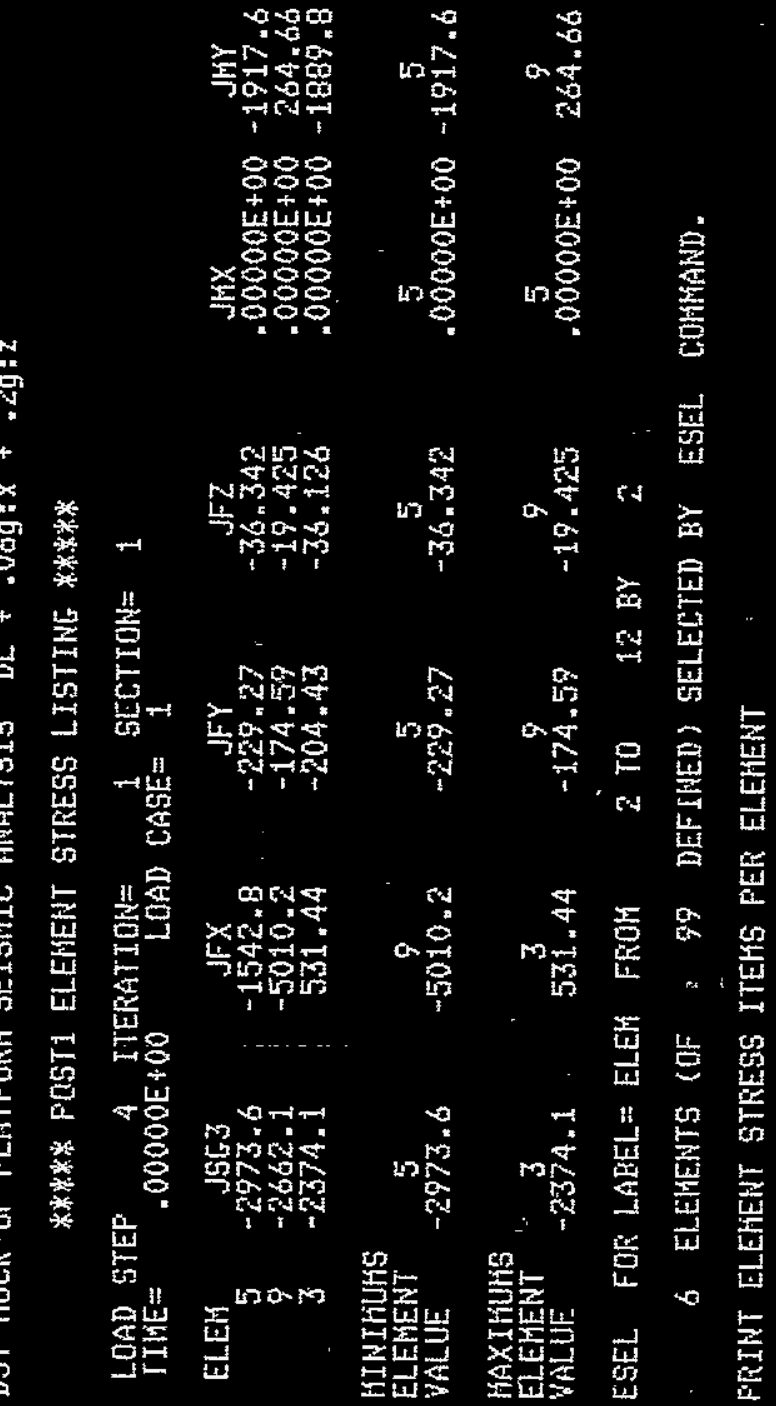

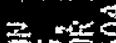

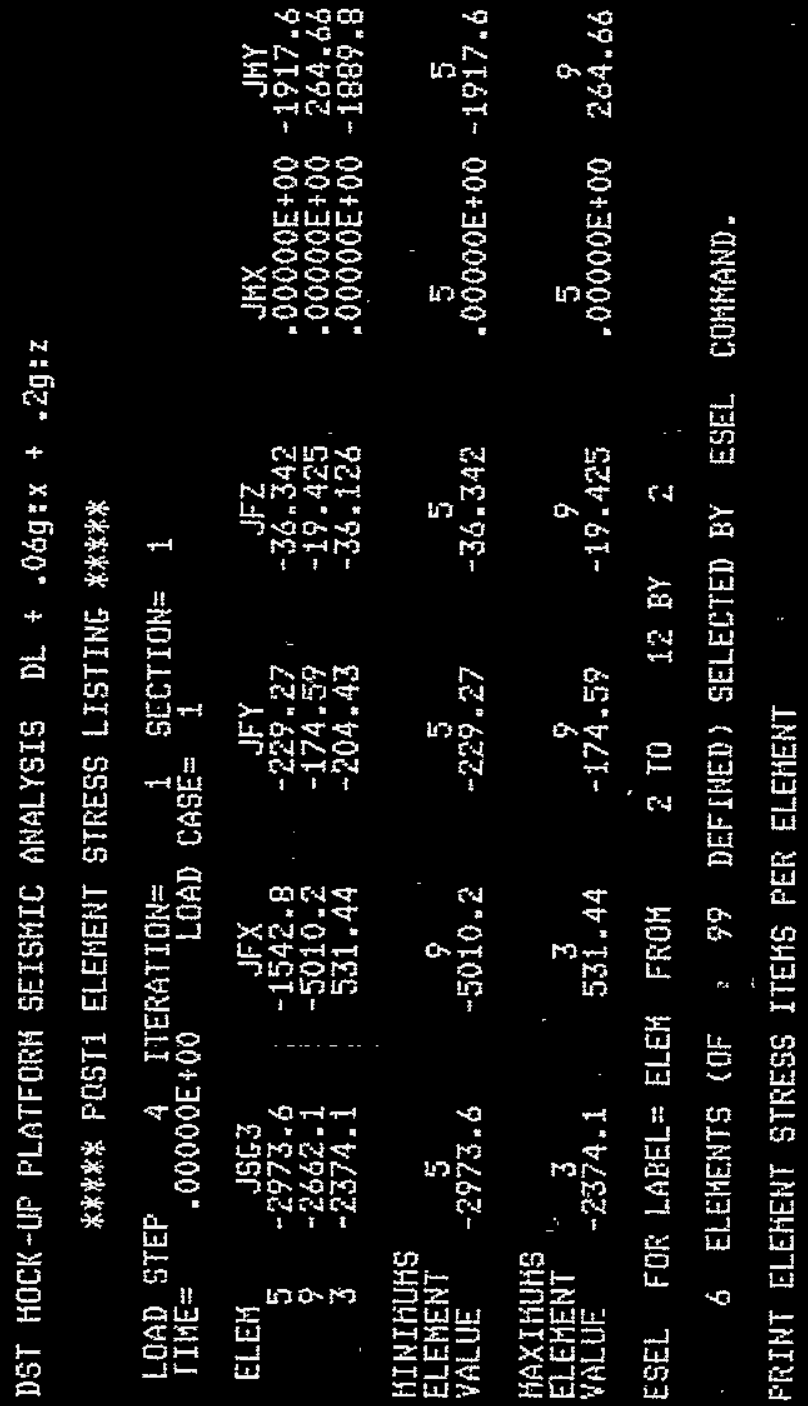

荧们?

atro

䒴一地的

जncing

$o^{n+1} \mathrm{u}^{2}$

thong

ing

蛋 $N$

서을

주을

采可

㟧的事

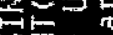

돈곤

证过

, 实密

the

然

工然

노의

空

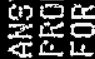


용

尔

运里

는

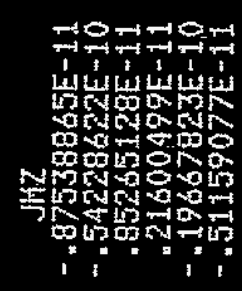

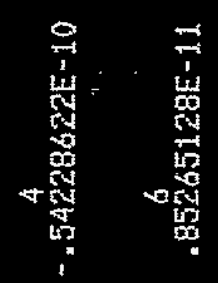

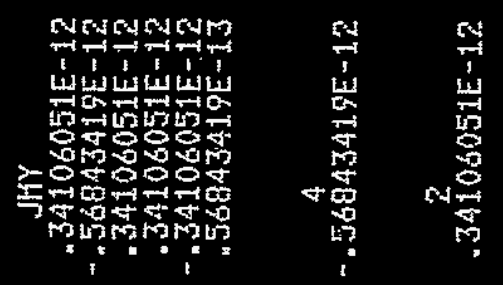

$\rho^{2}$

$\underset{2}{2}$

an

여의

$\rightarrow-\frac{5}{5}$

$\rightarrow \infty$

ais

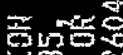

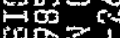

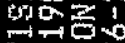

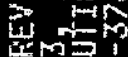

tasas

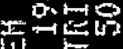

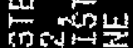

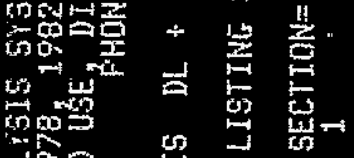

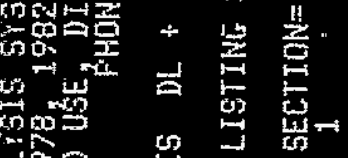

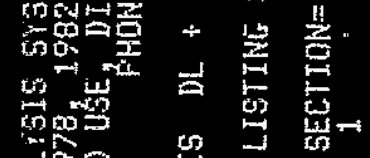

$1 \%$

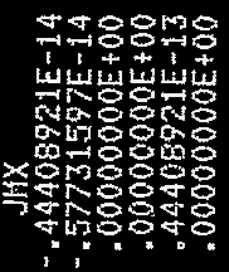



题

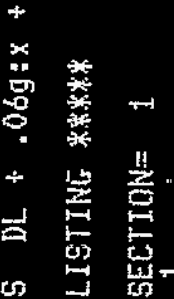

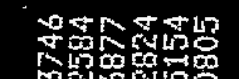

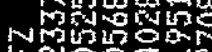

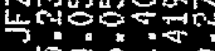

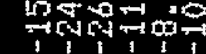

类然

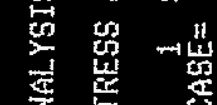

wosn

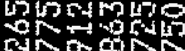

in

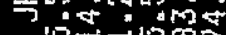

숭

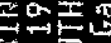

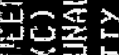

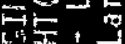

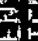

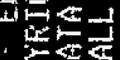

焉我

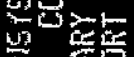

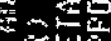

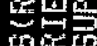

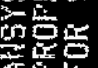

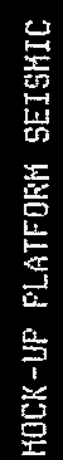

5

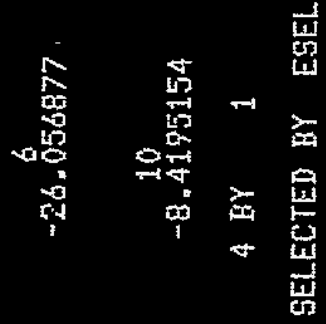

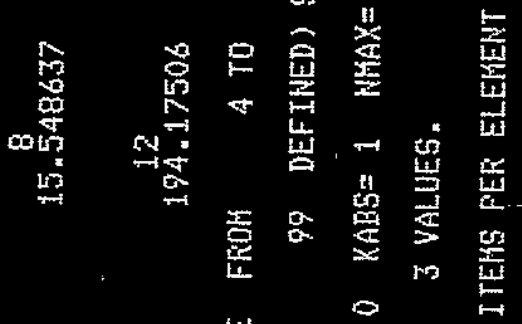

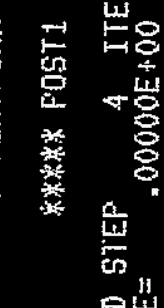

它

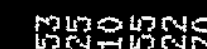

Mots

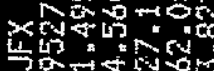

-

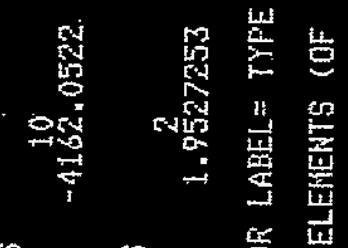

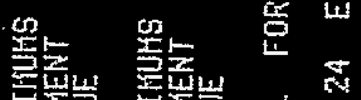

iI

5

$x^{5+400000}$

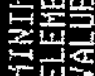
的部 称喜

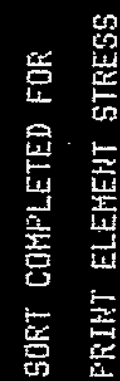

$$
A-142
$$


GHSYS - ENGIHEERIKG AHALYSIS STSTEH REVISIOH 4.4

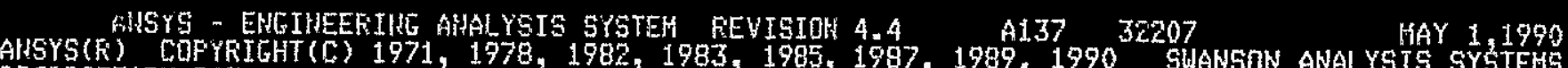

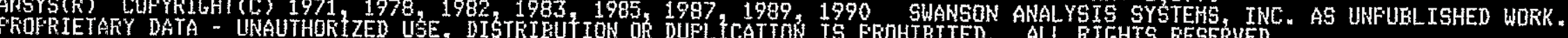
FOR SUFPORT CALL LarTy GanTON

DST HDCK-UP PLATFORH SEISHIC ANALYSIS DL $+.06 g: x+.2 \mathrm{~g} z$

****** POST1 ELEHENT STFESS LISTING *⿻一未:***

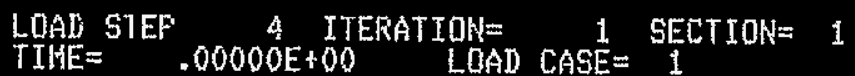
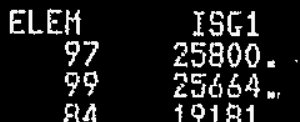

84 2.1964.

$\begin{array}{lll}\text { IFX } & \text { IFY } & \text { IFZ } \\ -95.916 & -1522.5 & 316.05 \\ 280.78 & -1.521 .5 & 286.95 \\ -470.60 & 1055.3 & 1.1823\end{array}$

IHX

79
10.353

$-24.801$

$\begin{array}{lr}\text { IMY } & \text { IHZ } \\ -6572.5 & -47702 . \\ -6590.3 & -47703 . \\ -127.99 & 39881 .\end{array}$

HINIHUHS

ELEHENT

84

84

$97 \quad 84$

1. 1.1823

84

HAXIMUHAS

ELENENT

25800 .

99
280.78

84
1055.3

316.05

$-24,801$

99
-6590.3

$-47703$.

GORT ON ISGZ DRDER $=0$ KABS= 1 NHAX=

DERT COHFLETED FOF 3 YALUES.

PRINT ELEHENT GTRESS ITEHS PEF ELEHENT 
AHSYS - EHGINEERTHE AHALYSIS SYSTEH PEEVISIOH 4.4

A137 32207

WAY 1,1990

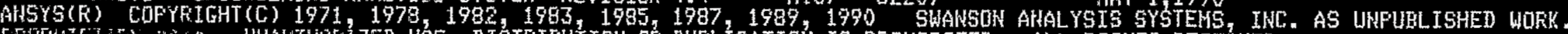

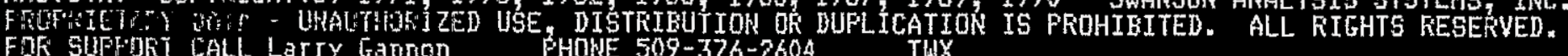

DST HBCK-UF FLATFORH SEISHIC ANALYSIS DL +.06g: $+.2 \mathrm{~g}: \mathrm{Z}$

15.126 ALG $1,1995 \mathrm{CP}=\quad 1.090$

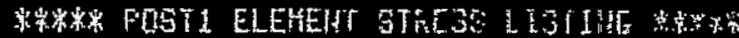

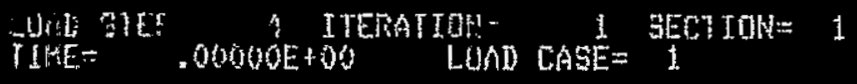

$\begin{array}{rr}\text { ELEH } & \text { TSL3 } \\ 99 & -25830 \\ 77 & -25726 \\ 84 & -18002\end{array}$
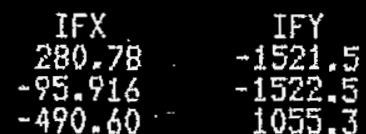

IF:

316.05

$-1524.5$

1.1823

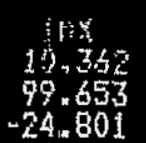

$15 Y$
-6590.3
-652

Itiz

$-47702$

$-127.99 \quad 39981$

tituIFiuite

ELEMENT

99
-25880

84
-490.60

97
-152.5

84
1.1923

84
-24.801

$-599$

99
-47703

\section{HAXIHUIHS}

ELEYENT

84
-18802

280.78

$\begin{array}{cc}84 & 97 \\ 1055.3 & 316.05\end{array}$

97.653

$-184$

394

SORT OH JSG1 'ORDEF= 0 KABS= 1 NWAX $=3$

SORT COHFLLETED FOR 3 VALLUES.

FRINT ELEKENT STRESS ITEHS PER ELENENT, 
AHSYS - ENGTHEEFYHG AHAL YSTS SYSTEH REVISIIH 4.4 1137 32207 MAY 1.1990

ALSYS (F) COFYKIGHT(C) $1971,1978,1982,1793,1995,1987,1989,1990$ SHANSON ANALYSIS SYSTEKS, INC. AS UNFUEL.ISHED WDKK.

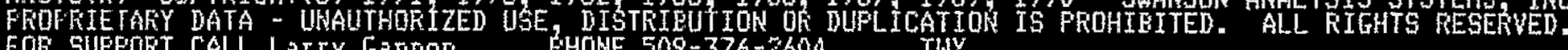

FOR SUFFORT CALL LATTY Gañon PHONE 509-376-2604 TWX

15.1261 ALG $1,1995 \mathrm{CP}=$

1.090

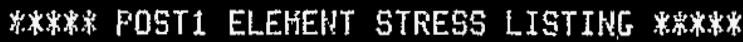

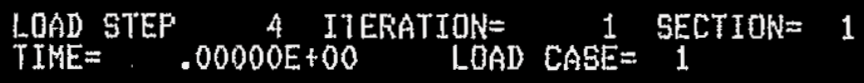

$\begin{array}{ll}\text { ELEH } & \text { JS61 } \\ 96 & 25870 \\ 98 & 25733 \\ 92 & 23653\end{array}$

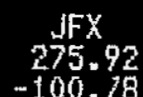

$-1477$

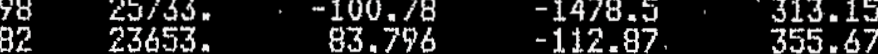

$\begin{array}{ccc}\text { NHX } & \text { MYY } & \text { JHZ } \\ -97.653 & 6572.5 & 47702 . \\ -10.362 & 6590.3 & 47703 . \\ .00000 \mathrm{E}+00 & 37784 . & 11977 .\end{array}$

HIHIMUTS5

ELEHERT

2365

$.00000 \mathrm{E}+00 \quad 37784$

11977.

\section{HAXIHUISG}

ELMEN

VALUE:

96

98
-100.78

98
-1479.5

96
283.95

$-99.653$

$\frac{96}{4572.5}$

11977.

SOIT ON JSG

96

92
-112.87

$3 \frac{82}{355} .67$

$.0200005+0013789$

47703.

SORT COHPLETED FOR " 3 VALUES.

PRTHT ELENENT STRESS ITENSS FER ELEHEHT 
$\stackrel{8}{\circ}$

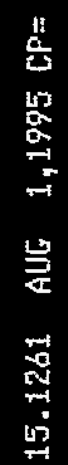

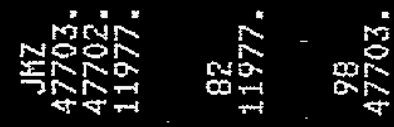

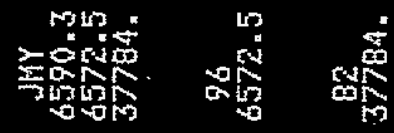

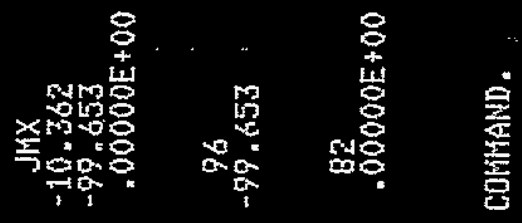

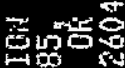

$N$ i i

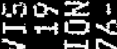

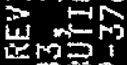

a

涪 $\rightarrow$

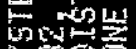

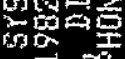

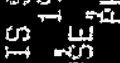

ing

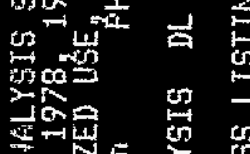

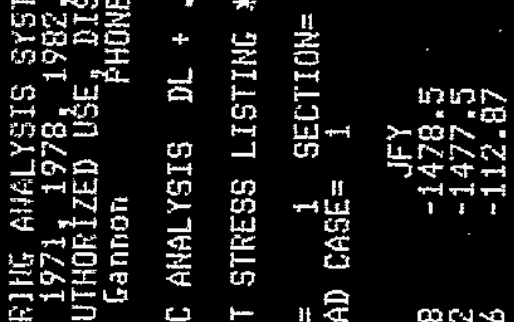

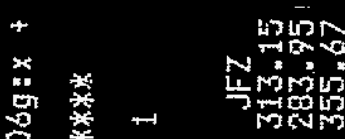

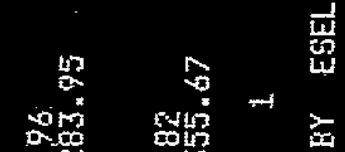

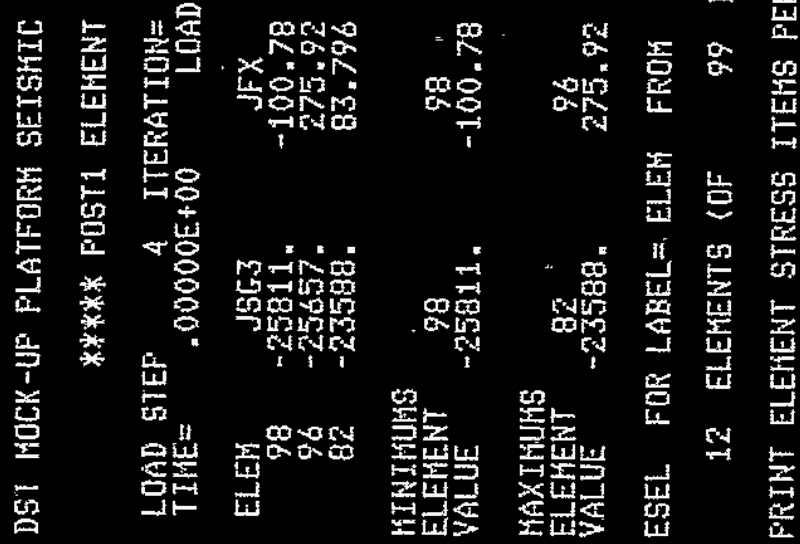

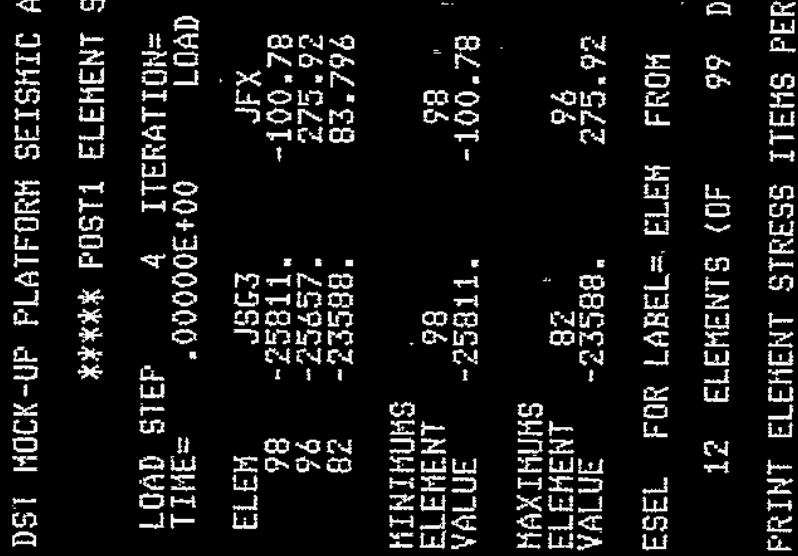
交

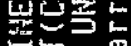

品, 包

岦

, 똔동

gis

牙察

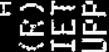

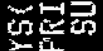

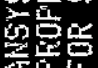


AHS TS - EHTIHEERIHG ANALYEIS GYSTEH REVISIOH +4

A137 32207

MAY 1,1990

ANSYS(F) COFYFIGHT(E) $1971,1978,1982,1983,1985,1987,1989,1990$ SHANSON ANALYSIS SYSTEHS, INC. AS UNFUELISHED WOKK.

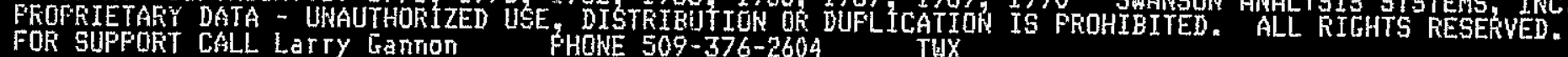

DST HOCK-UF FLATFOEH SEISHIC* AHALYSIS DL $+.06 \mathrm{~g}: \mathrm{x}+.2 \mathrm{~g}: 2$ TUX

****** POST1 ELEHENT STRESS LISTING $* * * * *$

15.1261 AUG $1,1995 \mathrm{CP}=$

1.100

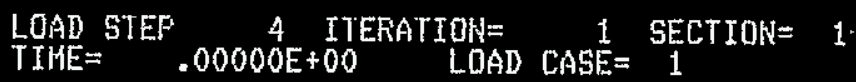

ELEY IFX
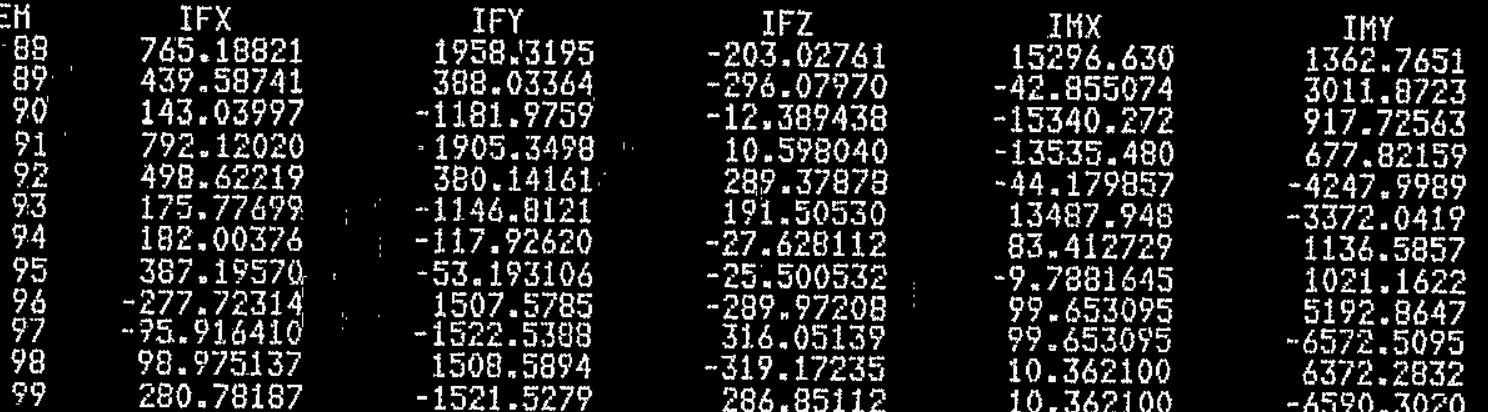

IIIZ

32063.666

$-14624.792$

$-24085.718$

31342.817

$-14273.675$

$-1457.678$

$-23558.075$

$-22.8 .2272$

$-6793.4375$

13491.300

$-47702.013$

13532.128

$-47702.634$

MINIHUMS

ELEMENT

96
-277.72314

97

98

VALUE

$-1522.5388$

90

$-319.17235$

$-15340.272$

99

$-6590.3020$

$-47702.634$

97

ELEHENT

91

1958.3195

VALUEE

792.12020

316.05139

15296.630

98

6372.2832

98

320633666 


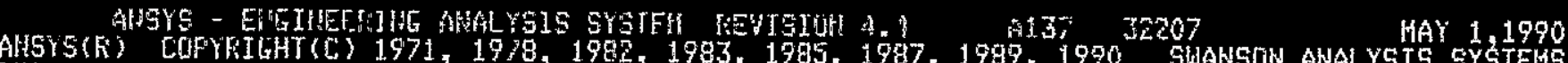

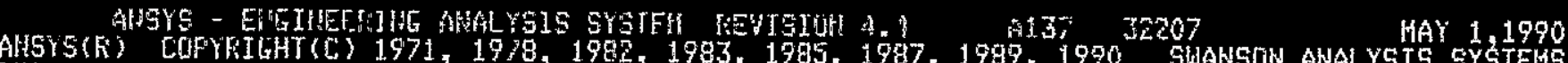

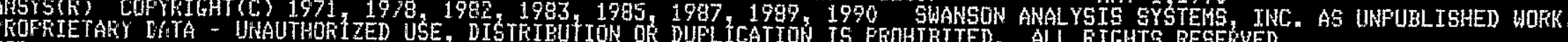
FOR SUFFORT CALL L LTTY GaNTON

DST HOCK-UP FLATFORH SEISHIC ANALYSIS DL +.06g: $+.2 \mathrm{~g}: \mathrm{z}$

***** FOST1 ELEHENT STRESS LISTING * * * * * *

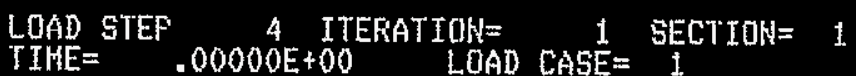

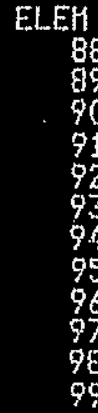

$$
\begin{gathered}
J F X \\
-761.66227 \\
-435.91456 \\
-139.51404 \\
-788.59427 \\
-494.94934 \\
-172.95105 \\
-178.39030 \\
-390.80916 \\
275.91641 \\
-74.109681 \\
-100.78167 \\
-282.58060
\end{gathered}
$$

$$
\begin{gathered}
\text { JFY } \\
-1940.6898 \\
-369.66940 \\
1199.6055 \\
-1887.7201 \\
-361.77737 \\
1164.4417 \\
57.691492 \\
113.42761 \\
-1477.4612 \\
1552.6562 \\
-1478.4721 \\
1551.6453
\end{gathered}
$$
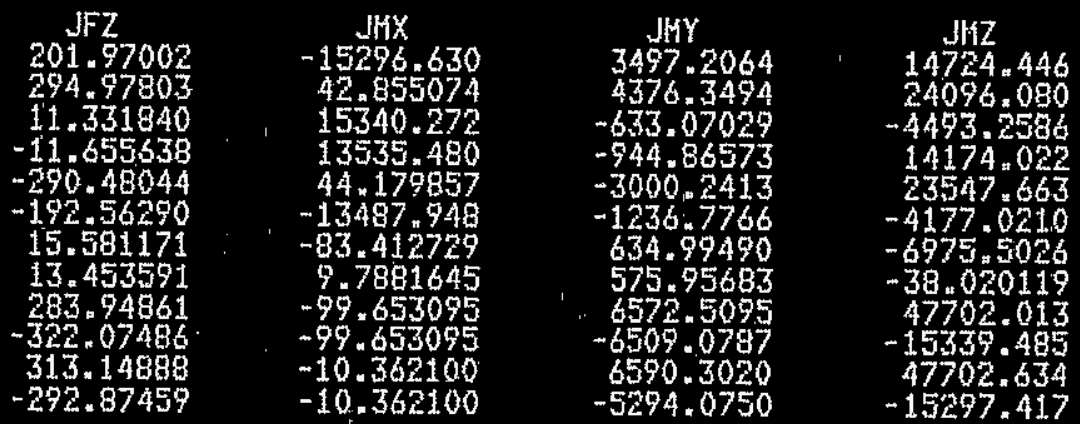

ELFHENT

$$
\begin{array}{cc}
71 & 88 \\
-788 \times 59427 & -1940.6898
\end{array}
$$

$$
97
$$

$-322.07486$

$-15276.630$

97
-6509.0787

97
-15730

MAXIMUHSS

ELEEKER

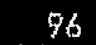

275.91641

552.6562

98

313.14888

90

15340.272

98

6590.3020

47702.634

ESEL FOR LABEL= ELEH FFöl " 7o to 82 BY 2
4 ELEHENTS (OF
99 DEFINED ) SELECTED RY
ESEL COHKAND.

FRTHT ELEHENT STRESS ITEHS PER ELEHENT 


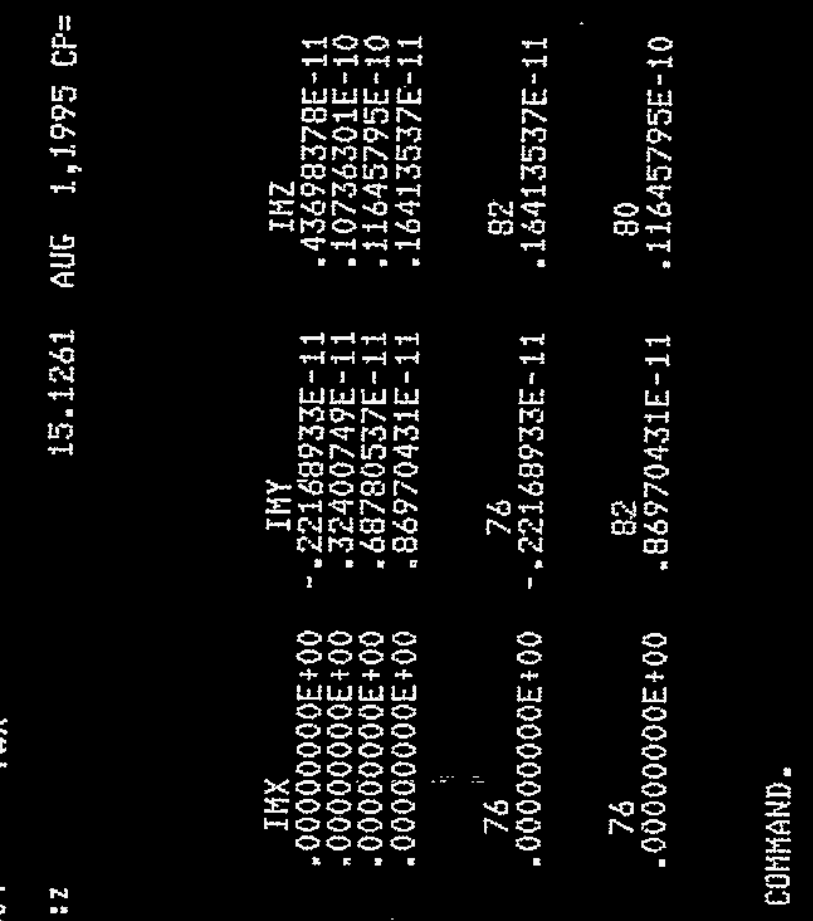

돌붕용

ํํำ

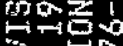

至

g象a

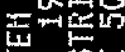

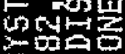
to

का

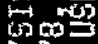

두요

N

gapra
$* \quad * \quad$ prag

量

* Nơs

जित्व

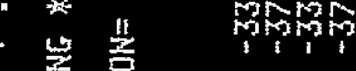

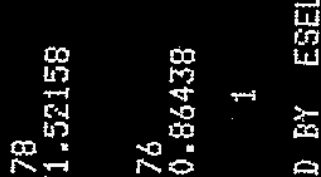

B

禺承

的案

봉도웅

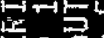

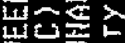

尌,

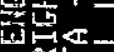

, 따욜

홍

点莺

농은

要

대눌룰

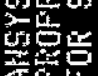

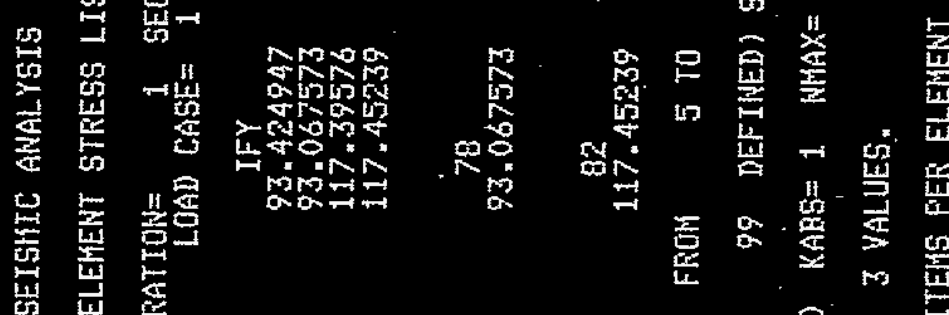

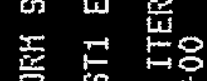

焉

结蓄

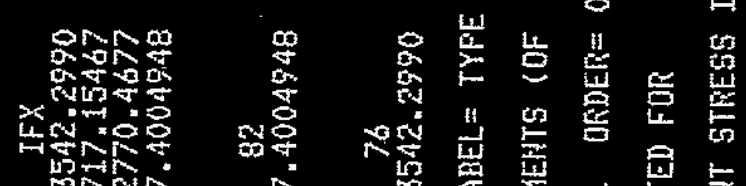

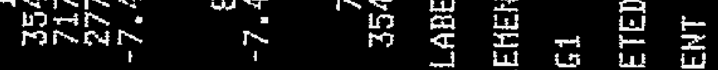

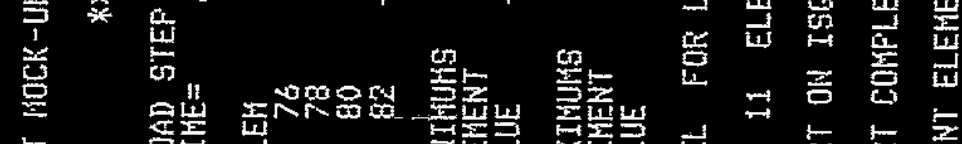

놇 옳

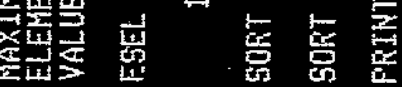

$$
A-149
$$


数

通高

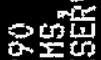

a

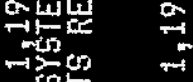

些

혼ㄷㄴㄴ

룽

空击

空

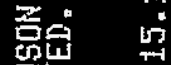

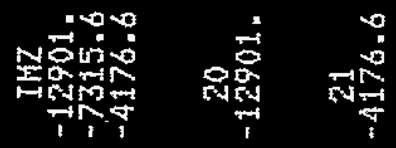

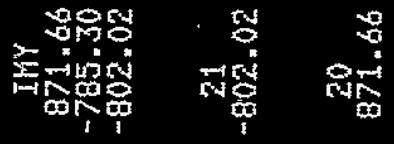

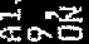

모으늘

술

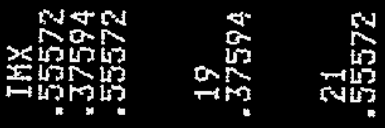

$+\cos$

뚠

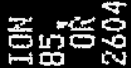

몬

$\rightarrow \rightarrow n$

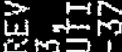

ara

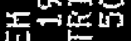

+
$x$
0
0

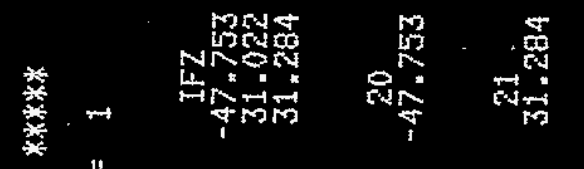

iscin

mat

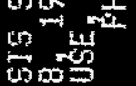

저눈

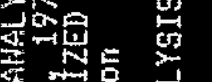

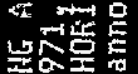

年心

+ 宸至

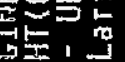

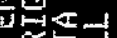

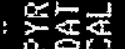

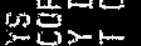

5 tris

동

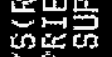

总语点

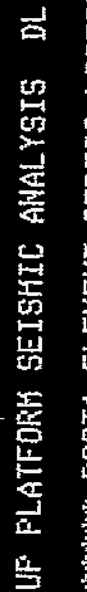

莺

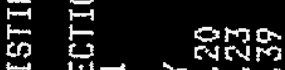

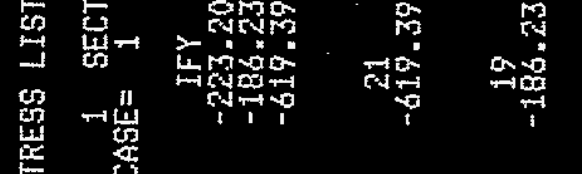

on

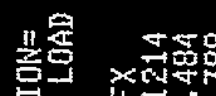

点

$\sum_{i=1}^{\infty}$

管

w

pis

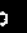

다용

ष

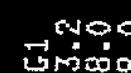

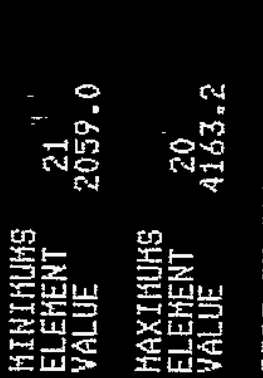

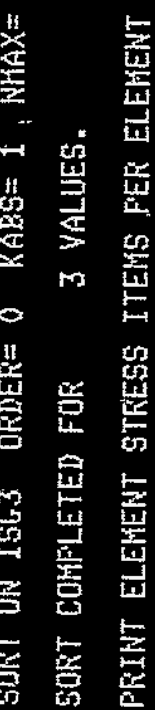


䠌

案

焉至

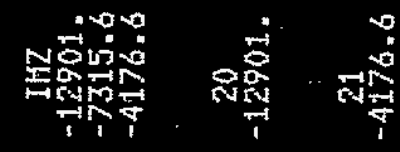

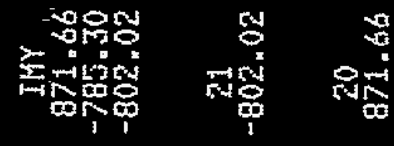

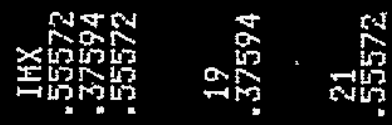

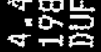

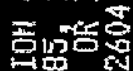

몬

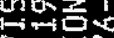

i

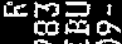

대눙

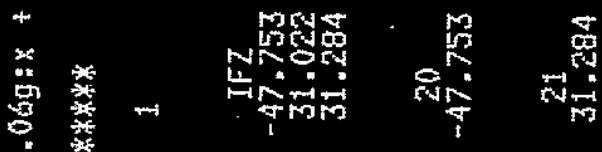

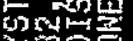

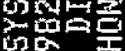

की

की

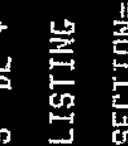

놀

䌉

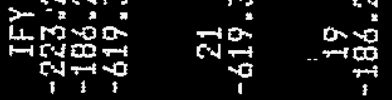

$m$

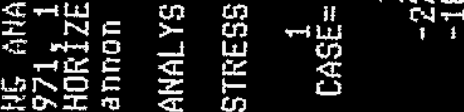

到和点

听

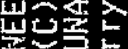

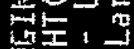

핸다

준도

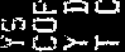

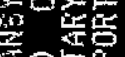

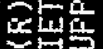

西它

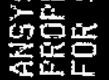

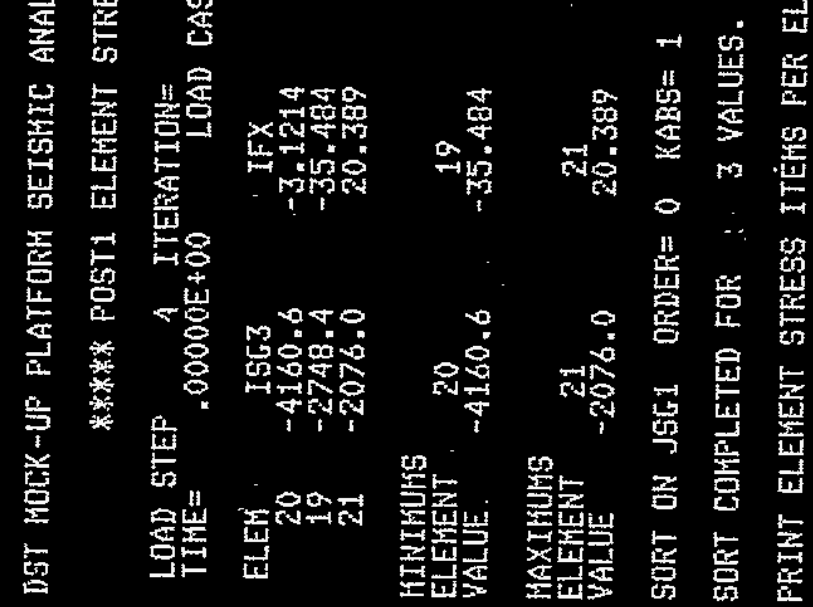


$\stackrel{9}{?}$

ga

究章

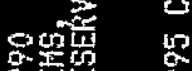

$x_{i=1}$

$-\frac{n}{c o n}$

的密

药数

萿势

营会 些

䍃

管禺

的

mis

政

$\rightarrow=$

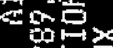

난랄

象

寸然育

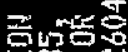

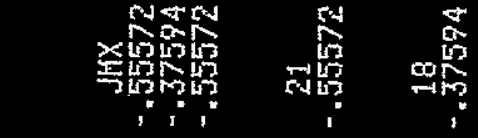

$\sin 2$

$\prod_{i=1}$

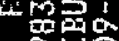

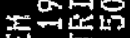

Wun

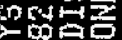

con 푸

wheng

结

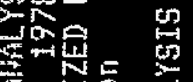

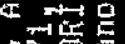

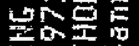

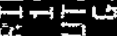

部新各

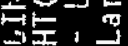

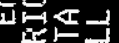

준

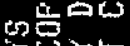

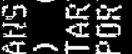

凯望

opers

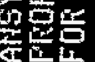

+
$\vdots$
0
0

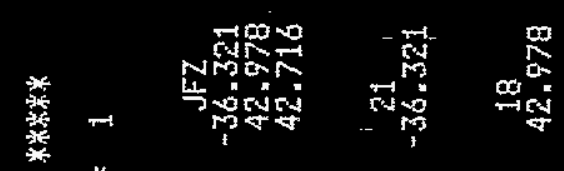

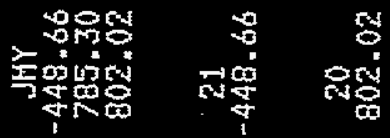

+ 至

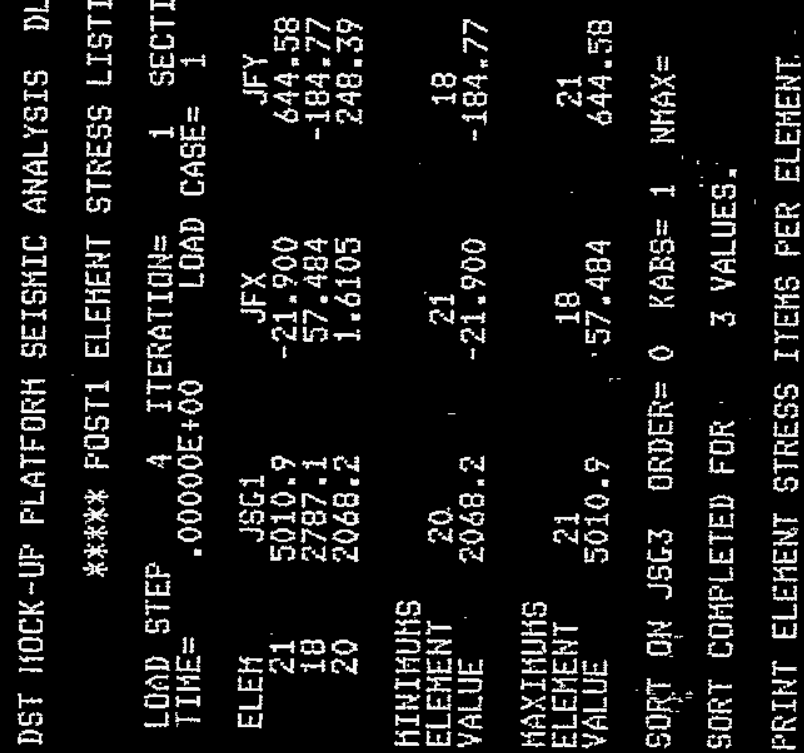


AAEY - EHEIHEERIHG AHAL YSIS EYSTEM GEUISIOH $4=4$

A137 32207

HAY 1,1990

AHSYS(F) COFYRIGHT(C) $1971,1979,1982,1983,1985,1987,1989,1990$ SWANSON ANALYSIS SYSTEHS, INC. AS UNFUELISHED WORK. FFOPKIETAFY DATA - LWALTHORIZED USE, DISTEIEUTION OR DUFLICATION IS FROHIBITED. ALL RIGHTS RESERVED.

FOR SUFPORT CALL LarTY GaTITON FHONE 509-376-2604 THX

DST HOCK-UF FLATFORH SEISHIC ANALYSIS DL $+.06 g: \mathrm{x}+.2 \mathrm{~g}: 2$

15.1261 AUG $1,1995 \mathrm{CP}=$

1.140

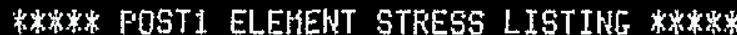

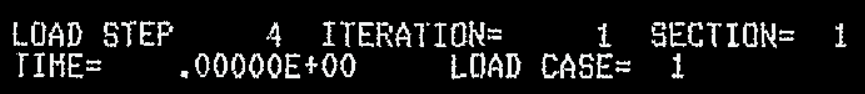

\begin{tabular}{|c|c|c|c|c|c|c|c|}
\hline $\begin{array}{r}\text { ELEH } \\
\begin{array}{r}21 \\
18 \\
20\end{array}\end{array}$ & $\begin{array}{c}5643 \\
-5029 \cdot 1 \\
-2739.2 \\
-2066.8\end{array}$ & $\begin{array}{c}\text { JFX } \\
-21.900 \\
57.494 \\
1.6105\end{array}$ & $\begin{array}{r}\text { JFY } \\
644 \times .58 \\
-184.77 \\
248.39\end{array}$ & $\begin{array}{c}J F Z \\
-36.321 \\
42.978 \\
42.716\end{array}$ & $\begin{array}{r}\text { JHX } \\
-55572 \\
-.37594 \\
-.55572\end{array}$ & $\begin{array}{r}\text { JHY } \\
-448.66 \\
785.30 \\
802.02\end{array}$ & $\begin{array}{c}\text { JHZ } \\
-19207 \\
7315.6 \\
4176.6\end{array}$ \\
\hline
\end{tabular}

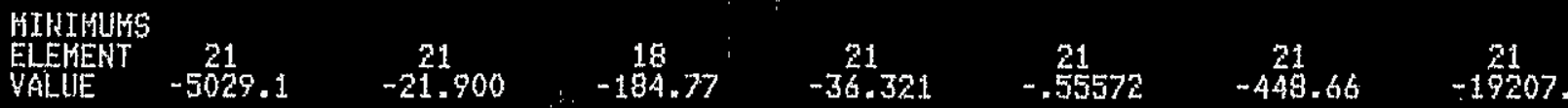

HAXIHIHE

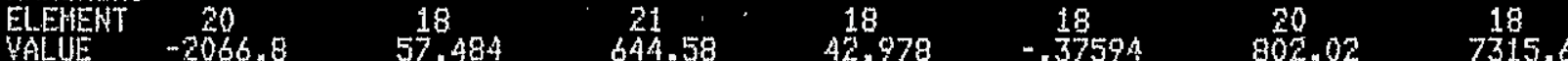

ESEL FOR LABEL= ELEH FFOM 18 TO $22 \mathrm{GY} \quad 4$

2 ELEHENTS (OF. 9 9 DEFINED) SELECTEB BY ESEL COHMAND.

PRINT ELEHENT STRESS ITEHS FER ELEHEHT 
AHEYS - EHGIREEFTHE AHALYCIS gYSTEH FEYTSTOH A A

A137 32207

HAY 1,1990

AMSYE(F) COFYFIGHT(E) 1971, 1778, $1982,1983,1985,1987,1989,1990$ SWANSON ANALYSIS SYSTEMS, INC. AS UNFUELISHED WORK.

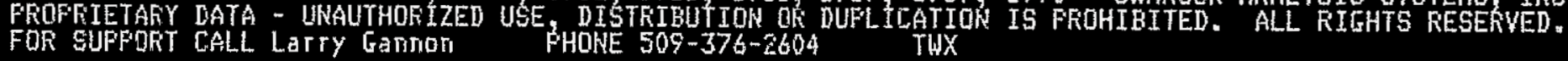

DST HOCK-UF PLATFORH SEIGHIC ANALYSIS DL $+.06 \mathrm{~g} \mathrm{x}+.2 \mathrm{~g}: \mathrm{z}$

15.1261 ALG $1,1995 \mathrm{CF}=\quad 1.140$ *

LOAD STEF $\stackrel{4}{\text { TITEFATIQH: }}=\stackrel{1}{1}$ SECTTOH: $=1$

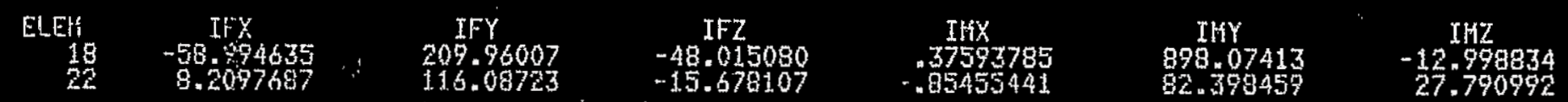

KINIHUHS

ELEKENT

$\frac{18}{-59.994635}$

VALUE

$11 \frac{22}{2} .08723$

$$
-48.015080
$$

$-.8 \frac{22}{855441}$

82.398459

18

MinEHS

$-22 \quad 5987$

$\frac{15}{2-6}=75$

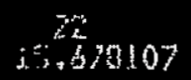

$\frac{18}{37593795}$

$\frac{18}{898.07413}$

$27=790992$

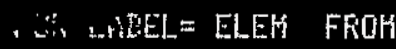

1970

23 11

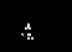

PRINT ELEGEHT GTRESS ITEHS FER ELEMENT 
量

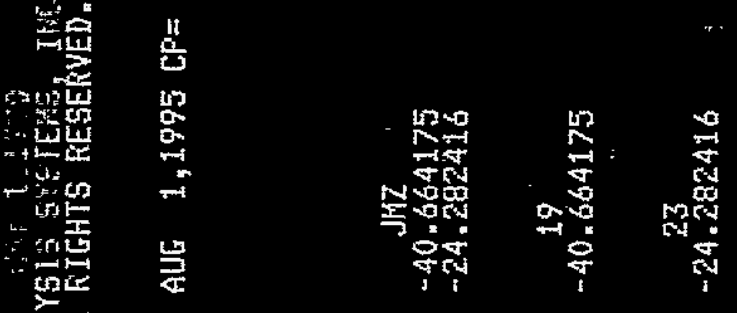

$\frac{1}{4}$

喜密

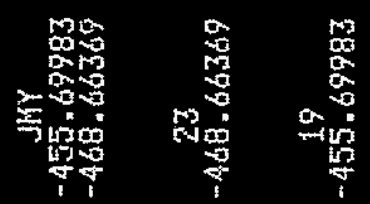

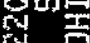

的

要的

mint

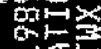

$\rightarrow$ 딴

大

$x_{i=1}$

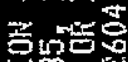

thos

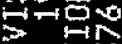

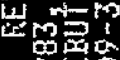

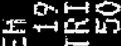

wntw

ing

$3^{-1} \omega^{5}$

tom

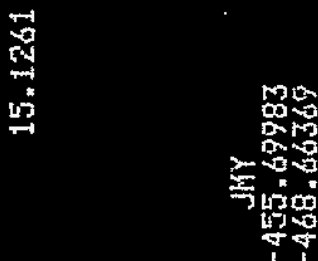

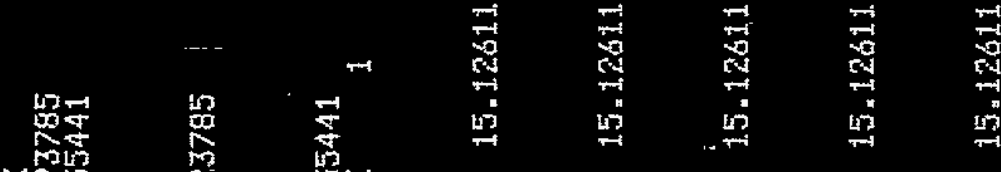

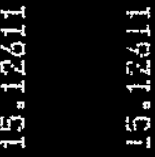

$\underbrace{\rightarrow-1}_{n=1}$

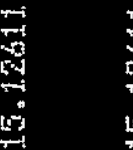

$\underset{7}{3}$

$\frac{x+y}{30}$

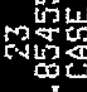

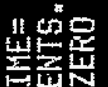

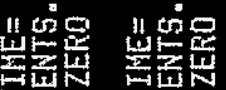

唩点点

若象

눌

몬

章的

논을

点的

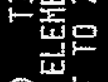

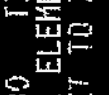

离巨

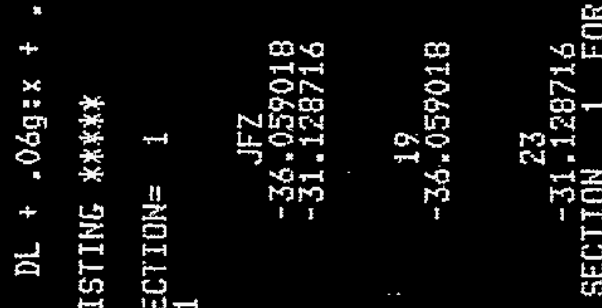

8 민

最十勧

过

-

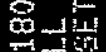

我最

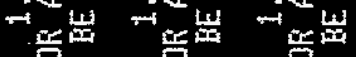

$\rightarrow$ 㟧

皆崖

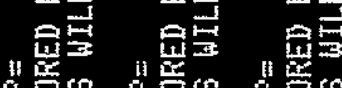

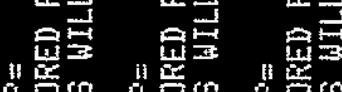

봉

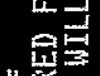

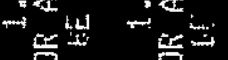

$\rightarrow \frac{1}{4}$

$+$

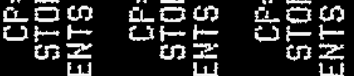

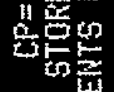

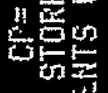

勧

荌空

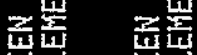

讧密

预

.

㔉

10

11.

和马四

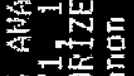

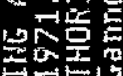

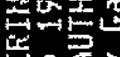

본돈

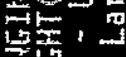

its

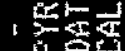

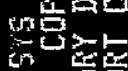

空

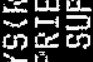

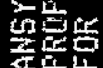

㟧焉

잉

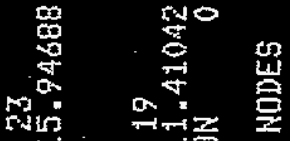

矛问

욜

空

列

논

욬ㅋㅋ

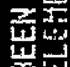

蛋:

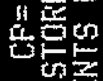

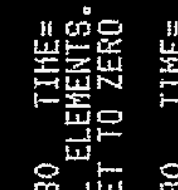

먼

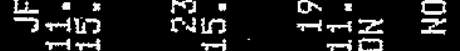

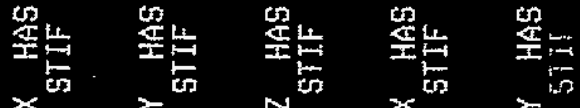

卢的

호웅

营安

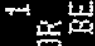

는

丝

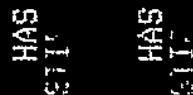

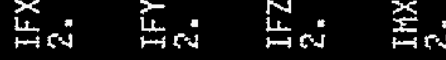

ind

Ni:

与: 50 兵

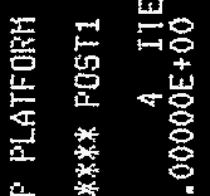

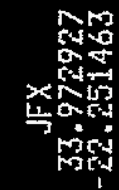

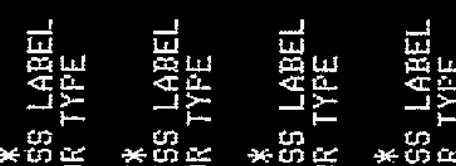

的它

*

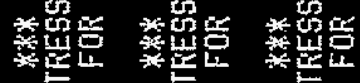

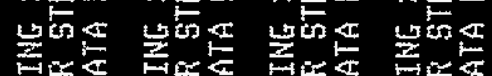

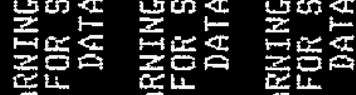

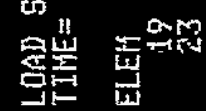

본동

焉

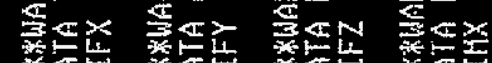

虰

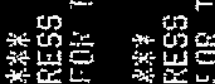

*质

wor was

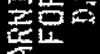

害山部

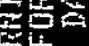

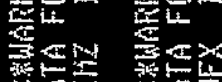

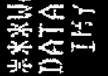

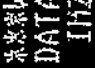

*as

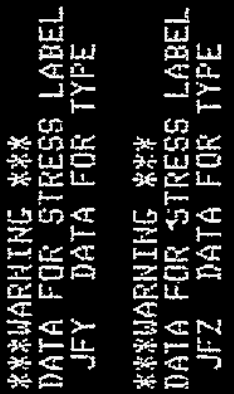




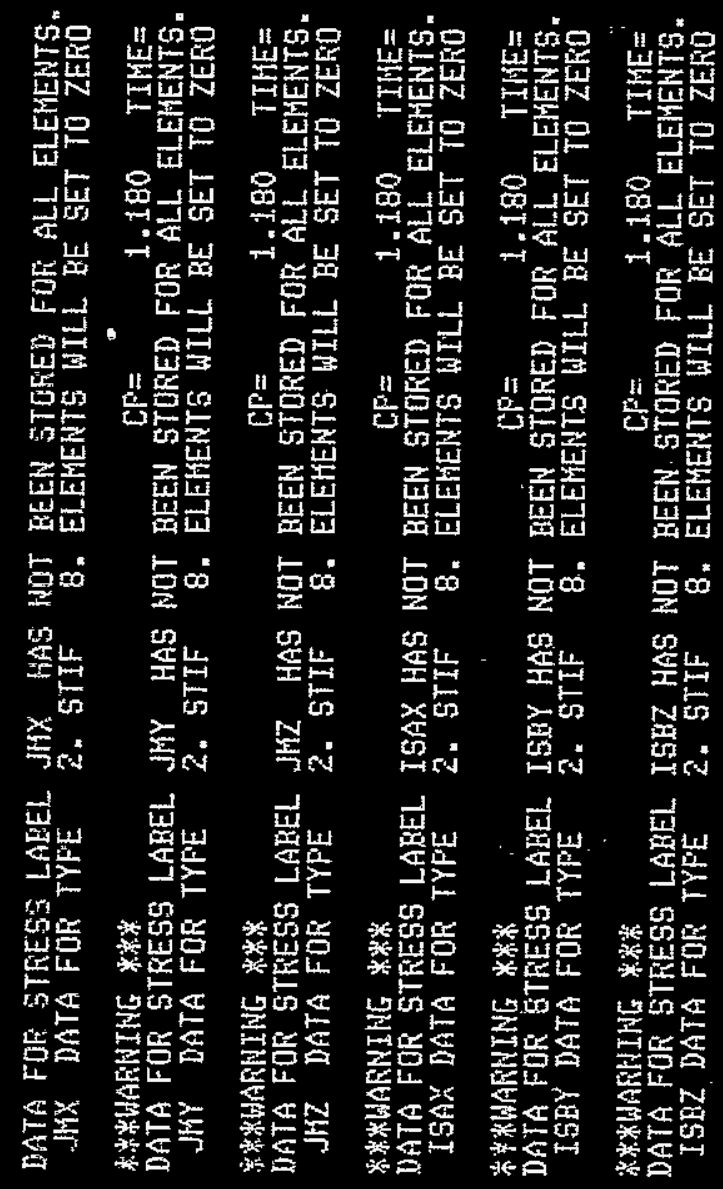


WWHAEHINE W

VATA FOR STRESS LABEL ISAX HAS NOT BEEN STORED FOR AI 1.50 TIHE= 15.13611

$\begin{array}{ll}\text { WSY DATA FOR TYFE } & \text { 2. STIF } \\ \text { B. ELEHENTS WILL BE SET TO ZEFO }\end{array}$

*P*UARNING ***

DATA FOR GTKESS LABEL JSBY HAS NUT BEEN STORED FOR ALL ELEHENTS.

JSBY DATA FOK TYFE 2 . STIF 8 . ELEHENTS UILL HE SET TO ZERO

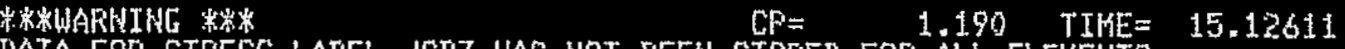

DATA FOR STRESS LAHEL JSHZ HAS NOT BEEN STORED FOR ALL ELEHENTS
ISEZ DATA FOK TYFE
2. STIF 8 . ELEMENTS WILL BE SET TO ZERO

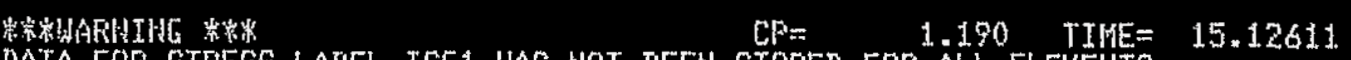

DATA FOR GTRESS LABEL ISEI HAS NOT BEEN GTORED FOR ALL ELEHENTS

T.G DATA FOR TYFE 2 , STIF 8 . ELEHENTS WILL BE SET TH ZEFO

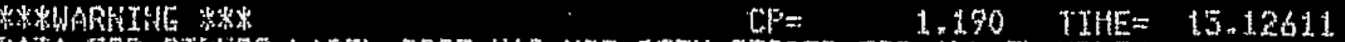

DATA FOR STRESS LABEL ISG3 HAS NOT BEEN STORED FOR ALL ELEHENTS.

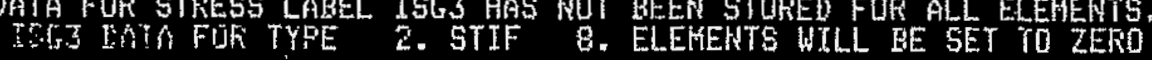

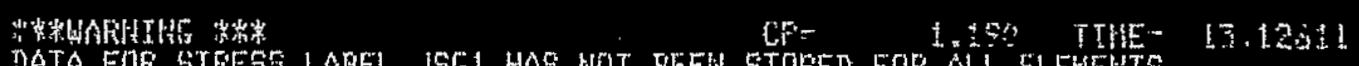

DATA FUR STKESS LABEL JGG 1 HNS NOT GEEH STORED FOR ALL ELEHEHTS.

WSG1 DATA FOR TYFE 2. STIF 8. ELEHENTS WILL BE SET TO ZEFO

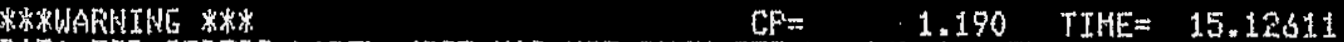

DATA FOR STRESS LABEL JSG3 HAS NOT BEEN STORED FOK ALL ELEMENTS

JSG 3 DATA FUK TYFE 2 . STIF 8 . ELEHENTS WILL BE SET TO ZERO

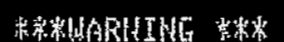

WATA FOF STFESS LAEEL SAX HAS NOT BEEN STORED FOR ALL ELEHENTS.

15.12611

SAX BATA FOR TYFE 1. STF

SAX DATA FOR TYPE 3, STIF 4. ELEHENTS UTLL BE SET TI ZERO

A. ELEMENTS UTLL WE GET TO ZENO

SAX DATA FOK TYFE ज̈. GTJF 4. ELEHENTS WIL BE SET TU ZERO

STRESSES STORED FOR 23 SELECTED ITEKS

ITERATIOH SUMMARY INFOHWATHON STOKED

HODAL FORCES STORED FOK 99 ELEHENTS

REACTIOHE STOKED FOR 18 REACTIONS

FOR LOAD STEF $=5$ ITEKATIOK= 1 SECTION= 1

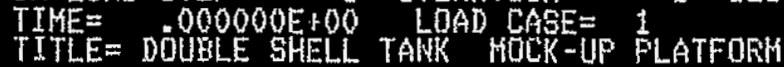

HEW TITLE= DST HOCK-UF FLATFORH SEISHIC AKALYSIS DL + .06g: $+.2 \mathrm{Z}:-z$

USE HAEKO FILE MTES

ESEL FOR LABEL= TYPE FRUH 1 TO 1 BY 1

36 ELEMENTS IOF 99 DEFINED) SELECTED BY ESEL COHHAWD.

SORT OH ISGI QRDER $=0$ KABS= 1 HWAX $=3$

SORT COHFLETED FOR 3 VALUES.

PRTHT ELEHENT GTFESS ITEHS FER ELEHENT

BEST RVAALABLE COPY 
AHSYS - ENGIHEERIHG AHAL YSIS SYSTEH REVISTOH 4.4

413735207 HAY 1,1990

ANSYE(K) COFYKTEHT(C) $1971,1979,1982,1983,1985,1987,1999,1990$ SHANSON AHALYSTS SYSTEHS, THC. AS UNFUELISHED WOFK.

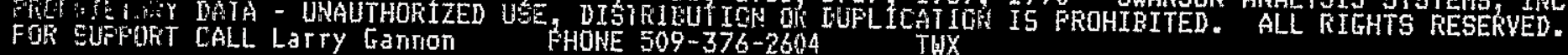

VST HOCW US FLATFORH SEISHIC AHALYSIS DL $+.060: x+.20:-1$

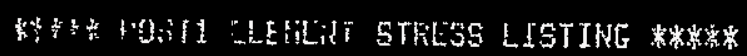

\subsection{AUG $1,1995 \mathrm{CP}=\quad 1.200$}

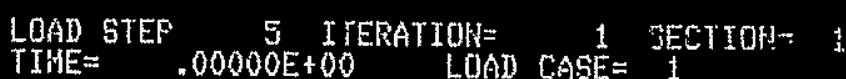

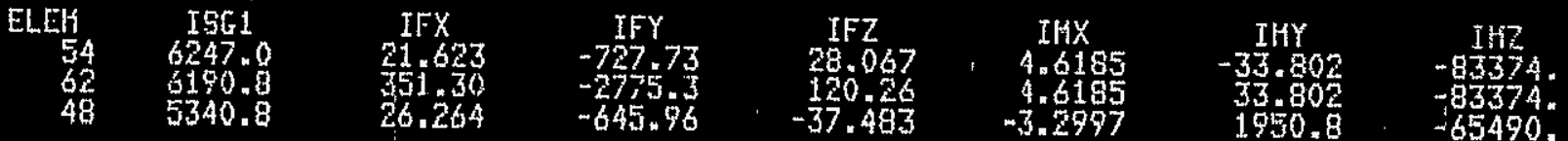

MTNTHUHS -

$\begin{array}{lccccccc}\text { ELEHENT } & 48 & 54 & 69 & 48 & 48 & 54 & 62 \\ \text { VALUE } & 5340.8 & 21.623 & -2775.3 & -37.483 & -3.2997 & -33.602 & -83374 .\end{array}$

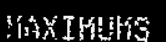

ELEHENT

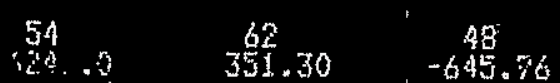

120.24

54.6189

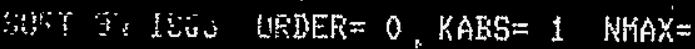

3

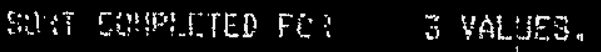

FRINT ELEHENT STRESS ITEF, : ? : : E'VEST 


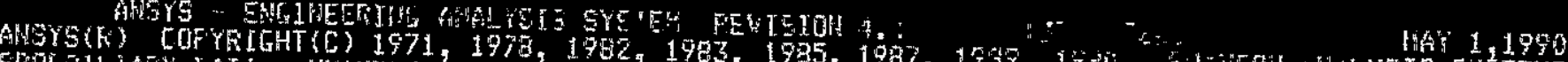

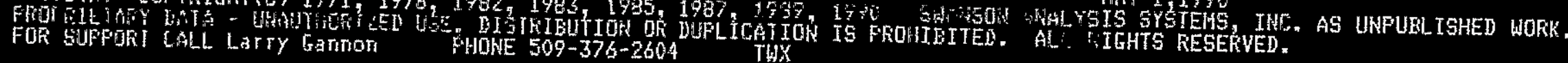

WOS NOCK-UP PLATFORH SEISHIC ALALYSIS DL $+.06 g: x+.29:-z$

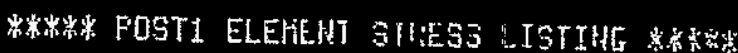

\subsection{AUG $1,1995 \mathrm{CP}=\quad 1,200$}

LOAD STEF 000 THERATION= 1 SECTION= 1

ELEH ISG3

$\begin{array}{ll}62 & -6310.5 \\ 54 & -6254.3\end{array}$

$\begin{array}{ll}54 & -6254.3 \\ 48 & -5349.8\end{array}$

$$
\begin{aligned}
& \text { IFX } \\
& 351.30 \\
& 21.623 \\
& 26.264
\end{aligned}
$$

$\begin{aligned} & \text { IFY } \\ &-2775,3 \\ &-727.73 \\ &-645.96\end{aligned}$

IFZ

28.067

$-37.483$

I

4,6185

4.6185

ITYY

$-33.802$

1950.8

bitrituks

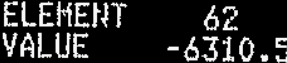

54.623

$-\frac{62}{-2775.3}$

48
-37.483

48
-3.2997

54

MAXIHUHS

62

$\begin{array}{lccc}\text { ELEKENT } & 48 & 62 & \\ \text { VALUE } & -5349.8 & 351.30 & -645.96 \\ \text { SORT ON JSG1 ORDER }=0 & \text { KABS }=1 & \text { HHAX }\end{array}$

120.26

4.54185

$-33.802$

$-83274$

SORT COMFLETED FOK 3 VALUES.

FRINT ELEHENT STRESS ITEMS FER ELEHENT

48
1 
望

$\frac{\mathrm{g}}{4}$

$\sum_{n=1}^{5}$

0 this

네는

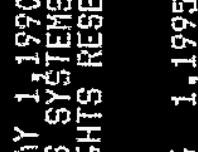

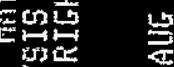

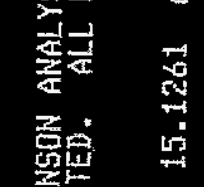

Nơic

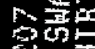

की

迹

总禺

중ำ

$\stackrel{4}{c}$

맘

的学

$+\sum_{0}^{+}$

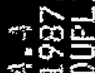

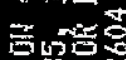

4
1
-1

: 1302

W

ins 91

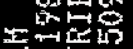

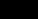

x

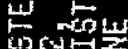

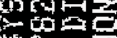

$\ln ^{-1} \frac{5}{2}$

$\lim _{n \rightarrow \infty}^{\infty}$ क क

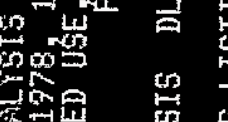

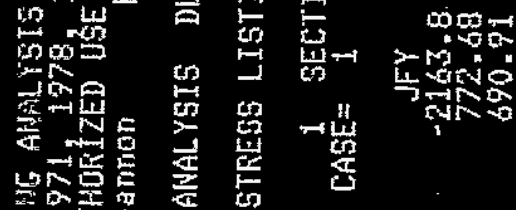

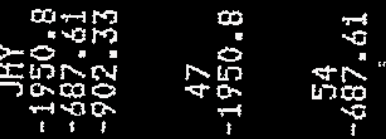

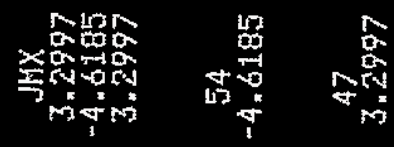

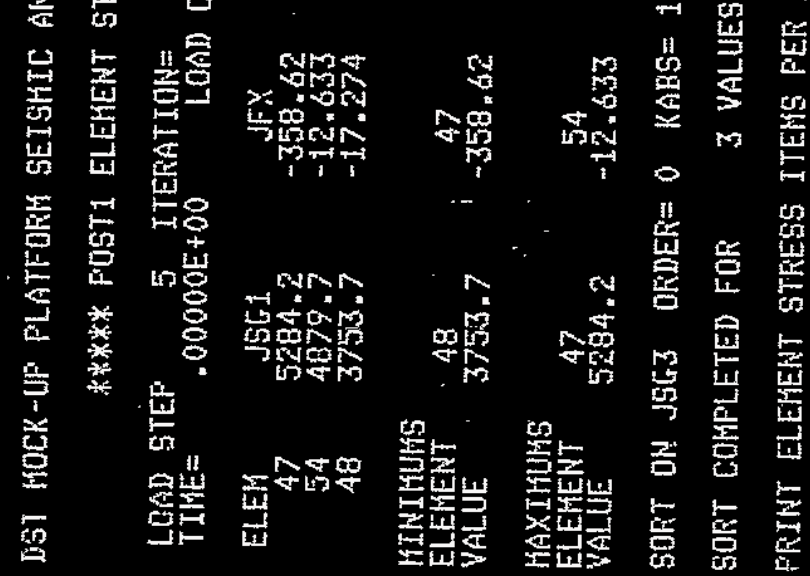

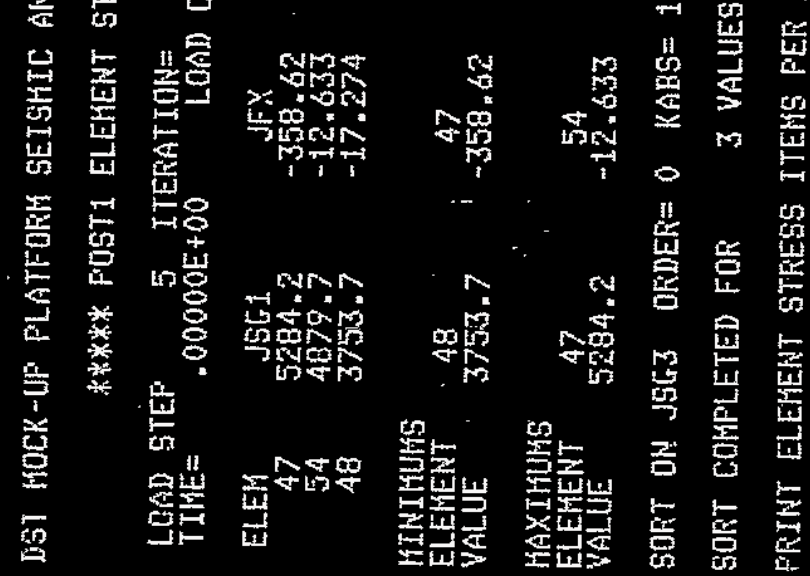

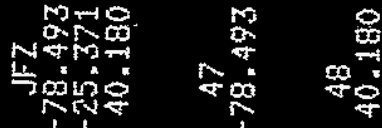

$x_{10}$

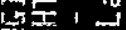

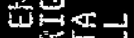

, 这要

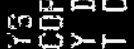

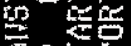

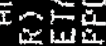

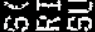

的管 
盖

案

窟

ogint

素些

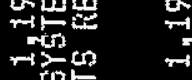

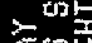

政赔

gre

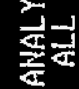

喜密

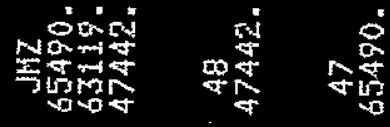

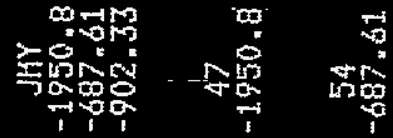

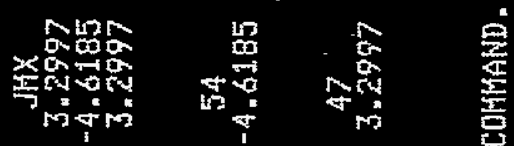

\section{标吉}

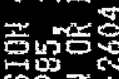

$\frac{41}{\frac{4}{5}}$

$\underset{20}{20}$

논

$\underset{n}{\rightarrow-1}$

$5 \rightarrow$ to

wivis

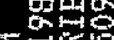

놉 $\rightarrow=5$

the

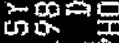

$w^{-1} \operatorname{win}^{-2}$

$\overrightarrow{b x} \cos$

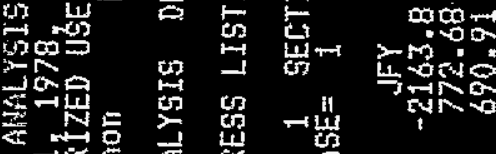

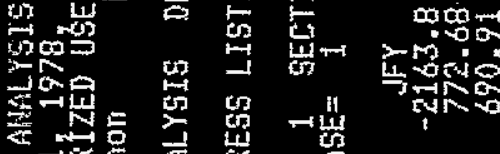

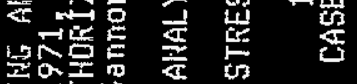

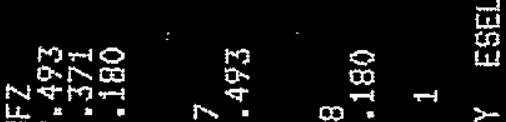

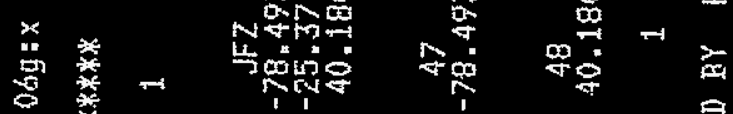

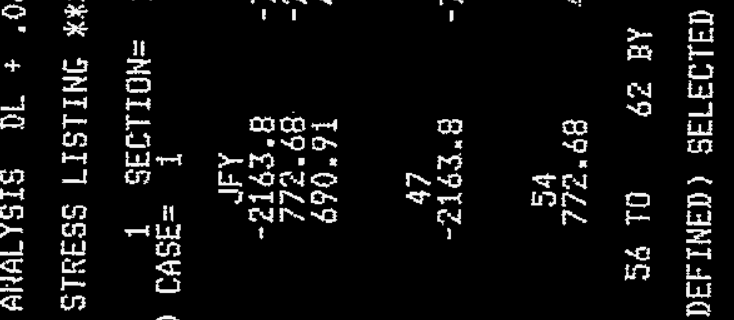

네네

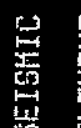

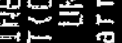

年, 要

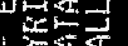

窟安

的密地

Syst

的 是要

然

窝怠

的总

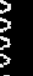

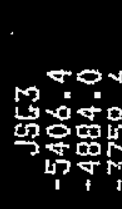

站

突

움본

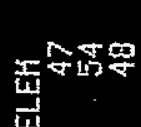

में

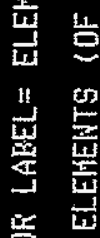

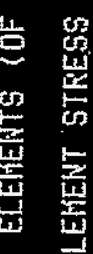

点

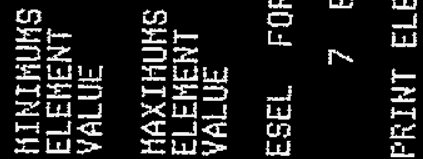


焉

哭

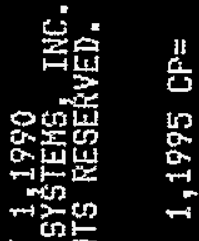

$>$ 工

空俚

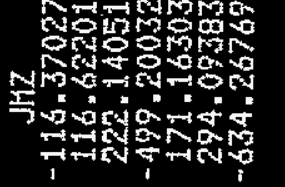

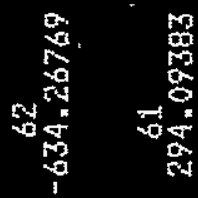

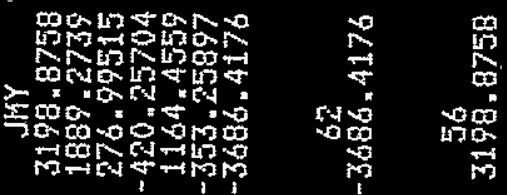

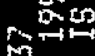

두용

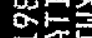

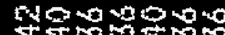

$8 \mathrm{cos}$

$\times 200500$

savin

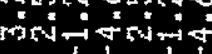

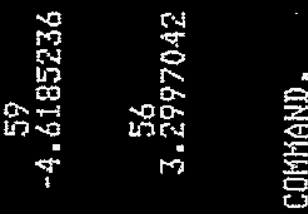

욜

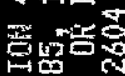

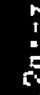

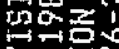

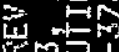

a롤요.

$x \rightarrow$

Encon

- wis

$\lim _{x \rightarrow 1}+$

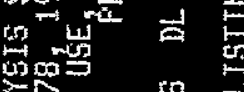

ita

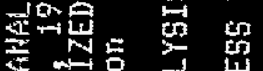

焉

군을

戠

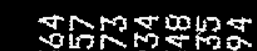

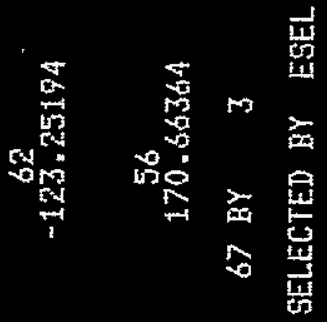

Nofr o of

Lotorim

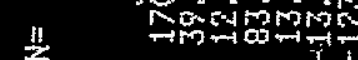

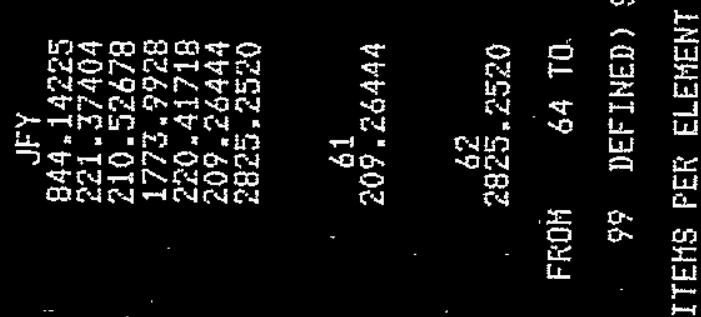

넌돈

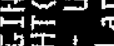

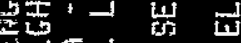

1 然管

of

분

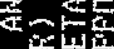

in

울응

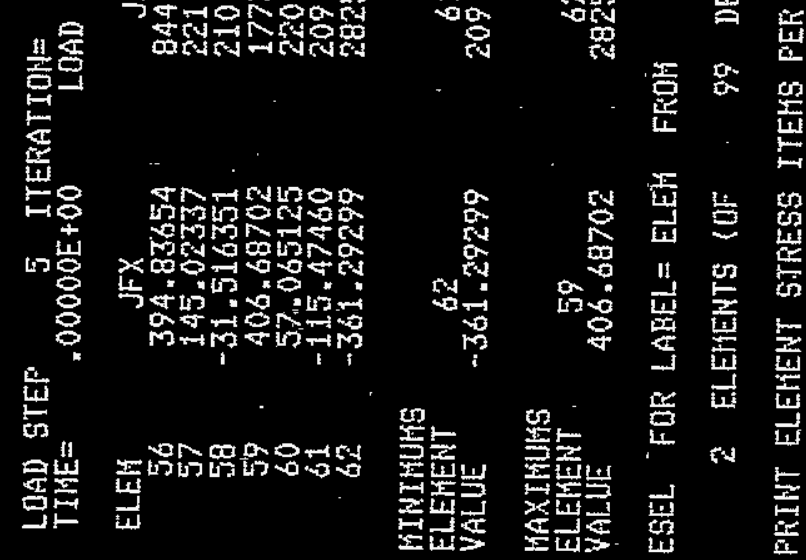


疍

온

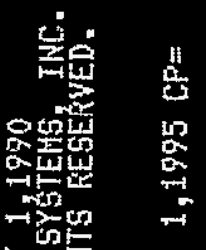

20

起我语

만다

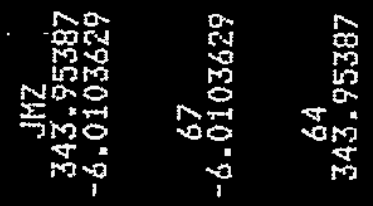

表

吉感嵒

要

人整

논

8

就

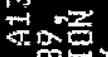

器此肴

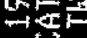

rity

일

골

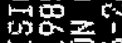

$\rightarrow$ 㣢

is

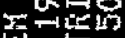

5

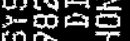

$\cos ^{-1}$

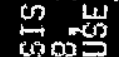

돈스

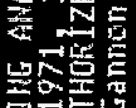

司鸟

可产点

武彺, 留

،需震

. 突乐乐

w它>1

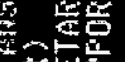

드는혹

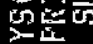

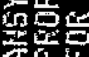

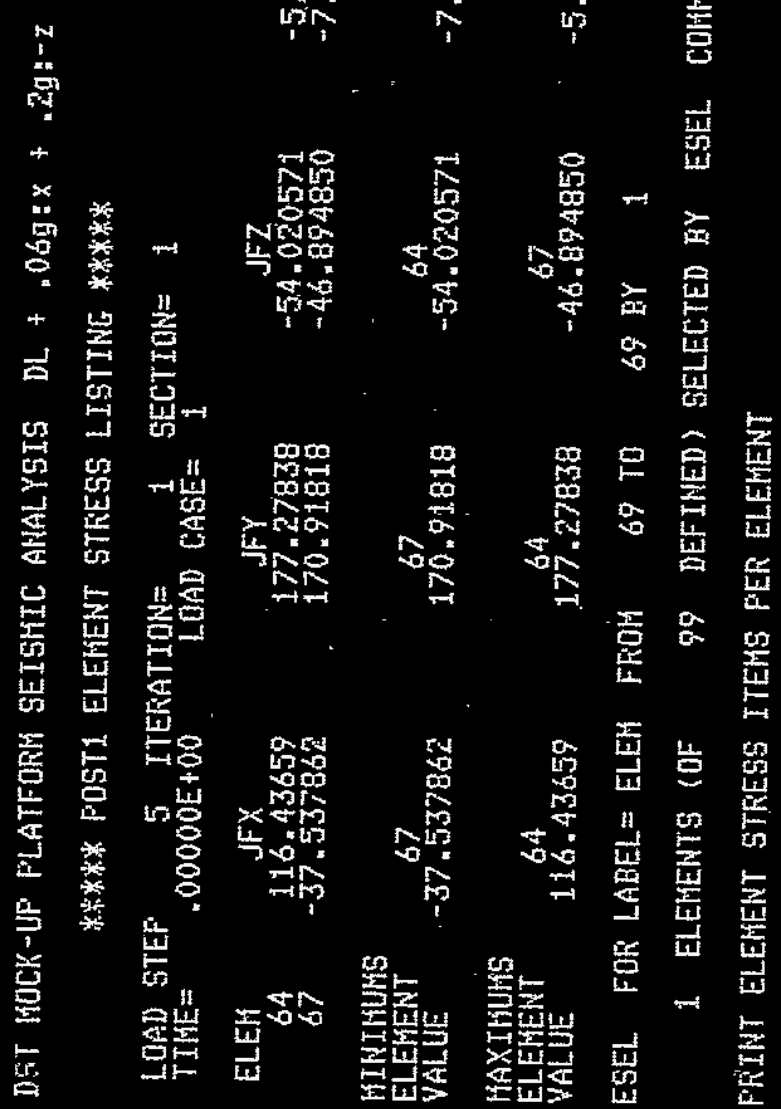

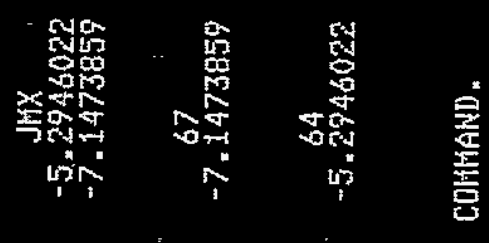


AHBYS - EHGINEERIHL AHALYSIE SYSTEH REVISIOH A.4

A137 32207

HAY 1,1990

ANSYS(F) COFYRIEHT(C) $1971,1978,1982,1993,1985,1987,1989,1990$ SWANSON ANALYSIS SYSTEMS, INC. AS UNPUELISHED WOEK.

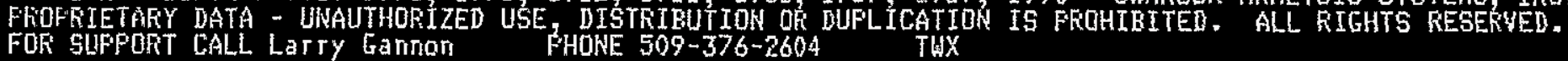

DST HOCK-UF PLATFORH SEISHIC AHALYSTS DL $+.06 \mathrm{~g}: \mathrm{x}+.2 \mathrm{~g}:-\mathrm{z}$

15.1261 AUG $1,1995 \mathrm{CP}=$

1.230

***** FOSTI ELEMENT STRESS LISTING *****

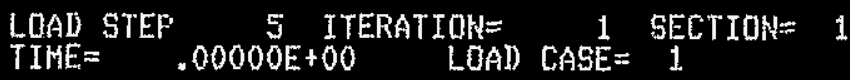
ELEH, 69 JFX
JFX 20.451391
122.37567
$-43 \cdot 057492$
6.89009043
$-2056.1896$
3. $\frac{\mathrm{dHZ}}{8510671}$

MININUSS

VALUE

69

69

$-20.451391$

122.37567

43.057492

6.8909043

69

MAXIKUHAS

ELEMENT

$-20.451391$

69

122.37567

69

$-43.057492$

6.89809043

$-2056.1896$

69

ESEL FOF LAEEL = ELEH FFOH

47 70 $\quad 47$ BY 1

$-2056.1896$

3.89510671

1 ELEMENTS (OF 99 DEFIHED') SELECTED BY ESEL COHMAHD.

PRINT ELEMENT STRESS ITEHS PER ELEHENT 
ANEYS - EHCIHEEFIWG AHALYSIS SYSTEH FEVISIOH 4,3

ANSYS(R) CUFYRIGHT(C) $1971,1975,1982,1983,1985,1987,1989,1990$ SWANSON ANALYSIS SYSTEMS, INC. AS UNFUBLISHED WOFK.

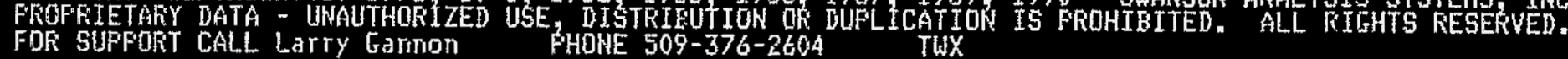

DST MOCK-UP FLATFEKH SEISKIC ANALYSIS DL +.04g: $+.2 \mathrm{~g}:-\mathrm{z}$

$1.5 \times 1261$ AUG $1,1995 \mathrm{CF}=\quad 1.240$

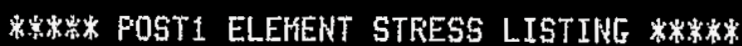

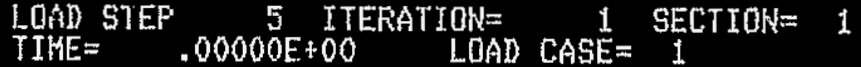

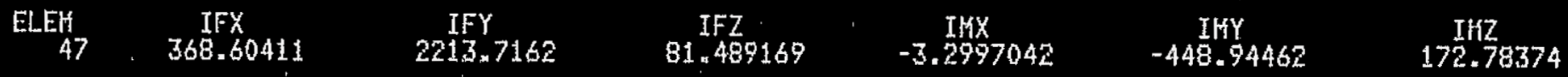

HINIHUHS 47

ELEMENT:
VALUE

$368 \times 60411$

47

2213.7162

47

81.489169

47

997042

47

MAXTHUMS

ELEHENT

47
368.60411

47
$2013 \times 716 \%$

81.489169

$-3.2797042$

$-448.9446 z$

47

VALUE

$63 \mathrm{TO}$

65 BY $?$

$-478.94462$

172.78374

$9 \%$ DFFIHED) SE!ECl:

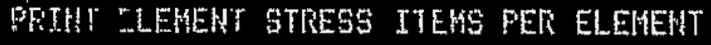

BEST AVAILABLE COPY 


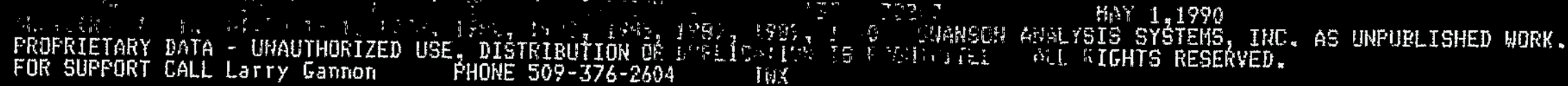

DST HOCK-UF PLATFORH SEISMIC ANALYSIS DL + .06g: $\mathrm{x}+.2 \mathrm{.}:-7$

$$
\text { 15.1261 AUG } 1,1995 \mathrm{CP}=\quad 1.240
$$

***** FOST1 ELEHENT STRESS LISTING ******

LOAD STEF 0000 ITEFATTON= 1 SECTION $=1$

ELEH

$63-187.3948$

$-187.3948$

IFY

178.51284
231.89302

IFZ
32.635062
10.421242

$\operatorname{IHX}_{-5 \times 294028}$

65

ning

VLEYGE

$-1.87 .39483$

178.51294

10.421262

$-17.2563549$

231.88302

32.635082

5.29346022

68 BY 1

ESEL. FOF LABEL = ELEH FFOH

$68 \mathrm{TO}$

1 ELEMENTS ROF 99 DEFINED) SELECTED BY ESEL CONHAND.

FRTNT ELEHENT STRESS ITEHS FER ELEHENT 
AHSYG - ENGIHECEIKG AKALYSIS SYSTEH FEVISIOH A A

A 13732207

HAY 1,1990

AHSYS(K) COFYFIGHT(C) $1971,1978,1982,1983,1985,1987,1989,1990$ SUANSON ANALYSIS SYSTEMS, INC. AS UNFUBLISHED WORK.

FROFRIETARY DATA - UWAUTHDRIZED USE, DISTRIEUTION OR DUFLICATION IS FFOHIBITED. ALL RIGHTS RESERVED*

DST HOCK-UP PLATFORH SEISHIC ANALYSIS DL $+.06 \mathrm{*}: \mathrm{x}+.2 \mathrm{~g}:-\mathrm{z}$

15.1261 AUT $1,1995 \mathrm{CP}=\quad 1.250$

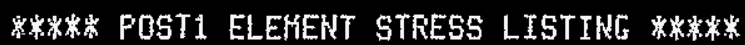

LOAD STEF $=005$ ITERATIOHE $\quad 1$ BECTION= 1

\begin{tabular}{|c|c|c|c|c|c|}
\hline $\begin{array}{l}\text { ELEF } \\
68\end{array}$ & $\begin{array}{c}\text { IFX } \\
-52.796113\end{array}$ & $\begin{array}{c}\text { IfY } \\
183.43970\end{array}$ & $\begin{array}{c}\text { IFZ } \\
36.718368\end{array}$ & $\operatorname{Inx}_{13.584320}$ & $\operatorname{IMY}_{-1767.9374}$ \\
\hline
\end{tabular}

HINTHUTS

ELEMENT

$-52.796113$

88

183943970

36.718368

68

13.584320

$-1769.9374$

68

MAXIMUKS

ELEHENT
VALUE

$-52.796113$

183.43970

36.718368

68

13.584320

$-1767.9374$

ESEL. FOR LAEEL = TYPE FFOH

2 To

2 BY 1

16 ELEMENTS (OF 99 DEFIHED) SELECTED BY ESEL COMHAND.

SORT OH SAX ORDER $=0$ KABS= 1 HHAX $=$

3

SORT COHPLETED FOR

3 VALUES.

PRTHT ELEHENT STRESS ITEMS PER ELEHENT 
垔

新

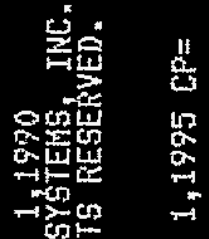

$>$

孯的鵕 늑

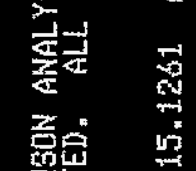

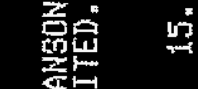

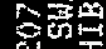

5.

踏

$\min ^{2}$

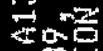

可的

삭

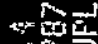

舟然要

춘

:

:

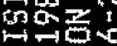

wit

9xa

전

照

50

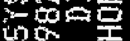

in

won

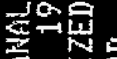

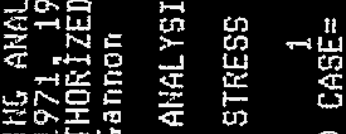

$5 \rightarrow 5$

분욜

哥高怠蒠

, 究重究

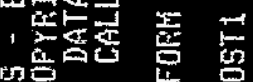

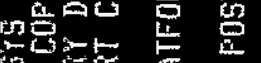

量

훈훌

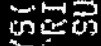

홀뭉

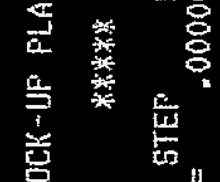

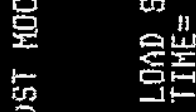

稟

som

=

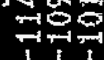

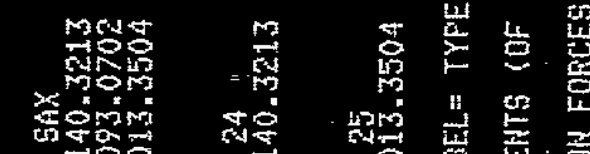

$\rightarrow \geqslant$

西喈

$m$

竞

蛿

㟧

뭉

-

$\therefore$ 㟧

동

总

岑

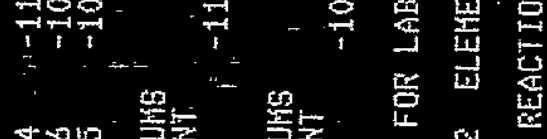

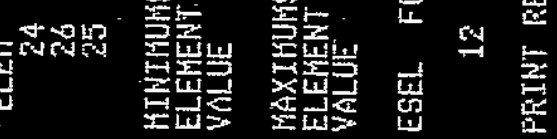


魚

架

运实

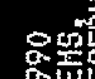

aw

$\min _{0 \rightarrow-1}^{m+\infty}$

范

$\underset{5}{4-13}$

的政

点

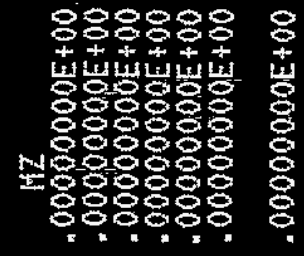

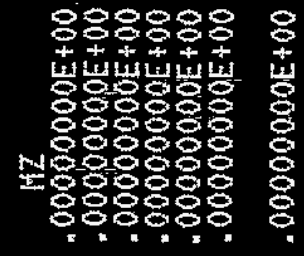

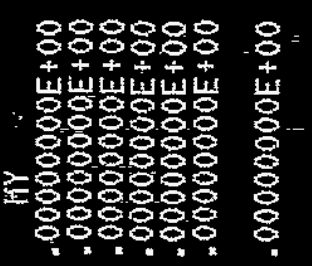

mo

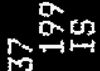

安语

898888

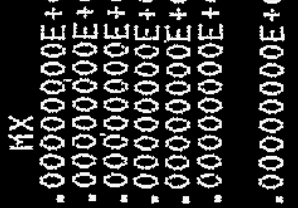

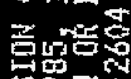

:

$\rightarrow \rightarrow{ }^{\circ}$

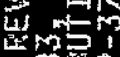

ga

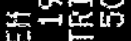

tron

son

की

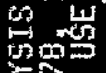

요

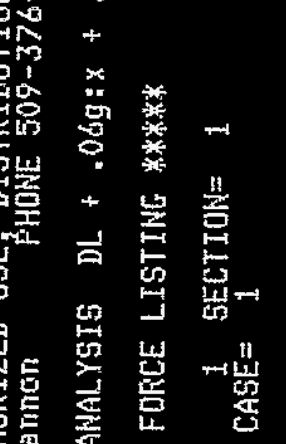

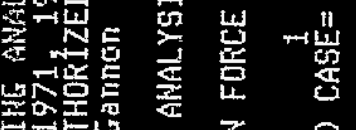

는

핀존난

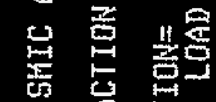

证, 骂

密密

1 눈

usto

대은

趾空

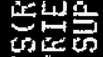

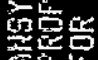

点变

哭 点。

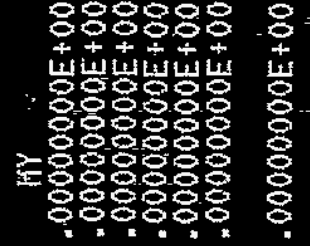

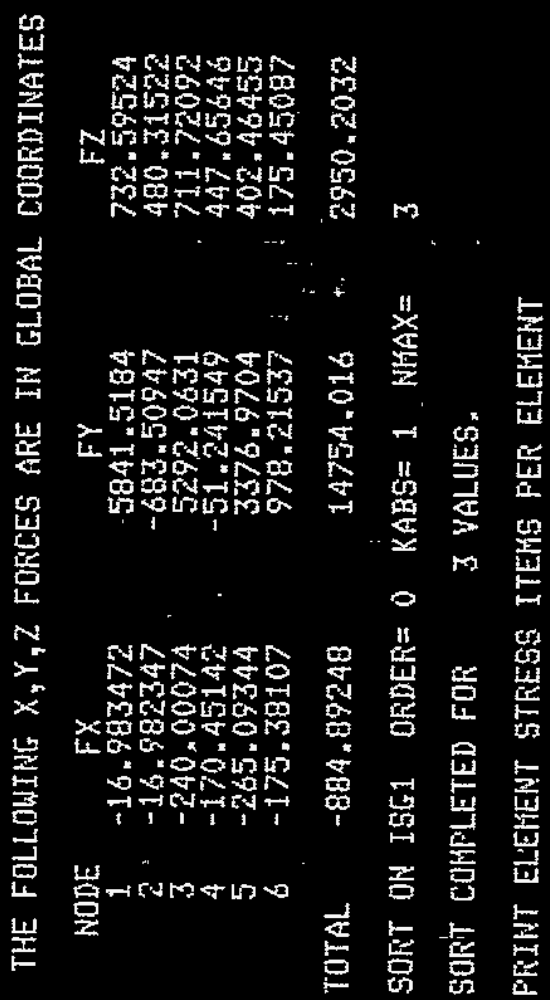

$$
A-169
$$


AHSYS - EHF IHEEETHG ALLALSIS SYSTEM FEVISTOH 4 A

A137 32207

HAY 1,1990

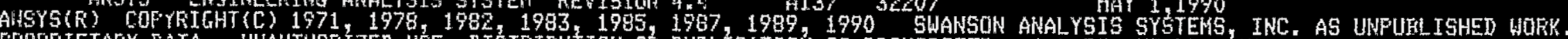

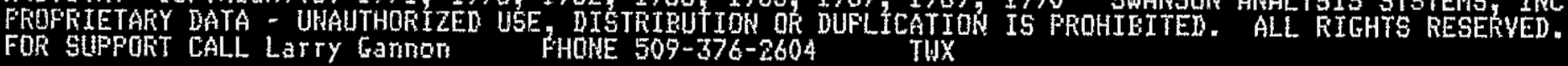

1GT HOCK-UP PLATFOKH SEISHIE ANALYSIS DL +.06g: $x+.2 \mathrm{~g}:-\mathrm{z}$

15.1261 AUG $1,1995 \mathrm{CP}=$

1.260

****** FOSTI ELEHENT STFESS LISTING $*$ *****

LIAE $=\stackrel{\text { STEF }}{\text { TIH }}$.00000E+00

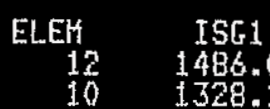

ISG1
1486.0
1328.1
1305.7

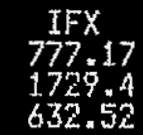

IFY
-157.07
-152.31
-80.780

IFZ

23.720

Ihx

.00000 E $+00-3$ IHY

$-.43521 E-13-730.17$

$.00000 \mathrm{E}+00-2079.7$

MINIHUHS

ELEKENT

VALUE

$1305.7 \quad 63 \frac{6}{6} \times 52$

$-157.07$

$\frac{12}{22.002}$

$\begin{array}{ll}10 & \\ -43521 E-13 & -2079.7\end{array}$

MAXINUTH

GAEME

$1 \frac{12}{486.0}$

1.729 .4

$-88^{6} .788$

37.731

12

3

SURT COHPLETED FUR 3 VALUES,

FRINT ELEHENT GTRESS ITENS PER ELENENT
$\operatorname{IHz}$

-20975 .

$-12590$.

$-21603$

$-1.2590$ 
量

문

空焉

竞

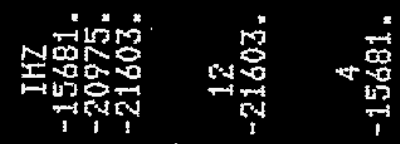

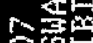

onify

$\mathrm{x}_{\rightarrow-1}^{\mathrm{m}}$

$\rightarrow \cos _{0 \rightarrow 5}=$

$\succ x$

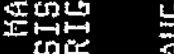

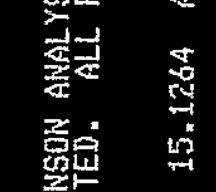

승

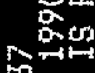

急为

㤎州幽

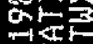

단

小战

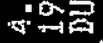

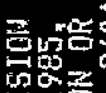

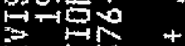

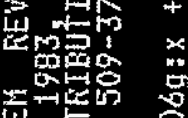

कालn

yyㅗㄹ

tion

㔖岁

留㟧

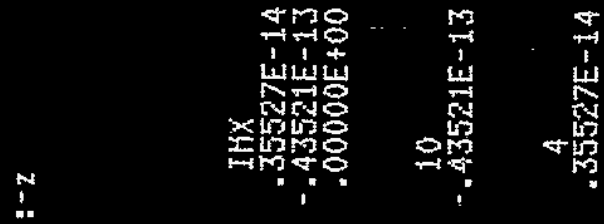

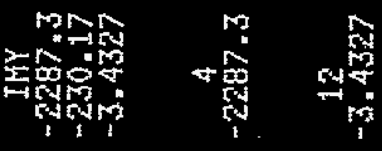

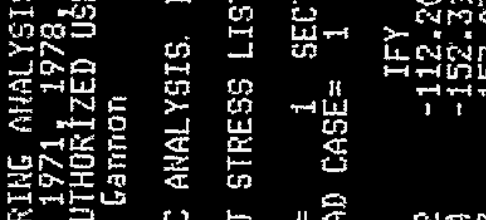

政入

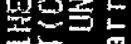

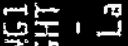

窝

, 它安震

氙

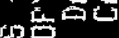

문

政象

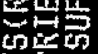

数密告

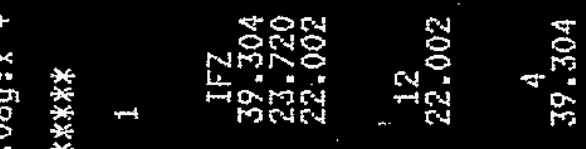

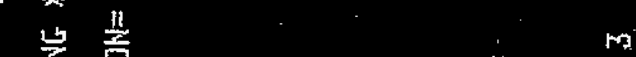

$F$

点

w峞

푤

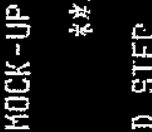

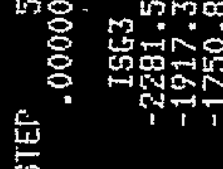

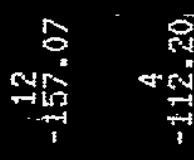

些

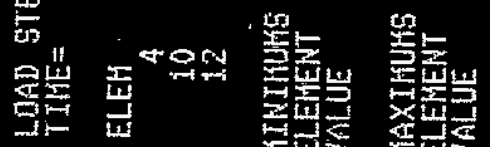

$\frac{11}{\frac{14}{2}}$

喜

영

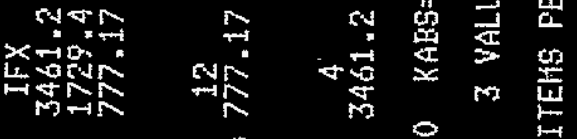

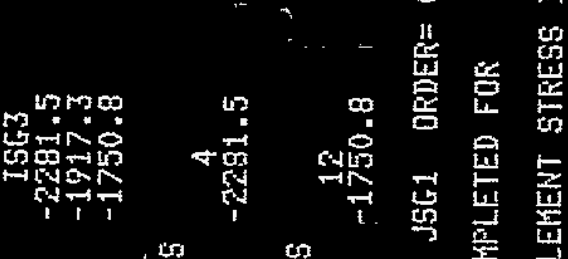

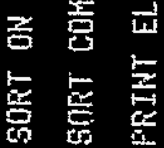


FHSYS - ENGIHEERIHG ALLLYSIS SYSTEH FEYTSIOH 4.4

$987.1989,1970^{32707}$ SWANSON ANALYSIS SYY 1990

作

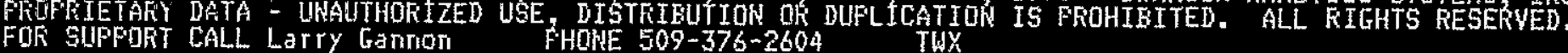

DST HOCK - IJF FLATFORH SEISMIC ANALYSIS DL +.06g:x $+.2 \mathrm{~g}:-\mathrm{z}$

\subsection{AUG $1,1995 \mathrm{CP}=\quad 1.270$}

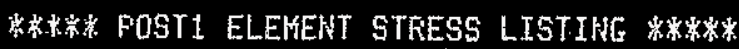

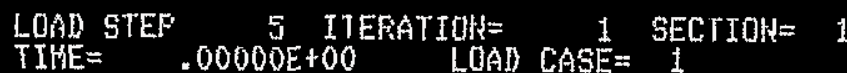

$\begin{array}{rr}\text { ELEM } & \text { J5G } 1 \\ 5 & 2198.7 \\ 7 & 1930.2\end{array}$

J5t JFX JFY

7
11
17730.2

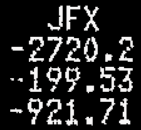

JFY

$-156.17$

15415

31.519

JHX $.00000 \mathrm{E}+00-781 \mathrm{JHY}$

$.00000 E+00$ 1.295.5

.00000E $+00 \quad 1379.5$

33199

$-161.12 \quad 32,173$

HINIMLIHS

ELEYENT

11173

$-2720.2$

$-250.84$

15.415

$.00000 E+00-781.87$

20960

Hax XI14

ELEYEN
VALUE:

2158.7 -

$-156.19 \quad 3 \frac{11}{32.173}$

$.00000 E+00 \quad \frac{11}{1379.9}$

33189.

SOKT ON JSE 3 OKDER $=0$ KABS $=1$ KHAX $=3$

SORT COMFLETED FOR 3 VALUES.

FRIRT ELEMENT STRESS ITEMS PER ELEMENT 
AHSYS - EHEIFEERIUG AHALYSIS SYETEH REYISTON 4 A A1372 32207 HAY 1,1990

AMSYS(F) COFYRIGHT(C) $1971,1978,1982,1983.1985,1987,1989,1990$ SWANSOK ANALYSTS SYSTEMS, INC. AS UNFUELISHED WOEK,

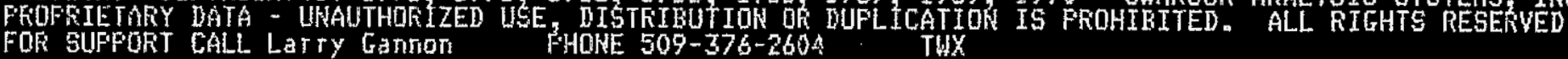

DST HOCK-UP FLATFORH SEISMIC ÁNALYSIS DL +.06g:x $+.2 \mathrm{~g}:-\mathrm{z}$

15.1264 AUE $1.1995 \mathrm{CF}=\quad 1.280$

***** FOSTI ELEHERT STRESS LISTIKG * $^{*}$ ***

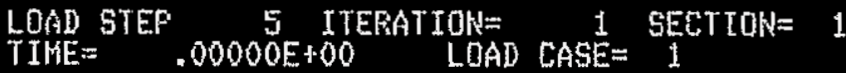

\begin{tabular}{|c|c|c|c|c|c|c|c|}
\hline LEK & $\begin{array}{r}\text { JEG } \\
-3168.9 \\
-3125.5 \\
-2087.9\end{array}$ & $\begin{array}{c}\text { JFX } \\
-4286 \cdot 1 \\
-2720,2 \\
-921.71\end{array}$ & $\begin{array}{l}\text { JFY } \\
-225.74 \\
-250.84 \\
-161.12\end{array}$ & $\begin{array}{l}J F Z \\
14.763 \\
15.415 \\
32.173\end{array}$ & $\begin{array}{l}\text { JHX } \\
.00000 E+00 \\
.00000 E+00 \\
.00000 E+00\end{array}$ & $\begin{array}{l}\text { JWY } \\
-866.02 \\
-791.97 \\
1379.9\end{array}$ & $\begin{array}{l}\text { J42 } \\
29952 . \\
33189 . \\
21616 .\end{array}$ \\
\hline
\end{tabular}

MINTMUHS

$\begin{array}{lcccccc}\text { ELEMEHT } & 3 & 3 & 3 & 3 & 3 & 3 \\ \text { VALLLE } & -3168.7 & -4286.1 & -250.84 & 14.763 & .00000 E+00-866.02 & 21616 *\end{array}$

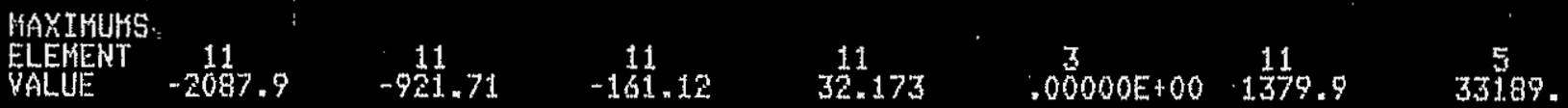

ESEL 'FOR LABEL= ELEH FFOM i2 TO $12 \mathrm{BY} \quad 2$

\& ELEMENTS (OF 99 DEFIMED) SELECTED BY ESEL COHMAND.

PRINT ELEHENT BTRESS ITEHS FER ELEHENT 
ALSYE - EHGINEERING AHALYEIS SYGTEH REYISIOH 4.4

A13\% 32207

HAY 1,1990

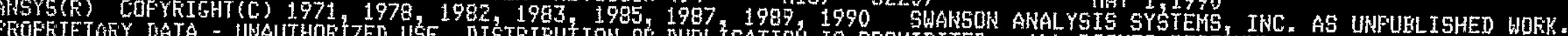

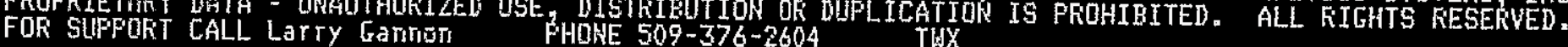

DST HOCK-UP PLATFORH SEISMIC AHALYGIS DL $+.06 \mathrm{~g}: \mathrm{x}+.2 \mathrm{~g}:-\mathrm{z}$

****** FOST1 ELEMENT STRESS LISTIHG *****

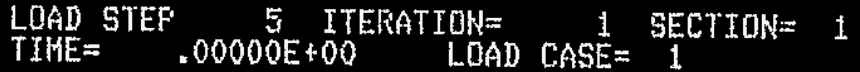

ELEH
2
4
6
8
10
12
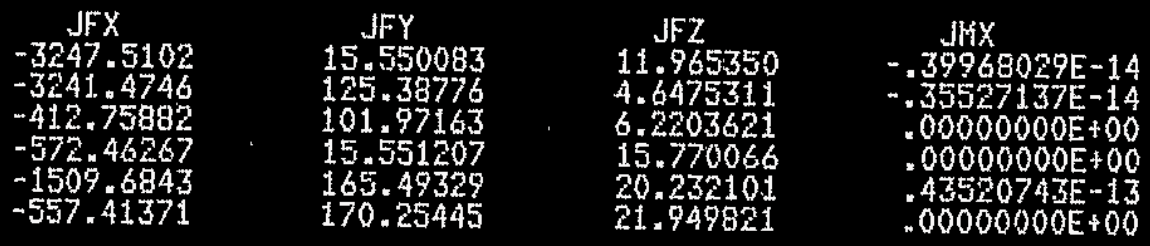

7 IHY

$.79580786 \mathrm{E}-12-121210855 \mathrm{E}-11$

-3273736 E $-12-32343905 E-10$

- $116064 \mathrm{E}-12$. $1273801 \mathrm{SE}-11$

$-.45474775 \mathrm{E}-1$ - $13130050 \mathrm{~K}-10$

$-.11368684 \mathrm{E}-12 \quad-.46895821 \mathrm{E}-10$

HILIHUHS

ELEYAENT

$-3247.5102$

$15.2_{5}^{5} 0083$

$\stackrel{4}{4} .6475311$

$-.39968029 E-14$

10

IAAXIHUHS

ELEMENT

$-412^{6} .75882$

1.70 .25445

$\frac{12}{21.749821}$

.4352

ESEL FOR LABEL = TYPE FROH

4 TO

4 BY

1

24 ELEMENTS (OF 99 DEFINED) SELECTED BY ESEL COHHAND.

$D$ SOFT GN ISGI ORDEF $=0$ KABS=1 NMAX $=3$

1 SORT COHPLETED FOR 3 VALUES.

$\sqrt{1}$

FRTHT ELEHENT STRESS ITEMS FER ELEHENT 
AHYYS - EHGIUEERTHG AHALYSIS SYSTEH REVISIOH 4.4

A137 32207

HAY 1,1990

AWSYS(F) COFYKIGHT(C) $1971,1978,1982,1983,1985,1987,1999,1990$ SWANSON ANALYSIS SYSTEHS, INC. AS UNFURLISHED WDKK.

FFGFFIETAFY DATA - LWAUTHORIZED USE, DISTRIEUTION OR DUFL ICATIOK IS FROHIBTED. ALL RIGHTS RESERVED.

DST HOCK-UF FLATFOFH SEISHIC ANALYSIS DL $+.06 \mathrm{~g}: \mathrm{x}+.2 \mathrm{~g}:-\mathrm{z}$

15.1264 AUG $1,1995 \mathrm{CP}=\quad 1.290$

****** POST1 ELEHENT STRESS LISTING $*$ ****

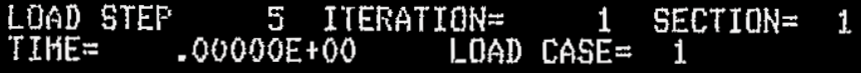

$\begin{array}{rr}\text { ELEH } & \text { IS61 } \\ 99 & 25800 \\ 97 & 35604\end{array}$

IFX
-95.919
390.78

IFY IFZ

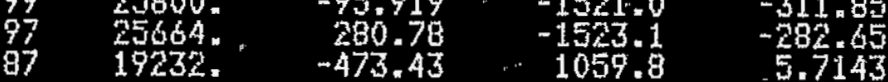

IKXX

63.635

$-32.683$

Iny

3590.3

$\frac{167}{47702}$
-47703

HININUKG

ELEFENT

87.987 .43

97
-1.523 .1

311.85

$\begin{array}{cc}87 & 87 \\ -32.683 & -130.31\end{array}$

97
-47703

KAXIKUHS

(9)

VALUE

25800

97
280.78

87
1059.8

57.7143

$\frac{77}{152.92}$

6790.3

87

SQRT ON ISG 3 ORDER $=0$ KAES $=1$ NHAX $=3$

SORT COHPLETED FOR 3 WALUES.

PRINT ELEHENT STRESS ITEHS PER ELEMENT 
$\stackrel{8}{8}$

哭

$\sum_{i=1}^{\infty}$

音

때대

$x_{n \rightarrow 1}$

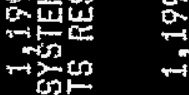

$>2$

皮证

在

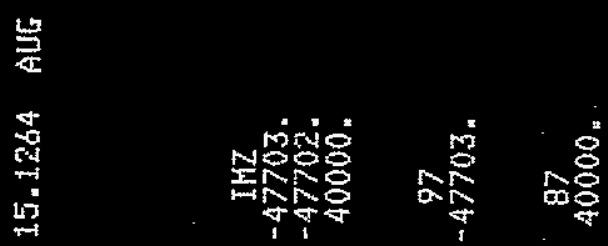

8

蛋

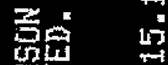

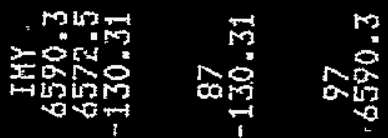

今䖵

的

$0^{2}$

and

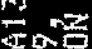

몬존

단

$\Rightarrow$ 항

$\lim _{x \rightarrow 0}$

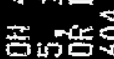

tho

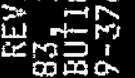

$0 \rightarrow 0$

蒠象的

tons

以s

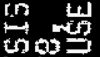

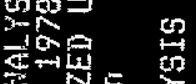

必溇昆

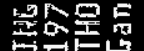

昰的展

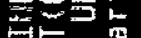

동,

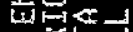

宕转

n형든

$\rightarrow$

旁

政要

믈

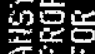

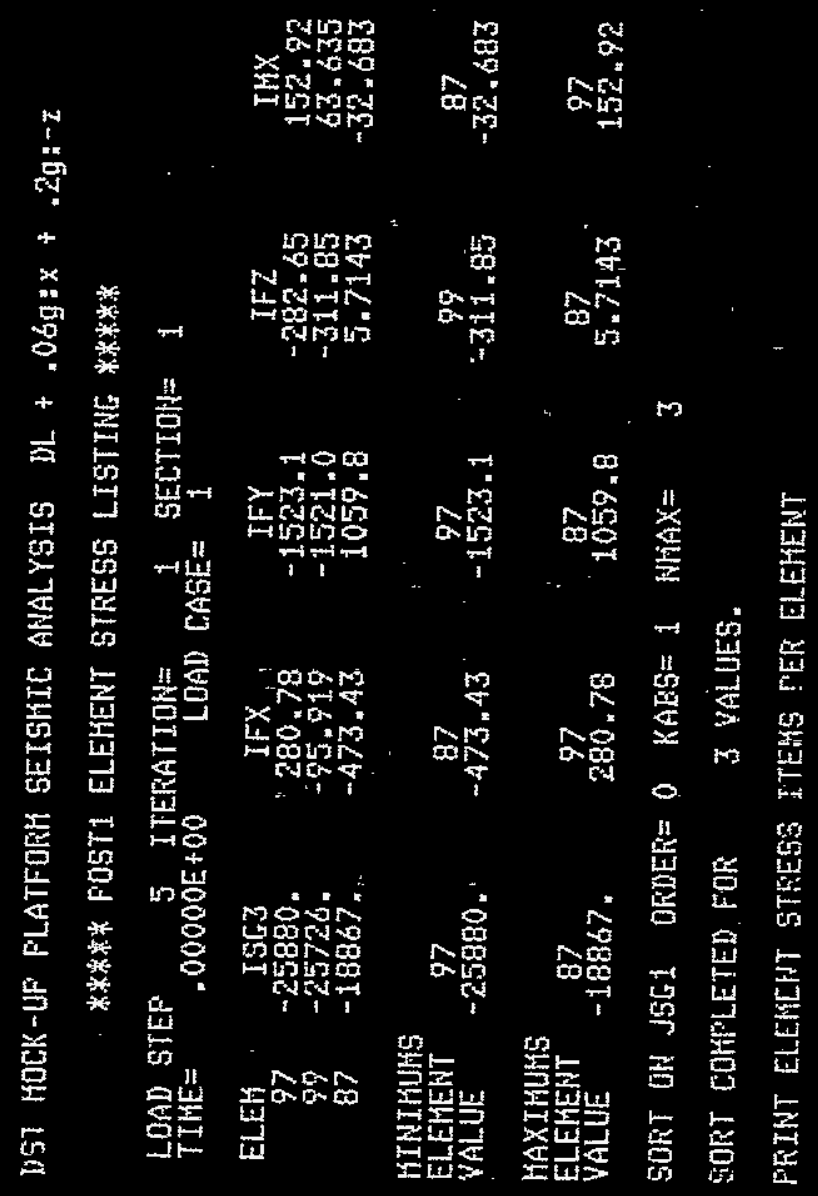




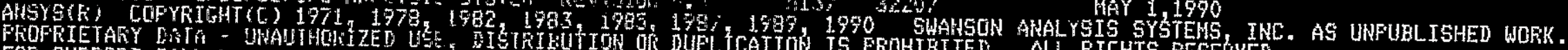

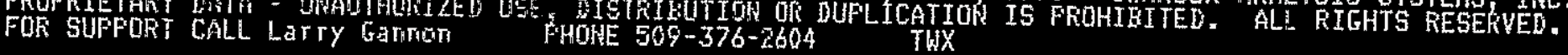

DST HOCK-UF FLATFORH SEISHIC AHALYSIS DL +.06g: $\mathrm{x}+.2 \mathrm{gt-7}$

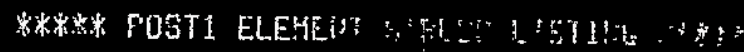

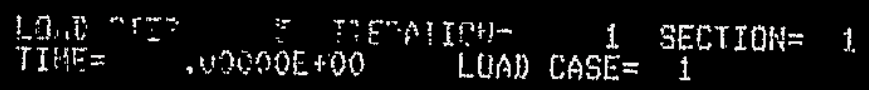

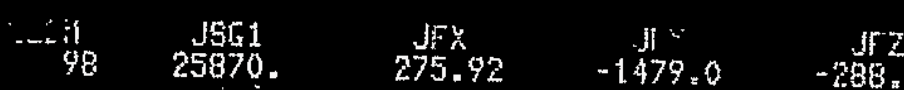

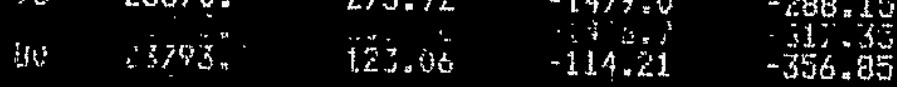

MINTHUKS

ELEHENT

$\begin{array}{ll}90 & 90 \\ 23793 . & -100.78\end{array}$

$\begin{aligned} & 98 \\ -1479.0 & -14\end{aligned}$

$-350.85$

HAXINUHS

ELEKENT

258

25670.

278.92

80

$-1.14 .2$

SORT OK JSGZ ORDER $=0$ KABS $=1$ WHAX $=$

98
-289.1 .5

SORT COHFLETED FOF

3 VALUES.

FRINT ELEHENT STRESS ITEHS PER ELEHENT

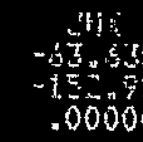

$-41 Y$
-6572.5
-6590.3

9590.3

JMZ

47703 .

12116.

$\begin{array}{rrr}96 & 90 \\ -152.92 & -37907 . & 1216 .\end{array}$

$80000 E+00-6572.5 \quad 97703$ 
ANGYS EHGINEETIHG AHALTSIS SHCTEH REUISIOH 4,4

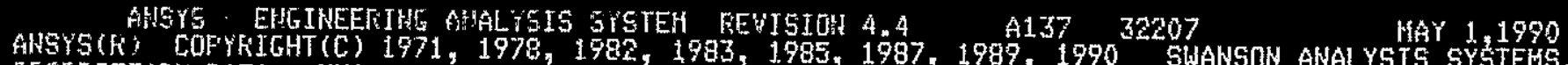

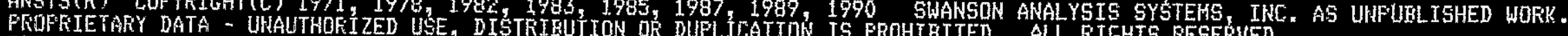
FOR SUPFUR DALL LaTry Gaminon FHONE 509-376-2604 TWX IS FROHIBITED* ALL RIGHTS RESERVED.

DST HOCK-UF FLATFOFH SEISHIC ANALYSIS DL $+.06 \mathrm{~g}: \mathrm{x}+.2 \mathrm{~g}:-\mathrm{z}$

****** POST1 ELEHENT STRESS LISTING $*$ *****

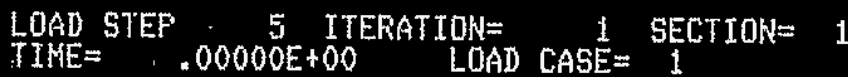

ELEF

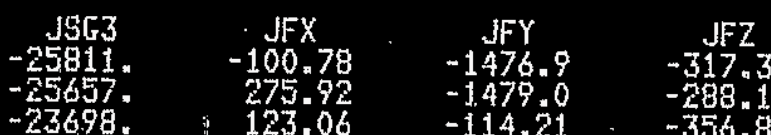

MIUITUHS

ELEMERT

VALIIE

$$
\begin{aligned}
& 96 \\
& -25811
\end{aligned}
$$$$
-100.78
$$

$-1479.0$

$\begin{aligned} & 80 \\ &-356.85\end{aligned}$

$-1.52 .72$

80
-37907

12116.

MAXIHIJHS

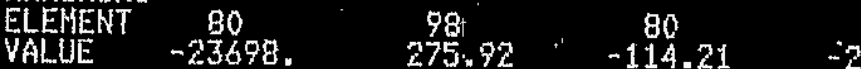

ESEL FOR LABEL = ELEH FROM 88 TO $99 \mathrm{EY}$
12 ELEHEHTS (OF
79 DEFIHED) SELECTED BY
ESEI

$.00000 E+00-6572.5$

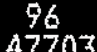

FRINT ELEMENT STRESS ITEHS PER ELEHENT 

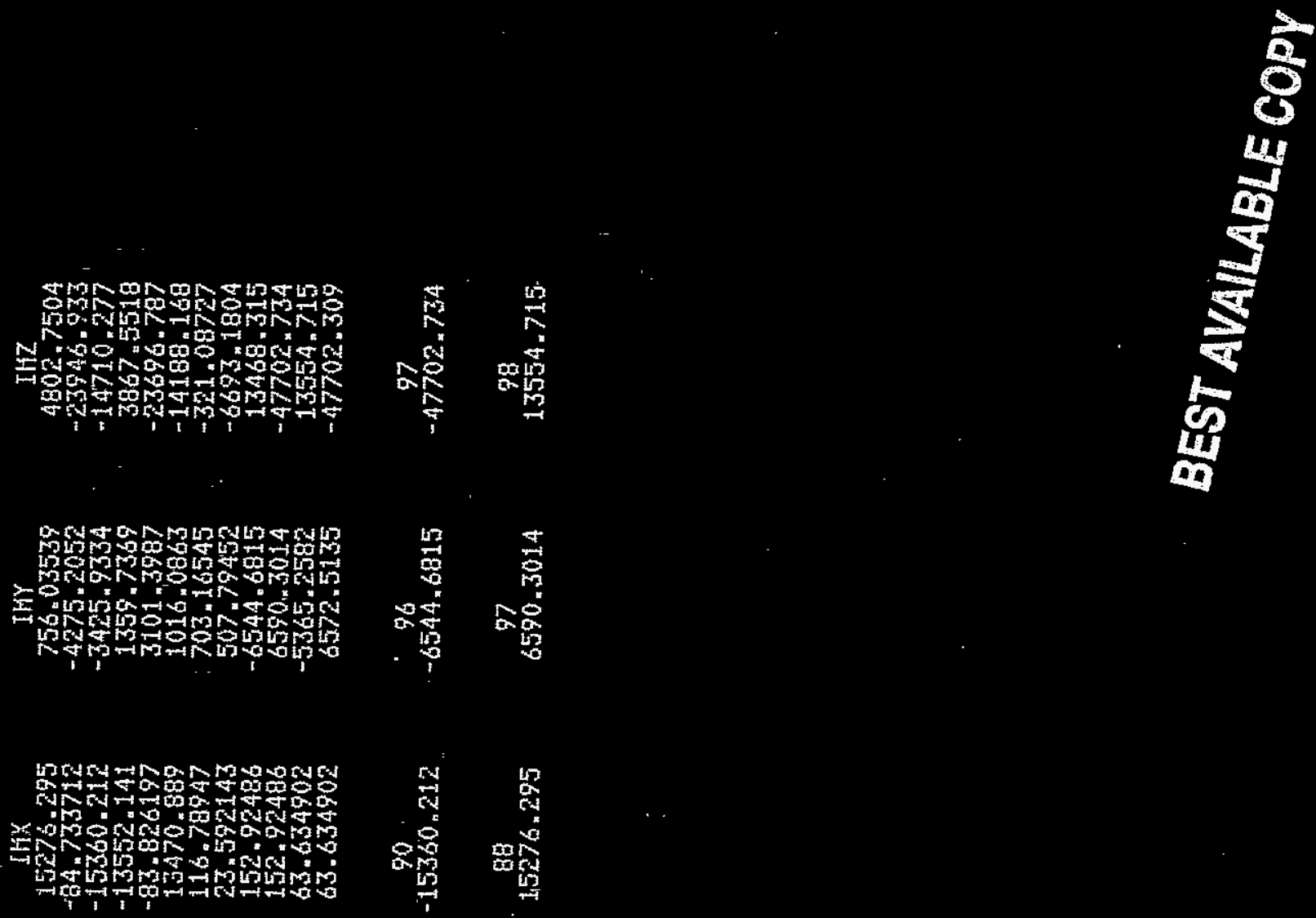

$\infty$

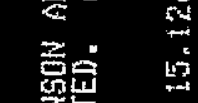

感

舟

悹里

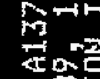

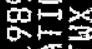

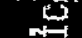

$\phi \alpha$

$+40$

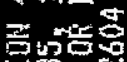

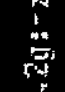

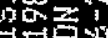

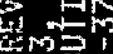

恕乘哭

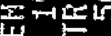

- $\quad$ mary

He

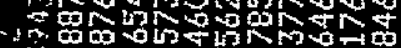

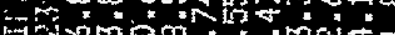

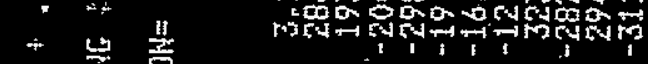

离

$\cos ^{-1}$

thoses

$\sum_{x \rightarrow \infty}$

$\sin ^{-1}$

낸돈

$\sum_{i=1} a_{i=1}$

证

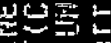

管:

너는

, 焉承

48

焉

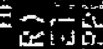

con

og

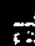

焉

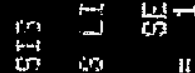

留

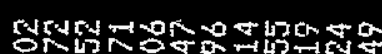

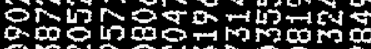

- N

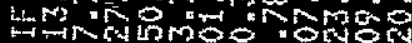

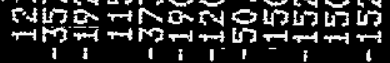

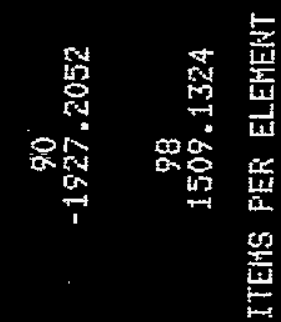

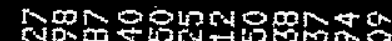

-

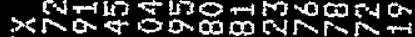

him

가의

菏

to

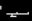

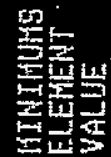

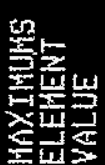

要 


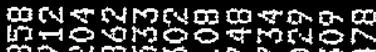
कD

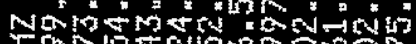
与on 은

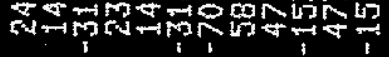

要

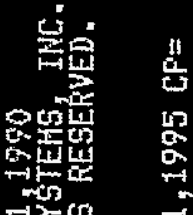

in

妾

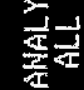

范

为

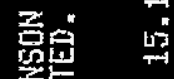

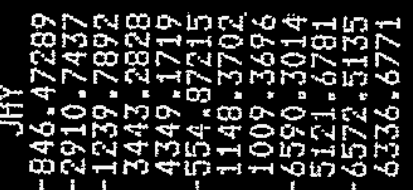

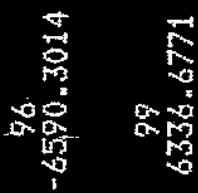

in

$\rightarrow$

atic

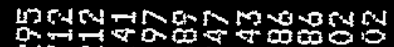

and

Xpo

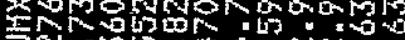

As

as

$\sigma_{n \rightarrow 1}$

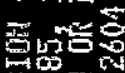

ìd

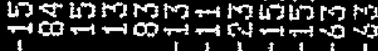

然

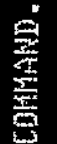

的它的

$\rightarrow \operatorname{lin}^{2}+$

wis

요요

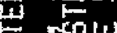

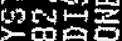

的政

$\cos ^{-14}$

$\log ^{2}=$

露

$\lim _{\rightarrow+\infty}$

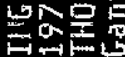

芭司

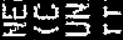

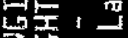

空

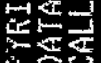

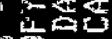

密喜

踏

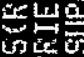

vaca

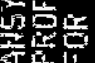

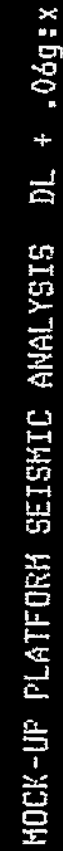

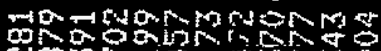

ano

Romoncombsans

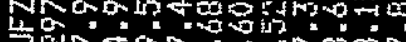

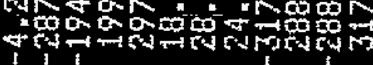

要

$8 \quad 8 \quad \frac{d}{6}$

$40050=04040$

-1

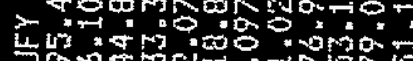

$7 a$ a mad

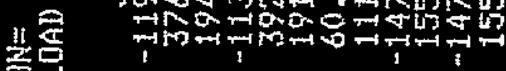

的焉

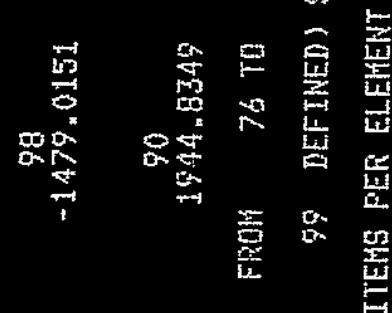

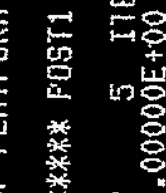

要

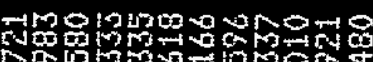

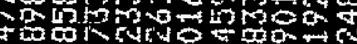

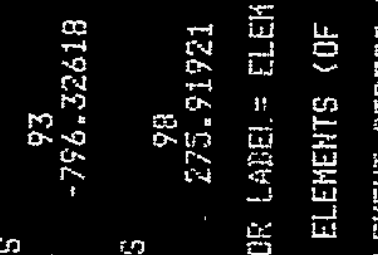

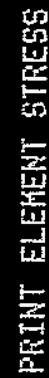

$A-180$ 


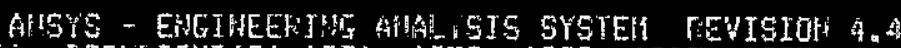
$413732 \% 07$ HAY 1,1790

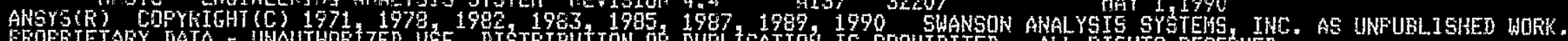

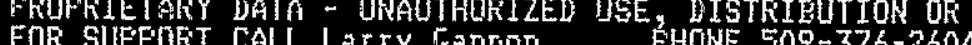
TWX

DGT HUCK-UP FLATFOFH SEISHIC AHALYSIS DL +.06g: $+.2 \mathrm{~g}:-\mathrm{x}$

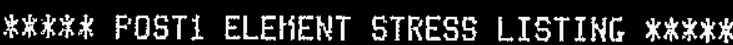

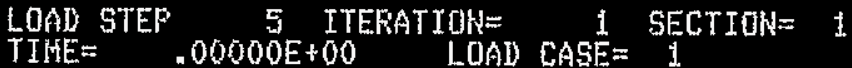

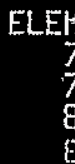

$\begin{array}{ccc}\text { IFX } & \text { IFY } & \text { IFZ } \\ 756.41837 & 94.384796 & 370.34088 \\ 3503.0353 & 92.189570 & 379.69098 \\ -46.664194 & 1.18 .79083 & 372.13150 \\ 26.9 .7314 & 115.97647 & 332.35091\end{array}$

HIXIMUIS?

ELEHENT

VALLUE

80
$-46+664154$

78
72.186570

327.68098

IHX

$.00000000 E+00$

$.00000000 E+00$

$.00000000 E+00$

$.00000000 E+00$

$-17791990 \mathrm{E}-10$

$-1051.6032 E-10$

$-10516032 \mathrm{E}-10$

$-.86970431 \mathrm{E}-11$

IHZ

(1817218E-11

$045 \mathrm{~F}=1 \mathrm{E}-11$

$.25437430 \mathrm{E}-11$

HAXIHUHES

ELEHEK

78
3503.0353

80
18.79633

$\frac{80}{3 i 2 n+300}$

76

76
$-17791990 E-10$

82

.00000000E+00

$25437430 \mathrm{E}-11$

ESEL FOR LABEL= TYFE FROK

570

5 BY 1

11 ELEHEHTS (OF 99 DEFINED) SELECTED BY ESEL CUHHAND.

SURT ON ISGI OFDER $=0$ KABS= 1 HHAX $=3$

GORT COKFLETED FOR 3 VALUES.

FRINT ELEHENT STRESS ITEHS PEF ELEHENT

X 


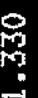

눈

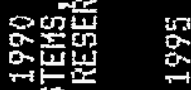

$\rightarrow$

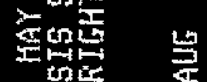

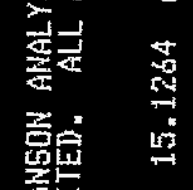

装

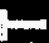

P

$n$

亭学

욜

的的

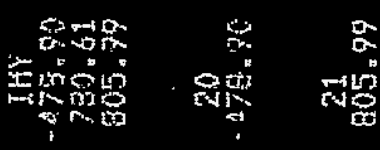

단돌

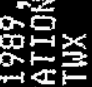

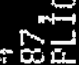

舟要

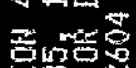

管

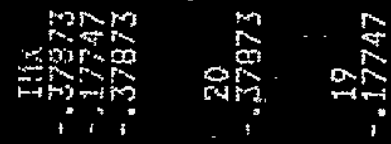

加要

$=m$

of.

$=7-2$

等

$\tan _{x \rightarrow 2}$

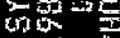

co

$\operatorname{tin} 2$

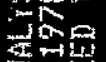

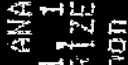

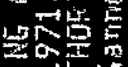

5

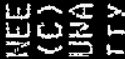

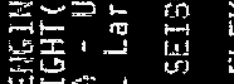

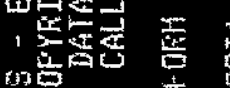

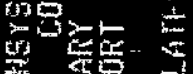

난

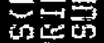

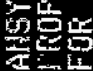

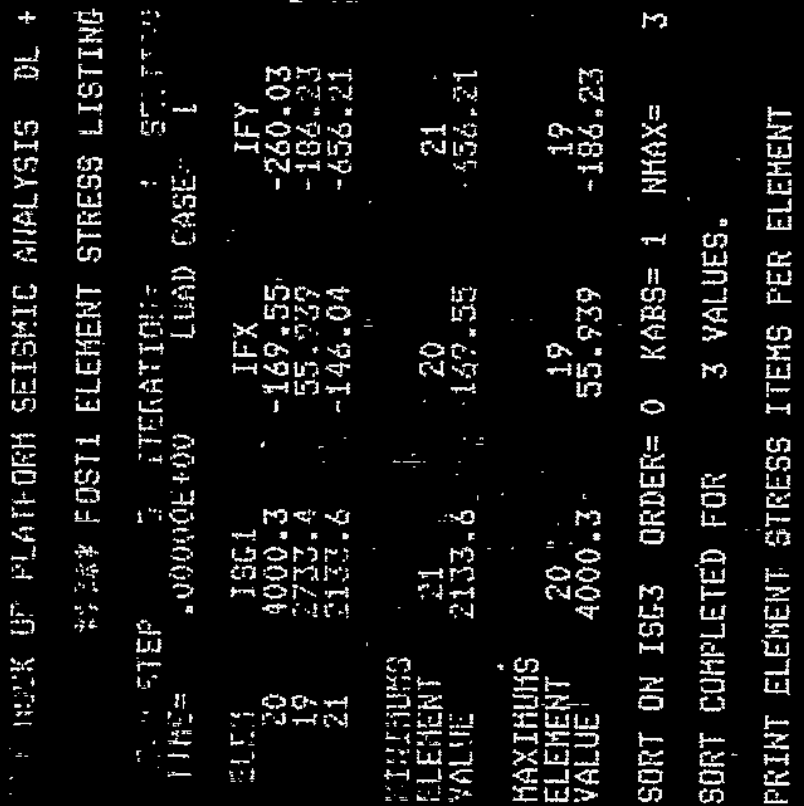

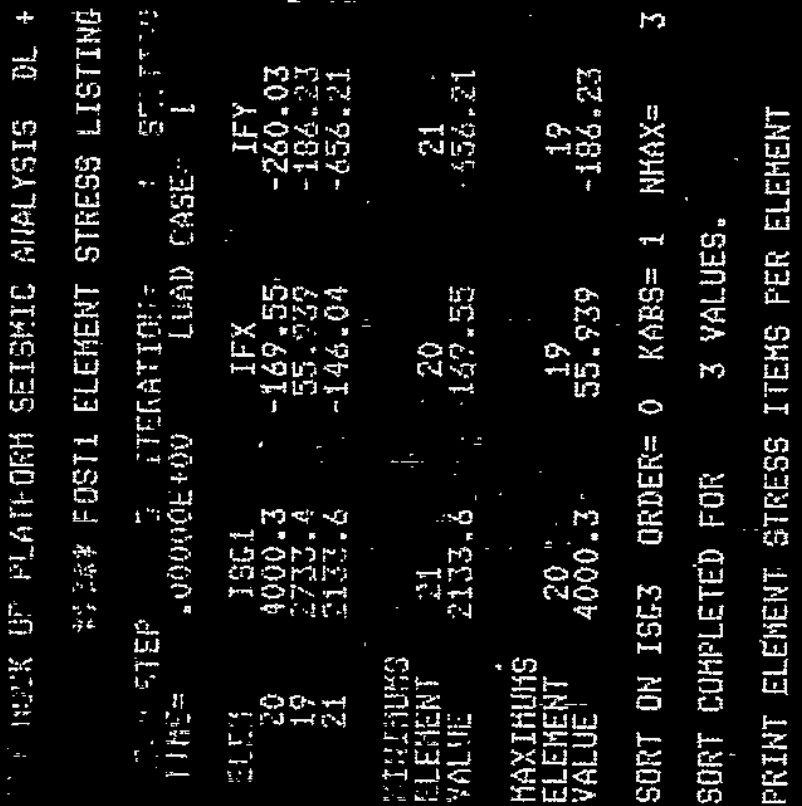


?

密离

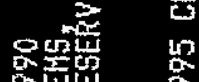

ind

mon

的话

点

空娄

不

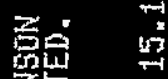

告

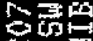

婇

욤

intin

急象

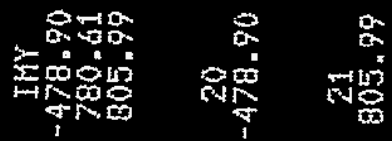

마말

是

$x$

$\rightarrow$

䆓语电

thas

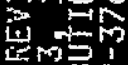

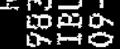

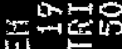

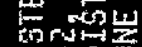

跔要

$\operatorname{lom}^{-1}$ mi

ติำ

$-5$

管岛

車点色

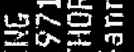

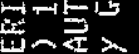

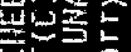

도엉

ist

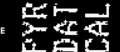

u竞然

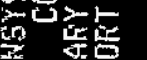

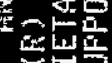

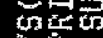

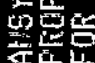

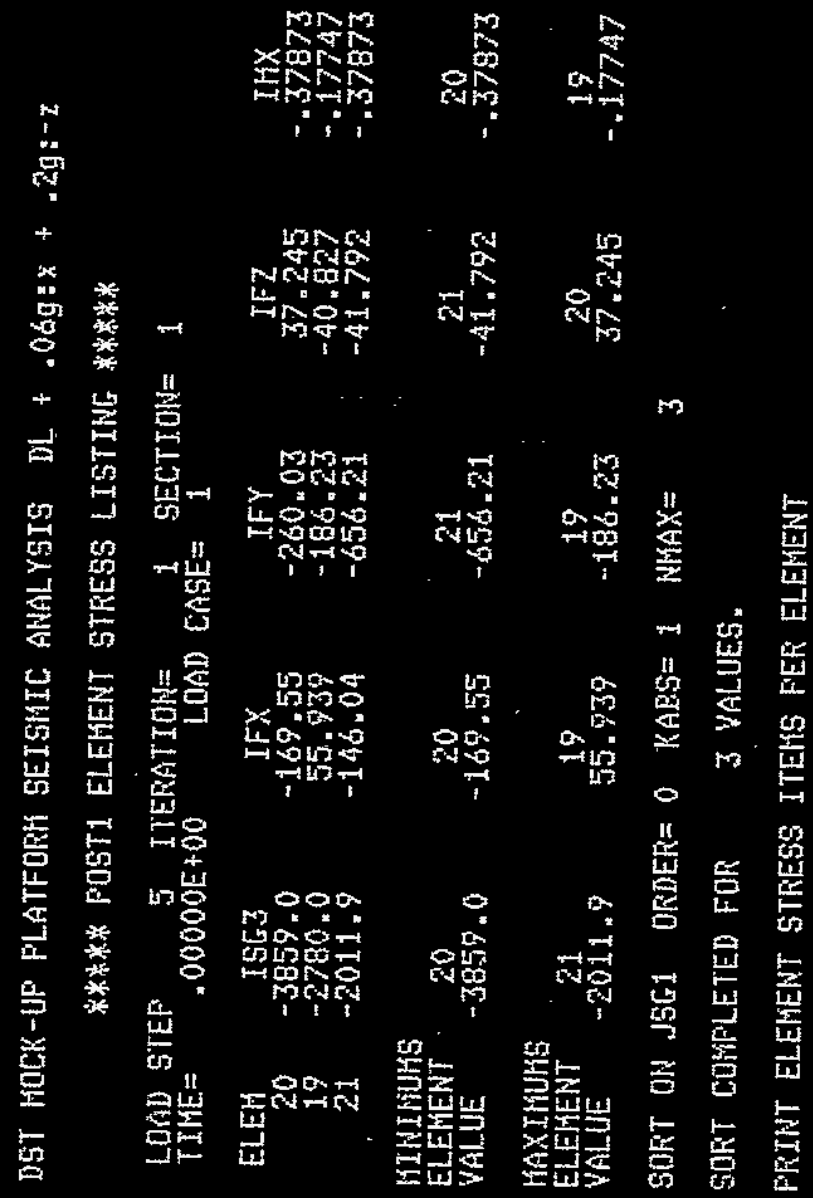


N

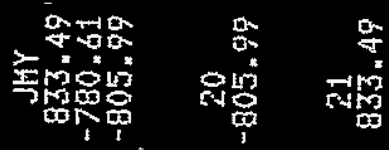

인준

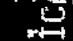

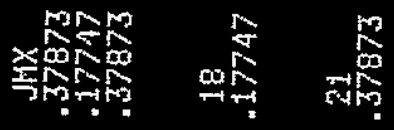

的要

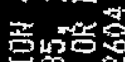

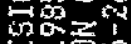

남

o횽.

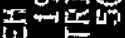

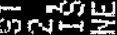

20훙

${ }_{3}{ }^{2}$

苟岁

管年

蛋梁

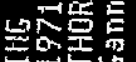

些定入

온돈돈

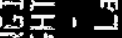

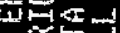

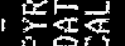

至四

出它

此背竞

ine

울돈

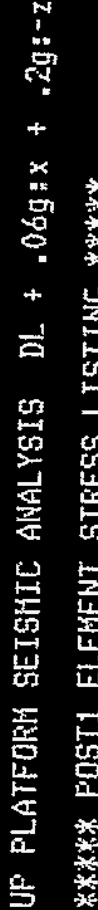

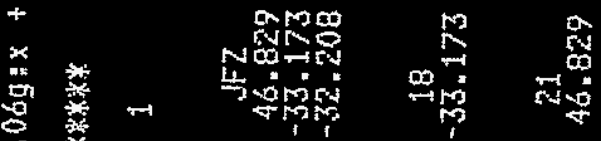

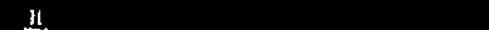

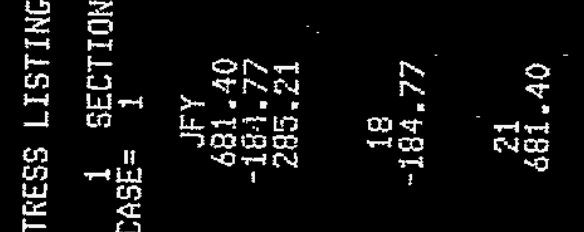

莣

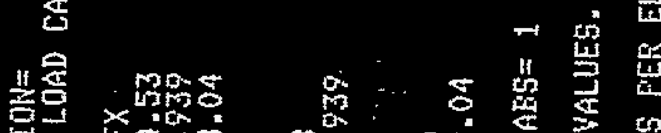

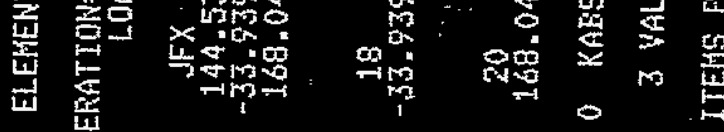

点

F

产

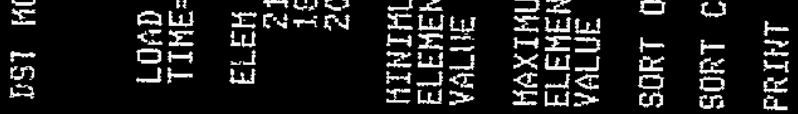

$\stackrel{\text { 湈 }}{6}$

mingas on

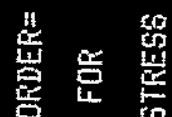

8 다

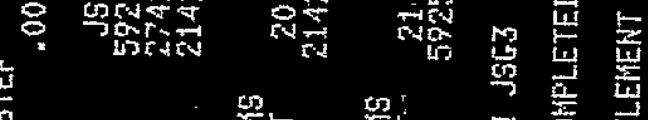

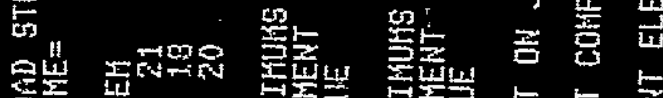




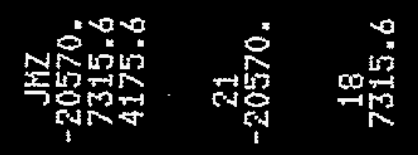

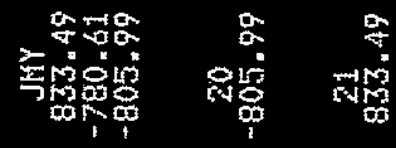

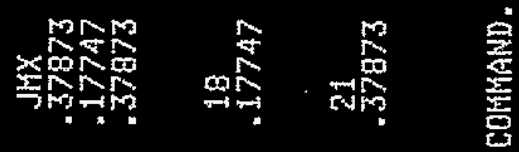

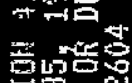

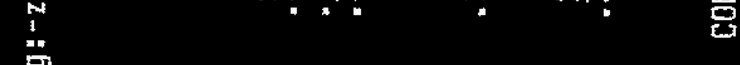

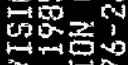

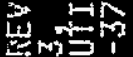

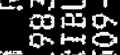

要然证

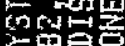

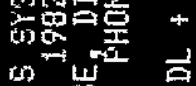

要菢

架留

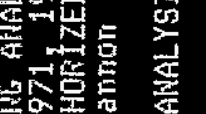

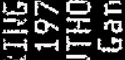

造一

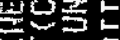

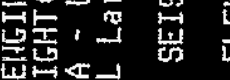

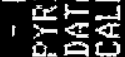

요의.

繁

可器

证是的

触量

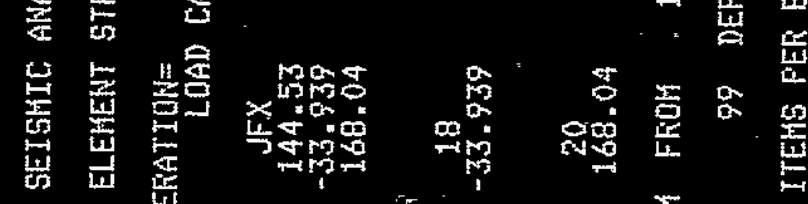

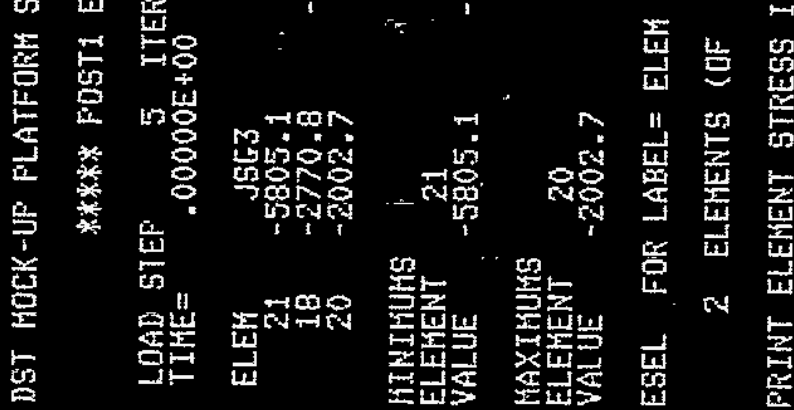


魚

tg

这望

oxf

g.

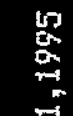

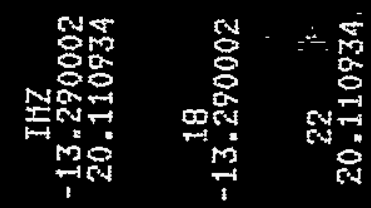

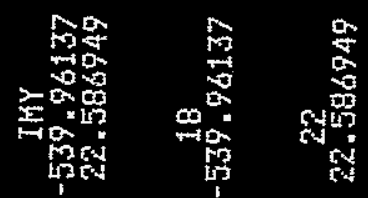

为

욤

लिखि

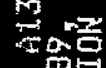

思此

负

$\underset{8}{8}$

$8+5$

唯

공

,

문당

i⿱

恕紫

대눈

ton -

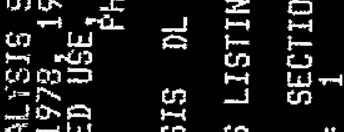

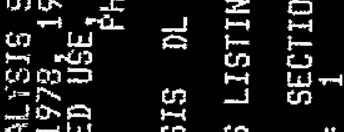

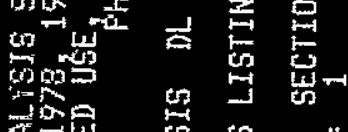

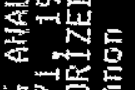

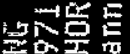

근연

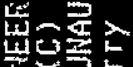

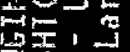

约地

. 紫焉

n두요

㧘管

或晋

ए人一叩

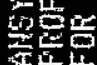

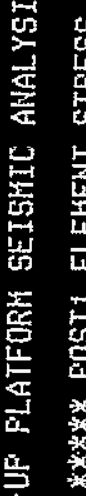

这

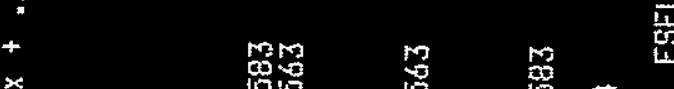

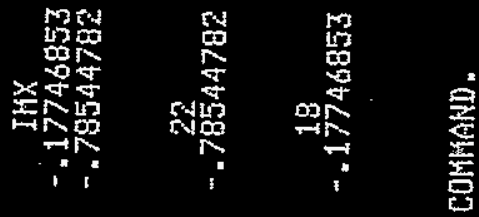

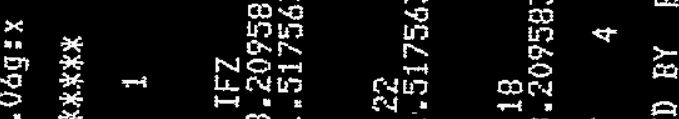

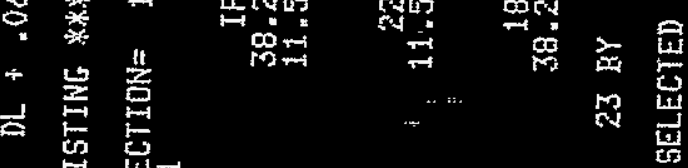

总

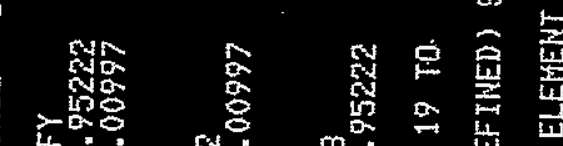

在 눈

总 的

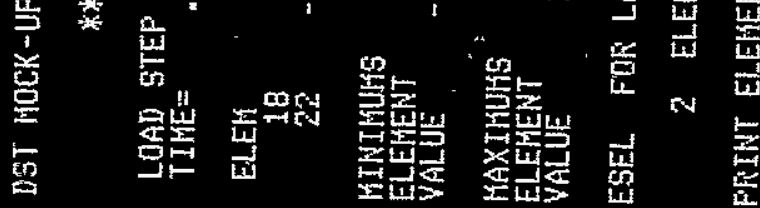

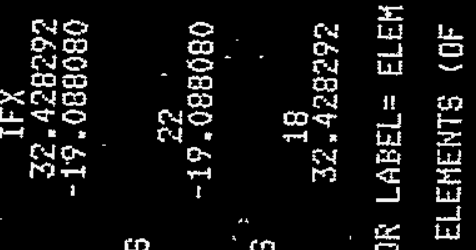

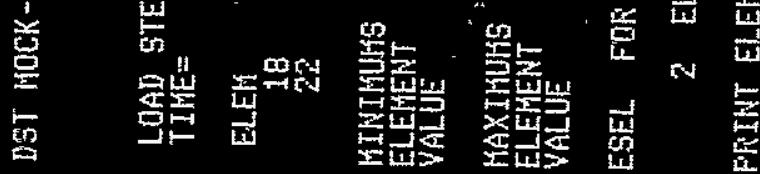

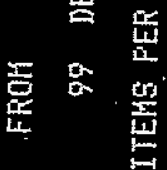


$m$

踏

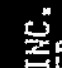

2

a

-150

雪的要

순

崖

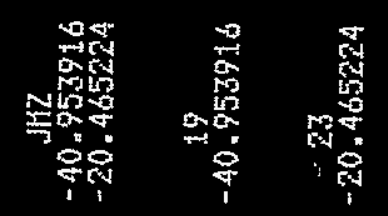



总

正

ma

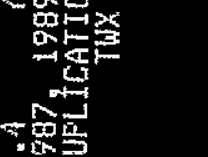

+4

声转定

品要,

in

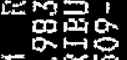

击

50

is

Sin

yage

正焉

논

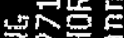

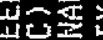

는

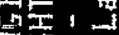

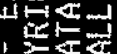

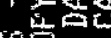

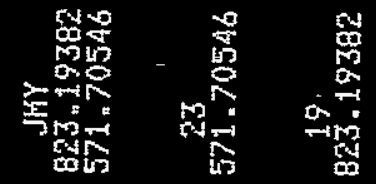
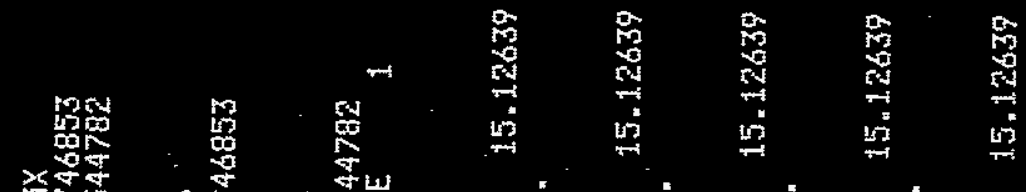

0
0
0
0

密

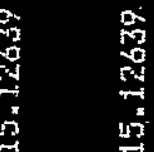<smiles>C1CCCC1</smiles>

然弯

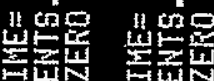

紫定

竞密

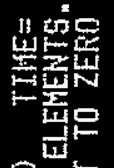

보룰

离的

商p

勇吊

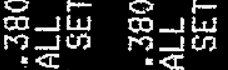

品指

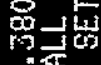

品出 0

我品

章的

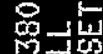

密

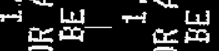

些出

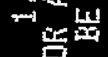

-

$=-1$

$\rightarrow-1$

렬

田

욜

词

可

it

量密

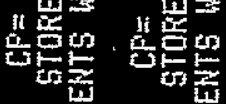

1

부요

, 보을

$\therefore$ 崖

눈

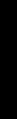

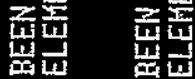

勇趟

崖国

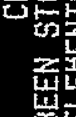

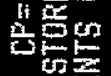

부롤

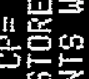

갰돈

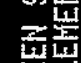

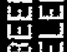

象㙂

占

군웅

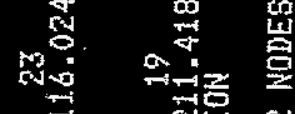

둔요

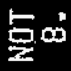

홍

计

包

$\rightarrow$ -

댄건

물

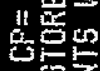

궀는

㪿

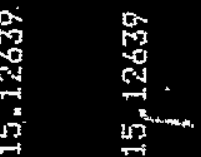

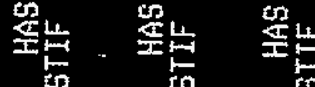

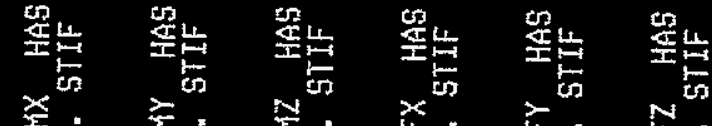

$N^{60}$

范

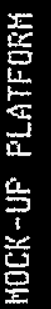

를ㅇㅇㅇ

8

मी

㩊传

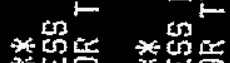

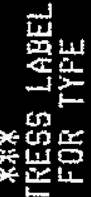

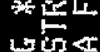

造地

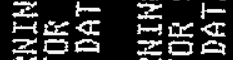

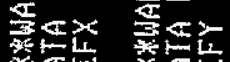

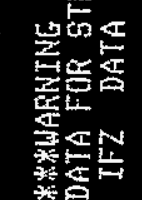

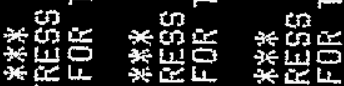

紫焉

*

는

물

홍

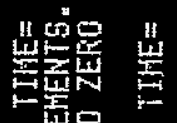

步早

嵒据

总

皆峲

멈

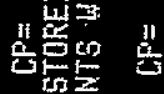

曹焉

恶四

$\infty^{*}$

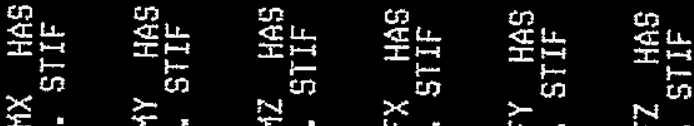

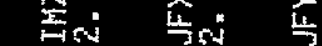

要

获点品

*

桃西

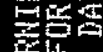

존지

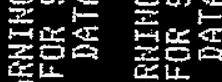

좇돈언

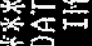

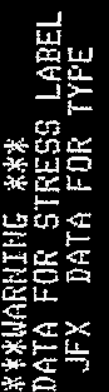

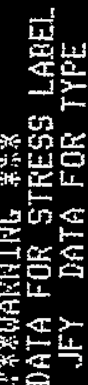

崖崖

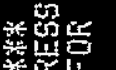

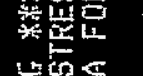

$$
A-1 \& 7
$$




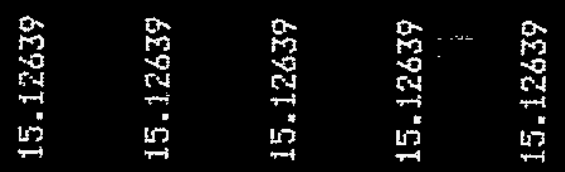

密里

ing

$\sin$

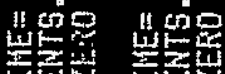

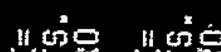

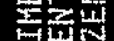

㟧

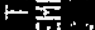

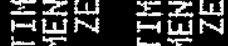

돈문

至

8 然 ${ }^{w}$

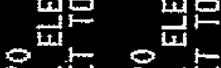

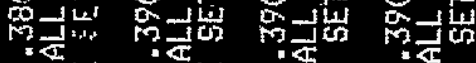

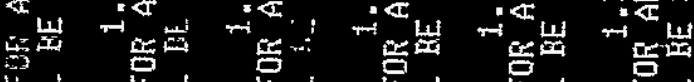

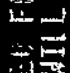

in

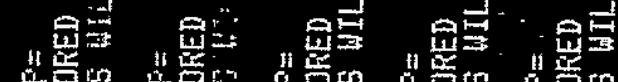

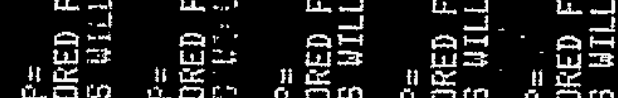

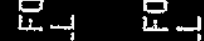

는

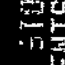

.

政

es

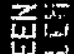

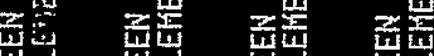

到

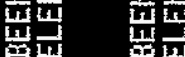

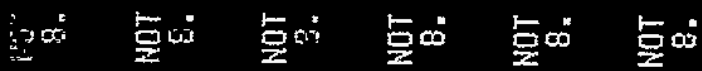

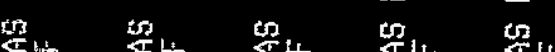

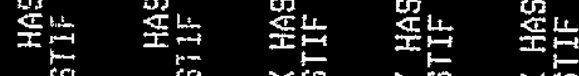

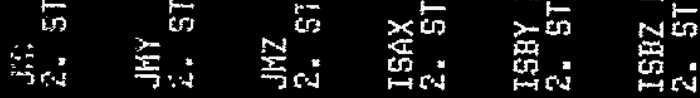

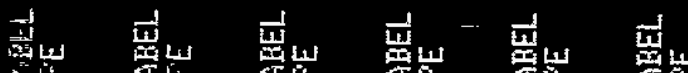

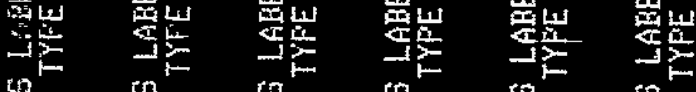

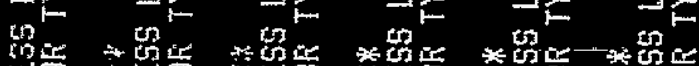

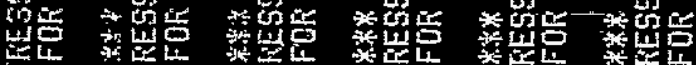

们

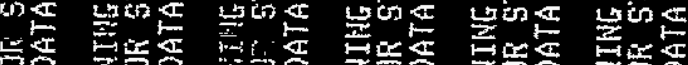

品 形品

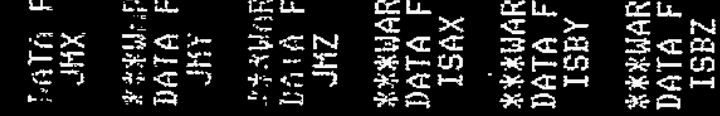


*ak FMIS View/Frint Document Cover Sheet tow

This document was retrieved from the Documentation and Records Manaqement (DRM) ISEARCH System. It is intended for Information only and may not be the most recent or updated version. Contact a Document Service Center (see Hanford Info for locations) if you need additional retrieval information.

Accession \#: D195067915

Document \#: SD-WM-ER-521

Title/Desc:

STRUCTRUAL ANALYSIS DESIGN \& EVALUATION OF MOCKUP PLATFORM MONORAIL \& TANK PLATE CUT OUT [SEC 3 OF 3]

Pages: 93 
This document was too large to scan as a whole document, therefore it required breaking into smaller sections.

\section{DOCUMENT NUMBER: $S D-\omega m-E R-521$}

\section{SECTION 3 OF 3}

TITLE:STrucTural Analysis Desigh: EValuation of mockup platform monorail I Tank PLaTe CUT-OUT

DATE: $12-19-95$

ORIGINATOR: I S. Hundal

CO:

RECIPIENT:

CO:

REFERENCES: 


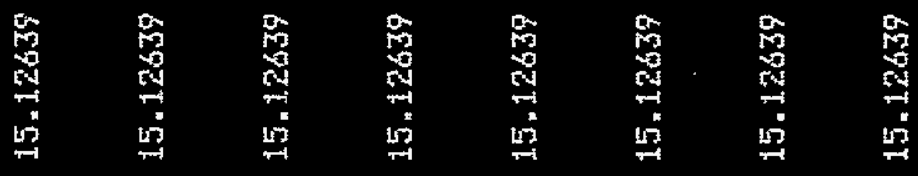

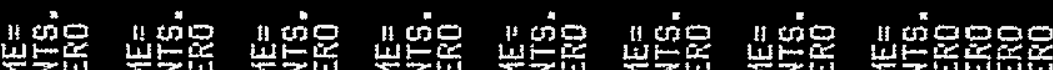

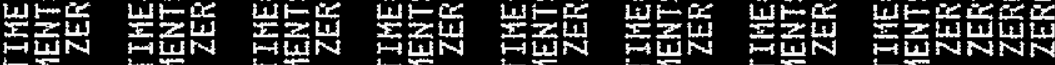

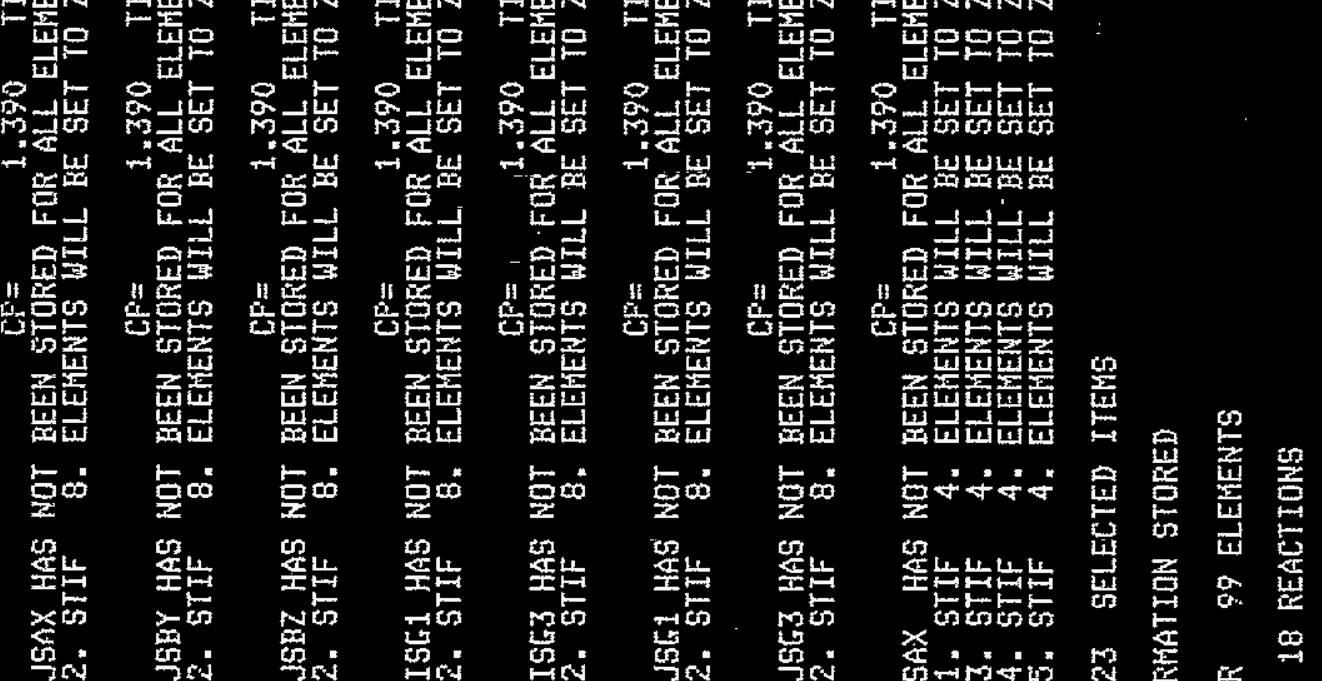

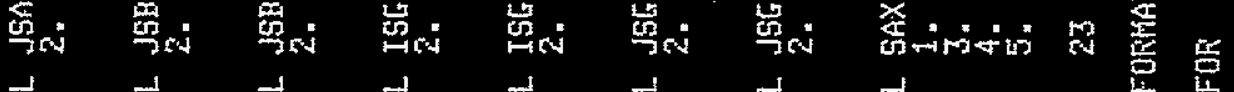

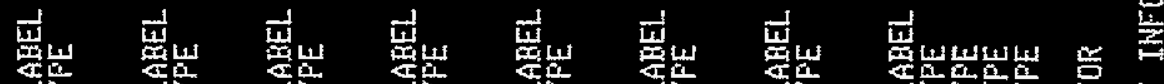

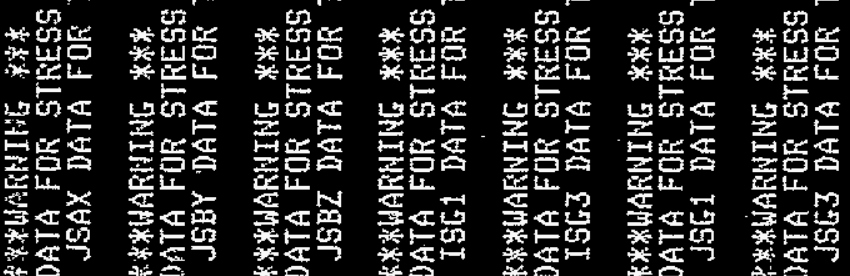

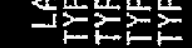

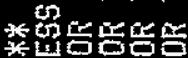

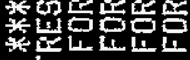

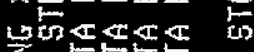

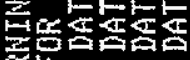

둔

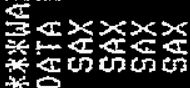

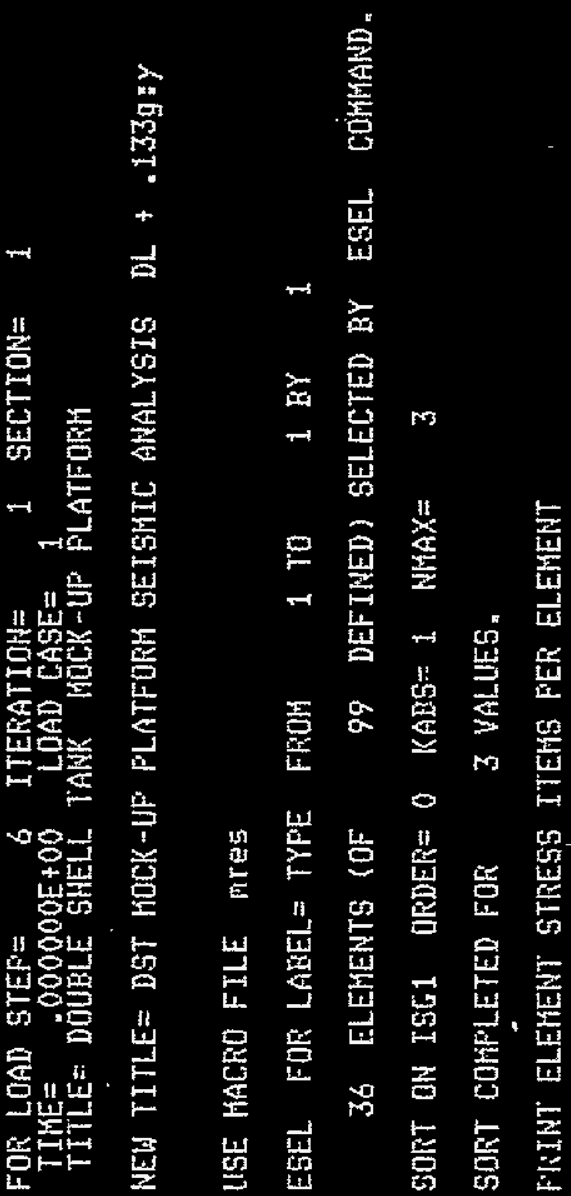

$A-189$ 
AWSYS - EHGIHEEETHG ANALYSIC SYSTEH FEVISION मे $^{2}$

A137 32207

HAY 1,1990

ANSYS(E) COFYRTGHT(C) $1971,1978,1982,1983,1985,1797,1999,1990$ SWANSON ANALYSIS SYSTEMS, INC, AS UNPUUELISHED WORK. FHOFFIETARY DATA - UHAUTHORTZED USE DISTRIBUT IDN OR DUFL ILATIO

DST HOCK-UP FLATFOFH SEISHIC AINALYSIS DL + .133g:y

15.1264 AUG $1,1995 \mathrm{CF}=$

1.400

******* FOSTI ELEHENT STKESS LISTIHG *******

LOAD STEF
TIHE $=\stackrel{6}{6}$ ITERATION=
LOAD
CASE $\frac{1}{1}$ SECTION= $=1$

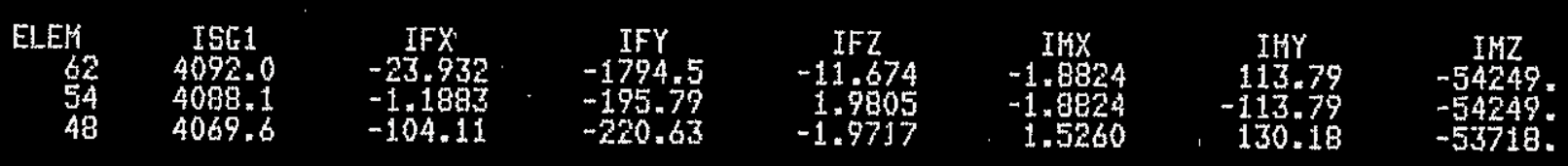

HINIKUMS

ELEHENT

VALUE

486

$-104.11$

$-1794.5$

62
-11.674

$-1.8824$

$-113.79$

$-54249$

ELETEE

$\frac{62}{4092.0} \quad-1.1893$

$-195.79$

1.94805

1.5260

130.18

48

SORT ON ISIS OKDER $=0$ KABS $=1$ NHAX $=3$

SORT COMPLETED FOR . 3 VALUES.

FRINT ELEKENT STRESS ITEHS FER ELEHENT 


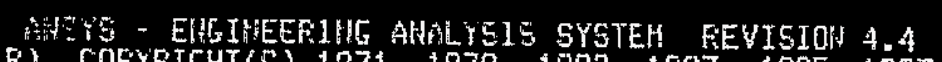

013732207

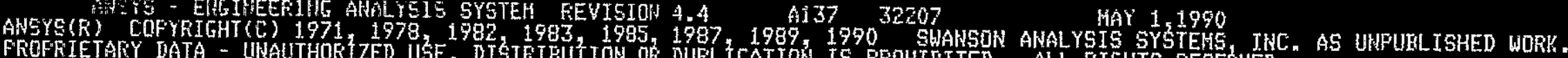
FDF SLFFORT CALL LarTy GannON USE, DISTEIEUTION OR DUFLICATION IS FROHIBITED. ALL RIGHTS RESERVED.

DST HOCK-UF PLATFERH GEISKIC AHALYSIS DL $+.13 \bar{g}$ g:

$$
15.1264 \text { Allt } 1,1995 \mathrm{CF}=\quad 1.410
$$

****** POST1 ELEKENT STRESS LISTING ******

LOAD STEF 0000 I ITERATION= $=1$ SECTIOK= 1

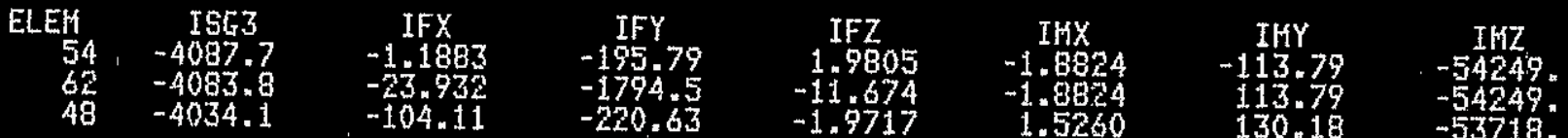

MTHTHUKS

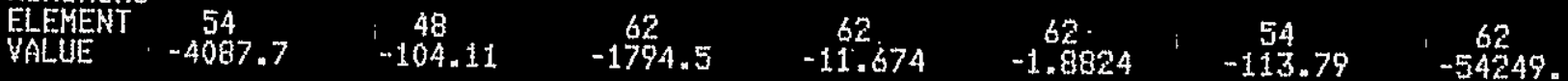

MAXIHULKS

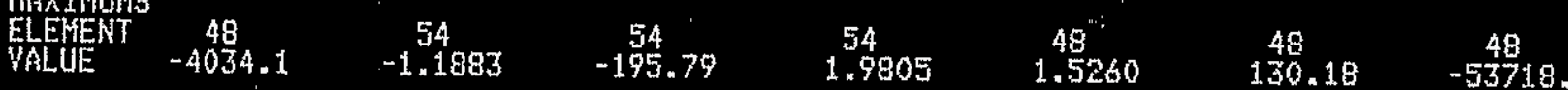

SORT ON JSGI ORDEF $=0 \quad$ KABS $=1$ NMAX $=3$

SORT COHFLETED FUR 3 VALUES.

PRINT ELEHENT STRESS TTEHS PER ELEHEHT 
晫

to

番监

步

80

at

$\rightarrow \operatorname{ton}^{2}$

는

近的焉

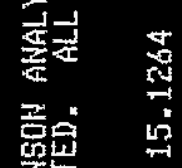

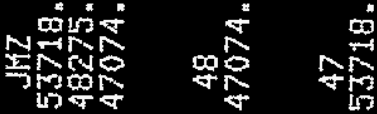

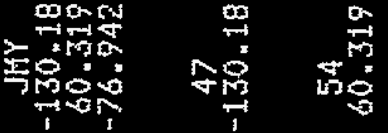

a

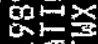

$\rightarrow$

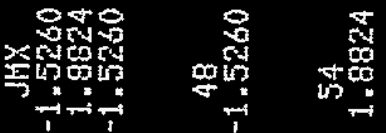

\&

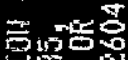

1

in

mas

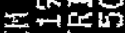

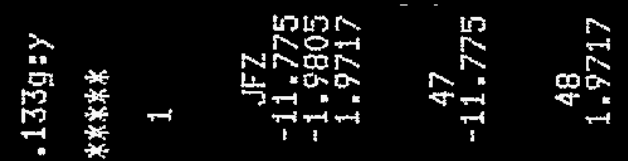

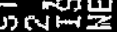

땜뭉

+ 崖焉

का

$\operatorname{trn}$

is

곤

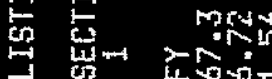

पर

$\int_{i}^{m}$ की

mat

象点夏

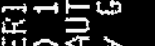

幽宾㱏

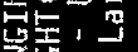

世넌

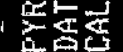

$5 \sum_{0}$

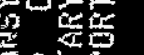

位至

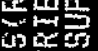

울은

案

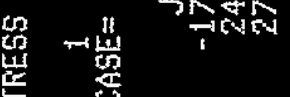

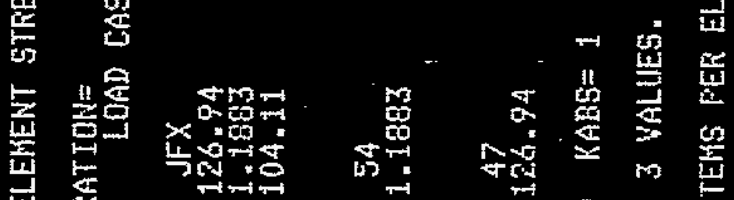

$m$ 竞

능

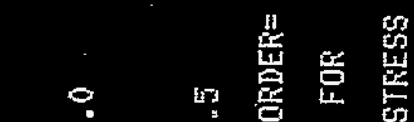

0 w

Finco

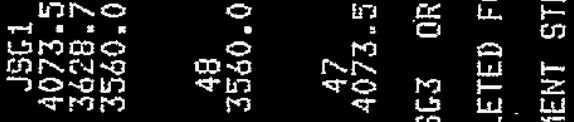

兽

पm

点

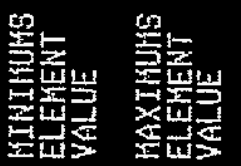

응

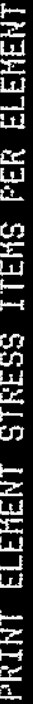


की

뭄름

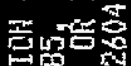

要要

복중명

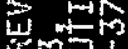

照泉向

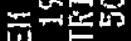

m

ons क力

$x_{i=1}^{-1,2}$

in

$\operatorname{lin}_{1}$

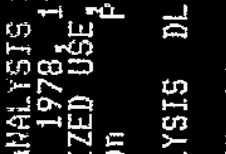

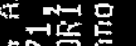

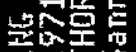

넌

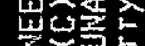

原, 要

눈

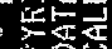

位舅

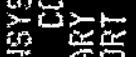

问这

뜨를

这密皆

홍

象要

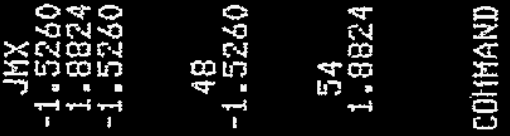

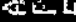

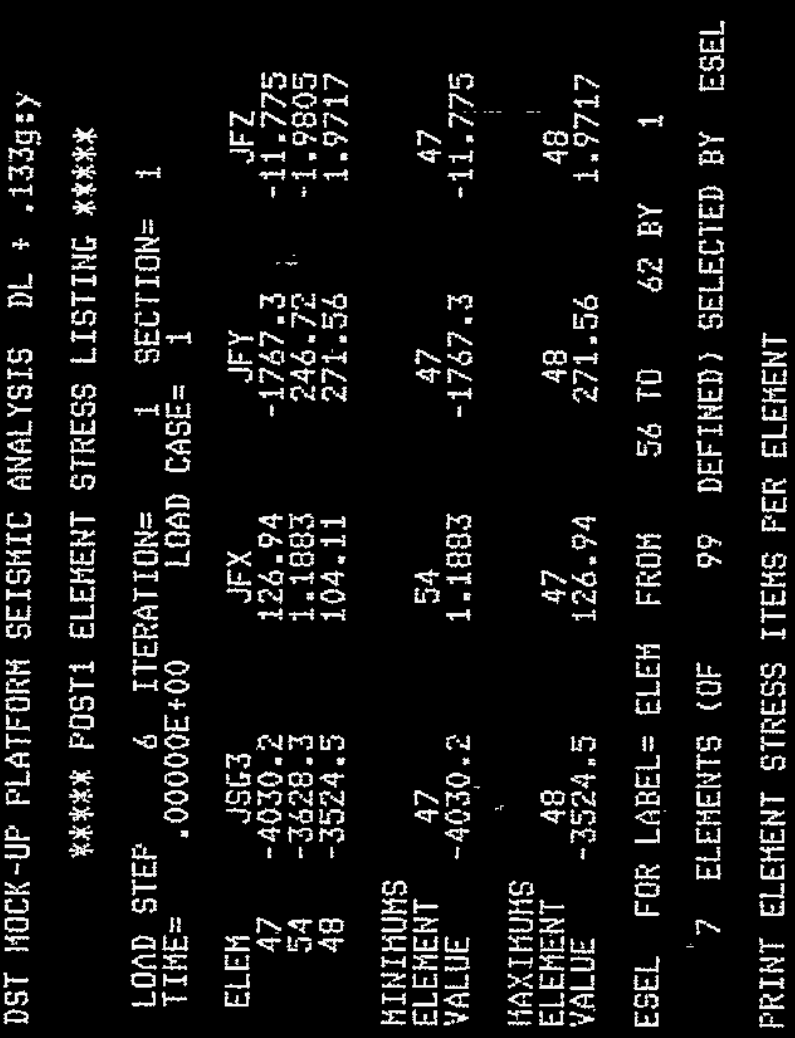


ANDYS - EHEIHEEFING AHALYSIS SYSTEK REVISION 4.4

A137 32207

KAY 1,1990

AHSYS(F) COFYKIGHT(C) $1971,1978,1982,1983,1985,1987,1989,1000$ SWANSON ANALYS IS SYSTEHS. THC. AS UHFUELISHED WORK FFOFRTETAFY DATA - UNAUTHDRIZED USE, DISTKTEUTTOH OR DUFL TCATION IS FROHIBITED. ALL RTEHTS RESERVED.

FOR SUPPORT CALL LarTy Gaminon PHOKE $509-376-2604$ TWX

DST KOCK-UF FLATFOFH SEISMIC ANALYSTS DL + .133g:Y

15.1264 AUG $1,1995 \mathrm{CF}=$

1.420

****** POST1 ELEHENT STRESS LISTING $* * * * *$

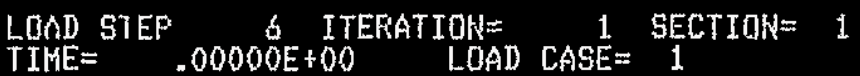

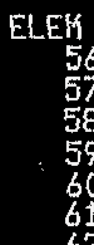

$$
\begin{gathered}
35 X \\
126.85410 \\
13.267052 \\
-2.7511181 \\
24.013928 \\
13
\end{gathered}
$$

$$
\begin{gathered}
\text { JFY } \\
2488.3528 \\
250.57241 \\
238.07824 \\
2512.2200 \\
250.46046 \\
237.95463 \\
1851.0654
\end{gathered}
$$

$$
\begin{array}{r}
J F Z \\
15.651208 \\
-.23501245 \\
-.20272044 \\
-15.752305 \\
-.250154 \\
-.20272044 \\
11.674161
\end{array}
$$
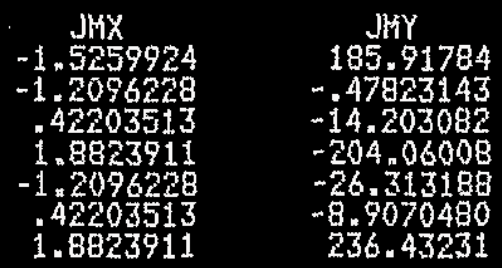

$$
\begin{array}{r}
\mathrm{JMZ} \\
-145.69591 \\
144.11405 \\
187.97145 \\
-409.82433 \\
150.49569 \\
195.01714
\end{array}
$$

MINIKUKS

ELEHENT

$-2.75811181$

237.95463

$-15.752305$

$-1.5659924$

59

63

HAXTHUHS

ELEHEHT

126.85410

2512.2200

15.651209

1. 8623911

2362.43231

18501714

ESEL FOR LABEL $=$ ELEM FROH

$64 \mathrm{TO} \quad 67 \mathrm{BY} \quad 3$

2 ELEHENTS (OF 99 DEFIHED) SELECTED BY ESEL' COMMAND.

$>$

FFINT ELEHEHT STRESS ITEHS FER ELEHEHT 


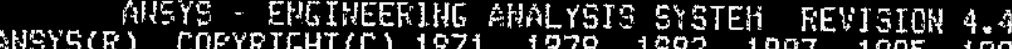

A137 32207

HAY 1,1990

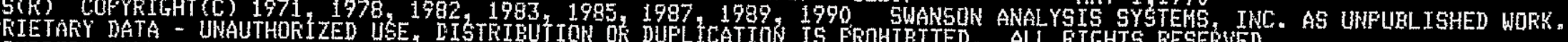

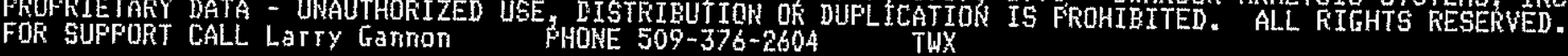

DST HOCK-IJP FLATFORH SEISHIC AHALYSIS DL + .133gry

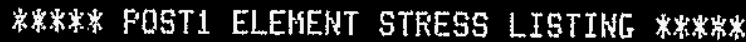

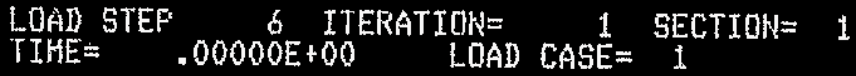

ELEH $\quad$ JFX $\quad$ JFY

$\begin{array}{lll}64 & 116.72473 & 201.4063\end{array}$

THTHUKS

ELEHENT

VALUE

$$
11.215114
$$

193.56882

.64755355

$201^{64} .40631$

1.6777086

$69 \mathrm{T0}$

69 BY 1

1 ELEMENTS (OF

99 DEFTNED S SELECTED BY

ESEL

HAXIKLHYS

VALUE

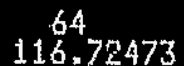

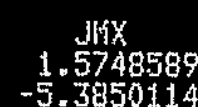

$-5.6785011 .4$

1.5448589

FRIHT ELEHENT STRESS ITEHS PER ELEHENT
.JMY

70.643091

$44.948667 \div-2.3666234$

$44.948667 \div-2.3668234$

$\mathrm{JHZ}$

237.80790

$-2.3666234$

70.643091

237.80790 
要

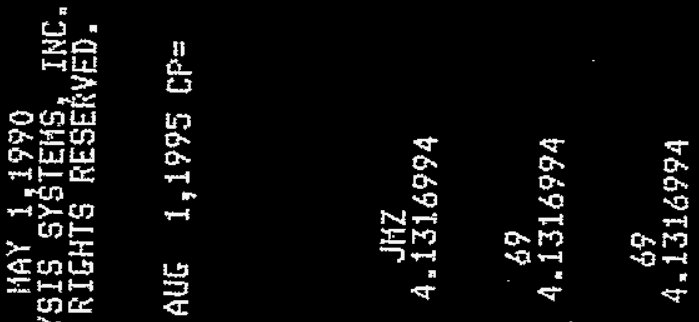

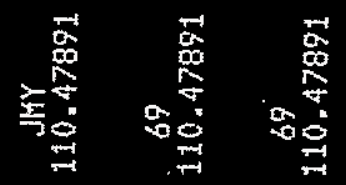

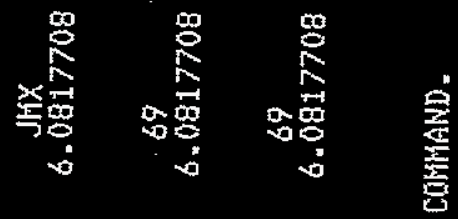

$+x$

过

㗊它 出

嗮

茨

运

Den

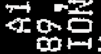

喿本承

(n)

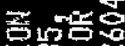

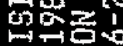

in

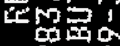

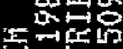

wisu

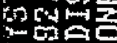

ing

$\omega_{4}^{n-4}$

32

문

on

皮家

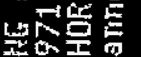

단단

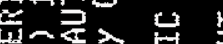

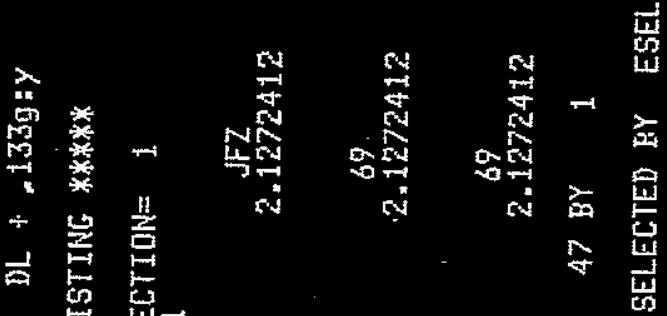

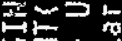

基霑门

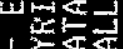

西空

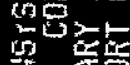

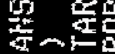

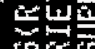

훌

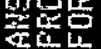

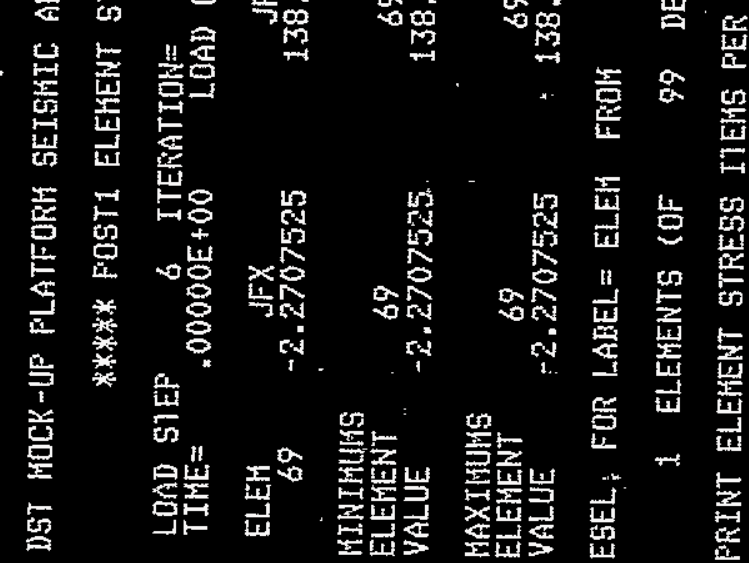


$\frac{8}{8}$

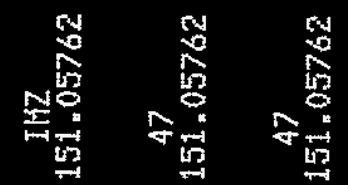

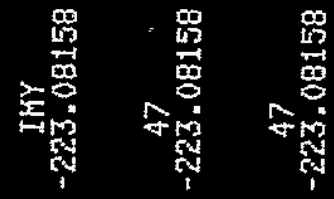

贾

䋑

묠

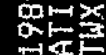

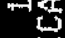

$\checkmark \mathrm{Din}$

楒

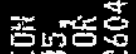

II

紫

峞

疍

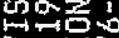

Wi

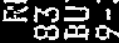

논요

논

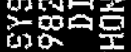

$n^{T-1} w^{2}$

thas

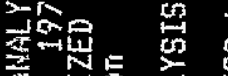

of co

넹둘

焉雪入

整谱,

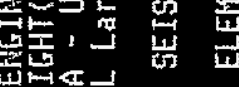

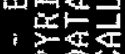

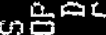

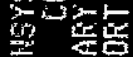

正园

数空

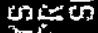

50동

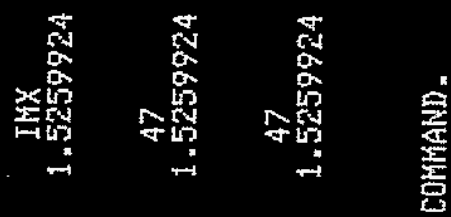

+ 崖

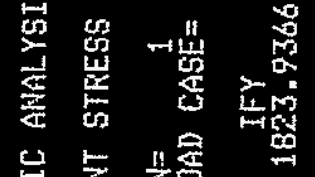

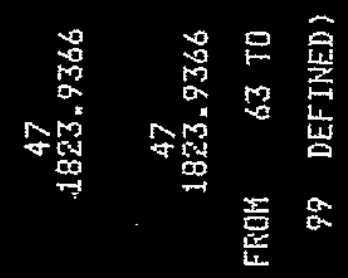

竞

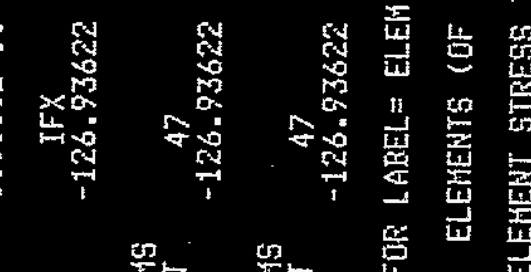

总皆

焉的点

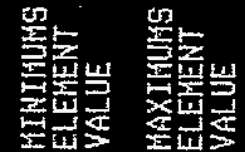

5

牙 


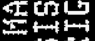

紧

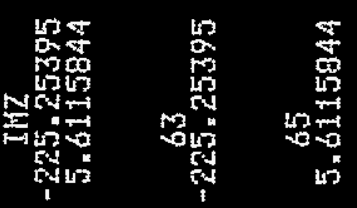

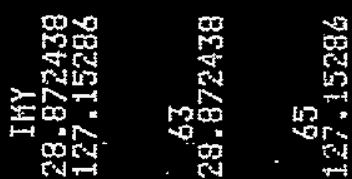

专热

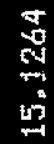

要

Buns

tint

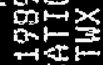

tim

$\rightarrow$ a

$\rightarrow$

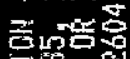

40

$\rightarrow$ 車

il

mo

눈요

Frow

on

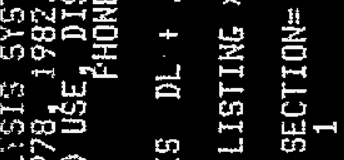

ond

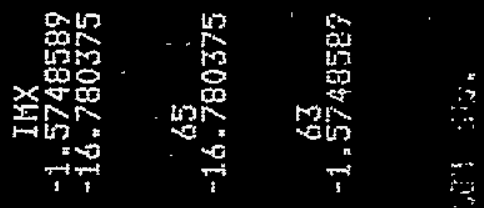

봉단,白

년전

, 毕正在 ๒9 运要 2늠 부웅 战家至

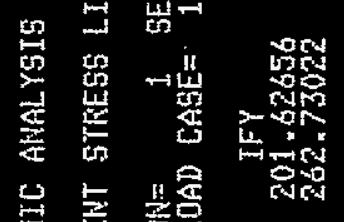

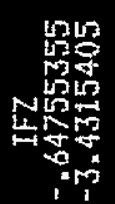

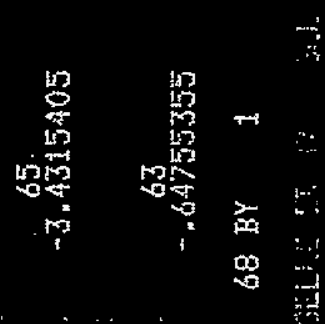

皆留竞

$\sum_{x=1}^{\infty}$

8

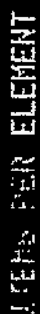

U w

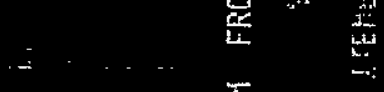

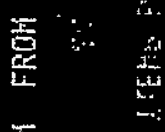

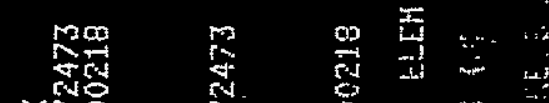

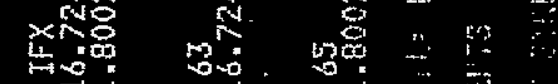

点

步

常

悹

总

남

运奶

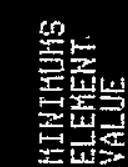

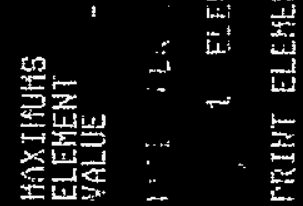


量

要

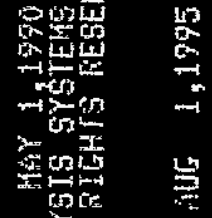

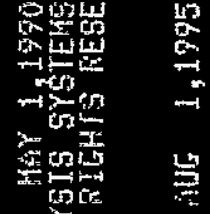

恋

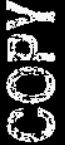

4⿴囗十

10

4

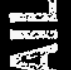

焉莎

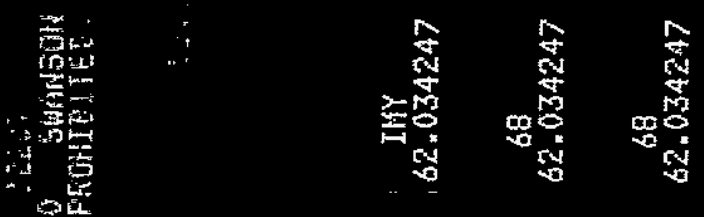

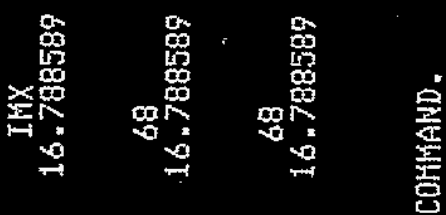

o

$\therefore=$

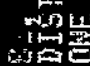

ind

$\frac{41}{201}$

事

点曷

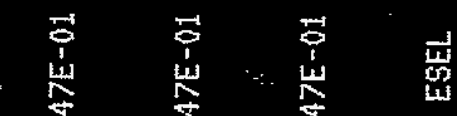

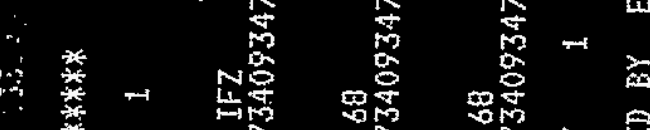

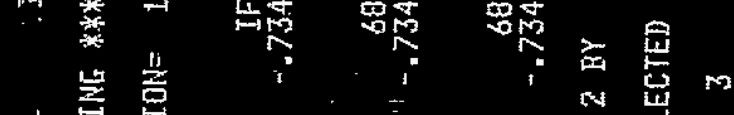

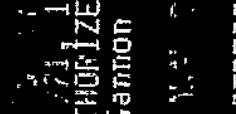

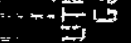

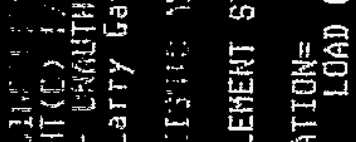

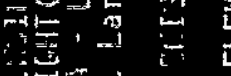

$\Rightarrow$ w

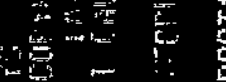

然

楚:

$\therefore=i$

$\because \quad \therefore \quad \dot{t}$

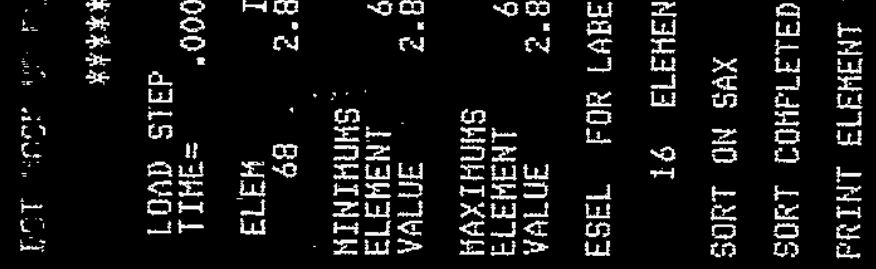


$\frac{8}{4}$

ts

远要

$\frac{14}{5}$

을

몬내

$\rightarrow$

$\rightarrow>0$

安的空

结造

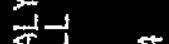

起考 品

D需至

N

$\mathrm{g}^{2}$

总暐

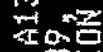

实在焉

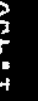

定

哭

晏

$+8$

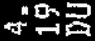

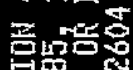

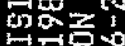

5 동

岸m?

gag

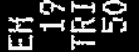

tor.ts

toxa寒

$a^{-1}+w^{2}$

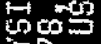

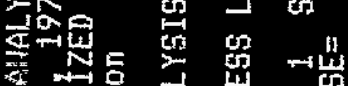

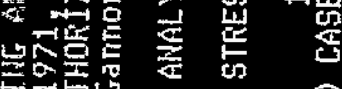

突

$5+5$

$\prod_{-1}^{m}$

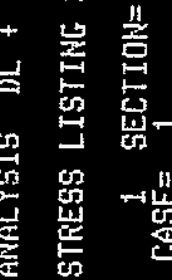

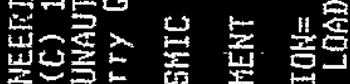

或,

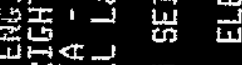

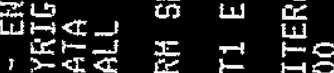

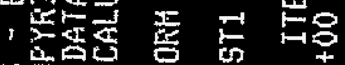

要焉

经遂

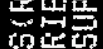

的的

훈항

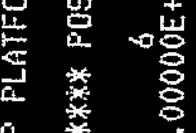

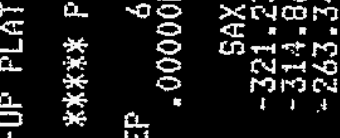

嘕

突

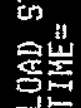

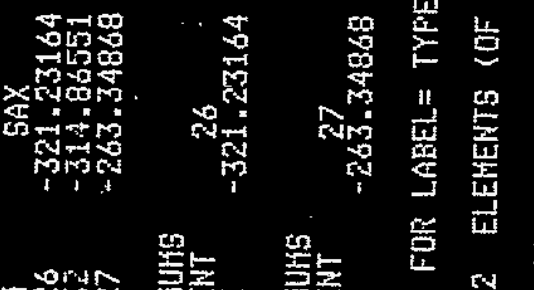

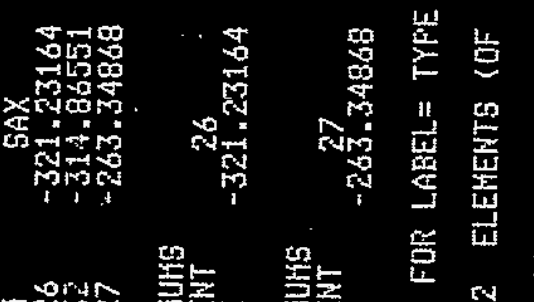

画

$\rightarrow$

量

M

离

을

동 $\frac{\text { 兽 }}{9}$

$\stackrel{5}{a-1}$

$A-200$ 
作

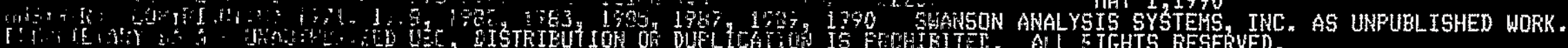

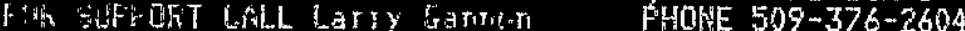

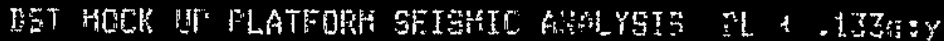
TWX

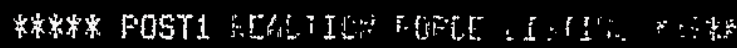

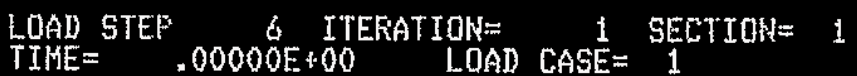

THE FOLLOWING $X, Y, Z$ FORCES ARE IN GLOBAL COORDINATES

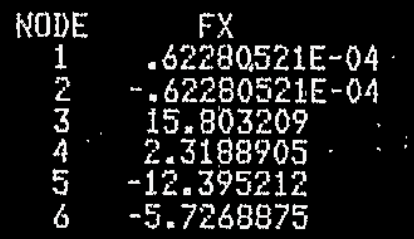

TUTAL.
FY

2950.4264 $3674+1418$ 3843.9026 4227.6729 1083.5757

936.20647

16715.926

SORT ON ISG1 ORDEF $=0$ KABS $=1$ NKAK $=$

SURT COMFLETED FOR

3 VALUES.

PRINT ELEHENT STRESS ITEHS PER ELEMENT

$F Z$
161.93357
-163.52626
211.57049
-202.57679
50.920817
-58.301839
$-.15285906 E-09$

3
15.225 AUS $1,1995 \mathrm{CF}=$

1.460
MX $.00000000 E+00$ $.00000000 E+00$ $.00000000 \mathrm{E}+00$ $.00000000 E+00$ $.00000000 \mathrm{E}+00$ .00000000 E +00

$.00000000 \mathrm{E}+00$
HYY

$.00000000 E+00$ $00000000 E+00$ $00000000 \mathrm{E}+00$ 00000000 E +00 $.00000000 \mathrm{E}+00$ $.00000000 E+00$

$.00000000 \mathrm{E}+00$

\section{Hiz}

$.00000000 E+00$ $.00000000 E+00$ $.00000000 E+00$ $00000000 \mathrm{E}+00$ $00000000 \mathrm{E}+00$ $.00000000 \mathrm{E}+00$

$.00000000 \mathrm{E}+00$

1
0
0 
告育

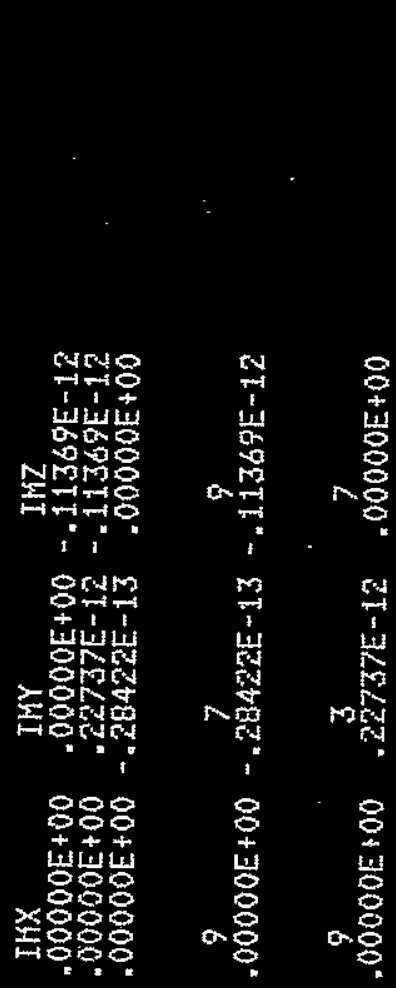

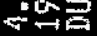

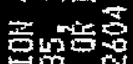

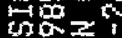

공

is

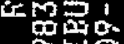

운요

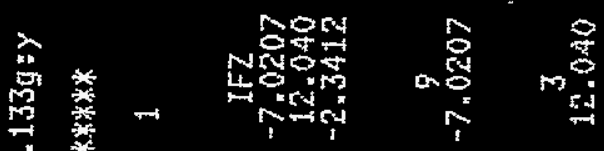

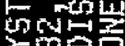

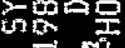

$\mathrm{cot}^{-1}$

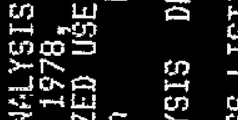

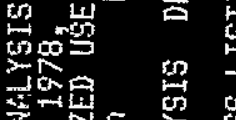

$+\frac{1}{5}$

吾 $\quad$ \&

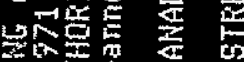

的家

它

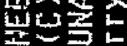

或:

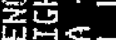

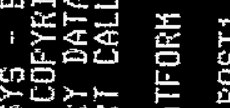

苍

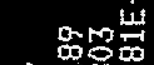

m啚㟧

tin

in

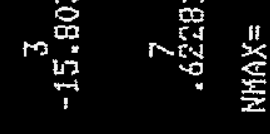

홍

18.

9

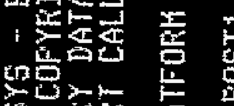

器要

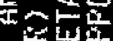

证空

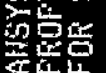

놉 坚完

누

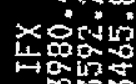

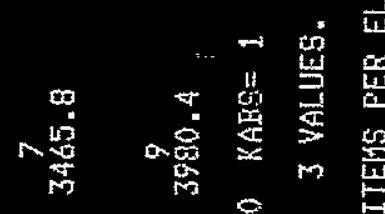

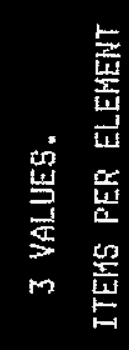

焉

돌

넌망

incis

音 *

$\frac{1}{3} * \frac{5}{5}$

a

空

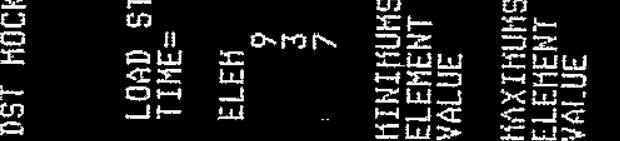

占 害

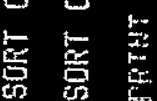


焉

g

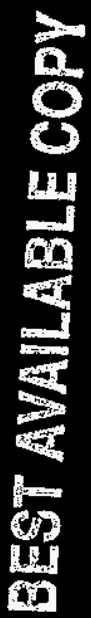

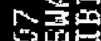

좀

mo

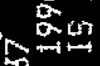

$\rightarrow+2$

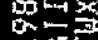

+4 F

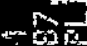

$\rightarrow \underset{x \rightarrow 1}{\infty}$

竞

45

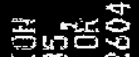

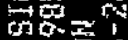

$\Rightarrow \rightarrow$

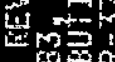

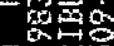

$x_{i=1}$

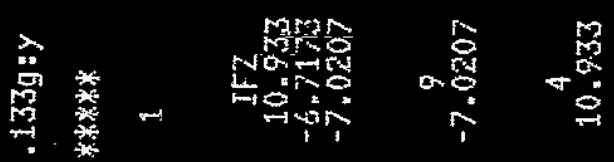

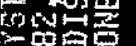

w的是

+ h

$u_{i=1}^{n-1} \frac{1}{w^{2}}$

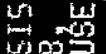

,

古点

畐

sha

$\sum_{i=1}^{2}=45$

过

希・紫

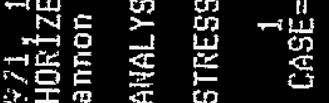

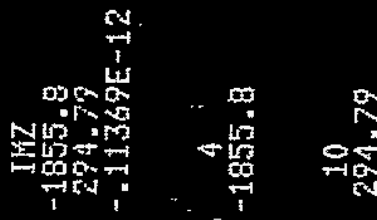

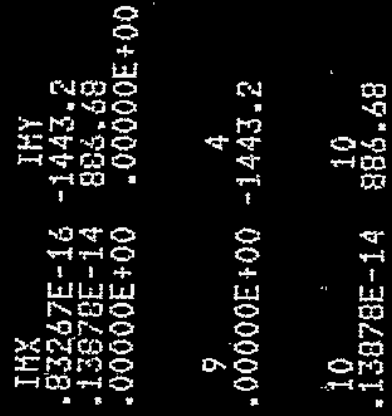

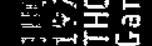

눈은

然:

总我

空焉

$3>0$

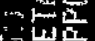

证仍

㩆管

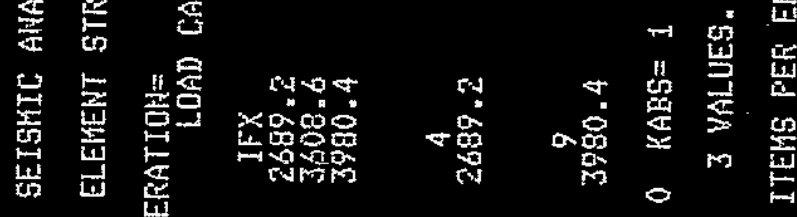

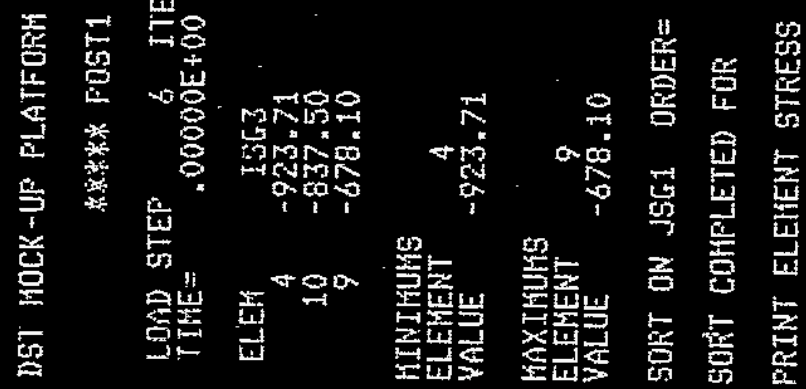




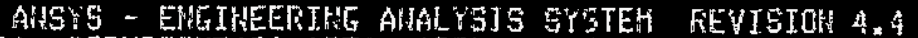
A137 32207 HAY 1,1990

AHSYS(F) COFYFIGHT (C) $1971,1978,1982,1993,1985,1967,1997,1990$ SWANGON ANALYSIS SYSTEHS INC, AS UNFUELISHED WOFK.

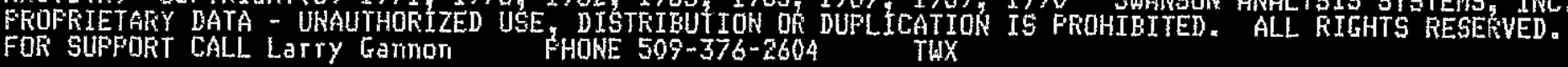

DST HOCK-UF FLATFORM SEISHIC ANALYSIS DL + .133g:y

15.1264 AUG $1.1995 \mathrm{CP}=\quad 1.470$ ****** FOST1 ELEMENT STRESS LISTIKE

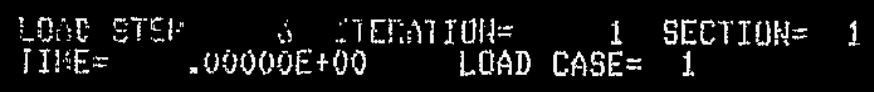

afi,

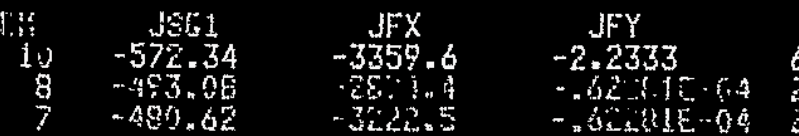

$\begin{array}{lll}\mathrm{JF} Z & \mathrm{JHX} & \mathrm{JHY} \\ .7 \mathrm{I} 73 & -.13879 \mathrm{E}-14 & 11369 \mathrm{E}-12\end{array}$

$00000 \mathrm{E}+00$

11369E-12 $20464 E-11$

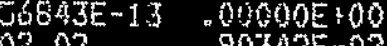

MINTHUHS

ELEHENT

$-572 \times 34$

$\begin{array}{cc}10 & 10 \\ -3359.6 & -2.2333\end{array}$

2. $\frac{8}{3230}$

10
$-13878 E-14-.56843 E-13$

8

MAXTHUHS

$\begin{array}{lcccc}\text { ELEHENT } & 7 & 8 & 8 & 10 \\ \text { VALUE } & -480.62 & -2894.4 & \sim .62281 E-04 & 6.7173\end{array}$

$.00000 E+00 \quad 302.02$

$.80342 E-02$

SOFT OH JSG3 ORDERE $=0$ KAES $=1$ HHAXX $=3$

SORT COHFLTED FEK : 3 VALLES.

in

PRTHT ELEHEHT STREGS ITEHE Thi LLEHEHT 


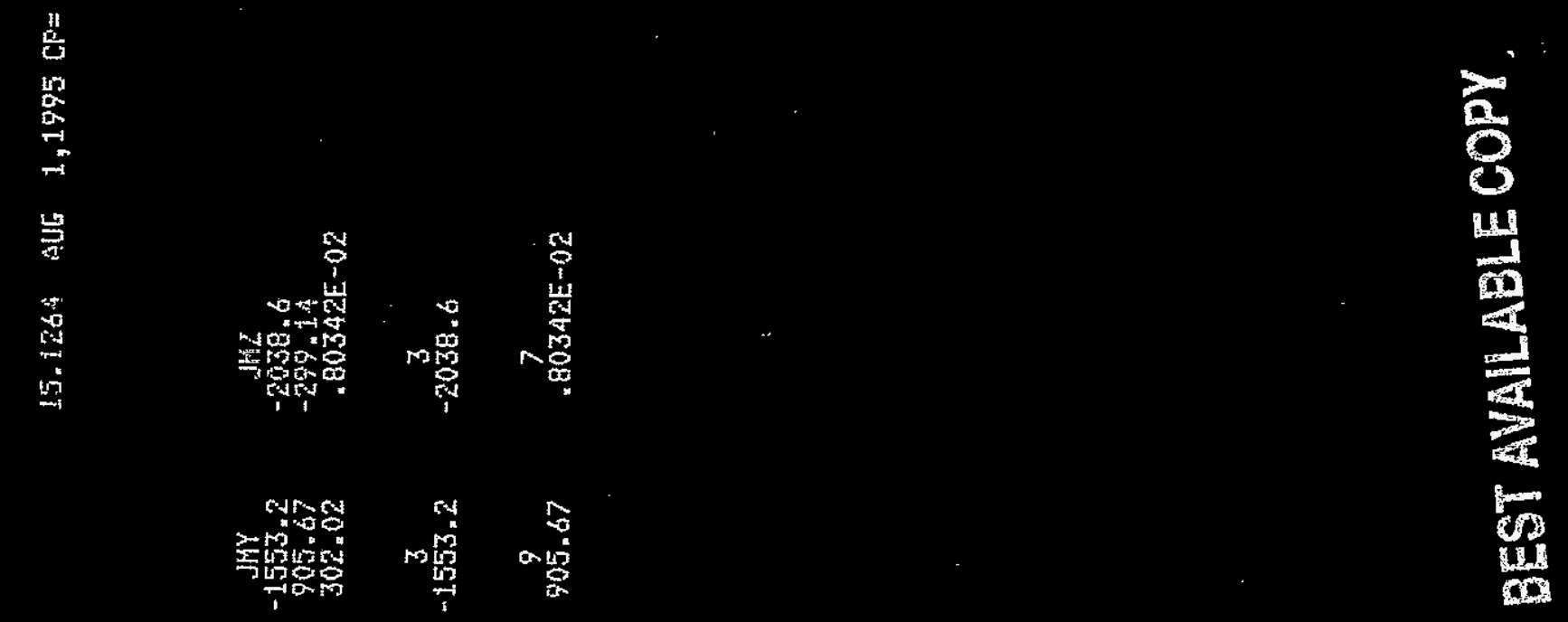

$-m$

留的肴

Tit

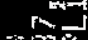

的部

$\therefore$ 我

$\stackrel{8}{8}$

贸

雚

on

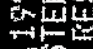

$x_{0}^{2}$

留

bot

起

沓白

$r=\frac{3}{5} x$

o

然o

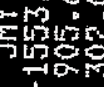

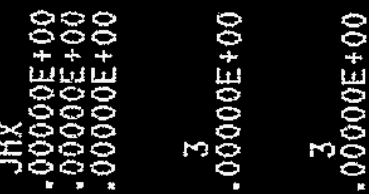

薏

$\lim _{1 \rightarrow 0}$

it

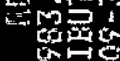

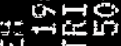

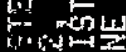

in

$\omega^{2}$

ing

5

순

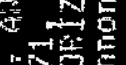

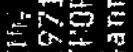

$\rightarrow$

过空

in

s.

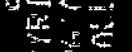

数到

in

证

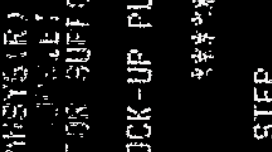

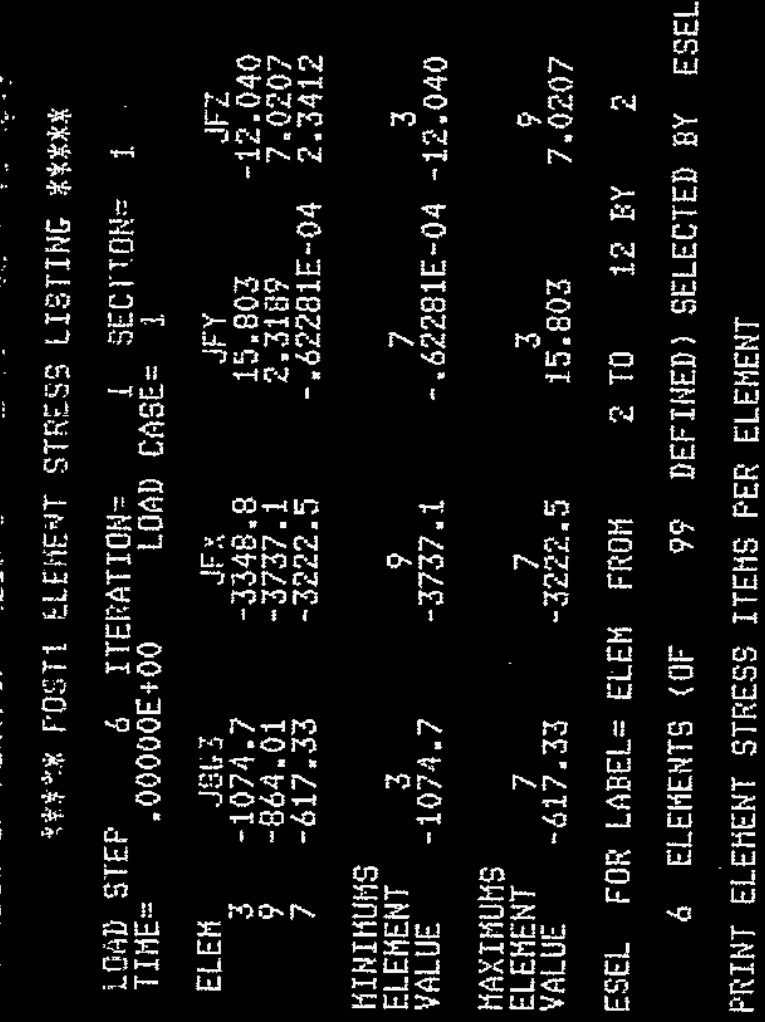




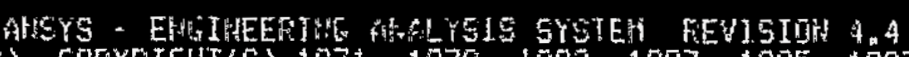

A137 32207

HAY 1,1990

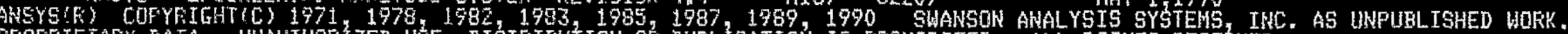
FFOFGIETAFY DATA - UNAUTHORIZED USE, DISTRIEUTION OR DUFL. ICATION IS FROHIBITED. ALL RIGHTS RESERVED.

DST MOCK-UP PLATFORH SEISMIC ANAL.YSIS DL + .133g:Y

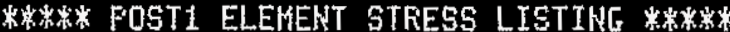

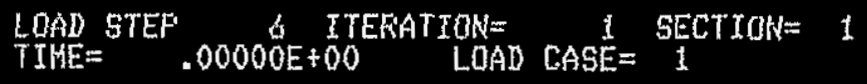

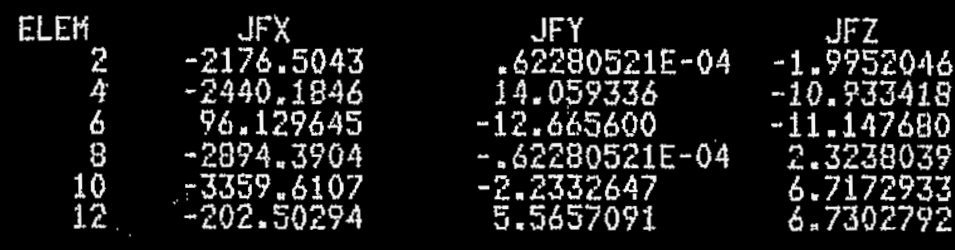

\section{HINIHUKS}

ELENENT

$$
-3359 \cdot 6107
$$

$-12.665600$

$-11.647680$

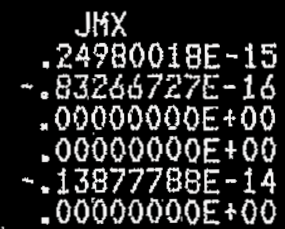

JWY

$-.14210855 E-13 \quad-.45474735 E-12$

$11369604 \mathrm{E}-12$

.

$1136048 \mathrm{E}-1$

$.00000000 E+00$

$45474735 \mathrm{E}-1$

$.00000000 \mathrm{E}+120$ (1) $.20463631 E-11$

ELEMENT

96.129645

14.059336

6. $\frac{12}{7302792}$

$-109789$

$-.11368684 E-12$

2

$.2498001 .8 E-1.5$

$.11368694 \mathrm{E}-12$

10
$-20463631 E-11$
$4 \mathrm{TO}$
$4 \mathrm{BY} \quad 1$

24 ELERENTS (OF 99 DEFINED) SELECTED BY ESEL COHHAND.

SORT ON ISG 1 ORDER $=0$ RABS $=1$ NHAX $=3$

SORT LOHFLETED FOF 3 VALUES.

$N$ FRINT ELEKENT STRESS ITEHS PEF ELEHENT

0

8 
4

i

opin

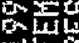

난

$\rightarrow$ in

㔭

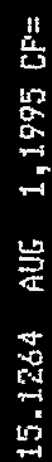

家卖

퐁:

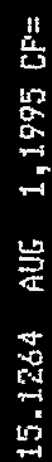

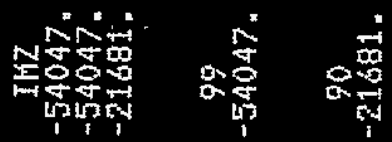

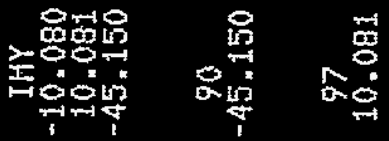

$\overrightarrow{x a}$

人is

폼

움

焉en

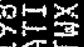

$\rightarrow$

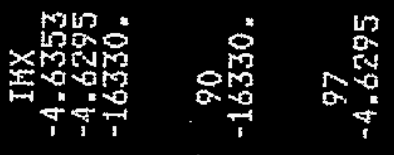

\&

돕

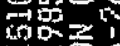

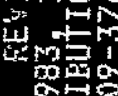

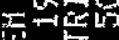

50

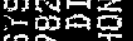

$\sin ^{2}$

inos

然需

전도

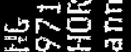

$\rightarrow+15$

분은존

논,

可迹

1 在焉

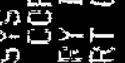

올

要

시웅

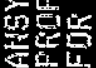

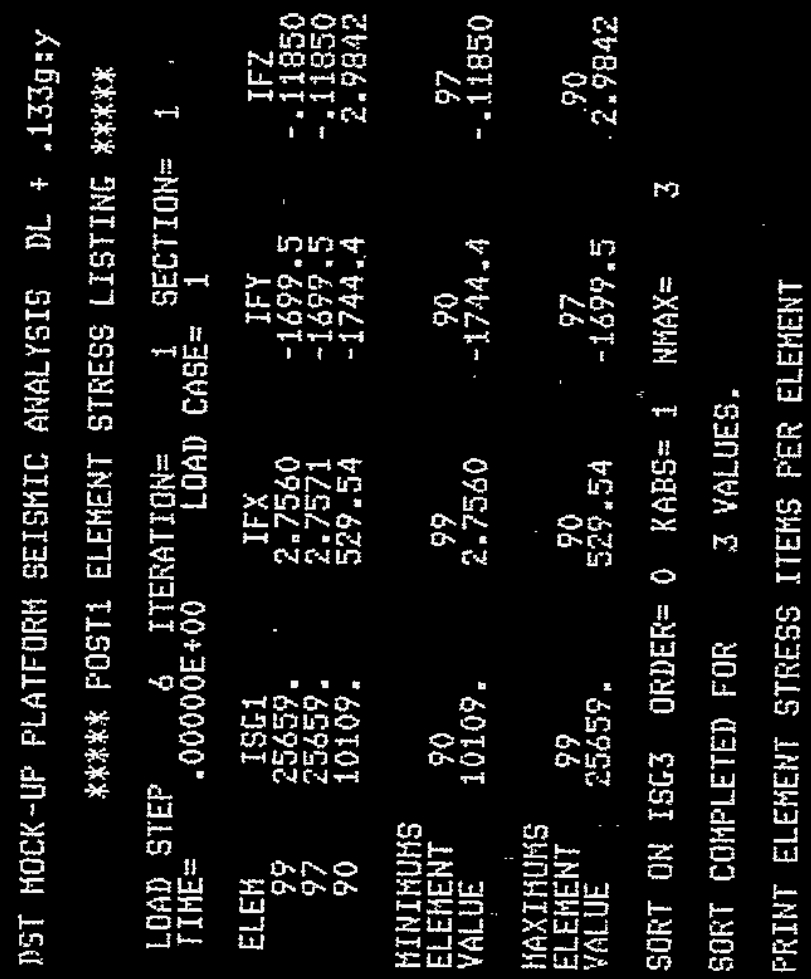


覀

$\underset{8}{8}$

ga

运望

욜ำ

댄단

$\rightarrow>0$

的

趾毞

군

䞨求

뭄

哥至

落

O虫出

为䨝

․․

紡出

준궁

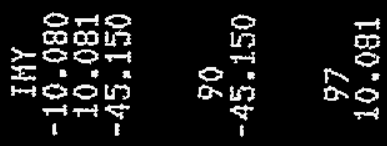

碞证希

다눌

政

桶

굼

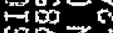

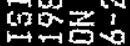

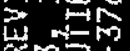

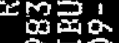

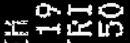

ำแ

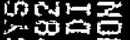

네

ts

$\cos =$

75

整至

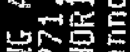

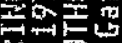

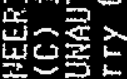

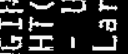

논

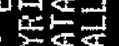

它氙

宊忞后

正

눈훙

通点战

永

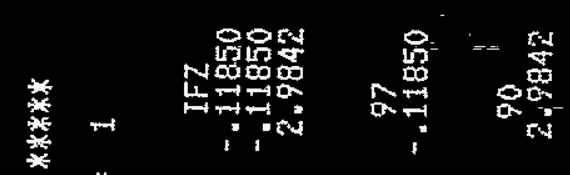

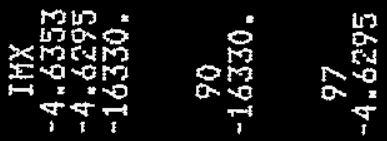

+ 宸量晋 m

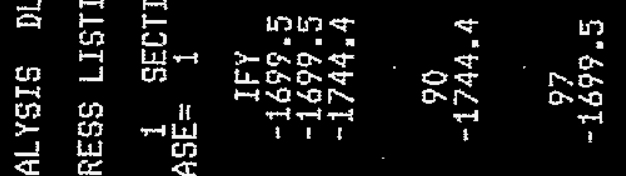

䒿高

丕卧密

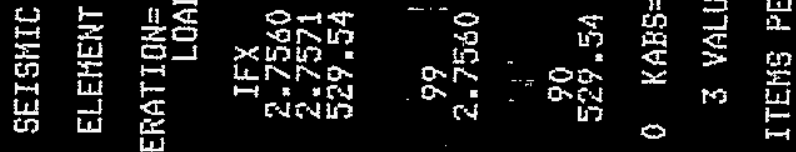

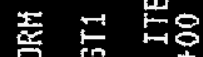

量

$\frac{i}{a}$

9

$\frac{1}{3} * \frac{5}{50}$

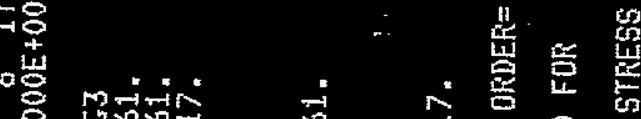

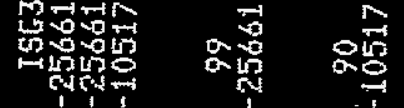

悹

窎

紫喜

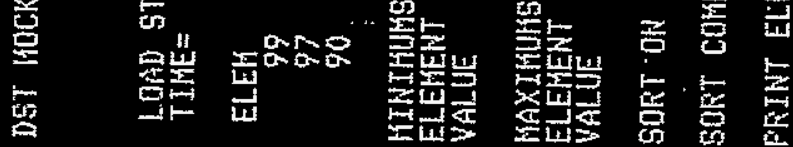


AHSYS - EHGTHEEFIHG ALALYSIS SYSTEH FEVISION 4.3

A 13732207

MAY 1,1990

ANSYS(F) COFYFTGHTCC) $1971,1970,1902,1983,1905,1987,1989,1990$ SWANSON ANALYSIS SYSTEHS, INC. AS UHFUBLISHED WOKK.

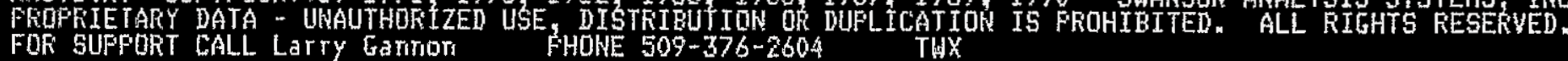

IIST HOCK-UF FLATFORK SEISKIC ALALYSIS DL + .133g:Y

15.1264 ALIG $1,1995 \mathrm{CP}=\quad 1.500$

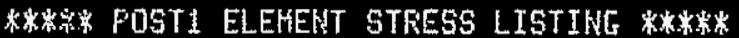

LOAW STEF $0000^{6}$ ITERATION=

$\begin{array}{cr}\text { ELEH } & \text { J561 } \\ 98 & 25659 \\ 96 & 25659 \\ 91 & 14939\end{array}$

$\begin{array}{cccc}\text { JSE. } & \text { JFX } & \text { JFY } & \text { JFZ } \\ 25659 . & -2.7560 & -1699.5 & \text {.11850 } \\ 25659 . & -2.7571 & -1699.5 & .11850 \\ 14939 . & -1790.7 & -325.49 & 529.13\end{array}$

$\begin{array}{lcr}\text { JMX } & \text { JHY } & \text { JWZ } \\ 4.6353 & 10.080 & 54047 . \\ 4.6295 & -10.081 & 54047 . \\ 6.9090 & 20422 . & -12513 .\end{array}$

HINIFUHS

ELEHENT

$\begin{array}{cccc}81 & 81 & 96 & 98 \\ 14937, & -1798.7 & -1697.5 & .11850\end{array}$

96
4.6275

96
-10.081

$-12513$

MAXIFUHS

ELEHENT

98

$-798$

81
$325.49 \quad 529.63$

6.9090

81

20422

598

SURT ON JSG3 ORUER $=0$ KABS $= \pm$ KHAK $=3$

SORT COMFLETED FOR 3 VALUES.

PRINT ELEMENT STRESS ITEHS FER ELEHENT 


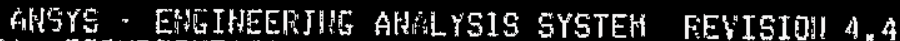

A 13732207

HAY 1,1990

AHSYS(F) COFYFILHT(C) $1971,1978,1992,1983,1989,1997,1989,1990$ SWANSON ANALYSIS SYSTEMS, INC. AS UHAUULLISHED WDEK.

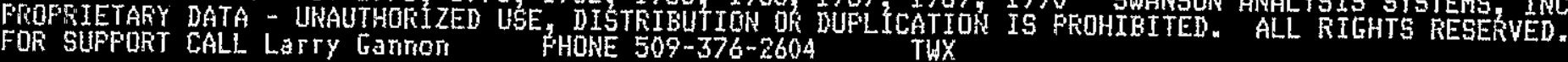

DST HOCK-UP PLATFORH SEISHIC ANALYSIS DL + .133g:y

15.1264 AUG $1,1995 \mathrm{CF}=\quad 1.500$

***** FOST1 ELEMENT STRESS LISTING ******

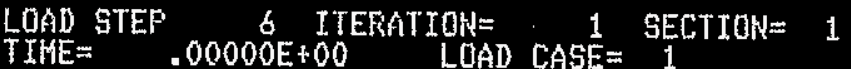

\begin{tabular}{|c|c|c|c|c|c|}
\hline $\begin{array}{r}\text { ELEH } \\
79 \\
96 \\
81\end{array}$ & $\begin{array}{l}5963 \\
-25661 . \\
-25661 . \\
-16328 .\end{array}$ & $\begin{array}{c}\text { JFX } \\
-2.7560 \\
-2.7571 \\
-1798.7\end{array}$ & $\begin{array}{c}\text { JFY } \\
-1699.5 \\
-1699.5 \\
325.49\end{array}$ & $\begin{array}{c}\sqrt{ }{ }^{2} \\
.11850 \\
.11850 \\
529.63\end{array}$ & $\begin{array}{c}\text { JHX } \\
4.6353 \\
4.6295 \\
6.9090\end{array}$ \\
\hline
\end{tabular}

HINIMUHS

ELEHENT 98 , 81 . 96 .

$\begin{array}{llll}\text { VALUE } & -25661 . & -1798.7 & -1699.5\end{array}$

$\begin{array}{ccc}9.6 & 96 & 81 \\ 4.6295 & -10.081 & -12513\end{array}$

HAXIMUHSS

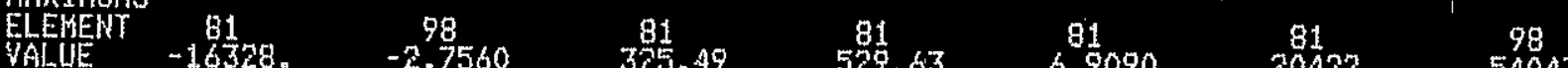

ESEL FQR LABEL.= ELEM FROH 88 TO 99 BY 1

12 ELEHEHTS SOF 99 DEFINED) SELECTED BY ESEL COHHAND.

PRINT ELEKENT STRESS ITEMS FER ELEHENT 
$\underset{\substack{1 \\ i=1}}{i=1}$

몬

II

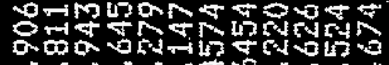

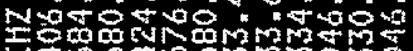

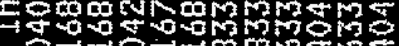

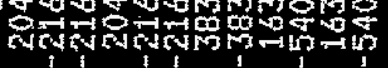

急

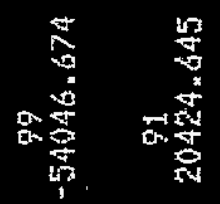

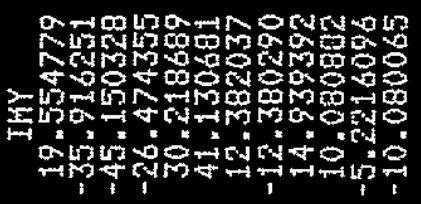

急

壱

究

总焉

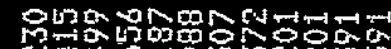

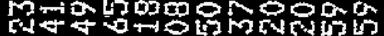
$\times$ 男o Empar

0 mon

要

然音

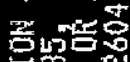

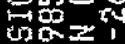

$>\rightarrow$

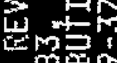

and

wen

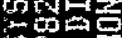

$\omega_{0 \rightarrow 1}$

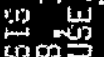

tong

通象

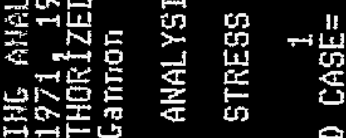

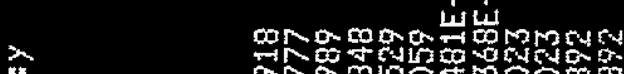

on

Nof tromont

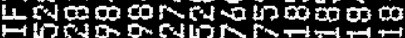

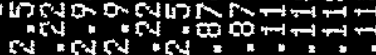

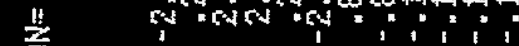

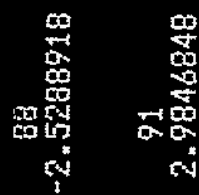

空学

뽈는돈

然,

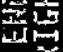

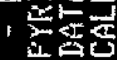

ung

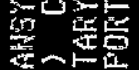

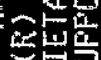

列居

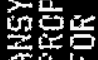

$+$

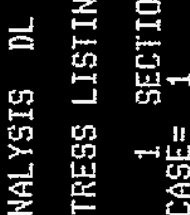

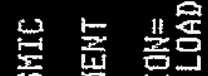

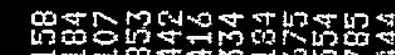

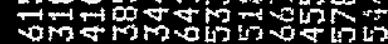

$>0$ -

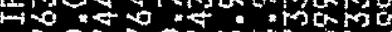

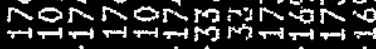

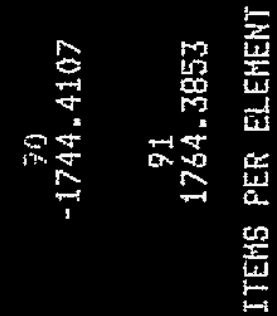

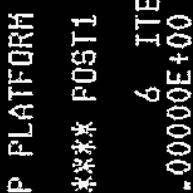

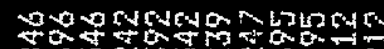

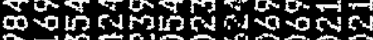

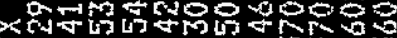

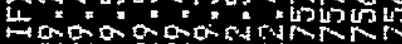

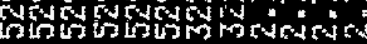

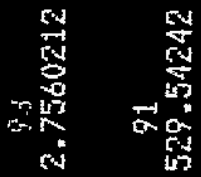

Q4.

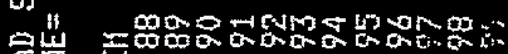


AHBYS - EHGTHEEFIHG AHALYSIS SYSTEH FEVISIOH 4.4

413732207 MAY 1,1970

ANSYE(E) COFYFIGHT (C) $1971,1978,1982,1983,1985,1987,1999,1990$ SWANSON ANALYSIS SYSTEHS. TNC. AS UNFUELISHED WOKK. FOR SUPFORT CALL L LITY GaIIOT

DST MOCK-UF FLATFORH SEIGHIC AKALYSIS DL + .133g:y

****** FOST1 ELEHENT STRESS LISTING ******

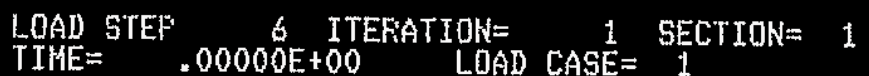

$\begin{array}{cc}\text { ELEH } & \text { JFX } \\ 88 & -529.29846 \\ 89 & -529.41696 \\ 90 & -529.53546 \\ 91 & -529.54242 \\ 92 & -529.42392 \\ 93 & -529.30542 \\ 94 & -322.50239 \\ 75 & -322.46247 \\ 96 & -2.7570695 \\ 97 & -2.750695 \\ 98 & -2.7560212 \\ 99 & -2.7560212\end{array}$

$$
\begin{gathered}
\text { JFY } \\
-1743.6415 \\
10.743042 \\
1764.3851 \\
-1744.4109 \\
10.063184 \\
1763.6160 \\
-34.320401 \\
34.320551 \\
-1699.5446 \\
1733.5783 \\
-1699.4556 \\
1733.6673
\end{gathered}
$$

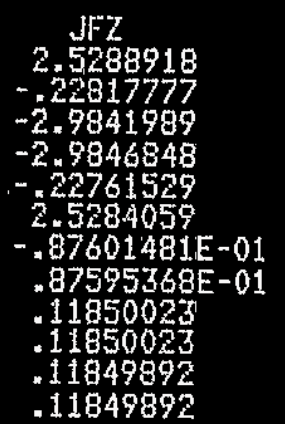

JHX

$-\frac{1}{6} 6334.230$

16330.499

16330.656

$-3.5641197$

16374000

$-16334.088$

3.0083507

3.0281372

4.6295201

4.6295201

4.6352591

4.6352591
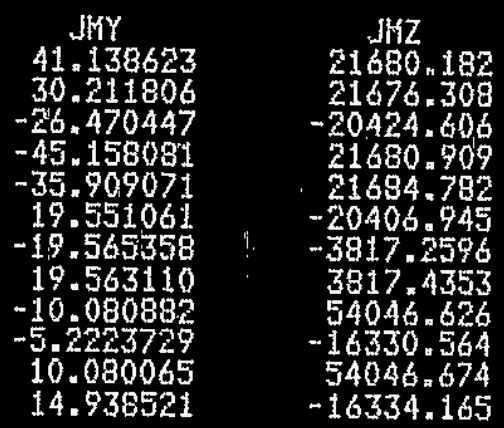

HTHIKUHS

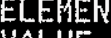

$$
\begin{array}{cc}
91 & 91 \\
-529.54242 \cdots & -1744.4109
\end{array}
$$

$-2.91846848$

$-16334,230$

91

$-45.158081$

90

MAXIMUHS

ELEHEN

\section{- 98}

1764.3851

2.5268918

16330.656

88

41.138623

4046.674

ESEL FOR LABEL = ELEH FROH $76 \mathrm{TO} \quad 92 \mathrm{BY} \quad 2$

$N 4$ ELEHENTS (OF 99 DEFINED) SELECTED BY ESEL COHHAKD.

$N$ PRTRT ELEHENT STRESS ITEHS FEF ELEHENT 
ALSYS - ENGTHEERTHE AHALYSTS SYSTEH REVISIG A A

A137 32207

HAY 1,1990

ANGYS(R) CDFYKIGHT(C) $1971,1978,1982,1993,1995,1987,1999,1990$ GWAHSON ANALYSIS SYSTEMS, INC. AS UNFUELISHED WOFK,

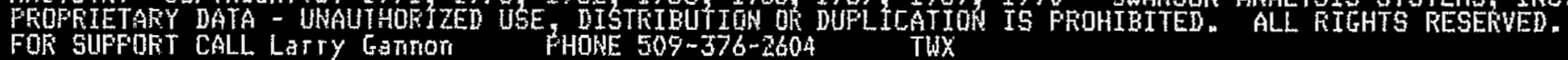

DST HOCK-UF PLATFORH SEISWTC ANALYSIS DL + .133g:Y

15.1264 ALG $1,1995 \mathrm{CF}=\quad 1.520$

W*W* POST1 ELEHENT STRESS LISTING

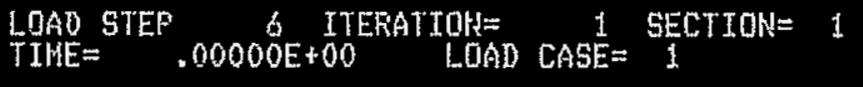

$\begin{array}{cccc}\text { ELEH } & \text { IFX } & \text { IFY } & \text { IFZ } \\ 76 & 1987.9824 & -13.771795 & 22.825427 \\ 78 & 1990.2741 & -13.654672 & -22.743510 \\ 80 & 1990.2741 & 13.679554 & 22.747805 \\ 82 & 1987.9024 & 13.746912 & -22.829722\end{array}$

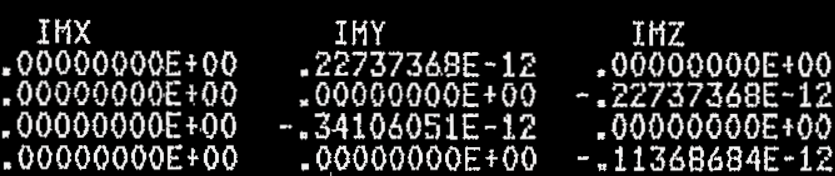

MITITHUHS

ELEHENT

$\begin{array}{cc}82 & 76 \\ 1987.9824 & -13.771795\end{array}$

82

MAXTHUHS

ELEHENT

YALUE

$1990.2741 \quad 13.746912$

$-2.829922$

$\begin{array}{ccc}76 & 80 & 78 \\ .00000000 E+00 & -.34106051 E-12 & -.22737368 E-12\end{array}$

ESEL FOR LAEEL = TYFE FKOM

510

22.825627

$.00000000 \mathrm{E}+00^{\circ}$

$.22737368 E-12$

76

5 BY 1

11 ELEKENTS (OF 99 DEFINED) SELECTED BY ESEL COHMAND.

SORT ON ISG1 ORDER $=0$ KABS $=1$ WHAX $=3$

D SORT COMFLETED FOR 3 VALUES.

N

PRINT ELEHEHT STRESS ITEKS PER ELEIENT

$\frac{N}{N}$ 
氙官

$\frac{4}{4}$

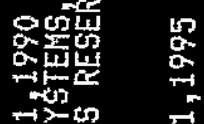

$>$

乐留它 象

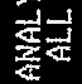

z⿸丆口⺕亅

분난

常

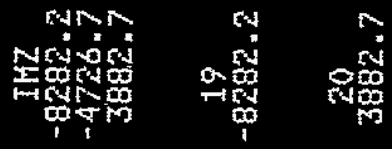

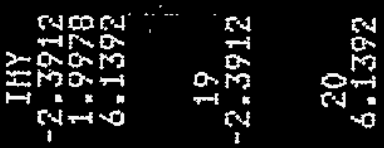

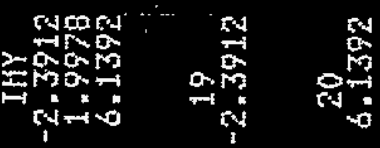

and

ID

融地

ती $\rightarrow$

?

स्य

$x$ ofax

我

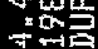

눕을

सिखे

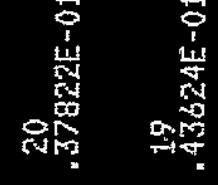

on

패에

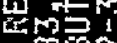

ogis

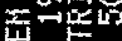

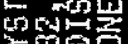

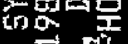

in ${ }^{7}$ is

ตึ่⿻日禸

슨

昰

我尊

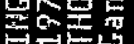

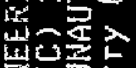

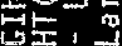

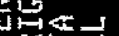

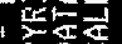

咅牙

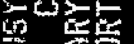

在政

计둥

綰点

值

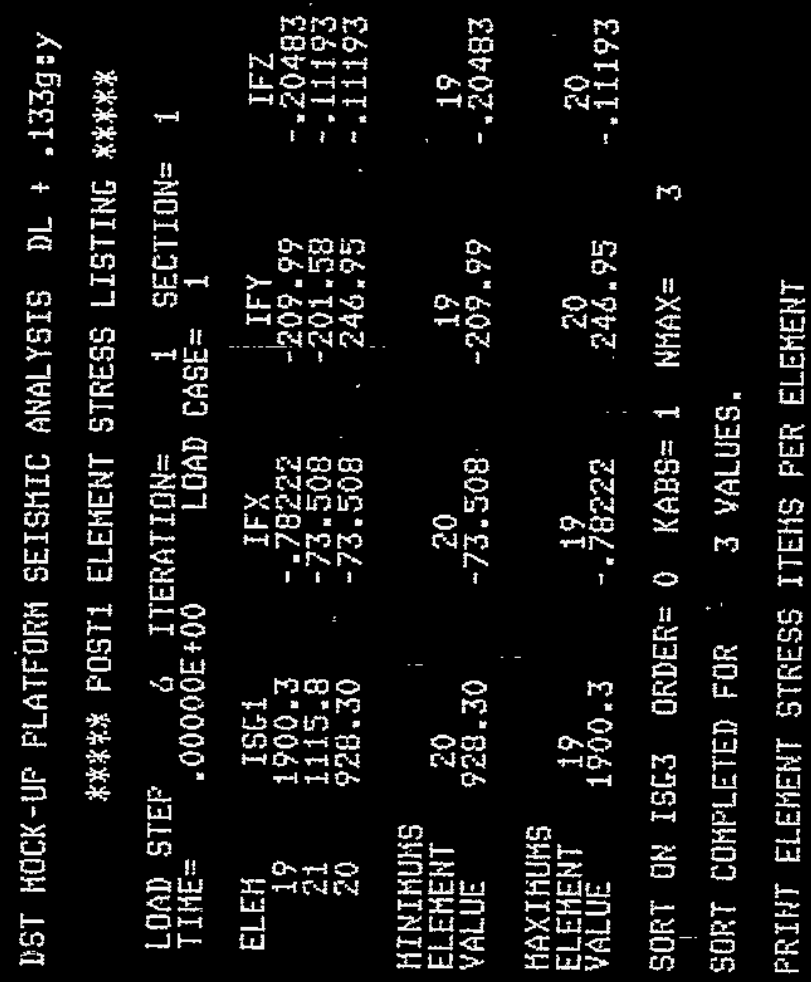


AHSYS - EMGIHEEFIHE AHALYSIS SYSTEH REVISIOH A

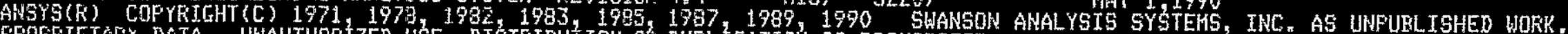
FROFFIETAFY DATA - UNAUTHORIZED USE, DISTRIRUT ION OK DUFLICATION IS FROHIEITED. ALL RIGHTS RESERUED.

DST HOCK-UF FLATFORH SEISHIC ANALYSIS DL + .133g;y

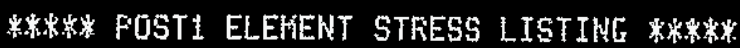

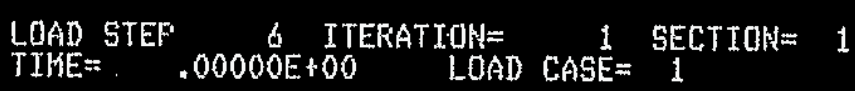

ELEH $19-1563$

$21-1054.6$

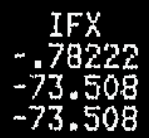

IFY
-209.99
-201.58

246.95

$$
\begin{gathered}
\text { IFZ } \\
-.20483 \\
-.11193 \\
-.11193
\end{gathered}
$$

$\begin{array}{ll}\text { IHK } & \text { IHY } \\ .43624 E-01 & -2.3912 \\ .37822 E-01 & 1.9978\end{array}$

$.37822 E-01 \quad 1.9978$

MINIMUHS

ELEHENT 19

$-730$

$-209.99$

$-.20483$

$.307822 E-01-2.3912$

HAXIHUHS

ELEMENT

$-820.05$

$-.7922$

246.95

$-20$

$\frac{19}{43}$

SORT COMFLETED FOR 3 VALUES:

FRIHT ELEHEHT STRESS ITEHS FEF ELEHEHT

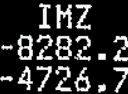

$-4726.7$

19

$-8282.2$

3082.7 
"

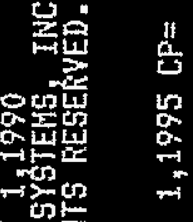

$>0$

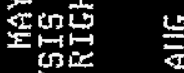

变妾

좀요

굽셔

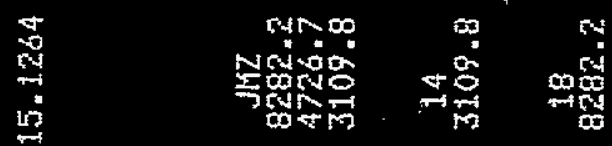

S聂

空

요

点

aㅛㄹ

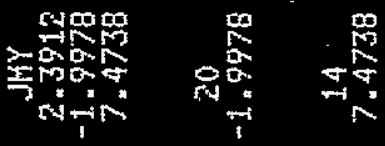

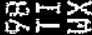

短

N"y

这密

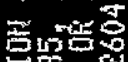

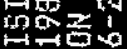

要

MP:

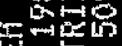

wisu

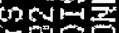

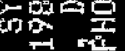

क

thing

구요

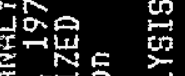

แN些点

눙우룰

意的空

뻐ㅇㅝㅗ은

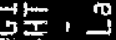

if

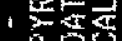

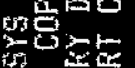

昶

둘

대눙

然家它

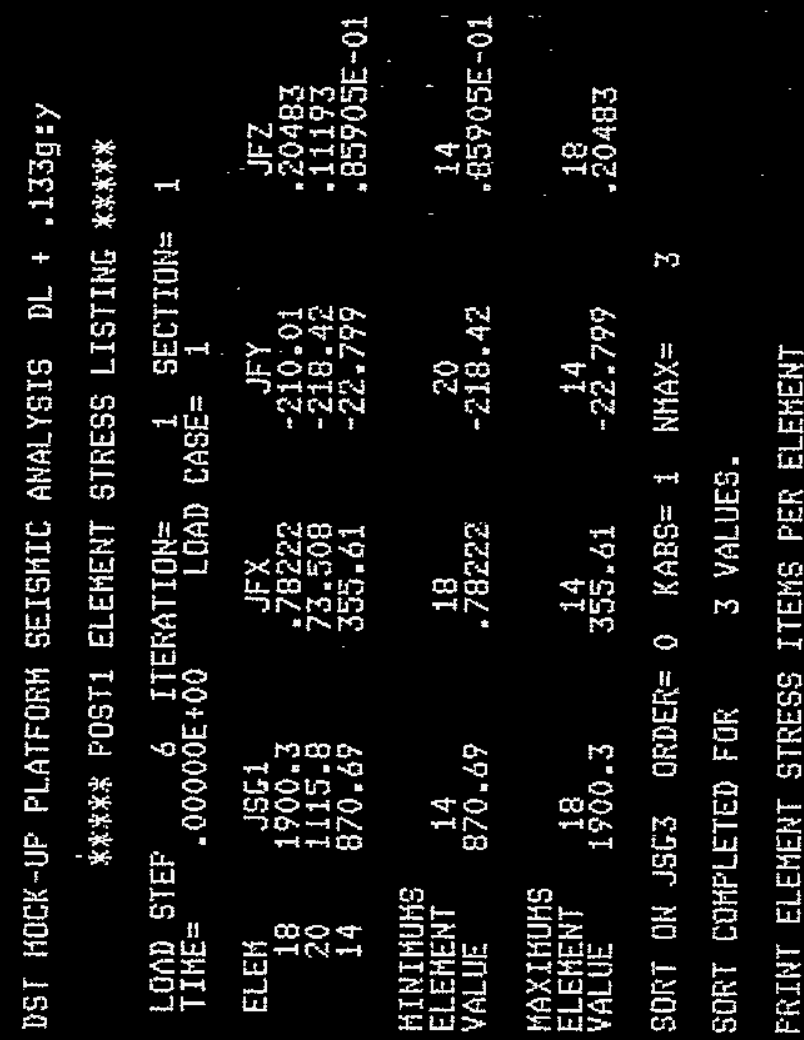


焉

se

我

空证

保幽

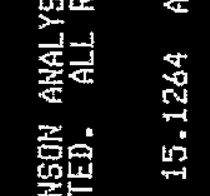

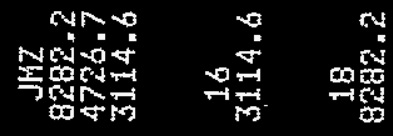

Sit

mo

的许

말

$\frac{8}{4}$

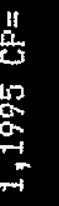

学

可店

勧

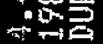

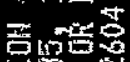

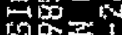

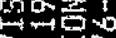

tis

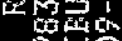

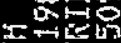

舟地

현동

on 0 T

tis

$=$

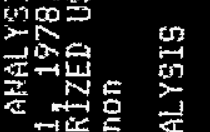

我紧

牙它以。

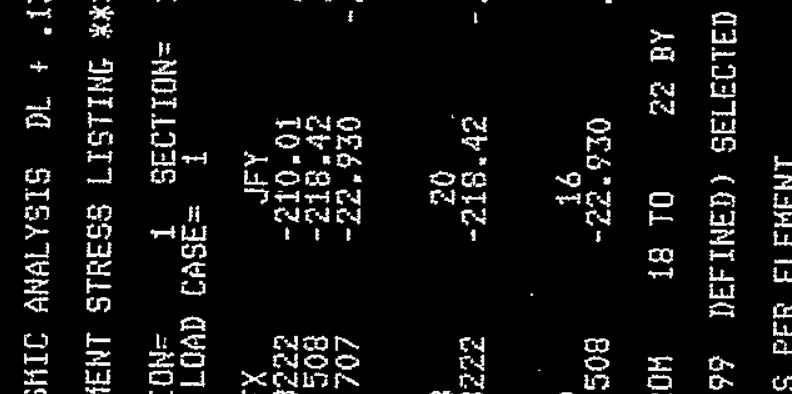

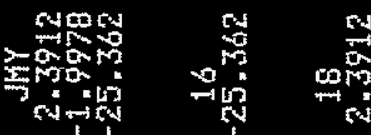

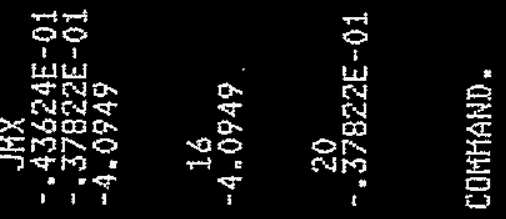

登些尔

空声我

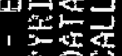

惫

Nong

* W M

4

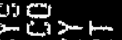

焉

出些

the

党㟧

童

$\frac{5}{1}$

迹

an

恶

$\stackrel{4}{=0}$

岁

密台

Mas

制畹

索

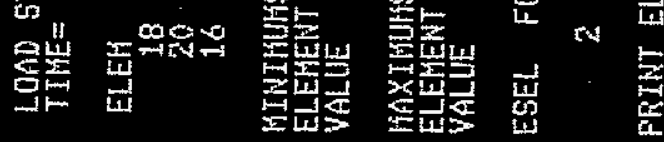


垔

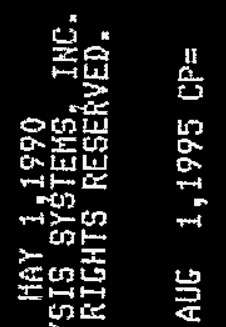

常

迹步

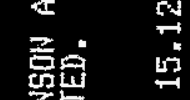

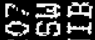

球

or

最

密果

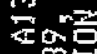

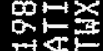

the

बक्षित्र

象语

골

ong

$\rightarrow \rightarrow 0 \infty$

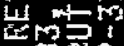

远年

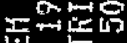

जonth 的思的量

$x^{-1}$ mit

约 崖

券的

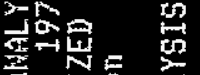

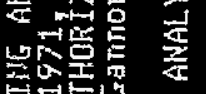

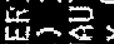

过造点

붕조. 목

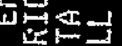

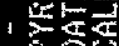

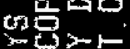

㛺

눈돈

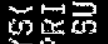

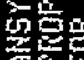

:

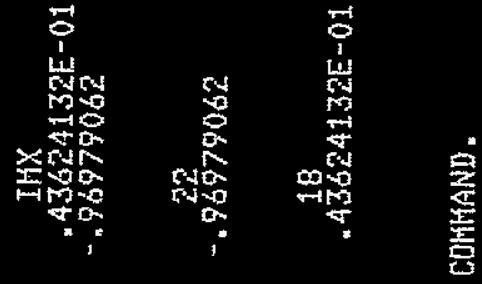

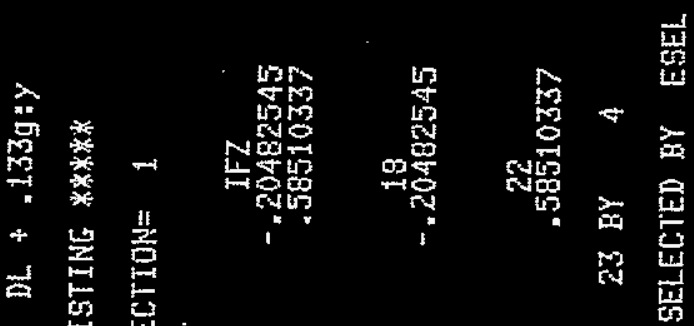

牙 㞻戠

量

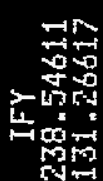

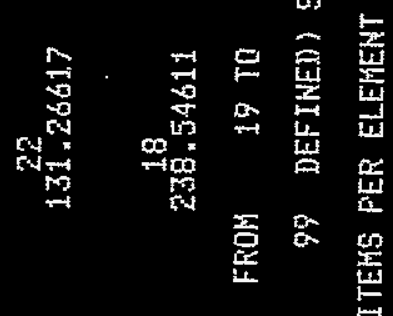

焉

농

$$
\frac{\dot{z}}{\frac{\pi}{5}}
$$

婮

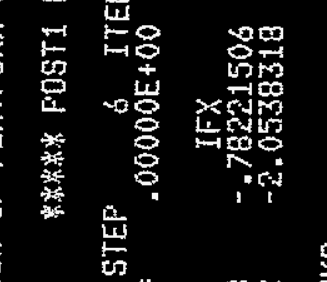

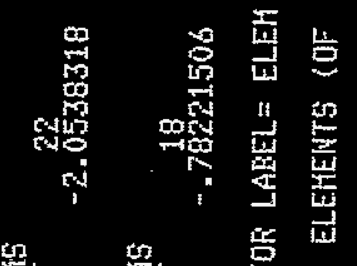

影热 焉

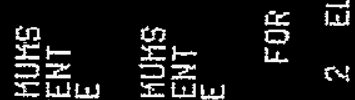

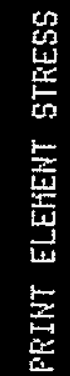


里

to

$\sum_{i=1}^{*}$

成至 㐘

造产

-

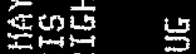

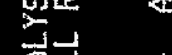

-

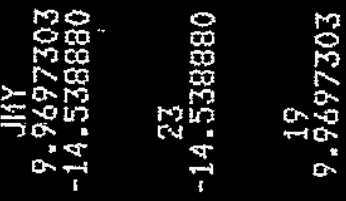

嵒品

당

따ㅇㅢㅜㄹ

dit

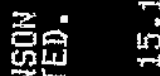

s橆

폰

要

$+\mathrm{cot}$

운

공묘용

본문?

un $N$

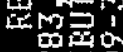

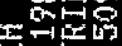

w叫地

50.

$y^{-1} w^{\text {in }}$

에에

in

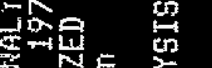

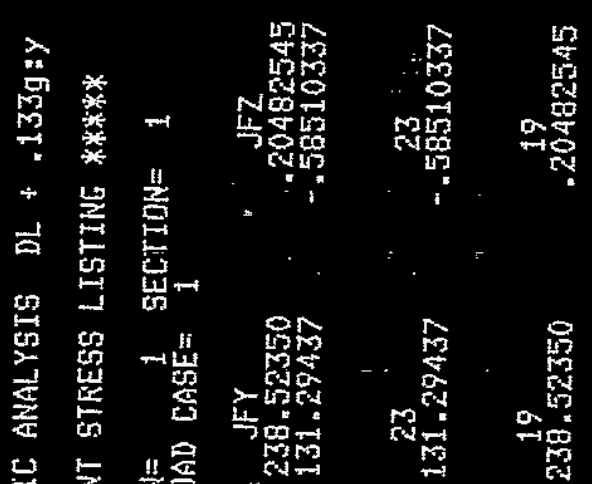

든든

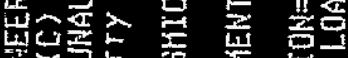

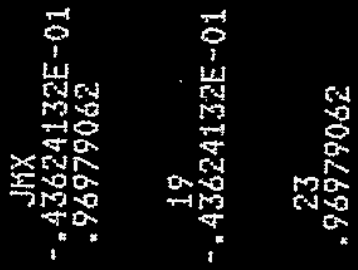

政,

氙

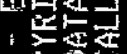

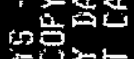

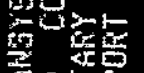

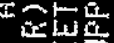

ตับ

的富点

西 焉

湈

단

点员

i.

喜

th

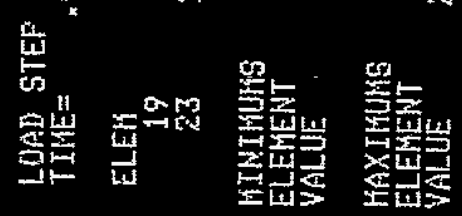




\section{A.4 SUMMARY OF OUYPUT RESULTS}

LOAD CASE NUMBERS: ( 1-6 ) Maximum Einveloped ForceValues

Ref. ANSYS Run (August 1, 1995)

MAXIMUM STRESS/FORCES (Element Coordinate System)

\begin{tabular}{|c|c|c|c|c|c|c|c|c|}
\hline Elem. & $\begin{array}{l}\text { Elem } \\
\text { Type }\end{array}$ & sig1/sig3 & $\begin{array}{l}\text { fx lbs } \\
\text { Sax * }\end{array}$ & $\begin{array}{l}\text { fy } \\
\text { libs }\end{array}$ & $\begin{array}{l}\text { fz } \\
\text { lbs }\end{array}$ & $\mathrm{mx}_{\mathrm{lb}-\mathrm{in}}$ & $\mathrm{my}_{\mathrm{lb}-\mathrm{in}}$ & $\mathrm{mz}_{\mathrm{lb}-\mathrm{in}}$ \\
\hline W6x20 Bm & 1 & 6311 & 864 & 3948 & 396 & 325 & 9174 & 83374 \\
\hline $\mathrm{L} 2 \times 2 \times 1 / 4$ & 2 & $---m$ & $-2410^{*}$ & - & ---- & - & --- & --- \\
\hline W6x20 Col & 3 & 9083 & 6332 & 781 & 39 & - & 2711 & 103500 \\
\hline \multirow[t]{2}{*}{$\mathrm{TS} 3 \times 3 \times 1 / 4$} & \multirow[t]{2}{*}{4} & \multirow{2}{*}{25880} & \multirow[t]{2}{*}{2095} & \multirow[t]{2}{*}{1745} & \multirow[t]{2}{*}{595} & 5 & 10 & 54047 \\
\hline & & & & & & 16330 & 37907 & 12116 \\
\hline C6x8.2 & 5 & 14435 & 356 & 1756 & 65 & 51 & 871 & 60348 \\
\hline
\end{tabular}

MAXIMUM CONNECTION FORCES (Element Coordinate System)

\begin{tabular}{|c|c|c|c|c|c|c|c|}
\hline Elem & $\begin{array}{l}\text { Elem } \\
\text { Type }\end{array}$ & $\begin{array}{l}\text { fx } \\
\text { lbs }\end{array}$ & $\begin{array}{l}\text { fy } \\
\text { lbs }\end{array}$ & $\begin{array}{l}\mathrm{fz} \\
\mathrm{lbs}\end{array}$ & $\mathbf{m x}_{\mathrm{Ib}-\mathrm{in}}$ & $\begin{array}{l}\text { my } \\
\text { lb-in }\end{array}$ & $\mathrm{mz}$ \\
\hline $\mathrm{W} 6 \times 20 \mathrm{Bm}$ & 1 & 565 & 3984 & 409 & 50 & 9174 & 604 \\
\hline $\mathrm{L} 2 \times 2 \times 1 / 4$ & 2 & -2261 & $-\cdots$ & - & --ב-- & ---- & ---. \\
\hline $\mathrm{TS} 3 \times 3 \times 1 / 4$ & 4 & 3503 & 1958 & 372 & 16334 & 6590 & 32063 \\
\hline C6x8.2 & 5 & 247 & 1756 & 170 & 51 & 898 & 59383 \\
\hline
\end{tabular}

MAXIMUM REACTIONS @ COLUMN BASES: (Nodes 1-6) (GLOBAL COORD. SYSTEM)

$\mathrm{Fx}=828 \mathrm{lbs}$

$\mathrm{Fy}=+7177 \mathrm{lbs},-4476 \mathrm{lbs}$

$\mathrm{Fz}=801 \mathrm{lbs}$

MAXIMUM FORCES @ TOP OF COLUMNS: (Nodes 13-18) J nodes of Elem. 2, 4,6,8,10,12

$\mathrm{fx}=-5623 \mathrm{lbs},+1624 \mathrm{lbs} \quad \mathrm{fy}=600 \mathrm{lbs} \quad \mathrm{fz}=26 \mathrm{lbs}$

MAXIMUM FORCES @ CMA BASE: (Nodes 63-66) I nodes of Elem. 76,78,80,82

$f x=3503 \mathrm{lbs} \quad f y=366 \mathrm{lbs} \quad f z=372 \mathrm{lbs}$ 
LOAD CASE NUMBER: ( 1 ) Static [ DL + LL ]

Ref. ANSYS Run (August 1, 1995)

MAXIMUM STRESS/FORCES (Element Coordinate System)

\begin{tabular}{|c|c|c|c|c|c|c|c|c|}
\hline Elem. & $\begin{array}{l}\text { Elem } \\
\text { Type }\end{array}$ & sig1/sig3 & $\begin{array}{l}\text { fx lbs } \\
\text { Sax * }\end{array}$ & $\begin{array}{l}\text { fy } \\
\text { lbs }\end{array}$ & $\begin{array}{l}\text { fz } \\
\text { lbs }\end{array}$ & $\operatorname{mx}_{\mathrm{lb} \text {-in }}$ & $\begin{array}{l}\mathrm{my} \\
\mathrm{lb} \text {-in }\end{array}$ & $\begin{array}{l}\mathrm{mz} \\
\mathrm{lb} \text {-in }\end{array}$ \\
\hline W6x20 Bm & 1 & 5559 & 154 & 2129 & 11 & - & 95 & 74000 \\
\hline$L 2 \times 2 \times 1 / 4$ & 2 & $\ldots$ & $-524^{*}$ & $-\cdots$ & --- & $\ldots$ & - & $-\cdots$ \\
\hline W6x20 Col & 3 & 1897 & 6221 & 30 & 21 & - & 2711 & 3903 \\
\hline $\mathrm{TS} 3 \times 3 \times 1 / 4$ & 4 & 22649 & 1592 & 1500 & 469 & 39 & 18068 & 47702 \\
\hline C6 68.2 & 5 & 3893 & 124 & 485 & - & 7 & 7 & 16973 \\
\hline
\end{tabular}

MAXIMUM CONNECTION FORCES (Element Coordinate System)

\begin{tabular}{|c|c|c|c|c|c|c|c|}
\hline Elem & $\begin{array}{l}\text { Elem } \\
\text { Type }\end{array}$ & $\begin{array}{l}\mathrm{fx} \\
\mathrm{lbs}\end{array}$ & $\begin{array}{l}\text { fy } \\
\text { libs }\end{array}$ & $\begin{array}{l}\mathrm{fz} \\
\mathrm{lbs}\end{array}$ & $\operatorname{mx}_{1 \mathrm{~b}-\mathrm{in}}$ & $\begin{array}{l}m y \\
1 b-\text { in }\end{array}$ & $\begin{array}{l}\mathrm{mz} \\
\mathrm{lb} \text {-in }\end{array}$ \\
\hline $\mathrm{W} 6 \times 20 \mathrm{Bm}$ & 1 & 207 & 2774 & 14 & 50 & 224 & 317 \\
\hline $\mathrm{L} 2 \times 2 \times 1 / 4$ & 2 & -491 & ---- & ---- & -..- & $-\ldots$ & -..- \\
\hline $\operatorname{TS} 3 \times 3 \times 1 / 4$ & 4 & 468 & 1560 & 4 & 14430 & 55 & 19138 \\
\hline C6x8.2 & 5 & 124 & 485 & - & 7 & 12 & 7709 \\
\hline
\end{tabular}

MAXIMUM REACTIONS @ () COLUMN BASES: (Nodes 1-6)

(GLOBAL COORDINATE SYSTEM)

$\mathrm{Fx}=30 \mathrm{lbs} \quad \mathrm{Fy}=6612 \mathrm{lbs} \quad \mathrm{Fz}=343 \mathrm{lbs}$

MAXIMUM FORCES @ TOP OF COLUMNS: (Nodes 13-18) J nodes of Elem.2,4,6,8,10,12

$\mathrm{fx}=-5623 \mathrm{lbs},+294 \mathrm{lbs}$

$\mathrm{fy}=29 \mathrm{lbs}$

$\mathrm{fz}=20 \mathrm{lbs}$

MAXIMUM FORCES @ CMA BASE: (Nodes 63-66) I nodes of Elem. 76,78,80,82

$f x=1765 \mathrm{lbs} \quad f y=12 \mathrm{lbs} \quad \mathrm{fz}=20 \mathrm{lbs}$ 
LOAD CASE NUMBER: ( 2 ) Seismic $[\mathrm{DL}+.2 \mathrm{~g}(\mathrm{x})+.06 \mathrm{~g}(\mathrm{z})]$

Ref. ANSYS Run (August 1, 1995)

MAXIMUM STRESS/FORCES (Element Coordinate System)

\begin{tabular}{|c|c|c|c|c|c|c|c|c|}
\hline Elem. & $\begin{array}{l}\text { Elem } \\
\text { Type }\end{array}$ & sig1/sig3 & $\begin{array}{l}\text { fx Ibs } \\
\text { Sax }^{*}\end{array}$ & $\begin{array}{l}\text { fy } \\
\text { los }\end{array}$ & $\begin{array}{l}\text { fzz } \\
\text { los }\end{array}$ & $\operatorname{mxx}_{\mathrm{lb} \text {-in }}$ & $\mathrm{my}_{\mathrm{b}-\mathrm{in}}$ & $\frac{m z}{1 b-i n}$ \\
\hline W6x20 Bm & 1 & 5769 & 864 & 3945 & 341 & 325 & 2426 & 69481 \\
\hline $\mathrm{L} 2 \times 2 \times 1 / 4$ & 2 & -..- & $-2410^{*}$ & - & -..-- & -.-- & - & $-\cdots$ \\
\hline W6 $620 \mathrm{Col}$ & 3 & 8920 & 5340 & 772 & 18 & - & 1546 & 102300 \\
\hline $\mathrm{TS} 3 \times 3 \times 1 / 4$ & 4 & 24201 & 2092 & 1576 & 594 & 476 & 23172 & 47703 \\
\hline C6x8.2 & 5 & 14234 & 128 & 1731 & 65 & 50 & 870 & 59383 \\
\hline
\end{tabular}

MAXIMUM CONNECTION FORCES (Element Coordinate System)

\begin{tabular}{|c|c|c|c|c|c|c|c|}
\hline Elem & $\begin{array}{l}\text { Elem } \\
\text { Type }\end{array}$ & $\begin{array}{l}\text { fx } \\
\text { lbs }\end{array}$ & $\begin{array}{l}\text { fy } \\
\text { lbs }\end{array}$ & $\begin{array}{l}\text { fzz } \\
\text { lbs }\end{array}$ & $\mathrm{mx}_{\mathrm{lb}-\mathrm{in}}$ & ${ }_{\mathrm{lb}-\mathrm{in}}^{\mathrm{my}}$ & $\begin{array}{l}m z \\
\mathbf{l b} \text {-in }\end{array}$ \\
\hline W6x20 Bm & 1 & 338 & 3984 & 405 & 7 & 8500 & 604 \\
\hline $\mathrm{L} 2 \times 2 \times 1 / 4$ & 2 & -2261 & -..-- & - & --- & ---- & ----- \\
\hline $\mathrm{TS} 3 \times 3 \times 1 / 4$ & 4 & 603 & 1765 & 359 & 17533 & 4046 & 23507 \\
\hline C $6 \times 8.2$ & 5 & 128 & 1731 & 65 & 50 & 803 & 59383 \\
\hline
\end{tabular}

MAXIMUM REACTIONS @ COLUMN BASES: (Nodes 1-6)

(GLOBAL COORDINATE SYSTEM)

$F x=819 \mathrm{lbs} \quad F y=+5868 \mathrm{lbs},-1251 \mathrm{lbs} \quad F z=389 \mathrm{lbs}$

MAXMUM FORCES @ TOP OF COLUMNS: (Nodes 13-18) J nodes of Elem.2,4,6,8,10,12

$f x=-2932 \mathrm{lbs} \quad f y=600 \mathrm{lbs} \quad f z=15 \mathrm{lbs}$

MAXIMUM FORCES @ CMA BASE: (Nodes 63-66) I nodes of Elem. 76,78,80,82

$f_{x}=3488 \mathrm{lbs} \quad f y=365 \mathrm{lbs} \quad f z=128 \mathrm{lbs}$ 
LOAD CASE NUMBER: ( 3 ) Sesmic [ DL $+.2 \mathrm{~g}(-\mathrm{x})+.06 \mathrm{~g}(\mathrm{z})]$ Ref. ANSYS Run (August 1,1995 )

MAXIMUM STRESS/FORCES (Element Coordinate System)

\begin{tabular}{|c|c|c|c|c|c|c|c|c|}
\hline Elem. & $\begin{array}{l}\text { Elem } \\
\text { Type }\end{array}$ & sig1/sig3 & $\begin{array}{l}\text { fx lbs } \\
\text { Sax * }\end{array}$ & $\begin{array}{l}\text { fy } \\
\text { lbs }\end{array}$ & $\begin{array}{l}\text { fz } \\
\text { lbs }\end{array}$ & $\operatorname{mx}_{\mathrm{lb} \text {-in }}$ & $\begin{array}{l}\text { my } \\
1 \mathrm{~b}-\text { in }\end{array}$ & $\begin{array}{l}m z \\
\mathrm{lb}-\mathrm{in}\end{array}$ \\
\hline W6×20 Bm & 1 & 5967 & 562 & 3948 & 396 & - & 9174 & 69064 \\
\hline $\mathrm{L} 2 \times 2 \times 1 / 4$ & 2 & $-\cdots$ & $-2356^{\star}$ & 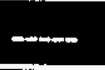 & - & $-\ldots$ & $-\cdots$ & --- \\
\hline W6×20 Col & 3 & 9083 & 6332 & 781 & 18 & - & 1504 & 103500 \\
\hline TS $3 \times 3 \times 1 / 4$ & 4 & 24235 & 2095 & 1576 & 595 & 475 & 1966 & 47702 \\
\hline C6x8.2 & 5 & 14435 & 249 & 1756 & 65 & 51 & 865 & 60348 \\
\hline
\end{tabular}

MAXIMUM CONNECTION FORCES (Element Coordinate System)

\begin{tabular}{|c|c|c|c|c|c|c|c|}
\hline Elem & $\begin{array}{l}\text { Elem } \\
\text { Type } \\
\end{array}$ & $\begin{array}{l}\mathrm{fx} \\
\mathrm{lbs}\end{array}$ & $\begin{array}{l}\text { fy } \\
\text { lbs }\end{array}$ & $\begin{array}{l}\mathrm{fz} \\
\mathrm{lbs}\end{array}$ & $\mathrm{mx}_{\mathrm{lb}-\mathrm{in}}$ & $\mathrm{my}_{\mathrm{lb} \text {-in }}$ & $\begin{array}{l}\mathrm{mz} \\
\mathrm{lb} \text {-in }\end{array}$ \\
\hline W6x20 Bm & 1 & 565 & 3966 & 409 & 10 & 9174 & 219 \\
\hline $\mathrm{L} 2 \times 2 \times 1 / 4$ & 2 & -2210 & --- & - & --.- & --.- & ---- \\
\hline $\mathrm{TS} 3 \times 3 \times 1 / 4$ & 4 & 603 & 1767 & 360 & 17543 & 3599 & 23554 \\
\hline C6x8.2 & 5 & 247 & 1756 & 65 & 51 & 828 & 54033 \\
\hline
\end{tabular}

MAXIMUM REACTIONS @ COLUMN BASES: (Nodes 1-6)

(GLOBAL COORDINATE SYSTEM)

$\mathrm{Fx}=828 \mathrm{lbs} \quad \mathrm{Fy}=+7177 \mathrm{lbs},-4476 \mathrm{lbs} \quad \mathrm{Fz}=550 \mathrm{lbs}$

MAXIMUM FORCES @ TOP OF COLUMNS: (Nodes 13-18) J nodes of Elem.2,4,6,8,10,12

$\mathrm{fx}=-4225 \mathrm{lbs},+1624 \mathrm{lbs} \quad \mathrm{fy}=588 \mathrm{lbs} \quad \mathrm{fz}=14 \mathrm{lbs}$

MAXIMUM FORCES @ CMA BASE: (Nodes 63-66) I nodes of Elem. 76,78,80,82

$\mathrm{fx}=3495 \mathrm{lbs} \quad \mathrm{fy}=366 \mathrm{lbs} \quad \mathrm{fz}=128 \mathrm{lbs}$ 
LOAD CASE NUMBER: ( 4 ) Seismic [ DL + .06g $(\mathrm{x})+.2 \mathrm{~g}(\mathrm{z})]$

Ref. ANSYS Run (August 1, 1995)

MAXIMUM STRESS/FORCES (Element Coordinate System)

\begin{tabular}{|c|c|c|c|c|c|c|c|c|}
\hline Elem. & $\begin{array}{l}\text { Elem } \\
\text { Type }\end{array}$ & sig1/sig3 & $\begin{array}{l}\text { fx lbs } \\
\text { Sax * }\end{array}$ & $\begin{array}{l}\text { fy } \\
\text { lbs }\end{array}$ & $\begin{array}{l}\text { fz } \\
\text { lbs }\end{array}$ & $\operatorname{mx}_{1 \mathrm{~b}-\mathrm{in}}$ & $\mathrm{my}_{\mathrm{lb}-\mathrm{in}}$ & $\mathrm{mz}_{\mathrm{lb}-\mathrm{in}}$ \\
\hline $\mathrm{W} 6 \times 20 \mathrm{Bm}$ & 1 & 3146 & 379 & 3547 & 379 & 201 & 4304 & 38678 \\
\hline$[2 \times 2 \times 1 / 4$ & 2 & - & $-1251 *$ & - & ---- & - & ----- & - \\
\hline W6×20 Col & 3 & 2974 & 5751 & 229 & 36 & - & 1918 & 30407 \\
\hline $\mathrm{TS} 3 \times 3 \times 1 / 4$ & 4 & 25870 & 491 & 1523 & 356 & 100 & 37784 & 47703 \\
\hline C6x8.2 & 5 & 5029 & 57 & 645 & 48 & - & 871 & 19207 \\
\hline
\end{tabular}

MAXMMUM CONNECTION FORCES (Element Coordinate System)

\begin{tabular}{|c|c|c|c|c|c|c|c|}
\hline Elem & $\begin{array}{l}\text { Elem } \\
\text { Type }\end{array}$ & $\begin{array}{l}\text { fx } \\
\text { lbs }\end{array}$ & $\begin{array}{l}\text { fy } \\
\text { lbs }\end{array}$ & $\begin{array}{l}\text { fz } \\
\text { lbs }\end{array}$ & $\operatorname{mx}_{\mathrm{lb}-\mathrm{in}}$ & $\mathrm{my}_{\mathrm{Ib} \text {-in }}$ & $\mathbf{m z}$ \\
\hline W6x20 Bm & 1 & 390 & 3560 & 154 & 9 & 4552 & 380 \\
\hline $\mathrm{L} 2 \times 2 \times 1 / 4$ & 2 & -1173 & ---.- & ----- & מבדיב- & 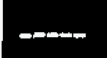 & --..- \\
\hline $\mathrm{TS} 3 \times 3 \times 1 / 4$ & 4 & 792 & 1958 & 322 & 15340 & 6590 & 32063 \\
\hline C6x8.2 & 5 & 59 & 645 & 48 & -- & 898 & 19207 \\
\hline
\end{tabular}

MAXIMUM REACTIONS @ COLUMN BASES: (Nodes 1-6)

(GLOBAL COORDINATE SYSTEM)

$\mathrm{Fx}=243 \mathrm{lbs} \quad \mathrm{Fy}=+6653 \mathrm{lbs},-1495 \mathrm{lbs} \quad \mathrm{Fz}=801 \mathrm{lbs}$

MAXMMUM FORCES @ TOP OF COLUMNS: (Nodes 13-18) J nodes of Elem.2,4,6,8,10,12

$f x=-4162 \mathrm{lbs} \quad f y=194 \mathrm{lbs} \quad \mathrm{fz}=26 \mathrm{lbs}$

MAXIMUM FORCES @ CMA BASE: (Nodes 63-66) I nodes of Elem. .76,78,80,82

$f x=3488 \mathrm{lbs} \quad f y=365 \mathrm{lbs} \quad \mathrm{fz}=128 \mathrm{lbs}$ 
LOAD CASE NUMBER: $(5)$ Seismic $[\mathrm{DL}+.06 \mathrm{~g}(\mathrm{x})+.2 \mathrm{~g}(-\mathrm{z})]$ Ref. ANSYS Run (August 1, 1995)

MAXIMUM STRESS/FORCES (Element Coordinate System)

\begin{tabular}{|c|c|c|c|c|c|c|c|c|}
\hline Elem. & $\begin{array}{l}\text { Elem } \\
\text { Type }\end{array}$ & sig1/sig3 & $\begin{array}{l}\text { fx lbs } \\
\text { Sax * }\end{array}$ & $\begin{array}{l}\text { fy } \\
\text { lbs }\end{array}$ & $\begin{array}{l}\mathrm{fz} \\
\mathrm{lbs}\end{array}$ & $\operatorname{mx}_{1 b-i n}$ & my & $\begin{array}{l}m z \\
\mathrm{lb} \text {-in }\end{array}$ \\
\hline W6x20 Bm & 1 & 6311 & 358 & 2164 & 170 & 7 & 1951 & 83374 \\
\hline$L 2 \times 2 \times 1 / 4$ & 2 & $\ldots$ & $-1140^{*}$ & $-\cdots$ & - & --- & - & - \\
\hline W6x20 Col & 3 & 3169 & 4286 & 251 & 39 & - & 2287 & 33189 \\
\hline $\operatorname{TS} 3 \times 3 \times 1 / 4$ & 4 & 25880 & 473 & 1523 & 357 & 153 & 37907 & 47703 \\
\hline C6x8.2 & 5 & 5926 & 170 & 681 & 47 & - & 833 & 20570 \\
\hline
\end{tabular}

MAXIMUM CONNECTION FORCES (Element Coordinate System)

\begin{tabular}{|c|c|c|c|c|c|c|c|}
\hline Elem & $\begin{array}{l}\text { Elem } \\
\text { Type }\end{array}$ & $\begin{array}{l}\mathrm{fx} \\
\mathrm{lbs}\end{array}$ & $\begin{array}{l}\text { fy } \\
\text { libs }\end{array}$ & $\begin{array}{l}\mathrm{fz} \\
\mathrm{lbs}\end{array}$ & $\operatorname{mx}_{1 \mathrm{~b}-\text { in }}$ & $\begin{array}{l}\text { my } \\
\mathrm{lb} \text {-in }\end{array}$ & $\begin{array}{l}m z \\
1 b-i n\end{array}$ \\
\hline W6x20 Bm & 1 & 407 & 2825 & 171 & 7 & 3686 & 344 \\
\hline $\mathrm{L} 2 \times 2 \times 1 / 4$ & 2 & -1069 & ---- & ---- & --- & --.. & --- \\
\hline $\mathrm{TS} 3 \times 3 \times 1 / 4$ & 4 & 3503 & 1945 & 372 & 13471 & 6545 & 31754 \\
\hline C6x8.2 & 5 & 57 & 211 & 170 & - & 823 & 20570 \\
\hline
\end{tabular}

MAXIMUM REACTIONS @ COLUMN BASES: (Nodes 1-6) (GLOBAL COORDINATE SYSTEM)

$\mathrm{Fx}=265 \mathrm{lbs} \quad \mathrm{Fy}=+5292 \mathrm{lbs},-684 \mathrm{lbs} \quad \mathrm{Fz}=732 \mathrm{lbs}$

MAXIMUM FORCES @ TOP OF COLUMNS: (Nodes 13-18) J nodes of Elem.2,4,6,8,10,12

$f x=-3248 \mathrm{lbs}$

$\mathrm{fy}=170 \mathrm{lbs}$

$\mathrm{fz}=22 \mathrm{lbs}$

MAXIMUM FORCES @ CMA BASE: (Nodes 63-66) I nodes of Elem. 76,78,80,82

$\mathrm{fx}=3503 \mathrm{lbs}$

$\mathrm{fy}=119 \mathrm{lbs}$

$\mathrm{fz}=372 \mathrm{lbs}$ 
LOAD CASE NUMBER: ( 6 ) Seismic [ DL + .133g (y) ] Ref. ANSYS Run (August 1, 1995)

MAXIMUM STRESS/FORCES (Element Coordinate System)

\begin{tabular}{|c|c|c|c|c|c|c|c|c|}
\hline Elem. & $\begin{array}{l}\text { Elem } \\
\text { Type }\end{array}$ & sig1/sig3 & $\begin{array}{l}\text { fx lbs } \\
\text { Sax * }\end{array}$ & $\begin{array}{l}\text { fy } \\
\text { lbs } \\
\end{array}$ & $\begin{array}{l}\text { fzz } \\
\text { los }\end{array}$ & $\mathrm{mx}_{\mathrm{lb}-\mathrm{in}}$ & $\mathrm{my}_{\mathrm{lb} \text {-in }}$ & $\begin{array}{l}\mathbf{m z} \\
\mathbf{l b}-\mathrm{in}\end{array}$ \\
\hline W6x20 Bm & 1 & 4092 & 127 & 1795 & 12 & 2 & 130 & 53718 \\
\hline $\mathrm{L} 2 \times 2 \times 1 / 4$ & 2 & ---- & $-321 *$ & --- & -- & - & ---- & ---- \\
\hline W6x20 Col & 3 & 1075 & 3980 & 16 & 12 & - & 1553 & 2039 \\
\hline $\mathrm{TS} 3 \times 3 \times 1 / 4$ & 4 & 25661 & 1799 & 1745 & 530 & 16330 & 20422 & 54047 \\
\hline C6x8.2 & 5 & 1900 & 356 & 247 & - & 4 & 25 & 8282 \\
\hline
\end{tabular}

MAXIMUM CONNECTION FORCES (Element Coordinate System)

\begin{tabular}{|c|c|c|c|c|c|c|c|}
\hline Elem & $\begin{array}{l}\text { Elem } \\
\text { Type }\end{array}$ & $\begin{array}{l}\text { fx } \\
\text { lbs }\end{array}$ & $\begin{array}{l}\text { fy } \\
\text { lbs }\end{array}$ & $\begin{array}{l}\mathrm{fz} \\
\mathrm{lbs}\end{array}$ & $\operatorname{mx}_{1 \mathrm{~b}-\mathrm{in}}$ & $\mathrm{my}_{\mathrm{lb}-\mathrm{in}}$ & $\begin{array}{l}\mathrm{mz} \\
\mathrm{lb} \text {-in }\end{array}$ \\
\hline W6x20 Bm & 1 & 127 & 2512 & 16 & 6 & 236 & 434 \\
\hline $\mathrm{L} 2 \times 2 \times 1 / 4$ & 2 & -301 & ---- & --.- & ---- & - & --- \\
\hline $\operatorname{TS} 3 \times 3 \times 1 / 4$ & 4 & 530 & 1764 & 3 & 16334 & 45 & 20424 \\
\hline C6x8.2 & 5 & 74 & 247 & - & - & 15 & 3883 \\
\hline
\end{tabular}

MAXIMUM REACTIONS @ COLUMN BASES: (Nodes 1-6) (GLOBAL COORDINATE SYSTEM)

$F x=16 \mathrm{lbs} \quad F y=4227 \mathrm{lbs} \quad F z=203 \mathrm{lbs}$

MAXIMUM FORCES @ TOP OF COLUMNS: (Nodes 13-18) J nodes of Elem.2,4,6,8,10,12 $f x=-3360 \mathrm{lbs}$ fy $=14 \mathrm{lbs} \quad \mathrm{fz}=11 \mathrm{lbs}$

MAXIMUM FORCES @ CMA BASE: (Nodes 63-66) I nodes of Elem. 76,78,80,82 $\mathrm{fx}=1990 \mathrm{lbs}$ fy $=14 \mathrm{lbs}$ $\mathrm{fz}=23 \mathrm{lbs}$ 
WHC-SD-WM-ER-521

Rev. 0

APPENDIX B

DESIGN EVALUATION OF MEMBERS AND JOINTS 
Page of

Subject DST MOCK-LP PLATEOKM

Originator

Checker

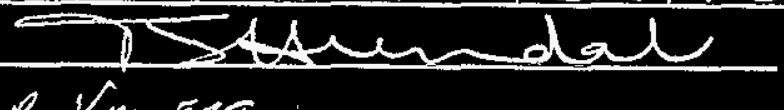

Date $8-10-95$

Date

$12-7-95$

SWMMMARY

LOAD CASE NUMBER: ( 1-6 ) Maximum Enveloped ForceValues Ref. ANSYS Run (August 1, 1995)

MAXIMUM STRESS/FORCES (Element Coordinate System)

\begin{tabular}{|c|c|c|c|c|c|c|c|c|}
\hline Elem. & $\begin{array}{c}\text { Elem } \\
\text { Type }\end{array}$ & sig1/sig3 & $\begin{array}{l}\text { fx lbs } \\
\text { Sax * }\end{array}$ & $\begin{array}{l}\text { fy } \\
\text { ibs }\end{array}$ & $\begin{array}{l}\mathrm{fz} \\
\mathrm{ibs}\end{array}$ & $\begin{array}{l}\mathrm{mx} \\
\mathrm{lb} \text {-in }\end{array}$ & $\begin{array}{l}\text { my } \\
\mathrm{lb} \text {-in }\end{array}$ & $\begin{array}{l}\mathrm{mz} \\
\mathrm{lb} \text {-in }\end{array}$ \\
\hline $\mathrm{W} 6 \times 20 \mathrm{Bm}$ & 1 & 6311 & 864 & 3948 & 396 & 325 & 9174 & 83374 \\
\hline $\mathrm{L} 2 \times 2 \times 1 / 4$ & 2 & ---- & $-2410^{*}$ & $-\cdots$ & ---- & -.-. & - & ---. \\
\hline W6 $\times 20 \mathrm{Col}$ & 3 & 9083 & 6332 & 781 & 39 & - & 2711 & 103500 \\
\hline \multirow[t]{2}{*}{$\operatorname{TS} 3 \times 3 \times 1 / 4$} & \multirow[t]{2}{*}{4} & \multirow[t]{2}{*}{25880} & \multirow[t]{2}{*}{2095} & \multirow[t]{2}{*}{1745} & \multirow[t]{2}{*}{595} & 5 & 10 & 54047 \\
\hline & & & & & & 16330 & 37907 & 12116 \\
\hline C6x8.2 & 5 & 14435 & 356 & 1756 & 65 & 51 & 871 & 60348 \\
\hline
\end{tabular}

*-psi

MAXIMUM CONNECTION FORCES (Element Coordinate System)

\begin{tabular}{|c|c|c|c|c|c|c|c|}
\hline Elem & $\begin{array}{l}\text { Elem } \\
\text { Type }\end{array}$ & $\begin{array}{l}\mathrm{fx} \\
\mathrm{lbs} \\
\end{array}$ & $\begin{array}{l}\text { fy } \\
\text { los }\end{array}$ & $\begin{array}{l}\mathbf{f z} \\
\mathrm{lbs}\end{array}$ & $\begin{array}{l}m x \\
\mathrm{lb} \text {-in }\end{array}$ & $\begin{array}{l}\mathrm{my} \\
\mathrm{lb} \text {-in }\end{array}$ & $\begin{array}{l}\mathrm{mz} \\
\mathrm{lb} \text {-in }\end{array}$ \\
\hline $\mathrm{W} 6 \times 20 \mathrm{Bm}$ & 1 & 565 & 3984 & 409 & 50 & 9174 & 604 \\
\hline $\mathrm{L} 2 \times 2 \times 1 / 4$ & 2 & -2261 & $-\cdots$ & - & ---- & ב--- & $-\cdots$ \\
\hline $\operatorname{TS} 3 \times 3 \times 1 / 4$ & 4 & 3503 & 1958 & 372 & 16334 & 6590 & 32063 \\
\hline C6x8.2 & 5 & 247 & 1756 & 170 & 51 & 898 & 59383 \\
\hline
\end{tabular}

MAXIMUM REACTIONS @ COLUMN BASES: (Nodes 1-6) (GLOBAL COORD. SYSTEM)

$\mathrm{Fx}=828 \mathrm{lbs}$

$\mathrm{Fy}=+7177 \mathrm{lbs},-4476 \mathrm{lbs}$

$\mathrm{Fz}=801 \mathrm{lbs}$

MAXIMUM FORCES @ TOP OF COLUMNS: (Nodes 13-18) J nodes of Elem.2,4,6,8,10,12

$f x=-5623 \mathrm{lbs},+1624 \mathrm{lbs} \quad f y=600 \mathrm{lbs} \quad f z=26 \mathrm{lbs}$

MAXIMUM FORCES @ CMA BASE: (Nodes 63-66) I nodes of Elem. 76,78,80,82

$\mathrm{fx}=3503 \mathrm{lbs}$

$f y=366 \mathrm{lbs}$

$\mathrm{fz}=\mathbf{3 7 2} \mathrm{lbs}$

$B-2$ 
Subject DST MOCK-LP PLATFORM

Originator

Checker

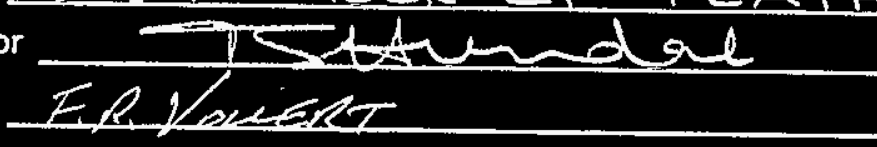

$$
\text { Date } \frac{8-10-95}{2-5-95}
$$
of

Page

LOAD CASE NUMBER: ( 1 ) Static [ DL + LL ] Ref. ANSYS Run (August 1,1995$)$

\begin{tabular}{|c|c|c|c|c|c|c|c|c|}
\hline Elem. & $\begin{array}{l}\text { Elem } \\
\text { Type } \\
\end{array}$ & sig1/sig3 & $\begin{array}{l}\text { fx lbs } \\
\text { Sax* }\end{array}$ & $\begin{array}{l}\text { fy } \\
\text { lbs }\end{array}$ & $\begin{array}{l}\text { fz } \\
\text { lbs }\end{array}$ & $\begin{array}{l}\mathrm{mx} \\
\mathrm{lb} \text {-in }\end{array}$ & $\begin{array}{l}\text { my } \\
\text { lb-in }\end{array}$ & $\begin{array}{l}\mathrm{mz} \\
\mathrm{lb} \text {-in }\end{array}$ \\
\hline $\mathrm{W} 6 \times 20 \mathrm{Bm}$ & 1 & 5559 & 154 & 2129 & 11 & - & 95 & 74000 \\
\hline $\mathrm{L} 2 \times 2 \times 1 / 4$ & 2 & --- & $-524^{*}$ & $-\cdots$ & -- & ---- & $-\cdots$ & - \\
\hline $\mathrm{W} 6 \times 20 \mathrm{Col}$ & 3 & 1897 & 6221 & 30 & 21 & - & 2711 & 3903 \\
\hline $\mathrm{TS} 3 \times 3 \times 1 / 4$ & 4 & 22649 & 1592 & 1500 & 469 & 39 & 18068 & 47702 \\
\hline $\mathrm{C} 6 \times 8.2$ & 5 & 3893 & 124 & 485 & - & 7 & 7 & 16973 \\
\hline
\end{tabular}

MAXIMUM STRESS/FORCES (Element Coordinate System)

\begin{tabular}{|c|c|c|c|c|c|c|c|}
\hline Elem & $\begin{array}{l}\text { Elem } \\
\text { Type } \\
\end{array}$ & $\begin{array}{l}\mathrm{fx} \\
\mathrm{lbs}\end{array}$ & $\begin{array}{l}\text { fy } \\
\mathrm{lbs}\end{array}$ & $\begin{array}{l}\text { fiz } \\
\text { lbs }\end{array}$ & $\begin{array}{l}\mathrm{mx} \\
\mathrm{lb} \text {-in }\end{array}$ & $\begin{array}{l}\text { my } \\
\mathrm{lb} \text {-in }\end{array}$ & $\begin{array}{l}\mathrm{mz} \\
\mathrm{lb} \text {-in }\end{array}$ \\
\hline $\mathrm{W} 6 \times 20 \mathrm{Bm}$ & 1 & 207 & 2774 & 14 & 50 & 224 & 317 \\
\hline $\mathrm{L} 2 \times 2 \times 1 / 4$ & 2 & -491 & --- & ---- & $-\cdots$ & --- & ---- \\
\hline $\mathrm{TS} 3 \times 3 \times 1 / 4$ & 4 & 468 & 1560 & 4 & 14430 & 55 & 19138 \\
\hline C6x8.2 & 5 & 124 & 485 & - & 7 & 12 & 7709 \\
\hline
\end{tabular}

MAXIMUM CONNECTION FORCES (Element Coordinate System)

MAXIMUM REACTIONS @ COLUMN BASES: (Nodes 1-6) (GLOBAL COORDINATE SYSTEM)

$\mathrm{Fx}=30 \mathrm{lbs}$

$$
\mathrm{Fy}=6612 \mathrm{lbs}
$$

$F_{Z}=343 \mathrm{lbs}$

MAXIMUM FORCES @ TOP OF COLUMNS: (Nodes 13-18) J nodes of Elem.2,4,6,8,10,12

$f x=-5623 \mathrm{lbs},+294 \mathrm{lbs}$

$\mathrm{fy}=29 \mathrm{lbs}$

$f z=20 \mathrm{lbs}$

MAXIMUM FORCES @ CMA BASE: (Nodes 63-66) I nodes of Elem. 76,78,80,82

$f x=1765 \mathrm{lbs}$

$$
\begin{gathered}
f y=12 \mathrm{lbs} \quad f z=20 \mathrm{lbs} \\
B-3
\end{gathered}
$$




\section{ANALYTICAL GALCULATIONS}

Page of

Subject DST MOCK-UP PLATFORM

Originator 1)tAundal Checker FiR Voust Date $8-10-95$ Date $12-5=95$

LOAD CASE NUMBER: (2) Seismic [ $\mathrm{DL}+.2 \mathrm{~g}(\mathrm{x})+.06 \mathrm{~g}(\mathrm{z})]$ Ref. ANSYS Run (August 1, 1995)

MAXIMUM STRESS/FORCES (Element Coordinate System)

\begin{tabular}{|c|c|c|c|c|c|c|c|c|}
\hline Elem. & $\begin{array}{l}\text { Elem } \\
\text { Type }\end{array}$ & sig1/sig3 & $\begin{array}{l}\text { fx lbs } \\
\text { Sax* }\end{array}$ & $\begin{array}{l}\text { fy } \\
\text { lbs }\end{array}$ & $\begin{array}{l}\mathrm{fz} \\
\mathrm{lbs}\end{array}$ & $\begin{array}{l}\mathrm{mx} \\
\mathrm{l} \mathrm{b} \text {-in }\end{array}$ & $\begin{array}{l}\text { my } \\
\mathrm{lb} \text {-in }\end{array}$ & $\begin{array}{l}\mathrm{mz} \\
\mathrm{lb}-\text { in }\end{array}$ \\
\hline $\mathrm{W} 6 \times 20 \mathrm{Bm}$ & 1 & 5769 & 864 & 3945 & 341 & 325 & 2426 & 69481 \\
\hline $\mathrm{L} 2 \times 2 \times 1 / 4$ & 2 & --- & $-2410^{*}$ & --- & - & --.- & - & --- \\
\hline $\mathrm{W} 6 \times 20 \mathrm{Col}$ & 3 & 8920 & 5340 & 772 & 18 & -- & 1546 & 102300 \\
\hline TS $3 \times 3 \times 1 / 4$ & 4 & 24201 & 2092 & 1576 & 594 & 476 & 23172 & 47703 \\
\hline C6x8.2 & 5 & 14234 & 128 & 1731 & 65 & 50 & 870 & 59383 \\
\hline
\end{tabular}

$$
\text { *-psi }
$$

MAXIMUM CONNECTION FORCES (Element Coordinate System)

\begin{tabular}{|c|c|c|c|c|c|c|c|}
\hline Elem & $\begin{array}{l}\text { Elem } \\
\text { Type }\end{array}$ & $\begin{array}{l}\mathrm{fx} \\
\mathrm{lbs}\end{array}$ & $\begin{array}{l}\text { fy } \\
\text { lbs }\end{array}$ & $\begin{array}{l}\mathrm{fz} \\
\mathrm{lbs}\end{array}$ & $\underbrace{\mathrm{mx}}_{\mathrm{lb}-\mathrm{in}}$ & $\begin{array}{l}\text { my } \\
\text { lb-in }\end{array}$ & $\begin{array}{l}\mathrm{mz} \\
\mathrm{lb}-\mathrm{in}\end{array}$ \\
\hline $\mathrm{W} 6 \times 20 \mathrm{Bm}$ & 1 & 338 & 3984 & 405 & 7 & 8500 & 604 \\
\hline $\mathrm{L} 2 \times 2 \times 1 / 4$ & 2 & -2261 & $-\cdots$ & $-\cdots$ & $-\cdots$ & $-\cdots$ & $-\cdots$ \\
\hline $\mathrm{TS} 3 \times 3 \times 1 / 4$ & 4 & 603 & 1765 & 359 & 17533 & 4046 & 23507 \\
\hline C6x8.2 & 5 & 128 & 1731 & 65 & 50 & 803 & 59383 \\
\hline
\end{tabular}

MAXIMUM REACTIONS @ COLUMN BASES: (Nodes 1-6) (GLOBAL COORDINATE SYSTEM)

$\mathrm{Fx}=819 \mathrm{lbs}$

$$
F y=+5868 \mathrm{lbs},-1251 \mathrm{lbs}
$$$$
\mathrm{Fz}=389 \mathrm{lbs}
$$

MAXIMUM FORCES @ TOP OF COLUMNS: (Nodes 13-18) J nodes of Elem.2,4,6,8,10,12

$f x=-2932 \mathrm{lbs}$

$f y=600 \mathrm{lbs}$ $\mathrm{fz}=15 \mathrm{lbs}$

MAXIMUM FORCES @ CMA BASE: (Nodes 63-66) I nodes of Elem. 76,78,80,82

$\mathrm{fx}=3488 \mathrm{lbs}$

$$
\text { fy }=365 \mathrm{lbs}
$$$$
\mathrm{fz}=128 \mathrm{lbs}
$$

$$
B-4
$$




\section{ANALYTICAL CALCULATIONS}

of

Subject DST MOCK-UP PLATFORM

Originator $12 d d a d a d$

Checker ER VolnerT

Date $\frac{8-10-95}{12-70}$

Date $1 z-7-95$

LOAD CASE NUMBER: ( 3 ) Sesmic [ DL $+.2 \mathrm{~g}(-\mathrm{x})+.06 \mathrm{~g}(\mathrm{z})$ ]

Ref. ANSYS Run (August 1, 1995)

MAXMMUM STRESS/FORCES (Element Coordinate System)

\begin{tabular}{|c|c|c|c|c|c|c|c|c|}
\hline Elem. & $\begin{array}{l}\text { Elem } \\
\text { Type }\end{array}$ & sig1/sig3 & $\begin{array}{l}\text { fx lbs } \\
\text { Sax* }\end{array}$ & $\begin{array}{l}\text { fy } \\
\text { lbs }\end{array}$ & $\begin{array}{l}\mathrm{fz} \\
\mathrm{lbs}\end{array}$ & $\begin{array}{l}\mathrm{mx} \\
\mathrm{lb} \text {-in }\end{array}$ & $\begin{array}{l}\mathrm{my} \\
\mathrm{lb}-\mathrm{in}\end{array}$ & $\begin{array}{l}\mathrm{mz} \\
\mathrm{lb} \text {-in }\end{array}$ \\
\hline $\mathrm{W} 6 \times 20 \mathrm{Bm}$ & 1 & 5967 & 562 & 3948 & 396 & - & 9174 & 69064 \\
\hline $\mathrm{L} 2 \times 2 \times 1 / 4$ & 2 & - & $-2356^{*}$ & ---- & $-\cdots$ & --.- & --- & --- \\
\hline $\mathrm{W} 6 \times 20 \mathrm{Col}$ & 3 & 9083 & 6332 & 781 & 18 & - & 1504 & 103500 \\
\hline $\operatorname{TS} 3 \times 3 \times 1 / 4$ & 4 & 24235 & 2095 & 1576 & 595 & 475 & 1966 & 47702 \\
\hline C6x8.2 & 5 & 14435 & 249 & 1756 & 65 & 51 & 865 & 60348 \\
\hline
\end{tabular}

MAXIMUM CONNECTION FORCES (Element Coordinate System)

\begin{tabular}{|l|l|l|l|l|l|l|l|}
\hline Elem & $\begin{array}{l}\text { Elem } \\
\text { Type }\end{array}$ & $\begin{array}{l}\mathrm{fx} \\
\mathrm{lbs}\end{array}$ & $\begin{array}{l}\mathrm{fy} \\
\mathrm{lbs}\end{array}$ & $\begin{array}{l}\mathrm{fz} \\
\mathrm{lbs}\end{array}$ & $\begin{array}{l}\mathrm{mx} \\
\mathrm{lb}-\mathrm{in}\end{array}$ & $\begin{array}{l}\mathrm{my} \\
\mathrm{lb}-\mathrm{in}\end{array}$ & $\begin{array}{l}\mathrm{mz} \\
\mathrm{lb}-\mathrm{in}\end{array}$ \\
\hline W6x20 Bm & 1 & 565 & 3966 & 409 & 10 & 9174 & 219 \\
\hline $\mathrm{L} 2 \times 2 \times 1 / 4$ & 2 & -2210 & --- & --- & --- & --- & -- \\
\hline $\mathrm{TS} 3 \times 3 \times 1 / 4$ & 4 & 603 & 1767 & 360 & 17543 & 3599 & 23554 \\
\hline $\mathrm{C} 6 \times 8.2$ & 5 & 247 & 1756 & 65 & 51 & 828 & 54033 \\
\hline
\end{tabular}

MAXIMUM REACTIONS @ COLUMN BASES: (Nodes 1-6)

(GLOBAL COORDINATE SYSTEM)

$F x=828 \mathrm{lbs} \quad F y=+7177 \mathrm{lbs},-4476 \mathrm{lbs} \quad F z=550 \mathrm{lbs}$

MAXIMUM FORCES @ TOP OF COLUMNS: (Nodes 13-18) J nodes of Elem.2,4,6,8,10,12

$\mathrm{fx}=-4225 \mathrm{lbs},+1624 \mathrm{lbs} \quad \mathrm{fy}=588 \mathrm{lbs} \quad \mathrm{fz}=14 \mathrm{lbs}$

MAXIMUM FORCES @ CMA BASE: (Nodes 63-66) I nodes of Elem. 76,78,80,82

$\mathrm{fx}=3495 \mathrm{lbs} \quad \mathrm{fy}=366 \mathrm{lbs} \quad \mathrm{fz}=128 \mathrm{lbs}$ 


\section{ANALYTICAL CALCULATIONS}

Page of

Subject DST MOCK-UP PLATFORM

Originator lstardad

Checker

FRV

$$
\text { Date } \frac{8-10-95}{12-7-95}
$$

LOAD CASE NUMBER: (4) Seismic [ DL + .06g $(\mathrm{x})+.2 \mathrm{~g}(\mathrm{z})]$

Ref. ANSYS Run (August 1, 1995)

\begin{tabular}{|c|c|c|c|c|c|c|c|c|}
\hline Elem. & $\begin{array}{l}\text { Elem } \\
\text { Type }\end{array}$ & sig1/sig3 & $\begin{array}{l}\text { fx Ibs } \\
\text { Sax* }\end{array}$ & $\begin{array}{l}\text { fy } \\
\text { los }\end{array}$ & $\begin{array}{l}\mathrm{fz} \\
\mathrm{lbs}\end{array}$ & $\begin{array}{l}\mathrm{mx} \\
\mathrm{lb} \text {-in }\end{array}$ & $\begin{array}{l}\text { my } \\
\text { lb-in }\end{array}$ & $\begin{array}{l}\mathrm{mz} \\
\mathrm{lb} \text {-in }\end{array}$ \\
\hline $\mathrm{W} 6 \times 20 \mathrm{Bm}$ & 1 & 3146 & 379 & 3547 & 379 & 201 & 4304 & 38678 \\
\hline $\mathrm{L} 2 \times 2 \times 1 / 4$ & 2 & $-\infty-$ & $-1251^{*}$ & $--\infty$ & --- & ---- & - & --- \\
\hline W6×20 Col & 3 & 2974 & 5751 & 229 & 36 & - & 1918 & 30407 \\
\hline $\operatorname{TS} 3 \times 3 \times 1 / 4$ & 4 & 25870 & 491 & 1523 & 356 & 100 & 37784 & 47703 \\
\hline C6x8.2 & 5 & 5029 & 57 & 645 & 48 & - & 871 & 19207 \\
\hline
\end{tabular}

MAXIMUM STRESS/FORCES (Element Coordinate System)

\begin{tabular}{|c|c|c|c|c|c|c|c|}
\hline Elem & $\begin{array}{l}\text { Elem } \\
\text { Type }\end{array}$ & $\begin{array}{l}\mathrm{fx} \\
\mathrm{lbs}\end{array}$ & $\begin{array}{l}\text { fy } \\
\text { lbs }\end{array}$ & $\begin{array}{l}\mathrm{fz} \\
\mathrm{lbs}\end{array}$ & $\mathbf{m x}_{\mathrm{lb}-\text { in }}$ & $\begin{array}{l}\text { my } \\
1 \mathrm{~b}-\mathrm{in}\end{array}$ & $\mathbf{m z}_{\mathrm{lb}-\mathrm{in}}$ \\
\hline $\mathrm{W} 6 \times 20 \mathrm{Bm}$ & 1 & 390 & 3560 & 154 & 9 & 4552 & 380 \\
\hline $\mathrm{L} 2 \times 2 \times 1 / 4$ & 2 & -1173 & $---\infty$ & ---- & $-\cdots$ & $-\infty--$ & ---- \\
\hline TS $3 \times 3 \times 1 / 4$ & 4 & 792 & 1958 & 322 & 15340 & 6590 & 32063 \\
\hline $\mathrm{C} 6 \times 8.2$ & 5 & 59 & 645 & 48 & - & 898 & 19207 \\
\hline
\end{tabular}

MAXIMUM CONNECTION FORCES (Element Coordinate System)

MAXMMUM REACTIONS @ COLUMN BASES: (Nodes 1-6) (GLOBAL COORDINATE SYSTEM)

$\mathrm{Fx}=243 \mathrm{lbs}$

$F y=+6653 \mathrm{lbs},-1495 \mathrm{lbs}$

$\mathrm{Fz}=801 \mathrm{lbs}$

MAXIMUM FORCES @ TOP OF COLUMNS: (Nodes 13-18) J nodes of Elem.2,4,6,8,10,12

$f x=-4162 \mathrm{lbs}$

$f y=194 \mathrm{lbs}$

$\mathrm{fz}=26 \mathrm{lbs}$

MAXIMUM FORCES @ CMA BASE: (Nodes 63-66) I nodes of Elem. 76,78,80,82

$\mathrm{fx}=3488 \mathrm{lbs}$

$f y=365 \mathrm{lbs}$

$\mathrm{f}=128 \mathrm{lbs}$

$B-6$ 
ANALYTICAL CALCULATIONS

Page of

Subject $D S T^{-} M O C K-U P$ PLATFORM

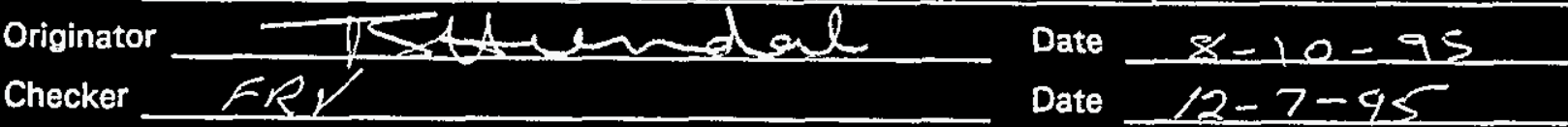

LOAD CASE NUMBER: ( 5 ) Seismic [DL + .06g $(\mathrm{x})+\cdot 2 \mathrm{~g}(-\mathrm{z})]$

Ref. ANSYS Run (August 1, 1995)

MAXIMUM STRESS/FORCES (Element Coordinate System)

\begin{tabular}{|c|c|c|c|c|c|c|c|c|}
\hline Elem. & $\begin{array}{l}\text { Elem } \\
\text { Type }\end{array}$ & sig1/sig3 & $\begin{array}{l}\text { fx lbs } \\
\text { Sax }\end{array}$ & $\begin{array}{l}\text { fy } \\
\text { lbs }\end{array}$ & $\begin{array}{l}\text { fz } \\
\text { lbs }\end{array}$ & $\begin{array}{l}\mathrm{mx} \\
\mathrm{lb}-\mathrm{in}\end{array}$ & $\begin{array}{l}\text { my } \\
1 b-i n\end{array}$ & $\begin{array}{l}\mathrm{mz} \\
\mathrm{lb} \text {-in }\end{array}$ \\
\hline W6x20 Bm & 1 & 6311 & 358 & 2164 & 170 & 7 & 1951 & 83374 \\
\hline $\mathrm{L} 2 \times 2 \times 1 / 4$ & 2 & - & $-1140^{*}$ & -.-- & ---- & --- & $--\infty$ & - \\
\hline W6x20 Col & 3 & 3169 & 4286 & 251 & 39 & - & 2287 & 33189 \\
\hline $\mathrm{TS} 3 \times 3 \times 1 / 4$ & 4 & 25880 & 473 & 1523 & 357 & 153 & 37907 & 47703 \\
\hline $\mathrm{C} 6 \times 8.2$ & 5 & 5926 & 170 & 681 & 47 & - & 833 & 20570 \\
\hline
\end{tabular}

MAXIMUM CONNECTION FORCES (Element Coordinate System)

\begin{tabular}{|c|c|c|c|c|c|c|c|}
\hline Elem & $\begin{array}{l}\text { Elem } \\
\text { Type }\end{array}$ & $\begin{array}{l}\mathrm{fx} \\
\mathrm{lbs}\end{array}$ & $\begin{array}{l}\text { fy } \\
\text { lbs }\end{array}$ & $\begin{array}{l}\text { fz } \\
\text { lbs }\end{array}$ & $\begin{array}{l}\mathrm{mx} \\
\mathrm{lb} \text {-in }\end{array}$ & $\begin{array}{l}\text { my } \\
\text { lb-in }\end{array}$ & $\frac{\mathrm{mz}}{\mathrm{Ib}-\mathrm{in}}$ \\
\hline $\mathrm{W} 6 \times 20 \mathrm{Bm}$ & 1 & 407 & 2825 & 171 & 7 & 3686 & 344 \\
\hline $\mathrm{L} 2 \times 2 \times 1 / 4$ & 2 & -1069 & --m-- -.- & $--\cdots$ & --- & $-\infty$ & ---- \\
\hline $\mathrm{TS} 3 \times 3 \times 1 / 4$ & 4 & 3503 & 1945 & 372 & 13471 & 6545 & 31754 \\
\hline C6 $6 \times 8.2$ & 5 & 57 & 211 & 170 & - & 823 & 20570 \\
\hline
\end{tabular}

MAXIMUM REACTIONS @ COLUMN BASES: (Nodes 1-6) (GLOBAL COORDINATE SYSTEM)

$\mathrm{Fx}=265 \mathrm{lbs} \quad \mathrm{Fy}=+5292 \mathrm{lbs},-684 \mathrm{lbs} \quad \mathrm{Fz}=732 \mathrm{lbs}$

MAXIMUM FORCES @ TOP OF COLUMNS: (Nodes 13-18) J nodes of Elem.2,4,6,8,10,12

$f x=-3248 \mathrm{lbs} \quad f y=170 \mathrm{lbs} \quad f z=22 \mathrm{lbs}$

MAXIMUM FORCES @ CMA BASE: (Nodes 63-66) I nodes of Elem. 76,78,80,82

$f x=3503 \mathrm{lbs} \quad f y=119 \mathrm{lbs} \quad f z=372 \mathrm{lbs}$ 
ANALYTICAL CALCULATIONS

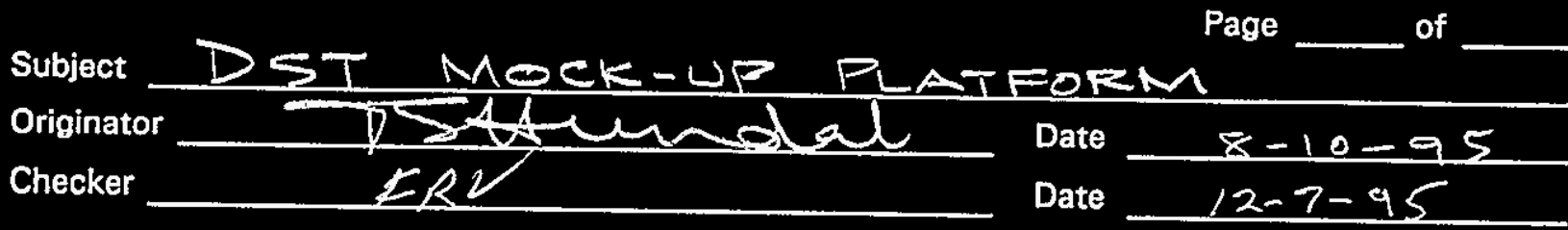

LOAD CASE NUMBER: ( 6 ) Seismic [DL + .133g (y) ]

Ref. ANSYS Run (August 1,1995 )

MAXIMUM STRESS/FORCES (Element Coordinate System)

\begin{tabular}{|c|c|c|c|c|c|c|c|c|}
\hline Elem. & $\begin{array}{l}\text { Elem } \\
\text { Type }\end{array}$ & sig1/sig3 & $\begin{array}{l}\text { fx lbs } \\
\text { Sax * }\end{array}$ & $\begin{array}{l}\text { fy } \\
\text { lbs }\end{array}$ & $\begin{array}{l}\text { fz } \\
\text { lbs }\end{array}$ & $\operatorname{mxx}_{\mathrm{lb}-\mathrm{in}}$ & $\begin{array}{l}\text { my } \\
\text { lb-in }\end{array}$ & $\begin{array}{l}\mathrm{mz} \\
\mathrm{lb}-\mathrm{in}\end{array}$ \\
\hline W6x20 Bm & 1 & 4092 & 127 & 1795 & 12 & 2 & 130 & 53718 \\
\hline $\mathrm{L} 2 \times 2 \times 1 / 4$ & 2 & $-\infty$ & $-321 *$ & ---- & --.- & $-\cdots$ & ---- & ---- \\
\hline $\mathrm{W} 6 \times 20 \mathrm{Col}$ & 3 & 1075 & 3980 & 16 & 12 & - & 1553 & 2039 \\
\hline $\mathrm{TS} 3 \times 3 \times 1 / 4$ & 4 & 25661 & 1799 & 1745 & 530 & 16330 & 20422 & 54047 \\
\hline C $6 \times 8.2$ & 5 & 1900 & 356 & 247 & - & 4 & 25 & 8282 \\
\hline
\end{tabular}

MAXIMUM CONNECTION FORCES (Element Coordinate System)

\begin{tabular}{|c|c|c|c|c|c|c|c|}
\hline Elem & $\begin{array}{l}\text { Elem } \\
\text { Type } \\
\end{array}$ & $\begin{array}{l}\mathrm{fx} \\
\mathrm{lbs}\end{array}$ & $\begin{array}{l}\text { fy } \\
\text { lbs }\end{array}$ & $\begin{array}{l}\text { fzz } \\
\text { lbs }\end{array}$ & $\begin{array}{l}\mathrm{mx} \\
\mathrm{lb} \text {-in }\end{array}$ & $\begin{array}{l}\text { my } \\
\text { lb-in }\end{array}$ & $\begin{array}{l}\mathrm{mz} \\
\mathrm{lb} \text {-in }\end{array}$ \\
\hline $\mathrm{W} 6 \times 20 \mathrm{Bm}$ & 1 & 127 & 2512 & 16 & 6 & 236 & 434 \\
\hline $\mathrm{L} 2 \times 2 \times 1 / 4$ & 2 & -301 & ---- & $-\cdots$ & -- & --- & $-\cdots$ \\
\hline $\mathrm{TS} 3 \times 3 \times 1 / 4$ & 4 & 530 & 1764 & 3 & 16334 & 45 & 20424 \\
\hline C6x8.2 & 5 & 74 & 247 & - & - & 15 & 3883 \\
\hline
\end{tabular}

MAXIMUM REACTIONS @ COLUMN BASES: (Nodes 1-6)

(GLOBAL COORDINATE SYSTEM)

$\mathrm{Fx}=16 \mathrm{lbs} \quad \mathrm{Fy}=4227 \mathrm{lbs} \quad \mathrm{Fz}=203 \mathrm{lbs}$

MAXIMUM FORCES @ TOP OF COLUMNS: (Nodes 13-18) J nodes of Elem.2,4,6,8,10,12

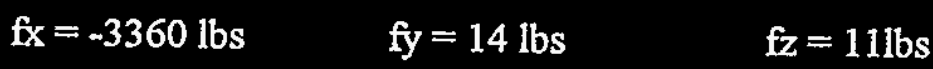

MAXIMUM FORCES @ CMA BASE: (Nodes 63-66) I nodes of Elem. 76,78,80,82

$\mathrm{fx}=1990 \mathrm{lbs} \quad \mathrm{fy}=14 \mathrm{lbs} \quad \mathrm{fz}=23 \mathrm{lbs}$

$B-8$ 
Subject DST Mock-2JP PLATFORM

Originator

Checker

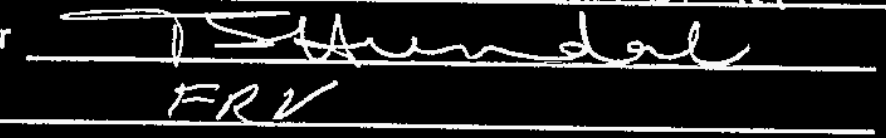

Date

Date $8-10-95$ $12-7-95$

NOTE: MEMBERS \& SOME CONNECTIONS WERE ALSO CHECKED BY ALTERNATE CALCULATIONS.

CHECK MEMBERS SEE PAGES B-21 THRU B-38

W6XZO BEAM

MAX. COMBINED STRESS $=6311$ pSi 221600 psi

$$
\therefore \text { ok }
$$

\section{$\underline{W 6 \times 20 \text { COLUMN }}$}

MAX. COMBINED STRESS $=9083$ psi $<21600 \mathrm{ppi}^{\circ}$ : . ol

$$
\begin{aligned}
& f_{a}=\frac{6332}{5.87}=1079 \mathrm{psi} \quad \mathrm{kl} / \mathrm{r}=264 / 2.66=99 \Rightarrow F_{a}=13.10 \mathrm{kri} \\
& f_{b}=\frac{10350.0}{13.4}=7,724 \mathrm{psi}
\end{aligned}
$$

\section{$\leq 2 \times 2 \times 4$}

MAX. STRESS, $f_{a}=2.410$ pri

MAX. UNBRACED LenGTH $=\sqrt{(114 / 2)^{2}+(132 / 2)^{2}}=87.2^{11}$ $\mathrm{kl} / \mathrm{r}=87.2 / .391=223>c_{c}=126.1$

$\therefore \quad F_{a}=\frac{12 \pi^{2} E}{23(K l / r)^{2}}=\frac{12 \pi^{2}\left(29 \times 10^{6}\right)}{23(223)^{2}}=3000$ pri

$=1.33(3000)$ for Seismic

$=3990$ psi $>$ fa

$c 6 \times 8.2$

$\therefore$ olc

MAX. COMBINED STRESS $=14435 \mathrm{fpri}<21600 \mathrm{pmi}$

$f_{a}=356 / 2.4=.148 \mathrm{ksi}$

$\therefore$ ole

$f_{b z}=60 / 4.38=13.70 \mathrm{ksi}, f_{b y}=9 / .492=1.8 \mathrm{ksi}$

ok

$B-9$ 
Subject DST MOCK-UP PLATFORM

Originator

Checker

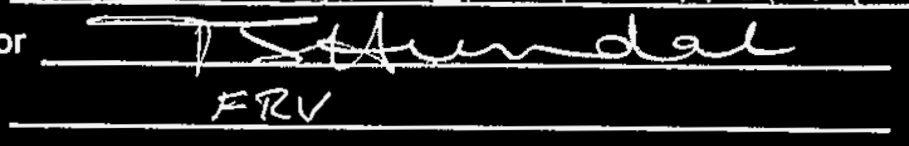

Date

$8-10-95$

$12-7-95$

\section{TS $3 \times 3 \times 14(A-1020) F_{y}=38^{\mathrm{ksi}}$}

$$
\begin{aligned}
& \text { MAX. LENGTH }=104^{\prime \prime} \\
& \text { MAX, COMB. STRESS }=25.9 \mathrm{kri} \\
& f_{x}=2095^{\#}, m_{y}=10^{\#-i n}, m_{z}=54047^{\# \text {-in }} m_{x}=5^{\#-\text { in }} \\
& f_{a}=2 / 5.87=.34 \mathrm{ksi} \quad \mathrm{kl} / \mathrm{r}=104 / 1.1=95, c_{c}=\sqrt{\frac{2 \pi^{2} E}{F_{y}}} \\
& \mathrm{ke} / \mathrm{r} / \mathrm{c}_{c}=95 / 122.74=-77 \Rightarrow c_{a}=.371 \\
& =\sqrt{\frac{2 \pi^{2}\left(29 \times 10^{6}\right)}{38000}} \\
& \therefore F_{a}=C_{a} F_{y}=.371 \times 38=14 \mathrm{ksi} \\
& =122.74 \\
& f_{a / F_{a}}=\cdot 34 / 14=.024<.15 \\
& \therefore \text { eheck } \\
& \begin{array}{c}
f_{a} / F_{a}+f_{b z / F_{b z}}+f_{b y / F_{b y}} \leqslant 1.0 \\
f_{b z}=54 / 2.1=25.7, f_{b y}=-01 / 2.1=.0 .05 \\
\therefore \quad 024+25.7 / 30.3+.005 / 30.3=.87<1.0 \therefore \text { ok }
\end{array} \\
& \begin{array}{c}
f_{a} / F_{a}+f_{b z / F_{b z}}+f_{b y / F_{b y}} \leqslant 1.0 \\
f_{b z}=54 / 2.1=25.7, f_{b y}=-01 / 2.1=.0 .05 \\
\therefore \quad 024+25.7 / 30.3+.005 / 30.3=.87<1.0 \therefore \text { ok }
\end{array} \\
& \begin{array}{c}
f_{a} / F_{a}+f_{b z / F_{b z}}+f_{b y / F_{b y}} \leqslant 1.0 \\
f_{b z}=54 / 2.1=25.7, f_{b y}=-01 / 2.1=.0 .05 \\
\therefore \quad 024+25.7 / 30.3+.005 / 30.3=.87<1.0 \therefore \text { ok }
\end{array} \\
& \begin{aligned}
F_{b z}=F_{b y} & =.6 \times 38=22.8^{\mathrm{ksi}} \\
& =1.33(22.8) \\
& =30.3 \mathrm{ksi}
\end{aligned}
\end{aligned}
$$


ANALYTICAL CALCULATIONS

HHC-SD-HM-ER-521
ReV. 0

Page 3 of 12

Subject IST MOCK-UP PLATFOFM

Originator

IStyundal

Date $8-10-95$

Checker

FR

Date

$12-7-95$

Check Connections

ANCHOR BOLTS (HILTIKWIKBOLTII)

$4-3_{4}^{\prime \prime} \phi \times 34^{\prime \prime}$ MIN. EMP.

Cone. $f_{c}^{\prime}=3000$ psi

Allow. LoAds: TEN. $T_{A}=2.17^{\mathrm{k}}$

$$
S_{A}=3.41 \mathrm{~K}
$$

$\frac{D L+S E I S M I C}{M A X . R E A C T I O N}$ @ BASE $\Rightarrow\left\{\begin{array}{l}\text { TENSION Fy }=4476 \mathrm{lbs} \\ \text { SHEAR: } F_{x}=828 \mathrm{lbs} \$ F_{z}=801 \mathrm{hs}\end{array}\right.$

TEN. PER BOLT $T=4476 / 4=1.12 \mathrm{k}$

SHEAR PER BOLT, $S=\sqrt{(.828)^{2}+(.801)^{2}} / 4=\cdot 3^{k}$

CHECK UNITY FORMULA

$$
\begin{aligned}
& \tau_{\tau_{A}}+5 / S_{A} \leq 1.0 \\
& \frac{1.12}{2.17}+\frac{.3}{3.41}=.60<1.0 \therefore \text { ok }
\end{aligned}
$$

BASE R

MAX. COMP. LOAD $=7177$ lbS (SEISMIC $+D L$ )

ALlOW. CONC. BEARINC PRESSURE, $F_{p}=.7 f_{c}^{\prime}=2100$ psi

REQ'D AREA $=7177 / 2100=4 \mathrm{in}^{2}$

$\left(\begin{array}{c}\text { AlSO MAN.) } \\ \text { P. } 5-79\end{array}\right.$

USE $18^{\prime \prime} \times 18^{\prime \prime} \times 1 / 2 \mathbb{R}$

$$
\begin{aligned}
& M=1.12^{k} \times 9 \times z=20.16^{k-1 n}
\end{aligned}
$$

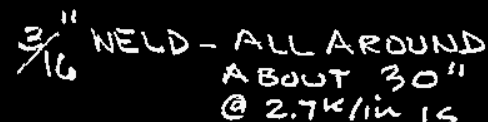

$$
\begin{aligned}
& \text { Q } 2.7 \mathrm{~K} / \mathrm{in} \text { is } \\
& t=\sqrt{\frac{2 M}{9 b}}=\sqrt{\frac{2 \times 20.16}{9 \times 18}}=.499^{\prime \prime}=1 / 2 \therefore \sigma k
\end{aligned}
$$

$B-11$

BD-6400-060.1 (07/93) 
Subject TST MOCK-UP PLATFORM

Originator

Checker

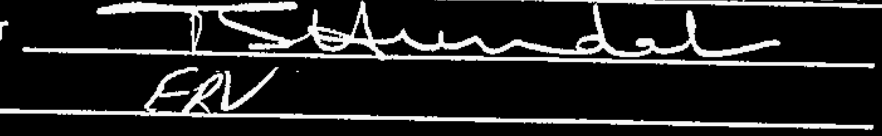

Date

Date

Page 4 of 12

$8-10-95$

$12-7-45$

BEAM/BEAM CONNECTION

$f_{x}=565, f_{y}=3984, f_{z}=409, m_{x}=50, m_{y}=9174, m_{z}=604$

$A_{\omega}=2\left(3 \frac{1}{2}+2 \times 21 / 2\right)=17$ in

$S_{z}=2(2.5) 3.5+3.5^{2} / 3=21.58 \mathrm{in}^{2}$

$S_{y}=5.5^{2} / 3=10 \mathrm{in}^{2}$

WELD (A)

$P_{x}=f_{x} / A_{\omega}+\frac{m z}{s_{z}}+\frac{m y}{s_{y}}$

$=\frac{-565}{17}+\frac{.604}{21.58}+\frac{9.174}{10}=.98 \mathrm{k} / \mathrm{in}$

$P_{y}=3.984 / 17=-23 \mathrm{k} / \mathrm{m}$

$P_{z}=.409 / 17=.024^{k} / \mathrm{in}$

$p_{r}=\sqrt{(.98)^{2}+(.23)^{2}+(.024)^{2}}=1^{k / i n}$

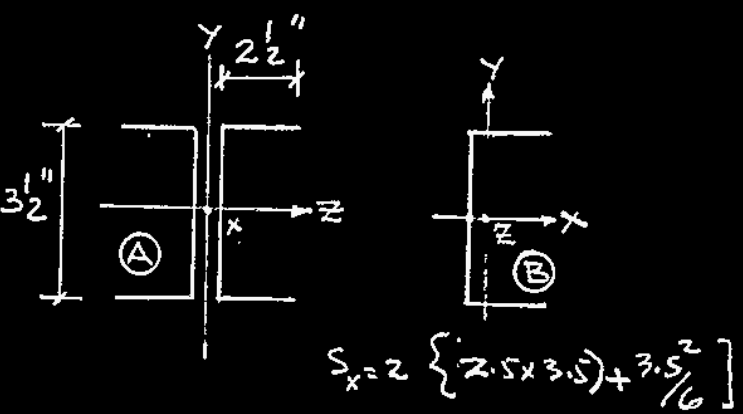

$=21.58 \mathrm{in}^{2}$

$S_{y}=2\left\{\frac{2 \times z^{1} 2 x^{3 / 2} / 2}{3}+2 \cdot 5^{2} / 3\right\}$

$=15.8 \mathrm{in}^{2}$ TOP

$=2\left\{\frac{2.5^{2}(2 \times 3.5+2.5)}{3(2.5+3.5)}\right\}$

$=6.6 \mathrm{in}^{2}$ BoT

51.173

$J=2\left\{\frac{3,5+2(2,5)]}{12}\right.$

$w_{\text {req } d}=\frac{1}{14.4}=.069^{\prime \prime}<3_{16}^{\prime \prime}=2.7 \mathrm{k} / \mathrm{in}$ $-(2 \cdot 5)^{2}(5.5+(2.5)\}$

$\therefore$ ole

WELD (B)

$$
\begin{aligned}
& P_{z}=409 / 17+9.174 / 6.6+050 / 21.58=1.42 \mathrm{k} / \mathrm{in} \\
& P_{z}=.565 / 17+\frac{m_{z}\left(c_{x}\right)}{J}=565 / 17+\frac{9.174 \times 1.75}{49.4}=.36 \mathrm{k} / \mathrm{in} \\
& P_{y}=3.984 / 17+\frac{m z\left(c_{y}\right)}{j}=-23+\frac{9.174 \times 1.76}{49.4}=.56 \mathrm{k} / \mathrm{in} \\
& p_{r}=\sqrt{(1.42)^{2}+(.36)^{2}+(.56)^{2}}=1.57 \mathrm{k} \text { in }<2.7 \mathrm{k} / \mathrm{in} \\
& c_{x}=1.75^{11} \\
& c_{y}=2.5-\frac{2.5^{2}}{2(2.5)+3.5} \\
& =2.5-74^{\prime \prime} \\
& =1.76^{\circ}
\end{aligned}
$$


WHC-SD-WM-ER-52]

ANALYTICAL CALCULATIONS

Rev, 0

Subject DST MOCK-UP PLATFORM

Page 5 of 12

Originator

Checker

E lV

Date $8-10-95$

Date $-12-2-95$

PLATFORM TO TOP OF COLUMN BOLTED CONNECTION

BEAM/COLUMN CONNECTION (4-5" \& A 325 BOLTS) (NODES 13-18)

MAX. FORCES@ TOP of COLUMNS (TENSION \& SHEAR FORCES) $f_{x}=-5623^{*}$ (COMP.), $1624^{\#}$ (TENSION) $\ll 13,2^{K} /$ BOLT

$$
\left.\begin{array}{l}
f_{y}=600^{*} \\
f_{z}=-26^{\#}
\end{array}\right\} \quad<<4.5^{k} / \text { BOLT }
$$

$\therefore \gamma k$

I/ZF WELDED Q COL, TOP

WITH MIN. of $12 "-3 " 16$ WELD OK BY INSPECTION

$\angle 2 \times 2 \times 14$ TO GUSSET $\mathbb{R}$ (WELD SIZE 3,16 )

MAX. FORCE $=2261^{\#}$

MIN. WELD LENGTH $=2\left(2_{8}^{5 \prime}\right)=5.25^{\prime \prime}$ TO ANGLE

$\therefore$ ALLOW, WELD LOAD $=5.25^{\prime \prime}\left(2.7^{k} /\right.$ in $\left.^{\prime}\right)=14.2^{k} \gg 2.26^{k}$

GUSSET RE TO COL. WELD LENGTH $=14^{\prime \prime}$ MIN, OK C6X8.2 TO COLUMNS (WELD CONN.)

MAX. FORCES:

$f_{x}=247^{\#}, f_{y}=1756^{\#}, f_{z}=170^{\#}$

$$
\begin{aligned}
A_{\omega} & =2(6+2 \times 2) \\
& =20 \mathrm{in} .
\end{aligned}
$$

$m_{x}=51^{\text {\#-in }}, m_{y}=898^{\text {Hin }}, m_{z}=59383^{\text {\#- in }}$

$p_{x}=-247 / 20+.898 / 4.66+59.4 / 36=1.86 \mathrm{k} / \mathrm{in}$

$P_{y}=1.76 / z_{0}=.09 \mathrm{k} / \mathrm{in} P_{z}=17 / 2_{0}=.01 \mathrm{k} / \mathrm{m}$

$P_{r}=\sqrt{(1.86)^{2}+(.09)^{2}+(.01)^{2}}=1.86 \mathrm{k} / \mathrm{im}$

$\therefore 3,16$ WELD $\therefore$ ob

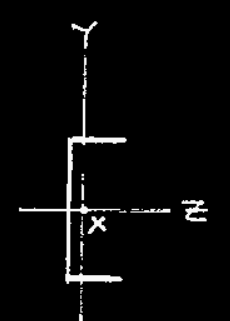

$$
\begin{aligned}
S_{z} & =2\left\{6 \times z+6^{2} / 6\right\} \\
& =36 \mathrm{in}^{2} \\
S_{y} & =\frac{z^{2}(2 \times 6+2)}{3(2+6)} \times 2 \\
& =4.66 \mathrm{in}^{2}
\end{aligned}
$$

$B-13$

kin

$00-6400-000.1(07 / 93)$ 
Subject DST MOCK-UP PLATFOEM

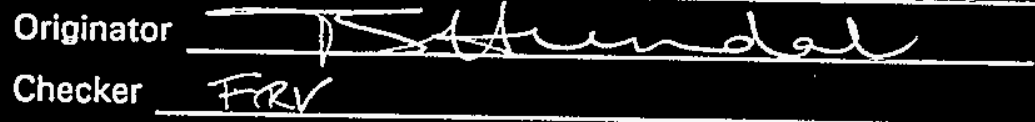

Date

Date

Page 6 of 12

Checker

$r R$

$x-10-95$

$12-7-95$

C6X8.2 TO COL. Flange (WELD CONN.)

LOAD CASE 2

ELEN 15

MAX, FORCES @ J NODE

$f_{x}=128, f_{y}=205, f_{z}=65$

$m x=50, m y=847, m z=2540$

$P_{x}=128 / 12+\frac{2.54 \times 3}{144}=.06 \mathrm{k} / \mathrm{in}$

$P_{y}=.205 / 12+\frac{2.54 \times 3}{144}=.07$

$P_{z}=.065 / 12+.05 / 36+.847 / 12=.08 \mathrm{k} / 1 \mathrm{~m}$

$P_{r}=\sqrt{(.06)^{2}+(.07)^{2}+(.08)^{2}}=.12 \mathrm{k} / \mathrm{in}$

$\ll 2.7^{k} /$ in

$\therefore$ ok
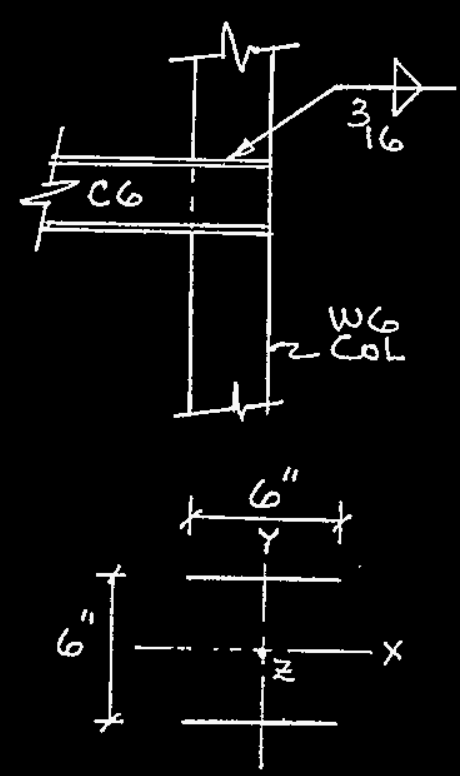

$S_{x}=6 \times 6=36$

$s_{y}=6^{2} / 3=12$

$I=\frac{6\left(3 \times 6^{2}+6^{2}\right)}{6}=144$

$A=2 \times 6=12$ 
ANALYTICAL CALCULATIONS

WHC-SD-WM-ER-521

Rev. 0

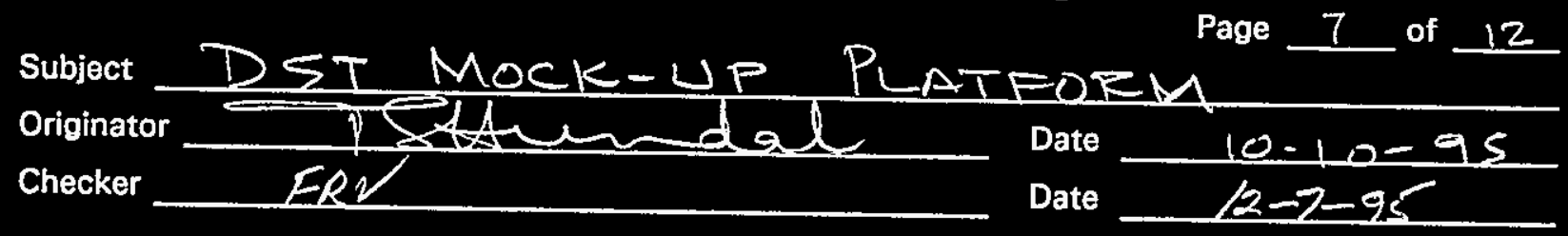

cMa base jacks attachment to ratForm

MAX. Forces e base (Nodes $63-66$ )

$f_{x}=3503^{\#}, f_{y}=366 \% f_{z}=128^{*} \Rightarrow f_{r}=388^{*}$

. COMPRESSION $)$ or $f_{y}=119 \#, f_{z}=372 \# \Rightarrow f_{r}=391 \#$

- NO TENSION QBASE

Allow. Jack LoAds

MAX. Allow. VERTICAL LOAD $=5000^{\#}>3503^{\#} \mathrm{Aok}$ MAX. SIDE LOAD IN RAISED POSITION $=400^{*}>391^{\#} \therefore$ ol

FOOT PAD NEEDS TO BE ANCHORED TO W6 BEAMS

PAD Re is 8 " $\phi \times 7$ GAGE SHEeT Metal $\left(t=-174^{\prime \prime}\right)$

COMMERcIAL QUALITY (LOW CARBON) SHEET METAL, F F $=27,500$ pun
(ASTM, A569) (ASTM, A 569)
(RYERSON)

TRY $z-1 / 2 " \phi A 307$ BOUTS/EACH PAD

ALLOW. SHEAR LOAD BOLT $=10 x \cdot 2=2^{k}>f_{r}=391^{\#} \therefore$ ok (NO TENSION ON BOLT, ONLY COMPRESSIVE LOAD @ BASE)

ALLOW. BEARING LOAD ON FOOT PAD $=1.5 \times 50 \times .5 \times .174=6.38^{\mathrm{K}} /$ BOLT

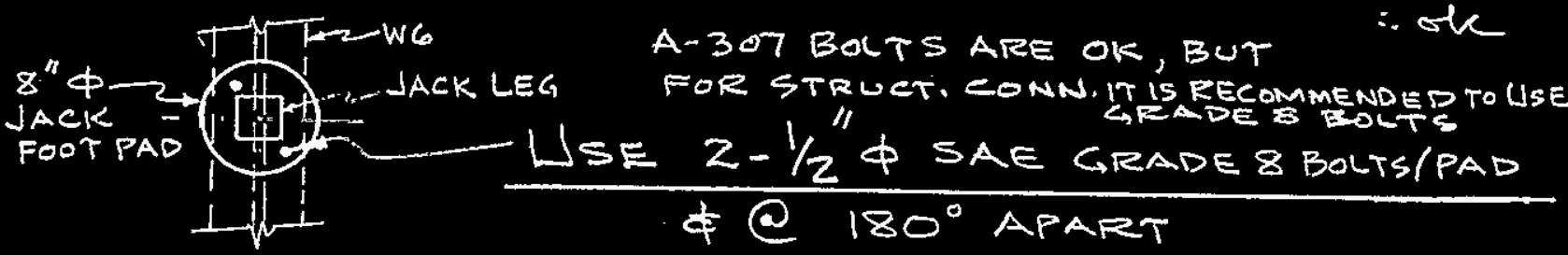

$B-15$ 
Subject DST MOCK-UP PLATFORM

Originator

Checker

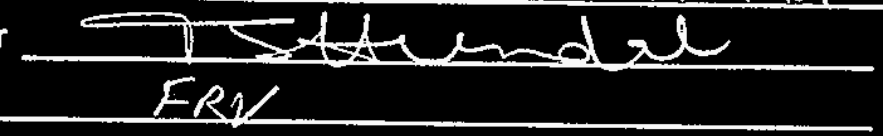

Date

Date

Page $\_.8$ of 12

$\frac{10-10-95}{12-7-95}$

TOP Two BEAMS SUPPORTING CMA (REEL) END SUPPORTS

REDZONE DWG. 9Z13-CMA-ASSY-ODI SHOWS THAT THESE

BEAMS ARE RESTING ON SIDE BEAMS, BUT ACTUALLY THE BEAMS AEE SEATED IN THREE SIDED CLAMPS AT BOTH ENOS, $W / 3_{Z}^{\prime \prime}$ Q REMMOVEABLE PIN.

SO RE-EVALUATE THESE BEAMS \& END SUPPORTS.

(i.e. SIMPLY SUPPOFTED)

BEAM ELEM, 96 THRU 99

$$
\begin{aligned}
m_{z} & =1500(1.133) 81(.333)=45,841 \cdot 16 \text {-in } \\
m_{y} & =1500(.2)(81)(.333)=809216 \text {-in } \\
f_{b z} & =\frac{45,841}{2.1}=21,829 \text { peri } \\
f_{b y} & =\frac{8092}{2.1}=3,853 \text { peri } \\
& 21.83 / 33.25+3.85 / 33.25=.77
\end{aligned}
$$

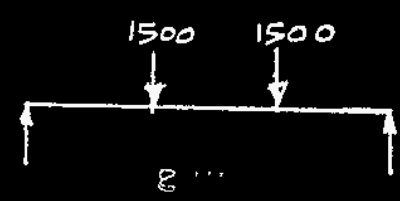

$$
\begin{aligned}
F_{b} & =.66(38) \\
& =25 \mathrm{ker}
\end{aligned}
$$

ForSEISMIC, $F_{b}=1.33 \times 25$ $=33.25 \mathrm{kei}^{1}$ $<1.0 \therefore$. ole

CHECK SUPPORT SEAT ( 4 "R)

REACTION, $R_{y}=1500(1.133)=1700 \mathrm{lbs}$

$$
R_{z}=1500(.2)=300 \mathrm{lbs}
$$

$\frac{\text { WELD }}{S_{y}}$

$$
S_{y}=2 \times 4=8 \text { in }^{2}, \quad S_{z}=2^{2} / 3=1.33 \mathrm{in}^{2}, A=4 \text { in }
$$

$f_{y}=1700 / 4=425 \% / \mathrm{in}$

$f_{x}=1700 \times 2 / 1.33=2556 \% /$ in

$f_{z}=300 \times 2 / 8=75$ \#/in

$F_{w}=1.33 \times 2.78$

$=3.7 \mathrm{Kiplin}$

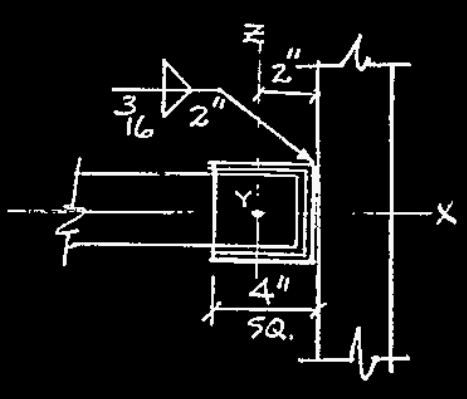

$f_{r}=\sqrt{(425)^{2}+(2.556)^{2}+(075)^{2}}=2.59 \mathrm{k} / \mathrm{in}<3.7 \mathrm{k} / \mathrm{in} \quad \therefore 8 \mathrm{~K}$

$$
B-16
$$




\section{ANALYTICAL CALCULATIONS}

WHC-SD-WM-ER-521

Rev. 0

Page 9 of 12

Subject DST MOCK UP PLAT FOEM

Originator $\frac{1 S A A v e d r l}{\text { ERecker }}$ Date $\frac{10-10-95}{22-7-95}$

CHECK SEAT R

$t=.25^{\prime \prime}$

2.74

$x_{1}=\frac{2(34 \times 4) \times 1.63+3 \times .25 \times 1.125}{2\left(3 \frac{1}{4}\right)(.25)+3 \times .25}$

$=1.15^{\prime \prime}$

2. 38

$X_{2}=3.25-1.15=2.1^{\prime \prime}$

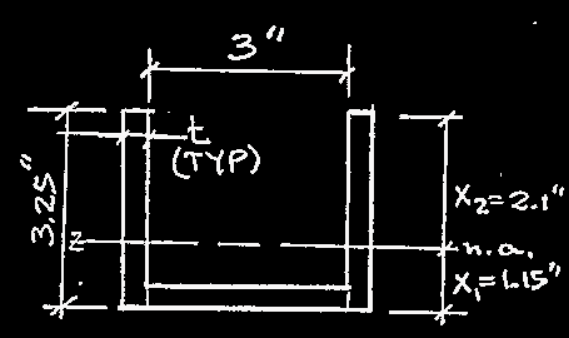

ELEV.

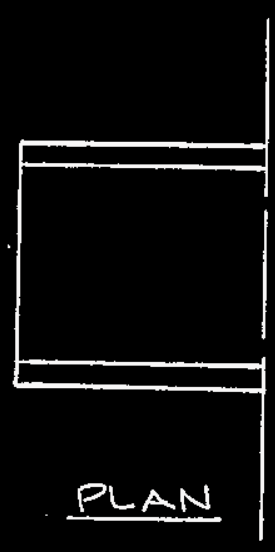

$I=2\left\{\left(3.25^{3} / 12\right)+3.25 \times .25 \times .47^{2}\right\}+3 \times .25(1.03)^{2}=6.88$ in $^{4}$

$s_{\min }=6.88 / 2.1=3.28 \mathrm{in}^{3}$

$f_{b z}=\frac{1700 \times 2}{3.28}=1036 \mathrm{psi}<27 \mathrm{ksi}$

$\therefore \delta$

$f_{b y}$ ok by insPEction

$\therefore$ BRACKET IS OK 


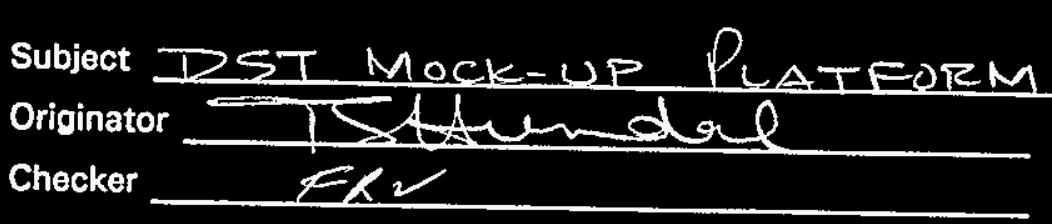

Page 10 of 12

Date $\frac{10-10-95}{12-7-95}$

\section{CMA TOP BEAM TO COLUMN CONN.}

because of cma reel beams are

SIMPLY SUPPORTED ON BACKETS.

CONAPLTER OWTPUT RESULTS

WONIT BE ISED.

NEW END FOREES

$m_{z}=M_{B}=\frac{1.133\left(1.5^{k}\right)(66)^{2} 7}{(73)^{2}}+\frac{1.133(1.5)(30)^{2} 43}{(73)^{2}}$

$m_{z}=z z k-i n$

$m_{y}=(1.5 \times, z) z^{\prime \prime} \times z=1.2^{k-1 i}$

WELD IS 316

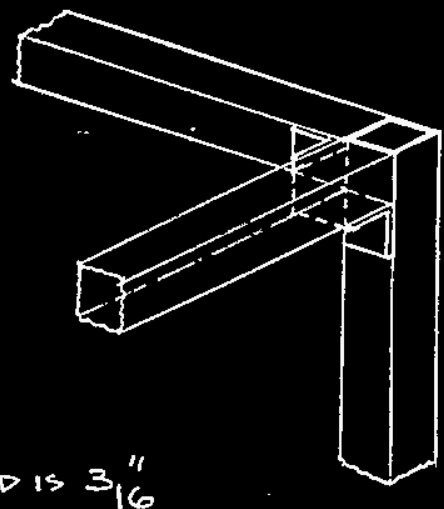

$m_{x}=1,2^{k-i n}$

$f_{x}=-3^{k}, f_{y}=197^{k}, f_{z}=.4^{k}$

WEL B

WELD AREA, $A_{1}=A_{2}=3 \times 3=9$ in

ALLOW.CAP. $=2.78 \mathrm{k} / \mathrm{in}$

ASSUME $m_{z}$ ARE TAKEN BY WELD(1)

$\begin{aligned} \therefore f_{x} & =\frac{m_{z}(1.5)}{J_{w_{1}}}+f_{x} / A_{1}=\frac{22(1.5)}{24.75}+.3 / 9=1.37^{\mathrm{k}} / \mathrm{in}\end{aligned}$

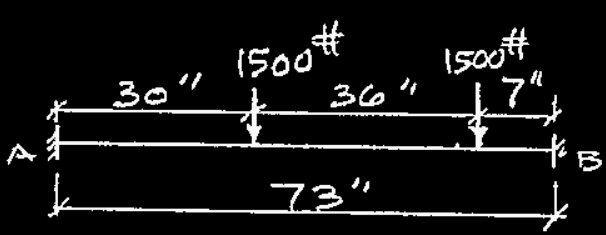

$f_{y}=f_{y / A_{1}}=1.97 / 9=-2 z^{k / 1 i n}$

$f_{x}+f_{y}=1.37+-2 z=1.59 \mathrm{k} / \mathrm{in}<2.78^{\mathrm{k}} / \mathrm{in}$

WELD (2) for $m_{x}, m_{y}+f_{z}$

$R_{A}=\frac{1.50(7+43)}{73}=1.03^{k}$

$R_{B}=3-1.03=1.97 \mathrm{~K}$

$\therefore f_{y}=m_{x / s_{x_{z}}}=1.2 / 10.5=.11 \mathrm{k} / \mathrm{in}$

$f_{x}=m_{y} / J_{w}^{(1.5)}=1.2(1.5) / 24.75=.07$

$\therefore$ ile

$f_{z}=m y / j \omega(1)+f_{z} / A_{2}=\frac{1.2(1)}{24.75}+4 / 9=.09$

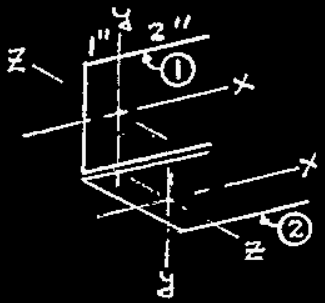

$f_{x}+f_{y}+f_{z}=.07+.11+.09=-27 \mathrm{k} / \mathrm{in}<2.78^{k} / \mathrm{in}$

$\therefore$ ol

$$
\begin{aligned}
J_{\omega_{1}} & =\frac{(3+2 \times 3)^{3}}{12}-\frac{3^{2}(3+3)^{2}}{(3+2 \times 3)} \\
& =24.75 \mathrm{in}^{3}
\end{aligned}
$$

$S_{z_{2}}=\frac{3^{2}(2 \times 3+3)}{3(3+3)}=4.5\left(R_{1} C_{H} H T\right)$

$S_{z_{2}}=\frac{2 \times 3 \times 3+3^{2}}{3}=9\left(\right.$ LEF $\left.T_{1}\right)$

$c_{y}=\frac{3^{2}}{3+2 \times 3}=1^{11}$

$S_{x_{2}}=3 \times 3+3 / 6=10.5$ in $^{2}$ 
Subject DS Mock-up plateren

Originator

Checker

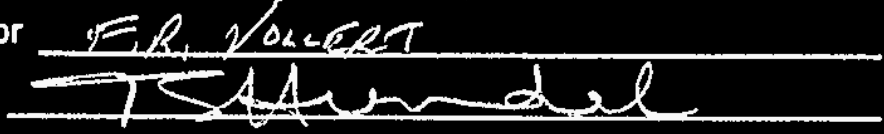

Date

Date $11-22-95$

CAMERA PLATFORM ATTACHMENT

TOTAL WT. $=750$ lbs

SEISMIC: VERT. $1.133 \times 750=850 \mathrm{lbs}=R_{\mathrm{V}}$

HORIz. $=.2(750)=150 \mathrm{lbs}$

HORIZONTAL REATION $\left(H_{B}\right)$

$\sum M_{A}=0$

For DL ONGY

$6 H_{B}=12 \times \frac{750}{2}$

$H_{B}=750 \mathrm{LB}^{2} /$ EACH TS

FOR SEISMIC + TO

$H_{B}=3504 B /$ ances 73

LOCAR STRESSES ON TS WALL

w 6 FCANGE, KTC DULTE

He DK BY INSPETTION

CAKCUCATE BENDING STresses IN Wh in $\& 24 \times 24$ WTH CAMERA -OAD

REP TMMOSHSWKO, PLATES

AND surecs Pb 147

PRESOME SMLLY SUPPORTED

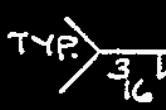

4

RECTHNGULAR $P$ CENTER WADED

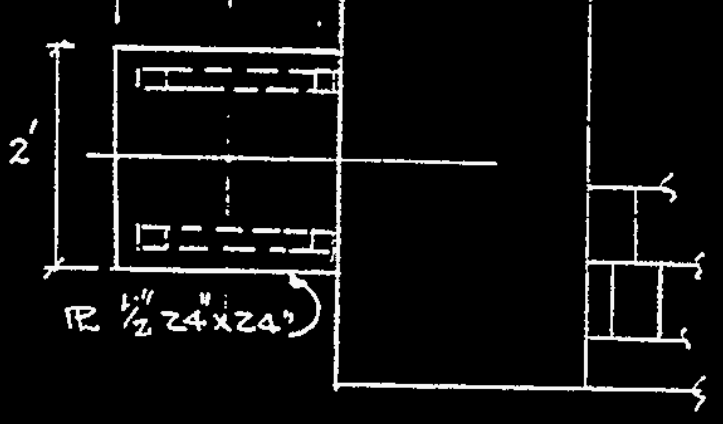

$$
\begin{aligned}
& a=24 \mathrm{~N}, \xi=\frac{a}{2}, \text { PoIsSONS RATIO } \rightarrow=0.3
\end{aligned}
$$

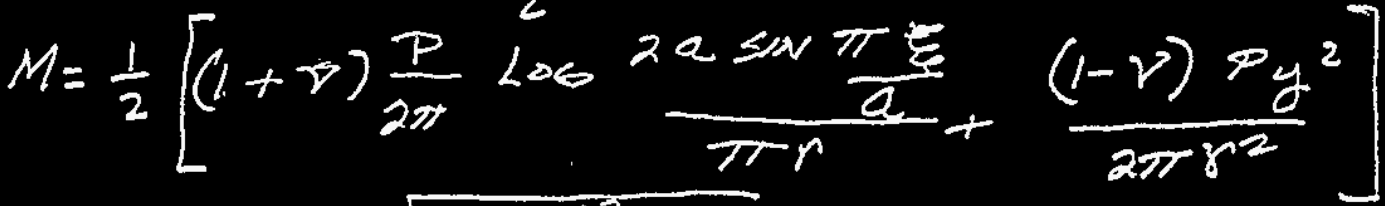

$$
\begin{aligned}
& y=\frac{a}{2}, r=\sqrt{(x-\xi)^{2}+y^{2}}, x=a / 2 \\
& r=y=\frac{a}{2} \\
& B-19
\end{aligned}
$$


ANALYTICAL CALCULATIONS

DHC-SD-WM-ER-521

Rev. 0

Page 12 of 12

Subject DeF Molk-up PLATEReM

Originator F.R.Vocecer

Date $11-22-95$

Checker OSALMrod

Date $11-2 z-95$

camera pLATFORM CONTIUUED

$$
\begin{aligned}
& M=\frac{1}{2}\left[(1+r) \frac{P}{2 \pi} \log \frac{2 a \sin \frac{\pi}{2}}{\frac{\pi a}{2}}+\frac{(1-r) P d^{2} / x}{2 \pi a^{2} / 4}\right] \\
& M=\frac{1}{2}\left[1.3 \times \frac{850}{2 \pi} \log \frac{48}{12 \pi}+\frac{0.7 \times 850}{2 \pi}\right] \\
& M=56.6 \mathrm{in-LE/NN}
\end{aligned}
$$

BENDING STRESS $\left(f_{b}\right)=M / s$

FOR $1 / 2 "$ UNIS LEXKTTH $S=\frac{0.5^{2}}{6}=0.04 \mathrm{iN}^{3}$

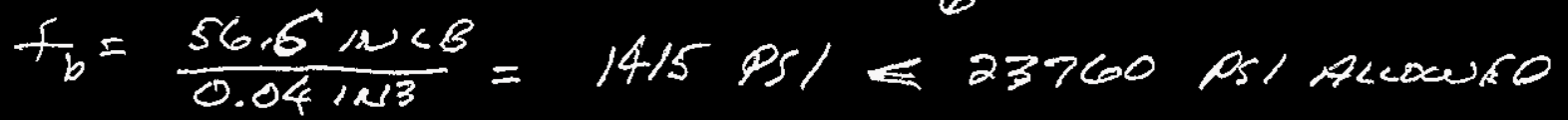

CALCULATE TORSION STRESS IN W $6 \times 20$ FOR TAMERA WEIGHT ONLY

$T=750 \times 12=9000 \mathrm{NL} \mathrm{LB}$

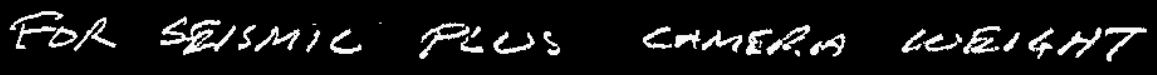

$T=850 \times 12=10200 \mathrm{Ne}$

TARSI SHEAR STRESS $(T)=\frac{3 T}{\varepsilon b t^{2}}$

FOR w6.20 $\sum b t^{2}=2 \times 6.02 \times 0.365^{2}+5.47 \times 0.26^{2}=1.97$

FOR $D L$ ency

$T^{2}=\frac{3 \times 5000}{1.97}=13706$ PSI $=14400$ PSI Allows

FOR SEISMIC

$$
Y=\frac{3 \times 10200}{1.97}=15.533 \mathrm{pl}<1.33 \times 14400 \mathrm{ps} \text { Headers }
$$

ToRsion in waxier $x$

$B-20$

8D-6400-060.1 (07/93) 
ANALYTICAL CALCULATIONS

WHC-SD-WM-ER-521

Rev. 0

ALTERNATE EALCS.

Page of 18

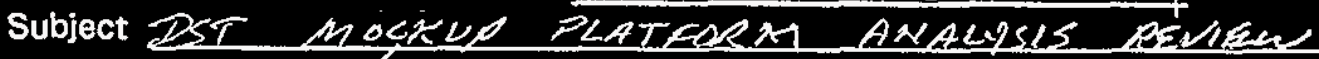
Originator EIRL YocLERT

Date $10 / 12 / 45$

Checker

Date

COLUMN STRESSES

$W 6 \times 20$ COLWMNS ARE CROSS BRACED IN THR GLOBAL $Z$ DIRCCTION OR THE WEAK (YM) DIRECTIOW THE UNORACEO LENGTH OF THE COSWMNAS W THE Yy DIRRLTION IS II FT OR 132 IV, $K=1.0$ EFFREIVE $4 N$

FOR THE DRACEO $r_{y}$ DIRECTION $\frac{K l}{r_{y}}=\frac{1.0 \times 132}{1.50}=88 \mathrm{~N}$

TWD OF THE W6 20 COLMNS ARE UNBRACED IN THE GuBAL $X$ DIREETION DR THE STRONGER $V_{x}$ DIRLTIOW

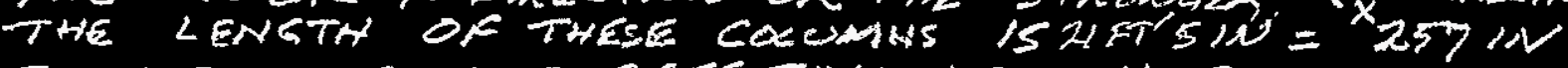
TO DETERMINE THE EFFELTIVE LENTTM FACTOR $K$ THE TOP CONNECTION TO THE PLATFORM WILC PROUIOE VERY LITTCE ROTATION RESTRANTT TOR ALTS AS A PINESO CONNELTION. THE COLUNN ANCHOREO TO THE CONCRETE FLOOR WITM 4- $3 / 4$ N HILTI KUIK BOCT DI . THE COLUMN IS RILET WELOEO

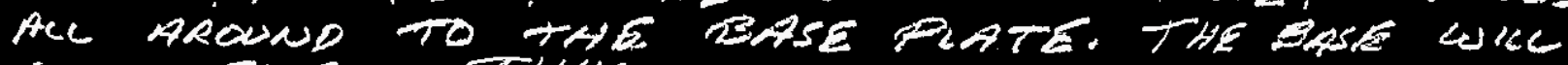
AT AI EIXED - THUS:

( $K=2.10 \quad$ (ASC 1989)

$$
\frac{k l}{r_{x}}=\frac{2.10 \times 25 l}{2.66}=\begin{array}{ll}
203 & \begin{array}{l}
\text { Exces } 200 \\
\text { BSy } 11 / 2 \% \\
\text { USE } \frac{k l}{r_{x}}=200
\end{array}
\end{array}
$$

$F_{R}=6.22 \mathrm{kS1}$ (ALCOLABCE, Alse /SP9)

$$
\begin{aligned}
& F_{b}=\frac{170 \times 10^{3}}{\left(l / r_{T}\right)^{2}} \quad \text { AND } \quad 156.7>\sqrt{\frac{510 \times 10^{3}}{F_{y}}}=119 \\
& F_{b}=\frac{170 \times 10^{3}}{(156.7)^{2}}=6.92 \mathrm{ks} /
\end{aligned}
$$

$$
B-21
$$

BD-6400-060.1(07/93) 
ANALYTICAL CALCULATIONS

WHC-SD-WM-ER-521

Rev. 0

Page 2 of 18

Subject DST MOCECU PLETFER ANALYSLE REVLW

Originator EN, Losheser

Date $10 / 12 / 25$

Checker

Date

COLUMN STRESIE, CONTINUEO

NOTE; THE UNBPALE GLOBR $\times$ DURETTON COCONCUS ARE ELEMENT NUMBERS $1,2,7,8$ w THE MOOEC.

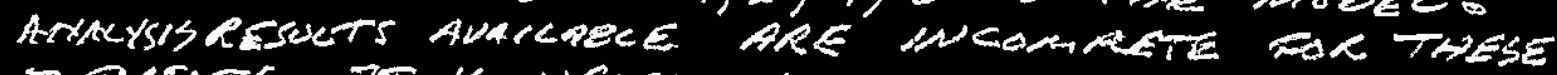
ELMUNTS. IT IS NGCESSACY TO USE THE MENBER FORLES FOR COCWMN ELEMENT 3 SORTEO DAS MAXMMUNS AND THEREROLE EXCEED THE OTMERS TO CRLUUTE CovenN STRESSES

FOR $D C+L C, \angle O C O M N$ ELERENT 3

$$
\begin{aligned}
& F_{x}=5819 \angle B, M z=3903 w \angle B \\
& F_{a}=\frac{F x}{5.87 / N^{2}}=\frac{5819}{5.87}=991 \mathrm{FI} \\
& f_{b}=\frac{M_{z}}{S_{z}}=\frac{3903}{13,4}=291 \mathrm{MI} \\
& \frac{f_{a}}{F_{a}}+\frac{f_{b}}{F_{b}}=\frac{991}{6220}+\frac{291}{6920}=0.16+0.04=0.20<1
\end{aligned}
$$

OF FOR DCWLC; Ca W6X20

FUR DL + 0.29 SEISMIC, COCUMM ELEMENT 3

$$
\begin{aligned}
& F_{X}=5826 \mathrm{LB}, M Z=103500 \\
& F_{a}=\frac{5826}{5.87}=992 \mathrm{ps} / \\
& F_{b}=\frac{103500}{13.4}=7724 \mathrm{ps} /
\end{aligned}
$$

FOR SEISMIC THE ALCOWABLE STRESSES F AND F MAY BE INCREASED BY 1/3.THUS:

$$
\begin{aligned}
& F_{a}=1.3 \times 6220=8086 \mathrm{FI}, \quad F_{b}=1.3 \times 6920=8996 \mathrm{ps} \\
& \frac{F_{a}}{F_{A}}+\frac{F_{b}}{F_{b}}=\frac{992}{8086}+\frac{7724}{8996}=0.12+0.86=0.98<1.0
\end{aligned}
$$

Cex WGX20 OK FOR DL + SEISMIC

$$
B-22
$$

BD-6400-060.1 (07/93) 
ANALYTICAL CALCULATIONS

Page 3 of 18

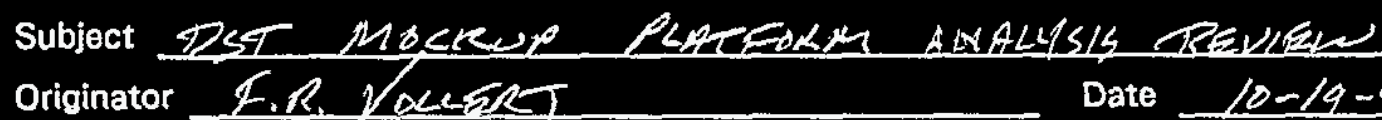
F.R. YOLST

Checker

Date $10-19-95$

$C 6 \times 8.2$ BEAM

BENDING STRESS GOUENNS

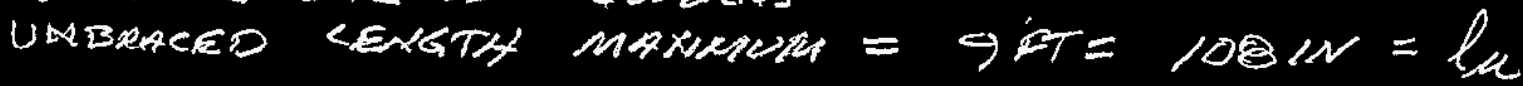
AISC 1.5.1.4,1 RERUIREMENT

$$
\ell_{\mu} \leq \frac{76 b_{4}}{\sqrt{F}}=\frac{76 \times 1.92}{6}=24 \mu<l_{\mu}
$$

so ABC 1.5 .1 .4 .5 , EQR $1.5-7$ \#PQLES

$$
F_{b}=\frac{12 \times 10^{3} c_{b}}{l d / A_{f}} \quad \begin{aligned}
& c_{b}=\text { uN/Ty } \\
& d=6.0, A_{f}=0.343 \times 1.92=0.654
\end{aligned}
$$

$F_{b}=\frac{12 \times 103}{108 \times 6 / 0.659}=12.2 \mathrm{ks} 1$

FOR SEISMK, $F_{b}=1.3 \times 12.2=5.9 \mathrm{ks}$

For sctice $f_{0}=\frac{16.973}{4.38}=3.9 \mathrm{ks} 1<12.251 \%$

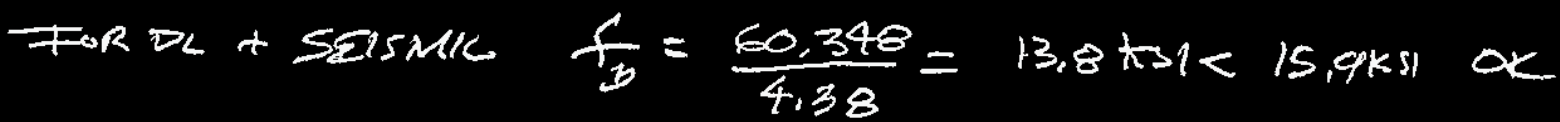


ANALYTICAL CALCULATIONS

WHC-SD-WM-ER-5? 1
ReV. 30

Subject DST MOCR-UP PLATGORM ANALWSL PSURE 4 of 18 Originator Fin, Lousier Checker Date poleglas Date

CROSS BRACING, ANGLE $2 \times 2 \times 14$ COMPRESSIVE STRESS, GOVERNS (MAXIMUM LENGTH).

MAX HORIZONTAl DISTANCE $l_{h}=114-2(6)=102 \mathrm{~N}$ MAX VERTICAL DISTANCE $l_{n}^{n}=138-18-9=111$ IN

$$
\begin{aligned}
& l_{\mu}=\sqrt{\left(\frac{102}{2}\right)^{2}+\left(\frac{11}{2}\right)^{2}}=754 \mathrm{~N} \ldots r_{z z}=r_{\min }=0.391 \mathrm{~N}
\end{aligned}
$$

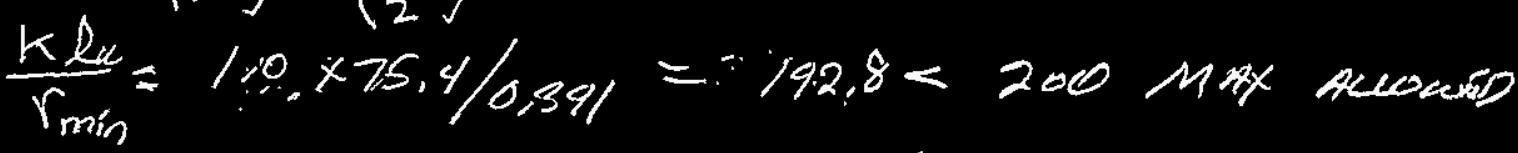$$
\frac{b}{t_{1}}=\frac{2}{0.25}=8<\frac{76}{\sqrt{F_{y}}}=12,67 \quad(A 15 \mathrm{C} 1.9,1,2)
$$$$
C_{c}=\sqrt{\frac{2 \pi r^{2} E}{F_{y}}}=126
$$

$\frac{K l_{0}}{r_{\text {man }}}>C_{c}$ AND $>120$ so:

$$
\begin{aligned}
& F_{a S}=\frac{F_{a}}{1,6-\frac{l}{200 r}} \\
& F_{q}=\frac{12 \pi^{2} E}{23(k l / r)^{2}}\left[\begin{array}{l}
\text { A1SC } \\
1,5-2 \\
1,5-3
\end{array}\right] \\
& F_{a}=4019 \mathrm{ps} \\
& \left.F_{a:}=\frac{4017}{1.6-\frac{192.8}{200}}=6316 \mathrm{Ps}\right)
\end{aligned}
$$

For seismic mas $=1.3 \times 6316=821195 /$ DuAL $f_{4}=524$ PS $<6316$ PSI OK $D C+$ SEISMIC $f_{a}=2410$ Psi< 8211 Ps/ OK

$$
B-24
$$

BD-6400-060.1 (07/93) 
ANALYTICAL CALCULATIONS

WHC-SD-WM-ER-5?1

Rev. 0

Page 5 of 18

Subject DST MOCK-UP PATFOPM ANALYS RENEW Originator $\in R_{1}$ /anterer

Checker Date $10 / 20 / 25$ Date

TS $3 \times 3 \times 1 / 4 \quad(A .5 T M A 1020, F y=38 \mathrm{ks})$

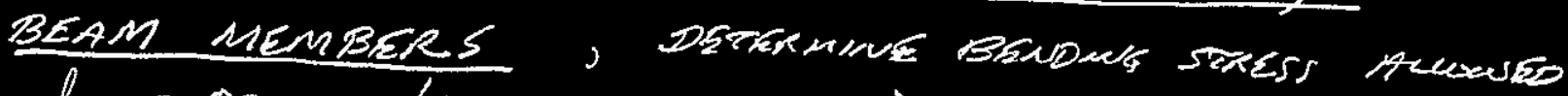
$l_{\mu}=82 \mathrm{~N}$ (MAXMON ACTONG)

$$
\begin{aligned}
& \frac{b}{t}=\frac{3}{0.25}=12 \quad<\frac{190}{\sqrt{F_{y}}}=\frac{190}{\sqrt{35}}=30.8 \\
& \frac{d}{t}=\frac{3}{0.25}=12<\frac{257}{\sqrt{F y}}=41.7 \\
& 1200\left(\frac{b}{F_{y}}\right)=\frac{1200 \times 3}{38}=.94 .7>l_{\mu}=82
\end{aligned}
$$

BEAM MFMBERS QUACIFY AS COMAACT

$$
F_{b}=0.66 F_{y}=25 \mathrm{ks} /
$$

For szismic $\sigma_{b}=1.3 \times 25=32.5 \mathrm{kS} 1$

FOR DC + LC. MAX MOMENT $=47702$ NCB

$$
f_{b}=\frac{M}{S}=\frac{47702}{2,1}=22715 \mathrm{ps}=25000 \mathrm{ps}=\mathrm{ok}
$$

FOR SEISMIC +DC MAX MOMEIT = 54047 MLS

$$
f_{b}=\frac{54047}{2.1}=25737 \mathrm{ps}<32500 \mathrm{ps} \text { ore }
$$
COCOMN MEMBERS PETERMINE AXIML AND BEUPRT

$$
\begin{aligned}
& l=104 \mathrm{~N}, \quad \frac{15 l}{r}=\frac{2 \times 104}{1,1}=189.1<200 \\
& c_{c}=\sqrt{\frac{2 \pi^{2} E}{F_{y}}}=\sqrt{\frac{2 \pi^{2} \times 29 \times 10^{3}}{38}}=122.7<\mathrm{kl} / \mathrm{r} \\
& F_{a}=\frac{12 \pi^{2} E}{23(k l / r)^{2}}=\frac{12 \pi^{2} \times 29 \times 10^{3}}{23(189.1)^{2}}=\begin{array}{l}
4.176 \mathrm{ks} \\
(4 \times 1,1 C)
\end{array}
\end{aligned}
$$

BD-6400-060.1 (07/93) 
ANALYTICAL CALCULATIONS

WHC-SD-WM-ER-521

Rev. 0

Page 6 of 18

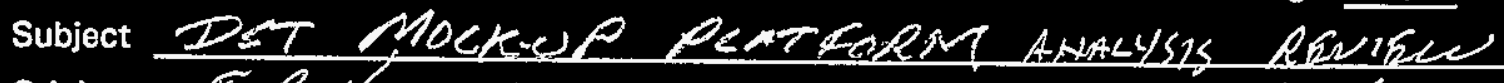

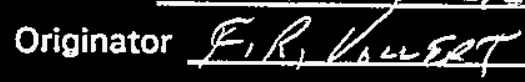

Checker

Date $10,60 / 95$

Date

TS $3 \times 3 \times 1 / 4$ contuses

(Columns, continued)

(AIs $1,5,1,4$ )

$1200 \frac{b}{F_{y}}=1200 \times \frac{3}{38}=94,7<l_{\mu}=104 \mathrm{kN}$

So $F_{b}=\frac{12 \times 10^{3} c_{b}}{l d / A_{f}}$ Not to Exceed $0.6 F_{y}$

$$
\begin{aligned}
& c_{b}=1 \\
& F_{b}=\frac{12 \times 10^{3}}{104 \times 3 / 0.75}=28.8 \mathrm{ks}, 0.6 \times F_{y}=0.6 \times 28=22.8 \mathrm{ks} \\
& F_{b}=22.8 \mathrm{ks} 1
\end{aligned}
$$

For $D C+C C$

$$
f_{a}=\frac{1765}{2.59}=681 \text { PSI }<F_{a}=4176 \text { AND }
$$

$\frac{F_{a}}{F_{a}}=0.16 \times 0.15$ BUT $4 \mathrm{HC}$ COMBINE STRESS

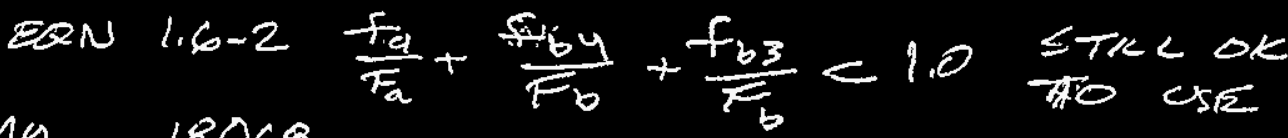

$F_{b y}=\frac{M y}{S}=\frac{18068}{2.1}=8604 \mathrm{ps}$

$$
F_{b_{3}}=\frac{M_{3}}{S}=\frac{11002}{2.1}=5282 \mathrm{~F} 1
$$

$\frac{615}{4176}+\frac{8604}{22800}+\frac{5282}{22800}=0.15+0.38+0.23=0.76$

TS $3 \times 3 \times 1 / 4$ coLumns OK FOR DC+ CC

$$
B-2 C
$$

BD-6400-060.1 (07/93) 
ANALYTICAL CALCULATIONS

WHC-SD-WM-ER-521

Rev. 0

Page 7 of 18

Subject DST MOCK-UP PLATCOLM ANALYSS REVIEW Originator Fo, hewser

Checker

Date $10 / 20 / 95$

Date

Is $3 \times 3 \times 1 / 4$ cantures

cocomas, cartenuso

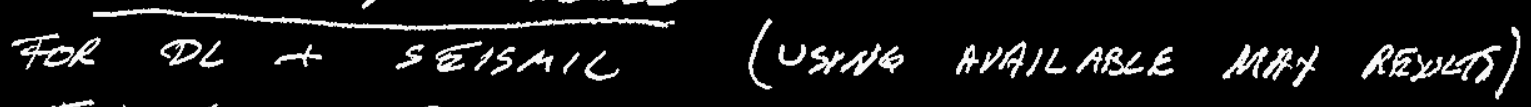

$F \times 3503 \angle B, M y=37907, M<8$

$$
\begin{aligned}
& M_{z}=12116 \text { w } \angle B \\
& f_{a}=\frac{3503}{2.59}=1353 \mathrm{ps}, \quad \frac{1353}{1.3 F_{a}}=\frac{1353}{3429}=0.2570 .15
\end{aligned}
$$

AlsC COMBMEO STRESS EQUATOU EEOMES

$$
\begin{aligned}
& \frac{F_{a}}{F_{a}}+\frac{0.85 F_{b 4}}{0.75 F_{b}}+\frac{0.85 f_{b}}{0.75 F_{b}}=1.0 \text { Fon } 1.6 .1 \mathrm{a} \\
& F_{b y}=\frac{37907}{2.1}=18051 \mathrm{Ps} \\
& F_{b 3}=\frac{12116}{2.1}=5.770 \mathrm{PS} \\
& \frac{1353}{5429}+\frac{0.85 \times 18051}{0.75 \times 1.3 \times 22800}+\frac{0.85 \times 5770}{0.75 \times 1.3 \times 22800}=0.25+0.71+0.22 \\
& =1.1871 .0
\end{aligned}
$$

COLwm TS $3 \times 3 \times 1 / 4$ COULD BE OUER

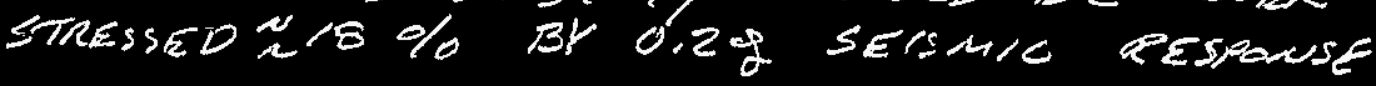
DESIGN CMNGE NOT RECOMMEPEO BASEO dU THE CRITERAA USAD, BASILAMY THIS IS A WERY NAN CONSERVATWE SEISMIL HNACYSIS FOR THE APPBR STRWTWRE BECNUSE IT IS A PENTHOUSE TYPE GTRWLTURE THAT WOED

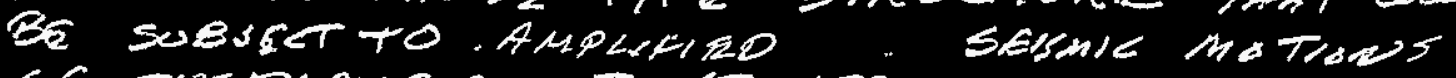
GA THEPLATFARM TT STANDS ON.

$$
B-27
$$

BD-6400-080.1(07/93) 
Subject DST MOCKUP PLATFORM ANRLVOS REMEN Page 8 of 18

Originator FLR V VueAT

Checker
Date

Date

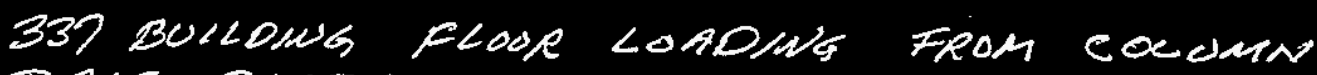
TBASE PLATES

FOR $(P L+\angle L)$ THE SUM DA ALL(G)COLUMN

LOADS $15 \quad 23,834<85$

ASSOMINA THIS TOTHL COAD IS PISTRIBUTED OVER THE AREA THE PLATEVM OCCUPES; THE ELOSR LOAD IS; (DC+CC) /AREA

AREA $=15,0^{\prime} \times 4,5^{\prime}=142,5 \dot{C}^{2}$

$F L=\frac{23834}{142.5}=167 \mathrm{pSt} \quad$ 250PR is the

THE UNIFOR FLODR LADD DUF TO THE $20 \mathrm{FT}$

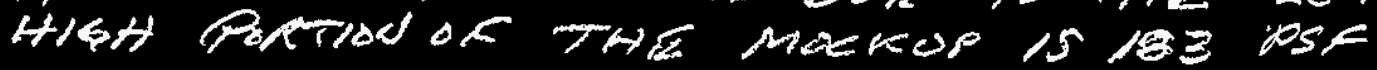
fOR THE MOCKUP BASE PLATE AREA THAT CONCIOES WITH THE PLATAORM TAE SUM OE THESE

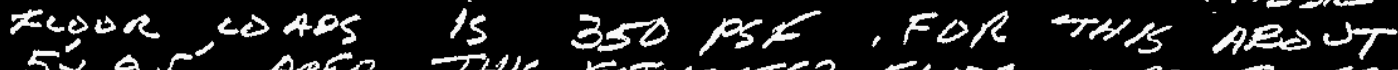

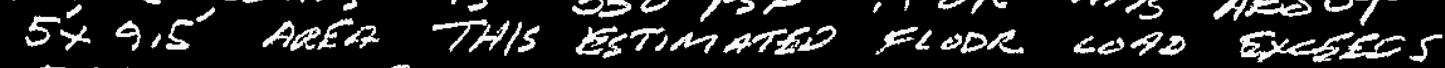
HIE 250 PSP MSTED CMIT. THE FLUW DESKN

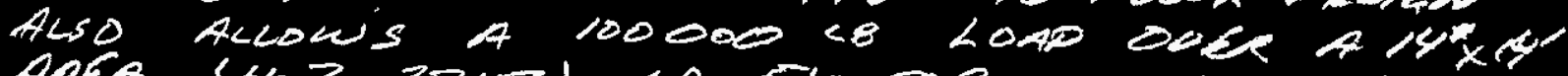

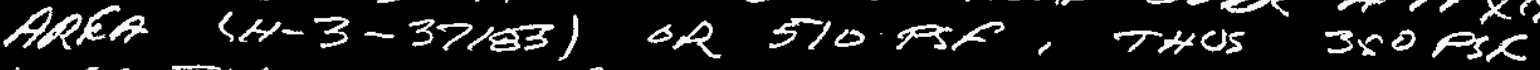

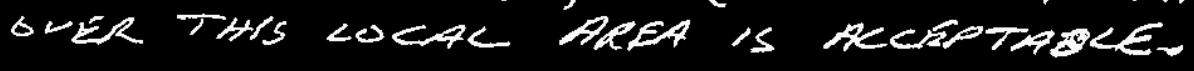

THE MAXIMOM SINGLE COLUMN LOAD (DUE TO DLTSEISMIC) IS $7177 \angle B, Y$ BASE th is $18^{\circ} \times 18=$

CONCRETE BEARMG STRESS $f_{b r}=\frac{717748}{18 \times 18}=22 \mathrm{ps} 1$ Accow $4 B C E=0.7 f_{C}^{\prime}, f_{C}^{\prime}=3000$ Ps, Min cancReTE

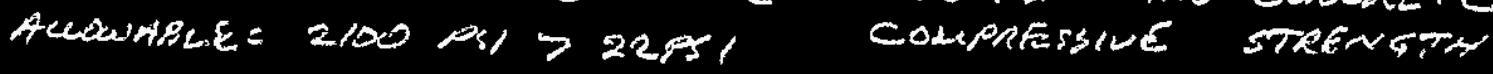
VERTICAC SHEAR STRESS IN THE FLOOR SCAO

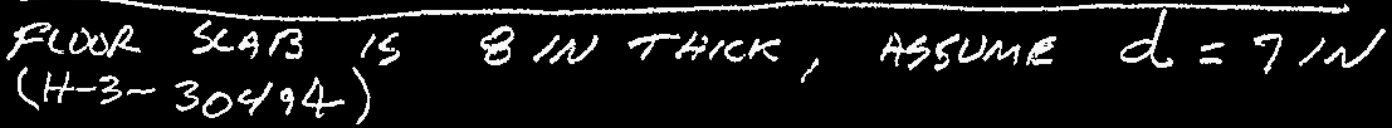

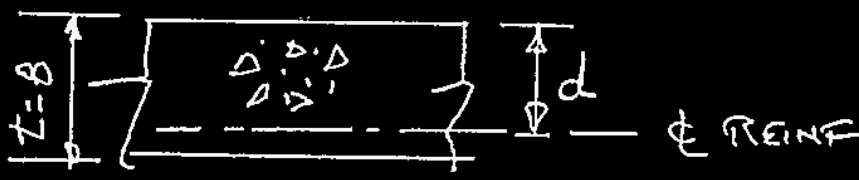


Subject DST MOCRWR PCATFERM ANACYSIS REVISW

Originator FR V $0<4$ RRT

Checker
Date

Date

\section{FLUUR SCAB SHEAR CONTINUED}

PUWCHAG SWEAR AT PERIMITER OF COLUMN BASE fL ALLOW ABLE $V_{L}=2 \sqrt{\frac{f_{L}}{6}} b_{0} d$

$b_{6}=$ PERMETKR, BASER $=4 \times 18=72 \mathrm{~m}$

$V_{c}=2 \sqrt{3000} \times 72 \times 7=55210487777 \mathrm{LB}$ OK

BEAM CONNECTIONS, WELDED

W6 620 PLATFORM BEAMS HAVE FAAMSO BEAM (SIMPUE)

CONINECTIONS. THESE TYPE CONMESTIONS BRE SHEAR RESISTING, AND DO NAT DENEWOX FUL END MOMBUTS. THUS, THESE MUST BEABCE TO CARRY THE AXIAC FORCES $(x, y, z)$ CALCUAATEO FOR THE CORCES PONDING END NODES

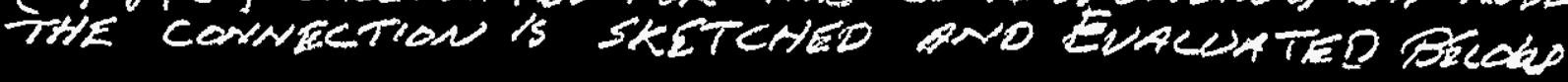

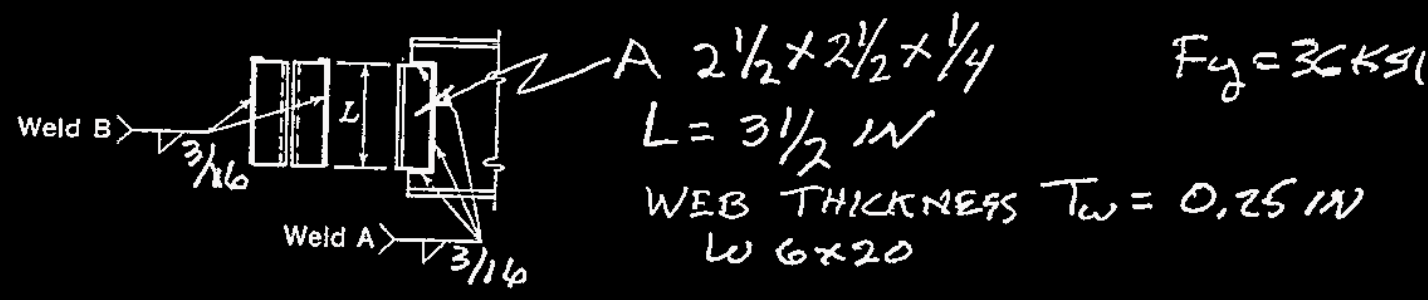

EVALUATE WELO CAPICITY FROM AISE 1989 PART 4 , TABE D.

WELD \& CAPACITY $F_{W B}=10,4 \mathrm{~K}$ IF $2=4, T_{W}=0.38 \mathrm{~N}$, AXD WELD THROAT $t_{W}=1 / 4 \mathrm{~W}, 50 !$ As is $F_{W B}=10.4 \times \frac{3.5}{4} \times \frac{0.25}{0.38} \times \frac{3}{16} \times \frac{4}{1}=4.5 \mathrm{~K}$ BUT TABCE IV $F_{U, B}=\frac{2.5}{3.0} \times 4.5=3.75 \mathrm{~K} \quad \frac{4}{4.38} \times \frac{3}{16} \times \frac{4}{1}=4.5 \quad \mathrm{~A} 3 \times 3$ so:

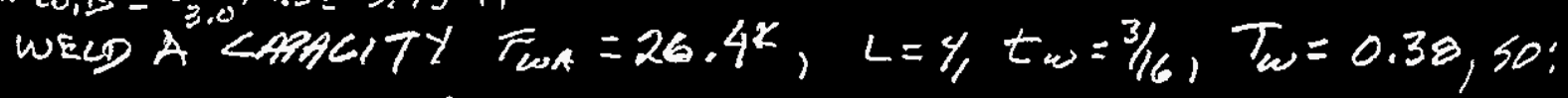
As is $F_{w, p}=26.4 \times \frac{3.5}{4} \times \frac{0.25}{0.38}=15.2 k, \frac{2.5}{3.0} \times 15.2=12.67=F_{2017}$ MAx $D C+$ SENSme $F_{x}=565 \mathrm{LB}, F_{4}=3984 \mathrm{CB}, F_{3}=409 \mathrm{CB}$ $F_{\omega}=\frac{\sqrt{565^{2}+3984^{2}+409^{2}}}{\operatorname{con}}=4.0^{K}+1.3 F_{W B}=4,88 \mathrm{~K}$
$B-29$ 
ANALYTICAL CALCULATIONS

WHC-SD-WM-ER-5?

Rev. 0

Page 10 of 18

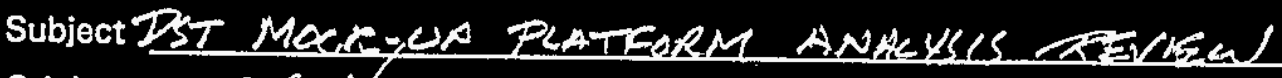

Originator $E R$ VoLe $2 R T$

Checker

Date $10 / 25 / 95$

Date

WELDED BEAM CONNECTIONS, CONTINUED

THE $C$ 6x8.2 PLATFER BEAM THAT SUPPOTS THE TOP OF THE STALWAY HAS WEE CLIP ANGLE SIMPLE CONNETTENS SEE SKETCH AW D EVALUA TROW BELOW

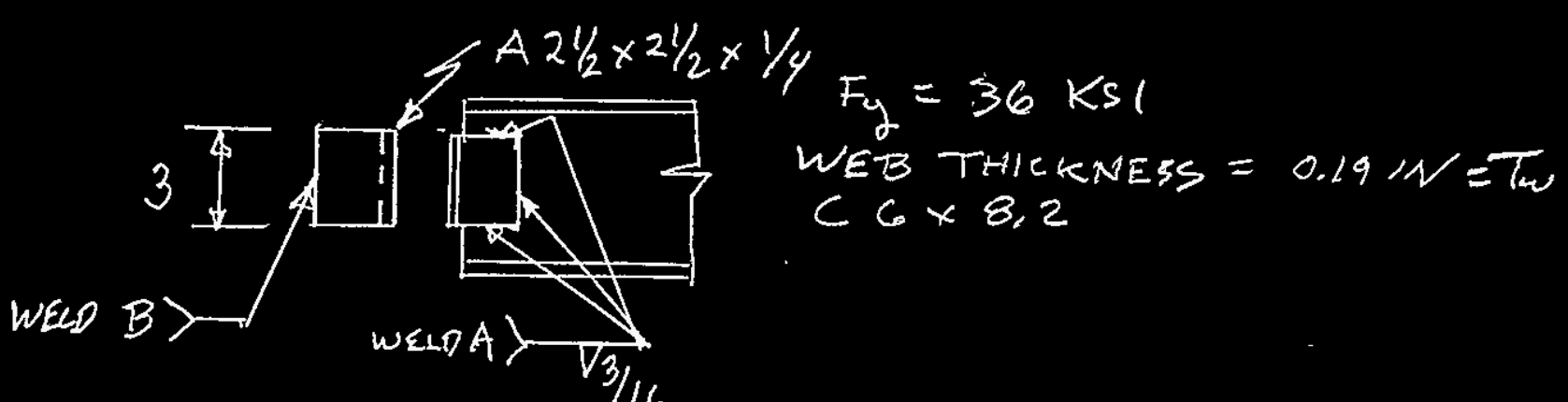

LARGEST CALCULATED FORCE ON CONNECTION IS $292 \angle B S$ DUE TO PL $L L$

FROM AlSO 7989 , PART 4, TABCE TV

$F_{w i 3}=\frac{10.4 k}{2}=5.2^{K}$ IF $L=4, T_{\omega w}=0.38, t_{\omega}=1 / 4 \mathrm{mo}$

FUR $3 \times 3$ CLIP ANGLE: SO:

$$
\begin{aligned}
& F_{\omega_{B}}=5.2 \times \frac{3}{4} \times \frac{3}{16} \times \frac{4}{1} \times \frac{0.19}{0.38} \times \frac{2.5}{3.0}=1.22 \mathrm{~K}>0.292 \mathrm{~K} \\
& F_{\omega_{4}}=\frac{26.4}{2} \times \frac{3}{4} \times \frac{0.19}{0.38} \times \frac{2.5}{3.0}=4.13 \mathrm{~K}
\end{aligned}
$$

CoNAkTHON OK

$B-30$

BD -6400-060.1(07/93) 
Subject IST Mockup PLATFearl ANACYSLS REVIEW/ Page 11 of 18 Originator FR. VOLLERT

Checker Date $9 / 29 / 95$ Date

THE ANSYS FINTE ELEMENT HODES FOR THIS STRUETURE HAS FIXED CONNECTIONS OR JOINTS BETWEEN MEMBERS EXCBPT FAR: PINAED COWUNN BHES, PUNED JOMTS FOR THE PLATFORM TO THE TOP OF THE COWMNS, AND

PINAED BASES FOR THE JACK LEGS. HOWENER, MOST OF THE ALTUAL DOINTS IN THE STRUETWRE ARE SMPLE GOMNECTIONS, SIMPLE CONMECTIOUS WILL DEVELOP AN INDETERMINATE AMOUNT OE ENO MOMENT, BUT GEUECXCY

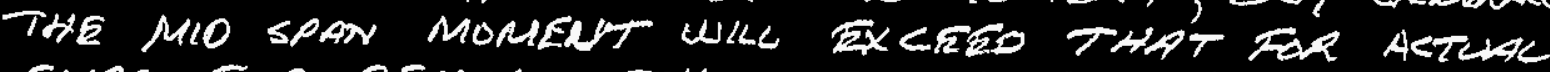

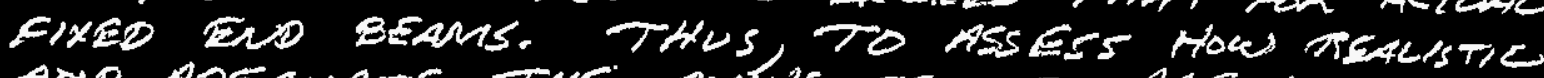
AND HOEQWATE THE ANSDS RESUCTS ARE LUTZR THE FXXD JOINT MODEL, SOME SELFCTED DETENMINATE ANALYES DF STRULTWRAL MEMBERS (BEAMS) WHC DE CONDWTED. THE RESULTS, SHEAP BND HOMENT

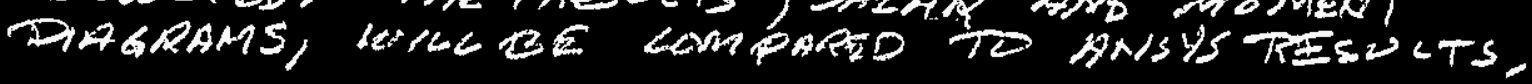




\section{ANALYTICAL GALCULATIONS}

$$
\text { Page } 12 \text { of } 18
$$

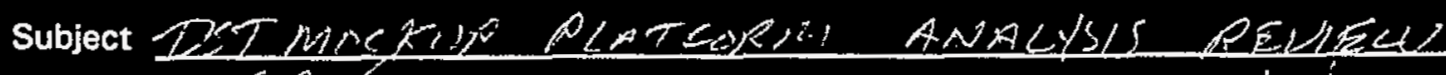

Originator fiR, VumereI

Checker

\section{Date clialqr}

Date

DO SELELTED DETERMINATE A NALYES DOR STRUCTURAC MEMBFES, COMPARE FOXCES KND MOMENTS CALCULATED WITH ANSAS 4.4 EEEUCTS,

PLATERMA EEAM THAT IS ELEMENTS 59,55, 54, 62 INANSYS WODEL, AL $6 \times 20$ EETION

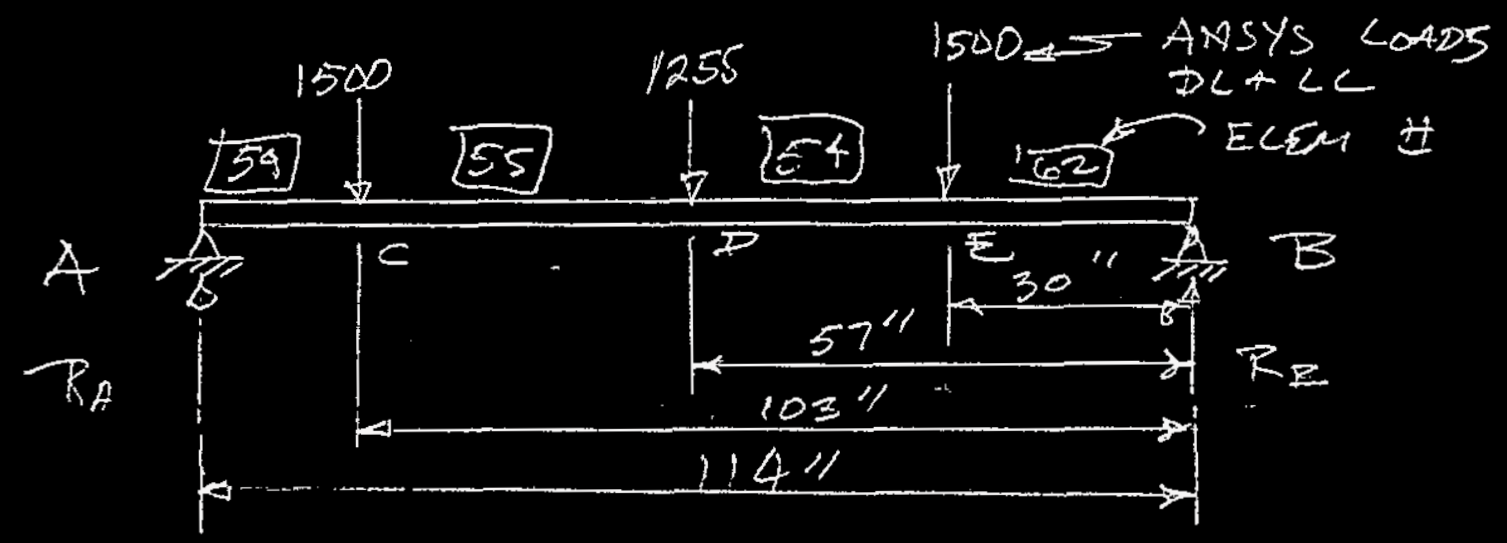

THAS MEMEFR IS INSTHCEO WITH SMPPLE CONAELTIONAS

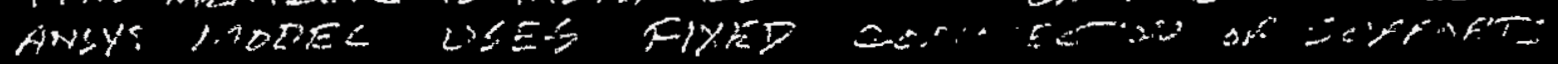

$\frac{a-10 \text { res }}{\angle M_{B}=0}$

$\therefore 4 R_{A}=1500 \times 103+1255 \times 50+1500 \times 30+\frac{50}{12} \times \frac{114^{2}}{2}$

$R_{A}=2.472 .5<8, R_{B}=1972,5<$

$M_{\text {MAX }}=M_{D}=57 R_{A}-1500 \times 46-\frac{20}{12} \times \frac{57^{2}}{2}=69225 \mathrm{NCB}$

$M_{C}=11 R_{A}-\frac{20 \times 11^{2}}{12 \times 2}=27007$ i $\angle E$

$M_{12}=30 f_{E}-\frac{20 r^{2}}{10.42}=58425 \mathrm{w} \mathrm{L}$ 


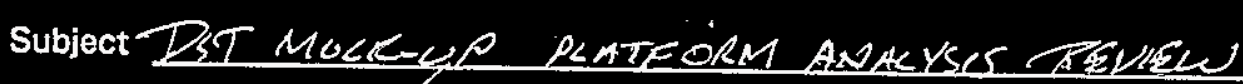

Page 13 of 18

Originator

Checker

Date $9119 / 9-$

Date

EACULATEP

SHEAR S MOKHWT DMGRAMS

$54,55,54,62$
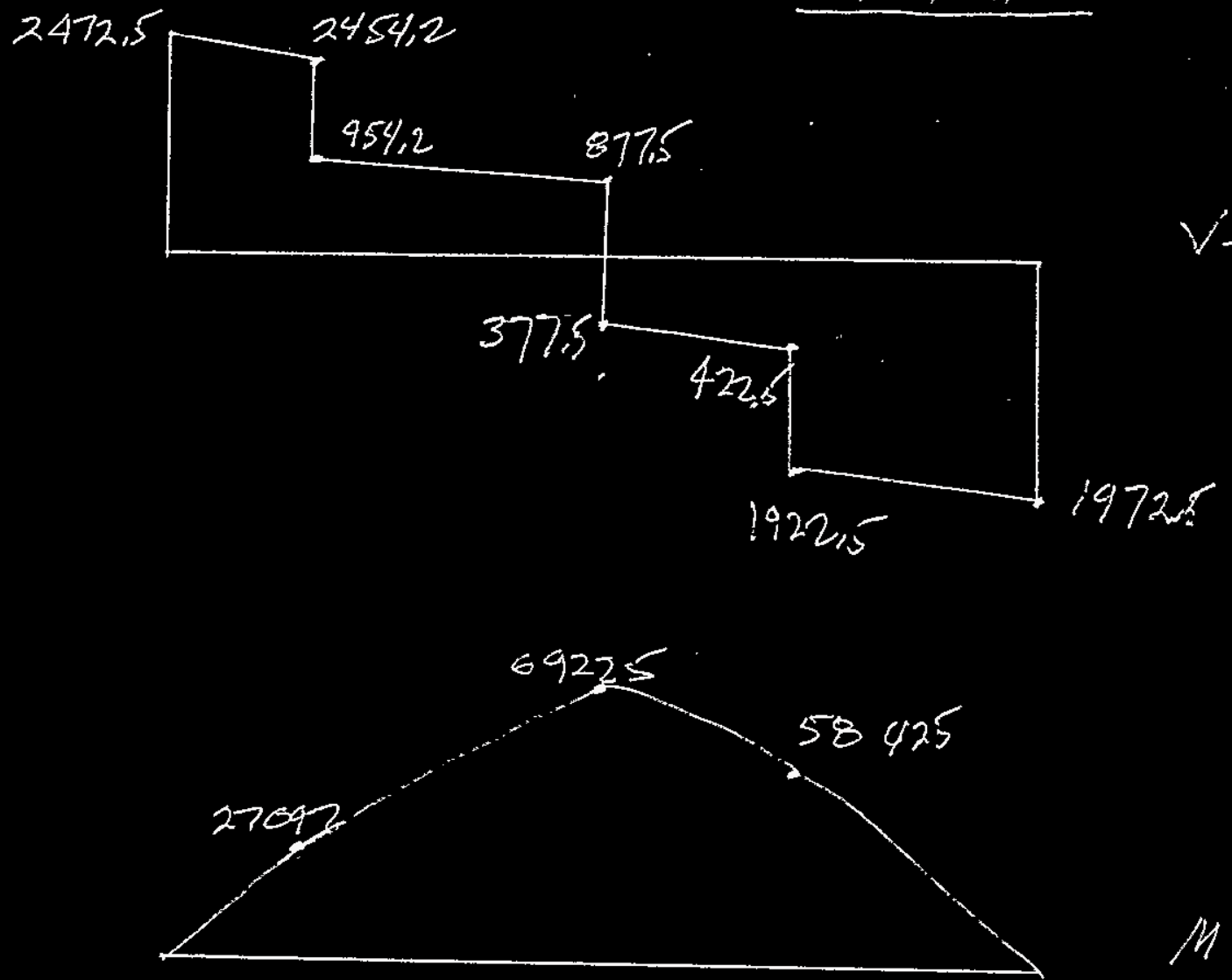

$M D I A C$

$B-33$ 


\section{ANALYTICAL CALCULATIONS}

Subject DST MOCK-US PLATFORM ANALYSK Page 14 of 18 Originator

Checker

\section{Date $91 / 2 / 95$}

Date

\begin{tabular}{|c|c|c|c|c|c|}
\hline ANS & $5+4$ & ANA & $30 x$ & 7 & $0 x+5$ \\
\hline El H & $I F Y$ & $d F y$ & $1 M Z$ & $\checkmark M Z$ & $D<x<4$ \\
\hline 59 & $x$ & 270,6 & K & -278 & \\
\hline 51 & -916.5 & $*$ & -74064 & * & \\
\hline 54 & 383,5 & -338.5 & $-6 \% 318$ & 74064 & \\
\hline 62 & $-2129,5$ & 2709.5 & -6438 & $-3 / 7$ & \\
\hline
\end{tabular}

* UAAUALABCE TESUCT

2.776 .6
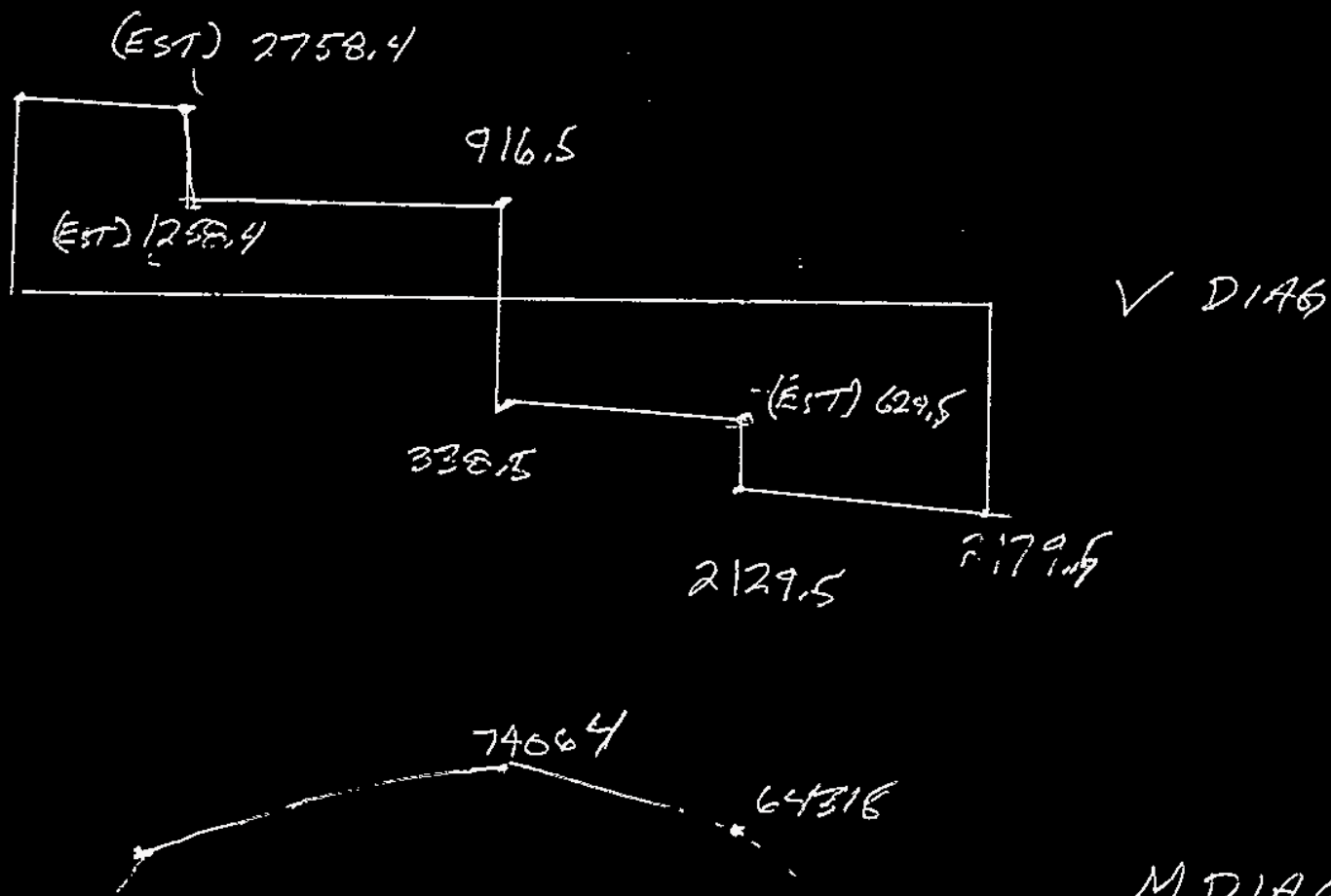

$M D A G$

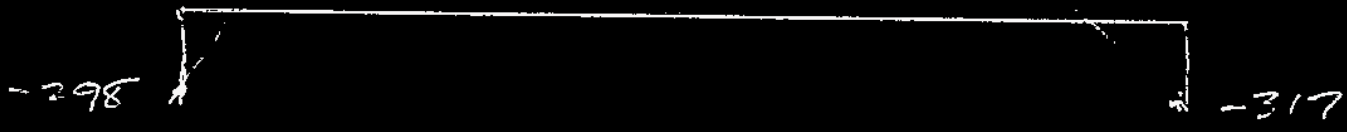

$B-34$ 


\section{ANALYTICAL CALCULATIONS}

Subject RT MNK-CP PLATEORM RNACW/S REUEN Originator F,R, Loc $E R T$ Checker Date $9 / 19 / 95$ Date

PLATFURM BEAM THAT SUPPORTS THE TON OF THE STAIRKAY, ELEMEATS 22 AND 2 ,

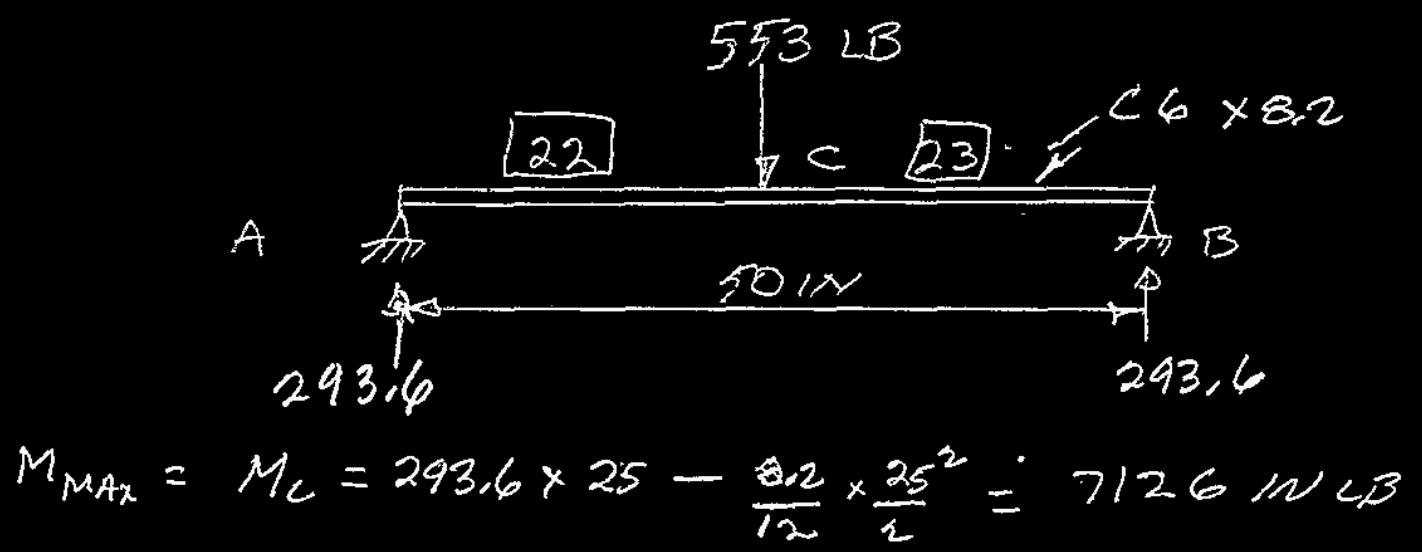

293,6

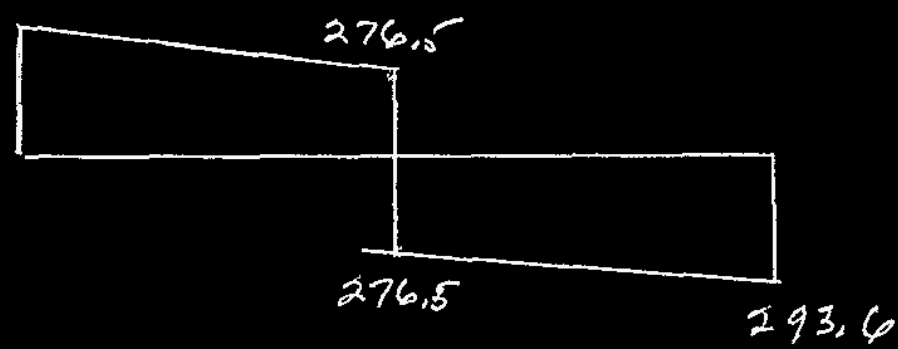

V.DISG

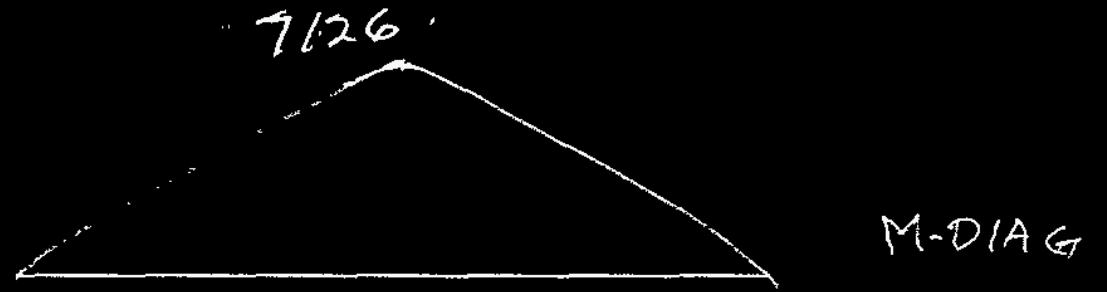


Page 16 of -18

Subjoct DST MOCK-UP PLATEURM ANALYSIS REMLEU

Originator FIR V QLLETT

Checker

\section{Date $9 / 10 / 95$}

Date

ANSYS SHEAR AND MOMIRNT DIAGPAMS

\begin{tabular}{c|c|c|c|c}
$E L$ & IFY & JFY & $1 M Z$ & JMZ \\
\hline 22 & 292,3 & 275.3 & 11.7 & 7084 \\
\hline 23 & $*$ & 294.7 & $*$ & -69.9
\end{tabular}

* un avallable

$$
2923
$$
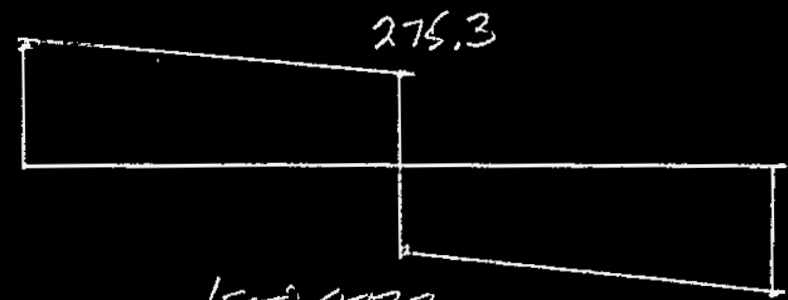

(Est) 197,7

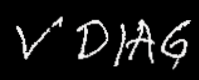

2947

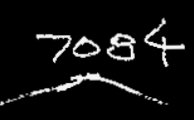

$M D / A G$

11.7

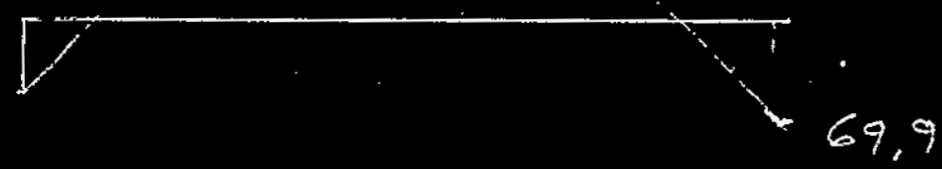

VEAY GoOD compars ax 


\section{ANALYTICAL CALCULATIONS}

Page 17 of 18

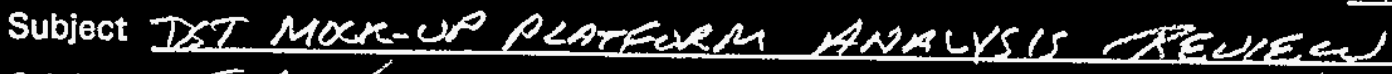

Originator F.K : 'scest

Checker

\section{Date $9 / 19 / 95$}

Date

STAIR LANDING BEAMS, ELEMIETTS 20, 21 . ANO
16,19

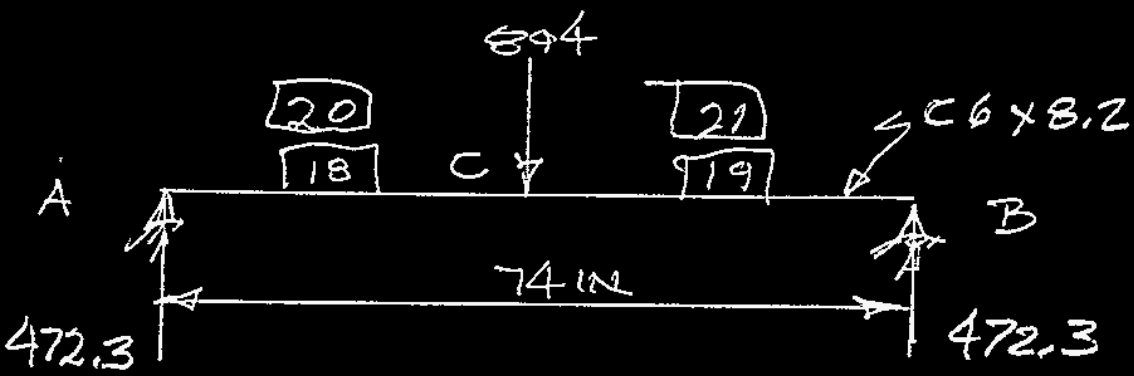

MMAXE $M_{C}=472.3 \times 37-\frac{3.2}{12} \times \frac{37^{2}}{2}=17,007 M-\angle B$

CALEMATEO VAND MDIAGRMMS ORUIOUS:

ANSYS SHEAR AND MDMERT DINGEMMS

\begin{tabular}{|c|c|c|c|c|}
\hline$E L$ & $1 F^{\prime}$ & IFY & $1 M z$ & JMZ \\
\hline 18 & 472,2 & 447.0 & 324 & .16973 \\
\hline 14 & $447=$ & 472,2 & 16973 & -31 \\
\hline$=0$ & 485. & 459,9 & 7709 & 9773 \\
\hline$=1$ & 434.1 & \# & 9773 & + \\
\hline
\end{tabular}

* UNAVAILABLE.
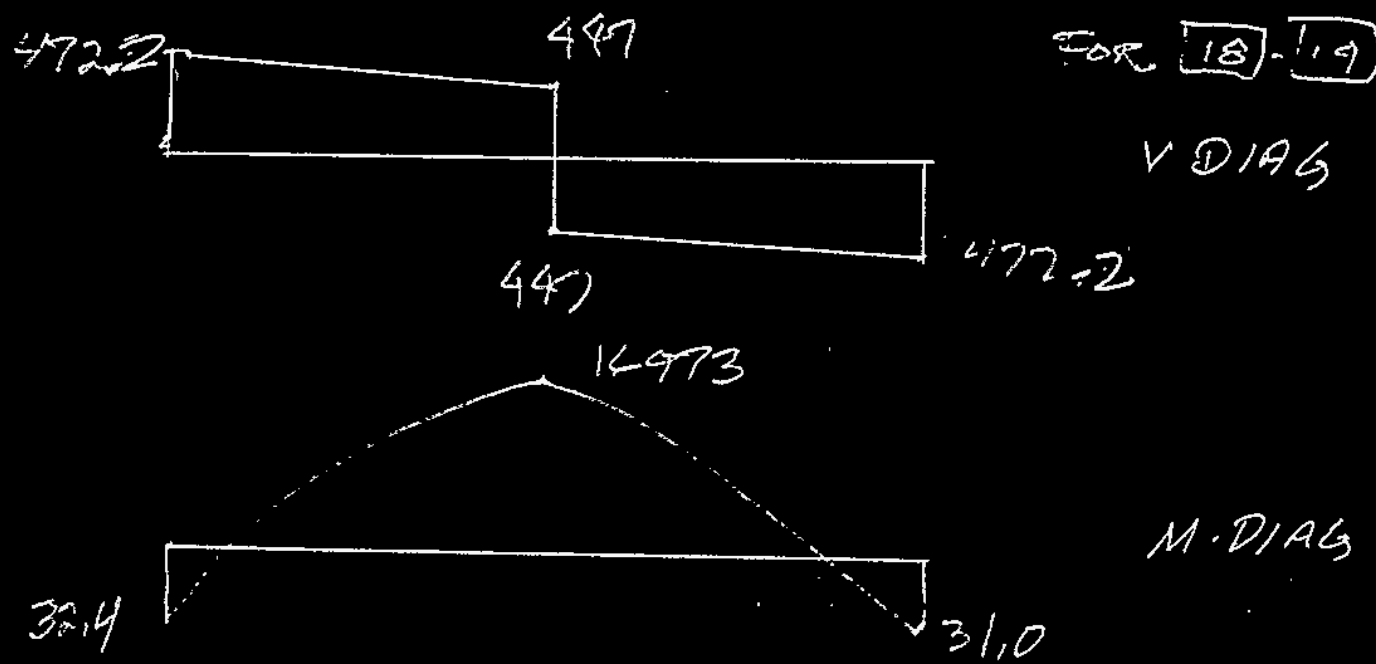

VERY GODD COMPAAISON $B-37$ 
ANALYTICAL CALCULATIONS

Page 18 of 18

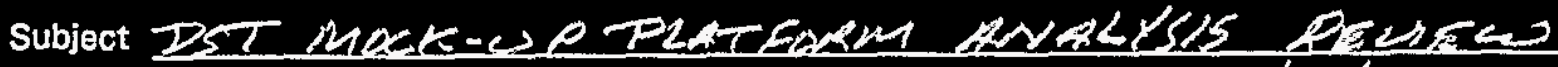

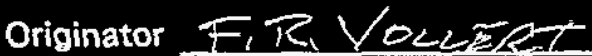

Checker

Date

9) 19195

Date

AMSYS, $20-21$

485.

459,9

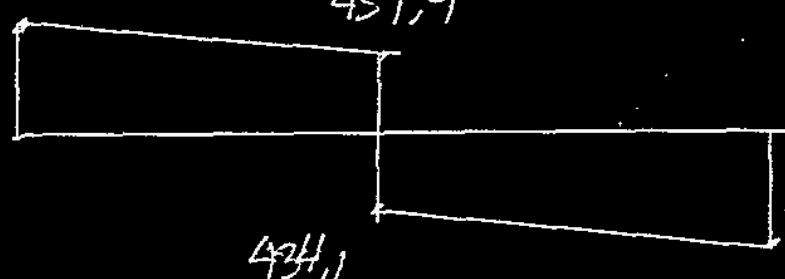

VD/AG

434,1

$485.1(85 T)$

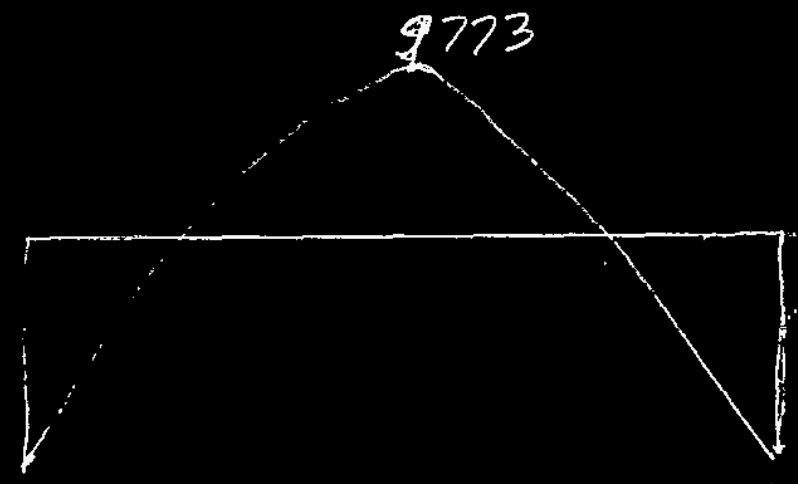

7709

$7709(55)$

POOR FOK $M$ DIAG COMPARISÓNIANSYS RESUCT'S FOR 20,21

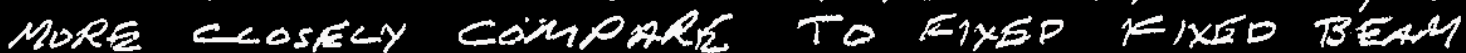
RESwLTS wHAH AREi

END MOMENTS $M_{E}=\frac{P l}{8}+\frac{\omega \lambda^{2}}{12}=\frac{894 \times 74}{8}+\frac{8.2}{12} \times \frac{74^{2}}{12}$

$$
M E=858 / N-\angle B
$$

CENTER MoMent $M=\frac{\rho l}{8}+\frac{\omega \ell^{2}}{24}=\frac{844 \times 74}{8}+\frac{8.2}{12} \times \frac{74^{2}}{24}$

$M_{L}=8425 \mathrm{NC}$

$$
B-38
$$


WHC-SD-WM-ER-521

Rev. 0

APPENDIX C

MONORAIL DESIGN AND DETAILS

C-1 
Subject DST Mock-Unp Tank (Monorail) Originator T.S.Fundal ad all

Page 1 of 8

Checker

Hacen 1

20

Date $10-25-95$

Date $11-2-95$

\section{INTRODUCTION:}

The DST mock-up section for Robot inspection needs to have a monorail/hoist system to catch the Robot in case it detaches from the DST walls. If the Robot is allowed to fall to the floor it may be extensively damaged and will hinder the actual testing in the field. To avoid this a trolley will be mounted on a monorail and a hoist arangement will be hung below it to catch the falling Robot. Allow one foot free fall of Robot.

\section{MATERTALS:}

Steel members: $\quad$ Materials used for Monorail and its support system will be A36

Welding: $\quad$ Electrodes to be E70XX.

Trolley and Hoist: Prepurchased from s vendor

\section{ALLOWABLES:}

Steel: $\quad$ As per ASIC Manual of Steel Construction, ninth edition.

Welds: $\quad$ As per AWS Structural Welding Steel Code D1.1.

Trolley and Hoist: As per manufacturer's recommendations.

\section{REFERDNCES:}

AISC, 1989 American Institute of Steel Construction, Manual of Steel Construction, 9th Edition.

AWS, 1994 American Welding Society, Structural Welding Steel Code, AWS D1.1.

Kleinlogel, I.A., Rigid Frame Formulas, 12th Edition. 


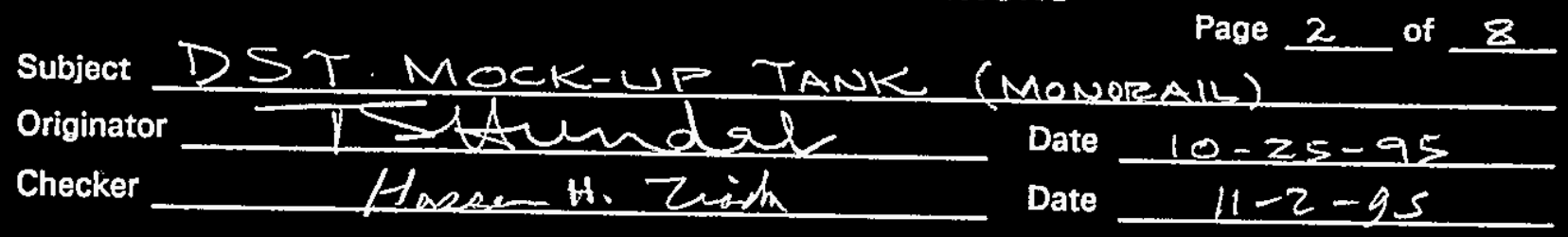

MONORAIL/HOIST DESIGN

- The purpose of this hoIst is to catch the fallina ROBOT OFF OF THE TANK WALL.

- HOIST WILl be HOOKED INDER trolley on the MONORAIL

\section{LOADS}

WT. OF ROBOT $=600 \#=W$

Assume it FALLS 1 FT. (FREE FALL)

$$
\begin{aligned}
\therefore v & =\sqrt{2 g h} \\
& =\sqrt{2 \times 32.2 \times 1}=8.03 \mathrm{fps}
\end{aligned}
$$

ASSUME IT TAKES O.I SECOND TO STOP IT COMPLETELY AFTER IMPACT i.e. IISTANT STOP. (DEACCELEATION)

$$
\therefore a=v / t=8.03 / 1=80.3 \mathrm{Ft} / \mathrm{sec}^{2}
$$

$\therefore$ Total force Exerted on the HoIsting MECHANISM

$$
\begin{aligned}
& F=w+(w / g) a \\
&=600+\left(\frac{600}{32.2}\right) 80.3 \\
&=600+1496 \quad=2100 \# \\
&=2096 \quad \text { USE } 3000 \# \\
& \therefore \quad \text { WSE I'TON CAPACITY TROLWEY } \text { HOIST MECHANISM } \\
& \text { C-3 }
\end{aligned}
$$


Subject DET Page 3 of 8

Subject DST MOCK-UP TANK (MONORAU)

Originator

Checker Haren H. Zidh

Date

Date

$100-25-95$ $11-2-95$

ANALYSIS

THE MONOFAIL CONFIGURATION DETAILS ARE SHOWN IN FIGURES I THRU 3.

MONORAIL

TRY WGXi6 w/10'0 MAX. SPAN, $L_{u}=12^{\prime} \Rightarrow F_{b}=21.6^{\mathrm{ksi}}$ $b_{f}=4^{\prime \prime} \quad t_{f}=3_{8}^{\prime \prime} \quad s=10.2 \mathrm{in}^{3} \quad M_{R}=20 \mathrm{~K}-f t$.

$d=6^{\prime \prime} 4^{\prime \prime} \quad t_{\omega}=4^{\prime \prime}$

$F=1 \frac{1}{2} \tau=3000 \#$

(Ref. AisC, 1989)

$M=\frac{3 \times 10}{4}=7.5^{k-f t} \angle M_{R}=.0 k$

TRY $W 4 \times 13$ TO SUPPORT THE MONORAIL @ $10^{\prime} \mathrm{C} / \mathrm{C}$

$M=\frac{3 \times 2.5}{4}=1.9^{k-1}<M_{k}=11^{k-1} \quad \therefore \sigma^{k}$

(ASSUME SIMPLY SUPPORTED) W4 $\times 13$

WELD TO DST WALLS (FIG.3)

$S=4 \times 4+(4)^{2} / 3=21.3 \mathrm{in}^{2}, A=4 \times 4$

$f_{a}=\cdot 75 / 16=\cdot 05 \mathrm{k} / \mathrm{in} \quad=16 \mathrm{in}$

$f_{b}=\frac{1.9 \times 12}{2 \times 21.3}=-54^{k} / \mathrm{in}$

$f=.05+.54=.59 \mathrm{k} / \mathrm{in} \quad<2.78^{k} /$ in

MONORAL TO $\begin{gathered}\text { W } \\ 4 \times 13\end{gathered}$ for 316 "WELD

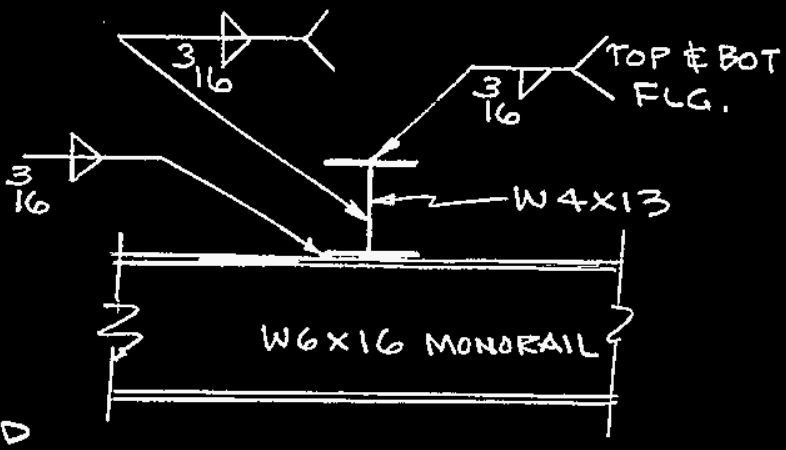

USE $3_{16} "$ WELD AS SHOWN $F_{A}=3 \times 70 \times .707 \times 3_{16}=2.78 \mathrm{k} / \mathrm{in}$ CAPACITY $=2 \times 2.78 \times 4^{\prime \prime}=22.2^{k}>>3^{k} \quad \therefore$ ok

MONORAIL STOP

USE $\angle 2 \times Z \times 4$ @ EA.END(BOTHSIDES OF WEB) OF MONORAIL

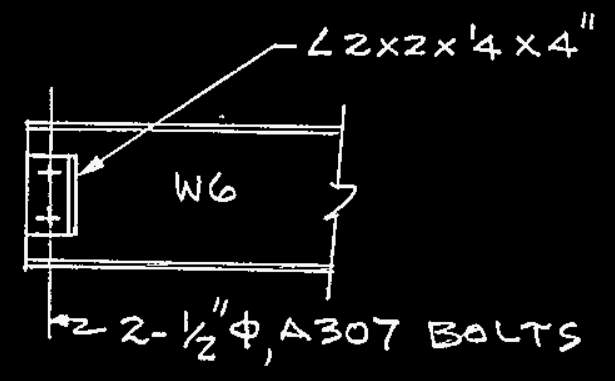

$$
c-4
$$




\section{ANALYTICAL CALCULATIONS}

Subject

Originator

Checker

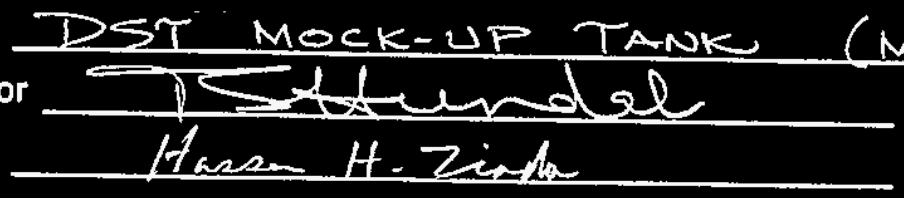

Page

4 of 8 MONOBAL)

Date

Date $11-2-95$

$10-25-95$

MONOEAIL CANTILEVER

$$
\begin{aligned}
M & =P l \\
M_{R} & \left.=20^{k-f t} \quad \text { (AISC } 1989\right)
\end{aligned}
$$

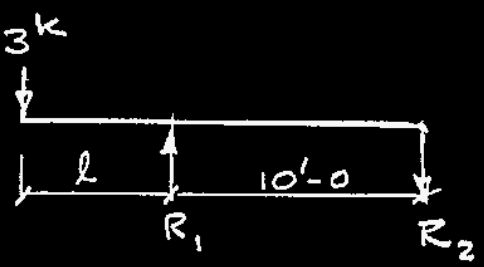

$3 l=20^{k-1}$

$$
\begin{aligned}
& l=6.67^{\prime} \text { LSE } l=5^{\prime}-0 \\
& \therefore R_{1} \times 10=3(15) \Rightarrow R_{1}=4.5^{k}+R_{2}=1.5^{k} \downarrow
\end{aligned}
$$

BENT SUPT. DESIGN @ 9'OHIGH SECTION (SEE FIG.Z)

REF. KLEINLOGEL ( $12^{\text {th }}$ EA.)

$$
\text { CASE } 41 / 3
$$

ASSUME FIXITY@A $\notin D$

$$
z=\frac{35 l}{8}
$$

$M_{A}=M_{D}=\frac{Z}{3 N_{1}}=\frac{3 \times 4.5 \times 2.83}{8(3 \times 2.71)}=.6^{k+P k}=7.2^{k-i n}$ $K=h / R=2 / 2.83=.71$

$M_{B}=M_{C}=-2 M_{A}=-14.4^{k-i n}$

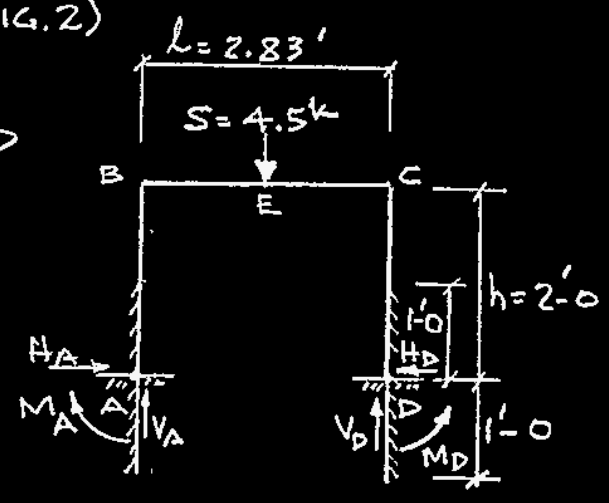

$M_{\text {MAX. }}$ @E, $M_{E}=\frac{4.5 \times 2.83 \times 12}{4}-14.4=23.8^{k-i n} \ll M_{R}=11 k-f t$

$H_{A}=H_{D}=3 M_{A} / \mathrm{h}=3 \times \cdot 6 / 2=.9 \mathrm{~K}$

$V_{A}=V_{D}=s / 2=4.5 / 2=2.25^{k}$ 


\section{ANALYTICAL CALCULATIONS}

WHC-SD-WM-ER-521

Rev. 0

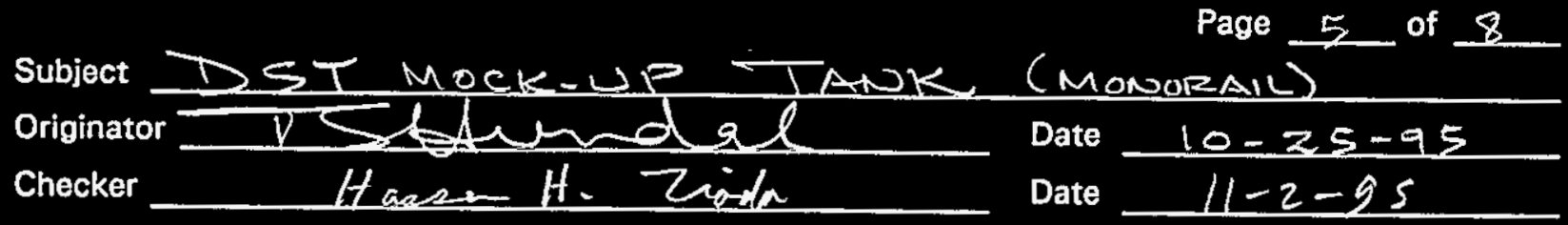

WELDS

HORIZ. MEME. TO VERT.

$S=4 \times 4+(4)^{2} / 3=21.3 \mathrm{in}^{2} \quad A=4 \times 4=16 \mathrm{in}^{2} \quad$ \# $\quad(3 / 16$ welos)

$f_{a}=2.25 / 16=-14 k /, i n$

$f=14.4 / 21.3=.68^{k} / \mathrm{in}$

$f_{r}=.14+.68=.82^{k} /$ in $<2.78^{k} /$ in for 3.16 WELD

VERT. MEMB, TO ES $\cdots$ (TRY 2 "wELD Q 6 " SPACING)

$A=5(z) z=20$ in $\quad M=7.2^{k-i n}$

$I=10(2)^{3} / 12+4\left\{2^{\prime \prime}(11)^{2}+2^{\prime \prime}(6)^{2}\right\}=1263 \mathrm{in}^{3}$

$f_{a}+f_{b}=2.25 / 20+7.2(12 / 1263)=.18^{k} / \mathrm{in}$

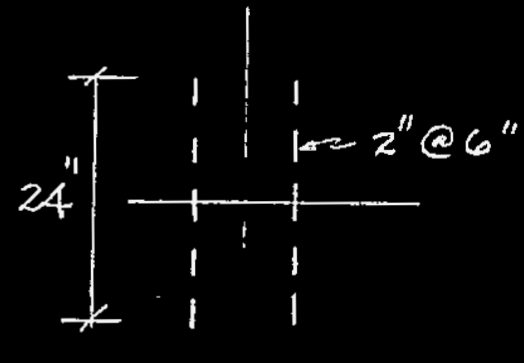

$<<2.78^{k} /$ in for 316 WEL

ALTERNATE METHOD

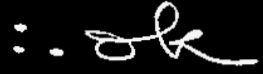

WELD EFFICIENCY $=2 / 6=.33 \Rightarrow$ ALLOW: $f_{V}=2.78 \times .33=.927 \mathrm{k} / \mathrm{in}$ $S=(24)^{2} / 3=192 \mathrm{in}^{2}$,

$f_{a}+f_{b}=2.25 / 20+7.2 / 192=.15 k / i n<.18^{k} / i n$

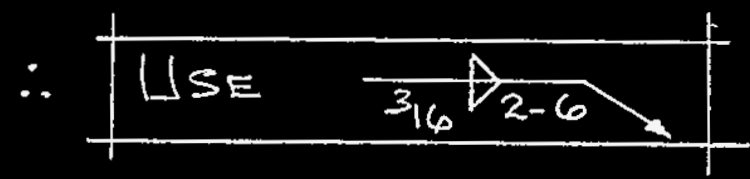

$c-6$ 
ANALYTICAL CALCULATIONS SEP 3195 TSFHAMHC-SD-WM-ER-521 Page 6 of 8

Subject DST MOCK-UP TANK A(MONORALL)

Originator

Checker

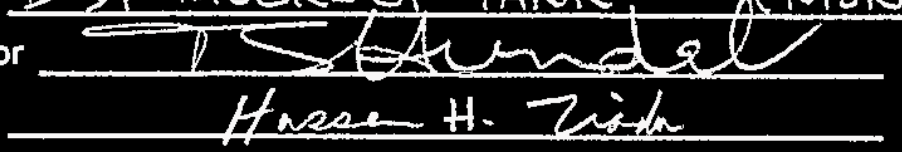

Date

Date

Rev. 0

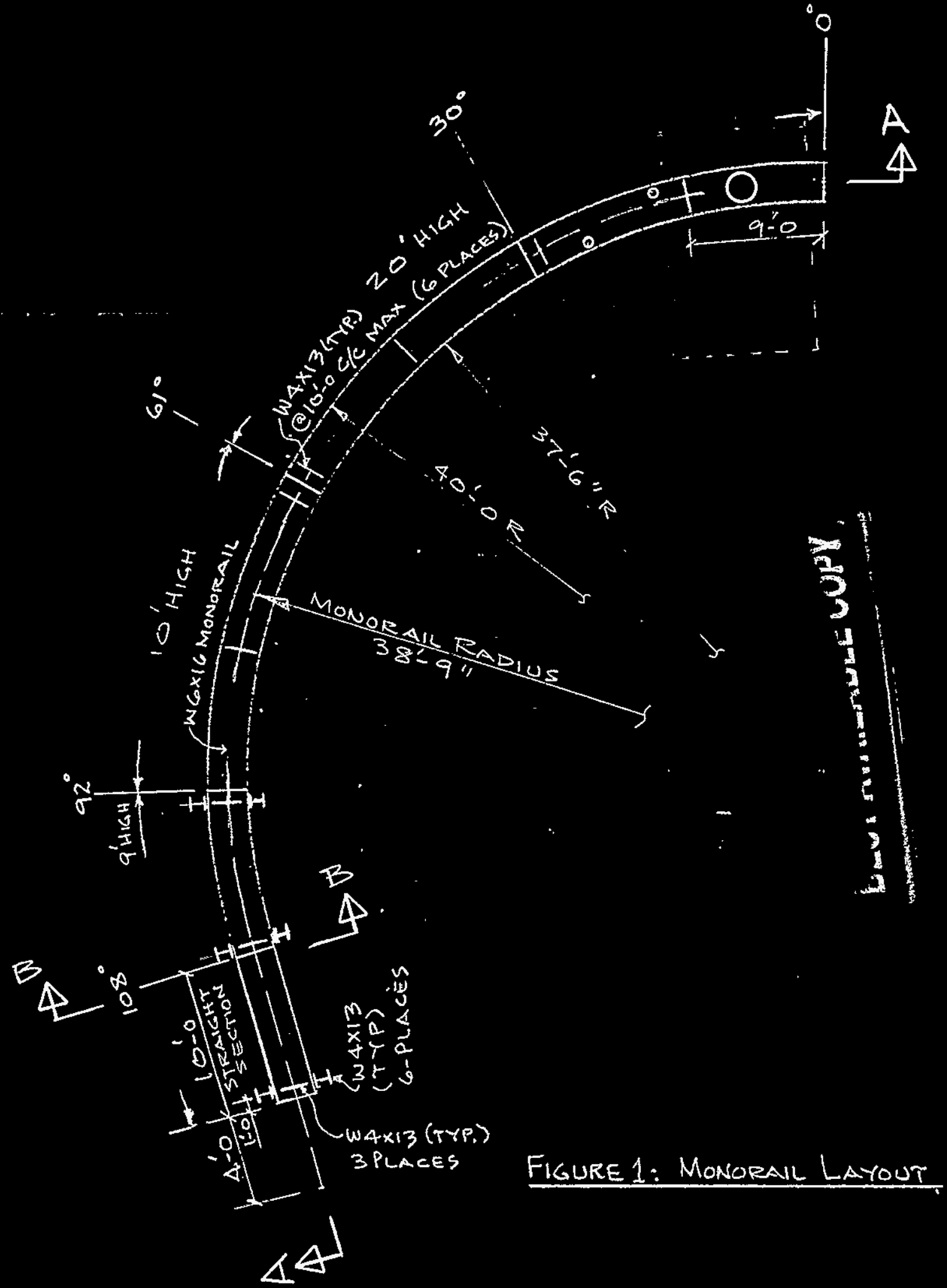

$c-7$

80-6400-060.1(07/93) 
Subject Originator Checker

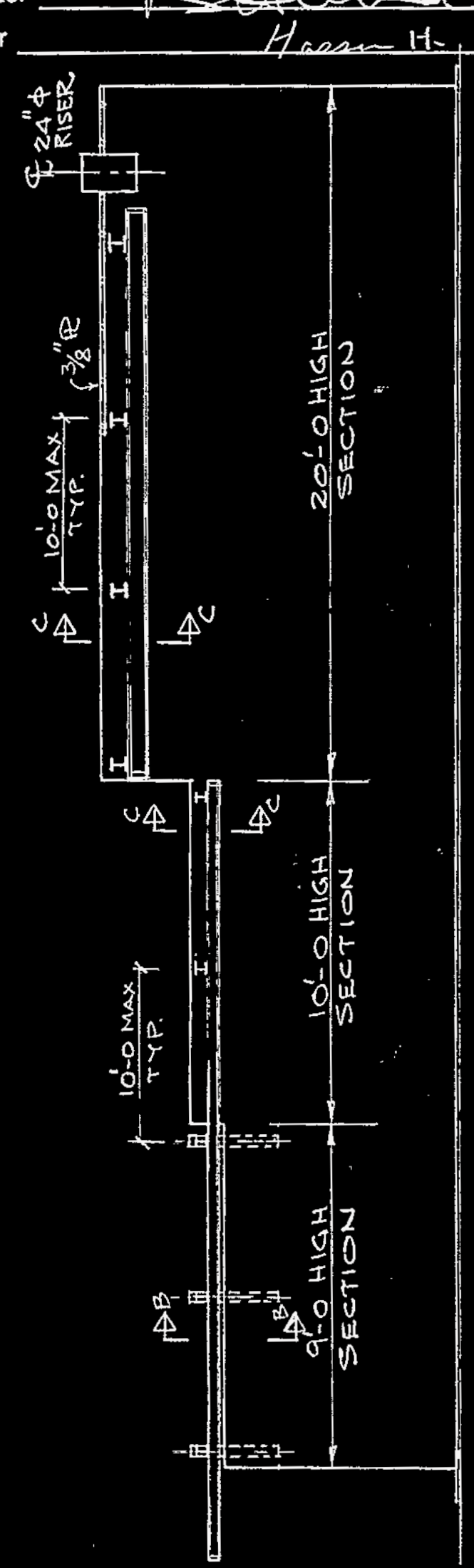
TANK (MONORAL)

Page 7 of 8 Date $10-25-95$ zisdw Date $11-2-95$

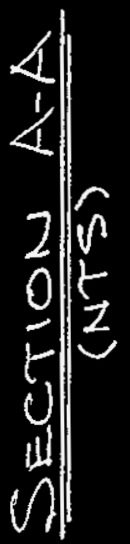

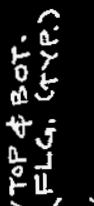
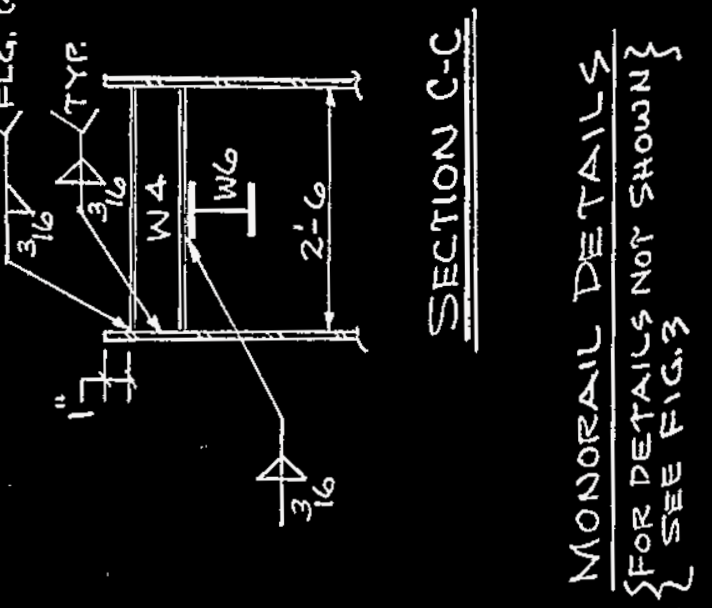

1
2
0
5
4
11

$\ddot{N}$

w

$\frac{N}{2}$

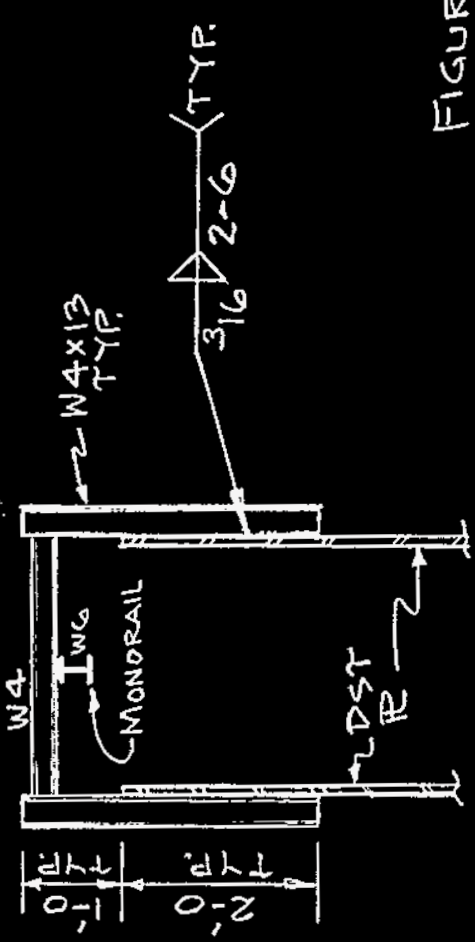

(1)

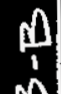




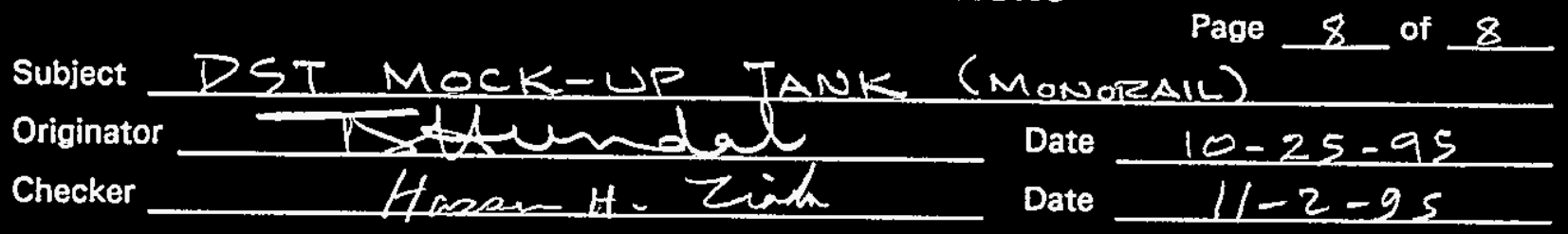

FIGURE 3

*TYP.@W4 \&DST WALL $\frac{\text { MONORAIL/HOIST DETAILS }}{\text { (TYP, FOR IO' } \$ 20^{\circ} \text { HEIGH SECTION) }}$

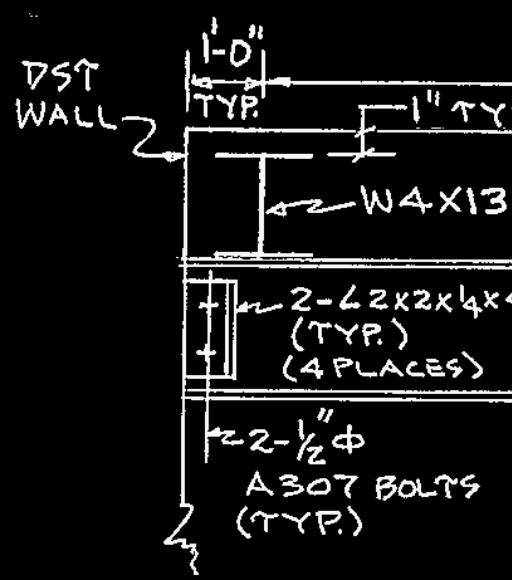

$12 \operatorname{TON}$ $10^{\prime}-0$ (max)

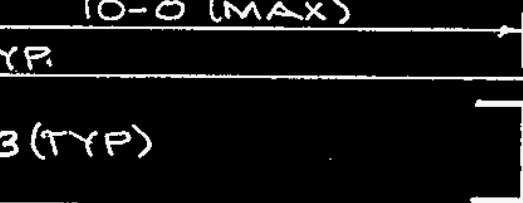

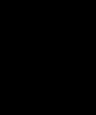

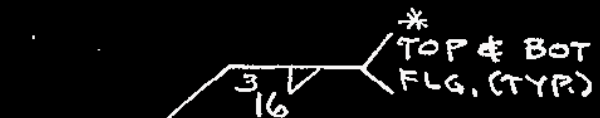


WHC-SD-WM-ER-521

Rev. 0

APPENDIX D

PRIMARY TANK PLATE CUT-OUT DESIGN AND DETAILS

D-1 
Subject DST Mock-up Tank (Primary Tank Plate Cut-out for Data Comparison) Originator T.S. Hundal PSAlendal Checker forsen $H . Z$ Z Date 10-30-95 Date $11-2-2 s$

\section{INTRODUCTION:}

It is required to demonstrate the accuracy of Robot inspection data report by comparing it to the known characteristics of exiting sample plates data. These sample plates are of various sizes (see data below) and with known defect/flaw characteristics such as stress corrosion cracks, pitting corrosion and wall thinning (uniform corrosion).

To accomplish this, a cut-out in the primary tank wall will be made and the opening will be detailed in such a way that the sample plates are mounted and held in place to be in flush with the primary tank wall on the annulus side.

A sample plate when mounted in the cut-out will be subject to an operational pressure exerted by the Robot foot during normal inspection of the annulus area. And this out of plane force on the sample plate is equal to $\mathbf{5 5 0} \mathrm{Ibs}$.

The analysis and evaluation will be in accordance with AISC 1989.

\section{EXISTING SAMPLE PLATES DATA}

\begin{tabular}{|c|c|c|}
\hline Length & Width & Thick \\
\hline 20.153" & $12.225^{\prime \prime}$ & $.875^{\prime \prime}$ \\
\hline 21.631 & 12.005 & .500 \\
\hline 21.619 & 14.511 & .875 \\
\hline 21.613 & 14.507 & .875 \\
\hline 21.596 & 14.500 & .875 \\
\hline 21.610 & 14.500 & .880 \\
\hline 21.604 & 14.495 & .499 \\
\hline 21.6 & 14.5 & .880 \\
\hline 21.610 & 14.505 & .505 \\
\hline 21.610 & 14.530 & .887 \\
\hline 12.0 & 12.0 & .875 \\
\hline
\end{tabular}

Minimum Plate Dimensions

Maximum Plate Dimensions

$12^{\prime \prime} \times 12^{\prime \prime}$

$21.631^{\prime \prime} \times 14.530^{\prime \prime}$

The cut-out needs to be made at about $5 \mathrm{ft}$. up from the floor, for ease of mounting sample plates. The arrangement should be made in such a way that it is easy to mount/dismount the sample plates at the cut-out and be able to accommodate all plate sizes.

Reference: AISC 1989, Manual of Steel Construction, 9th Edition.

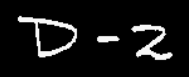




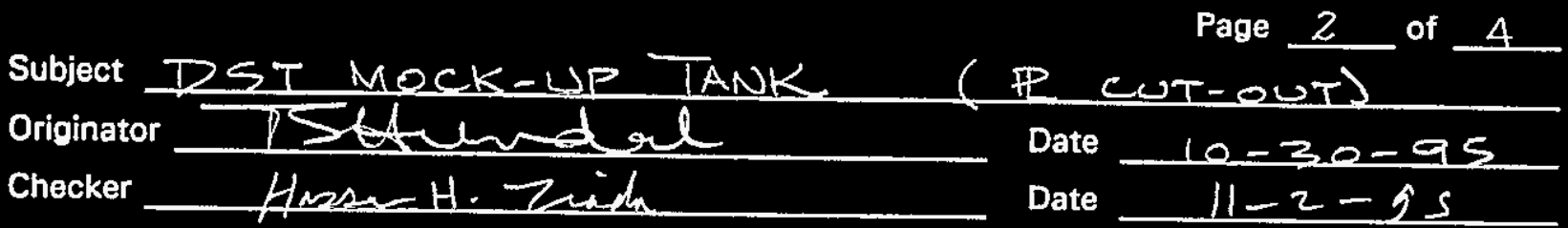

\section{$\frac{\text { Cut-olt Arrangement Detalls }}{\text { (SEE FIGURE } 1 \text { ) }}$}

MAX. OUTWARD FORCE FROM ROBOT FOOT $=550 \mathrm{lbS}$

* Note: the holding bolts resting against the specimen plate

ARE HAND TIGHT \& WILL NOT IMPOSE APPRECIABLE LOAD ON HOLOING RS ON ANULLUS SIDE.

CHECK R $t^{\prime \prime} \times 3 \times 16^{\prime \prime}$ "W/HOLDING BOLTS*

ASSUME FOOT LOAD, @ TOP BOLT \& ONLY

$$
M_{A}=\frac{.55(11)^{2}(5)}{16^{2}}=1.3^{k-i n}
$$$$
M_{B}=\frac{.55(5)^{2}(11)}{16^{2}}=.591 \mathrm{k}-\mathrm{in}
$$

$t_{\text {reqd }}=\sqrt{\frac{6 M}{F_{b} b}}=\sqrt{\frac{6 M}{27(b)}}$

$$
M_{c}=\frac{2(.55)(5)^{2}(11)^{2}}{16^{3}}=.81 \mathrm{k-in} \quad s=b t^{2} / 6
$$

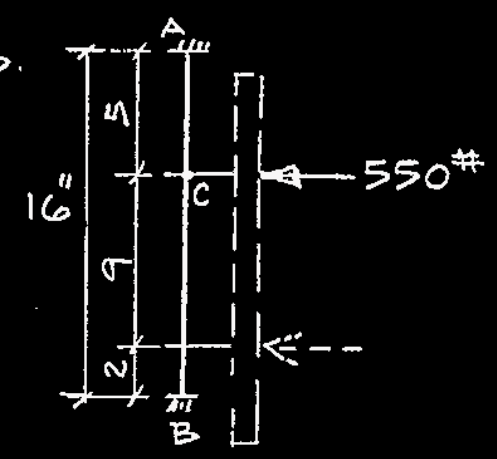

BENDING STRESS\} $F_{b}=.75 F_{Y}$ (RECTRER E E WEIS)

$$
=\sqrt{\frac{2 M}{a b}}
$$

$=.75(36)=27^{\mathrm{ksi}}$

$$
\begin{aligned}
& t_{A}=\sqrt{\frac{2(1.3)}{9(3)}}=.31^{\prime \prime} \\
& t_{B}=\sqrt{\frac{2 \times(591)}{9(3)}}=-21^{\prime \prime}
\end{aligned}
$$

NOW ASSUME FOOT @ BOTTOM BOLT

$$
\begin{aligned}
& M_{A}=\frac{.55(2)^{2}(14)}{16^{2}}=.12 \mathrm{k}-\text { in } \\
& M_{B}=\frac{.55(14)^{2}(2)}{16^{2}}=.842^{\mathrm{k}-i n} \\
& M_{C}=\frac{55(14)^{2}(2)^{2}}{16^{3}}=.11 \mathrm{k-in} \\
& t_{B}=\sqrt{\frac{2(842)}{9(3)}}=-25^{\prime \prime} .
\end{aligned}
$$

$$
\frac{\text { WSE } \mathbb{R} 3_{8}^{\prime \prime} \times 3^{\prime \prime}}{D-3}
$$


Subject IVST MOCK-UP (R CUT-OUT)

Originator

Checker

Date

Date

$10-30-95$

Hanen H. $2 i x$

$11-2-95$

WELD $3_{8}^{\prime \prime} \times 3 "$ R TO $\angle 1 \frac{1}{2} \times 1 \frac{1}{2}$ (FILLET WELDON SIDES) MAX. $M=.842 k$-in Allow. Stress for $3{ }^{3}$ "weLs $=14.85 x_{10}^{3}=2.78 \mathrm{k} / \mathrm{in}$

DIRECT TENSION $=550$ \#

$$
\begin{aligned}
& S=(1.5)^{2} / 3=.75 \mathrm{in}^{2} \quad A=2(1.5) \\
& f=.550 / 3+.842 / .75=1.31 \mathrm{k} / \mathrm{in}
\end{aligned}
$$

$$
\therefore \text { USE 3" WELD SIZE }
$$

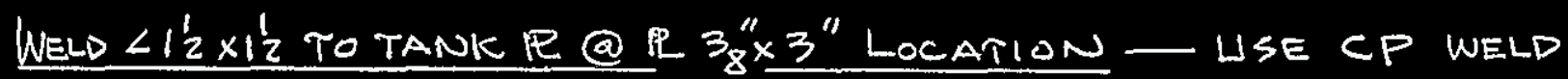
(a) OTHER LOCATION - WSE $316-2 @ 6 "$

WELD R $3_{8}^{\prime \prime} \times 3^{\prime \prime} \times 16_{4}^{\prime \prime}$ TO E $3_{8}^{\prime \prime} \times 1{ }^{\prime \prime} \times 3^{\prime \prime}$ - WSE CP

"R $\frac{3 "}{8} \times 1 \frac{1}{2} \times 3^{\prime \prime}$ TO TANK WALL - " "

CHECK NUT TO $38^{\prime \prime} \mathbb{E}$ (SIDE = 7.6)

USE MAX. FORCE $=550 \mathrm{lbs}$

WELd 3 aLternate sides WITH 3 "WELd

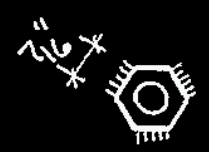

$$
\text { WELP CAP. }=2.7 \times(7 / 16 \times 3)=3.54^{k}>>50^{\#}
$$

2 HOLDING RS (NO LOADS)

USE R ${ }^{\prime \prime} \times 2^{\prime \prime} \times 5^{\prime} 2^{\prime \prime} Q$ BOTTOM $\frac{1}{4}$

$\mathbb{R} 4^{\prime \prime} \times z^{\prime \prime} \times 8 z^{\prime \prime} Q$ TOP

$z-1 / z " \phi x z " A 307$ BolTs@EA.R 


\section{ANALYTICAL CALCULATIONS}

Rev. 0

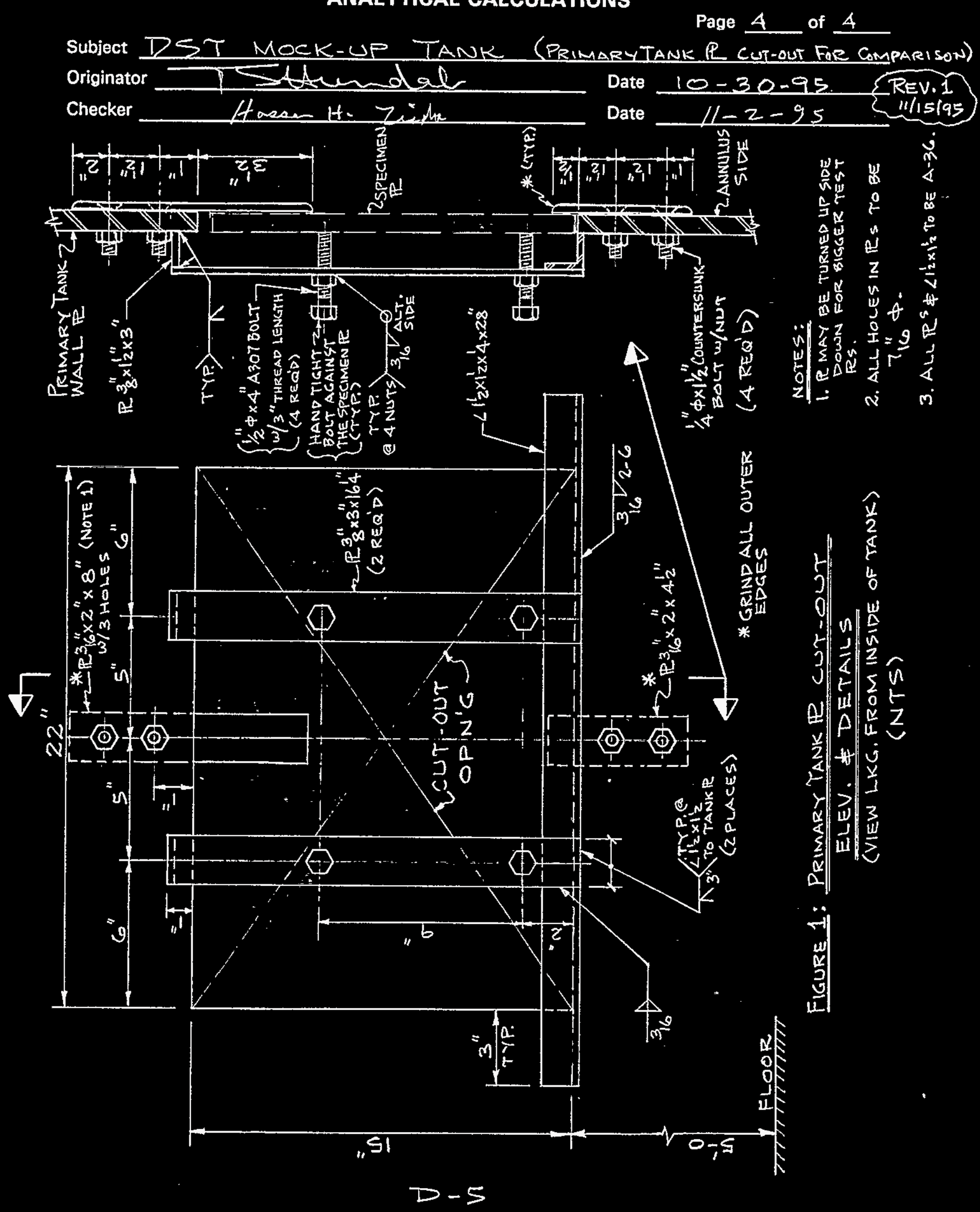




\section{DISTRIBUTION SHEET}

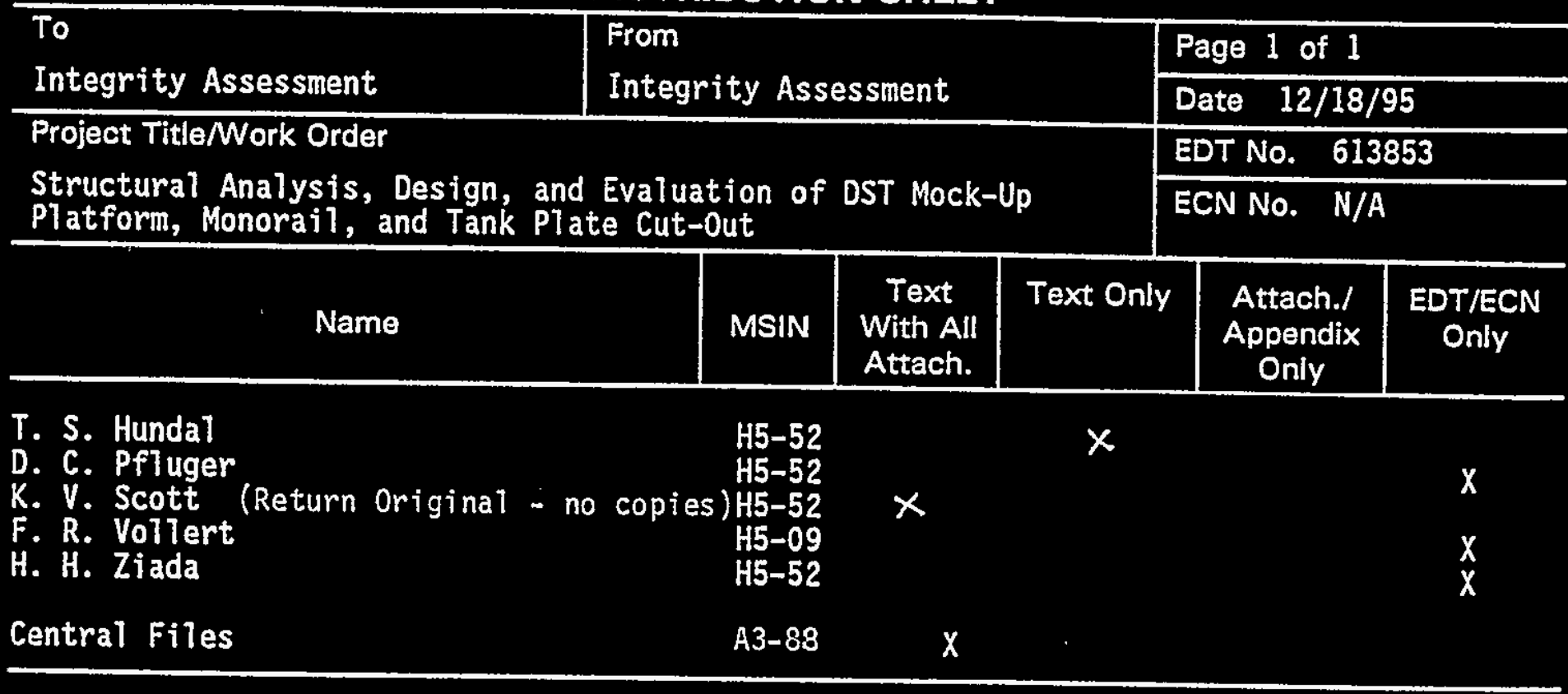

